IDKD Springer Series

Series Editors: Juerg Hodler · Rahel A. Kubik-Huch · Gustav K. von Schulthess

Juerg Hodler

Rahel A. Kubik-Huch

Gustav K. von Schulthess

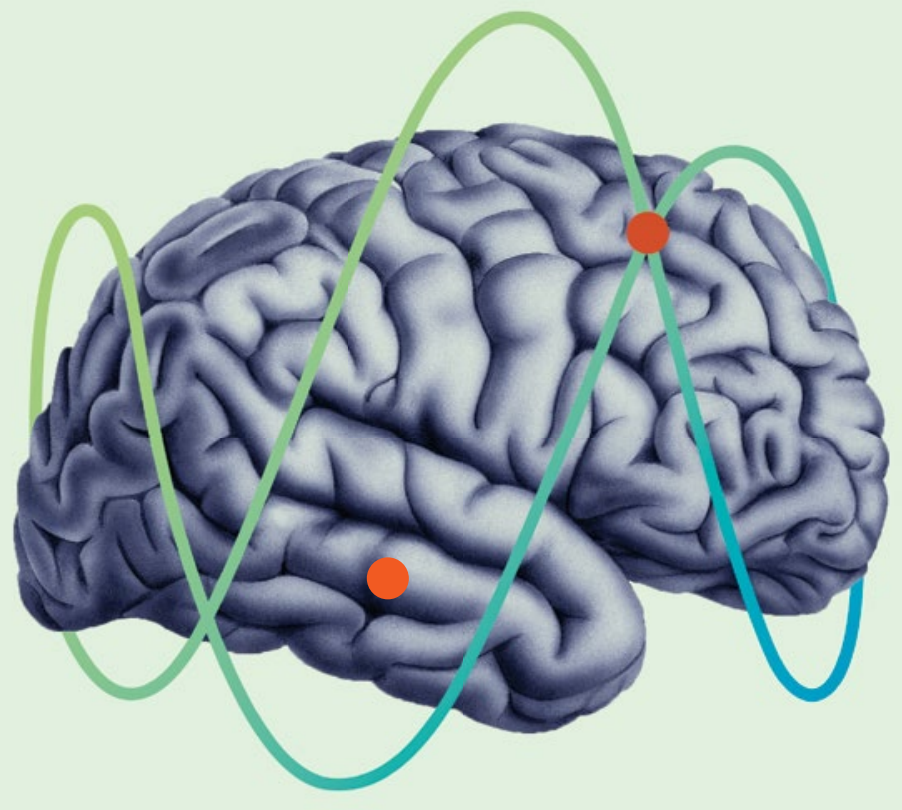
Editors

Diseases of the Brain,

Head and Neck, Spine

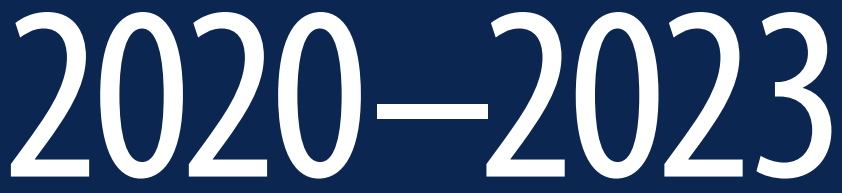

Diagnostic Imaging 


\section{IDKD Springer Series}

\section{Series Editors}

Juerg Hodler

Department of Radiology

University Hospital of Zürich

Zurich, Switzerland

Rahel A. Kubik-Huch

Department of Radiology

Kantonsspital Baden

Baden, Switzerland

Gustav K. von Schulthess

Department of Nuclear Medicine

University Hospital of Zurich

Zürich, Switzerland 
The world-renowned International Diagnostic Course in Davos (IDKD) represents a unique learning experience for imaging specialists in training as well as for experienced radiologists and clinicians. IDKD reinforces his role of educator offering to the scientific community tools of both basic knowledge and clinical practice. Aim of this Series, based on the faculty of the Davos Course and now launched as open access publication, is to provide a periodically renewed update on the current state of the art and the latest developments in the field of organbased imaging (chest, neuro, MSK, and abdominal).

More information about this series at http://www.springer.com/series/15856 
Juerg Hodler • Rahel A. Kubik-Huch

Gustav K. von Schulthess

Editors

Diseases of the Brain, Head
and Neck, Spine 2020-2023

Diagnostic Imaging

SpringerOpen 


\section{Editors}

\section{Juerg Hodler}

Department of Radiology

University Hospital of Zürich

Zürich

Switzerland

Gustav K. von Schulthess

Nuclear Medicine

University Hospital of Zürich

Zürich

Switzerland
Rahel A. Kubik-Huch

Department of Radiology

Kantonsspital Baden

Baden

Aargau

Switzerland

\section{(c) (1)}

This book is an open access publication.
ISSN 2523-7829
ISSN 2523-7837 (electronic)
IDKD Springer Series
ISBN 978-3-030-38489-0 ISBN 978-3-030-38490-6 (eBook)
https://doi.org/10.1007/978-3-030-38490-6

(C) The Editor(s) (if applicable) and The Author(s) 2020

Open Access This book is licensed under the terms of the Creative Commons Attribution 4.0 International License (http://creativecommons.org/licenses/by/4.0/), which permits use, sharing, adaptation, distribution and reproduction in any medium or format, as long as you give appropriate credit to the original author(s) and the source, provide a link to the Creative Commons license and indicate if changes were made.

The images or other third party material in this book are included in the book's Creative Commons license, unless indicated otherwise in a credit line to the material. If material is not included in the book's Creative Commons license and your intended use is not permitted by statutory regulation or exceeds the permitted use, you will need to obtain permission directly from the copyright holder.

The use of general descriptive names, registered names, trademarks, service marks, etc. in this publication does not imply, even in the absence of a specific statement, that such names are exempt from the relevant protective laws and regulations and therefore free for general use.

The publisher, the authors, and the editors are safe to assume that the advice and information in this book are believed to be true and accurate at the date of publication. Neither the publisher nor the authors or the editors give a warranty, expressed or implied, with respect to the material contained herein or for any errors or omissions that may have been made. The publisher remains neutral with regard to jurisdictional claims in published maps and institutional affiliations.

This Springer imprint is published by the registered company Springer Nature Switzerland AG

The registered company address is: Gewerbestrasse 11, 6330 Cham, Switzerland 


\section{Preface}

The International Diagnostic Course in Davos (IDKD) is a unique learning experience for radiologists, nuclear physicians, and clinicians. The course is useful for various levels of experience, from residents preparing for their Board's examination to experienced imaging experts. Clinicians wishing to update their current state of the art of imaging appreciate the course as well.

The workshop teachers of the IDKD are internationally renowned experts. They are all contributing to this issue of the IDKD book series with the current topic of diseases of the brain, head and neck, and spine. It includes relevant pediatric aspects. All relevant imaging modalities are covered, with an emphasis on MR imaging.

The IDKD books were started as a syllabus for the IDKD courses but have developed into well-structured publications of excellent quality over the years. The IDKD book series has found a large number of readers from all over the world.

Great emphasis has been put on the design of this book in order to improve readability and for quick orientation. Relevant aspects are highlighted in the form of learning objectives, key points, tables, take home messages, and summaries.

Additional information on IDKD courses can be found on the IDKD website: www.idkd.org.

Zürich, Switzerland

Baden, Switzerland

Juerg Hodler

Zürich, Switzerland

Rahel A. Kubik-Huch

Gustav K. von Schulthess 


\section{Contents}

\section{Part I Brain, Skull Base, and Head}

1 Diseases of the Sella Turcica and Parasellar Region $\ldots \ldots \ldots \ldots \ldots \ldots \ldots$ Walter Kucharczyk and Chip L. Truwit

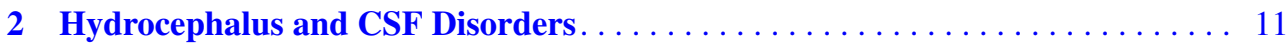
Richard Farb and Àlex Rovira

3 Stroke and Stroke Mimics: Diagnosis and Treatment ............... 25 Howard Rowley and Achala Vagal

4 Cerebral Neoplasms . . . . . . . . . . . . . . . . . . . . . . . . . . . . 37 G. Fatterpekar and E. Knopp

5 Nontraumatic Intracranial Hemorrhage . . . . . . . . . . . . . . . . 45 Pedro Vilela and Martin Wiesmann

6 Intracranial Infection and Inflammation . . . . . . . . . . . . . . . . . . . . 59 Majda M. Thurnher and Pia C. Sundgren

7 Traumatic Neuroemergency: Imaging Patients with Traumatic Brain Injury-An Introduction. . . . . . . . . . . . . . . . . . . . . . . . . 77 Paul M. Parizel and C. Douglas Philips

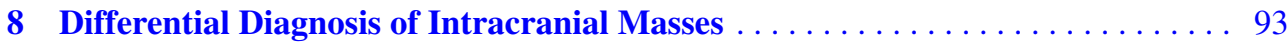
James G. Smirniotopoulos and Hans Rolf Jäger

9 Toxic and Metabolic Disorders: Metabolic Diseases . . . . . . . . . . . . . . 105 Marco Essig

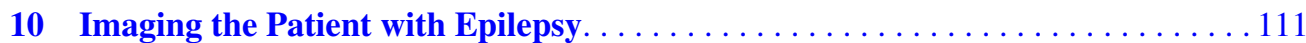
Charlie Chia-Tsong Hsu, Lars Stenberg, and Timo Krings

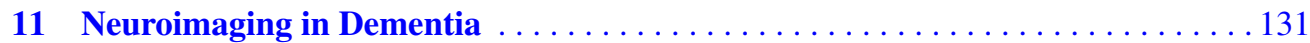
Meike W. Vernooij and Mark A. van Buchem

12 Imaging Evaluation of Patients with Cranial Nerve Disorders Alexandre Krainik and Jan W. Casselman 


\section{Part II Brain and Spine}

13 Demyelinating Diseases of the CNS (Brain and Spine) 165

Frederik Barkhof and Kelly K. Koeller

\section{Part III Pediatric and Fetal}

14 Children with Acute Neurological Emergency . . . . . . . . . . . . . . . . . . . . . . . . 179 Gunes Orman, Andrea Rossi, Avner Meoded, and Thierry A. G. M. Huisman

\section{Part IV Head and Neck}

15 Evaluation of Tinnitus and Hearing Loss in the Adult 193 Jenny K. Hoang and Laurie A. Loevner

16 Approach to Masses in Head and Neck Spaces . . . . . . . . . . . . . . . . 203 Ashley H. Aiken and Deborah R. Shatzkes

17 Head and Neck Squamous Cell Cancer: Approach to Staging and Surveillance ...

Christine M. Glastonbury

Part V Spine, Spinal Cord, Plexus, and Nerves

18 Low Back Pain. . . . . . . . . . . . . . . . . . . . . . . . . . . 225 Luigi Manfrè and Johan Van Goethem

19 Spinal Trauma and Spinal Cord Injury $(\mathbf{S C I}) \ldots \ldots \ldots \ldots \ldots \ldots \ldots \ldots \ldots \ldots$ Luc van Den Hauwe, Pia C. Sundgren, and Adam E. Flanders

20 Brachial and Lumbosacral Plexus and Peripheral Nerves . 241 Roberto Gasparotti and Lubdha Shah 


\section{Part I}

Brain, Skull Base, and Head 


\title{
Diseases of the Sella Turcica and Parasellar Region
}

\author{
Walter Kucharczyk and Chip L. Truwit
}

\section{Learning Objectives}

- Acquire knowledge of the micro-anatomy of the parasellar region.

- Learn the basic physiology of the hypothalamicpituitary axis.

- Gain an understanding of the diagnostic features of the most common lesions arising from each structure in this region.

- Become proficient in establishing a concise differential diagnosis.

\section{Key Point}

- Knowledge of the anatomy of the pituitary gland, pituitary stalk, cavernous sinuses, suprasellar cistern, optic chiasm, and arteries of the central skull base, and their relationship to one another, is essential for accurate diagnosis.

\section{$1.1 \quad$ Introduction}

A large variety of disorders affect the pituitary gland and surrounding region. By far the commonest disorders are pituitary adenomas and Rathke's cleft cysts. Magnetic resonance imaging (MRI) is the imaging modality of choice in the investigation of pituitary pathology. Patients are referred for MR imaging of the pituitary most commonly on the basis of clinical and laboratory findings, although given the frequency of MR imaging of the brain, it is also not uncommon to discover

W. Kucharczyk $(\bowtie)$

University of Toronto, Toronto, ON, Canada

e-mail:w.kucharczyk@utoronto.ca

C. L. Truwit

University of Minnesota, Minneapolis, MN, USA lesions in this region as incidental findings. Computed tomography (CT) is used in occasional cases where intra-lesional calcifications or bone destruction is suspected.

\subsection{Pituitary Adenomas}

Despite the many advances in MR imaging over the past decade, the imaging work-up of pituitary disease remains largely unchanged. T1-weighted images are obtained in the sagittal and coronal plane as well as a T2-weighted sequence in the coronal (occasionally sagittal). Contrast-enhanced sequences are also done in many, but not all, centers on a routine basis. Many investigators also perform dynamic contrastenhanced T1-weighted imaging, and occasionally delayed enhanced images, especially if the patient is a surgical candidate and conventional imaging is negative or equivocal.

Pituitary microadenomas commonly exhibit hypointensity to the normal adenohypophysis on T1-weighted images. The lesions are round or oval in shape; more commonly than not, the pituitary infundibulum is deviated away from the side of the lesion. Occasionally, pituitary adenomas may exhibit hyperintensity on T1-weighted images, generally reflecting internal hemorrhage. Such hemorrhage is more common in prolactinomas, oftentimes spontaneous or following pharmacotherapy.

The signal intensity on T2-weighted images of microadenomas is variable, although if focal hyperintensity to the normal gland is seen, a microadenoma is likely. Hypointensity on T2-weighted images is also a helpful feature. In the clinical and laboratory scenario of excess growth hormone, a mass of focal T2 hypointensity is highly correlated with a diagnosis of a densely granulated $\mathrm{GH}$-secreting adenoma.

In many cases of prolactinoma, unenhanced $\mathrm{T} 1-$ and T2-weighted imaging is normal. If the lesion is not obvious, contrast enhancement is indicated. As the pituitary is a circumventricular organ and not subject to the blood-brain barrier, a half dose of paramagnetic contrast is generally sufficient. Following the administration of contrast, a hypoin- 
tense lesion either surrounded by, or superiorly capped by normal avidly enhancing pituitary tissue will reveal a microadenoma or other lesions in the differential diagnosis. As well, delayed contrast-enhanced imaging may inadvertently obscure microadenomas by virtue of their slower "wash-in" curve, relative to normal pituitary. Uncommonly, a small tuft of intra-lesional enhancement may be seen within an otherwise hypointense (lesser enhancing) microadenoma. Dynamic contrast-enhancing imaging may be useful for some microadenomas, especially ACTH-secreting tumors.

In contradistinction to the microadenoma, pituitary macroadenomas (defined as tumors greater than $10 \mathrm{~mm}$ in size) often extend beyond the confines of the sella. Most commonly, such tumors extend cephalad toward the optic chiasm and hypothalamus or laterally into the cavernous sinus. While the delineation of such extension has always been important to the surgeon, with the increasing adoption of transnasal endoscopic surgical approaches to the sella and parasellar regions, the description of such extension has become a routine component of the preoperative neurosurgical checklist.

Macroadenomas that extend cephalad are commonly bilobed in shape, constrained at the waist by the diaphragma sellae. Unlike their microadenoma counterparts which are more commonly of one signal intensity, macroadenomas usually are of multiple signal intensities due to the mixed solid and cystic nature of these lesions. Solid components tend to be slightly hypointense on T2 and T2-weighted images and exhibit some contrast enhancement. Cystic and/ or necrotic components exhibit variable signal, but often contain proteinaceous debris which may show some hyperintensity on T1-weighted images. On T2-weighted images, hyperintensity within the cystic or necrotic components of an adenoma is common. Occasionally, an intra-tumoral, intracystic component may reveal a fluid-fluid level, perhaps reflecting intra-lesional hemorrhage.

Usually the posterior lobe hyperintensity on T1-weighted images is positioned slightly behind the adenohypophysis, often within a cup-shaped depression of the dorsum sellae. With a macroadenoma the normal neurohypophysis may be compressed, laterally displaced, or completely absent from the normal location. In many cases compression of the infundibulum is disruptive enough that the neurohypophyseal function is displaced upstream, with a "new" bright spot more cephalad along the infundibulum.

Cavernous sinus involvement may be due to compressive growth and consequent medial and occasionally lateral dural reflection or due to true invasion of the sinus. On imaging, invasion is suggested by tumor appearing to encircle the cavernous internal carotid artery. Invasion is excluded if normal pituitary tissue is seen between the mass and the sinus.

There is a correlation between prolactin levels and adenoma size. However, given two prolactinomas of equal size, the hypointense tumor on T2-weighted images usually secretes more prolactin than its counterpart. Medical treatment based on bromocriptine decreases adenoma volume drastically; occasionally, bromocriptine-induced hemorrhage can be seen.

While prolactinomas and growth hormone $(\mathrm{GH})$-secreting adenomas are usually located laterally in the sella turcica, ACTH-secreting adenomas in Cushing's disease, usually smaller in size and more difficult to detect, are more often located in the midline. Because of the severe prognosis of this disease and the surgical possibilities, ACTH-secreting lesions require the most detailed and exhaustive imaging.

$\mathrm{GH}$-secreting adenomas have the unique characteristic of exhibiting hypointensity on T2-weighted images in twothirds of cases, usually the densely granulated subtype. Spontaneous infarction or necrosis of GH-secreting adenomas is far from exceptional. Some cases of acromegaly that were detected late in the course of the disease exhibited an enlarged, partially empty sella turcica, lined with adenomatous tissue that proved difficult to analyze. Medical treatment based on octreotide analogs (somatostatin) decreases the size of the adenoma by an average of $35 \%$ and brings the level of somatomedin $\mathrm{C}$ back to normal in $50 \%$ of cases.

Macroadenomas can be nonfunctioning, but they can also be prolactin-secreting adenomas, gonadotroph adenomas, and growth hormone-secreting adenomas. The greater their size, the more heterogeneous they are, as areas of cystic necrosis are caused by poor tumoral blood supply. Gonadotroph adenomas are often massive and have a strong tendency to recur.

Hemorrhage occurs in all or parts of $20 \%$ of all pituitary adenomas, but it is usually occult. Pituitary apoplexy, with severe headache, cranial nerve paralysis, and severe hypopituitarism, is generally caused by massive hemorrhage within a pituitary macroadenoma. Smaller scale hemorrhage occurs much more often and can be seen within pituitary adenomas.

Surgery of the sella and parasellar region is currently usually undertaken via the transsphenoidal and transnasal endoscopic. The transnasal endoscopic approach to the sella has been more widely adopted for a number of reasons. Multiple instruments can be utilized, including angled endoscopes. A transnasal mucosal flap can be created at the time of surgery. This flap has been very successful at limiting complication of CSF leak. And the exposure can be lateralized to extend to parasellar lesions, as well as extended posteriorly through the clivus to reach the prepontine cistern, the basilar artery, and the anterior brainstem.

Preoperative CT is often routinely performed in some centers. CT will reveal bony anatomy including the number and location of intra-sphenoidal septae and their insertions, the presence of Onodi cells, and the degree of aeration of the sphenoid sinus, all of which may influence the surgical approach, patient safety and ultimately, the patient outcome. 


\subsection{Postoperative Sella Turcica and Pituitary Gland}

The surgical cavity immediately after surgery is often filled with packing material after transsphenoidal resection of a pituitary adenoma. Surgicel is frequently used and is impregnated with blood and secretions. The presence of packing material, secretions, and periadenomatous adhesions usually keeps the cavity from collapsing in the days and weeks that follow surgery. Blood, secretions, and packing material slowly involute over the following 2-3 months. Even after a few months, fragments of blood-impregnated Surgicel can still be found in the surgical cavity. If the diaphragm of the sella turcica is torn in the course of surgery, fat or muscle implants are inserted by the surgeon to prevent the occurrence of a cerebrospinal fluid fistula. Their resorption takes much longer. Implanted fat involutes slowly and may exhibit hyperintensity on the T1-weighted image up to 2-3 years after surgery. Postoperative MRI 2-3 months after surgery is useful to monitor further development of a resected adenoma. An earlier MRI examination performed 48 hours after surgery checks for potential complications and may visualize what appears to be residual tumor, i.e., a mass of intensity identical to that of the adenoma before surgery that commonly occupies a peripheral portion of the adenoma. This early investigation is extremely helpful to interpret the follow-up MR images. At this stage, the remaining normal pituitary tissue can be characterized: it is usually asymmetrical, and a hyperintense area is frequently observed at the base of the deviated hypophyseal stalk, due to an ectopic collection of neurohypophyseal secretory vesicles. The 2-month follow-up MRI examination is essential to check for residual tumor. Late follow-up MRI, after 1-2 years or more, usually demonstrates adenoma recurrence as a rounded or convex progressively enlarging mass.

\section{$1.4 \quad 3 T$ MRI and DWI for Pituitary Imaging}

The improved SNR of $3 \mathrm{~T}$ scanners relative to $1.5 \mathrm{~T}$ can be traded off for thinner image slices and smaller voxels, thereby offering improved spatial resolution at comparable SNR. Hence, some microadenomas may be detected at 3T that are invisible at 1.5T. Also, the cavernous sinus wall can be depicted more consistently. These facts favor the performance of pituitary exams on 3T MRI. DWI and ADC images have been applied to pituitary imaging as aid to determining tumor consistency and thereby aiding surgical planning. Early evidence suggests that soft adenomas with high cellularity and scant fibrous stroma have low ADCs, whereas firm adenomas, with low cellularity and abundant fibrous stroma, have high ADCs. DWI obtained without echo planar imaging offers improved imaging at the skull base. Such TSE-based DWI is increasingly supplanting echo planar DWI in pituitary imaging.
Key Point

- The most common lesions in the sella turcica are pituitary adenomas and Rathke's cleft cysts. Most of these are visible on non-contrast MRI.

\subsection{Craniopharyngioma}

Craniopharyngiomas are epithelial-derived neoplasms that occur exclusively in the region of the sella turcica and suprasellar cistern or in the third ventricle. Craniopharyngiomas account for approximately $3 \%$ of all intracranial tumors and show no gender predominance. Craniopharyngiomas are hormonally inactive lesions, although compression of the stalk may result in diabetes insipidus. They have a bimodal age distribution; more than half occur in childhood or adolescence, with a peak incidence between 5 and 10 years of age; there is a second smaller peak in adults in the sixth decade. The tumors vary greatly in size, from a few millimeters to several centimeters in diameter. The center of most is in the suprasellar cistern. Infrequently, the lesions are entirely within the sella or in the third ventricle.

Most discussions of craniopharyngiomas in the literature are confined to the most frequent form, the classic adamantinomatous type, but a distinct papillary type is also recognized. Typically, adamantinomatous craniopharyngiomas are identified during the first two decades of life. These children most often present with symptoms and signs of increased intracranial pressure: headache, nausea, vomiting, and papilledema. Visual disturbances due to compression of the optic apparatus are also frequent but difficult to detect in young children. Others present with pituitary hypofunction because of compression of the pituitary gland, pituitary stalk, or hypothalamus. Rarely, adamantinomatous craniopharyngiomas are found outside the suprasellar cistern, including the posterior fossa, pineal region, third ventricle, and nasal cavity (sphenoid sinus).

Adamantinomatous tumors are almost always grossly cystic and usually have both solid and cystic components. Calcification is seen in the vast majority $(\sim 90 \%)$ of these tumors. Commonly, these calcifications can be identified on MR scans as low signal nodular excrescences of the wall of the primary lesion. Occasionally, the calcifications are difficult to discern; in these cases, CT will prove helpful. Extensive fibrosis and signs of inflammation are often found with these lesions, particularly when they are recurrent, so that they adhere to adjacent structures, including the vasculature at the base of the brain. Optic tract edema on T2-weighted images is a common associated finding that is not commonly seen with other suprasellar masses. Due to the inflammatory 
and fibrotic nature of this lesion, recurrence is common, typically occurring within the first 5 years after surgery.

The most characteristic MRI finding is a suprasellar mass that is heterogeneous and contains a cystic component that is well defined, internally uniform, and hyperintense on both T1- and T2-weighted images. Almost always, an adamantinomatous craniopharyngioma that presents with large cystic components in the middle cranial fossa and elsewhere can be traced back to the suprasellar region, where a more solid, enhancing component of the lesion can be seen. On rare occasions, the cyst is absent and the solid component is completely calcified. These calcified types of tumors can be entirely overlooked on MRI unless close scrutiny is paid to subtle distortion of the normal suprasellar anatomy. Contrast medium administration causes a moderate degree of enhancement of the solid portion of the tumor, which otherwise may be difficult to see.

Papillary craniopharyngiomas are typically found in adult patients. These lesions are solid, without calcification, and may be found within the third ventricle. Although surgery remains the definitive mode of therapy for all craniopharyngiomas, as papillary variants are encapsulated and are readily separable from nearby structures and adjacent brain, they are generally thought to recur much less frequently than the adamantinomatous type.

In distinction from their adamantinomatous counterpart, MRI typically shows papillary craniopharyngiomas as solid lesions. Occasionally, cysts may be seen, although they are unlikely to be dominant cysts as in the adamantinomatous variety. These lesions demonstrate a non-specific signal intensity pattern, without the characteristic hyperintensity on T1-weighted images of the cystic component of adamantinomatous tumors. Like all craniopharyngiomas, papillary lesions typically enhance.

\subsection{Rathke's Cleft Cyst}

Symptomatic cysts of Rathke's cleft are less frequent than craniopharyngiomas, although asymptomatic Rathke's cysts are a common incidental finding at autopsy. In a recent evaluation of 1000 non-selected autopsy specimens, 113 pituitary glands (11.3\%) harbored incidental Rathke's cleft cysts. These cysts are predominantly intrasellar in location. Of incidental Rathke's cysts larger than $2 \mathrm{~mm}$ in a large autopsy series, $89 \%$ were localized to the center of the gland, whereas the remaining $11 \%$ extended to show predominant lateral lesions. In that series, of all incidental pituitary lesions localized to the central part of the gland, $87 \%$ were Rathke's cysts. Others may be centered in the suprasellar cistern, usually midline and anterior to the stalk. Rathke's cysts are found in all age groups. They share a common origin with some craniopharyngiomas in that they are thought to origi- nate from remnants of squamous epithelium from Rathke's cleft. The cyst wall is composed of a single cell layer of columnar, cuboidal, or squamous epithelium on a basement membrane. The epithelium is often ciliated and may contain goblet cells. The cyst contents are typically mucoid, less commonly filled with serous fluid or desquamated cellular debris. Calcification in the cyst wall is rare.

Most Rathke's cleft cysts are small and asymptomatic, incidentally discovered at autopsy. Symptoms occur if the cyst enlarges sufficiently to compress the pituitary gland or optic chiasm and rarely, secondary to hemorrhage. The cysts with mucoid fluid are hyperintense on T1- and T2-weighted images. Those with serous cysts match the signal intensity of cerebrospinal fluid (CSF). Those containing cellular debris pose the greatest difficulty in differential diagnosis for they resemble solid nodules. Rathke's cleft cysts do not typically enhance. However, occasionally there may be thin marginal enhancement of the cyst wall. This feature can be used to advantage in difficult cases to separate these cysts from craniopharyngiomas.

\subsection{Meningioma}

Approximately $10 \%$ of meningiomas occur in the parasellar region. These tumors arise from a variety of locations around the sella including the tuberculum sellae, clinoid processes, medial sphenoid wing, and cavernous sinus. Meningiomas are usually slow-growing lesions that present because of compression of vital structures. Patients may suffer visual loss because of ophthalmoplegia due to cranial nerve involvement, proptosis due to venous congestion at the orbital apex, or compression of the optic nerves, chiasm, or optic tracts.

Meningiomas are most frequently isointense-and less commonly hypointense - to gray matter on unenhanced T1-weighted sequences. Approximately 50\% remain isointense on the T2-weighted sequence, whereas $40 \%$ are hyperintense. Since there is little image contrast to distinguish meningiomas from brain parenchyma, indirect signs such as a mass effect, thickening of the dura, buckling of adjacent white matter, white matter edema, and hyperostosis are important diagnostic features. Other diagnostic signs include visualization of a cleft of CSF separating the tumor from the brain (thus denoting that the tumor has an extra-axial location) and a clear separation of the tumor from the pituitary gland (thus indicating that the tumor is not of pituitary gland origin). The latter sign is particularly well assessed on sagittal views of planum sphenoidale meningiomas. A peripheral black rim occasionally noted at the edges of these meningiomas is thought to be related to surrounding veins. Hyperostosis and calcification are features that may be apparent on MRI but are better assessed with CT. Vascular encasement is not uncommon, particularly with meningio- 
mas in the cavernous sinus. The pattern of encasement is of diagnostic value. Meningiomas commonly constrict the lumen of the encased vessel. This is rare with other tumors. As on CT, the intravenous administration of contrast medium markedly improves the visualization of basal meningiomas. They enhance intensely and homogeneously, often with a trailing edge of thick surrounding dura (the "dural tail sign").

\subsection{Chiasmatic and Hypothalamic Gliomas}

The distinction between chiasmatic and hypothalamic gliomas often depends on the predominant position of the lesion. In many cases, the origin of large gliomas cannot be definitively determined, as the hypothalamus and chiasm are inseparable; therefore, hypothalamic and chiasmatic gliomas are discussed as a single entity. The vast majority (75\%) of these tumors occur in the first decade of life, with equal prevalence in males and females. There is a definite association of optic nerve and chiasmatic gliomas with neurofibromatosis, more so for tumors that arise from the beginning of optic nerve rather than from the chiasm or hypothalamus.

Tumors of chiasmal origin are also more aggressive than those originating from the optic nerves and tend to invade the hypothalamus and floor of the third ventricle and cause hydrocephalus. Patients suffer from monocular or binocular visual disturbances, hydrocephalus, or hypothalamic dysfunction. The appearance of the tumor depends on its position and direction of growth. It can be confined to the chiasm or the hypothalamus; however, because of its slow growth, the tumor usually attains a considerable size by the time of presentation, and the site of origin is frequently conjectural. Smaller nerve and chiasmal tumors are visually distinct from the hypothalamus, and their site of origin is more clear-cut. From the point of view of differential diagnosis, these smaller tumors can be difficult to distinguish from optic neuritis, which can also cause optic nerve enlargement. The clinical history is important in these cases (neuritis is painful, tumor is not) and, if necessary, interval follow-up of neuritis will demonstrate resolution of optic nerve swelling.

On T1-weighted images, the tumors are most often isointense, while on T2-weighted images they are moderately hyperintense. Calcification and hemorrhage are not features of these gliomas but cysts are seen, particularly in the larger hypothalamic tumors. Contrast enhancement occurs in about half of all cases. Because of the tumor's known propensity to invade the brain along the optic radiations, T2-weighted images of the entire brain are necessary. This pattern of tumor extension is readily evident as hyperintensity on the T2-weighted image; however, patients with neurofibromatosis (NF) present a problem in differential diagnosis. This relates to a high incidence of benign cerebral hamartomas and atypical glial cell rests in NF that can exactly mimic glioma. These both appear as areas of high signal intensity on T2-weighted images within the optic radiations. Lack of interval growth and possibly the absence of contrast enhancement are more supportive of a diagnosis of hamartoma, while enhancement suggests glioma.

\subsection{Metastases}

Symptomatic metastases to the pituitary gland are found in $1-5 \%$ of cancer patients. These are primarily patients with advanced, disseminated malignancy, particularly breast and bronchogenic carcinoma. The vast majority will succumb to their underlying disease before becoming symptomatic of pituitary disease. Autopsy series have demonstrated a much higher incidence, but these by and large are small and asymptomatic lesions. Intrasellar and juxtasellar metastases arise via hematogenous seeding to the pituitary gland and stalk, by CSF seeding, and by direct extension from head and neck neoplasms. There are no distinctive MRI characteristics of metastases, although infundibular involvement is common, and bone destruction is a prominent feature of lesions that involve skull base. Occasionally, leptomeningeal enhancement of posterior fossa sulci may be visualized on the postcontrast images which lends credence to the diagnosis in those cases of CSF tumor seeding, although this finding also invokes the differential diagnosis of sarcoidosis and tuberculosis.

\subsection{Infections}

Infection in the suprasellar cistern and cavernous sinuses is usually part of a disseminated process, or occurs by means of intracranial extension of an extracranial infection. The basal meninges in and around the suprasellar cistern are susceptible to tuberculous and other forms of granulomatous meningitis. The cistern may also be the site of parasitic cysts, in particular (racemose and subarachnoid) neurocysticercosis. In infections of the cavernous sinus, many of which are accompanied by thrombophlebitis, the imaging findings on CT and MRI consist of a convex lateral contour to the affected cavernous sinus with evidence of a filling defect after contrast administration. The intracavernous portion of the internal carotid artery may also be narrowed secondary to surrounding inflammatory change.

Infections of the pituitary gland itself are uncommon. Direct viral infection of the hypophysis has never been established and bacterial infections are unusual. There has been speculation that cases of acquired diabetes insipidus may be the result of a select viral infection of the hypothalamic supra-optic and paraventricular nuclei. Tuberculosis and 
syphilis, previously encountered in this region because of the higher general prevalence of these diseases in the population, are now uncommon. Gram-positive cocci are the most frequently identified organisms in pituitary abscesses. Pituitary abscesses usually occur in the presence of other sellar masses such as pituitary adenomas, Rathke's cleft cysts, and craniopharyngiomas, indicating that these mass lesions function as predisposing factors to infection.

There are a few reports on CT of pituitary abscesses. These indicate that the lesion is similar in appearance to an adenoma. As a result of the frequent coincidental occurrence of abscesses with adenomas, and because of their common clinical presentations, the correct preoperative diagnosis of abscess is difficult and rarely made. Non-contrast MRI demonstrates a sellar mass indistinguishable from an adenoma. With intravenous administration of contrast medium, there is rim enhancement of the mass with persistence of low intensity in the center. Occasionally, pituitary abscesses are unrelated to primary pituitary lesions. In these cases, erosion of the bony sella from an aggressive sphenoid sinusitis may be the route of infection.

\subsection{Noninfectious Inflammatory Lesions}

Lymphocytic hypophysitis is a rare, noninfectious inflammatory disorder of the pituitary gland. It occurs almost exclusively in women and particularly during late pregnancy or in the post-partum period. The diagnosis should be considered in a peripartum patient with a pituitary mass, particularly when the degree of hypopituitarism is greater than that expected from the size of the mass. It is believed that, if untreated, the disease results in panhypopituitarism. Clinically, the patient complains of headache, visual loss, failure to resume menses, inability to lactate, or some combination thereof. Pituitary hormone levels are depressed. CT and MRI demonstrate diffuse enlargement of the anterior lobe without evidence of any focal abnormality or change in internal characteristics of the gland. The distinction between simple pituitary hyperplasia and lymphocytic hypophysitis may be difficult on MRI alone.

Sarcoid afflicting the hypothalamic-pituitary axis usually manifests itself clinically as diabetes insipidus, or occasionally as a deficiency of one or more anterior lobe hormones. Low signal intensity on T2-weighted images is one finding that occurs in sarcoid with some frequency, but rarely in other diseases, with few exceptions (other granulomatous inflammatory diseases, lymphoma, some meningiomas). This low signal finding may aid in differential diagnosis. Also, the presence of multiple, scattered intra-parenchymal brain lesions should raise the possibility of the diagnosis, as should diffuse or multifocal lesions of the basal meninges. The latter are best defined on coronal contrast-enhanced T1-weighted images.
Tolosa-Hunt syndrome (THS) refers to a painful ophthalmoplegia caused by an inflammatory lesion of the cavernous sinus that is responsive to steroid therapy. Pathologically, the process is similar to orbital pseudotumor. Imaging in this disorder is often normal, or may show subtle findings such as asymmetric enlargement of the cavernous sinus, enhancement of the prepontine cistern, or abnormal soft tissue density in the orbital apex. The lesion resolves promptly with steroid therapy. Hypointensity on T2-weighted images may be observed; since this observation is uncommon in all but a few other diseases (e.g., meningioma, lymphoma, and sarcoid), it may be helpful in diagnosis. Clinical history allows further precision in differential diagnosis: meningioma does not respond to steroids, while lymphoma and sarcoid have evidence of disease elsewhere in almost all cases.

\subsection{Vascular Lesions}

Saccular aneurysms in the sella turcica and parasellar area arise from either the cavernous sinus portion of the carotid artery or its supra-clinoid segment. These are extremely important lesions to identify correctly. Confusion with a solid tumor can lead to surgical catastrophes. Fortunately, their MRI appearance is distinctive and easily appreciated. Aneurysms are well defined and lack any internal signal on spin echo (SE) images, the so-called signal void created by rapidly flowing blood. This blood flow may also cause substantial artifacts on the image, usually manifest as multiple ghosts in the phase-encoding direction, and in itself is a useful diagnostic sign.

Thrombus in the aneurysm lumen fundamentally alters these characteristics, the clot usually appearing as multilamellated high signal on T1-weighted SE images, partially or completely filling the lumen. Hemosiderin from superficial siderosis may be visible in the adjacent brain, evident as a rim of low signal intensity on T2-weighted SE images, or on gradient echo (GE) or susceptibility-weighted images. If confusion exists as to the vascular nature of these lesions, MR or CT angiography is used to confirm the diagnosis, define the neck of the aneurysm, and establish the relationship of the aneurysm to the major vessels.

Carotid cavernous fistulas are abnormal communications between the carotid artery and cavernous sinus. Most cases are due to trauma; less frequently they are "spontaneous." These spontaneous cases are due to a variety of abnormalities, including atherosclerotic degeneration of the arterial wall, congenital defects in the media, or rupture of an internal carotid aneurysm within the cavernous sinus. Dural arteriovenous malformations (AVMs) of the cavernous sinus are another form of abnormal arteriovenous (AV) communication in this region.

On MRI, the dilatation of the venous structures, in particular the ophthalmic vein and cavernous sinus, is usually clearly vis- 
ible. The intercavernous venous channels dilate in both direct and indirect carotid cavernous fistulae and may also be seen on MR images. Furthermore, the internal character of the cavernous sinus is altered; definite flow channels become evident secondary to the arterial rates of flow within the sinus. The fistulous communication itself is most often occult on MRI. The pituitary gland has been noted to be prominent in cases of dural arteriovenous fistula without evidence of endocrine dysfunction. The exact mechanism of pituitary enlargement is not known; however, venous congestion is a postulated cause.

Cavernous hemangiomas are acquired lesions and not true malformations. However, there have been reports of extra-axial cavernous hemangiomas occurring in the suprasellar cistern. Of importance is that one of these hemangiomas did not have the features usually associated with, and so highly characteristic of, cavernous hemangiomas in the brain. The atypical appearance of extra-axial cavernous hemangiomas indicates that some caution must be exercised in the differential diagnosis of parasellar masses, because even though cavernous hemangiomas in this location are rare, failure of the surgeon to appreciate their vascular nature can lead to unanticipated hemorrhage. Cavernous hemangiomas should at least be considered in the differential diagnosis of solid, suprasellar masses that do not have the classic features of more common lesions, in particular craniopharyngiomas or meningiomas. Furthermore, T2-weighted images should be a routine part of the MRI protocol for suprasellar masses because visualization of a peripheral dark rim may be the only sign of the nature of the lesion.

Other vascular abnormalities of the sella include unilateral tortuous or bilateral "kissing" internal carotid arteries, and medial trigeminal artery. While the former are relatively straightforward on imaging, the medial trigeminal artery is worth remembering. Much like with the intrasellar aneurysm, with the medial trigeminal artery, neurosurgical catastrophes can occur if the presence of an intrasellar artery is not identified. This artery will arise from the medial aspect of the cavernous carotid artery and will course directly posteriorly through the gland and through the dorsum sellae to reach the basilar artery. Approximately $40 \%$ of trigeminal arteries arise medially. In addition, patients with trigeminal arteries are at increased risk of associated intracranial aneurysm. Finally, congenital absence of the internal carotid artery and asymmetric pneumatization of the sphenoid and sella can pose confusing images.

\section{Key Point}

- Carotid artery aneurysms and anomalies can mimic intrasellar and parasellar mass lesions. Arterial lesions must be considered in the differential diagnosis of lesions in this area. MRA and/or CTA can be used to confidently confirm or exclude the presence of arterial lesions.

\subsection{Other Conditions}

Many other lesions may involve the sella turcica and parasellar region. These include mass lesions such as germinoma, epidermoid, dermoid, teratoma, schwannoma, chordoma, ecchordosis, choristoma, arachnoid cyst, hamartoma, IgG4related disease, and Langerhans cell histiocytosis. Also, there are several important metabolic conditions that may cause pituitary dysfunction or MRI-observable abnormalities in and around the sella. These include diabetes insipidus, growth hormone deficiency, hemochromatosis, hypermagnesemia, and hypothyroidism. Space limitations preclude their further discussion in this synopsis.

\subsection{Conclusion}

There are nine or ten important structures in and around the sella turcica. There are only 3-4 types of pathology that arise from each of these structures, except in rare circumstances. Knowledge of these structures and their relationship to one another, and familiarity with these pathologies results in high probability of correct diagnosis.

\section{Take Home Messages}

- The vast majority of lesions arising in the sella turcica are pituitary adenomas or Rathke's cleft cysts.

- The most important lesions to not misdiagnose are aneurysms and arterial anomalies.

- Anatomic relationships are key to diagnosis.

- Knowledge of basic physiology helps with diagnosis.

Open Access This chapter is licensed under the terms of the Creative Commons Attribution 4.0 International License (http://creativecommons. org/licenses/by/4.0/), which permits use, sharing, adaptation, distribution and reproduction in any medium or format, as long as you give appropriate credit to the original author(s) and the source, provide a link to the Creative Commons license and indicate if changes were made.

The images or other third party material in this chapter are included in the chapter's Creative Commons license, unless indicated otherwise in a credit line to the material. If material is not included in the chapter's Creative Commons license and your intended use is not permitted by statutory regulation or exceeds the permitted use, you will need to obtain permission directly from the copyright holder. 


\title{
Hydrocephalus and CSF Disorders
}

\author{
Richard Farb and Àlex Rovira
}

\section{Learning Objectives}

- To know CSF physiology and the different types of hydrocephalus

- To recognize the radiological features of the different types of hydrocephalus

- To be able to recognize the imaging features that distinguish normal pressure hydrocephalus from other causes of ventriculomegaly in the adult population

- To recognize imaging findings in disorders of intracranial pressure and how they impact direct subsequent management

\subsection{Introduction}

Modern imaging techniques play an essential role for understanding of the anatomy of the cerebrospinal fluid (CSF) spaces and ventricular system, as well as the hydrodynamics of CSF flow. These principles are important to the understanding of pathological processes affecting the ventricles and CSF spaces, and abnormalities induced by changes in the intracranial pressure.

The classical model of CSF hydrodynamics presumes that CSF is produced primarily in the choroid plexus epithelium (and to a lesser degree in the ependymal cells) at a rate of approximately $0.2-0.6 \mathrm{~mL} / \mathrm{min}$ and $400-600 /$ day. However, numerous evidences indicate that CSF is also produced throughout the entire CSF-interstitial fluid functional unit across the walls of central nervous system (CNS) blood capillaries [1].

\section{R. Farb}

Division of Neuroradiology, Toronto Western Hospital, University

Health Network, University of Toronto, Toronto, ON, Canada

e-mail: richard.farb@uhn.ca

À. Rovira $(\bowtie)$

Section of Neuroradiology, Hospital Universitari Vall d'Hebron,

Barcelona, Spain

e-mail: alex.rovira@idi.gencat.cat
Since the total volume of CSF in the ventricles and subarachnoidal spaces (SAS) averages approximately $150 \mathrm{~mL}$, a threefold turnover of CSF occurs daily, explaining the presence of a bulk flow of CSF from the sites of origin to the sites of absorption. This bulk flow of CSF starts in the lateral ventricles, passes through the third and fourth ventricles and, via the foramina of Luschka and the foramen of Magendie into the cerebellopontine angle and prepontine cisterns and the vallecula of the cisterna magna. From the basilar cisterns, the bulk flow of CSF continues cephalad along two major routes: a ventral route through the interpeduncular and pre-chiasmatic cisterns and a dorsomedial route through the ambient cisterns and the cisterna magna. The bulk flow also extends downward into the spinal canal dorsal to the cord to the lumbar thecal sac and upward in front of the cord to the basilar cistern.

CSF is absorbed not only via the arachnoid villi that are ideally situated to drain CSF from the SAS into the major dural sinuses, but also through the lymphatic system, and through the glymphatic system in the brain parenchyma, (extracellular fluid spaces that are in communication with the brain capillaries). In fact, it is widely accepted that the vast majority of CSF is absorbed through the capillary network of the CNS [1,2].

There is an increasing body of evidence indicating that superimposed on the low-velocity bulk flow of CSF, which is almost insignificant $(4 \mathrm{~mL} / \mathrm{min})$, there is a high-velocity oscillatory CSF motion $(700 \mathrm{~mL} / \mathrm{min})$, whose main driving force is the cardiac-related intracranial arterial expansion. MRI is well suited for demonstrating this oscillatory high-velocity craniospinal CSF flow in a noninvasive manner. Cardiac-gated flow-sensitive phase-contrast cine MRI (PC-MRI) is probably the method of choice, since it allows qualitative and quantitative analysis of oscillatory CSF movement in normal and abnormal situations. This MRI technique uses a two-dimensional (2D) cine phase-contrast sequence that sensitizes the images to velocity changes in a specific direction while canceling signals from stationary protons and from motion in other directions [3] (Fig. 2.1). 

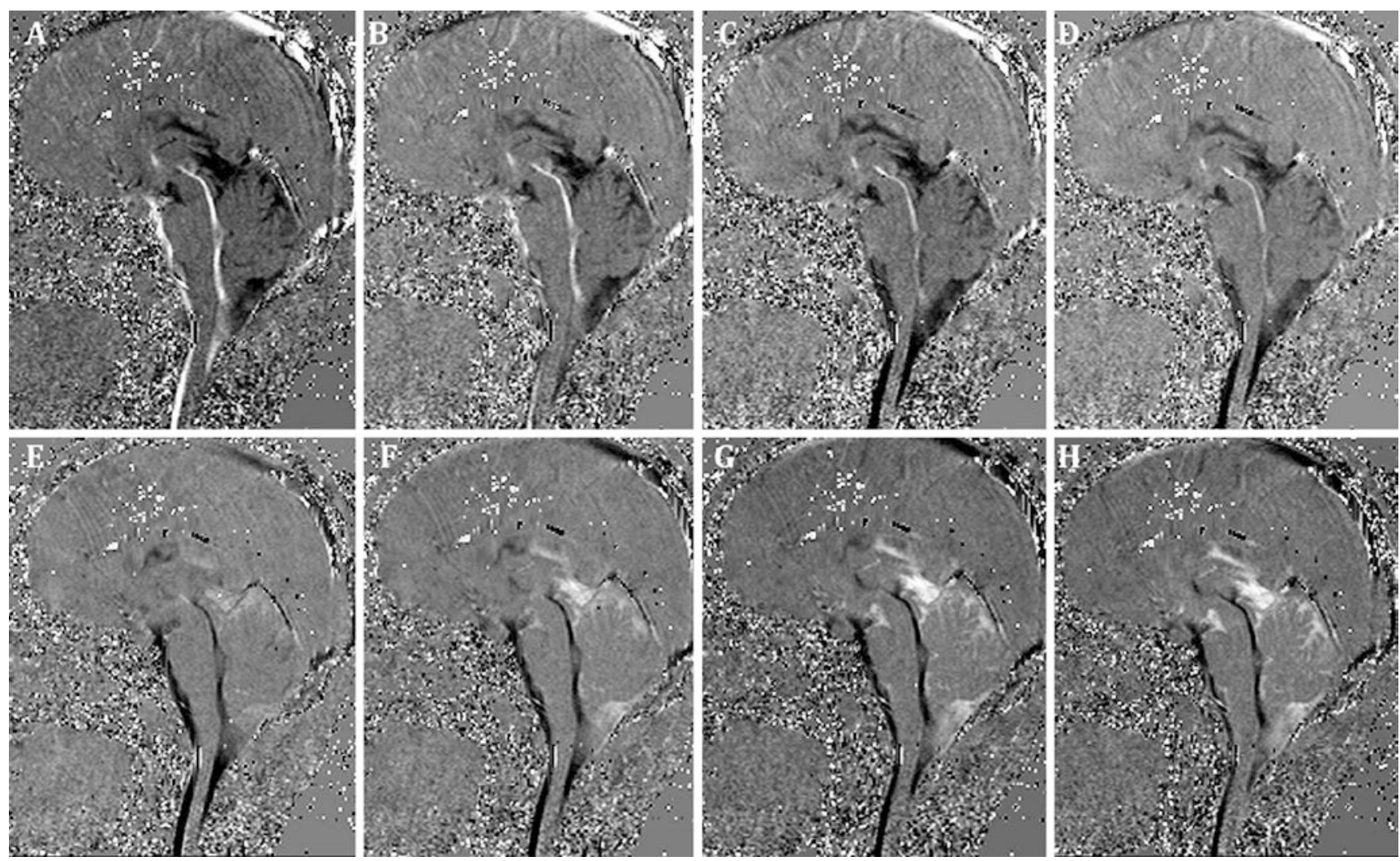

Fig. 2.1 Cine phase-contrast MRI in a normal subject. (a-h) Normal intracranial and upper cervical spine cerebrospinal fluid oscillatory flow pattern: Midsagittal phase-contrast flow-sensitive images showing the whole cardiac cycle. The flow is sensitized craniocaudally. Caudocranial flow is represented in black, and craniocaudal in white. Antegrade flow is initiated from the lower fourth ventricle and cisterna magna. Caudal flow begins in the

posterior third ventricle, aqueduct of Sylvius, and upper fourth ventricle. As pulsation through the aqueduct continues caudally, pulsation in the cisterna magna becomes quiescent, and then reverses to pulse cephalically. All flow shows cephalic pulsation. Reproduced from Rovira A. Communicating Hydrocephalus. Normal Pressure Hydrocephalus. In F. Barkhof et al. (eds.), Clinical Neuroradiology, @ Springer Nature Switzerland AG 2019

In this chapter we will review the clinical and imaging features of the different types of hydrocephalus, both in adults and in children, and other idiopathic disorders associated with an abnormal intracranial pressure, such as idiopathic intracranial hypertension and spontaneous intracranial hypotension.

\section{Key Points}

- The production of CSF mostly takes place within the CNS capillaries: filtration of water volume through the capillary walls into the surrounding brain tissue. The choroid plexuses are a less relevant place for this process.

- CSF absorption takes place at different sites, and its capacity far exceeds CSF production.

- A high-velocity oscillatory CSF motion driven by cardiac-related intracranial arterial expansion can be identified and quantified non-invasively with different MRI techniques.

\subsection{Obstructive Hydrocephalus in Adults}

The term obstructive hydrocephalus usually indicates noncommunicating hydrocephalus, and occurs when the flow of CSF is blocked along one or more of the narrow apertures connecting the ventricles [5].

Usually CT is the first-line imaging method, especially in the emergency setting. Although this technique is able to show signs of acute as well as chronic hydrocephalus, MRI is the method of choice for detailed assessment of this condition. Contrastenhanced T1-weighted imaging is not required for the diagnosis unless there is a suspicion of a neoplastic lesion or an inflammatory process. The main purpose of the diagnostic management of the patient with obstructive hydrocephalus is to find an obstacle impeding the CSF flow within the ventricular system [6].

When conventional MRI sequences are not able to clearly find the obstructive lesion, some new MR techniques may help for determining the mechanism producing the hydrocephalus. These new techniques include PC-MRI and 3D heavily T2-weighted steady-state sequences (3DT2WSS) [5]. 

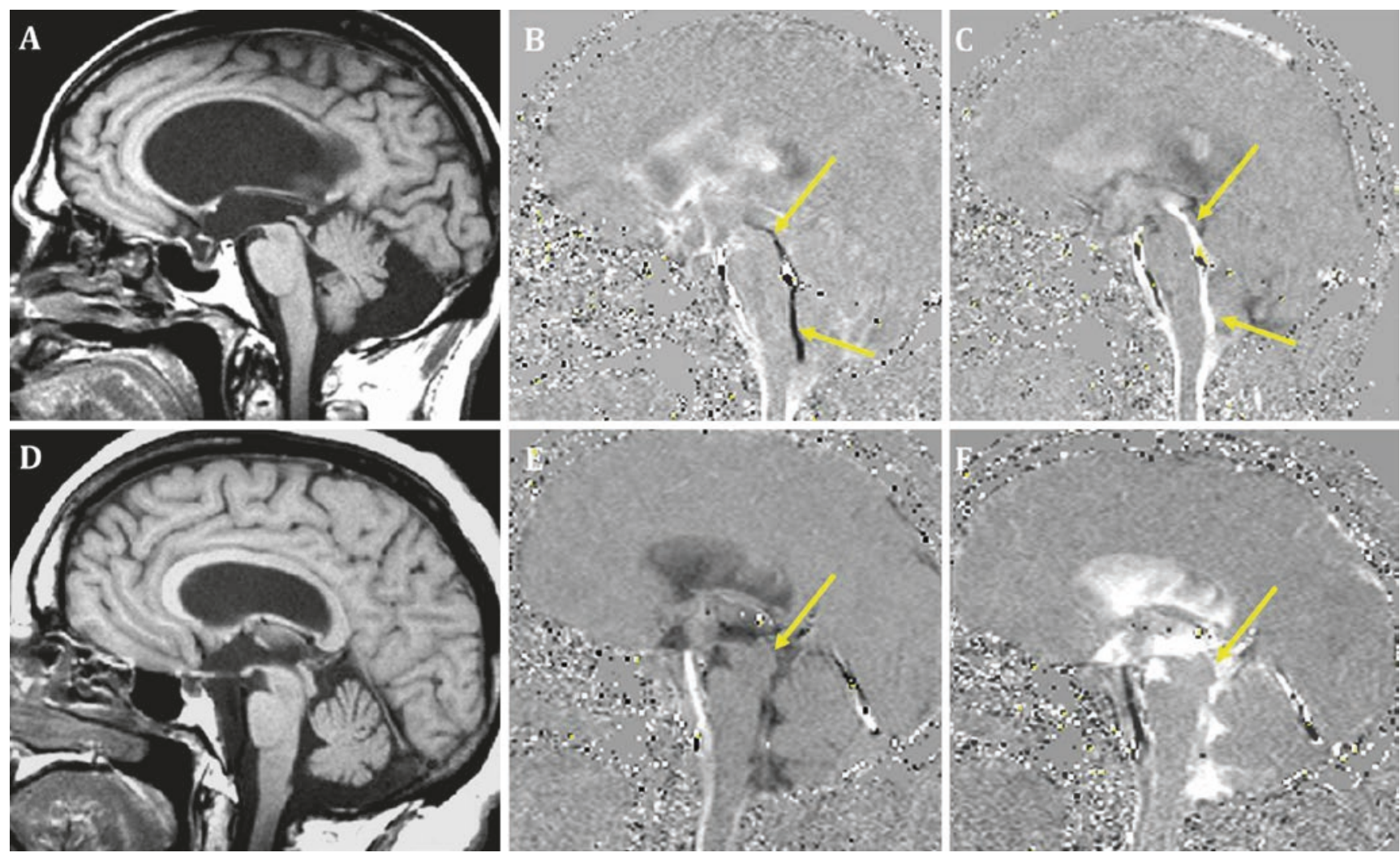

Fig. 2.2 Brain MRI in a patient with communicating hydrocephalus $(\mathbf{a}-\mathbf{c})$ and in a patient with a non-communicating hydrocephalus secondary to aqueductal stenosis (d-f). Midsagittal T1-weighted and two representative frames of the diastolic and systolic phases of a phasecontrast flow-sensitive MRI sequence $(\mathbf{b}, \mathbf{c}, \mathbf{e}, \mathbf{f})$. Observe the presence of a normal craniocaudal cerebrospinal fluid flow through the aqueduct

PC-MRI enables quantitative and qualitative assessment of the CSF circulation (Fig. 2.2), while 3DT2WSS sequences such as DRIVE (Philips), CISS (Siemens), and FIESTA (GE) are useful for accurate anatomic evaluation and are highly recommended in patients with suspicion of aqueductal pathology or intraventricular adhesions. Alternatively, to PC-MRI, 3D T2-weighted turbo spin-echo sequences with variable flip angle (3DT2WTSE) can be used (Siemens: SPACE, GE: CUBE, Philips: VISTA) for detecting the high-velocity CSF bulk flow, as are strongly susceptible to CSF flow effects. This technique is noninvasive, less sensitive to artifacts, and enables scanning of the whole skull using isotropic voxels with an acceptable acquisition time [5] (Figs. 2.3 and 2.4).

\subsubsection{Radiological Features of Hydrocephalus}

The typical radiological features of any kind of hydrocephalus are:

- Enlargement of the ventricles (Evans index $>0.3$ ) out of proportion of the size of SAS (cortical sulci appear dis- in the patient with a communicating hydrocephalus, and the absence of the CSF flow in the patient with aqueductal stenosis. Reproduced from Rovira A. Communicating Hydrocephalus. Normal Pressure Hydrocephalus. In F. Barkhof et al. (eds.), Clinical Neuroradiology, (C) Springer Nature Switzerland AG 2019

proportionately narrowed), with ballooning of the frontal and posterior ventricular horns

- Dilatation of temporal ventricular horns

- Dilatation of the third ventricular recesses

- Reduced mamillopontine distance

- Upward bowing and thinning of the corpus callosum

- Hyperintense on MRI (hypodense on CT) areas in the periventricular white matter, suggestive features of acute hydrocephalus.

Typical obstacle sites in obstructive hydrocephalus are the foramen of Monro, the cerebral aqueduct, the fourth ventricular outlets (Luschka and Magendie foramina), and the foramen magnum.

\subsubsection{Foramen of Monro Obstruction}

Several lesions growing in the region adjacent to Monro foramina might produce obstructive hydrocephalus. These include intra- and periventricular cysts such as arachnoid, colloid, dermoid, epidermoid cysts, tumors (ependymomas, 

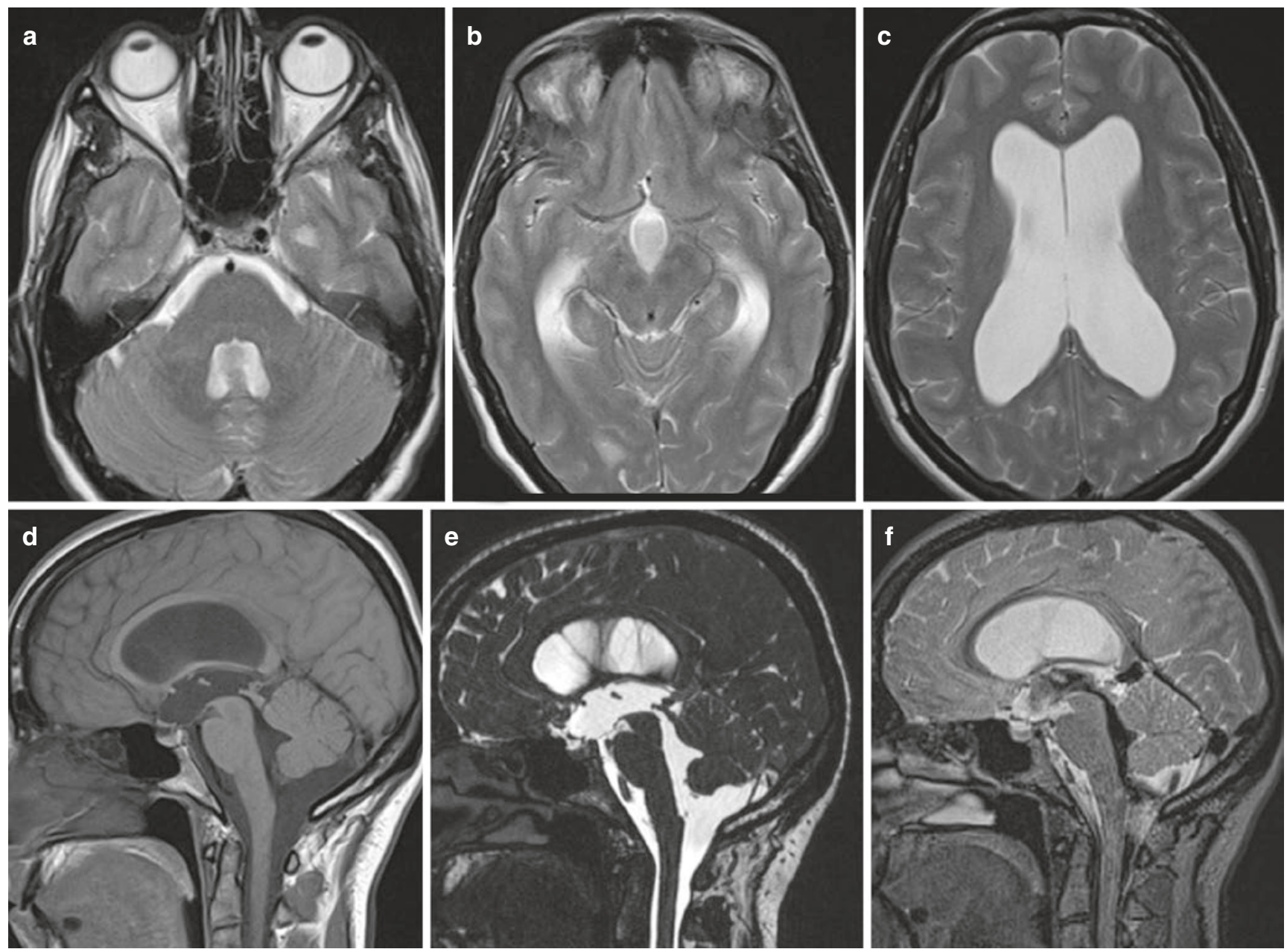

Fig. 2.3 Brain MRI in a patient with communicating hydrocephalus. Axial T2-weighted images (a-c) show ventriculomegaly involving the supratentorial and infratentorial ventricular cavities. Observe the presence of flow voids inside the aqueduct and fourth ventricle. Midsagittal T1-weighted (d) 3DT2WSS sequence (CISS) (e) and 3DT2WTSE
(SPACE) (f) images show a morphological normal aqueduct with marked flow void, better seen on the SPACE sequence. Reproduced from Rovira A. Communicating Hydrocephalus. Normal Pressure Hydrocephalus. In F. Barkhof et al. (eds.), Clinical Neuroradiology, (C) Springer Nature Switzerland AG 2019 subependymomas, central neurocytomas, or glial tumors), as well as adhesions. Not infrequently these lesions may lead to unilateral ventriculomegaly. Also, unilateral mass lesions producing midline shift might eventually obstruct the contralateral foramen of Monro causing contralateral ventricular dilatation as well as compression of the ipsilateral lateral ventricle.

The most common pathology located in this area and responsible for hydrocephalus is a colloid cyst, which are slow-growing, benign cystic lesions, typically located in the anterior part of the third ventricle. These usually oval or round lesions are mostly hyperdense on unenhanced $\mathrm{CT}$ in comparison to the gray matter, although in one-third of cases are hypo- or isodense. On MRI, the signal intensity is quite variable, depending on their protein content, but approxi- mately $50 \%$ of them are hyperintense on T1-weighted sequences and hypointense on T2-weighted sequences.

Subependymal giant cell astrocytomas (SEGAs) are the most common brain tumors in tuberosus sclerosis complex occurring in about one out of every ten patients. These tumors are low-grade (World Health Organization [WHO] grade 1), mixed, glioneuronal, and highly vascularized neoplasms predominantly located near the foramen of Monro, and frequently with bilateral distribution. They represent a significant cause of morbidity and mortality because of the risk of sudden death from acute hydrocephalus, the risk of which is directly proportional to their volume. On CT, SEGAs appear as iso/hyperdense masses showing frequent calcification. On MRI, they have hypo/isointense signal on T1-weighted sequences and 
Fig. 2.4 Brain MRI in a patient with communicating hydrocephalus $(\mathbf{a}, \mathbf{b})$ and in a patient with a noncommunicating hydrocephalus secondary to aqueductal stenosis (c, d). Midsagittal 3DT2WSS sequence (CISS) (a, c) and 3DT2WTSE sequence (SPACE) (b, d) images. Observe the presence of a normal morphology of the aqueduct and normal cerebrospinal fluid flow through the aqueduct in the patient with a communicating hydrocephalus (arrows), and the thickened mesencephalic tectum (probable tectum glioma) and absence of the CSF flow in the patient with aqueductal stenosis (arrows). Note the presence of normal CSF flow through the Magendie foramen (short arrow). Reproduced from Rovira A. Communicating Hydrocephalus. Normal Pressure Hydrocephalus. In F. Barkhof et al. (eds.), Clinical Neuroradiology, (C) Springer Nature Switzerland AG 2019
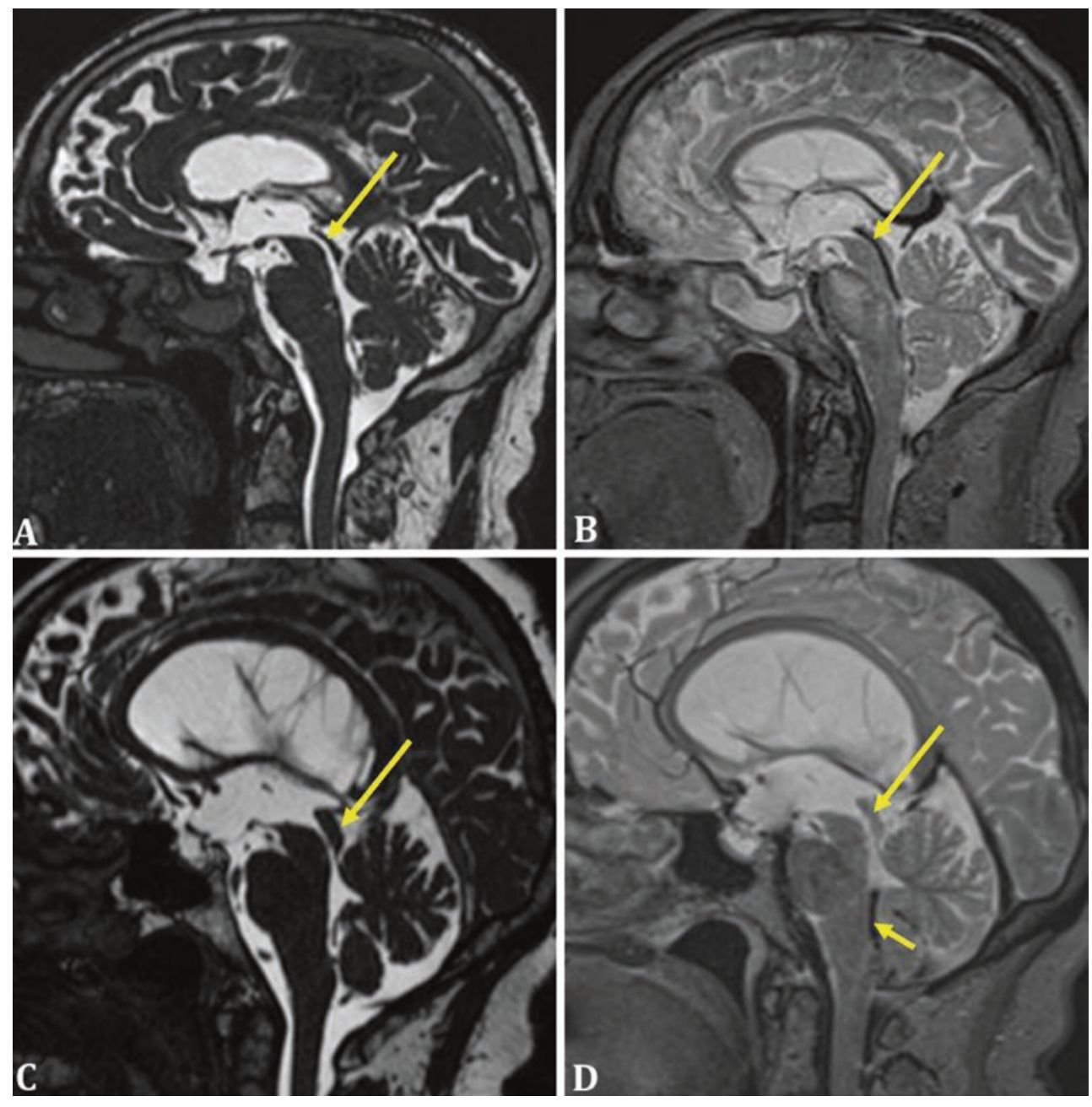

hyperintense signal on T2-weighted sequences, presenting intense, but inhomogeneous, contrast enhancement.

\subsubsection{Cerebral Aqueduct}

Aqueductal stenosis, one of the most common causes of obstructive hydrocephalus both in children and adults, can be divided into congenital and acquired. The most frequent acquired lesion resulting in aqueductal stenosis includes pineal region tumors (pineal gland tumors, tectum-tegmentum gliomas, tentorial meningiomas, metastasis), cerebral vascular malformations (e.g., vein of Galen aneurysm), adjacent intracranial hemorrhage, and infections (meningitis/ventriculitis) (Fig. 2.4). The congenital causes may be aqueductal webs or diaphragms. The aqueductal web is a membrane formation whose origin may be either congenital or inflammatory. It has been hypothesized that it might be caused by a small glial occlusion of the caudal aqueduct with the subsequent development of a sheet of tissue due to extended pressure and dilatation of the canal above. On MRI sequences, enlargement of the lateral and third ventricles, without dilatation of fourth ventricle is a key imaging feature. The aqueduct may show funneling superiorly.

\subsubsection{Trapped Fourth Ventricle}

Obstruction of all CSF pathways of the fourth ventricle, including foramina of Luschka and Magendie, as well as aqueduct, produces and isolated enlargement of this cavity known as the trapped fourth ventricle. This is a rare clinical condition most often associated with previous ventricular shunting for hydrocephalus [7], infection, intraventricular hemorrhage, and postinflammatory changes after posterior fossa surgery (Fig. 2.5). The clinical symptoms include nausea, vomiting, and cranial nerves palsy as a result of cerebellar and brain stem compression. Treatment options include placement of a fourth ventriculoperi- 
Fig. 2.5 Sagittal T1- (a) and T2-weighted (b), and coronal T2-FLAIR (c) brain MRI in an 8-year-old girl with congenital hydrocephalus secondary to prenatal infection treated with ventricular shunting. Observe the presence of web-like membranes (arrows in b) at the level of the aqueduct and Magendie foramen, producing a trapped fourth ventricle. (Courtesy of Antoni Rovira. Parc Tauli. Sabadell. Spain)
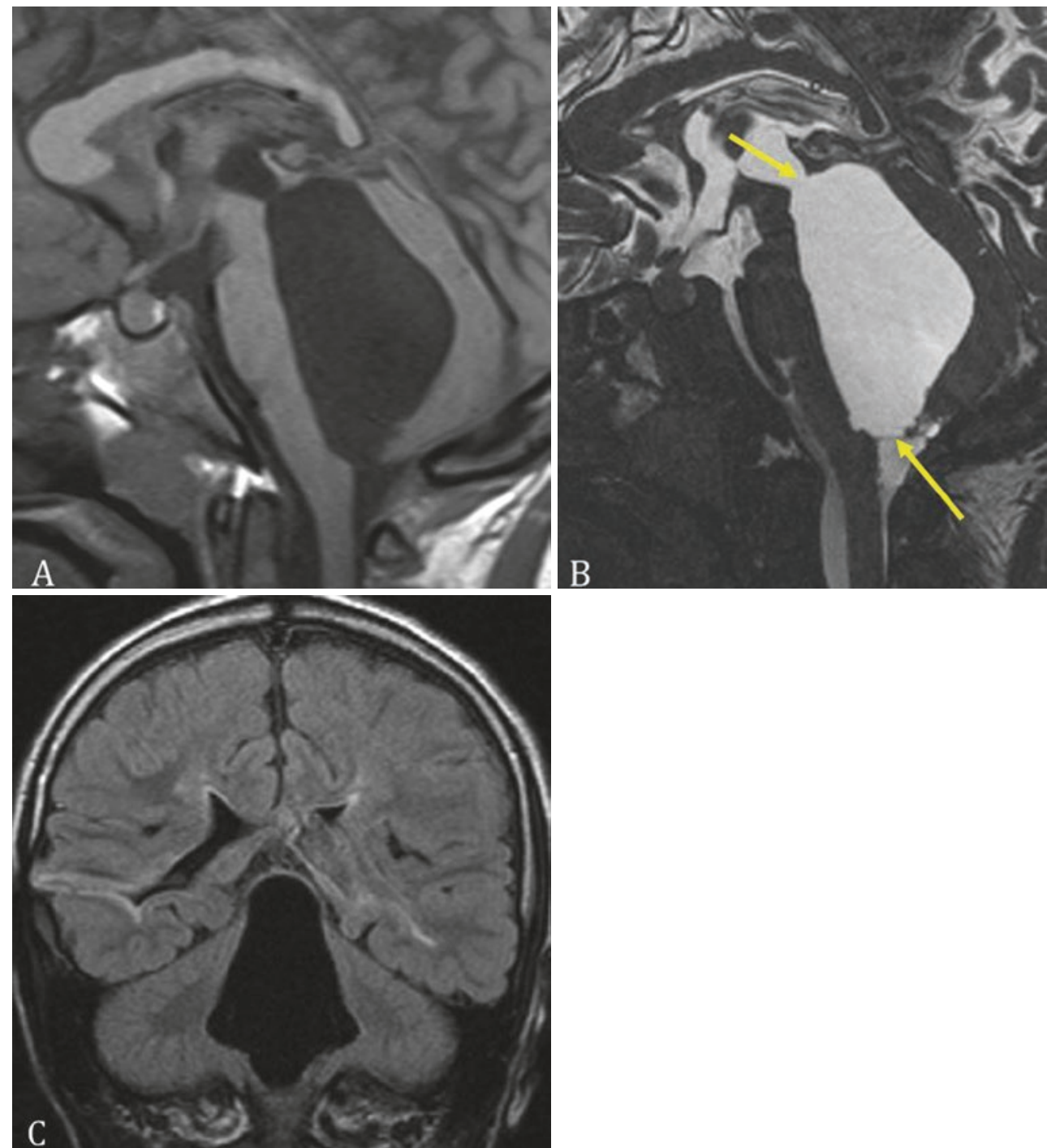

toneal shunt, endoscopic aqueductoplasty, as well as interventriculostomy or open fenestration via suboccipital craniotomy.

\subsubsection{Fourth Ventricular Outlets and Foramen Magnum}

Obstruction of the fourth ventricle outlets (foramina of Luschka and Magendie) and foramen magnum are caused by hemorrhage, meningitis, and extrinsically compressing lesions including tumors (cerebellar metastases, meningiomas, hemangioblastomas, astrocytomas, medulloblastomas) and craniocervical developmental malformation involving the cranio-cervical junction (osteochondrodysplasia, metabolic diseases, developmental abnormalities, and Chiari malformations) [5]. Acute/subacute cerebellar infarcts are also a common cause in the adult population [5]. Primary obstruction of the foramina of Magendie and Luschka by the presence of web-like membranes is an uncommon cause of non-communicating hydrocephalus. 3DT2WSS sequences are particularly useful in the identification of these thin webs.

\section{Key Points}

- 3DT2WSS sequences are useful for accurate anatomic evaluation of the ventricular system, and are highly recommended in patients with suspicion of non-communicating hydrocephalus.

- The inclusion in the imaging protocol of CSF-flow sensitive sequences, such as PC-MRI and3D-T2WTSE, is highly recommended for detecting the normal highvelocity CSF flow through narrower segments of the ventricular system.

- Contrast-enhanced T1-weighted imaging should be obtained when there is a suspicion of a neoplastic lesion or an inflammatory process as the causative mechanism of non-communicating hydrocephalus. 


\subsection{Hydrocephalus in Children}

The etiology and prevalence of pediatric hydrocephalus varies widely throughout the world. In the developing world, neonatal infections account for most cases of hydrocephalus, while in developed regions the more commonly seen etiologies of hydrocephalus include parenchymal hemorrhage, congenital aqueduct stenosis, myelomeningocele, and posterior fossa tumors. The prevalence of hydrocephalus has been estimated to be at least 1 in 1000 births [8, 9].

Etiologies for hydrocephalus in the pediatric populations can also be generally categorized as either congenital or acquired entities. Congenital etiologies include aqueduct stenosis, neural tube defects, and posterior fossa malformations (e.g., vein of Galen malformation obstructing flow at the aqueduct). Acquired etiologies include: subarachnoid or intraventricular hemorrhage or infection, brain tumors, choroid plexus papilloma resulting in overproduction of CSF, and extrinsic venous obstruction.

In the infant, hydrocephalus may present with macrocephaly, irritability, and bulging of the fontanelles. In the older child hydrocephalus typically presents with signs and symptoms of elevated intracranial pressure including headache, vomiting, and delay in developmental milestones.

Management of hydrocephalus consists of treatment of the underlying primary abnormality such as tumor or hemorrhage and CSF diversion procedures with placement of ventriculoperitoneal shunting devices. Newer methods of endoscopic third ventriculostomy (ETV) and choroid plexus cauterization (CPC) have developed since the 1990s and represent an alternative treatment for chronic hydrocephalus. A third ventriculostomy involves a small craniotomy usually through the right frontal region and placement of an endoscope into the right frontal horn, through the foramen of Monro toward the floor of the third ventricle where a small opening is cut in the floor of the third ventricle allowing direct communication with the prepontine cistern. ETV along with CPC via the same endoscopic procedure has shown promising results in early trials.

Complications of long-term shunting include perioperative morbidity and mortality, shunt infection (5-9\% mostly occurring within 3 months of surgery) and shunt malfunction (occurring in $40 \%$ of children within the first 2 years after shunt placement). Interestingly use of valvular apparatus coupled to the silastic catheter has been shown to be ineffective in randomized control trials to decrease the rate of shunt failure.

MRI is the current modality of choice for assessing pediatric hydrocephalus and the effects that might produce in the developing brain. This includes evaluation for size and contour of the ventricles and SAS, presence or absence of interstitial periventricular edema, and identification of the normal CSF aqueductal flow void. Imaging is obviously also indispensable in evaluation for the complications associated with surgical management of hydrocephalus. And, following ETV, can also demonstrate patency of flow across the surgical defect, mainly using flow-sensitive MRI sequences, such as PC-MRI and 3DT2WTSE [10].

\section{Key Point}

- Neonatal infections account for most cases of hydrocephalus in developing regions, while in developed regions the most common causes include parenchymal hemorrhage, congenital aqueduct stenosis, myelomeningocele, and posterior fossa tumors.

\subsection{Communicating Hydrocephalus}

Communicating hydrocephalus is defined as a CSF flow circulation abnormality outside the ventricular system that produces an increase in the ventricular size. Most cases are secondary to obstruction of CSF flow between the basal cisterns and brain convexity, and include common conditions such as subarachnoid hemorrhage, bacterial and aseptic meningitis, and leptomeningeal carcinomatosis. This type of hydrocephalus could be better classified as communicating with obstruction, to differentiate them from idiopathic normal pressure hydrocephalus (iNPH), in which there is not objective obstruction of CSF circulation and absorption [4].

\subsubsection{Communicating Hydrocephalus with Obstruction}

- Subarachnoid hemorrhage: Acute and chronic hydrocephalus is one of the major complications after subarachnoid hemorrhage, with a reported incidence of $20-30 \%$. The hydrocephalus is produced as a consequence of blockage of the SAS that produces impairment of CSF circulation and absorption. The acute form is more common and usually develops within few days after the hemorrhage.

- Meningitis: Bacterial meningitis may be complicated with communicating hydrocephalus, which is associated with poor neurologic outcome. The causative mechanism includes the blockage of SAS by exudates and high protein levels at basal cisterns or convexity that prevent normal CSF circulation and absorption. Less frequently, exudates can block the CSF flow at fourth ventricle exits (Magendie and Luschka foramina), resulting in a noncommunicating type of hydrocephalus. Aseptic meningitis, which could be infective (viral or fungal) or secondary to systemic conditions such as autoimmune diseases or neurosarcoidosis, might produce a communicating hydrocephalus induced by similar mechanisms, but usually 
develops subacutely over a period of weeks to months, and is associated with a lower morbi-mortality compared to the most common hydrocephalus secondary to bacterial meningitis. CT or MRI is rarely indicated for the diagnosis of meningitis, but should be immediately performed in those patients with clinical suspicious of complications (e.g., signs of increased intracranial pressure).

- Leptomeningeal carcinomatosis: Both primary (medulloblastoma, ependymoma, glioblastoma, and oligodendroglioma) and secondary tumors (lymphoma, lung and breast cancers, melanoma) may spread through the SAS (leptomeningeal carcinomatosis). In some cases, the clinical presentation is secondary to impairment of CSF flow with development of communicating hydrocephalus. Contrast-enhanced T1-weighted and T2-FLAIR sequences should be added in patients with communicating hydrocephalus of unknown origin, particularly when the clinical symptoms (e.g., headache) developed acutely or in patients with a known history of cancer.

- Vestibular schwannomas: Hydrocephalus is relatively common in patients with vestibular schwannomas. Although obstructive hydrocephalus due to mechanical compression of the fourth ventricle or cerebral aqueduct by the tumor is demonstrated in some patients, communicating hydrocephalus is more frequent. This latter type of hydrocephalus could be explained by a compromise of CSF absorption by protein leakage from the tumor.

\section{Key Points}

- The term communicating hydrocephalus, better defined as communicating with obstruction, must be differentiated from iNPH, in which there is not objective obstruction of CSF circulation and absorption.

- Common causes of this form of hydrocephalus in the adult population include subarachnoid hemorrhage, bacterial and aseptic meningitis, and leptomeningeal carcinomatosis.

\subsubsection{Normal Pressure Hydrocephalus}

Normal pressure hydrocephalus (NPH) is a syndrome initially identified in 1957 by Solomon Hakim characterized by the triad of gait disturbance, mental deterioration, and urinary incontinence, which are associated with enlargement of the ventricular system and normal CSF pressure. It is difficult to establish the prevalence of NPH due to the non-specific nature of the symptoms, but it has been estimated to be 181.7 per 100,000 people in the 70-79 years age group. About 50\% of cases with NPH have a known cause (secondary or symptomatic NPH [sNPH]), such as meningitis, subarachnoid hemorrhage, or cranial trauma, while the remaining $50 \%$ of cases are idiopathic (iNPH), usually presenting in the seventh decade of life.

The classical concept that NPH is simply a form of CSF circulation disorder, involving an imbalance between CSF production, and reabsorption, is probably not valid. In fact, unlike other forms of hydrocephalus, the CSF pressure is not abnormally raised, which implies that such a simplistic hydrodynamic theory would be insufficient to explain the pathophysiology of this condition. Other factors might contribute to the development of NPH. Chronic hypertension and white matter disease (which may naturally occur in the aging brain) may lead to periventricular ischemia that increases the compliance of the ventricular wall and causes gradual ventricular enlargement. Alternatively, periventricular ischemia may also lead to locally increased venous resistance that may lead to decreased CSF absorption and ventricular enlargement. Finally, diminished vascular (arterial) compliance that frequently occurs in the aging brain might produce a redistribution of systolic vascular pulsations, which are then transmitted directly into the ventricular system, progressively increasing its size.

iNPH is characterized clinically by gait and balance impairment, cognitive deterioration, and urinary incontinence, and radiologically by a communicating ventricular enlargement (Evans Index $>0.3$ ), in the context of a CSF opening pressure of $<24 \mathrm{~cm}$ of $\mathrm{H}_{2} \mathrm{O}$ (in recumbent position). However, the complete triad appears only in 50-60\% of iNPH patients.

The diagnosis of NPH is a challenging process. Initially the diagnosis is suspected on the basis of the history, neurological examination, and interpretation of CT and/or MRI scans. MRI is particularly useful for establishing that a communicating hydrocephalus is present, but may also be effective in identifying patients who will respond to shunting. Exclusion of a noncommunicating hydrocephalus is the first radiological objective in the diagnosis of iNPH. Combination of 3DT2WSS such as CISS (constructive interference steady state) that offer superb anatomical information, and 3DT2WTSE sequences such as SPACE (sampling perfection with application optimized contrast using different flip angle evolutions), which is highly sensitive to CSF flow, provides an easy evaluation of CSF pathway and circulation to establish the patency of CSF flow [11, 12].

A simple way to establish the diagnosis of ventriculomegaly is by means of the Evans index (EI), an indirect linear measurement of ventricular size. It has been established that in normal adults, the EI is $<0.3$, and therefore values $>0.3$ has been used for an objective form to diagnose ventriculomegaly. $\mathrm{EI}$ is not specific for iNPH, as increases with ventriculomegaly independently of its cause. Other morphological features of the ventricular system such as bilateral enlargement of the temporal horns not attributable to hippocampal atrophy, and a dilated third ventricle, have been described as typical findings in both iNPH and obstructive hydrocephalus, but it is not clear the value of these features both for establishing the diagnosis of iNPH or to predict shunt response. 
The callosal angle measured on MRI is determined as the angle between the medial superior borders of the left and right lateral ventricles, from a coronal image obtained at the level of the posterior commissure, perpendicular to a plane that intersected the anterior and posterior commissure. The CA in patients with iNPH is significantly smaller than those with Alzheimer disease or healthy controls, and a value $<90$ $100^{\circ}$ has been used to discriminate between these two groups of subjects with a high accuracy. This accuracy is improved when combining both the CA and the EI $(>0.30-0.32)$. The $\mathrm{CA}$ has also been used to predict shunt-responders, as these patients have a significantly smaller mean preoperative CA compared with non-responders, using $63^{\circ}$ as the cut-off value to achieve the best prognostic accuracy [13].

Narrow cortical sulci at the high convexity (parietal high convexity and medial surface) is a typical finding in patients with NPH. This finding, better identified on coronal MRI or CT scans, is frequently associated with wide Sylvian fissures. This widening, although not uncommon in Alzheimer's disease, is more pronounced and frequent in iNPH. These structural brain changes have been grouped under the term "disproportionately enlarged subarachnoid space hydrocephalus" (DESH), which refers to the combination of ventriculomegaly with narrow high convexity and medial subarachnoid spaces and enlarged Sylvian fissures (disproportionate distribution of the CSF between the inferior and superior subarachnoid spaces), and is present in a high proportion of patients with a final diagnosis of iNPH (Fig. 2.6).

The DESH pattern is extensively used for iNPH diagnosis and predicting shunt response, without the need of a CSF tap test or other invasive procedures [14].
Fig. 2.6 Idiopathic normal pressure hydrocephalus with radiological features of disproportionately enlarged subarachnoid space hydrocephalus (DESH). A 78-year-old woman with one-year history of gait disturbances, cognitive impairment, and urinary incontinence. Observe the normal CSF flow across the aqueduct on the 3DT2WTSE (SPACE) midsagittal image (a), the enlarged Sylvian fissures associated with narrowing of the sulci over the high convexity, and low callosal angle on the coronal T2-FLAIR image (b). Ventriculomegaly associated with extensive white matter and some focal sulcal dilation changes is seen on $\mathrm{T} 2$ and T2-FLAIR images $(\mathbf{c}, \mathbf{d})$. Reproduced from Rovira A. Communicating Hydrocephalus. Normal Pressure Hydrocephalus. In F. Barkhof et al. (eds.), Clinical Neuroradiology, (C) Springer Nature Switzerland AG 2019
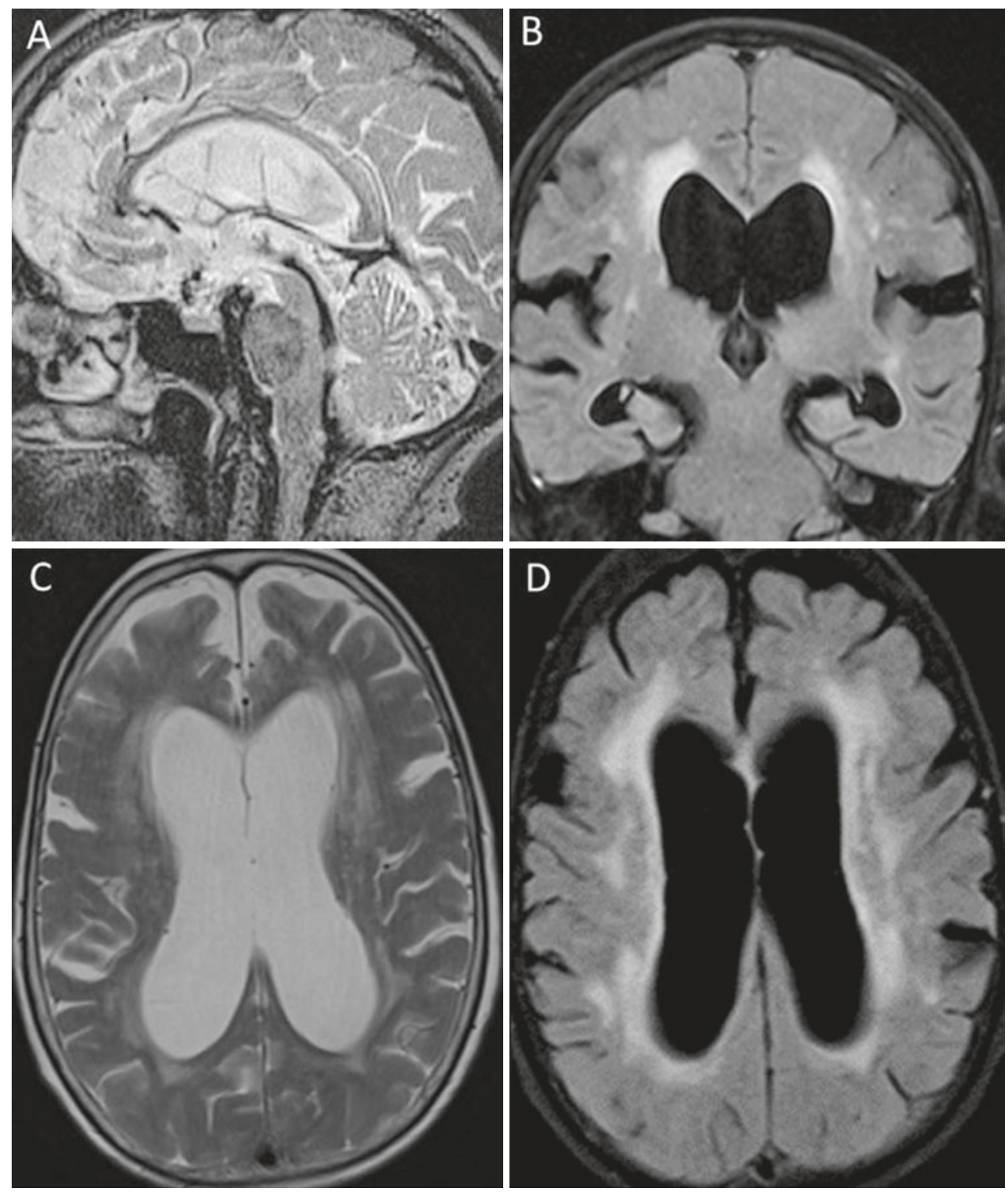
Focal sulcal dilation also occurs in iNPH and should not be mistaken for focal cortical atrophy. These enlarged sulci, which have been considered reservoirs of CSF, usually disappear partially or completely after shunting,

White matter abnormalities (leukoaraiosis) on T2-weighted images, involving both the periventricular and subcortical white matter are a common finding in patients with iNPH, and correlated with a higher frequency of vascular risk factors, such as hypertension. These signal changes are probably related to chronic ischemia as a consequence of cerebral hypoperfusion, and can decrease or even disappear after successful shunt surgery, indicating that at least partially correspond to interstitial edema, as a consequence of an impairment of the glymphatic circulation. The extension of leucoaraiosis in patients with iNPH cannot be used to predict shunt responsiveness.

PC-MRI studies, with a slice positioned in the midsagittal or axial planes, sensitive to CSF movement in the craniocaudal direction (in plane or through plane CSF flow) is a simple way to assess the patency of the aqueduct and to assess the intensity of the pulsatile movement across it. However, the identification and quantification of this CSF movement is not completely specific for the diagnosis of iNPH and does not predict shunt response.

Diversion of CSF is the standard of surgical care for iNPH. Ventriculoperitoneal (VP) shunts are the most popular, while the ventriculoatrial (VA) shunts are rarely implanted because of their more frequent long-term complications. Gait impairment is the symptom that is most responsive to shunting, while cognitive impairment may improve if it is not very severe at the time of intervention, while urinary incontinence improves in 36-90\% of patients.

On CT/MRI studies performed few months after shunt surgery, a mild decrease of the ventricular size is usually seen, associated with CA increased, partial resolution of white matter changes and expansion of sulci over the high convexity sulci (resolution of convexity CSF block).

Not every patient with possible or probable iNPH will be a candidate for shunt surgery, and the risk to benefit ratio has to be assessed on individual bases. Diagnostic test used for selecting patients for shunt surgery includes those based on surgical invasive procedures (CSF dynamics and intracranial pressure monitoring), and those based on morphological or functional MRI studies.

\section{Key Points}

- NPH is characterized by the triad of gait disturbance, mental deterioration, and urinary incontinence, which are associated with enlargement of the ventricular system and normal CSF pressure.

- Structural brain changes grouped under the term "disproportionately enlarged subarachnoid space hydro- cephalus" (DESH), defined as the combination of ventriculomegaly with narrow high convexity and medial subarachnoid spaces and enlarged Sylvian fissures is a key feature to establish the diagnosis of NPH

\subsection{Idiopathic intracranial hypertension (IIH)}

Idiopathic intracranial hypertension (IIH) also known as pseudotumor cerebri or benign intracranial hypertension is a syndrome of elevated intracranial pressure for which there is no identifiable underlying cause. It is most commonly associated with headache and papilledema and more commonly affects females. The symptom of tinnitus is also commonly encountered in patients with IIH. The syndrome typically presents in obese females of child bearing age but can be seen in just about any male or female regardless of body habitus or age. Several medications have been implicated as a cause of secondary $\mathrm{IIH}$; these include tetracycline, minocycline, vitamin A, and retinoids.

The most recently revised diagnostic criteria for the diagnosis of IIH employ a combination of clinical and imaging criteria for diagnosis [15]. IIH has traditionally been a diagnosis of exclusion where neuroimaging was used to simply "rule out" an intracranial mass, hydrocephalus, or dural sinus thrombosis as a cause of elevated intracranial pressure. Many important signs of IIH on MRI and MR venography are now recognized that have dramatically improved our ability to identify the disease in patients independent of clinical suspicion for elevated intracranial pressure.

Diagnostic clinical criteria for IIH include papilledema, normal neurologic examination (except cranial nerve abnormality), normal imaging of the brain parenchyma and meninges, normal CSF composition, and elevated CSF-opening pressure ( $>25 \mathrm{~cm} \mathrm{H}_{2} \mathrm{O}$ ). The diagnosis of $\mathrm{IIH}$ can be considered probable even if there is no papilledema or CSF opening is not elevated provided supportive neuroimaging signs are present.

The neuroimaging signs of elevated intracranial pressure (IP) include empty sella, optic globe deformation seen as flattening of its posterior aspect, papillary protrusion (appreciable optic nerve head protrusion [ \pm enhancement] into the globe correlating with papilledema), tonsillar descent, CSF distention of optic nerve sheath, vertical tortuosity of the intraorbital optic nerve, and stenosis of the distal transverse sinuses. These signs are highly variable in their sensitivity and specificity for predicting the presence of elevated intracranial pressure. Several signs with low sensitivity for increased IP such as papillary enhancement (less than 20\%) have been shown to have very high specificity (99\%), while another such as empty sella has a relatively high sensitivity $(80 \%$ ) but low specificity (less than $80 \%$ ) since it is com- 
monly seen in normal subjects [16]. Stenosis of the distal transverse sinuses remains the most valuable sign for the neuroimaging evaluation of IIH since it has consistently and reproducibly shown the highest (greater than 93\%) sensitivity and specificity. With the advent of gadolinium-enhanced MR venography [17] the transverse sinuses were, for the first time, reliably visualized free from flow artifacts commonly encountered on time-of-flight and phase-contrast MR venographic techniques. Gadolinium-MR venography techniques allowed for the visualization and characterization of the narrowing of the distal transverse sinuses seen in IIH [18]. These most commonly appear as collapse of the dural venous sinuses at or proximal to the transverse sigmoid junction. Alternatively, the stenosis in this region can occasionally be seen due to an enlarged intraluminal arachnoid granulation intrinsically narrowing the lumen.

It must be stressed that the imaging signs described above relate to elevated intracranial pressure and are not exclusive to IIH, as can be present with all other conditions causing elevated IP. The diagnosis of IIH is one of exclusion and all other etiologies must be ruled out. A careful drug history and CSF analysis is mandatory in the work-up of these patients. Moreover, these signs will diminish or resolve following treatment of the elevated pressure [19].

Management of IIH is directed primarily at preserving vision and diminishing the headaches associated with the disease. Treatment typically begins with strategies to improve body habitus and medication (most commonly acetazolamide) and progresses from there as required utilizing surgical procedures of optic nerve fenestration, CSF diversion procedures of ventriculoperitoneal or lumbo-peritoneal shunting, and more recently transverse venous sinus stenting [20]. Since the cause of IIH is not known the treatments can only be directed at the phenotypic or characteristic expression of the disease, i.e., drugs are given which may decrease CSF production or continually remove CSF through extracranial pathways and thus lower IP.

The narrowed transverse sinuses seen in IIH are thought to be a combination of secondary effects arising from elevated IP as well as subsequent superimposition of a positive feedback loop further worsening the IP with venous hypertension. In this way the stenoses are thought to exacerbate the increased IP. Stenting of the stenosed segments breaks the cycle and normalizes venous pressures providing symptomatic relief and protects patients from visual loss. Unfortunately, to date, no randomized control trials exist for evaluation of the efficacy of the surgical options and, therefore, their true efficacy is unknown [21]. Each of the surgical and endovascular options has been shown to have success in treating headache, papilledema, visual loss, diplopia, and tinnitus. Each of these options also carries the risk of complication or need for revision surgery.

Regardless of the cause, the neuroimaging signs of elevated IP (which may relate to IIH) should not be overlooked or overdiagnosed. A diagnosis of IIH cannot be made on imaging grounds alone, and clinical correlation is mandatory to rule out other causes of elevated IP masquerading as IIH.

\section{Key Points}

- IIH has traditionally been a diagnosis of exclusion where neuroimaging is used to simply "rule out" an intracranial mass, hydrocephalus, or dural sinus thrombosis as a cause of elevated IP.

- Stenosis of the distal transverse sinuses remains the most valuable sign for the neuroimaging evaluation of IIH since it has consistently and reproducibly shown the highest sensitivity and specificity for disease.

\subsection{Spontaneous Intracranial Hypotension (SIH)}

Spontaneous intracranial hypotension (SIH) is commonly caused by leakage of CSF from the thecal sac at some point along the spinal canal. The leakage of CSF causes variable symptoms, the most characteristic of which is a positional headache and is commonly associated with typical intracranial MRI features, such as venous distension, pachymeningeal thickening and enhancement, pituitary enlargement, effacement of the suprasellar cistern, subdural fluid collection hygroma or hemorrhage, and sagging deformity of the brainstem with or without tonsillar herniation [22-25] (Fig. 2.7).

In patients being considered for a possible diagnosis of SIH imaging of the entire spine with high-resolution T2-weighted sagittal sequences is mandatory. While many SIH patients present with spinal longitudinal extradural collections (SLECs) some do not [26]. These SLECs have a typical appearance and are seen exclusively in SIH patients with dural mechanical tears along the thecal sac. Conversely, patients with more laterally placed CSF leakage beyond the epidural compartment either from a CSF-venous fistula (CVF) or from a distal root sleeve tear do not display SLECs. The presence or absence of a SLEC on MRI is used to proscribe for the prone versus lateral decubitus positioning of the subsequent dynamic myelography [26, 27]. Digital subtraction myelography (DSM) and ultrafast CT myelography [28] are techniques of dynamic myelography which allow for imaging of gravity dependent contrast media as it descends within the thecal sac from site of injection within the lumbar spine into the cervical spinal canal. By obtaining images in this manner the site at which intrathecal contrast first escapes from the thecal sac and enters the epidural space can be identified with certainty.

Imaging and management of patients with suspected SIH has been a difficult arena for diagnostic neuroradiology. Until 
Fig. 2.7 Spontaneous intracranial hypotension. A 35-year-old woman with a 3-month history of positional headache. Observe the typical intracranial MRI features of this condition: sagging deformity of the brainstem on the sagittal T1-weighted image (a), pachymeningeal thickening and enhancement on axial T2 and contrastenhanced T1-weighted images (b, c), and pituitary enlargement on coronal contrast-enhanced T1-weighted images (d)
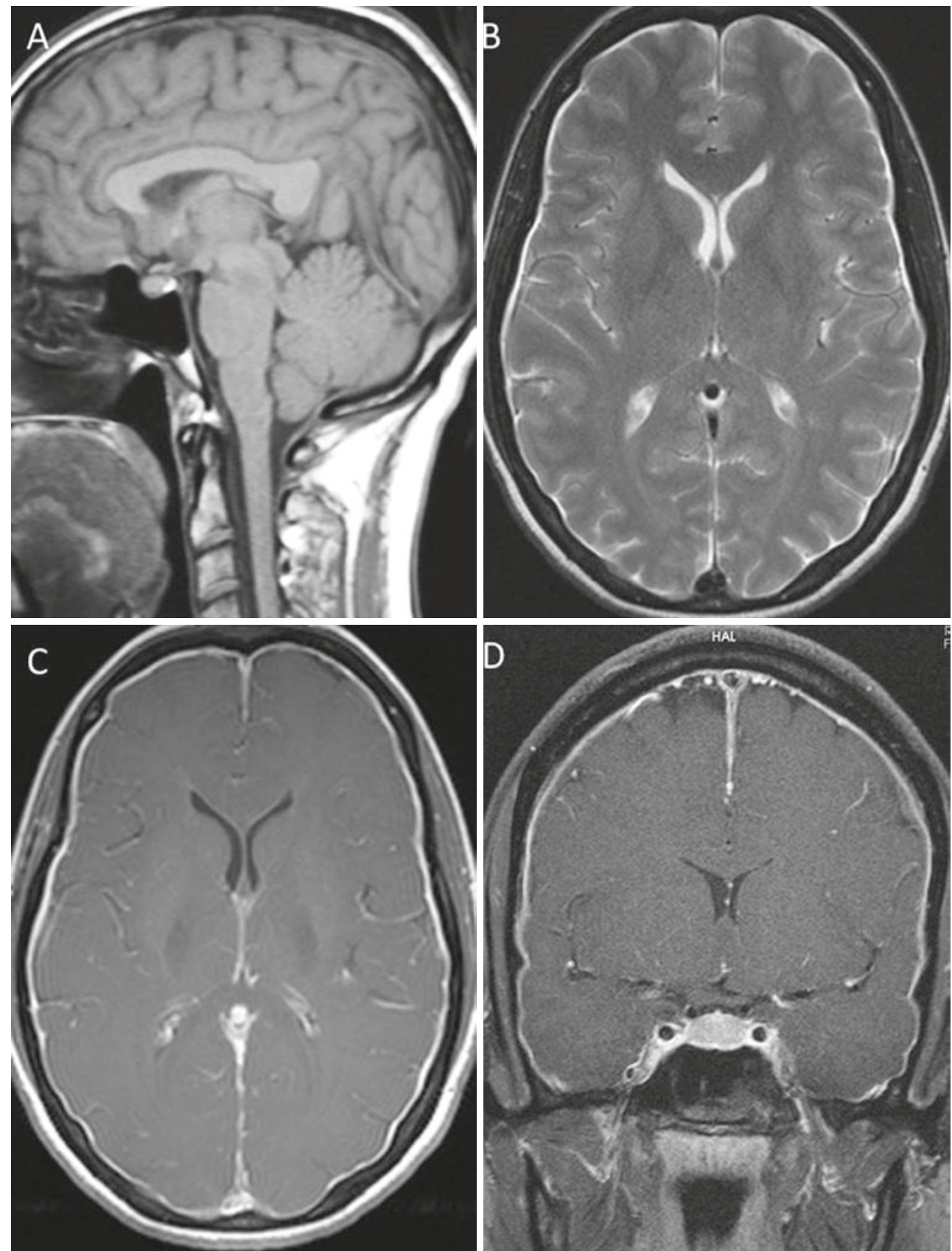

recently there has been a paucity of reliable knowledge disseminated about this syndrome. SIH has only recently been recognized as a syndrome that requires an organized approach by knowledgeable experienced neuroradiologists [26, 29, 30].

In the past, this syndrome has be poorly understood and was thought to be a result of several potential causes including compliance and laxity problems of the thecal sac, low CSF production, and possibly accelerated physiologic loss of CSF. It now appears these leaks are predominantly due to mechanical tears, leaking root sleeves, and CVF. For practicality and ease of description CSF leaks have been classified into four types based on distance from midline [26]. Type 1 is caused by degenerative disc disease creating a mechanical tear in the ventral dura. Patients with these ventral dural punctures are now well recognized as comprising a large population of patients with SIH. All of these patients are SLEC-positive on MRI with or without intracranial findings and show a typical ventral hole in the thecal sac at DSM or dynamic CT. The site of CSF leakage is occasionally seen associated with a calcific "microspur" at the site of a degenerated disk. Unless imaged in a dynamic fashion where the initial flow of contrast is imaged as it travels down the spine the site of leakage (which is commonly microscopic) will be obscured by mixing of contrast in the intradural and extradural compartments above 
and below the hole. A type 2 CSF leak is a less common cause of SIH and is due to a posterolateral dural dehiscent lesion or tear. Type 2 leaks are also associated with SLECs and are not associated with degenerative disk disease. These SLECs may tend to be more posteriorly positioned and their site of origin is commonly incorrectly visualized as a diverticulum adjacent to the root sleeve. In our experience these are more commonly seen in females and appear to be more likely successfully treated with multiple epidural blood patches (EBPs) and bedrest. These are thought to arise from the base of the nerve root (within the axilla) possibly relating to a predisposed thinned or dehiscent area of the dura [29].

If the tear occurs further along the root sleeve adjacent to an arachnoid granulation, where the root sleeve is in close proximity to a venous plexus, then the extravasated fluid is more likely to find its way into a venous channel. In this manner an embryonic pseudomeningocele drains into the venous system and heals aberrantly creating a CVF-a type 3 leak. The type 4 leak is a rare root sleeve leak that does not fistulize into the venous system but rather tracks into and dissipates into the adjacent facial planes. Type 3 and type 4 occur more laterally along the root sleeve beyond the epidural compartment and therefore (1) do not result in a SLEC on spinal MRI and (2) do not respond to nondirected EBP. Focal fibrin patching may have some slowing effect on these leaks, although in our experience has not consistently provided durable cure.

MRI of the brain and spine in patients with SIH allows us to dichotomize patients into those which may benefit from initial EBP prior to dynamic myelography, i.e., SLEC-positive patients (predicting a type 1 or type 2 leak) from those patients in whom nondirected EBP is likely not beneficial, i.e., patients with SLEC-negative imaging (who harbor a type 3 or type 4 leak). SLEC-positive patients (who fail initial EBP) should then undergo DSM in the prone position. This will find over $80 \%$ of the offending type 1 or type 2 tears. If this initial prone DSM is negative, then left and right decubitus DSM should be performed until a leak is found. Conversely SLEC-negative patients should undergo DSM in lateral decubitus position (left then right) to find the offending type 3 or type 4 leaks. Using this type of imaging paradigm an overall success rate of definitively locating the site of CSF leak can be expected in over $90 \%$ of cases.

Dynamic CT myelography (DCTM) is an alternative method to DSM for dynamic myelography [25]. Several authors have reported success in identifying these types of CSF leaks particularly the type 1 and type 2 [26] leaks. It has also been reported on the success of DCTM for type 3 leaks by positioning these patients in the decubitus position to search for CVF. Regardless of the modality chosen it is the spinal MRI which defines these patients as SLEC-positive or SLEC-negative, and proscribes the most efficacious positioning for the subsequent dynamic myelogram. Once found, management can then be directed at the site of CSF leakage. Variable success has been reported with targeted epidural fibrin injections for all types of leaks. The most definitive curative results for treatment of CSF leaks are obtained with surgical repair of the dural tears (types 1 and 2 and 4) and with surgical clipping of the CVF (type 3).

\section{Key Points}

- SIH is commonly caused by leakage of CSF from the thecal sac at some point along the spinal canal.

- In patients being considered for a possible diagnosis of SIH imaging of the entire spine with high-resolution T2-weighted sagittal sequences is mandatory.

- Presence or absence of SLECs on spine MRI is highly useful for guiding dynamic myelographic studies, which are highly sensitive for detecting dural tears.

\subsection{Concluding Remarks}

Structural and functional MRI plays an essential role for understanding the anatomy of the CSF spaces and ventricular system, as well as the hydrodynamics of CSF flow. These principles are important to the comprehension of pathological processes affecting the ventricles and CSF spaces, mainly the different types of hydrocephalus, and disorders associated with abnormal intracranial pressure, such as IIH and SIH. Combination of 3DT2WSS sequences that offer superb anatomical information, and 3DT2WTSE sequences that are highly sensitive to CSF flow, provides an easy evaluation of CSF pathway and circulation to establish the patency of CSF flow and therefore in distinguishing non-communicating from communicating hydrocephalus, which is the first radiological objective in patients with ventriculomegaly.

Diagnosis of IIH and SIH cannot be made exclusively on imaging features, and clinical correlation is mandatory to rule out other causes of elevated or decreased intracranial CSF pressure. In patients being considered for a possible diagnosis of $\mathrm{SIH}$, in addition to brain MRI, imaging of the entire spine with high-resolution T2-weighted sagittal sequences is mandatory, as it provides imaging features that not only support the diagnosis but also guide the subsequent dynamic myelography.

\section{Take Home Messages}

- MRI is the imaging modality of choice in the diagnosis and management of hydrocephalus and other CSF-related diseases.

- Conventional MRI sequences associated with 3DT2WTSE sequences seem to be most efficient MRI strategy for evaluation of hydrocephalus. 
- In complex cases, PC-MRI and 3DT2WSS sequences may add relevant diagnostic information.

- Many signs of elevated intracranial pressure on MRI may suggest the diagnosis of IIH. However, this diagnosis should only be made after careful clinical correlation and exclusion of other etiologies.

- When evaluating patients for a possible diagnosis of SIH, a systematic approach, with proper application of MRI of the brain and spine allows for subsequent efficient work-up and management.

\section{References}

1. Chikly B, Quaghebeur J. Reassessing cerebrospinal fluid (CSF) hydrodynamics: a literature review presenting a novel hypothesis for CSF physiology. J Bodyw Mov Ther. 2013;17:344-54.

2. Chen L, Elias G, Yostos MP, Stimec B, Fasel J, Murphy K. Pathways of cerebrospinal fluid outflow: a deeper understanding of resorption. Neuroradiology. 2015;57:139-47.

3. Kelly EJ, Yamada S. Cerebrospinal fluid flow studies and recent advancements. Semin Ultrasound CT MR. 2016;37:92-9.

4. Agarwal A, Bathla G, Kanekar S. Imaging of communicating hydrocephalus. Semin Ultrasound CT MR. 2016;37:100-8.

5. Kartal MG, Algin O. Evaluation of hydrocephalus and other cerebrospinal fluid disorders with MRI: an update. Insights Imaging. 2014;5:531-41.

6. Maller VV, Gray RI. Noncommunicating hydrocephalus. Semin Ultrasound CT MR. 2016;37:109-19.

7. Barami K, Chakrabarti I, Silverthorn J, et al. Diagnosis, classification and management of fourth ventriculomegaly in adults: report of 9 cases and literature review. World Neurosurg. 2018;116:e709-22.

8. Kahle KT, Kulkarni AV, Limbrick DD, Warf BC. Hydrocephalus in children. Lancet. 2016;387(10020):788-99.

9. Krishnan P, Raybaud C, Palasamudram S, Shroff M. Neuroimaging in pediatric hydrocephalus. Indian J Pediatr. 2019;86:952-60.

10. Dincer A, Ozek MM. Radiologic evaluation of pediatric hydrocephalus. Childs Nerv Syst. 2011;27:1543-62.

11. Bradley WG Jr. CSF flow in the brain in the context of Normal pressure hydrocephalus. AJNR Am J Neuroradiol. 2015;36:831-8.

12. Bradley WG Jr. Magnetic resonance imaging of normal pressure hydrocephalus. Semin Ultrasound CT MR. 2016;37:120-8.

13. Virhammar J, Laurell K, Cesarini KG, Larsson EM. Preoperative prognostic value of MRI findings in 108 patients with idiopathic normal pressure hydrocephalus. AJNR Am J Neuroradiol. 2014;35:2311-8.

14. Mori E, Ishikawa M, Kato T, et al. Japanese Society of Normal Pressure Hydrocephalus. Guidelines for management of idiopathic normal pressure hydrocephalus: second edition. Neurol Med Chir (Tokyo). 2012;52:775-809.

15. Friedman DI. Contemporary management of the pseudotumor cerebri syndrome. Expert Rev Neurother. 2019;19:881-93.

16. Agid R, Farb RI, Willinsky RA, Mikulis DJ, Tomlinson G. Idiopathic intracranial hypertension: the validity of cross-sectional neuroimaging signs. Neuroradiology. 2006;48:521-7.

17. Farb RI, Scott JN, Willinsky RA, Montanera WJ, Wright GA, ter Brugge KG. Intracranial venous system: gadolinium-enhanced three-dimensional MR venography with auto-triggered elliptic centric-ordered sequence-initial experience. Radiology. 2003;226:203-9.

18. Farb RI, Vanek I, Scott JN, et al. Idiopathic intracranial hypertension: the prevalence and morphology of sinovenous stenosis. Neurology. 2003;60:1418-24.

19. Bidot S, Saindane AM, Peragallo JH, Bruce BB, Newman NJ, Biousse V. Brain imaging in idiopathic intracranial hypertension. J Neuroophthalmol. 2015;35:400-11.

20. Friedman DI, Liu GT, Digre KB. Revised diagnostic criteria for the pseudotumor cerebri syndrome in adults and children. Neurology. 2013;81:1159-65.

21. Kalyvas AV, Hughes M, Koutsarnakis C, et al. Efficacy, complications and cost of surgical interventions for idiopathic intracranial hypertension: a systematic review of the literature. Acta Neurochir. 2017;159:33-49.

22. Schievink WI, Maya MM, Jean-Pierre S, Nuno M, Prasad RS, Moser FG. A classification system of spontaneous spinal CSF leaks. Neurology. 2016;87:673-9.

23. Holbrook J, Saindane AM. Imaging of intracranial pressure disorders. Neurosurgery. 2017;80:341-54.

24. Kranz PG, Amrhein TJ, Choudhury KR, Tanpitukpongse TP, Gray L. Time-dependent changes in Dural enhancement associated with spontaneous intracranial hypotension. AJR Am J Roentgenol. 2016;207:1283-7.

25. Mokri B. Spontaneous intracranial hypotension. Continuum (Minneap Minn). 2015;21(4 Headache):1086-108.

26. Farb RI, Nicholson PJ, Peng PW, et al. Spontaneous intracranial hypotension: a systematic imaging approach for CSF leak localization and management based on MRI and digital subtraction Myelography. AJNR Am J Neuroradiol. 2019;40:745-53.

27. Schievink WI, Maya MM, Moser FG, et al. Lateral decubitus digital subtraction myelography to identify spinal CSF-venous fistulas in spontaneous intracranial hypotension. J Neurosurg Spine. 2019;31(6): 1-4.

28. Thielen KR, Sillery JC, Morris JM, et al. Ultrafast dynamic computed tomography myelography for the precise identification of high-flow cerebrospinal fluid leaks caused by spiculated spinal osteophytes. J Neurosurg Spine. 2015;22:324-31.

29. Dobrocky T, Mosimann PJ, Zibold F, et al. Cryptogenic cerebrospinal fluid leaks in spontaneous intracranial hypotension: role of dynamic CT Myelography. Radiology. 2018;289:766-72.

30. Dillon WP. Challenges in the diagnosis and treatment of spontaneous intracranial hypotension. Radiology. 2018;289:773-4.

Open Access This chapter is licensed under the terms of the Creative Commons Attribution 4.0 International License (http://creativecommons. org/licenses/by/4.0/), which permits use, sharing, adaptation, distribution and reproduction in any medium or format, as long as you give appropriate credit to the original author(s) and the source, provide a link to the Creative Commons license and indicate if changes were made.

The images or other third party material in this chapter are included in the chapter's Creative Commons license, unless indicated otherwise in a credit line to the material. If material is not included in the chapter's Creative Commons license and your intended use is not permitted by statutory regulation or exceeds the permitted use, you will need to obtain permission directly from the copyright holder.

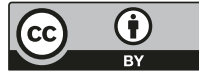




\title{
Stroke and Stroke Mimics: Diagnosis and Treatment
}

\author{
Howard Rowley and Achala Vagal
}

\section{Learning Objectives}

- To review recent evidence based guidelines for triage in acute ischemic stroke

- To describe the imaging findings, design of CT and MRI protocols, and optimal workflow for ischemic stroke triage

- Recognize stroke mimics and offer differential considerations for emergency "Code Stroke" cases

\subsection{Introduction}

Advanced CT and MRI imaging is critical to best practice in clinical stroke care, particularly in the era of intravenous thrombolysis and intra-arterial thrombectomy treatments for appropriately selected candidates. The optimal imaging strategy will vary from site to site but needs to harness the best modality (CT versus MRI), workflow, communication, staff and provider education, expertise, and cost for the right patient at the right time. This requires a coordinated systemwide approach from regional emergency providers to inhospital care.

The over-arching goal of imaging is to establish the right diagnosis, not "just" to help direct critical triage decisions for IV thrombolysis or endovascular therapy. The following three key areas should be confidently and expeditiously evaluated in every acute stroke imaging protocol:

H. Rowley $(\varangle)$

Department of Radiology, University of Wisconsin,

Madison, WI, USA

e-mail: hrowley@uwhealth.org

A. Vagal

Department of Radiology, University of Cincinnati,

Cincinnati, $\mathrm{OH}$, USA

e-mail: vagala@ucmail.uc.edu
1. Hemorrhage: Exclude acute intracranial hemorrhage, including parenchymal hemorrhage and subarachnoid hemorrhage, as this is a critical triage branch point and exclusion for revascularization therapy.

2. Ischemia: When acute ischemic stroke signs are identified, both the extent of baseline ischemic damage and the underlying cause should be determined during the initial triage phase if possible.

3. Mimics: Stroke mimics, including seizures, tumor, infection, migraine, and other acute neurologic conditions should be detected by imaging, or at least suggested in the differential diagnosis.

\subsection{Evidence Based Guidelines for Imaging in Acute Ischemic Stroke}

There has been a recent paradigm shift in the treatment of acute ischemic stroke. Imaging is a central component in the diagnosis, triage, and selection criteria. This is reflected in the updated 2018 guidelines from American Heart Association (AHA)/ American Stroke Association (ASA) [1] and combined 2019 European Stroke Organization (ESO) and the European Society for Minimally Invasive Neurological Therapy (ESMINT) [2].

Endovascular treatment (EVT) is now established as standard of care for patients with acute ischemic stroke with large vessel occlusion (LVO) involving the anterior circulation. Large vessel occlusion is defined as occlusion involving internal carotid artery and proximal middle cerebral artery. In 2015, five randomized controlled trials (MR CLEAN, ESCAPE, REVASCAT, SWIFT-PRIME, and EXTEND-IA) [3-7] demonstrated benefit for patients with LVO within $6 \mathrm{~h}$ from symptom onset as compared to medical therapy. These patients had moderate to severe stroke deficits (NIHSS score 6) and absence of widespread established infarction on brain imaging. In 2018, two additional randomized controlled trials (DAWN and DEFUSE $3[8,9]$ ) were positive in showing benefit of EVT treatment for up to $24 \mathrm{~h}$ from the symptom onset. The late onset 
(6-24 h) trials used automated perfusion imaging including volumetric core, penumbra, and mismatch criteria for imaging selection. These trials provided level 1a evidence for benefit of advanced imaging to predict good outcomes after thrombectomy, by selecting patients with a small estimated core infarct (CBF $<30 \%$ of normal) compared to a larger symptomatic putative penumbra (clinical deficit or perfusion time prolonged $>6$ s). The DAWN trial used clinical-imaging mismatch as a selection criterion between 6 and $24 \mathrm{~h}$ from symptom onset. The DEFUSE 3 trial used mismatch and core up to $70 \mathrm{~mL}$ for patients from 6 to $16 \mathrm{~h}$ from symptom onset. An example of advanced multimodal $\mathrm{CT}$ imaging selection for thrombectomy is shown in Fig. 3.1.

Recently, two major trials have been published for extending the use of intravenous alteplase (IV tissue plasminogen activator, tPA). The WAKE-UP trial [10] used mismatch between diffusion-weighted imaging (DWI) and fluid-attenuated inversion recovery (FLAIR) on MRI and showed benefit of IV tPA versus placebo in patients with unknown time of onset and for whom mechanical thrombectomy was not performed. The EXTEND trial [11] evaluated an extended thrombolytic time window from 4.5 to $9 \mathrm{~h}$ or patients with a wake-up stroke with an uncertain onset and again showed better outcomes for IV tPA versus placebo. EXTEND used automated perfusion imaging selection (core $<70 \mathrm{~mL}$ and a mismatch $>10 \mathrm{~mL}$ ). It is expected that the guidelines will be updated soon to include the benefit of imaging selection for extended times of thrombolytic treatment (similar to the current extended EVT times).

\subsection{Protocol Strategies: Choice of Modality}

Investing in acute stroke imaging protocols yields dividends for all types of acute neurologic emergency care. With rapidly improving hardware and software, automated post-

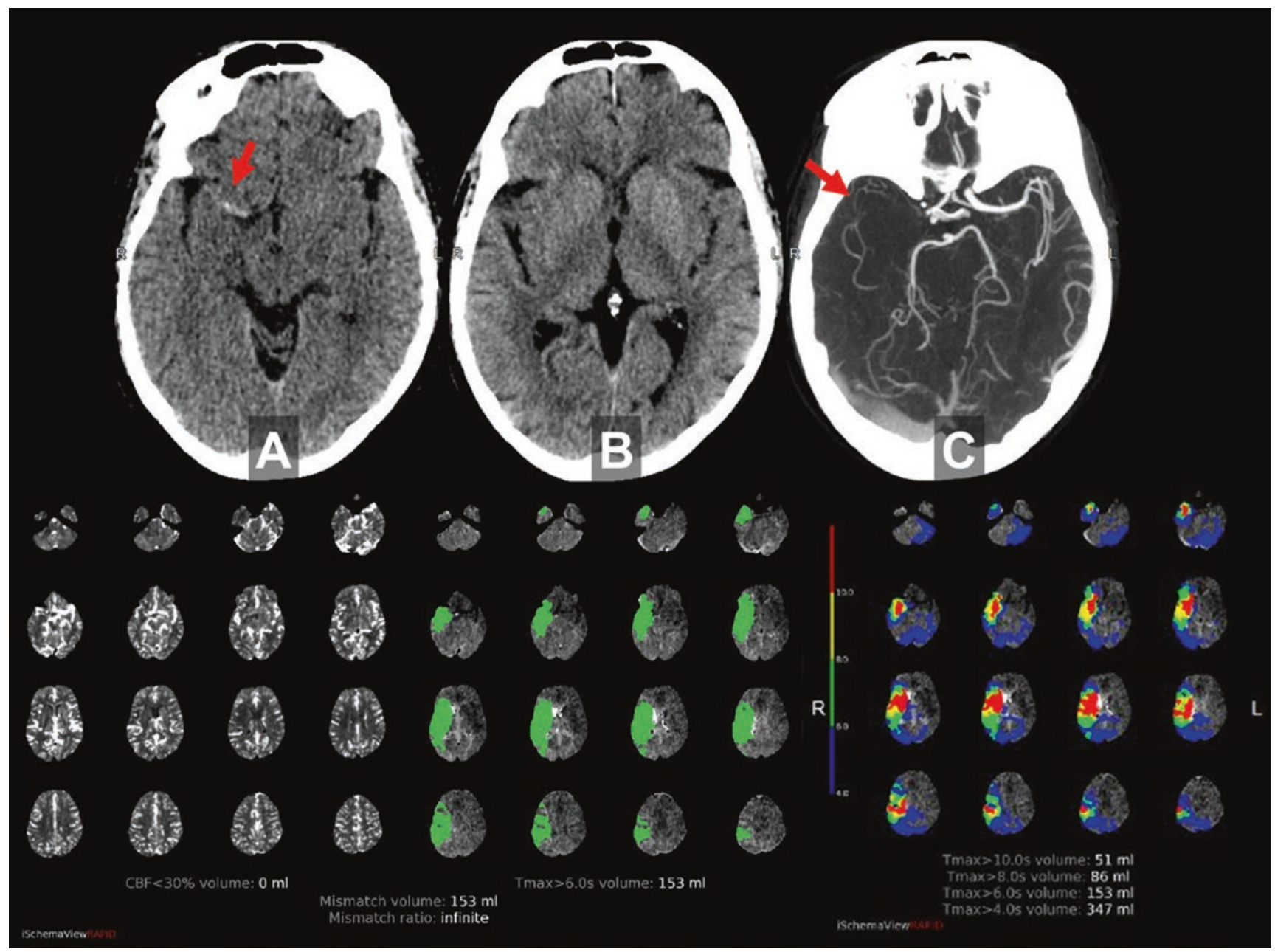

Fig. 3.1 A 75-year-old woman with acute right M1 MCA occlusion due to cardiac embolism. (a) hyperdense MCA sign (arrow); (b) normal CT $($ ASPECTS $=10)$; (c) CTA confirms right MCA occlusion with poor distal collaterals (arrow). Quantitative CT perfusion (RAPID software, lower panel) shows no estimated core infarct $(\mathrm{CBF}<30 \%)$ but a
$153 \mathrm{~mL}$ area of poor perfusion (Tmax $>6 \mathrm{~s}$ ), therefore essentially completes "target mismatch." Gradations in Tmax are shown in rainbow color. The patient underwent urgent thrombectomy with excellent recovery. (Reprinted with permission, Springer) 
processing, and artificial intelligence, rapid scanning and diagnosis increasingly facilitate emergency triage care, both in the emergency department and the inpatient setting. Either CT or MRI-based protocols work very well in acute stroke triage. However, the details are critical: these protocols require careful workflow adjustments, training, and ongoing education for rapid analysis and accurate interpretation.

Each modality has its advantages and disadvantages. $\mathrm{CT}$ is favored in most institutions world-wide due to widespread availability, inherently rapid imaging, relatively lower cost, and comfort level for referring providers and interpreting physicians; the only major potential disadvantages are radiation exposure and lack of sensitivity for brainstem/lacunar strokes and very early ischemia. MRI is arguably better, since, for example, diffusion-weighted imaging is more sensitive for detection of acute ischemia than CT, but this typically comes at higher cost, lower availability, need for MRI safety screening, and usually longer imaging exam times.

\subsection{Advanced Stroke Triage: 3C's or 4P's?}

Practical and efficient protocols have successfully been built with both $\mathrm{CT}$ and MR, ensuring accurate characterization of parenchymal injury and relevant vascular anatomy using CTA or MRA. With CT-based protocols, several groups have convincingly argued inclusion of multimodal CTA to assess for collaterals which can be particularly helpful in triage selection in the setting of large vessel occlusion. A more controversial component is perfusion imaging for either modality, particularly whether it is required for triage within $6 \mathrm{~h}$ of symptom onset. However, there is less debate for the 6- to 24-h time window, where perfusion confers valuable selection refinement based on level $1 \mathrm{~A}$ evidence from clinical trials. Mnemonics capturing these strategies, without or with perfusion, are summarized by either the 3C's: Core, Clot, and Collaterals [12]; vs the 4P's: Parenchyma, Pipes, Perfusion, and Penumbra [13]. Beyond selection for intravenous or intra-arterial therapies, perfusion imaging also enables the proper diagnosis in cases of TIA without visible parenchymal injury and can assist the reader in CTA or MRA interpretation, pointing to areas for additional scrutinywhich might have otherwise escaped detection.

\subsection{CT: Findings, Protocols, and Cases}

For most stroke centers around the world, CT is the workhorse of stroke imaging given its speed and ubiquitous availability. A CT-based stroke workup includes a non-contrast CT, CT angiogram (CTA), and CT perfusion (CTP). A non-contrast CT (NCCT) head study is the initial imaging test in a suspected case of acute ischemic stroke, most importantly to exclude hemorrhage. The early findings of acute ischemic stroke include insular hypodensity/loss of insular ribbon, hypodensity in the basal ganglia with obscuration of gray-white matter and gyral swelling. The Alberta Stroke Programme Early CT Score (ASPECTS) is a widely used objective way of assessing early ischemic changes in the middle cerebral artery distribution and uses a negative ordinal scale of 0-10 (Fig. 3.2). Low ASPECTS scores (approximately 7 or less) have been associated with increased morbidity and mortality. The recent endovascular trials used ASPECTS as a selection criterion, a score of 6 or higher suggesting a small ischemic core. Although an objective scale, ASPECTS has multiple limitations including poor interrater reliability, poor performance in setting of extensive white matter disease, and applicability only to middle cerebral arterial infarcts. Currently, there are automated ASPECTS tools using artificial intelligence (AI) that may be complimentary to human readers. The hyperdense vessel sign is another important finding on NCCT to assess intracranial thrombus. This is best visualized on thin section NCCT with MIP reconstructions which can improve sensitivity and specificity.

A CT angiogram (CTA) is now standard of care in a CT-based stroke workup to detect large vessel occlusion (LVO) and global assessment of the vascular system, including the neck. CTA has distinct advantages; it is quick, readily available, provides a comprehensive view of the arterial system, and is highly effective at identifying LVO, with an accuracy of 99\% [14]. (Ref) An LVO defined as occlusion of the ICA or MCA-M1 segment prompts consideration for EVT. An "M1 like" proximal M2 occlusion also may be considered as LVO for possible EVT, although the M2 occlusions have not been adequately studied in the EVT trials. CTA is also helpful in guiding EVT and procedural planning with depiction of extracranial vasculature for occlusions, dissections, aneurysms, or anatomic variations. Anatomic coverage of a CTA should begin at the origins of the great vessels at the aortic arch and extend through the vertex. A CTA can be performed as a single or multiphase study. A multiphasic CTA (with arterial, early venous, and late venous phases) technique can provide temporal information of collateral circulation.

An additional important piece of information that can be obtained in a CTA study is collateral circulation. Collaterals are now an established independent predictor of outcomes $[15,16]$. Good collaterals can sustain the ischemic tissue for a longer time with slower infarct growth (slow progressors), smaller final infarcts, and better outcomes. Poor collaterals are associated with faster progressors, larger infarcts, and worse outcomes in spite of successful reperfusion. Overlapping, thick section maximal intensity projection (MIP) images can be easily reconstructed on the CT scanners or on PACS stations and are helpful for LVO detection and for collateral grading (Fig. 3.1). There are numerous collateral grading scores with no consensus on optimal score; however, for practical purposes, a "good" versus "bad" dichotomized collateral information of the affected arte- 


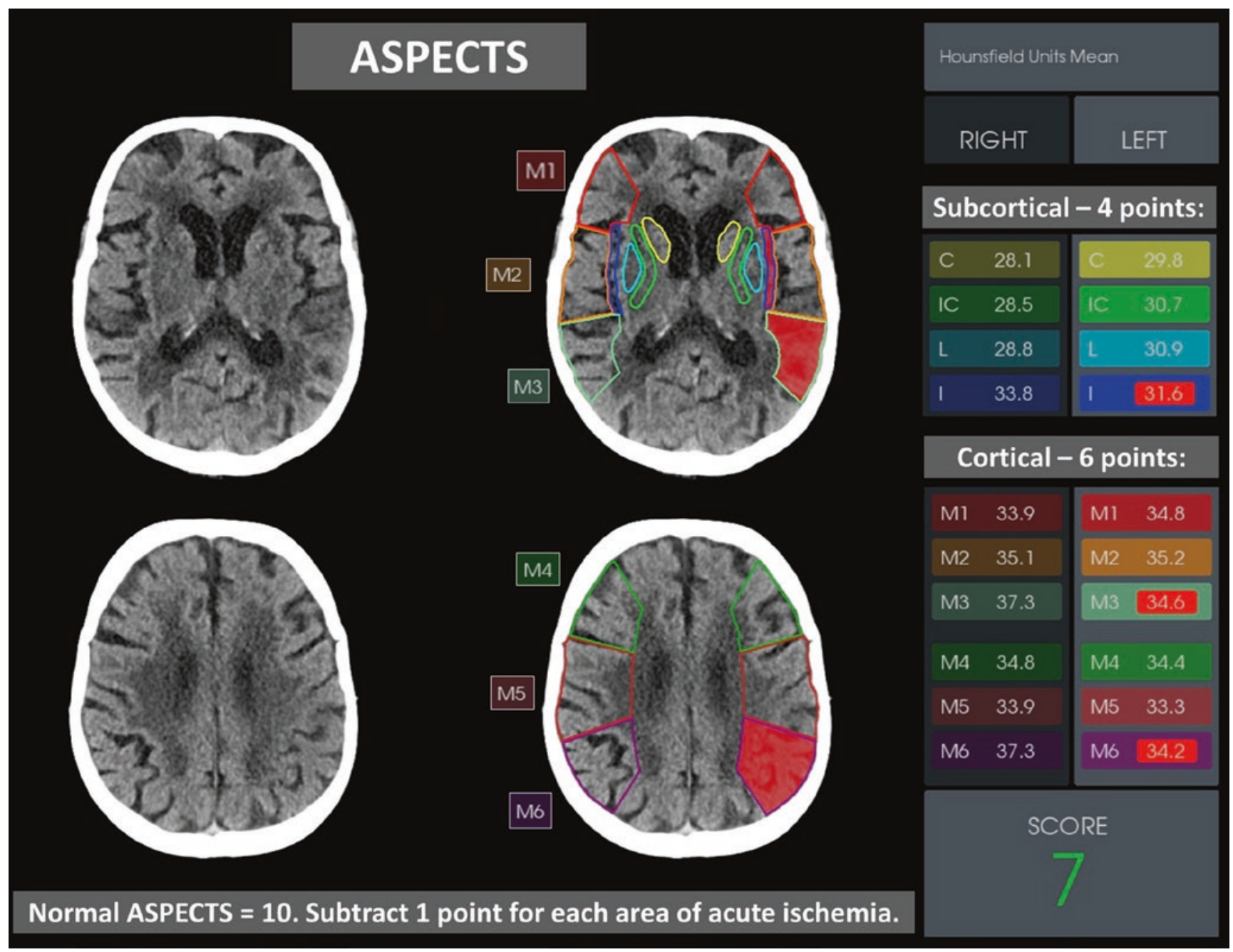

Fig. 3.2 ASPECTS (Alberta Stroke Programme Early CT Score), acute left MCA ischemia. On all $5 \mathrm{~mm}$ non-contrast axial images from basal ganglia to the centrum semiovale, four subcortical areas (caudate, internal capsule, lentiform nucleus, and insula) and six MCA sectors (M1-M6) are reviewed for signs of low attenuation suggesting acute MCA ischemia. A normal scan or one with only old lesions receives ASPECTS $=10$. A point is subtracted for each of the ten areas showing

rial territory as compared to the contralateral side is sufficient. Color coded collateral circulation displays using AI are being introduced which may aid accurate estimation of the collateral circulation, thus helping in timely patient triage.

CT Perfusion (CTP) is now increasingly used in stroke centers as part of the imaging workup, performed in conjunction with NCCT and CTA. CTP gives important information about the extent of the ischemic core (irreversibly injured tissue) and penumbra (at risk but salvageable tissue). CTP is performed in a cine mode by sequentially imaging a defined section of tissue after a single high-flow bolus of contrast material is administered. The images are post-processed using automated or semiautomated perfusion software using deconvolution algorithms to create multiple CTP maps including cerebral blood flow $(\mathrm{CBF})$, cerebral blood volume $(\mathrm{CBV})$, mean transit time acute changes, with low scores predicting less favorable outcome. Software packages are being developed to automate ASPECTS scoring, as shown here (ASPECTS $=7$ ). (RAPID software images modified from PD Dr. med. Carlo Cereda Medico Caposervizio, Neurocentro (EOC) della Svizzera Italiana, Stroke Center, Servizio di Neurologia, Ospedale Civico, Lugano, Switzerland.) (Reprinted with permission, Springer)

(MTT), time to peak (TTP), time to maximum (Tmax), and delay maps. The commonly used parameter for measuring ischemic core volume is $\mathrm{CBF}$ using threshold of $\mathrm{CBF}<30 \%$ as compared to contralateral tissue. The penumbra (hypoperfused area) volume is commonly measured using the threshold of Tmax $>6 \mathrm{~s}$. Mismatch ratios can be generated using core and penumbral volumes. CTP and CTA results are complimentary, facilitating both accurate interpretation and diagnosis (Fig. 3.3).

There has been a recent surge in the use of automated perfusion software, which is critical when triage and treatment decisions need to be made urgently and every minute counts. At the same time, it is important to be aware of the pitfalls of automated CTP processing. The pitfalls include motion artifact, poor signal-to-noise ratio from a suboptimal contrast material bolus, skull base artifacts, inadequate arterial and 


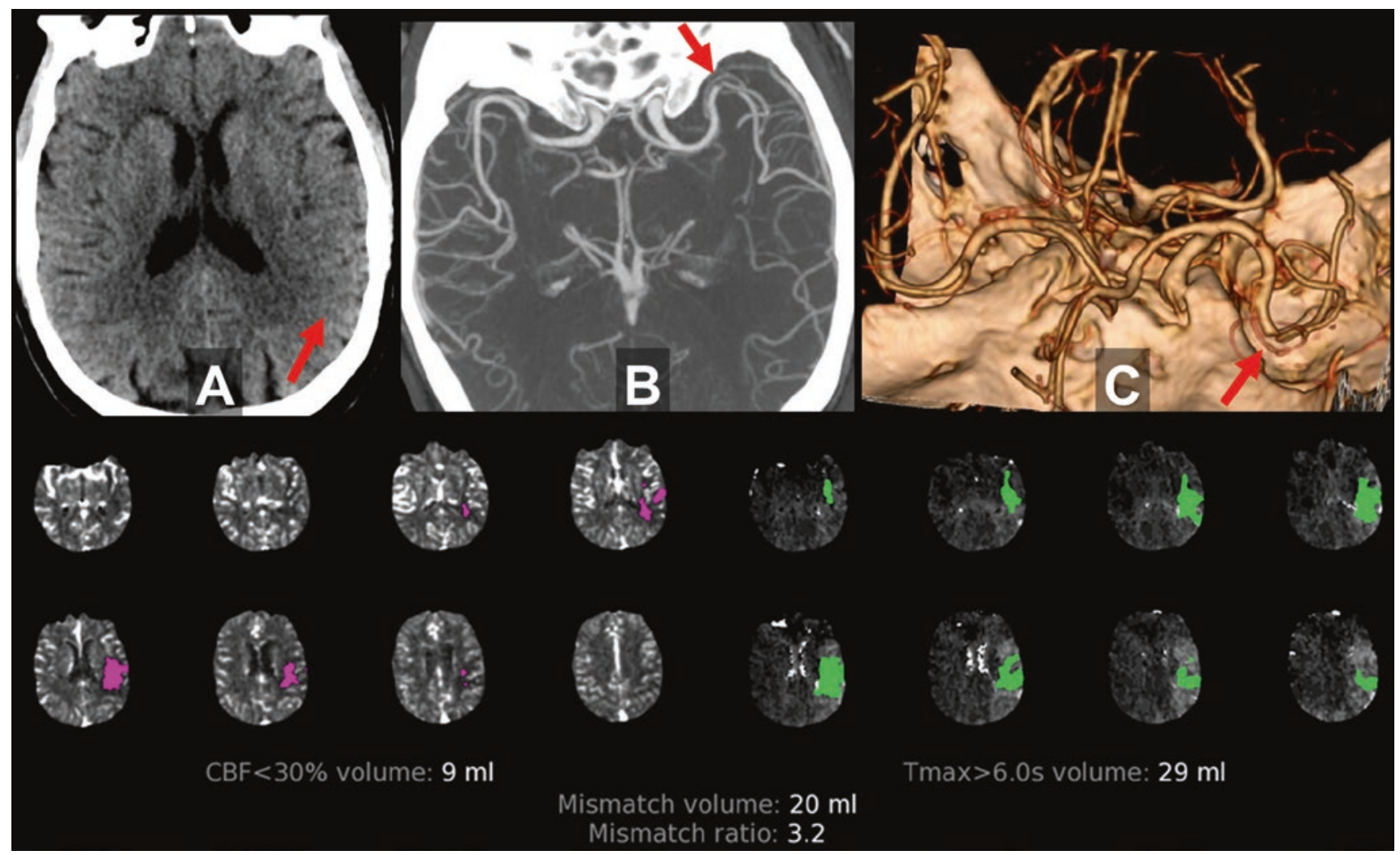

Fig. 3.3 A 74-year-old man 4 days after cardiac bypass surgery with abrupt aphasia and right hemiparesis. (a) non-contrast CT has ASPECTS $=9$, with subtle loss of gray-white distinction in the left angular region (arrow). (b) a subtle filling defect is seen on thick multiplanar reconstructions from the CTA (arrow). CT perfusion (below) shows a large area of ischemia and small mismatch, suggesting a more

venous input selections, and truncated time density attenuation curves. Poor cardiac output and atrial fibrillation can also result in poor contrast bolus. In addition, chronic carotid occlusion can result in false positive penumbra. Furthermore, the thresholds $(\mathrm{CBF}<30 \%)$ may not always capture the true ischemic core, especially for ultra-early acute infarcts and in cases of partial reperfusion. One must be aware of these pitfalls and evaluate the NCCT and CTA in conjunction with CTP for correct interpretation. It is also essential to be cognizant of the fact that there is inherent variation in the different perfusion software packages and post-processing platforms. Even with all of these limitations, CTP is an important tool to assess the ischemic core, penumbra, and mismatch.

\subsection{MRI: Findings, Protocols, and Cases}

An MRI-based approach to stroke imaging offers both advantages and disadvantages compared to CT. Key advantages include maximal sensitivity to ischemia using diffusion MRI, whole brain coverage with dynamic susceptibilitybased perfusion, and lack of ionizing radiation. Disadvantages include availability, need for safety screening, and typically significant stenosis or occlusion is likely present. (c) CTA was postprocessed in 3D to show a proximal M2 occlusion (arrow), missed on initial CTA review due to tortuous overlapping vessels on 2D images. The occlusion site was proven during successful thrombectomy procedure, with good clinical recovery. (Reprinted with permission, Springer)

longer imaging times for similar information. A fully functional MRI triage system requires $24 / 7$ availability near the point of care. Recognizing the urgent nature of stroke triage and to compete with CT, protocols need to be designed to run with a room time of about 10 min or less (Figs. 3.4 and 3.5). Although these requirements can be hard to meet in many centers, MRI should be considered front line in several scenarios (see below Key Points).

\section{Key Points}

Clinical Scenarios Favoring MR over CT

- Wake-up stroke/unknown time of onset

- Late transfer

- Transient ischemic attack

- Posterior fossa/brainstem localization

- Pediatric stroke

- Concern for amyloid or hemosiderosis

- Known or expected complex lesions (e.g., MoyaMoya, stroke vs tumor)

- Iodine allergy 


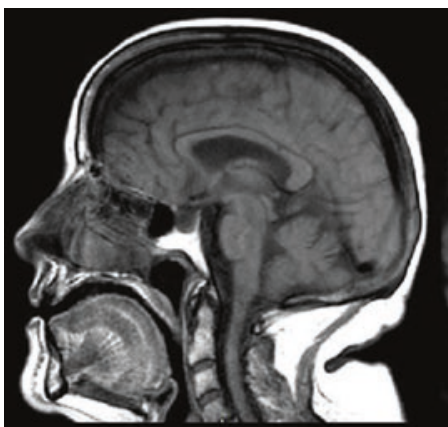

T1

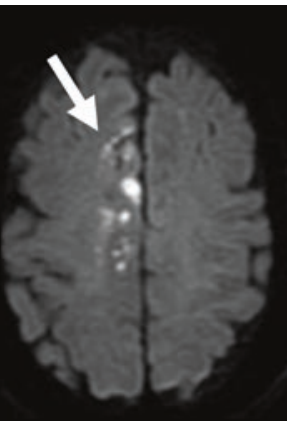

DWI

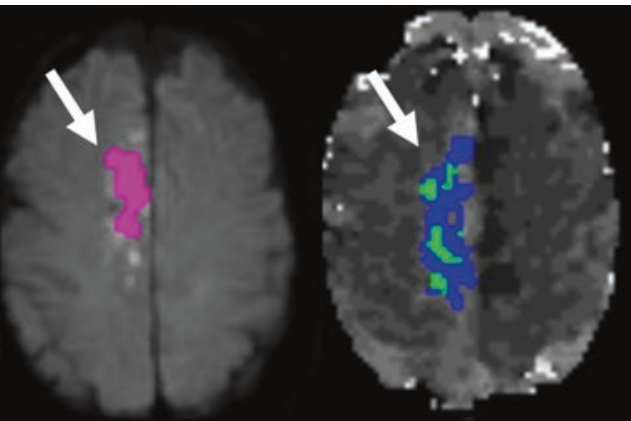

ADC $<620$
Tmax

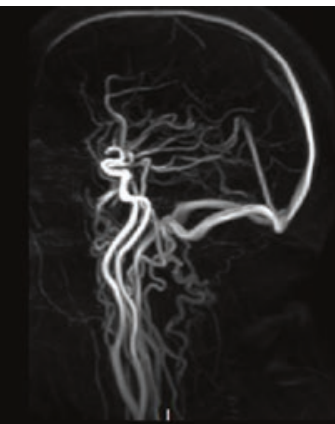

3DPC-MRA

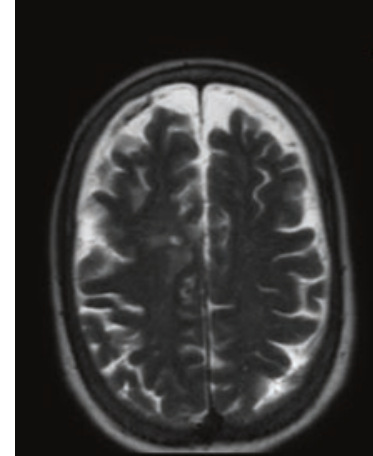

SSFSE-T2

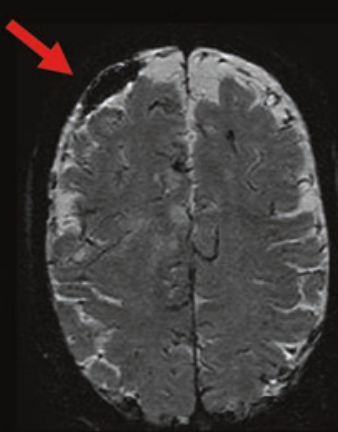

EPI-T2*
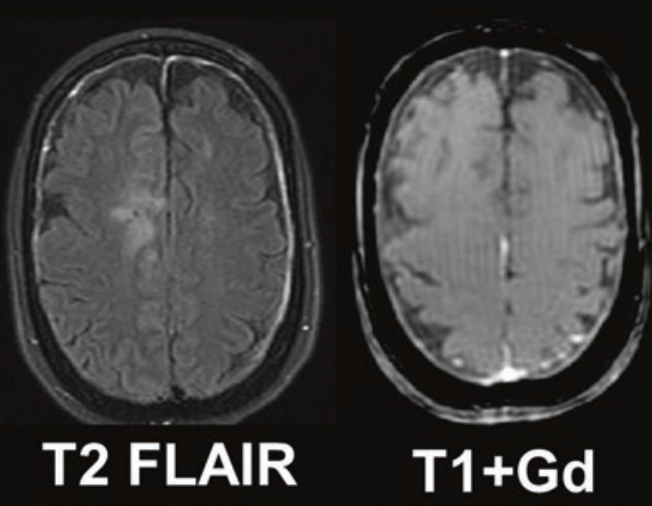

$\mathrm{T} 1+\mathrm{Gd}$

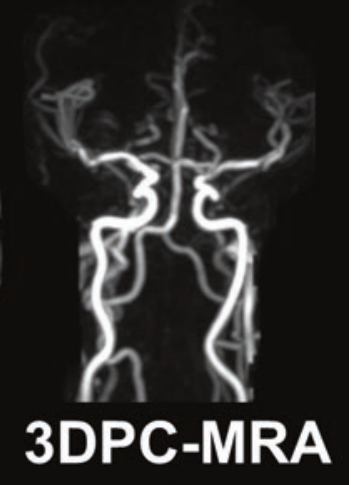

Fig. 3.4 Fast Stroke MRI Screening Protocol. This 64-year-old woman with right anterior cerebral artery stroke 5 days earlier was referred due to falls and worsening leg weakness. The screening protocol shown took $8 \mathrm{~min}$ of total room time, including, in order: sagittal T1 (30 s), DWI (30 s), and dynamic perfusion (75 s). Once completed these were sent to RAPID, with automated mismatch maps constructed (thresholded ADC and Tmax) which returned to PACS in about 3 min, while the post-contrast sequences finished up. Post-contrast images included SSFSE T2 (15 s), EPI-GRE T2* (25 s), 2D FLAIR (70 s), and a 3D phase contrast MRA sequence (130 s) using velocity encoding $50 \mathrm{~cm} / \mathrm{s}$ to capture large arterial or venous occlusions. The MRA sequence was

\subsection{Neurocognitive Approach to Acute Stroke Imaging: Thinking, Fast and Slow}

Whether CT or MRI is used, the time pressure and complexity of large stroke protocols is a challenge for radiologists and neurovascular specialists. In the rush to make key decisions, we run the risk of "having blinders on"-looking for just what we think should be there, and overlooking other stroke territories or mimics. Other members of the stroke team are typically focused on their own key portions of workflow, so it is the radiologist who must help see the big picture and make both an accurate diagnosis and recommendations. A disci- designed with large field of view to capture the carotid bifurcations in the neck, then post-processed to produce both targeted MIP vascular reconstructions from the phase components, and also nominally T1-weighted images in three planes from the magnitude components (only axial shown). There is an early subacute right ACA infarction without large vessel occlusion or significant penumbra (white arrows), as well as acute on chronic hemispheric subdural hematomas, right larger than left (red arrow). Careful construction of fast protocols can refine both stroke diagnosis and capture other significant lesions, including stroke mimics plined search pattern and brief pause at the time of reporting will add value and help achieve these goals. This philosophy is inspired by Daniel Kahneman's book "Thinking, Fast and Slow [17]." In our adaptation of Kahneman's construct, radiologists can harness their innate fast, reflexive thinking patterns to very quickly find and report the key findings of an exam, but they must also impose a slower, orderly approach to drive a less biased, more disciplined exam search pattern and conclusions. Quick action is needed in stroke evaluation but it must always be tempered by a (slightly) slower phase to find unexpected lesions and consider stroke mimics. This essentially boils down to: "Look for what you are supposed to look for-but don't forget about everything else!" 


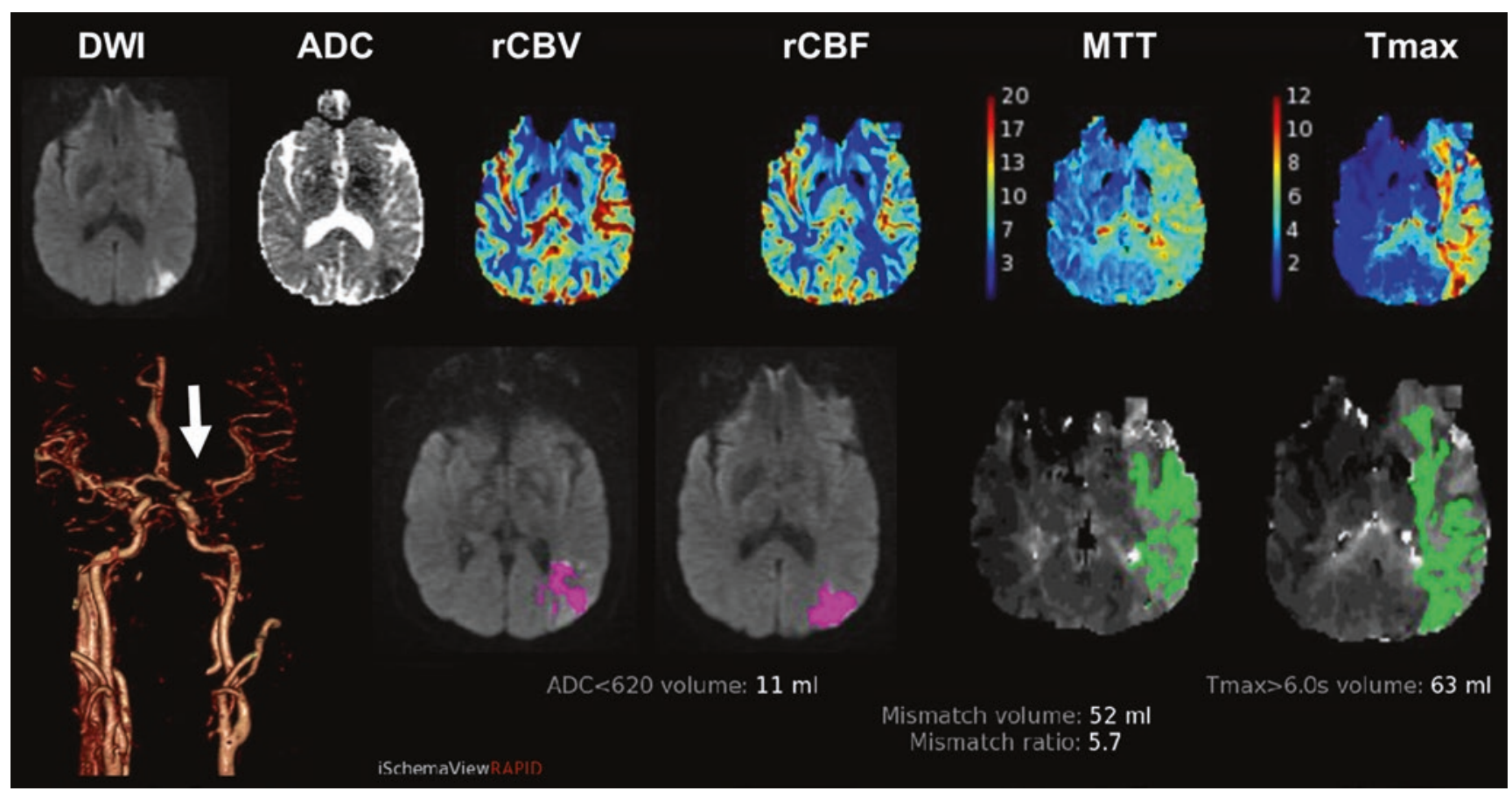

Fig. 3.5 MRI-based triage in a 46-year-old man with global aphasia and right hemiparesis for more than $4.5 \mathrm{~h}$. Upper row shows a small diffusion lesion with low ADC, with much larger perfusion defect by qualitative "eyeball" method. Lower panel shows left carotid terminus near-total occlusion on TRICKS MRA (arrow), with good access via patent cervical carotid. Quantitative thresholded maps made using
RAPID software show a favorable "target mismatch" pattern: a small estimated core infarct (ADC $<620=11 \mathrm{~mL}$, in pink) but large significant perfusion defect (Tmax $>6 \mathrm{~s}=63 \mathrm{~mL}$, in green), for an estimated mismatch volume/penumbra $=52 \mathrm{~mL}$. He underwent successful endovascular therapy and made a complete clinical recovery. (Reprinted with permission, Springer)

\section{Key Points}

Acute Stroke Imaging: Thinking, Fast and Slow

CT/MR exam analysis Search pattern $\rightarrow$ Findings

Fast Hemorrhage Ischemia/infarction Vascular occlusion Perfusion defect

Slow Size/extent/ASPECTS Core and penumbral pattern

Collateral status Non-MCA territories Atherosclerotic disease Second lesions

Stroke mimics

"Corner shot" incidentals Address clinical question Recheck history for clues
Report impression/ conclusion Summary $\rightarrow$ Synthesis Hemorrhage-present or not

Ischemia: location and extent

Vascular occlusion details, site(s) Mismatch and penumbral pattern if any

Differential diagnosis

- What else could it be?

- Pause and consider mimics by category

Mechanistic implications of pattern

Etiology and prevention of recurrent stroke (e.g., non-calcified plaque or

carotid web)

Recommendations

\subsection{Optimizing Imaging Workflow}

Imaging is increasingly integrated into the workup of an acute stroke case and it is critical to establish time efficient imaging protocols. Even though the treatment time windows have been extended for up to $24 \mathrm{~h}$, time remains a crucial variable for good clinical outcomes. A few key elements of an optimal workflow in acute stroke are highlighted below.

1. Ideally, it is important to notify the imaging team as soon as a stroke patient is identified (for example, using a "Code Stroke" pager), which can be during transportation or in the emergency department. Serial group pages or web-based applications serve to update the entire team simultaneously, following the patient's location, streamlining communication, and coordinating care during triage. Patients can be taken straight to CT from ambulance, bypassing the ER evaluation to minimize "door to needle' and "door to groin" times.

2. Imaging including CT angiogram and perfusion studies should not delay the administration of intravenous thrombolysis or EVT. A parallel workflow should be utilized; 
IV tPA can be started in the CT scanner for qualified patients after a non-contrast CT is obtained, as CTA/CTP is being obtained.

3. It is not necessary to delay contrast administration to check renal function. As per the AHA/ASA guidelines, vascular imaging can be obtained without creatinine/GFR check in patients without a history of renal impairment. There is now convincing evidence that in the absence of a history of renal impairment, there is a very low risk of transient, contrast-induced nephropathy.

4. Patient triage decisions are mostly made on the scanner console and hence it is important for the radiologist to be involved in quick interpretation, for example, a NCCT images or DWI sequences should be sent to PACS workstation without waiting for perfusion or angiographic images.

5. Automated perfusion imaging is becoming standard of care and can expedite the workflow. All recent EVT trials used automated software for post-processing of the perfusion studies giving volumetric core, penumbra, and mismatch according to methods used in clinical trials. Processing of the perfusion images on a separate workstation can produce delays along with user variability.

6. The use of automated ASPECTS and automated LVO detection using artificial intelligence (AI) algorithms is increasingly being used by stroke teams, requiring the current and future generation of radiologists to adapt to this new workflow. Multiple expanded uses of AI are in development for use in stroke triage (see below Key Points).

7. It is important for the radiologists to be at the helm for designing efficient workflow at their institution. It is equally important to work in close collaboration with the technologists and stroke team to organize this workflow.

\section{Key Points}

Stroke Imaging of the Future: Opportunities for Artificial Intelligence

- Robust communication systems optimizing regional triage/ambulance workflow and transfer decisions

- Data-driven individualized treatment options

- Radiology reading worklist prioritization based on ordering context, clinical status, and lesion detection algorithms

- Automated radiology exam screening/scoringASPECTS, large vessel occlusion detection, collateral scoring, volumetric core, and penumbral calculations

- Functional deficit risk predictions based on clinical features, lesion location, and volume (at triage and follow-up recovery phase)

\subsection{Stroke Mimics}

Stroke mimics or non-ischemic etiologies represent up to one-third of cases of new neurological deficits [18]. It is important to identify these mimics correctly to avoid unnecessary acute treatment. MRI has a distinct advantage in identifying stroke mimics and narrows the differential as compared to CT-based workup. Multiple etiologies can mimic acute or subacute ischemic stroke including vascular, metabolic, medication-related, infectious, inflammatory, neoplastic, and traumatic etiologies [19]. Common stroke mimics include seizures, migrainous aura, venous thrombosis, PRES, and neoplasms [20].

Seizures: Seizures are one of the most frequent stroke mimics, particularly in patients with presenting with Todd's paresis or postictal aphasia/dysphasia. Seizure related cortical signal abnormalities may have associated DWI abnormality. The distinguishing features from an arterial infarct include a nonvascular distribution, gyral or leptomeningeal enhancement, and absence of vascular occlusion. Signal changes are usually reversible but may progress to cortical laminar necrosis or focal atrophy. There may be mild hyperperfusion or a normal perfusion pattern in the epileptic region.

Migraine: An acute migrainous aura may mimic an acute stroke including motor symptoms (hemiplegic migraine). The neuroimaging is usually normal but uncommonly may show restricted diffusion. The perfusion abnormalities are frequent in these cases, usually with hypoperfusion at onset, sometimes followed by hyperperfusion. An important point to note is that usually more than one vascular territory is affected, in contrast to the hypoperfusion pattern of a single vascular territory in an acute infarct [21].

Neoplasms: Patients with neoplasms may present with sudden attacks of "stroke like" symptoms. This is important to recognize not only to avoid unnecessary thrombolytic treatment but also not to delay recognizing and managing a brain tumor. A common misinterpretation occurs when tumors are small, cortical, in an arterial distribution and have varying enhancement pattern (mimicking a subacute infarct-see Fig. 3.6). Furthermore, DWI can show varying signal characteristics depending on the cellularity of the tumor. Perfusion maybe be helpful especially if there is increased perfusion including increased CBV in a high-grade glioma (as compared to the expected low $\mathrm{CBV}$ in an acute infarct).

Venous infarction: Cerebral venous thrombosis is a lifethreatening neurological condition which should not be missed. A venous infarction has a different topography as compared to arterial infarct; distribution depends on the location of the venous thrombosis but usually lacks an arterial distribution. The DWI abnormality can be variable in a venous infarct. Venous infarcts can also demonstrate a flame shaped hemorrhage. Most of the CTA studies have an adequate venous opacification and evaluation of the 


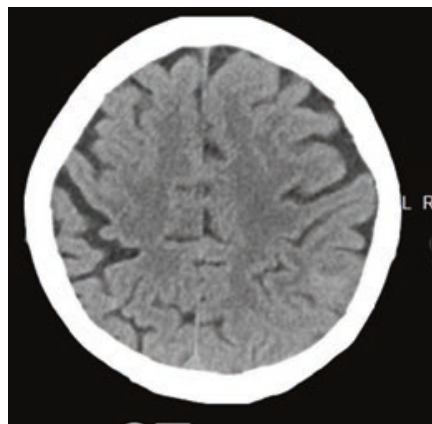

CT -

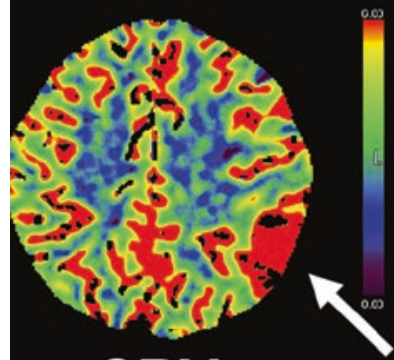

CBV
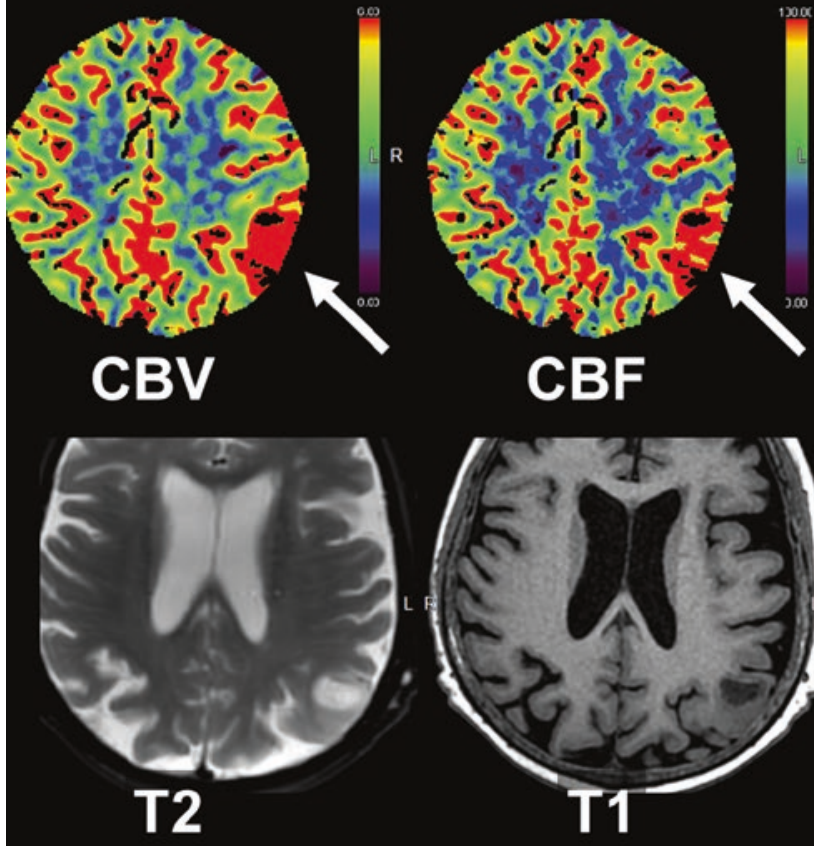

CBF

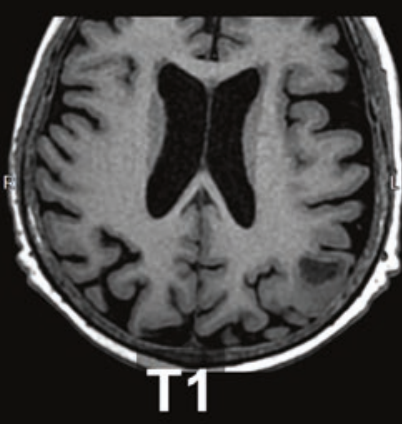

Fig. 3.6 Stroke mimic: tumor. This 83-year-old woman presented to an outside hospital with dysarthria. The emergency stroke CT protocol showed a focal left parietal lesion with curvilinear cortical enhancement and focal elevation of blood volume and blood flow (white arrows), with normal to fast perfusion transit times. This was inter-

venous sinuses should be part of the check list of a CTA interpretation (Fig. 3.7). MR can have variable signal depending on the age of the thrombus. Cortical venous thrombosis can present with focal convexity subarachnoid hemorrhage and is best seen on gradient-echo T2* or susceptibility-weighted sequences.

Posterior reversible encephalopathy syndrome (PRES): PRES is an under recognized entity in the ED with presenting symptoms of headaches, seizures, altered mental status, and visual changes or loss. It is a clinical radiological diagnosis characterized by transient failure of vascular autoregulation leading to multifocal vasogenic edema. Patients with malignant hypertension, eclampsia, on chemotherapy, or taking post-transplant drugs seem particularly susceptible. Typically, the PRES lesions are bilateral, cortical-subcortical, non-enhancing and involve predominantly the parietal-

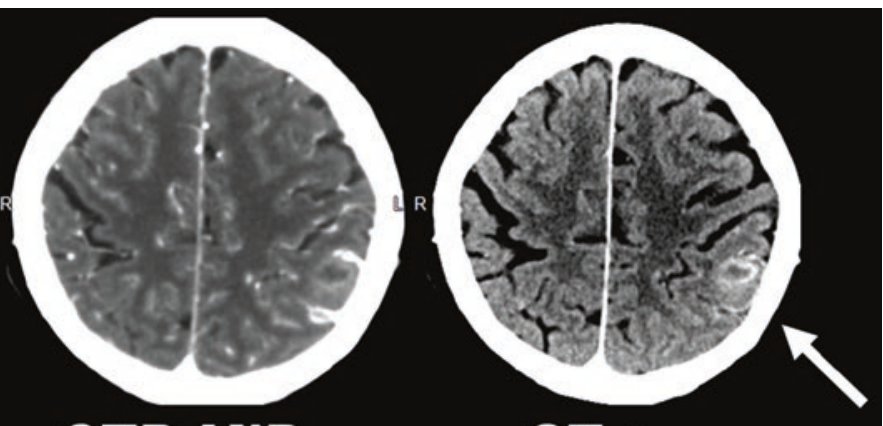

CTP-MIP
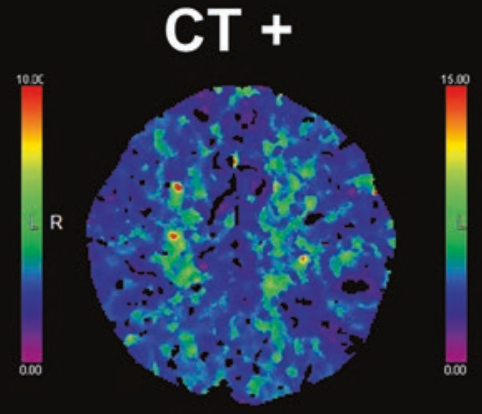

TTD

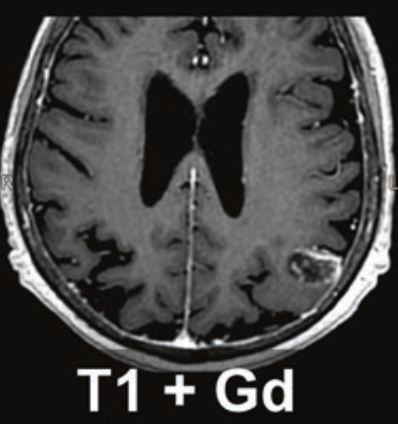

FLAIR + Gd preted as subacute infarction with gyral enhancement and "luxury perfusion." As symptoms progressed, a follow-up MRI 1 month later showed an expansile heterogeneously enhancing lesion, pathologically proven as glioblastoma

occipital regions. Uncommon PRES patterns include posterior fossa involvement, diffusion restriction, enhancement, and hemorrhage (Fig. 3.8).

Subdural hematoma: Patients with subacute to chronic subdural hematoma can present with clinical symptoms masquerading as stroke including confusion, ataxia, and hemiparesis. These are easy to recognize on CT and MRI given their characteristic imaging findings (Fig. 3.9).

Additional entities mimicking acute arterial ischemic stroke include infection (encephalitis, cerebritis, abscess, meningitis, sepsis), toxic-metabolic abnormalities (hypoglycemia, hepatic encephalopathy, medications, and illicit drugs), demyelinating disease, and MELAS (mitochondrial encephalopathy, lactic acidosis, and stroke-like events). Knowledge of these mimics is imperative to minimize incorrect or delayed diagnosis. 


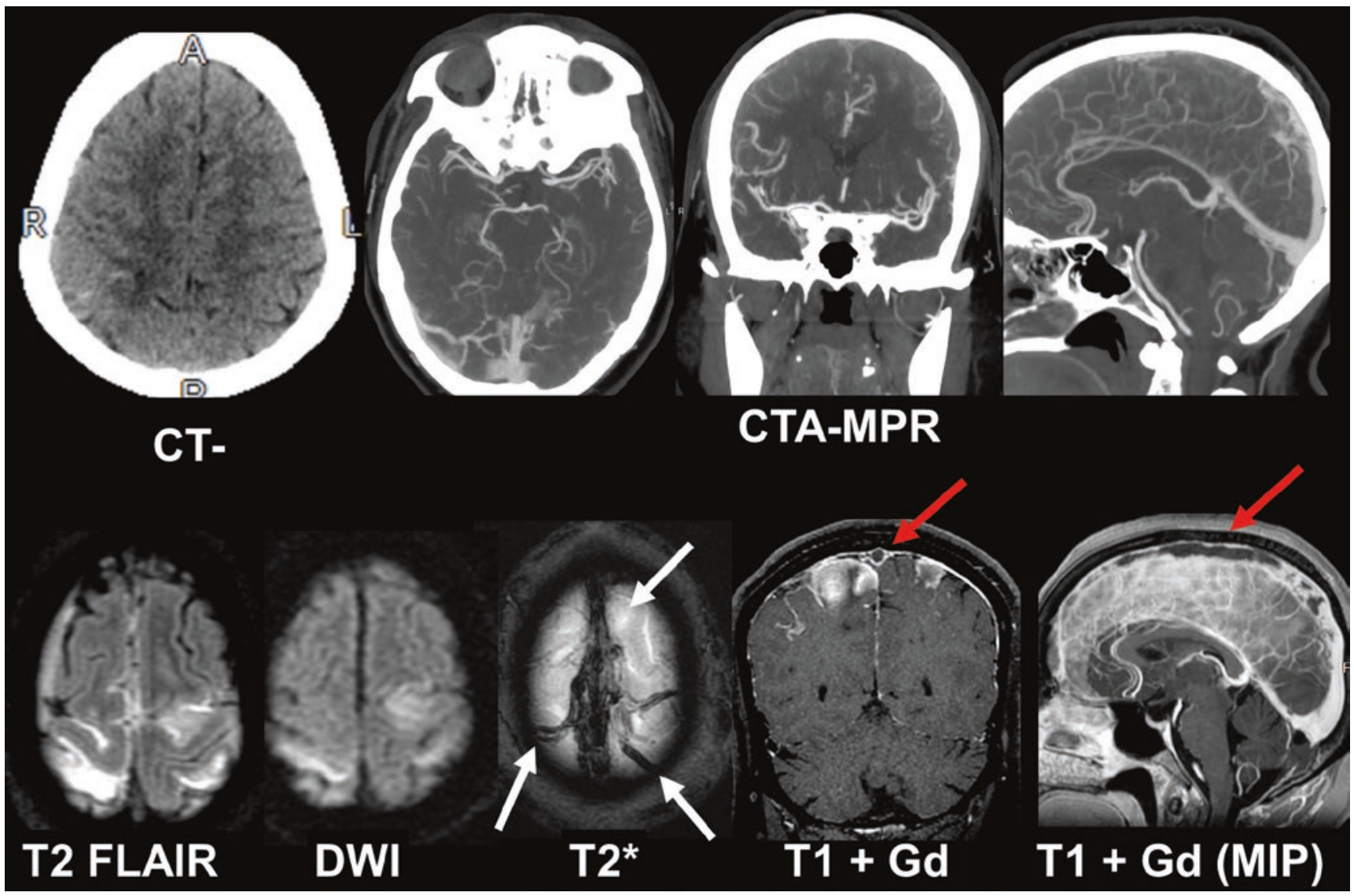

Fig. 3.7 Arterial stroke mimic: venous infarction. This 48-year-old woman with a 2-day history of headache presented with sudden left arm paresthesias. An emergency non-contrast CT shows heterogeneous density and swelling in the right parietal lobe, with linear intrasulcal density suggesting subarachnoid blood. CT perfusion was symmetrical and the CTA was initially interpreted as normal. She worsened and so was referred for MRI $6 \mathrm{~h}$ later (lower panel), showing signs of bilateral hemorrhagic venous infarction, thrombosed superior sagittal sinus, and cortical veins (white arrows), and corresponding dural sinus filling defects on post-contrast images (red arrows). In retrospect, an "empty delta" sign was clearly present in the sagittal sinus on the initial coronal and sagittal CTA reconstructions, but missed due to a focused review done in haste

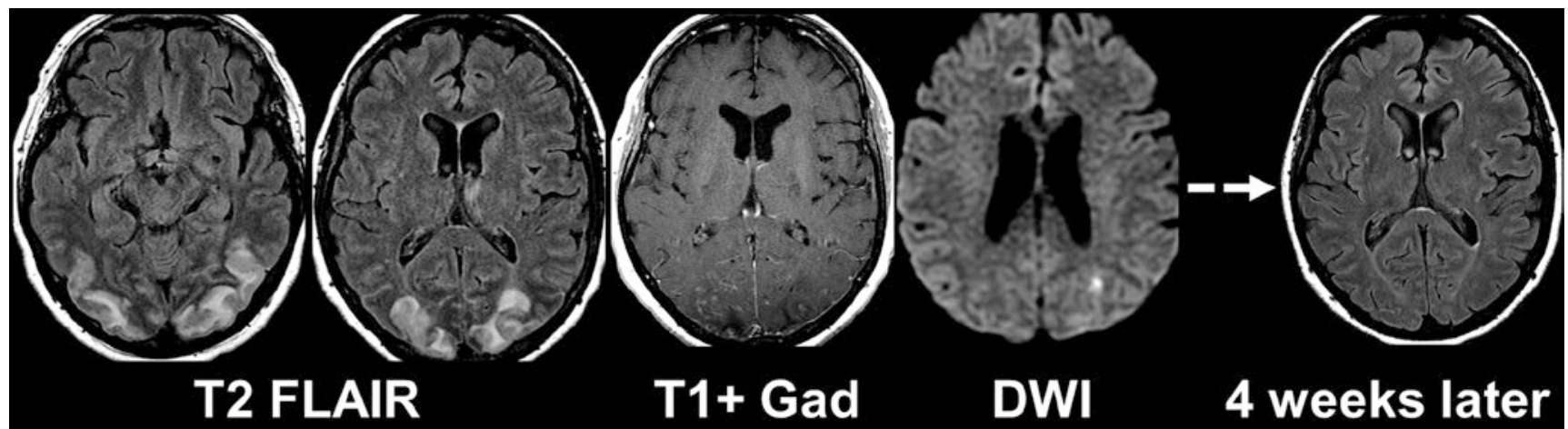

Fig. 3.8 Stroke mimic: PRES. This 55-year-old presented with acute headaches and visual disturbances. MRI demonstrates bilateral, symmetric signal abnormalities consistent with vasogenic edema in the parieto-occipital region, in a classic PRES-like pattern. There is lepto- meningeal congestion but no abnormal parenchymal enhancement, and only a small focus of diffusion restriction. Follow-up FLAIR at 4 weeks shows complete resolution of the signal abnormalities 


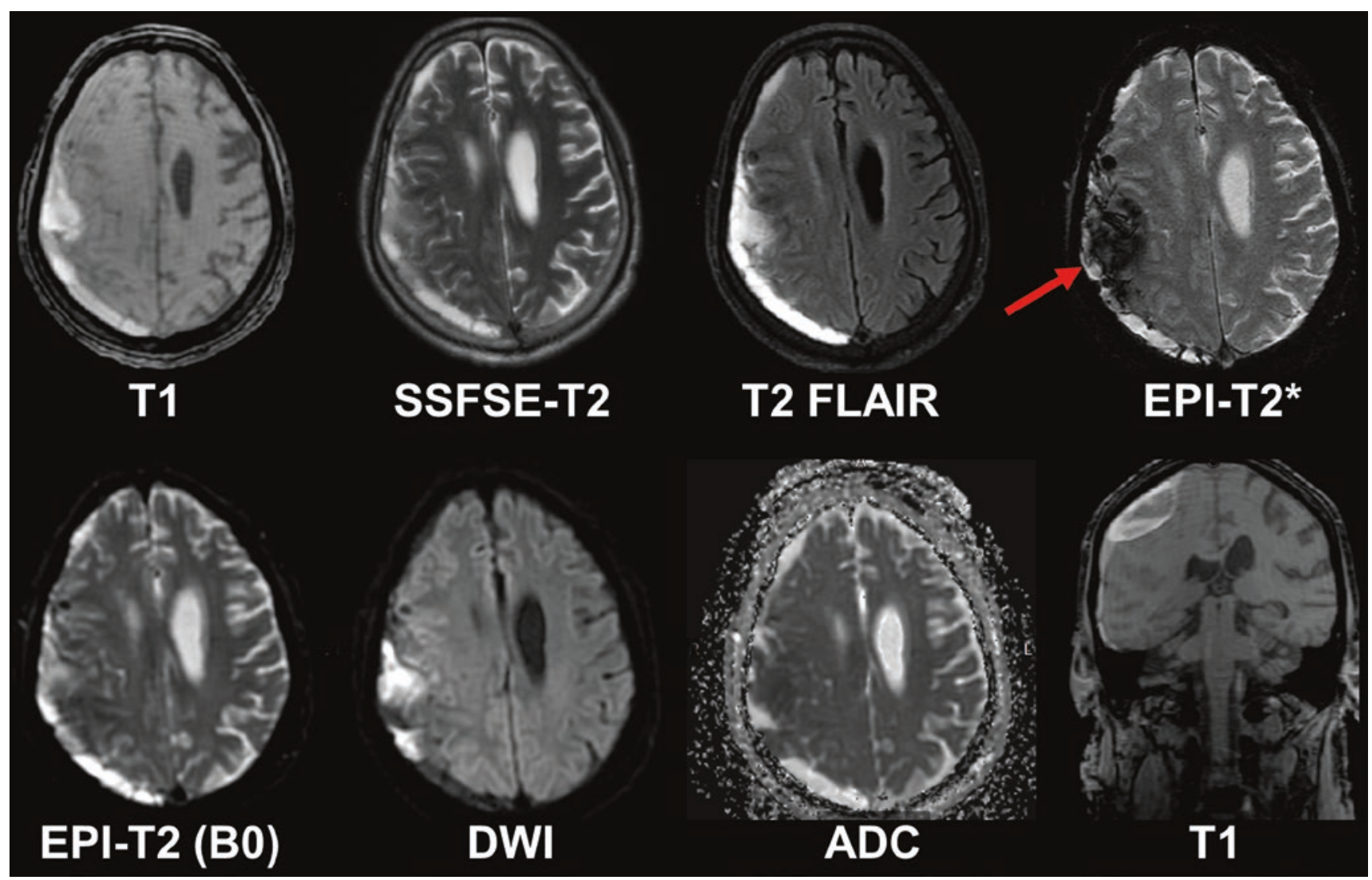

Fig. 3.9 Stroke mimic: subdural hematoma, diagnosed by fast screening MRI protocol. This 58-year-old man presented with abrupt left hemiparesis and confusion. A stroke code was called in the emergency department due to concern for right MCA occlusion. A non-contrast fast screening MRI protocol was obtained in $<7$ min of imaging time

\section{Take-Home Messages}

- Streamline and coordinate stroke triage across your facility

- Build fast yet comprehensive imaging protocols

- Identify extent of acute ischemia, large vessel occlusions, and mismatch/penumbral patterns if any

- Get the right diagnosis - distinguish true strokes from stroke mimics

\subsection{Concluding Remarks}

Rapid stroke imaging triage is challenging but is key to improving outcomes for patients with acute ischemic stroke. Radiologists are at the helm of the changing landscape of (including normal MRA-MRV, not shown). An unexpected right convexity subdural hematoma causes mass effect at the fronto-parietal junction. Predominantly T1 hyperintense signal is consistent with methemoglobin, with focal blooming on $\mathrm{T} 2 *$ (arrow) indicative of an acute intracellular component

acute stroke. Streamlined protocols, workflow, and analysis focus on getting the right diagnosis, adding value not just for acute stroke but also for stroke mimics.

\section{References}

1. Powers WJ, Rabinstein AA, Ackerson T, et al. 2018 guidelines for the early management of patients with acute ischemic stroke: a guideline for healthcare professionals from the American Heart Association/ American Stroke Association. Stroke. 2018;49(3):e46-e110.

2. Turc G, Bhogal P, Fischer U, et al. European stroke organisation (ESO)- European Society for Minimally Invasive Neurological Therapy (ESMINT) guidelines on mechanical thrombectomy in acute ischemic stroke. J Neurointerv Surg. 2019;11(6): $535-8$.

3. Berkhemer OA, Fransen PS, Beumer D, et al. A randomized trial of intraarterial treatment for acute ischemic stroke. N Engl J Med. 2015;372(1):11-20. 
4. Goyal M, Demchuk AM, Menon BK, et al. Randomized assessment of rapid endovascular treatment of ischemic stroke. N Engl J Med. 2015;372(11):1019-30.

5. Jovin TG, Chamorro A, Cobo E, et al. Thrombectomy within 8 hours after symptom onset in ischemic stroke. N Engl J Med. 2015;372(24):2296-306.

6. Saver JL, Goyal M, Bonafe A, et al. Stent-retriever thrombectomy after intravenous t-PA vs. t-PA alone in stroke. N Engl J Med. 2015;372(24):2285-95.

7. Campbell BC, Mitchell PJ, Kleinig TJ, et al. Endovascular therapy for ischemic stroke with perfusion-imaging selection. N Engl J Med. 2015;372(11):1009-18.

8. Nogueira RG, Jadhav AP, Haussen DC, et al. Thrombectomy 6 to 24 hours after stroke with a mismatch between deficit and infarct. N Engl J Med. 2018;378(1):11-21.

9. Albers GW, Marks MP, Kemp S, et al. Thrombectomy for stroke at 6 to 16 hours with selection by perfusion imaging. N Engl J Med. 2018;378(8):708-18.

10. Thomalla G, Simonsen CZ, Boutitie F, et al. MRI-guided thrombolysis for stroke with unknown time of onset. N Engl J Med. 2018;379(7):611-22.

11. Ma H, Campbell BCV, Parsons MW, et al. Thrombolysis guided by perfusion imaging up to 9 hours after onset of stroke. N Engl J Med. 2019;380(19):1795-803.

12. Rowley HA, Vilela P. Brain ischemia: CT and MRI techniques in acute stroke. In: Hodler J, Kubik-Huch RA, von Schulthess GK, editors. Diseases of the brain, head and neck, spine 2016-2019: diagnostic imaging. Cham: Springer International Publishing; 2016. p. 37-47.
13. Rowley HA. The four Ps of acute stroke imaging: parenchyma, pipes, perfusion, and penumbra. AJNR Am J Neuroradiol. 2001;22(4):599-601.

14. Lev MH, Farkas J, Rodriguez VR, et al. CT angiography in the rapid triage of patients with hyperacute stroke to intraarterial thrombolysis: accuracy in the detection of large vessel thrombus. J Comput Assist Tomogr. 2001;25(4):520-8.

15. Miteff F, Levi CR, Bateman GA, Spratt N, McElduff P, Parsons MW. The independent predictive utility of computed tomography angiographic collateral status in acute ischaemic stroke. Brain. 2009;132(Pt 8):2231-8.

16. Menon BK, Smith EE, Modi J, et al. Regional leptomeningeal score on $\mathrm{CT}$ angiography predicts clinical and imaging outcomes in patients with acute anterior circulation occlusions. AJNR Am J Neuroradiol. 2011;32(9):1640-5.

17. Kahneman D. Thinking, fast and slow. New York: Farrar, Straus and Giroux; 2011.

18. Merino JG, Luby M, Benson RT, et al. Predictors of acute stroke mimics in 8187 patients referred to a stroke service. J Stroke Cerebrovasc Dis. 2013;22(8):e397-403.

19. Liu X, Almast J, Ekholm S. Lesions masquerading as acute stroke. J Magn Reson Imaging. 2013;37(1):15-34.

20. Adam G, Ferrier M, Patsoura S, et al. Magnetic resonance imaging of arterial stroke mimics: a pictorial review. Insights Imaging. 2018;9(5):815-31.

21. Floery D, Vosko MR, Fellner FA, et al. Acute-onset migrainous aura mimicking acute stroke: MR perfusion imaging features. AJNR Am J Neuroradiol. 2012;33(8):1546-52.

Open Access This chapter is licensed under the terms of the Creative Commons Attribution 4.0 International License (http://creativecommons. org/licenses/by/4.0/), which permits use, sharing, adaptation, distribution and reproduction in any medium or format, as long as you give appropriate credit to the original author(s) and the source, provide a link to the Creative Commons license and indicate if changes were made.

The images or other third party material in this chapter are included in the chapter's Creative Commons license, unless indicated otherwise in a credit line to the material. If material is not included in the chapter's Creative Commons license and your intended use is not permitted by statutory regulation or exceeds the permitted use, you will need to obtain permission directly from the copyright holder. 


\title{
Cerebral Neoplasms
}

\author{
G. Fatterpekar and E. Knopp
}

\section{Learning Objectives}

- To familiarize radiologists with the revised classification of CNS tumors in terms of certain important mutation profiles including IDH (isocitrate dehydrogenase) mutation, 1p/19q-codeletion status, TP53 (tumor protein 53) mutation, BRAF mutation, and histone H3K27M-mutation and their influence on improving diagnostic accuracy, treatment strategies, and overall survival.

- To provide an overview of the imaging phenotypes for the different glioma genotypes.

\subsection{Introduction}

The World Health Organization (WHO) Classification of Tumors of Central Nervous System (CNS) provided an update in 2016 nearly 10 years after the 2007 version to help more systematically categorize brain tumors. The revised system for the first time uniquely included molecular and genetic parameters of the individual tumor types, in addition to the histological features. Accordingly, each tumor is now identified by both its phenotype (based on histology), and genotype (based on its molecular and genetic parameters) [1].

\subsection{Goals of the Revised Classification}

The goals are multi-fold: (1) To resolve some of the confusion created by classifying brain tumors based only on histology, (2) Provide greater diagnostic accuracy, (3) Aid better

G. Fatterpekar $(\bowtie)$

NYU Langone Medical Center, New York, NY, USA

e-mail: girish.fatterpekar@nyulangone.org

E. Knopp

Northport VA Medical Center, Northport, NY, USA treatment strategies, and (4) Allow for an improved assessment of the prognosis based on the specific tumor type.

\subsection{Background}

A classic example of uncertainty created in the past where tumors were classified based only on histology included the group "oligoastrocytoma." These were tumors which exhibited features of both oligodendroglioma and astrocytoma on histology, and were therefore lumped together as oligoastrocytomas [2-4]. Accordingly, their management was not definitive which in turn influenced their prognosis. Categorizing this tumor group based on the underlying $1 \mathrm{p} / 19 \mathrm{q}$ codeletion (genetic mutation) status allows them to be clearly distinguished almost always into either $(1 \mathrm{p} / 19 \mathrm{q}-$ codeleted) oligodendroglioma or (1p-19q-intact) astrocytoma [5-8]. Only a few tumors cannot be categorized into either group, and are known as oligoastrocytoma, NOS (not otherwise specified) $[1,9,10]$. This clear distinction allows more accurate diagnosis, which therefore influences more appropriate tumor specific treatment strategies, and a better predictor for the overall prognosis. Another perplexing prognostic feature was noted in terms of the overall survival of certain low grade gliomas, which surprisingly were noted to perform much worse than Grade III astrocytomas. This can now be explained based on their IDH-mutation status, with IDH-wildtype Grade I Gliomas performing much poorer than IDH-mutant Grade III astrocytomas [5, 6]. Thus, it is the mutation status which influences the overall prognosis much more than the histology.

Utilizing the background above, the discussion below will mention the salient features of how the Revised 2016 classification influences our understanding of brain tumors focusing on infiltrating gliomas in adults, gliomas in children, and the introduction of certain new tumor types. A description of all CNS tumor types included in the Revised 2016 Classification of CNS tumors is beyond the scope of this text. 


\subsection{Infiltrating Gliomas in Adults}

Several mutations are associated with infiltrating gliomas in adults. Of these, some of the important ones include IDH mutation, $1 \mathrm{p}-19 \mathrm{q}$ codeletion, and TP53 mutation status [11].

The primary deterministic mutation includes the IDHmutation status-presence suggests IDH-mutant, and absent an IDH-wildtype tumor [12].

\subsection{IDH-Mutant gliomas}

There are two types of IDH mutation, IDH1- and IDH2mutated tumors. An overwhelming majority of such tumors are IDH1 mutated. Less than 3\% of IDH-mutated tumors are exclusively IDH2-mutant tumors [13].

\subsubsection{Clinical Relevance and Prognosis}

IDH-mutant tumors are seen more commonly in the middleaged population (30-60 years of age), than IDH-wildtype tumors which are more frequently seen in the older population (>60-65 years of age). The overall survival of IDHmutant tumors is far better than IDH-wildtype tumors. In fact, as mentioned previously, low grade (grade I by histology) IDH-wildtype gliomas have an overall survival close to that of grade IV IDH-wildtype glioblastomas, but much worse than grade III IDH-mutant gliomas. It is the IDHmutation status which is the driving force in terms of overall prognosis, much more than the histological grade. Furthermore, even among the Grade IV glioblastomas, it has been noted that IDH-mutant glioblastomas have an overall survival much better than IDH-wildtype glioblastomas. Supporting this is the fact that most IDH-mutant glioblastomas are the secondary type, while most IDH-wildtype glioblastomas are the de novo or primary type [14].

It is a known fact in glioma surgery that wider the resection, better is the overall survival. Knowing preoperatively that the tumor is an IDH-mutant type can influence the surgeon for a more complete surgical resection, including the FLAIR signal abnormality surrounding the enhancing mass [15]. The radiologist by suggesting the genetic profile preoperatively can accordingly influence the extent of surgical resection.

\section{Key Point}

- IDH-mutant tumors, seen more commonly in the middle-aged population (third to sixth decade of life), have a far better overall survival than IDH-wildtype tumors, which are seen more commonly in the older patients ( $>60-65$ years of age).

\subsubsection{Radiological Features}

Both IDH1 and IDH2 mutations change the role of IDH in the citric acid cycle. This results in accumulation of 2-HG within tumor cells. 2-hydroxyglutarate (2-HG) can be detected on MR spectroscopy and is therefore considered to be the imaging hallmark of all IDH-mutant tumors [16]. However, reliable detection is challenging, and is possible only at some select centers with special MR spectroscopists on site [17-19].

It has been noted that most IDH-mutated tumors occur in a single lobe, frontal lobe being the most common, followed by temporal, parietal, and occipital lobes [20]. Most such tumors demonstrate a sharp margin and inhomogenous but mild contrast enhancement. In contrast, IDH-wildtype tumors are frequently multilobar in location. Preferred site involves the insula with extension into the adjacent temporal, frontal, and parietal lobes $[20,21]$. In terms of their morphological appearance, these IDHwildtype tumors demonstrate ill-defined margins with the adjacent brain. Necrosis and moderate to intense heterogenous, especially peripheral enhancement is seen in the IDH-wildtype tumors (Fig. 4.1). The presence of necrosis, more intense enhancement, and ill-defined margins suggests more oxygen demand, more robust neoangiogenesis, and infiltrative nature of the wildtype tumors than their IDH-mutant counterparts.

\subsection{1p/19q-Codeletion}

IDH-mutant gliomas can subsequently be classified into those which are $1 \mathrm{p} / 19 \mathrm{q}$-codeleted tumors or $1 \mathrm{p} / 19 \mathrm{q}$-intact tumors. Of these, those gliomas which are $1 \mathrm{p} / 19 \mathrm{q}$-codeleted are the oligodendrogliomas, while those which are $1 \mathrm{p} / 19 \mathrm{q}$-intact are astrocytomas. Astrocytomas typically also show TP53 mutation, a mutation which is never seen in oligodendrogliomas, another distinguishing feature that separates these two entities. As mentioned previously, this $1 \mathrm{p} / 19 \mathrm{q}$-codeleted status and TP53 mutation help clearly separate the confusing oligoastrocytoma group into either oligodendroglioma or astrocytoma (which was not possible based on histological features alone), which helps better manage these patients.

\section{Key Point}

- $1 \mathrm{p} / 19 \mathrm{q}$-codeletion status in an IDH-mutant tumor is diagnostic of oligodendroglioma; $1 \mathrm{p} / 19 \mathrm{q}$-intact status with TP53 mutation is diagnostic of astrocytoma.

\subsubsection{Clinical Relevance and Prognosis}

It has been shown in two large randomized control trials that chemotherapeutic agents including procarbazine, lomustine, 

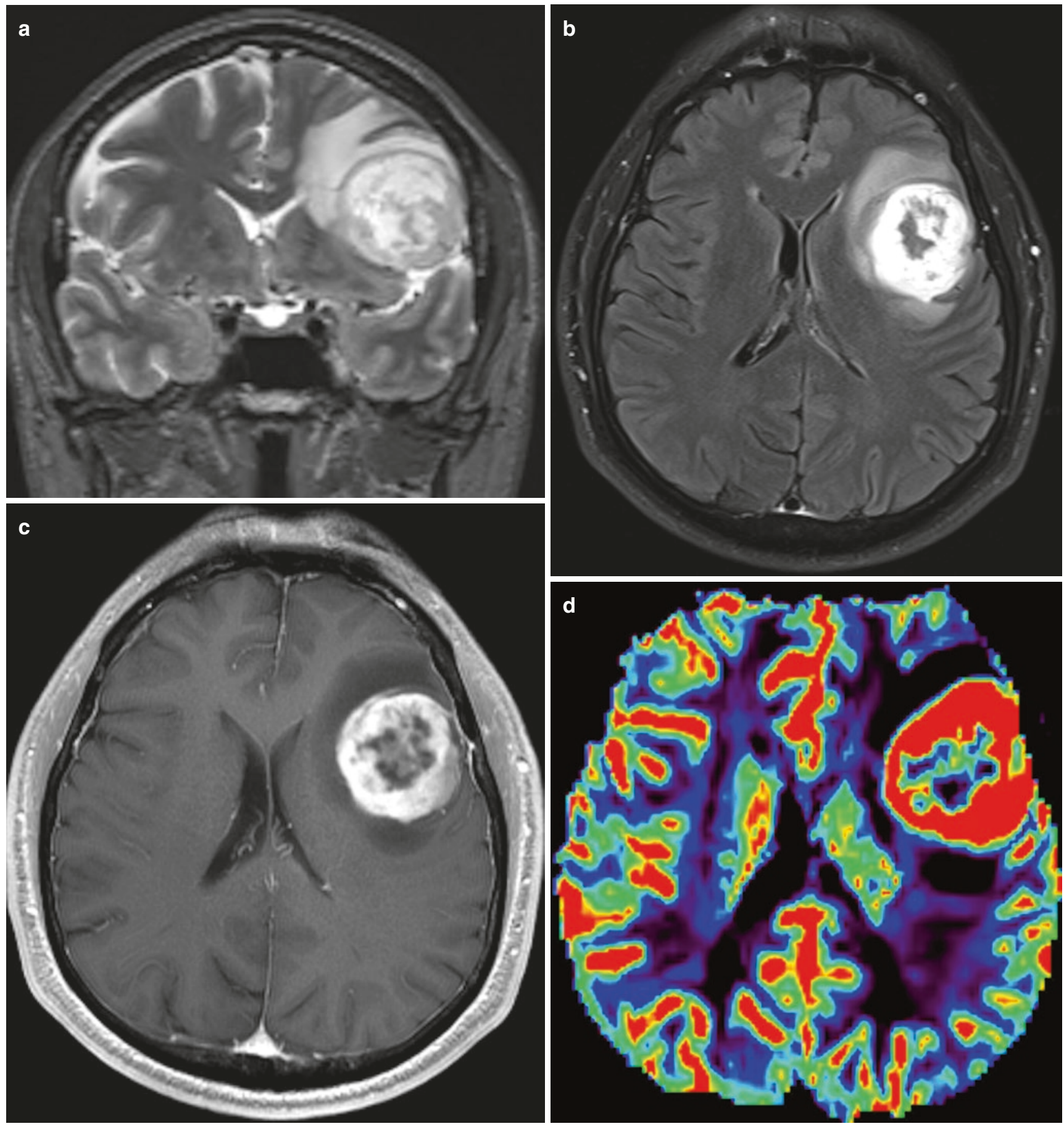

Fig. 4.1 A 69-year-old male with change in mental status. (a) Coronal T2WI demonstrates a heterogenous centrally necrotic mass in the left insular region extending to involve the frontal lobe. (b) Axial FLAIR image demonstrates FLAIR signal abnormality surrounding this lesion which shows indistinct margin with the adjacent brain. (c) Axial T1

post-contrast image demonstrates heterogenous but predominantly peripheral intense enhancement. (d) Corresponding axial DSC (dynamic susceptibility contrast) perfusion map demonstrates increased relative blood volume from the enhancing component of this lesion. Diagnosis: IDH-wildtype glioblastoma

and vincristine (PCV) when added to radiation therapy significantly improve the overall survival in patients with $1 \mathrm{p} / 19 \mathrm{q}$-deleted tumors when compared with radiation therapy alone $[22,23]$. This is now the standard of care for all $1 \mathrm{p} / 19 \mathrm{q}$-codeleted oligodendrogliomas.

\subsubsection{Radiological Features}

Frontal lobe is the most common location of $1 \mathrm{p} / 19 \mathrm{q}-$ codeleted tumors. Other common sites include the parietal and occipital lobes. In contrast, 1p/19q-intact tumors are 
seen most often in the temporal lobes and the insular cortex. In terms of their morphological appearance, $1 \mathrm{p} / 19 \mathrm{q}-$ codeleted tumors demonstrate a more heterogenous appearance. Also, calcification is a common feature of such tumors (Fig. 4.2). In fact, presence of florid calcification and enhancement favors a higher grade (Grade III) oligodendro-
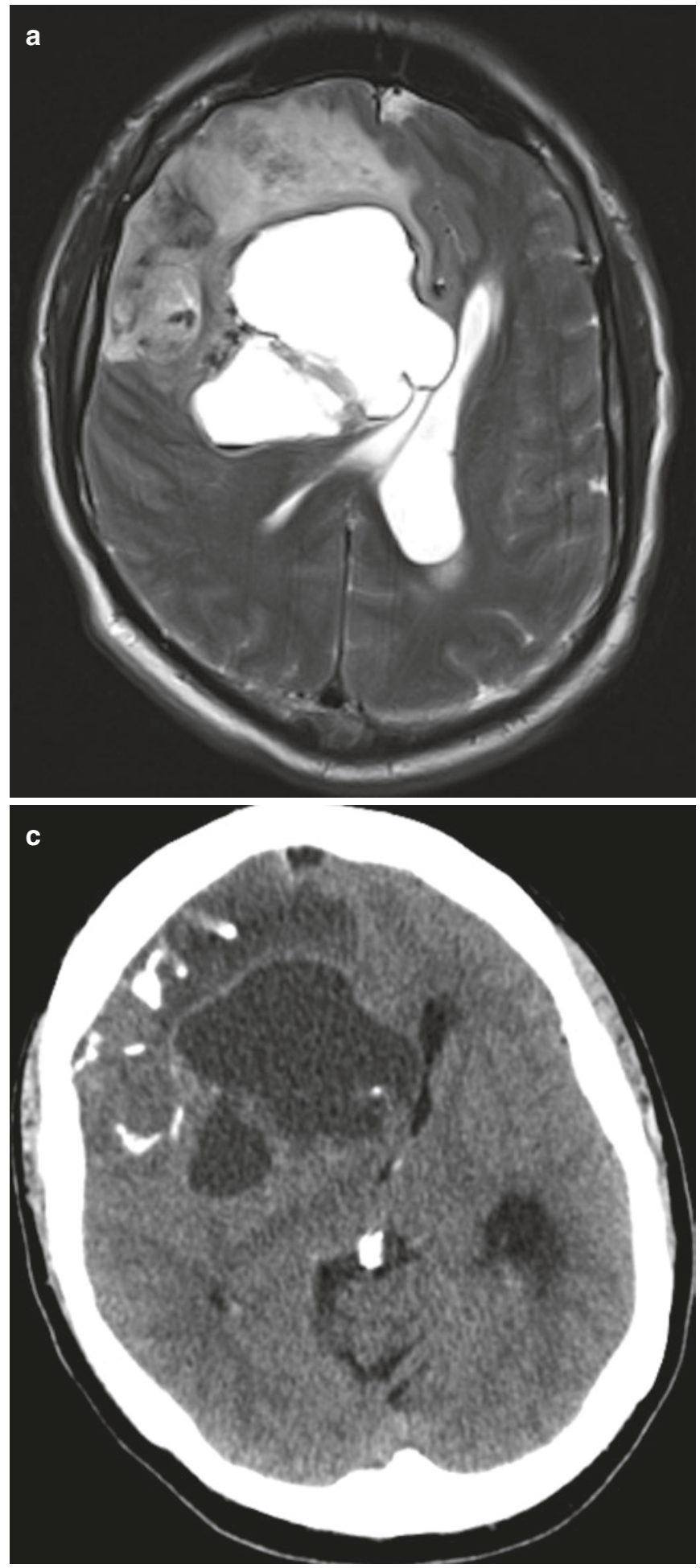

Fig. 4.2 A 48-year-old man with seizures. (a) Axial T2WI demonstrates a heterogenous mass involving the right frontal lobe. (b) Corresponding axial $\mathrm{T} 1$ post-contrast image demonstrates heteroge- glioma [24, 25]. An intact margin favors a 1p/19q-intact tumor, while ill-defined margins can be seen in both types. T2-FLAIR mismatch sign corresponding to a predominantly hyperintense signal of the mass on T2WI and corresponding hypointense signal on FLAIR has a high positive predictive value for 1p/19q-intact tumors (Fig. 4.3) [26].

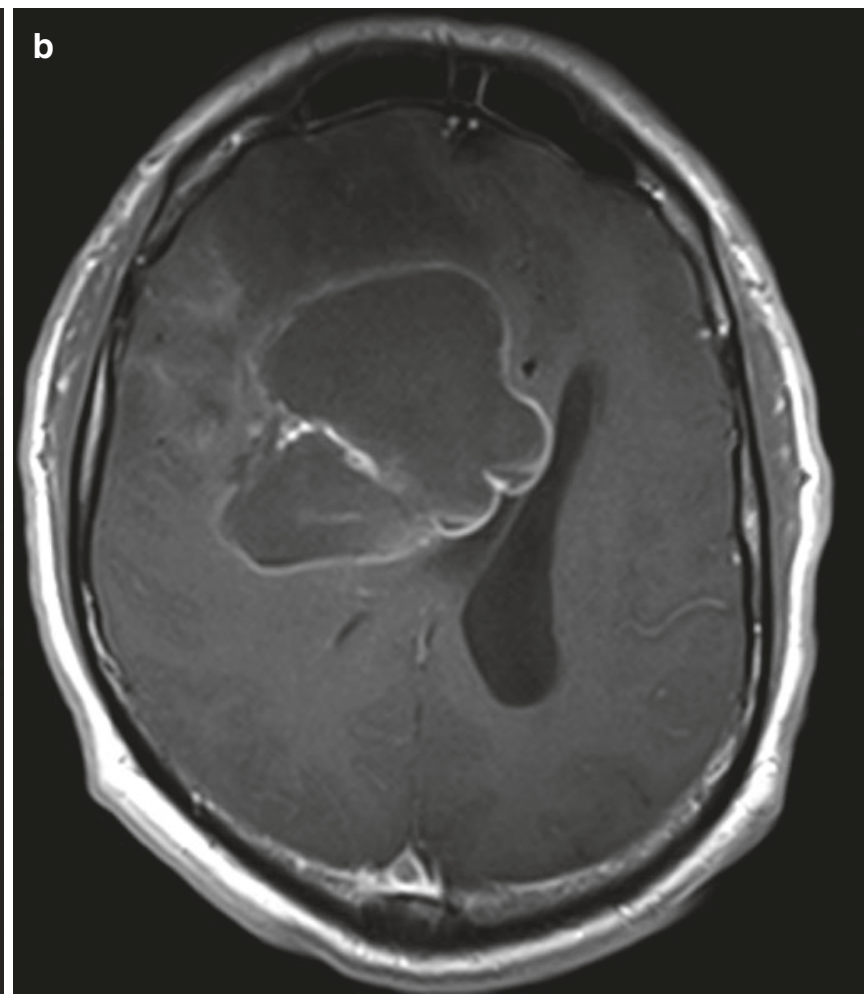

nous but minimal enhancement. (c) Axial CT scan from the same patient demonstrates multiple arcs of calcification within this mass. Diagnosis: Oligodendroglioma, IDH-mutant, 1p/19a codeleted tumor 


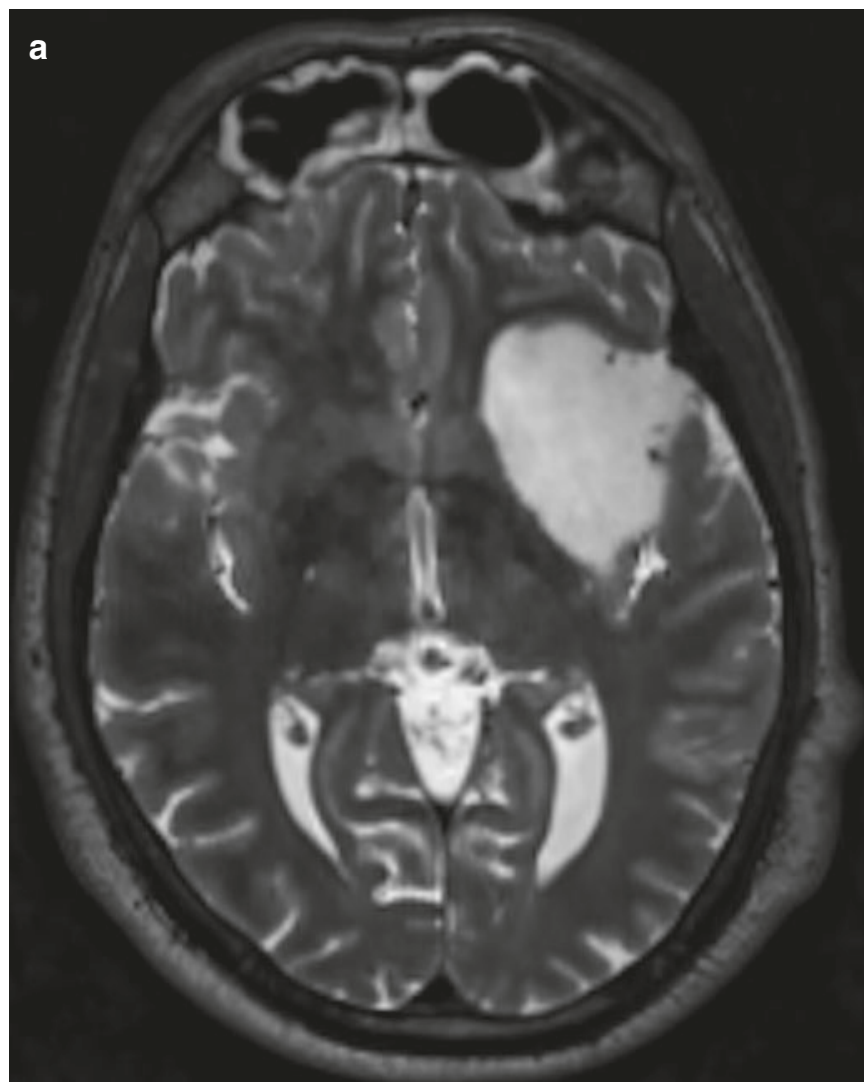

Fig. 4.3 A 34-year-old man with headache. (a) Axial T2WI demonstrates a well-defined expansile mass involving the left insula, which appears predominantly bright in its signal intensity when compared to

\subsection{Gliomatosis Cerebri}

Gliomatosis cerebri as a specific tumor subtype was included in the 2007 version of the WHO Classification of CNS tumors. This term is deleted from the 2016 Update. A diffusely infiltrating non-enhancing tumor extending to involve three or more lobes is no longer to be considered as gliomatosis cerebri. It is recognized as a diffuse glioma type, with its subtype dependent on further genetic, molecular testing and histological evaluation [1].

\section{Key Point}

- Gliomatosis cerebri as a tumor type is no longer recognized.

\subsubsection{Gliomas in Children}

Gliomas in children have been known to behave differently than those seen in the adult population. This is related to the fact that mutations seen commonly in gliomas in adults including IDH mutation and $1 \mathrm{p} / 19 \mathrm{q}$-codeletion occur only uncommonly in children [27]. In fact, the two common

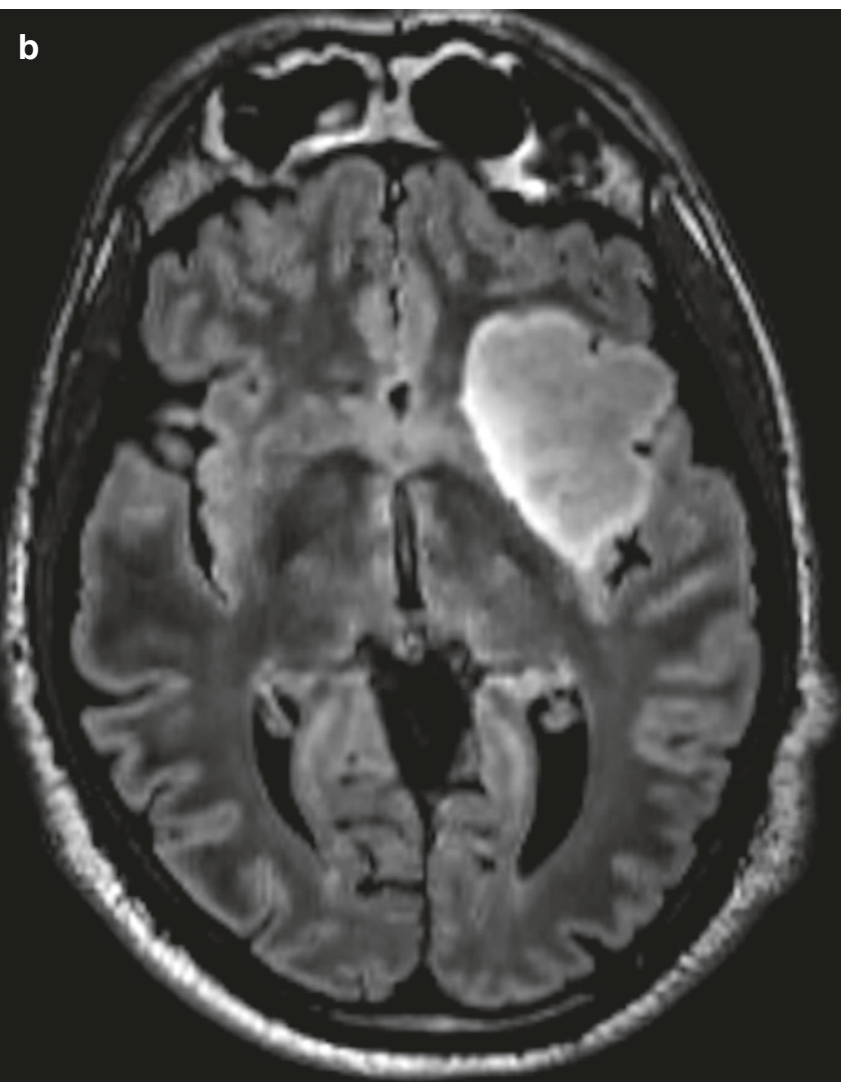

the gray matter. (b) Corresponding axial FLAIR image demonstrates the mass to be predominantly hypointense to the gray matter. Diagnosis: Diffuse astrocytoma, IDH-mutant, 1p/19q-noncodeleted (intact) tumor

mutation types commonly seen in children, include BRAF mutation and histone $\mathrm{H} 3-\mathrm{K} 27$ m-mutation.

\subsection{BRAF Mutation}

These tumors are usually well-circumscribed and carry an excellent prognosis.

\subsection{Radiological Features}

Cystic lesion with a mural enhancing nodule are common imaging features (Fig. 4.4). This mutation type encompasses pilocytic astrocytoma, pilomyxoid astrocytoma, and ganglioglioma $[5,11]$.

\section{Key Point}

- BRAF mutation is one of the most common mutations seen in the pediatric population, and includes tumor types such as pilocytic astrocytoma, pilomyxoid astrocytoma, and ganglioglioma. 


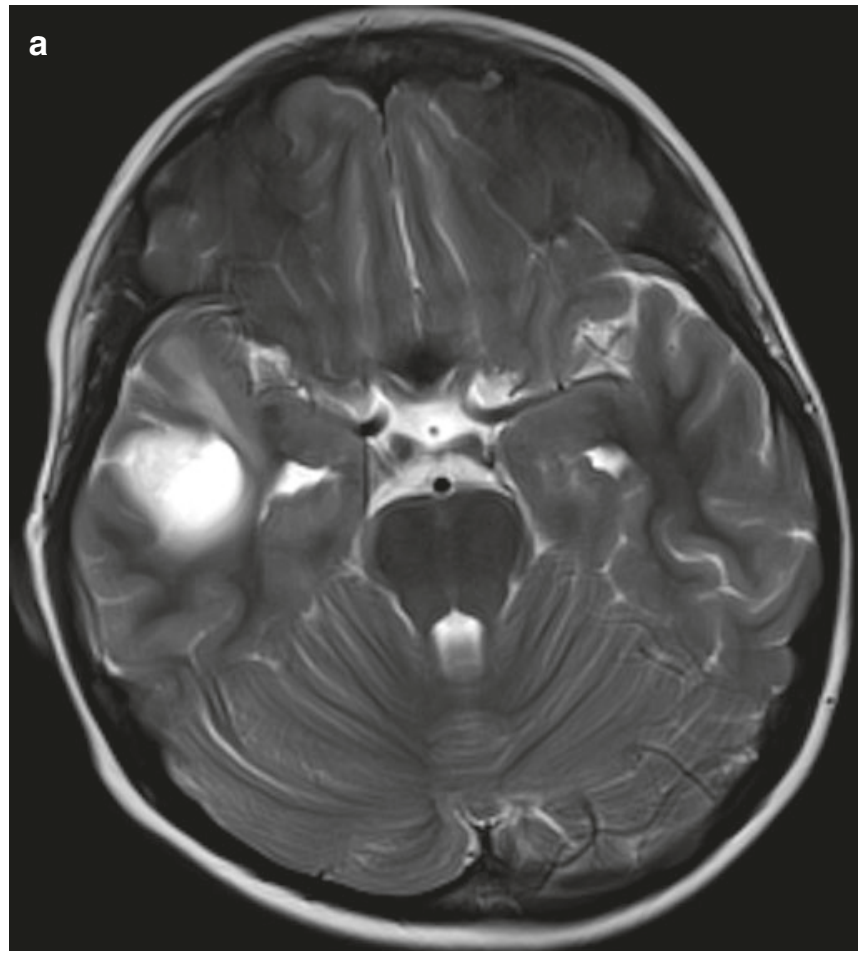

Fig. 4.4 A 18-year-old boy with seizures. (a) Axial T2WI demonstrates a well-defined cystic appearing lesion in the right temporal lobe. (b) Corresponding axial $\mathrm{T} 1$ post-contrast image demonstrates a mural

\subsection{Histone H3-K27m-Mutant Tumors}

These are diffuse midline gliomas (previously known as diffuse infiltrating pontine glioma) and carry an extremely poor prognosis. Location of this tumor type makes it difficult to biopsy these tumors or attempt a surgical resection [28, 29]. Radiation and chemotherapy are not particularly helpful.

\subsubsection{Radiological Features}

Brainstem (pons) is the most common location. Other common locations include thalami and spinal cord. As previously described this is a diffusely infiltrating lesion which results in secondary expansion of the structure involved. Only minimal enhancement is typically seen. Occasionally however, heterogenous enhancement and cyst(s) can be seen [30]. Leptomeningeal dissemination is seen in about one-third of all such patients.

\section{Key Point}

- H3K27M-mutant glioma now includes the previously known diffuse infiltrating pontine glioma, and carries a dismal prognosis.

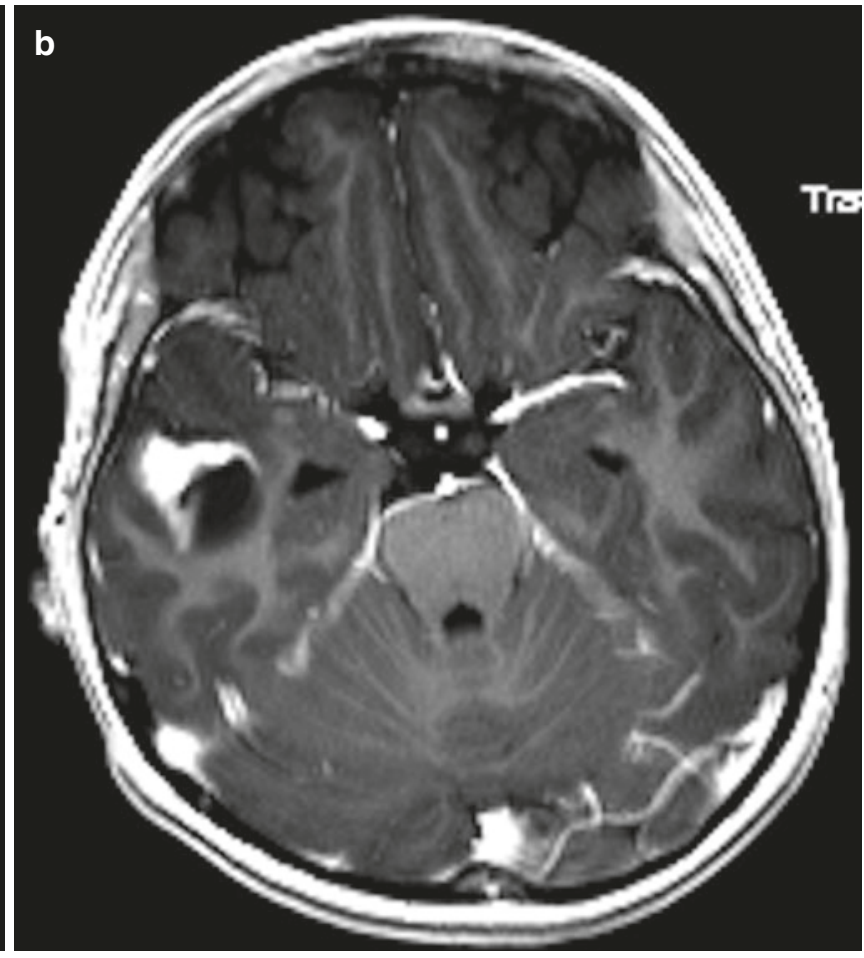

enhancing nodule along the lateral aspect of this lesion. Diagnosis: Pleomorphic xanthoastrocytoma, BRAF-mutant tumor

\subsection{Solitary Fibrous Tumor (SFT) and Hemangiopericytoma (HPC)}

Both these tumors share the same genetic feature which includes genomic inversion at the $12 q 13$ locus, fusing the NAB2 and STAT6 genes. Hence, these two previously distinct tumors are now combined as SFT/HPC tumor. Of these, the SFT/HPC Grade I is a slowly growing tumor with excellent prognosis, while SFT/HPC Grade II and III have a slightly poor prognosis, carry a high risk to recur following resection, and are associated with metastasis [27].

\subsection{New Tumors and Patterns}

\subsubsection{Multinodular and Vacuolating Neuronal Tumor}

This rare entity has received mention in the 2016 Revised CNS Tumor Classification. It is unclear if it is a distinct tumor or in the tumor-dysplasia category. It carries an excellent prognosis and is believed to be a "Leave-me-alone" lesion. 


\subsubsection{Radiological Features}

It is known to occur anywhere in the brain, but commonly in the supratentorial compartment and especially in the frontal and temporal lobes. On morphological appearance, the lesion is seen as a cluster of FLAIR and T2 bright lesions typically in the subcortical white matter. Involvement of the overlying cortex and periventricular white matter has been reported. The lesion appears dark on T1WI and does not demonstrate contrast enhancement or diffusion restriction. No susceptibility is seen [31].

\subsection{Diffuse Leptomeningeal Glioneuronal Tumor}

This is a rare glioneuronal neoplasm mainly seen in children. It is largely localized to the leptomeningeal compartment. Oligodendroglioma like tumor cells are seen on histology.

\subsubsection{Radiological Features}

Diffuse, somewhat heterogenous leptomeningeal enhancement is noted. Frequently the basal cisterns are involved with associated extensive involvement of the subarachnoid space along the surface of the cord. Secondary hydrocephalus is commonly noted [32]. Parenchymal involvement can also be seen. When present, it is seen to involve the spinal cord and the brain stem.

\subsection{Conclusion}

The Revised 2016 classification of CNS tumors by including the genetic profile improves diagnostic accuracy of brain tumors. This allows neuro-oncologists and the surgeons to optimize treatment strategies targeted to the specific tumor type, thus allowing for a better prognosis, and improved overall survival. The neuroradiologist by identifying the imaging phenotype of the particular glioma genotype plays an important role in guiding the clinical team in their treatment planning.

\section{Take Home Messages}

- IDH-mutated tumors are more solid in their imaging profile, and demonstrate less enhancement than IDH-wildtype counterparts.

- $1 \mathrm{p} / 19 \mathrm{q}$-codeleted tumors are more heterogenous in their imaging appearance, and exhibit calcification more frequently than their $1 \mathrm{p} / 19 \mathrm{q}$-intact (noncodeleted) counterparts.

- BRAF-mutant tumors are seen more commonly in the pediatric population. These include pilocytic astroctyoma, pilomyxoid astrocytoma, and ganglioglioma in their molecular profile spectrum.

- H3K27M-mutant tumors are also seen more commonly in the pediatric population, but are more diffuse and aggressive. These mutant tumors encompass the previously described diffuse infiltrating pontine glioma spectrum of tumors.

\section{References}

1. Louis DN, et al. The 2016 World Health Organization classification of tumors of the central nervous system. Acta Neuropathol. 2016;131(6):803-20.

2. Louis DN, et al. The 2007 WHO classification of tumours of the central nervous system. Acta Neuropathol. 2007;114(2):97-109.

3. Giannini C, et al. Oligodendrogliomas: reproducibility and prognostic value of histologic diagnosis and grading. J Neuropathol Exp Neurol. 2001;60:248-62.

4. van den Bent MJ. Interobserver variation of the histopathological diagnosis in clinical trials on glioma: a clinician's perspective. Acta Neuropathol. 2010;120:297-304.

5. Brat DJ, Cancer genome atlas research network, et al. Comprehensive integrative genomic analysis of diffuse lower-grade gliomas. N Engl J Med. 2015;372:2481-98.

6. Metellus P, et al. Absence of IDH mutation identifies a novel radiologic and molecular subtype of WHO grade II gliomas with dismal prognosis. Acta Neuropathol. 2010;120(6):719-29.

7. Sahm F, et al. Farewell to oligoastrocytoma: in situ molecular genetics favor classification as either oligodendroglioma or astrocytoma. Acta Neuropathol. 2014;128:551-9.

8. Wiestler B, et al. Integrated DNA methylation and copy-number profiling identify three clinically and biologically relevant groups of anaplastic glioma. Acta Neuropathol. 2014;128:561-71.

9. Huse JT, et al. Mixed glioma with molecular features of composite oligodendroglioma and astrocytoma: a true "oligoastrocytoma"? Acta Neuropathol. 2015;129:151-3.

10. Wilcox P, et al. Oligoastrocytomas:throwing the baby out with the bathwater? Acta Neuropathol. 2015;129:147-9.

11. Appin CL, et al. Molecular pathways in gliomagenesis and their relevance to neuropathologic diagnosis. Adv Anat Pathol. 2015;22(1):50-8.

12. Cohen AL, et al. IDH1 and IDH2 mutations in gliomas. Curr Neurol Neurosci Rep. 2013;13(5):345.

13. Smits $\mathrm{M}$, et al. Imaging correlates of adult glioma genotypes. Radiology. 2017;284:316-31.

14. Yan H, et al. IDH1 and IDH2 mutations in gliomas. N Engl J Med. 2009;360(8):765-73.

15. Beiko J, et al. IDH1 mutant malignant astrocytomas are more amenable to surgical resection and have a survival benefit associated with maximal surgical resection. Neuro-Oncology. 2014;16(1): 81-91.

16. Esmaeili M, et al. 2-hydroxyglutarate as a magnetic resonance biomarker for glioma subtyping. Transl Oncol. 2013;6(2):92-8.

17. Andronesi OC, et al. Detection of 2-hydroxyglutarate in IDHmutated glioma patients by in vivo spectral-editing and 2D cor- 
relation magnetic resonance spectroscopy. Sci Transl Med. 2012;4(116):116ra4.

18. Bertolino N, et al. Accuracy of 2-hydroxyglutarate quantification by short-echo proton-MRS at 3T:a phantom study. Phys Med. 2014;30(6):702-7.

19. Pope WB, et al. Non-invasive detection of 2-hydroxyglutarate and other metabolites in IDH1 mutant glioma patients using magnetic resonance spectroscopy. J Neurosci. 2012;107(1):197.

20. Wang Y, et al. Anatomical localization of isocitrate dehydrogenase 1 mutation: a voxel-based radiographic study of 146 low-grade gliomas. Eur J Neurol. 2015;22(2):348-54.

21. Smits $\mathrm{M}$, et al. Imaging of oligodendrogliioma. $\mathrm{Br} \mathrm{J}$ Radiol. 2016;89(1060):20150857.

22. van den Bent MJ, et al. Adjuvant procarbazine, lomustine, and vincristine chemotherapy in newly diagnosed anaplastic oligodendroglioma: long-term follow up on EORTC brain tumor group study 26951. J Clin Oncol. 2013;31(3):344-50.

23. Cairncross G, et al. Phase III trial of chemotherapy for anaplastic oligodendroglioma: long-term results of RTOG 9401. J Clin Oncol. 2013;31(3):337-43.

24. van den Bent MJ, et al. Diffuse infiltrating oligodendroglioma and astrocytoma. J Clin Oncol. 2017;35(21):2394-401.

25. Khalid L, et al. Imaging characteristics of oligodendrogliomas that predict grade. AJNR Am J Neuroradiol. 2012;33(5):852-7.
26. Patel SH, et al. T2-FLAIR mismatch, an imaging biomarker for IDH and $1 \mathrm{p} / 19 \mathrm{q}$ status in lower-grade gliomas: a TCGA/TCIA project. Clin Cancer Res. 2017;23(20):6078-85.

27. Johnson DR, et al. 2016 updates to the WHO brain tumor classification system: what the radiologist needs to know. Radiographics. 2017;37:2164-80.

28. Khuong-Quang DA, et al. K27M mutation in histone H3.3 defines clinically and biologically distinct subgroups of pediatric diffuse intrinsic pontine gliomas. Acta Neuropathol. 2012;124(3):439-47.

29. Solomon DA, et al. Diffuse midline gliomas with histone H3-K27M mutation: a series of 47 cases assessing the spectrum of morphologic variation and associated genetic alterations. Brain Pathol. 2016;26(5):569-80.

30. Aboian MS, et al. Imaging characteristics of pediatric diffuse midline gliomas with histone H3K27M mutation. AJNR Am J Neuroradiol. 2017;38(4):795-800.

31. Nunes RH, et al. Multinodular and vacuolating neuronal tumor of the cerebrum: a new "leave me alone" lesion with a characteristic imaging pattern. AJNR Am J Neuroradiol. 2017;38(10):1899-904.

32. Preuss M, et al. Disseminated oligodendroglial-like leptomeningeal tumors: preliminary diagnostic and therapeutic results for a novel tumor entity. J Neuro-Oncol. 2015;124(1):65-74.

Open Access This chapter is licensed under the terms of the Creative Commons Attribution 4.0 International License (http://creativecommons. org/licenses/by/4.0/), which permits use, sharing, adaptation, distribution and reproduction in any medium or format, as long as you give appropriate credit to the original author(s) and the source, provide a link to the Creative Commons license and indicate if changes were made.

The images or other third party material in this chapter are included in the chapter's Creative Commons license, unless indicated otherwise in a credit line to the material. If material is not included in the chapter's Creative Commons license and your intended use is not permitted by statutory regulation or exceeds the permitted use, you will need to obtain permission directly from the copyright holder. 


\title{
Nontraumatic Intracranial Hemorrhage
}

\author{
Pedro Vilela and Martin Wiesmann
}

\section{Learning Objectives}

- To understand typical imaging characteristics of intracranial hemorrhage in CT and MR imaging

- To know the most likely differential diagnosis of pathologies responsible for the hemorrhage with reference to anatomical location and patient characteristics

- To know how to triage patients suffering from an intracranial hemorrhage for further imaging and therapy

\subsection{Introduction}

Stroke is a major global health problem with different expressions among the globe but with a significant impact in the countries. The global prevalence of stroke varies from 339 to 1184 per 100,000, being higher among the more developed countries that face the problem of aging of the population. The prevalence of hemorrhagic strokes also varies among countries from 32-270 per 100,000 [1].

Spontaneous intracranial hemorrhage (IH) accounts for up to $10-15 \%$ of all strokes and is an important cause of neurological morbidity and mortality. The intracranial hemorrhage incidence is estimated in 10-30/100,000, increasing with age (doubles each decade after 35 years of age) [2].

The 1-year survival is only about $30 \%$, ranging the 30-day mortality between 35 and $52 \%$, with approximately half of the deaths occurring during the first 2 days after presentation [2]. The prognosis of IH mainly depends on the severity of intracra-

P. Vilela

Neuroradiology Department, Hospital Beatriz Angelo, Loures, Portugal

Neuroradiology Department, Hospital da Luz, Lisbon, Portugal

M. Wiesmann $(\bowtie)$

Department of Diagnostic and Interventional Neuroradiology, University Hospital, Technical University of Aachen RWTH,

Aachen, Germany

e-mail:mwiesmann@ukaachen.de nial hypertension and associated complications such as brain herniation, the development of hydrocephalus, and for subarachnoid hemorrhage on the development of arterial vasospasm.

Spontaneous intracranial hemorrhage may be parenchymal, intraventricular, subarachnoid, subdural, and epidural, the former being the most common location and the latter two being uncommon and usually associated with trauma.

Computed tomography $(\mathrm{CT})$ remains the standard method to detect intracranial hemorrhage although radiologists need also to be familiar with the appearance of hematomas using magnetic resonance imaging (MRI). In this paper the authors suggest a structured imaging diagnostic approach for spontaneous intracranial hemorrhage and review the imaging findings of the most common causes.

\subsection{Hemorrhage Imaging}

In the presence of a stroke with acute neurological deficit an emergent imaging study is warranted to evaluate the presence of ischemic or hemorrhagic stroke. Generally, CT is the initial imaging method used, due to its wider availability, rapid acquisition, lack of contraindications, and high accuracy to depict intracranial hemorrhage.

Imaging has a crucial role on intracranial hemorrhage evaluation. Firstly, it defines the type of stroke (ischemic or hemorrhagic), which have completely different managements. It is also essential to identify the anatomic compartment the lesion is located in, and approximate the age of the hemorrhage. The next step is to triage patients into those likely to have an underlying cause (Table 5.1) which requires urgent diagnosis and treatment, and those who do not require urgent interventions. To do this, neuroimaging findings need to be considered in combination with the age and medical history of the patient. In the setting of acute intracranial hemorrhage, CT angiography (or MR angiography) can be used to screen for the presence of vascular abnormalities such as intracranial aneurysmas, arteriovenous malformations, or 
Table 5.1 Most common etiologies of nontraumatic intracranial hemorrhage by age group and anatomic location

\begin{tabular}{|c|c|c|c|c|}
\hline & \multicolumn{2}{|l|}{ Adult } & \multicolumn{2}{|l|}{ Pediatric } \\
\hline & Elderly & Young/middle aged & Adolescent/child/infant & Newborn \\
\hline Parenchymal & $\begin{array}{l}\text { - Hypertensive hemorrhage } \\
\text { - Cerebral amyloid angiopathy (CAA) } \\
\text { - Hemorrhagic transformation of acute } \\
\text { ischemic infarction } \\
\text { - Dural AVF } \\
\text { - Venous thrombosis } \\
\text { - Coagulopathies } \\
\text { - Neoplasms }\end{array}$ & $\begin{array}{l}\text { - } \text { Brain AVM } \\
\text { - Cavernomas } \\
\text { - Venous thrombosis } \\
\text { - Hypertensive } \\
\text { encephalopathies/PRES } \\
\text { - RVCS } \\
\text { - Drug abuse } \\
\text { - Neoplasms }\end{array}$ & $\begin{array}{l}\text { - Brain AVM } \\
\text { - Cavernomas } \\
\text { - Venous thrombosis } \\
\text { - Tumors } \\
\text { - Coagulopathies } \\
\text { (congenital/acquired) }\end{array}$ & $\begin{array}{l}\text { - Hypoxic-ischemic } \\
\text { injury }\end{array}$ \\
\hline Subarachnoid & $\begin{array}{l}\text { - Aneurysm } \\
\text { - Cerebral amyloid angiopathy (CAA) } \\
\text { - Perimesencephalic hemorrhage } \\
\text { - Bleeding diathesis (e.g., excessive } \\
\text { anticoagulation) }\end{array}$ & $\begin{array}{l}\text { - Aneurysm } \\
\text { - Arterial dissection } \\
\text { - Brain AVM }\end{array}$ & \multicolumn{2}{|c|}{$\begin{array}{l}\text { - Aneurysm } \\
\text { - Sickle cell disease (in children) } \\
\text { - Coagulopathies (congenital/acquired) }\end{array}$} \\
\hline $\begin{array}{l}\text { Epidural/ } \\
\text { subdural }\end{array}$ & \multicolumn{2}{|c|}{$\begin{array}{l}\text { - Dural AVF } \\
\text { - Aneurysm } \\
\text { - Moyamoya syndrome } \\
\text { - Dural metastases } \\
\text { - Bleeding diathesis/hematological disorders }\end{array}$} & & \\
\hline
\end{tabular}

Table 5.2 CT and MR characteristics of intracranial hemorrhage

\begin{tabular}{|c|c|c|c|c|c|}
\hline Time & NECT & CECT & T1-weighted & T2-weighted & $\mathrm{T} 2 * \mathrm{~W}$ \\
\hline Hyperacute $(<6$ h) & Hyperdense & & Iso & Hyper & Hypo \\
\hline Acute (6 h-3 days) & Hyper & & Iso & Нypo & Нypo \\
\hline \multirow[t]{2}{*}{ Early subacute (3-7 days) } & \multirow{2}{*}{ Hyper/iso } & & Iso (center) & Нyро & Нypo \\
\hline & & & Hyper (rim) & & \\
\hline Late subacute (1-4 weeks) & Iso/hypo & Rim enhancement & Hyper & Hyper & Hypo \\
\hline Chronic (months-years) & Нyро & & Нуро & Нуро & Нyро \\
\hline
\end{tabular}

Density/signal intensity compared to cortex. NECT non-enhanced CT, CECT contrast enhanced CT

dural arteriovenous fistulas. Thus, imaging is essential to evaluate critical situations, to establish the prognosis, and to guide the treatment.

It is important to highlight that the blood imaging signs will vary over time in both computed tomography (CT) and magnetic resonance imaging (MRI), as shown in Table 5.2. Evolution over time will vary according to the location of the hemorrhage, with a faster clearance in the CSF spaces.

To understand the appearance of blood clots on CT and MRI, some knowledge on clot formation is helpful.

Primary acute intracerebral hemorrhages appear as lesions of homogenous hyperattenuation (Figs. 5.1 and 5.2) on (noncontrast enhanced) CT. The hyperattenuation results from the increasing protein density within the hematoma. As the hematoma matures, there is progressive clot formation and retraction with fluid loss, which then causes the hyperattenuation to increase from a range of 40-80 HU to $80-100 \mathrm{HU}$ [3]. In the acute phase, the presence of fluid-fluid levels and/or hypoattenuated areas inside the hematoma may represent a hyperacute hematoma, active bleeding (with the new incoming blood corresponding to these hypoattenuating areas), and/or the presence of coagulation disorder (acquired or congenital). The "swirl sign" characterized by the mixture of different densities or MR signal intensities in an acute hemorrhage suggest an active bleeding. Typically, clots become isodense to brain on CT images at 8-14 days after hemorrhage, then progressively hypodense after 2-4 weeks and, finally, at the late subacute and chronic phases, they are usually hypodense approaching the attenuation of cerebrospinal fluid (CSF).

For MRI it is also important to understand the type of hemoglobin present at each stage of the hematoma. This allows the estimation of the age of the hematoma. Initially, an intracerebral hematoma consists of intact red blood cells (RBCs) containing mainly oxygen-saturated hemoglobin (oxyhemoglobin, $\mathrm{OxyHb}$ ). The hemoglobin is then gradually deoxygenated. At $48 \mathrm{~h}$ after hemorrhage the clot consists almost entirely of deoxyhemoglobin (DeoxyHb). At this stage, the hematoma is surrounded by edema. In the early subacute phase (3-7 days) DeoxyHb is gradually converted to methemoglobin (MetHb). These changes begin at the periphery of the clot and then progress towards the center. In the late subacute phase (2-4 weeks) the RBCs lyse and MetHb is released from the intracellular into the extracellular space. In this stage the edema gradually dissolves. Later on, in the chronic phase ( $>4$ weeks) the hematoma 



Fig. 5.1 Hypertensive hematoma. Axial (a) and reformatted coronal (b), and sagittal (c) CT images showing an acute hypertensive hematoma at the basal ganglia (putamen) dissecting into the corona radiata. The 10 day follow up CT images (d) shows the expected peripheral

hyperattenuation decrease and the presence of edema. The late follow up (1 year) CT image (e) shows the hematoma scar as a small area of calcification with peripheral hypoattenuation 


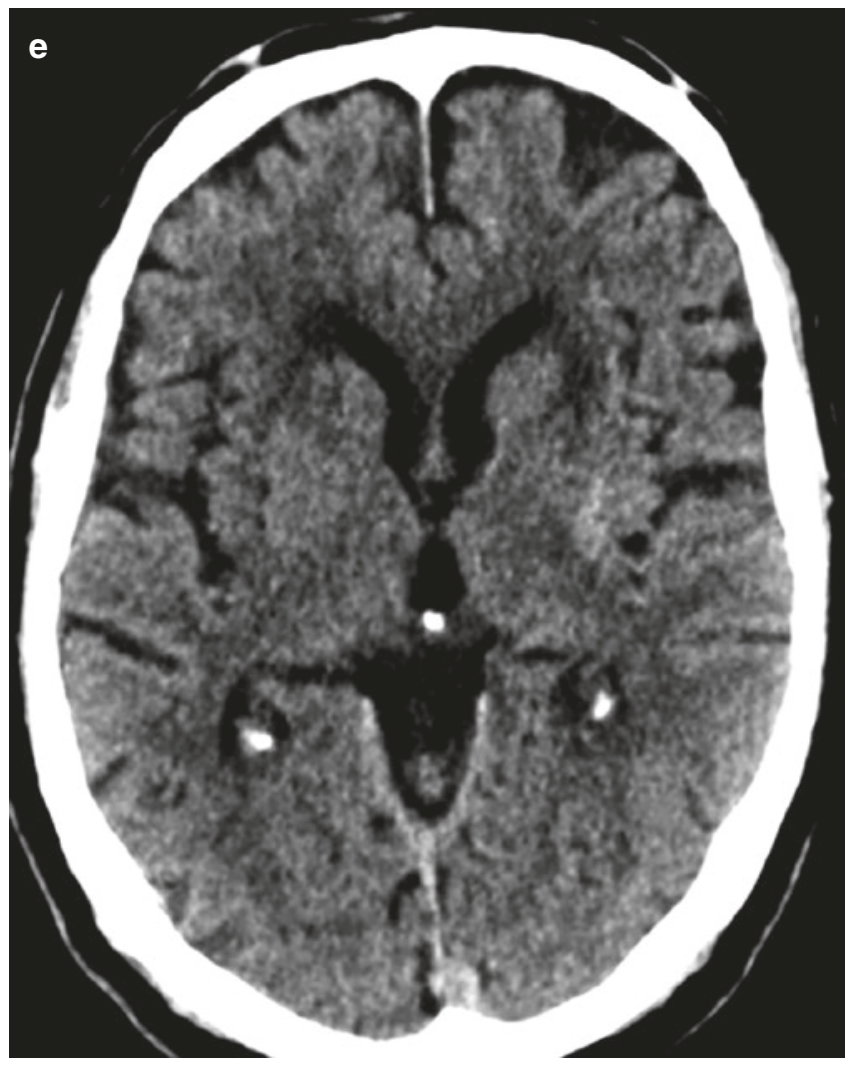

Fig. 5.1 (continued)
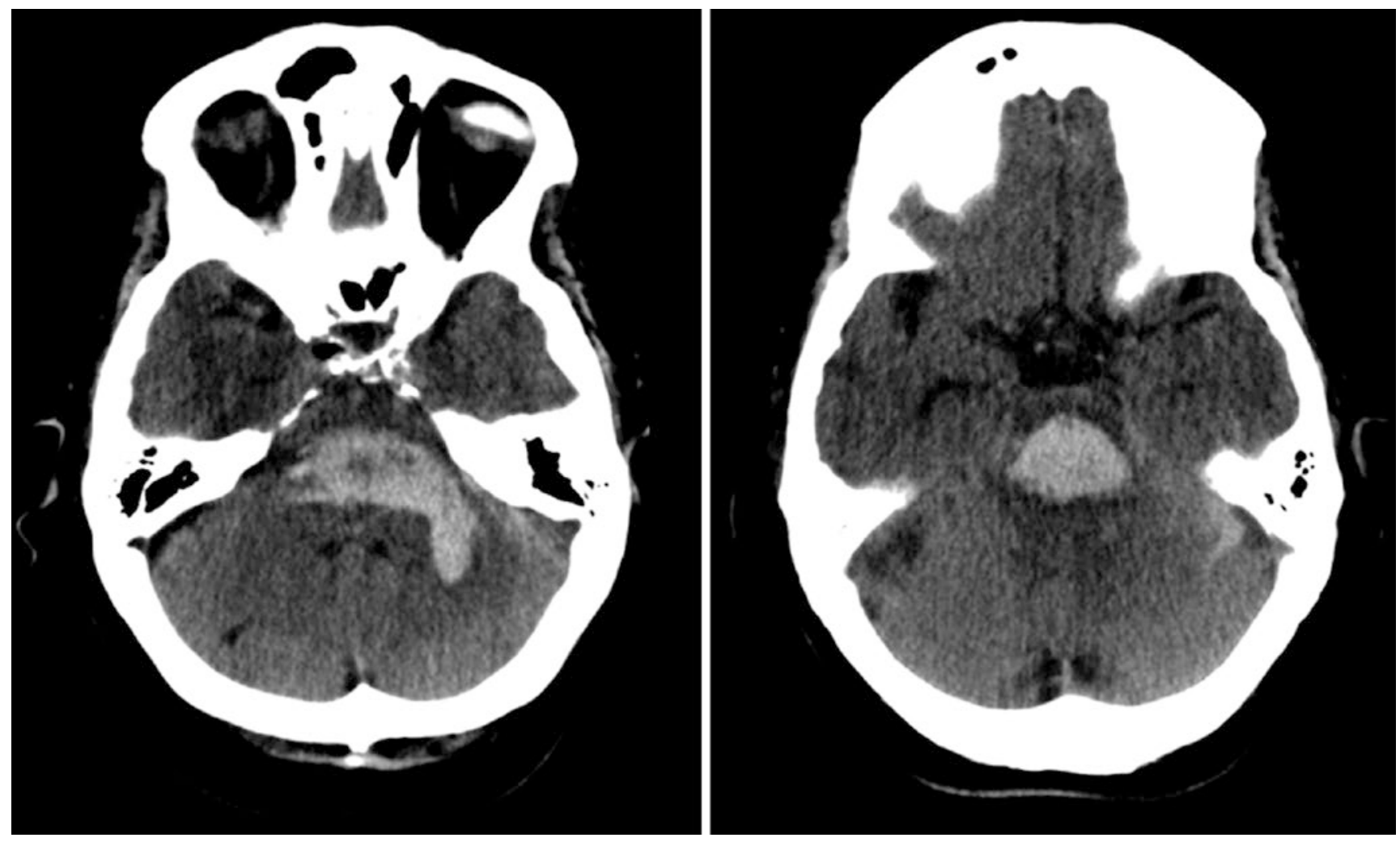

Fig. 5.2 Hypertensive hematoma. Axial CT images showing an acute (hyperattenuating) pontine hypertensive hematoma dissecting into the middle cerebellar peduncle and left medulary center 
with brain atrophy with sulcal and/or ventricular enlargement, and may exhibit focal calcification and peripheral gliosis.

Microhemorrhages are chronic small punctate brain hemorrhages only depicted by MRI using T2*W susceptibility imaging. They are depicted as small $(<5 \mathrm{~mm})$ rounded low $\mathrm{T} 2 *$ lesions which result from presumed ruptures of tiny vessels with diameters smaller than $200 \mu \mathrm{m}$. They are commonly associated with sporadic small vessel disease (systemic hypertension) and CAA, but can also be found in patients with nonsporadic small vessel disease, such as CADASIL and vasculitis, among others. They are also seen in ageing with increasing prevalence with older age and hypertension. The differential diagnosis of microbleeds includes non-hemorrhagic subcortical mineralization areas; pneumocephalus and hemorrhagic lesions such as cavernomas, hemorrhagic diffuse axonal injury, and microhemorrhagic brain metastasis.

The microhemorrhage pattern associated with chronic hypertension has a central distribution, located at the deep gray structures (basal ganglia and thalamus), cerebellum, and less importantly at the cerebral cortico-subcortical junction (Fig. 5.3). The CCA pattern has a peripheral distribution at the cerebral lobar cortico-subcortical areas with a posterior predominance (Fig. 5.4).

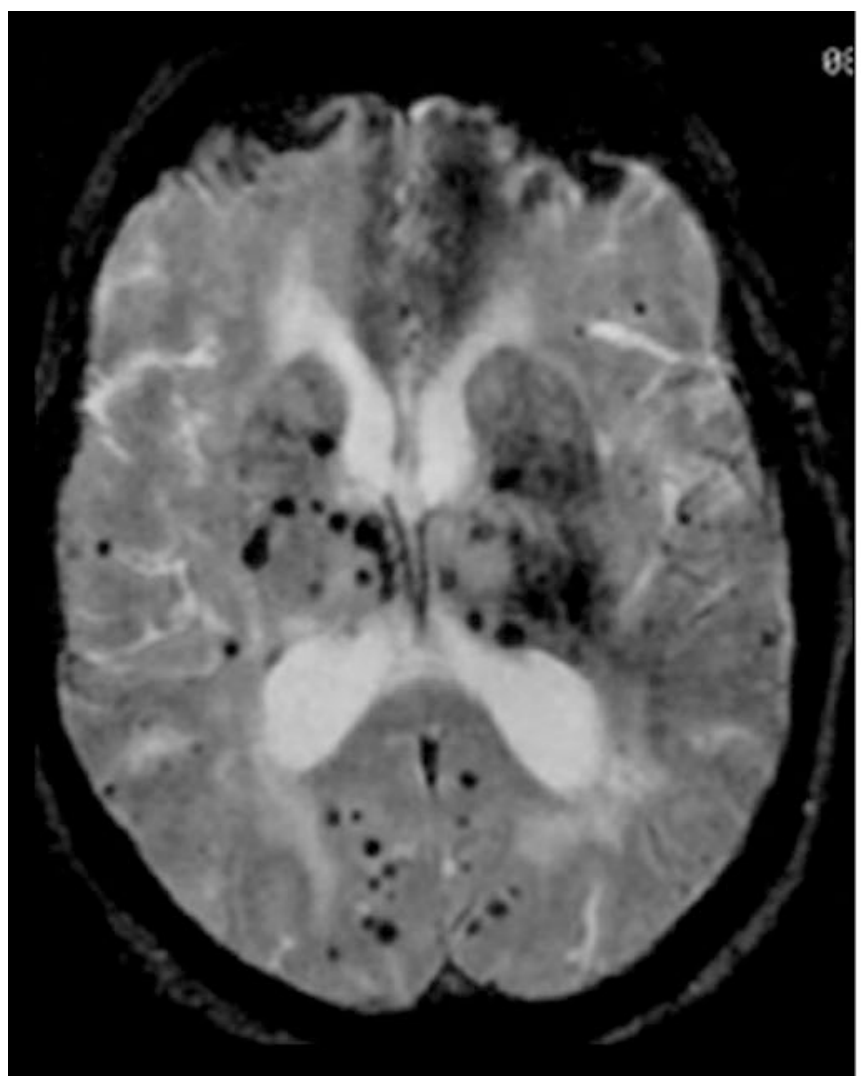

Fig. 5.3 Hypertensive microhemorrhages. Axial T2*-weighted MR images demonstrating the central pattern of microbleeds (small punctuate low intensity signal lesions) distribution, with thalamic, lenticular,
Subarachnoid and intraventricular hemorrhage manifest with hemorrhage into the CSF spaces presenting with increased attenuation on CT images (blood). The hemorrhage attenuation and MRI signal intensity follow the abovementioned evolution.

\section{Key Points}

- CT is the method of choice to detect acute intracranial hemorrhage

- CT and CTA allow the detection of active intracranial bleeding

- In the subacute stage MRI is superior to CT in the detection of subarachnoid hemorrhage

CT is the gold standard imaging method to depict acute SAH $[6,7]$. The sensitivity of CT to depict SAH during the first $6-12 \mathrm{~h}$ is $98-100 \%[6,7]$. The same diagnostic accuracy may be assumed for intraventricular hemorrhage. Over days, especially after the fifth day, the accuracy of CT decreases, and in the subacute phase a lumbar puncture may be needed to make the diagnosis [6].

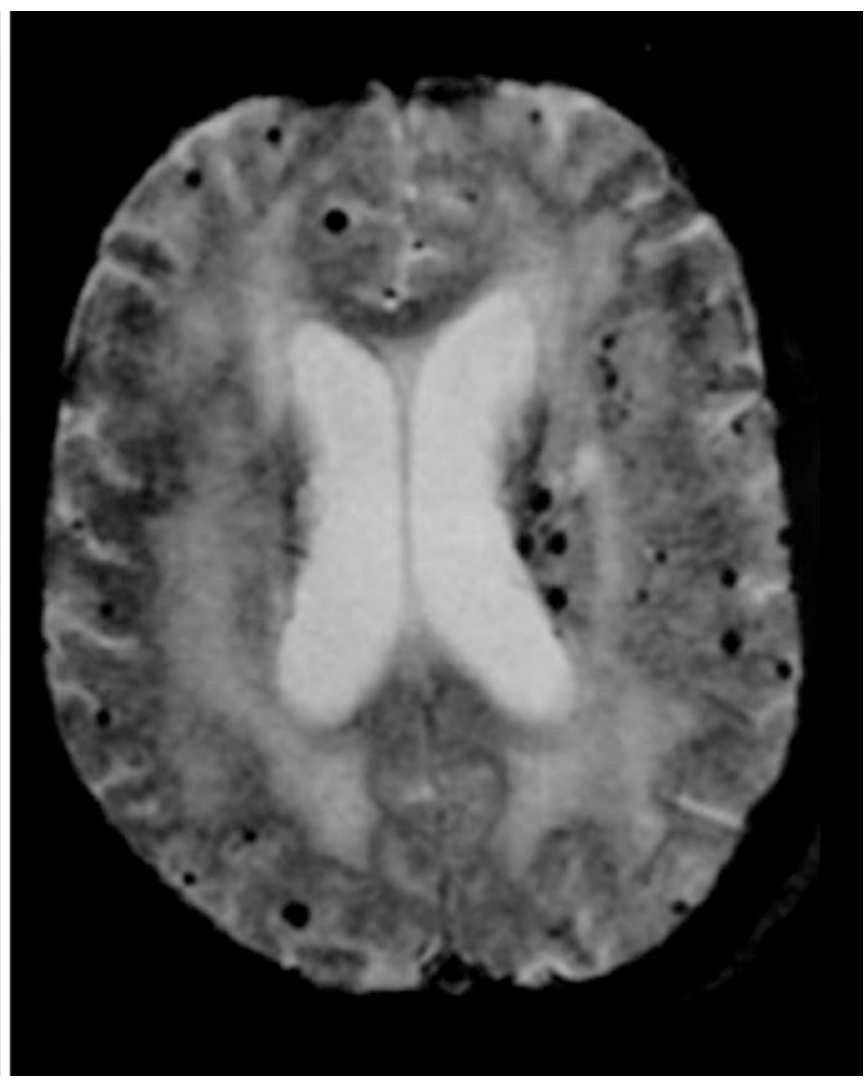

caudade, capsular and corona radiata locations, in association with a peripheral distribution at the lobar cortical-subcortical junction 

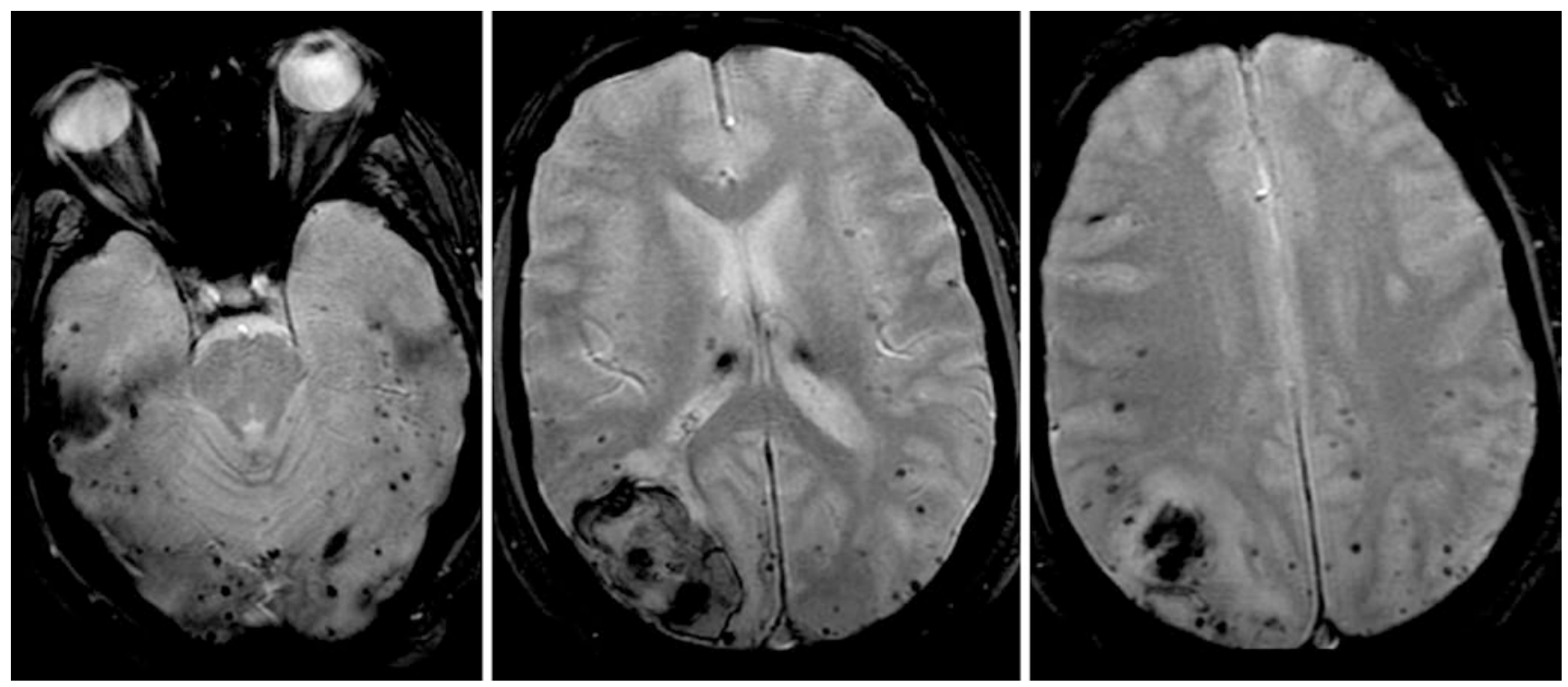

Fig. 5.4 Cerebral amyloid angiopathy (CAA) microhemorrhages. Axial T2-weighted MR images demonstrating the isolated peripheral pattern of microbleeds (small punctuate low intensity signal lesions)

Table 5.3 Modified Fisher grade

Grade 1 Minimal or diffuse thin SAH without IVH

Low risk for symptomatic vasospasm

Grade 2 Minimal or thin SAH with IVH

Grade 3 Thick cisternal clot without IVH

Grade 4 Cisternal clot with IVH

Intermediate risk for symptomatic vasospasm

High risk for symptomatic vasospasm

Recently, it has been shown that MRI has a very high accuracy, similar to $\mathrm{CT}$, to depict SAH in the acute phase, using FLAIR/DF sequences, and a higher accuracy than CT in the subacute and chronic phases (using FLAIR/DF and susceptibility weighted sequences) $[8,9]$. MRI is especially sensitive to chronic SAH, depicting the presence of superficial cortical hemosiderosis. This is an important diagnostic sign since its presence can help to suspect prior subarachnoid hemorrhage, or to substantiate the differential diagnosis of amyloid angiopathy.

The severity of the SAH is measured by the (modified) Fisher grade (Table 5.3). The pattern of blood distribution, namely basal cistern, superficial, or perimesencephalic distribution, allows the prediction of the etiology.

\subsection{Intracranial Hemorrhage Etiology}

There are multiple potential causes of hemorrhagic stroke, which can be primarily categorized according to the hemorrhage location and patient age, facilitating the imaging differential diagnosis approach, as summarized in Table 5.1. distribution with distribution at the lobar cortical-subcortical junction with posterior cerebral predominance, in association to a right occipital lobar hematoma

Additional imaging to evaluate an intracranial hemorrhage includes $\mathrm{CT}$ angiography to look for underlying vascular lesions and MRI to rule out other etiologies of ICH.

Vascular imaging is indicated whenever a vascular lesion is suspected, such as arterial aneurysms, pial arteriovenous malformations and dural arteriovenous fistula, or vasculitis. $\mathrm{CT}$ or MR venograms are very helpful to depict the presence of cerebral venous thrombosis, especially dural sinus thrombosis. CTA and MRA are the initial imaging methods. DSA is reserved for inconclusive cases, and for the treatment of some of the vascular lesions. The spot sign on CTA, reflecting contrast extravasation, depicted as an enhancing focus within the acute hematoma, deserves special attention since is associated with a higher risk of growth of the hematoma [10-14].

Brain hemorrhage accounts for up to $15 \%$ of all strokes [15]. Among the adult population brain hemorrhage is most commonly associated with systemic hypertension (Figs. 5.1, 5.2, and 5.3), followed by cerebral amyloid angiopathy (CAA) (Figs. 5.4 and 5.5). In younger patients and in children, vascular malformations are an important cause of brain hemorrhage. Cerebral venous thrombosis is also an important cause of brain hemorrhage in the young patient especially in females and during the pregnancy and puerperium. Hemorrhagic transformation of acute ischemic stroke and hemorrhagic brain tumors are also important causes of brain hemorrhage. Other potential causes of brain hemorrhage, representing $<10 \%$ of cases, include reversible cerebral vasoconstriction syndrome (RVCS), posterior reversible encephalopathy syndrome (PRES), vasculitis, coagulopathy disorders, drugs consumption (sympathomimetics, cocaine, amphetamine, ecstasy, among others), moyamoya disease, infections, and pre-eclampsia/eclampsia, among others. 

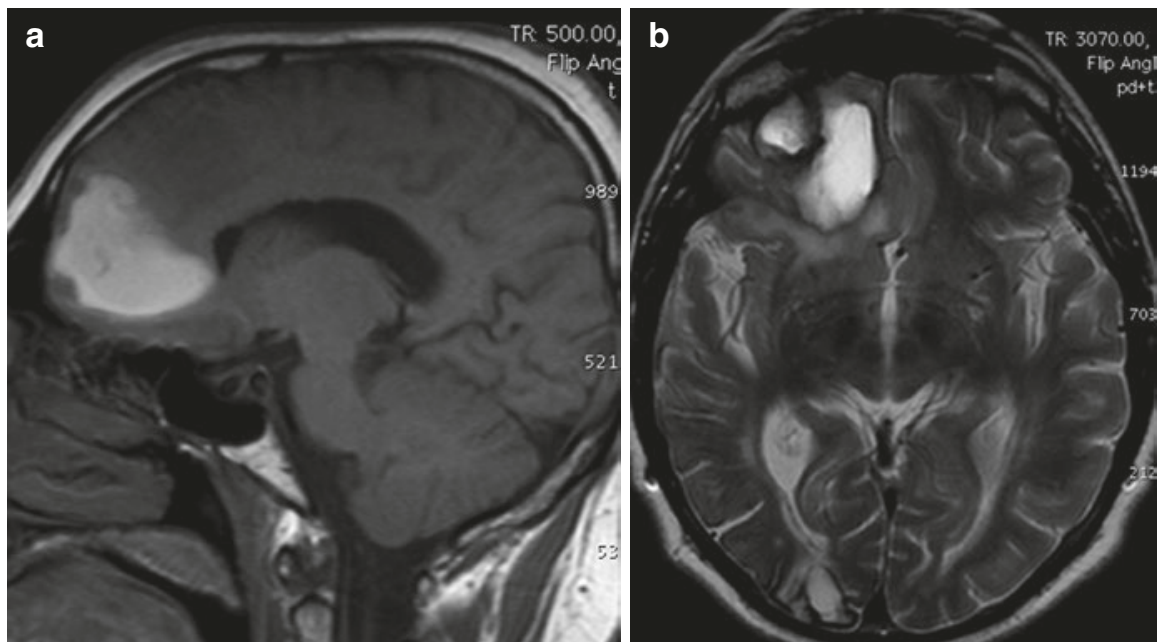

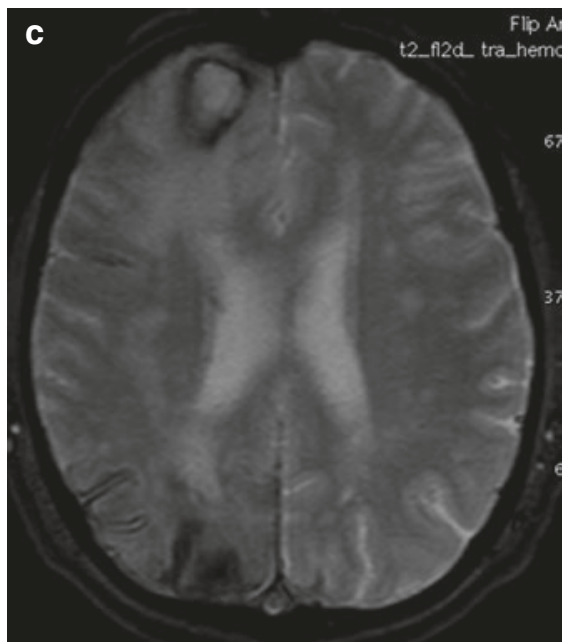

Fig. 5.5 Cerebral amyloid angiopathy (CAA). Axial T1-weighted MR image (a) depicting a frontal lobar hematoma in the subacute stage (extracellular methemoglobin). Axial T2-weight (b) and T2*-weighted

Hypertensive hemorrhage is the leading cause of cerebral hematomas in the adult population, being secondary to a long-standing hypertensive vasculopathy characterized by lipohyalinosis of small-to-medium penetrating arteries. Hematomas result from the rupture of small arterioles and have a rebleeding rate of up to $2 \%$ per year [16]. Typical locations for hypertensive hemorrhage (Figs. 5.1 and 5.2) include basal ganglia (in $62-65 \%$ of cases), thalamus (in $20-26 \%$ of cases), pons (in 5-10\% of cases), cerebellum (in approximately $6 \%$ of cases), and subcortical-cerebral lobar (in 1-2\%) of cases $[17,18]$. The association of leucoaraiosis, lacunar infarcts, and microbleeds with a central distribution pattern supports the diagnosis.

Cerebral amyloid angiopathy (CAA) is the second most common cause of spontaneous intracranial hemorrhage in adults and results from the vessel wall deposition of amyloid-b peptide in small leptomeningeal and cortical vessels. CAA is responsible for up to $50 \%$ of spontaneous hemorrhages in older patients ( $>65$ years) and its incidence increases with patient age, being present in up to one third of brain autopsies in patients above 60 years old, which reflects that in an ageing population it will be a very important cause of hemorrhage. The most common type of CAA is the sporadic type occurring in the elderly, but there are hereditary types that may present earlier, such as the HCHWA-Dutch type and HCHWA-Icelandic type with onset in the third/forth decade, and other reported familiar types. Imaging is an important criterion for the diagnosis of CAA according to the Boston diagnostic criteria (Table 5.4) [19]. CAA should be considered in cases of older patients with multiple lobar, cortical, and/or subcortical hemorrhages; superficial/cortical subarachnoid hemorrhage (superficial siderosis); and peripheral distributed microhemorrhages. Hemorrhages rarely involve the cerebellum and (c) images demonstrate additional cortical (superficial) subarachnoid hemorrhage at the right brain convexity and a chronic occipital lobar hematoma

Table 5.4 The modified Boston criteria for cerebral amyloid angiopathy (CAA)

\begin{tabular}{|c|c|c|}
\hline $\begin{array}{l}\text { Definite } \\
\text { CAA }\end{array}$ & $\begin{array}{l}\text { Full post-mortem } \\
\text { examination }\end{array}$ & $\begin{array}{l}\text { - Lobar, cortical, or cortical/ } \\
\text { subcortical hemorrhage } \\
\text { - Pathological evidence of } \\
\text { severe cerebral amyloid } \\
\text { angiopathy } \\
\text { - Absence of other diagnostic } \\
\text { lesion }\end{array}$ \\
\hline $\begin{array}{l}\text { Probable } \\
\text { CAA with } \\
\text { supporting } \\
\text { pathology }\end{array}$ & $\begin{array}{l}\text { Clinical data and } \\
\text { pathological tissue } \\
\text { (evacuated } \\
\text { hematoma or cortical } \\
\text { biopsy specimen) }\end{array}$ & $\begin{array}{l}\text { - Lobar, cortical, or } \\
\text { corticosubcortical hemorrhage } \\
\text { - Some degree of CAA in } \\
\text { specimen } \\
\text { - Absence of other diagnostic } \\
\text { lesion }\end{array}$ \\
\hline \multirow[t]{2}{*}{$\begin{array}{l}\text { Probable } \\
\text { CAA }\end{array}$} & Clinical data & $\begin{array}{l}\text { - Patient } 55 \text { years or older } \\
\text { - Absence of other diagnostic } \\
\text { lesion } \\
\text { - Absence of other cause of } \\
\text { hemorrhage or superficial } \\
\text { siderosis }\end{array}$ \\
\hline & $\begin{array}{l}\text { MRI or CT } \\
\text { demonstrating }\end{array}$ & $\begin{array}{l}\text { - Multiple hemorrhages } \\
\text { restricted to lobar, cortical, or } \\
\text { corticosubcortical regions } \\
\text { (cerebellar hemorrhages } \\
\text { allowed) of varying sizes/ } \\
\text { agesOr } \\
\text { - Single lobar, cortical, or } \\
\text { corticosubcortical hemorrhage } \\
\text { and focal (three or less sulci) } \\
\text { or disseminated (more than } \\
\text { three sulci) cortical superficial } \\
\text { siderosis }\end{array}$ \\
\hline \multirow[t]{2}{*}{$\begin{array}{l}\text { Possible } \\
\text { CAA }\end{array}$} & Clinical data & $\begin{array}{l}\text { - Patient } 55 \text { years or older } \\
\text { - Absence of other cause of } \\
\text { hemorrhage or superficial } \\
\text { siderosis }\end{array}$ \\
\hline & $\begin{array}{l}\text { MRI or CT } \\
\text { demonstrating }\end{array}$ & $\begin{array}{l}\text { - Single lobar, cortical, or } \\
\text { corticosubcortical } \\
\text { hemorrhageOr } \\
\text { - Focal or disseminated } \\
\text { superficial siderosis }\end{array}$ \\
\hline
\end{tabular}


the microbleeds spare the basal ganglia and brain stem (Fig. 5.4) [19].

\section{Key Points}

- CAA shows a different lesion pattern than hypertensive hemorrhage pattern and can be recognized by the presence of superficial siderosis

- In intracerebral hematomas early hematoma expansion, intracranial hypertension, brain herniation, and the presence of hydrocephalus need to be recognized early to allow for specific treatment

For brain hematomas, there are some red-flag imaging findings that should prompt our special attention. These cases may require early surgical treatment. It is essential to depict early hematoma expansion, intracranial hypertension and/or signs of mass effect, such as brain herniation, and the presence of hydrocephalus.

Predictors for worst outcome include hemorrhage volume, infratentorial location (brain stem and/or cerebellum), presence of intraventricular hemorrhage, and/or hydrocephalus $[15,20]$.

Hematoma volume can be measured from multiplanar reconstruction as the ratio $\mathrm{ABC} / 2$, being $\mathrm{ABC}$ the three orthogonal diameters (the diameters are multipliedlength $\times$ width $\times$ height—and divided by 2). It is also essential to depict intraventricular extension, since it represents a higher risk for hydrocephalus, especially if the clot is inside the aqueduct and/or IV ventricle. Brain herniation is associated with a dismal outcome, and may necessitate surgical decompression.

Other unfavorable factors of brain hematomas include advanced age, and high scores on the Glasgow Coma Score (GCS) and National Institutes of Health Stroke Scale (NHIHSS) at admission.

The ICH score allows an early estimation of the patient prognosis [20]. Higher scores are associated with higher 30 days mortality rates, namely of $13 \%, 26 \%, 72 \%$, and $97 \%$ corresponding to scores of $1,2,3$, and 4 , respectively (Table 5.5) [20]. Another brain hemorrhage prognostic score,

Table 5.5 ICH score

\begin{tabular}{|l|l|l|}
\hline ICH Score & & Points \\
\hline GCS score & $3-4$ & 2 \\
& $5-12$ & 1 \\
& $13-15$ & 0 \\
ICH volume $\left(\mathrm{cm}^{3}\right)$ & $\geq 30$ & 1 \\
& $<30$ & 0 \\
Intraventricular hemorrhage & Yes & 1 \\
& No & 0 \\
Infratentorial origin & Yes & 1 \\
\hline \multirow{2}{*}{ Age } & No & 0 \\
Total & $\geq 80$ & 1 \\
\hline & $<80$ & 0 \\
\hline & & $0-6$
\end{tabular}

that estimates the functional independence at 90 days, is the Functional Outcome in Primary Intracerebral Hemorrhage (FUNC) score [21].

It is also worthwhile to stress that up to $30 \%$ of brain hematomas will increase in size during the first $24 \mathrm{~h}$, especially during the first $3 \mathrm{~h}$ after presentation, which may be associated with neurological decline [22]. Repeat CT scans at $24 \mathrm{~h}$ or whenever there is a neurological decline is recommended [22].

Intracranial venous thrombosis (IVT) is an important cause of intracranial hemorrhage, especially in young patients and children. There is also a higher incidence during pregnancy and puerperium and in females under oral contraception. IVT results from the formation and propagation of a venous clot leading to the occlusion of the intracranial venous system components. Venous thrombosis features a wide spectrum of clinical and imaging presentation. Headache is the most common symptom. The diagnosis of IVT includes the demonstration of the thrombus/venous occlusion (direct signs), depicted by CT (which depicts only one third of cases as hyperdense thrombus inside the dural sinus—dense triangle sign-or cortical vein-cord sign-) or by MRI by the loss of the normal venous flow void by a clot that follows the same signal intensity progression previously described for parenchymal hematomas. MRV or CTV is also helpful to establish the diagnosis. It is also important to depict the presence of intracranial lesions (indirect signs), such as brain swelling, brain edema, venous infarction, intracranial hemorrhage, which are better defined by MRI [23].

Vascular malformations are the leading cause of spontaneous intracranial hemorrhage in children and young patients, including cavernomas and brain arteriovenous malformations (AVMs).

Brain AVMs are composed by abnormal arteriovenous shunts, occurring in a central nidus, which is the central area towards multiple feeding arteries converge and from which (early and enlarged) draining veins start. Most of the cases are sporadic, but they may be associated with HHT/ROW and RASA 1 mutation or being a part of metameric syndromes. Brain AVMs appear as a tangled cluster of abnormal vessels, dilated arteries and veins, which can be sulcal, parenchymal, and/or intraventricular. Brain AVMs are constituted by feeding arteries, with the nidus corresponding to the shunting area and veins that drain the malformation. The vessels present slightly hyperdense on $\mathrm{CT}$ and with flow voids on MRI. Small AVMs may only be depicted by DSA which is the gold standard imaging method. They may be associated with surrounding brain changes such as edema, gliosis, previous hemorrhage, and/or mass effect. The most common brain AVM classification used is the MartinSpetzler classification evaluating the size, location, and venous drainage of the brain AVM predicting the surgical risk for treatment. The hemorrhagic risk is higher for those AVM that have previously bled and for those with infratentorial, deep location, and deep venous drainage. 
Cavernomas are venous vascular malformations that are seen on CT as isodense or hyperdense lesions with calcifications in up to $40-60 \%$ of cases. On MRI their appearance is variable. Most commonly they have a central core with mixed signal from blood in different stages ("popcorn like"), being hyperintense on T1WI and hyper-hypointense on T2 WI. There is generally a completely $\mathrm{T} 2$ hypointense rim of hemosiderin and the contrast-enhancement is variable. The $\mathrm{T} 2 *$ /susceptibility sequences are the most sensitive imaging methods to depict cavernomas (Fig. 5.6). Cavernomas may appear only as small punctate lesions (more common in multiple cavernomas) that are poorly or not seen on $\mathrm{T} 1$ and $\mathrm{T} 2$ and only demonstrated on $\mathrm{T} 2 * /$ susceptibility sequences.
DSA is negative for the detection of cavernomas. Most of the cavernomas are sporadic but there are some familiar forms, such as CCM 1-3, for which cases multiple cavernomas are more common and an entire evaluation of the neuraxis is suggested. The most common presentation of cavernomas and brain AVM are hemorrhage, seizures, and neurological deficits.

Dural arteriovenous fistulae (AVF) tend to present in older patients. They are acquired vascular malformations in which there are abnormal connections between arteries that normally feed the meninges, bone, or muscles, but not the brain, and the dural sinuses or small venules within the dura mater. They are difficult to depict since there is no evident

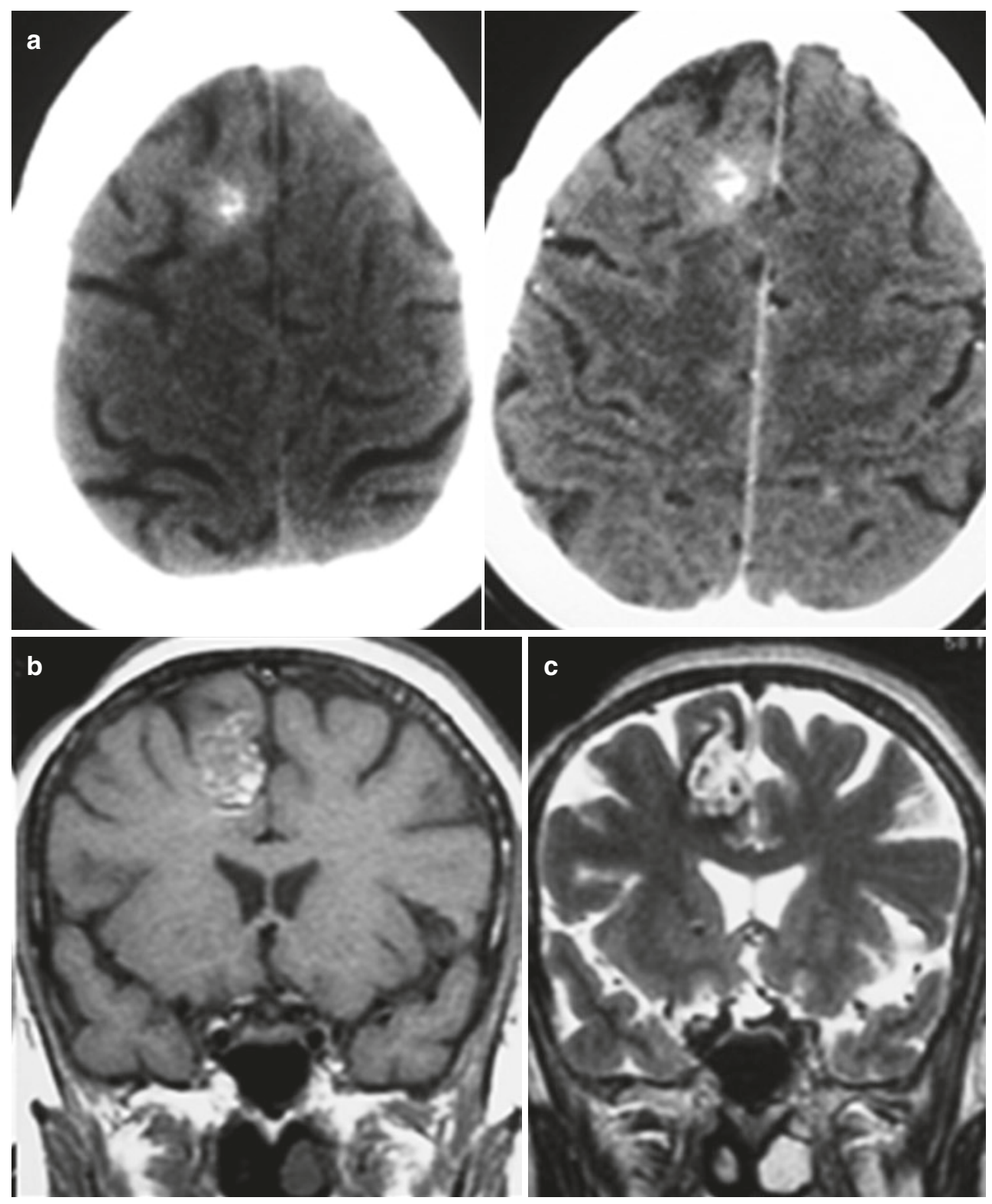

Fig. 5.6 Cavernoma. Axial CT images (a) showing a slight hyperattenuating superior frontal gyrus lesion with central calcifications. The coronal T1-weighted (b) and T2-weighted (c) MR images demonstrate the "popcorn-like" appearance with intralesional hyper- and hypoin- tense components in both sequences corresponding to blood in different stages. The axial T2*-weighted images (d) confirms the hemorrhagic nature of the lesion demonstrating the predominance of low signal with blooming effect 

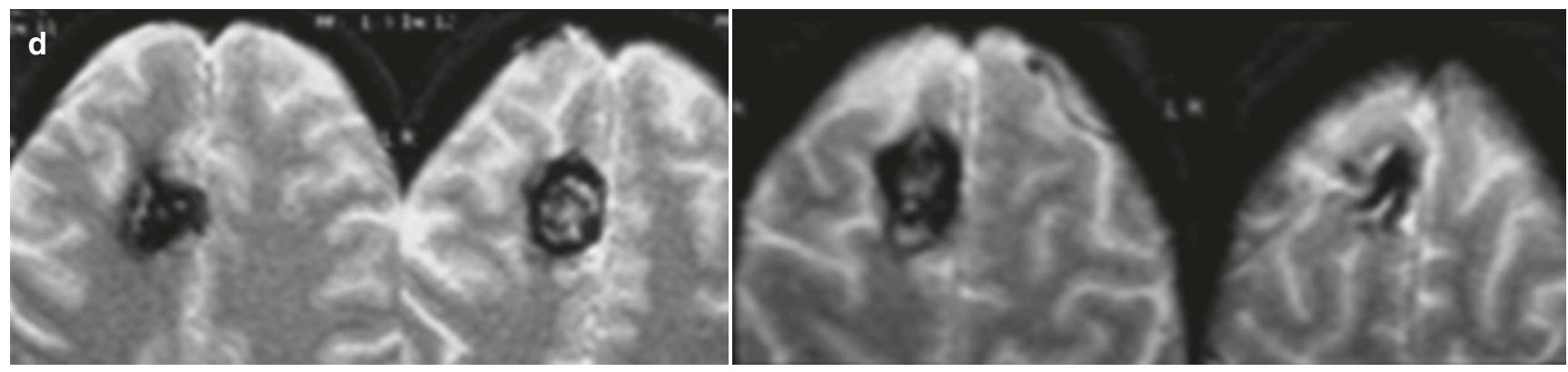

Fig. 5.6 (continued)

nidus and the feeding arteries are not as dilated as in brain AVM. The dilatation of meningeal arteries, presence of small arteries at the dura sinus and/or arterialized dural sinus, diffuse venous congestion and/or dilated veins should raise the suspicion of dural AVF. The hemorrhage risk of dural AVF is higher when a cortical venous reflux is present. The most common classifications, the Cognard and the Borden classifications, address the type of venous drainage and predict the future risk for hemorrhage.

Brain tumors are responsible for approximately $10 \%$ of intracranial bleedings. Early diagnosis allows for specific treatment. The most common causes of hemorrhage associated with brain tumors are intratumoral and/or extratumoral hemorrhage from the tumoral mass, generally in hypervascularized tumors. Other causes of hemorrhagic stroke may exist in neoplastic patients, such as malignancy induced coagulopathy, side effects from chemotherapy and/or radiotherapy, hemorrhagic infarcts resulting from thrombotic coagulopathy, arterial tumoral emboli (in association with heart myxoma), or from venous thrombosis in thrombotic coagulopathy or in association with venous compression/ invasion/thrombosis.

The tumors more commonly associated with brain hemorrhage are metastases, especially from breast and lung (bronchogenic) carcinoma, melanoma, choriocarcinoma, renal cell carcinoma, thyroid carcinoma, and primary highly vascularized tumors, such as high-grade gliomas.

There are some imaging findings that should raise the suspicion of the presence of an underlying tumor, such as atypical hematoma location; the presence of multiple hemorrhagic foci within the lesion; heterogeneous hematoma with different hemorrhage stages not following the usual chronological evolution or having a delayed evolution (due to hypoxic environment); the presence of fluid-fluid levels, that may represent blood-blood levels, blood-cystic/necrotic levels; the presence of an incomplete hemosiderin rim; the presence of irregular margins; the presence of a disproportionately larger edema; and/or the presence of non-hemorrhagic enhancing areas (Fig. 5.7). In the follow-up studies the presence of non-hemorrhagic enhancing areas may be more con- spicuous and there is generally a persisting mass effect and edema that does not subside completely.

Congenital or acquired coagulation disorders are important causes of spontaneous brain hemorrhages, since the number of patients under anticoagulation/antiplatelet therapy has been increasing over the years. These hemorrhages can occur in any intracranial compartment, even simultaneously. The presence of large or multiple and synchronous (same age/stage bleedings at CT/MRI) hemorrhages, and/or the presence of fluid-fluid hemorrhagic levels should raise the suspicion of coagulation disorder (Fig. 5.8).

It is not common to have brain hemorrhages associated with intracranial infections. Some of the typical examples include hemorrhagic encephalitis, such as herpes encephalitis, dengue fever, Crimean hemorrhagic fever-Congo virus, Epstein-Barr virus, and chikungunya virus, among others. Hemorrhage may also be a presentation of infectious vasculitis, such as varicella-zoster vasculitis. Fungal infection with vessel wall invasion may cause hemorrhage, e.g. in cases of aspergillosis and mucormycosis. Actinomycosis, paragonimiasis, cerebral malaria (due to hemorrhagic infarcts) may also present with devastating hemorrhagic lesions. Other causes of hemorrhage include infective endocarditis with septic emboli, hemorrhage associated with sepsis-induced coagulopathy and with infection treatment as in toxoplasmosis. In cases of meningitis, cerebral venous thrombosis and hemorrhagic transformation of ischemic infarcts may also lead to brain hemorrhage.

\subsection{Subarachnoid Hemorrhage and Intraventricular Hemorrhage}

The overall incidence of SAH is in between 9 and 20 per 100,000 person-years, accounting for up to $5 \%$ of all stroke cases [24-26]. The most common cause of spontaneous subarachnoid hemorrhage (SAH) is the rupture of an intracranial aneurysm, accounting for up to $80-85 \%$ of cases [24-26]. Ruptured saccular aneurysms commonly present with SAH located in the basal cisterns. 

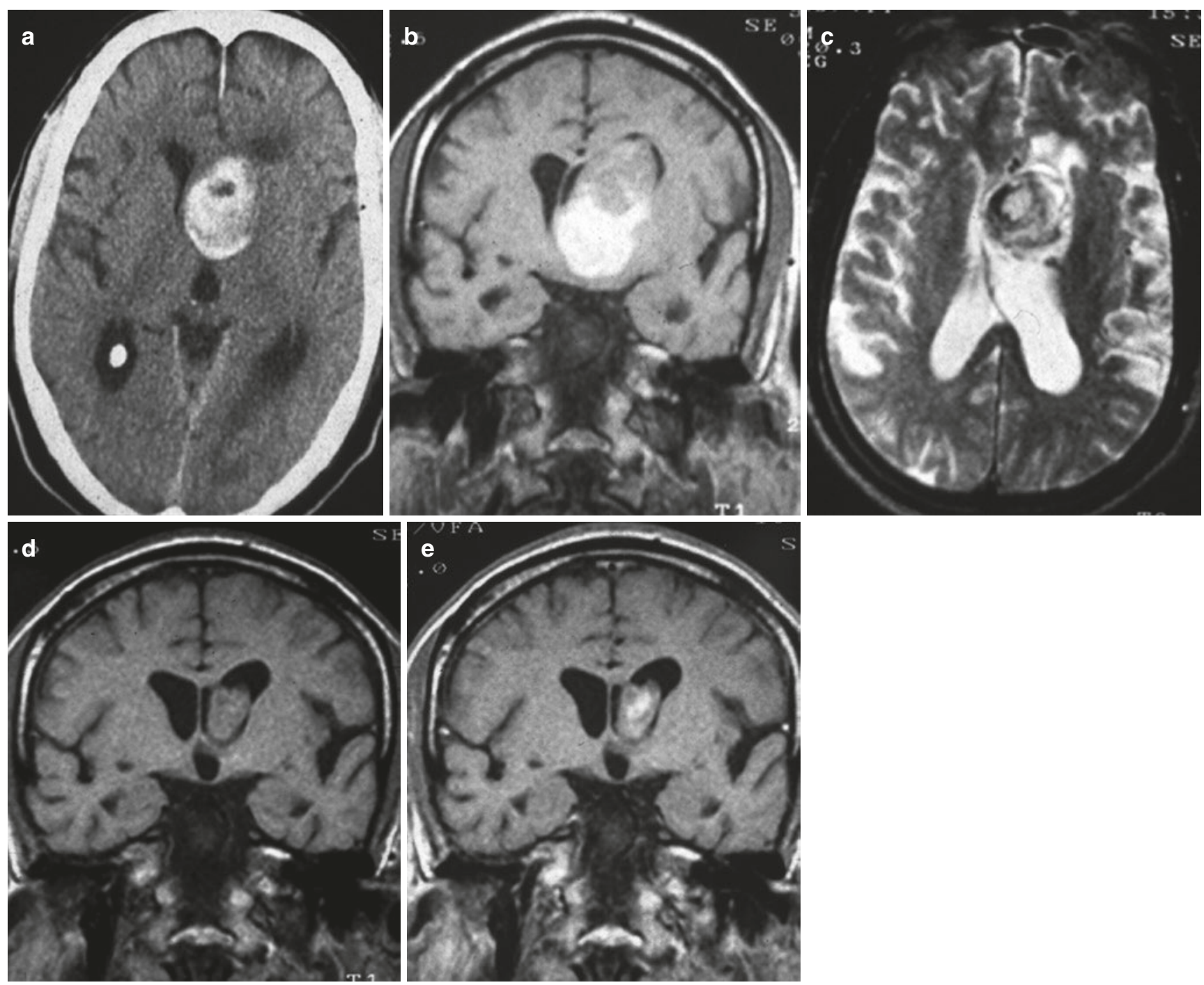

Fig. 5.7 Hemorrhagic tumor. Axial CT image (a) shows an acute hemorrhagic lesion (hyperattenuating) with intraventricular location (left frontal horn). The coronal T1-weighted (b) and axial T2-weighted (c) MR images demonstrate the heterogeneity of the signal intensities within the lesion not following the expected layered pattern of a pri- mary hemorrhage. The coronal T1-weighted (d) and coronal T1-weighted (e) post contrast images of follow up MRI performed after 3 weeks demonstrate the absorption of the hemorrhage and demonstrate the underlying intraventricular contrast-enhancing lesion corresponding to a high grade glioma
Additional causes of SAH include intracranial dissections, and for the cases with superficial/cortical subarachnoid hemorrhage, CAA, cortical venous thrombosis, vasculitis, reversible vasoconstriction syndrome (RVCS), distal arterial aneurysms, and dural arteriovenous fistulae (dAVF) should be considered.

Benign perimesencephalic SAH is a subgroup of SAH with an excellent prognosis. It accounts for up to $10 \%$ of cases and is believed to result from rupture of veins. Typically, the blood is collected at the perimesencephalic and prepontine cisterns and CTA and DSA are negative for the presence of aneurysm/vascular malformation.

\section{Key Points}

- DSA is the method of choice to screen for multiple aneurysms in all cases of SAH

- In cases of typical perimesencephalic SAH followup DSA studies are not required

Isolated intraventricular hemorrhage is an uncommon location for spontaneous hemorrhage. It generally results from the direct expansion of a parenchymal hematoma or the recirculation of blood in a SAH case. 

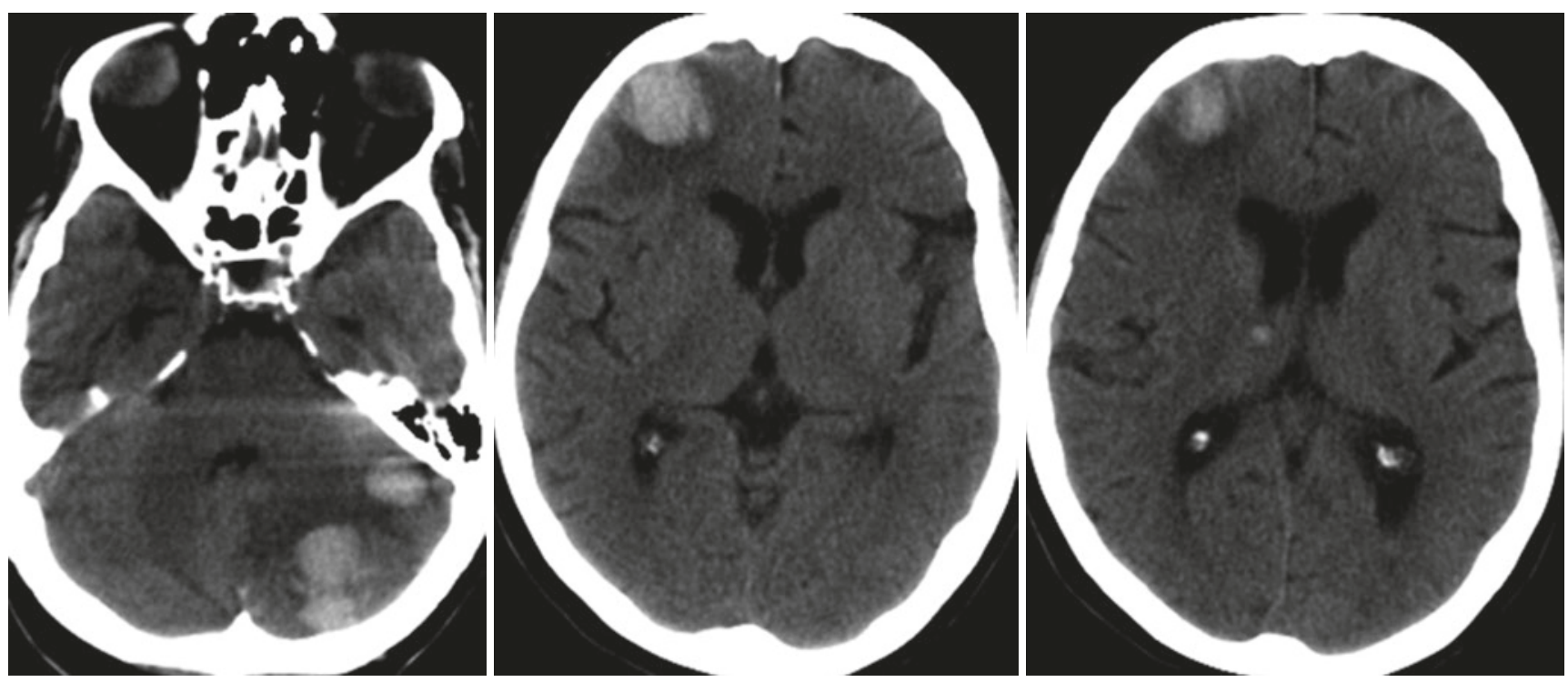

Fig. 5.8 Acquired coagulopathy associated brain hemorrhages. Axial CT images demonstrating multiple (left cerebellar, right inferior frontal and thalamic) acute hemorrhages with associated intraventricular hem- orrhage (blood level at the left atrium) in a patient with aplastic anemia and low platelets count $(12,000 / \mathrm{mcL})$

\subsection{Epidural/Subdural Hemorrhage}

Epidural and subdural hematomas are generally associated with head trauma. Exceptionally they may occur spontaneously in patients under anticoagulation or antiplatelet therapy. Isolated spontaneous EDH and SDH have been described most commonly in association with coagulopathies, dural and osteodural arteriovenous fistula, intracranial hypotension, and dural/bone tumors, especially metastases.

\subsection{Conclusions}

Imaging is essential for the management of intracranial hemorrhage. It allows for the depiction of the bleeding, the classification of the hemorrhage according to its size and location, to detect and/or exclude an underlying disorder, such as a vascular malformation, tumor, infection, among others. It also establishes the prognosis and guides the treatment.

\section{Take Home Messages}

- The imaging characteristics of intracranial hemorrhages vary over time for both CT and MRI

- In intracerebral hemorrhages the spot sign on CTA, reflecting contrast extravasation, deserves special attention since it is associated with a higher risk of growth of the hematoma
- CAA shows a different lesion pattern than hypertensive hemorrhage pattern and can be recognized by the presence of superficial siderosis

\section{References}

1. Feigin VL, et al. Global burden of stroke and risk factors in 188 countries, during 1990-2013: a systematic analysis for the Global Burden of Disease Study 2013. Lancet Neurol. 2016;15(9):913-24.

2. Broderick J, et al. Guidelines for the management of spontaneous intracerebral hemorrhage in adults: 2007 update: a guideline from the American Heart Association/American Stroke Association Stroke Council, High Blood Pressure Research Council, and the Quality of Care and Outcomes in Research Interdisciplinary Working Group. Circulation. 2007;116(16):e391-413. https://doi. org/10.1161/CIRCULATIONAHA.107.183689.

3. Parizel PM, et al. Intracranial hemorrhage: principles of CT and MRI interpretation. Eur Radiol. 2001;11(9):1770-83.

4. Venkatasubramanian $C$, et al. Natural history of perihematomal edema after intracerebral hemorrhage measured by serial magnetic resonance imaging. Stroke. 2011;42(1):73-80. https://doi. org/10.1161/STROKEAHA.110.590646.

5. Urday $\mathrm{S}$, et al. Targeting secondary injury in intracerebral haemorrhage - perihaematomal oedema. Nat Rev Neurol. 2015;11(2):11122. https://doi.org/10.1038/nrneurol.2014.264.

6. Bederson JB, et al. Guidelines for the management of aneurysmal subarachnoid hemorrhage: a statement for healthcare professionals from a special writing group of the Stroke Council, American Heart Association. Stroke. 2009;40(3):994-1025.

7. Perry JJ, et al. Sensitivity of computed tomography performed within six hours of onset of headache for diagnosis of subarachnoid haemorrhage: prospective cohort study. BMJ. 2011;343:d4277. 
8. Mitchell P, et al. Detection of subarachnoid haemorrhage with magnetic resonance imaging. J Neurol Neurosurg Psychiatry. 2001;70(2):205-11.

9. U-King-Im JM, et al. Current diagnostic approaches to subarachnoid haemorrhage. Eur Radiol. 2005;15(6):1135-47.

10. Safatli DA, et al. Predictors of 30-day mortality in patients with spontaneous primary intracerebral hemorrhage. Surg Neurol Int. 2016;7(Suppl 18):S510-7.

11. Kothari RU, et al. The ABCs of measuring intracerebral hemorrhage volumes. Stroke. 1996;27(8):1304-5.

12. Brouwers HB, et al. CT angiography spot sign in intracerebral hemorrhage predicts active bleeding during surgery. Neurology. 2014;83(10):883-9.

13. Delgado Almandoz JE, et al. The spot sign score in primary intracerebral hemorrhage identifies patients at highest risk of inhospital mortality and poor outcome among survivors. Stroke. 2010;41(1):54-60.

14. Fujii Y, et al. Multivariate analysis of predictors of hematoma enlargement in spontaneous intracerebral hemorrhage. Stroke. 1998;29(6):1160-6.

15. Broderick JP, et al. Intracerebral hemorrhage more than twice as common as subarachnoid hemorrhage. J Neurosurg. 1993;78(2):188-91.

16. Arakawa $S$, et al. Blood pressure control and recurrence of hypertensive brain hemorrhage. Stroke. 1998;29(9):1806-9.

17. Kranz PG, et al. Approach to imaging in patients with spontaneous intracranial hemorrhage. Neuroimaging Clin $\mathrm{N}$ Am. 2018;28(3):353-74.
18. Linn J, et al. Subarachnoid hemosiderosis and superficial cortical hemosiderosis in cerebral amyloid angiopathy. Am J Neuroradiol. 2008;29(1):184-6.

19. Greenberg SM, Charidimou A. Diagnosis of cerebral amyloid angiopathy: evolution of the Boston criteria. Stroke. 2018;49(2):491-7.

20. Hemphill JC 3rd, et al. The ICH score: a simple, reliable grading scale for intracerebral hemorrhage. Stroke. 2001;32(4): $891-7$.

21. Rost NS, et al. Prediction of functional outcome in patients with primary intracerebral hemorrhage: FUNC score. Stroke. 2008;39(8):2304-9. https://doi.org/10.1161/STROKEAHA.107. 512202 .

22. Brott T, et al. Early hemorrhage growth in patients with intracerebral hemorrhage. Stroke. 1997;28(1):1-5.

23. Vilela P. Imaging of cerebral venous and sinus thrombosis. In: Barkhof F, Jager R, Thurnher M, Rovira CA, editors. Clinical neuroradiology. Cham: Springer; 2019.

24. van Gijn J, Rinkel GJ. Subarachnoid haemorrhage: diagnosis, causes and management. Brain. 2001;124(2):249-78.

25. de Rooij NK, et al. Incidence of subarachnoid haemorrhage: a systematic review with emphasis on region, age, gender and time trends. J Neurol Neurosurg Psychiatry. 2007;78(12): 1365-72.

26. Zacharia BE, et al. Epidemiology of aneurysmal subarachnoid hemorrhage. Neurosurg Clin N Am. 2010;21(2):221-33.

Open Access This chapter is licensed under the terms of the Creative Commons Attribution 4.0 International License (http://creativecommons. $\mathrm{org} /$ licenses/by/4.0/), which permits use, sharing, adaptation, distribution and reproduction in any medium or format, as long as you give appropriate credit to the original author(s) and the source, provide a link to the Creative Commons license and indicate if changes were made.

The images or other third party material in this chapter are included in the chapter's Creative Commons license, unless indicated otherwise in a credit line to the material. If material is not included in the chapter's Creative Commons license and your intended use is not permitted by statutory regulation or exceeds the permitted use, you will need to obtain permission directly from the copyright holder. 


\title{
Intracranial Infection and Inflammation
}

\author{
Majda M. Thurnher and Pia C. Sundgren
}

\section{Learning Objectives}

- To explain how to use imaging to distinguish between infectious and non-infectious meningitis

- To introduce diagnostic algorithm for ring-enhancing brain lesions

- To discuss imaging findings in autoimmune encephalitis

- To review imaging features of CNS vasculitis

- To learn about IgG-related CNS disease

\section{Key Points}

- Thin, linear, diffuse meningeal enhancement suggests infectious meningitis, whereas in thick, nodular enhancement neoplastic origin should be suspected

- Perfusion MR imaging has a central role in differentiation of infectious and neoplastic ring-like enhancing brain lesions

- Use vessel wall MR imaging for evaluation of CNS vasculitis

- In unilateral/bilateral thick, dural enhancement suspect IgG-related pachymeningitis
M. M. Thurnher $(\varangle)$

Section of Neuroradiology and Musculoskeletal Radiology, Department of Biomedical Imaging and Image-guided Therapy,

University Hospital Vienna, Vienna, Austria

e-mail: majda.thurnher@meduniwien.ac.at

P. C. Sundgren

Institution for Clinical Sciences/Radiology, Lund University,

Lund, Sweden

e-mail: pia.sundgren@med.lu.se

\subsection{Meningitis}

CT and MR imaging findings in meningitis are nonspecific, with MR being superior to CT in the detection of meningeal pathology. Imaging findings in meningitis include: high signal intensity of subarachnoid spaces on FLAIR, leptomeningeal enhancement, subdural effusions, ventricular debris, and hydrocephalus, as well as high signal changes on diffusion-weighted MR imaging (DWI), and infarcts [1].

\section{Key Points}

- Use FLAIR and postcontrast 3D T2-FLAIR

- Be aware of different enhancement patterns

- Look for DWI lesions in suspected meningitis

\subsubsection{MR Imaging}

On fluid-attenuated inversion-recovery (FLAIR) sequences, a high signal will be present in the subarachnoid spaces, which reflects the high protein content in the cerebral spinal fluid (CSF). Differential diagnosis includes metastatic disease, subarachnoid hemorrhage, with the administration of oxygen and some sedation agents. Leptomeningeal enhancement can be best depicted with post-contrast 3D T2-FLAIR (Fig. 6.1a-c) [2].

\subsubsection{Enhancement Pattern}

Intracranial meningeal enhancement patterns are pachymeningeal (dura-arachnoid) or pia-subarachnoid (leptomeningeal). In a pachymeningeal enhancement pattern, the dura and the outer layer of the arachnoid will enhance. This pattern will be seen in intracranial hypotension, after 

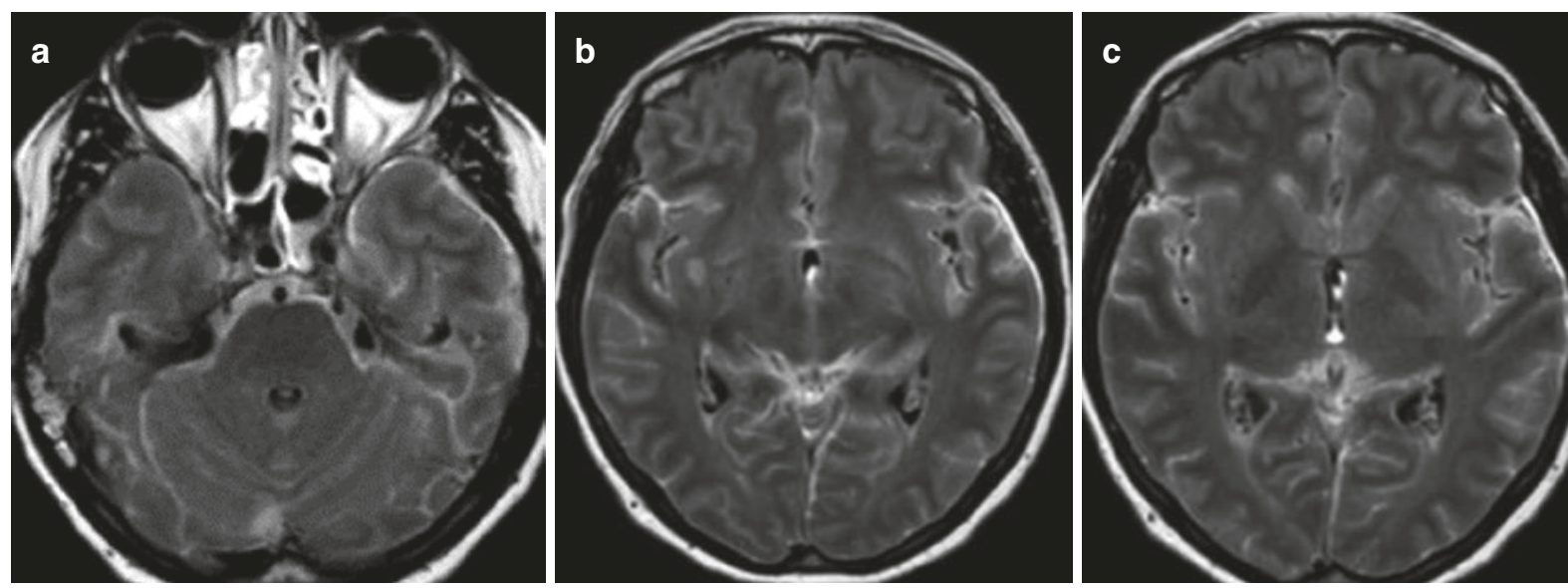

CE-FLAIR
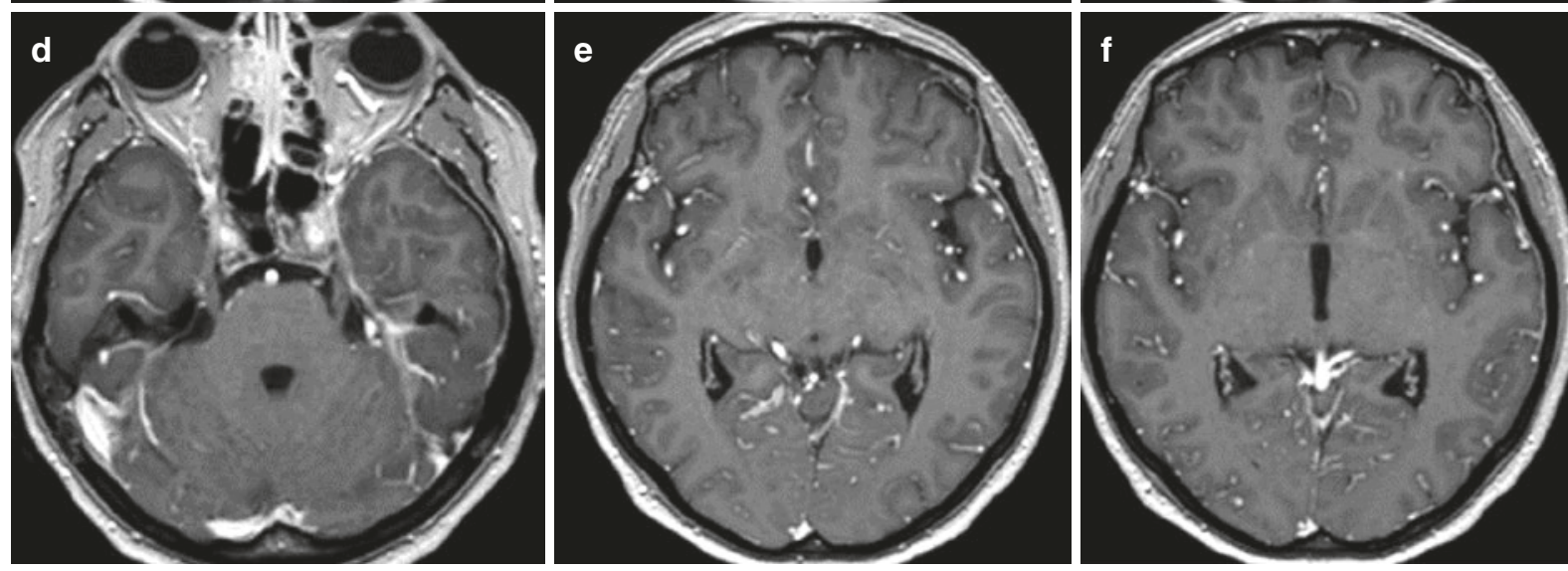

CE-T1WI

Fig. 6.1 Superiority of post-contrast 3D T2-FLAIR (a-c) to post-contrast T1WI $(\mathbf{d}-\mathbf{f})$ in the detection of leptomeningeal enhancement

surgery, granulomatous diseases, meningioma, and metastatic disease. Leptomeningeal enhancement follows the pial surface of the brain and fills the subarachnoid spaces of the sulci and cisterns. This pattern suggests an infectious origin (bacterial, viral, fungal), or, in some cases, carcinomatous meningitis. Thin, linear enhancement will be seen in meningitis, whereas thick, lumpy, nodular enhancement suggests carcinomatous origin (Figs. 6.2 and 6.3). Unilateral, thin, leptomeningeal enhancement may be due to non-infectious diseases, such as neurosarcoidosis, vasculitis, IgG-related pachymeningitis, and SturgeWeber syndrome (Fig. 6.2).

\subsubsection{Diffusion-Weighted MR Imaging (DWI) in Meningitis}

Hyperintense DWI lesions may be seen in several locations: (a) in the ventricles representing purulent fluid; (b) in the cortical sulci; and (c) in the Virchow-Robin spaces (VRSs) (Fig. 6.4).

\subsubsection{Tuberculous Meningitis}

\section{Key Points}

- Bilateral basal ganglia infarctions

- Thick leptomeningeal enhancement of the basal cisterns

- Hydrocephalus

- Multiple ring-enhancing lesions (tuberculomas)

- Cranial nerve enhancement and perineural spread

In tuberculous meningitis (TBM), FLAIR will show hyperintense subarachnoid spaces and the cisterns at the base of the brain (interpeduncular fossa, suprasellar, perimesencephalic, and pontine cisterns) with intense leptomeningeal enhancement on post-contrast T1WI or post-contrast FLAIR. In addition, surrounding brain edema will be recognized as a high signal on T2WI and FLAIR. In children, communicating or non-communicating hydrocephalus may occur. Spread of the infection to small vessels with arteritis 


\section{Enhancement pattern}

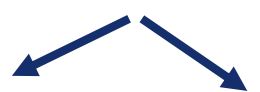

Pachymeningeal enhancement

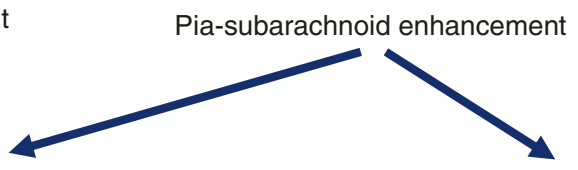

Thin, linear enhancement

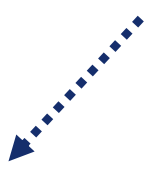

Bilateral, diffuse

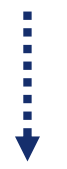

Meningitis

(bacterial, viral, fungal)

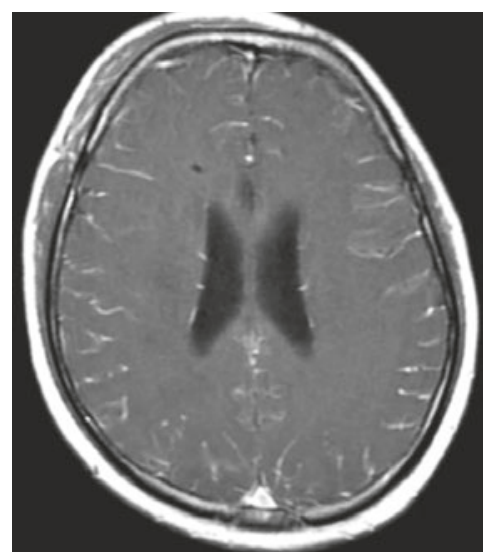

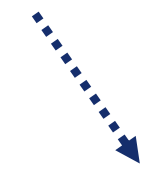

Unilateral

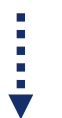

IgG-related pachymeningitis Sturge-Weber syndrome Neurosarcoidosis Vasculitis

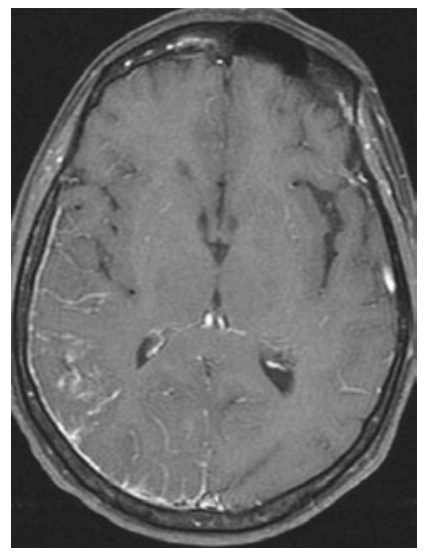

Thick, lumpy, nodular

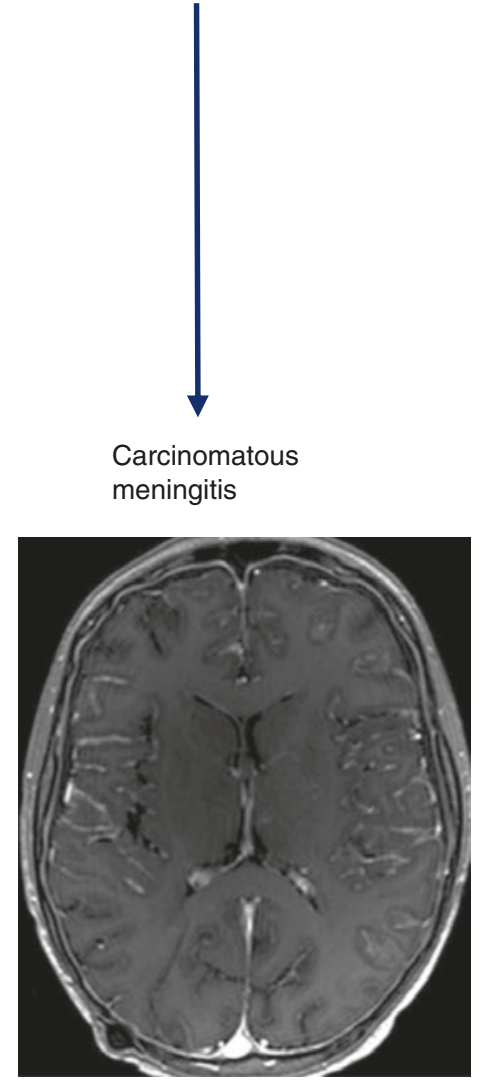

Fig. 6.2 Different enhancement patterns in meningeal diseases

and subsequent thrombosis will lead to arterial infarctions. Bilateral infarctions in the basal ganglia region and in the internal capsule on DWI should raise the suspicion of tuberculous meningitis. Narrowing or occlusion of the arteries may be seen on MR angiography (MRA). Cranial nerve involvement with thickened enhancing nerves is a common finding (Fig. 6.5).

\subsection{Brain Abscess}

A brain abscess is a focal infection of the brain that begins as a localized area of softening of the brain parenchyma (cerebritis), and develops into a collection of pus surrounded by a capsule.

\section{Key Points}

- Use MR perfusion to differentiate neoplastic from non-neoplastic, ring-enhancing brain lesions

- Use DWI \& SWI to further differentiate pyogenic from fungal abscesses

- Use MRS to differentiate tuberculomas from pyogenic abscesses and neoplasms

\subsubsection{MR Imaging of Brain Abscesses}

\subsubsection{T2-Wi}

Bacterial brain abscesses have a T2 high-signal-intensity center, a T2 low-signal-intensity capsule, prominent perifo- 
Fig. 6.3 Thin, linear enhancement $(\mathbf{a}, \mathbf{b})$ suggests infectious origin, whereas a thick, nodular enhancement will favor neoplastic diseases $(\mathbf{c}, \mathbf{d})$
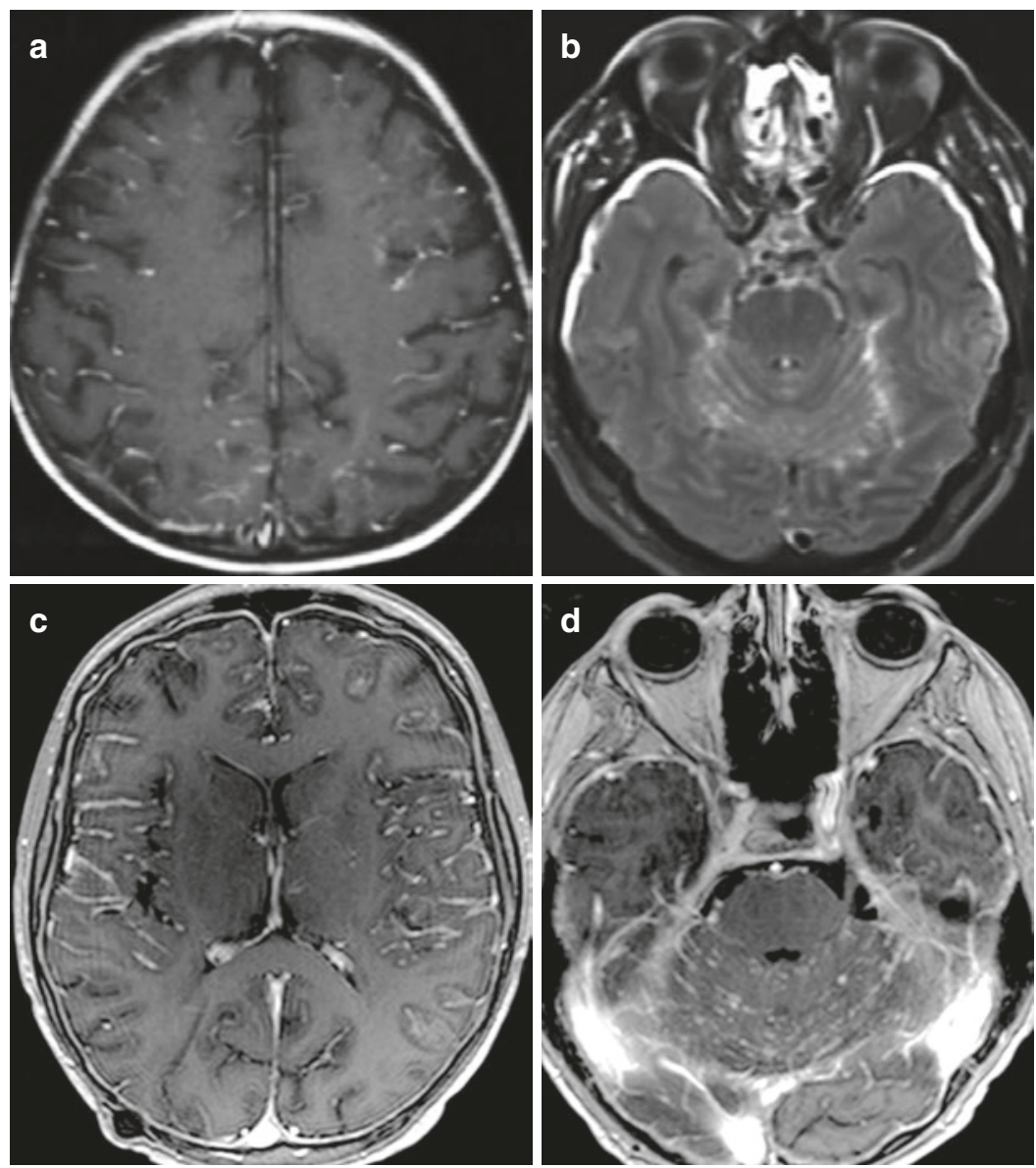

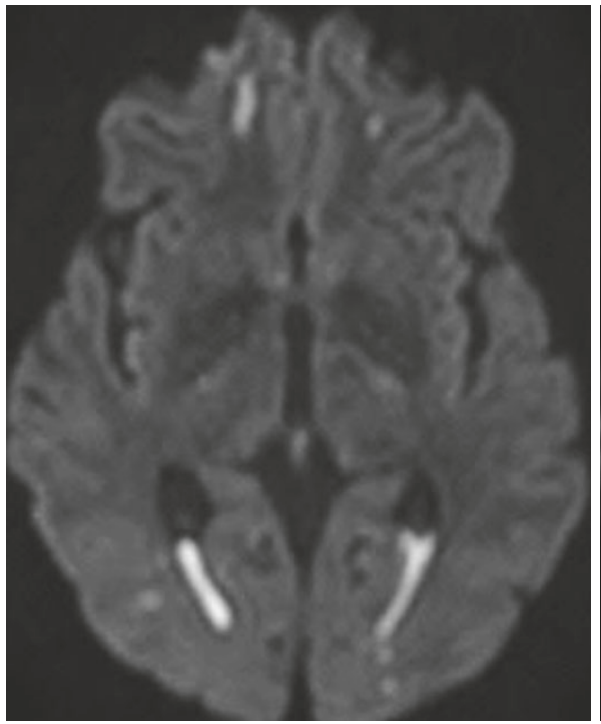

High signal in the ventricles (purulent fluid)

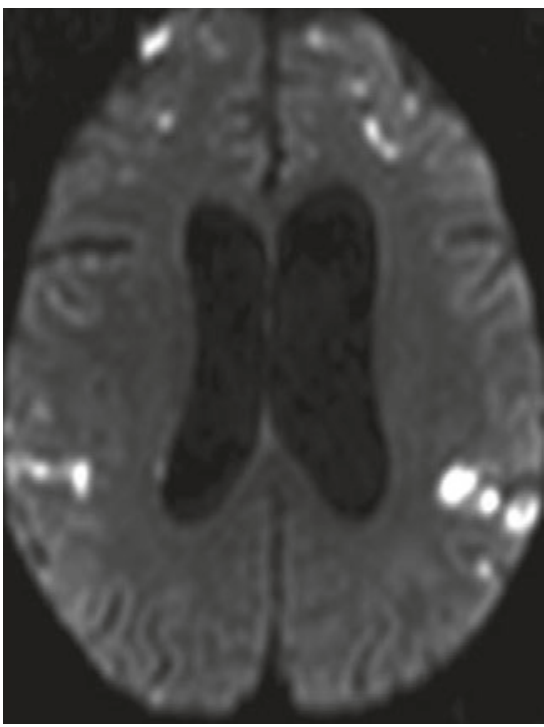

High signal in the subarachnoid spaces

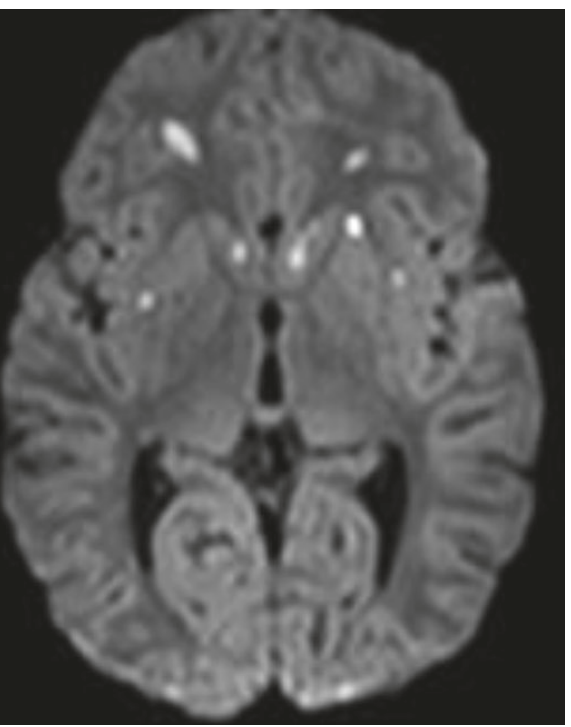

High signal in VR spaces

Fig. 6.4 Diffusion-weighted MRI abnormalities in meningitis

cal edema, and ring-like enhancement on post-contrast T1WI. Fungal abscesses usually demonstrate low T2 signal in the center. Parasitic abscesses, such as toxoplasma abscesses, may show centrally high or low signal.

\subsubsection{Diffusion-Weighted MR Imaging}

A high signal on DWI, with low ADC values in the pyogenic abscess cavity, is consistent with restricted diffusion due to the high viscosity pus. Fungal abscesses may show high, 

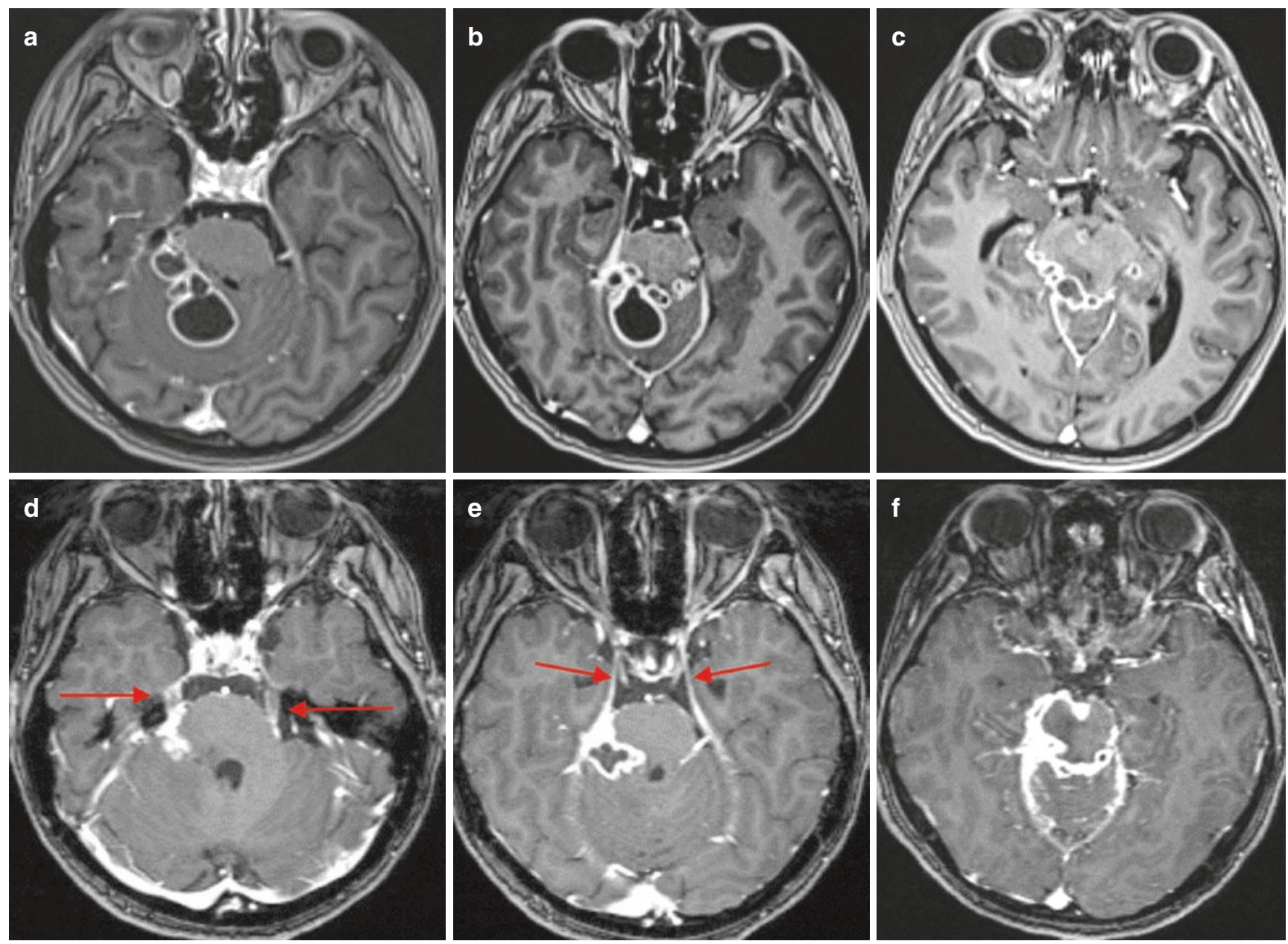

Fig. 6.5 Proven tuberculous meningitis with multiple ring-enhancing tuberculomas in the basal cistern (a-c). With disease progression, there was perineural spread with thickened and enhancing cranial nerves (d, e, red arrows)

low, or intermediate signals on trace DWI in the abscess cavity. Restricted diffusion may be seen in the walls of the fungal abscess called "intracavitary projections." High signal on DWI (with low ADC values) is due to fungal organisms located at the periphery of the lesions and mucoid material (Fig. 6.6).

\subsubsection{Susceptibility-Weighted MR Imaging (SWI)}

On susceptibility-weighted MR imaging (SWI), pyogenic brain abscesses show the "dual rim sign," (hypointense outer layer and hyperintense inner layer) [3]. Fungal abscesses do not show the dual rim sign, but rather, are recognized by the thick dark rim on SWI. In addition, fungal abscesses may have multiple, punctate, intralesional dark signals indicative of hemorrhage (common in Aspergillus infection) (Fig. 6.6) [4, 5].

In demyelinating lesions, which present as ring-enhancing lesions, SWI will demonstrate linear dark structures that run through the lesion, representing dilated veins. SWI can also be used to differentiate glioblastoma that presents as a peripheral or ring-enhancing mass from an infectious abscess. Glioblastoma shows dark dots and lines on SWI due to hemorrhage and neoangiogenesis.

\subsubsection{Perfusion MRI}

Perfusion MRI demonstrates low regional cerebral blood flow (rCBV) in all infectious abscesses regardless of etiological agents. Very rarely, in bacterial brain abscesses, high rCBV in the enhancing part will be detected due to the vascularization present in the early capsular stage. Neoplastic lesions (glioblastoma, metastases), as well as demyelinating lesions, may present as ring-like enhancing brain lesions, and can mimic a brain abscess. Perfusion will be useful as it will show increased rCBV in the enhancing part of neoplastic lesions, but low rCBV in demyelinating lesions (Fig. 6.6) $[1,5]$.

\subsubsection{MR-Spectroscopy}

To distinguish between aerobic and anaerobic pyogenic abscesses, MR spectroscopy (MRS) can be used. Succinate and acetate signal resonances suggest an anaerobic agent. In fungal abscesses lipids, lactate, amino acids, and trehalose (3.6-3.8 ppm) are commonly detected. 
Ring-like enhancing lesion
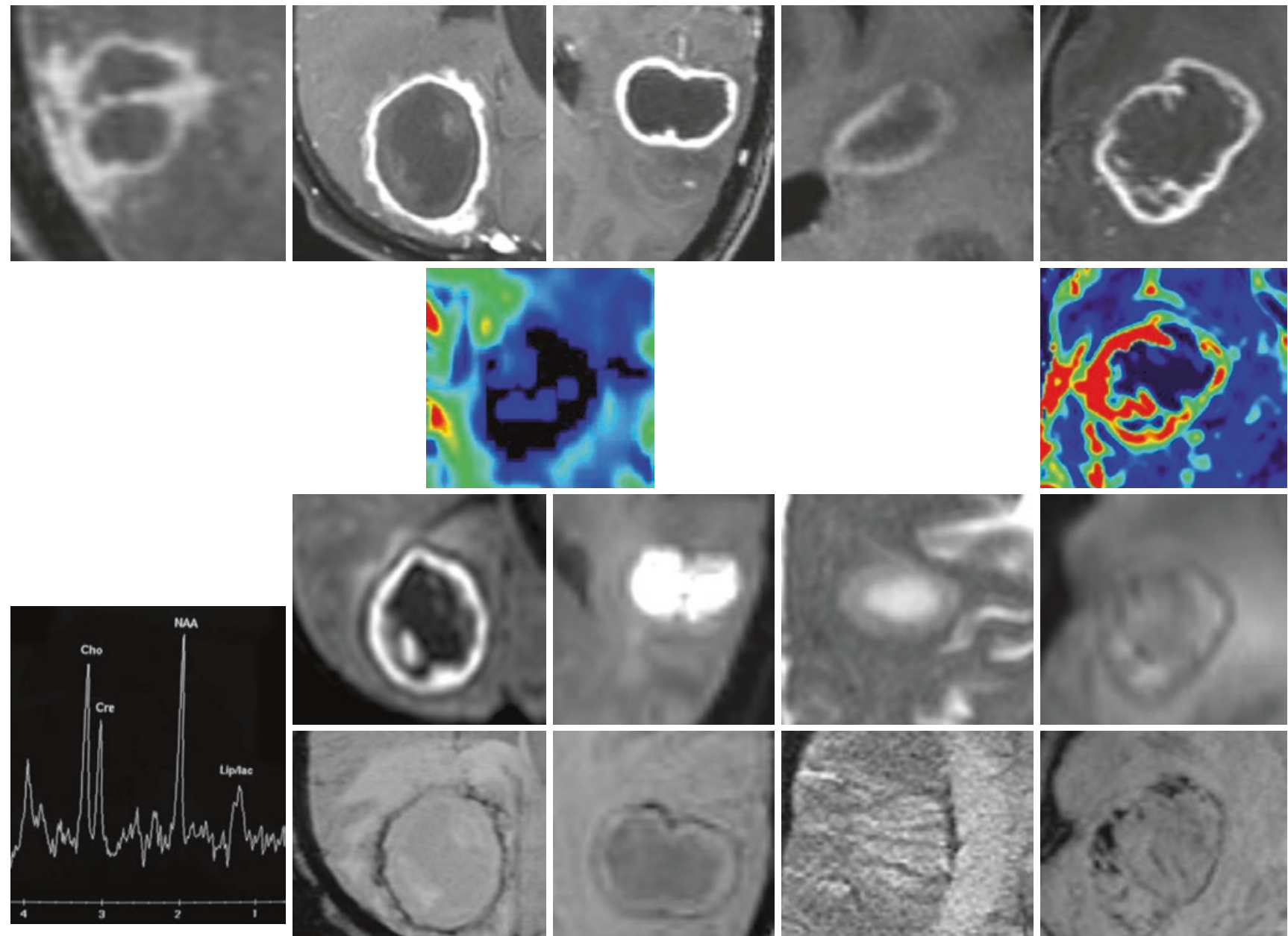

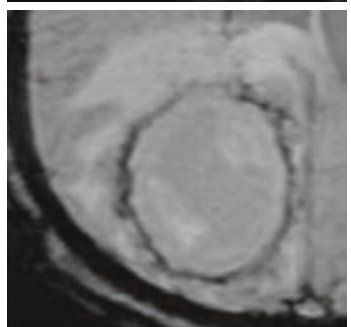

Fungal

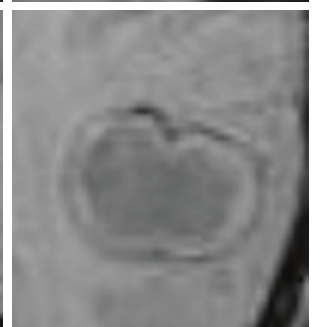

Bacterial

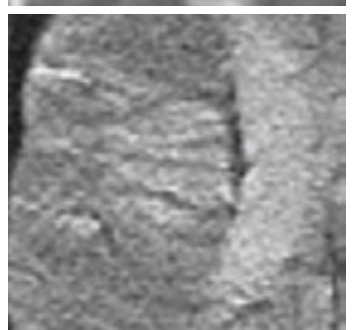

MS

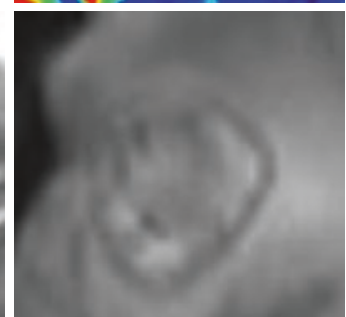

Tuberculosis

Fig. 6.6 Diagnostic scheme for differentiation of ring-enhancing brain lesions

\subsubsection{Tuberculoma and Tuberculous Abscesses}

Tuberculous granuloma (tuberculoma) is the most common parenchymal form of CNS TB. The imaging features depend on the stage of infection. Noncaseating tuberculomas have a low T1WI and a high T2WI signal and show nodular enhancement. Caseating tuberculomas demonstrate a low T1- and T2-WI signal with ring-like enhancement. DWI characteristics will also depend on the stage and content; caseating tuberculomas with a T2 high-signal-intensity center show restricted diffusion. Caseating tuberculomas with a T2-lowsignal-intensity center have elevated diffusion. MRS can also be used to differentiate pyogenic abscesses from tuberculous lesions (a large lipid peak will be found in T2-hypointense caseating tuberculomas, and large choline and lipid peaks will be detected in T2-hyperintense tuberculomas) (Fig. 6.6).

Tuberculous abscesses are large, multiloculated masses, with thick enhancement and edema with mass effect.

\subsection{Autoimmune Encephalitis}

The term "autoimmune encephalitis" generally refers to a family of closely related disease processes that share overlapping clinical features and neuroimaging findings, but are ultimately differentiated by the specific antibody subtypes driving the underlying immune-mediated attack on different CNS structures [6-8].

Autoimmune encephalitis can be classified into two major groups: paraneoplastic and non-paraneoplastic.

\section{Key Points}

- New-onset altered mental status-consider an autoimmune cause!

- LOOK at the limbic system, basal ganglia, cerebellum, brainstem

- Contrast enhancement or diffusion restriction is rarely present

- No hemorrhage 
A subset of patients with autoimmune encephalitis will have no neuroimaging findings despite profound neuropsychiatric dysfunction. Serum antibody testing will ultimately lead to the diagnosis of autoimmune encephalitis.

${ }^{18}$ FDG-PET could be a helpful alternative with which to narrow the diagnosis when MRI and biological tests are negative. FDG-PET was reportedly more sensitive for AE compared to EEG, MRI, or routine CSF findings. Hypometabolism was found in the cortex, whereas hypermetabolism was seen in the basal ganglia.

$N$-methyl D-aspartate receptor encephalitis (NMDAr) is one of the most common and best characterized subtypes of autoimmune encephalitis, classically seen in young women and children. It can be paraneoplastic (associated with ovarian cancer) or non-paraneoplastic. Over half of MRIs performed in anti-NMDA receptor encephalitis show NO abnormalities. T2/ FLAIR hyperintensity of the medial temporal lobe (Fig. 6.7),
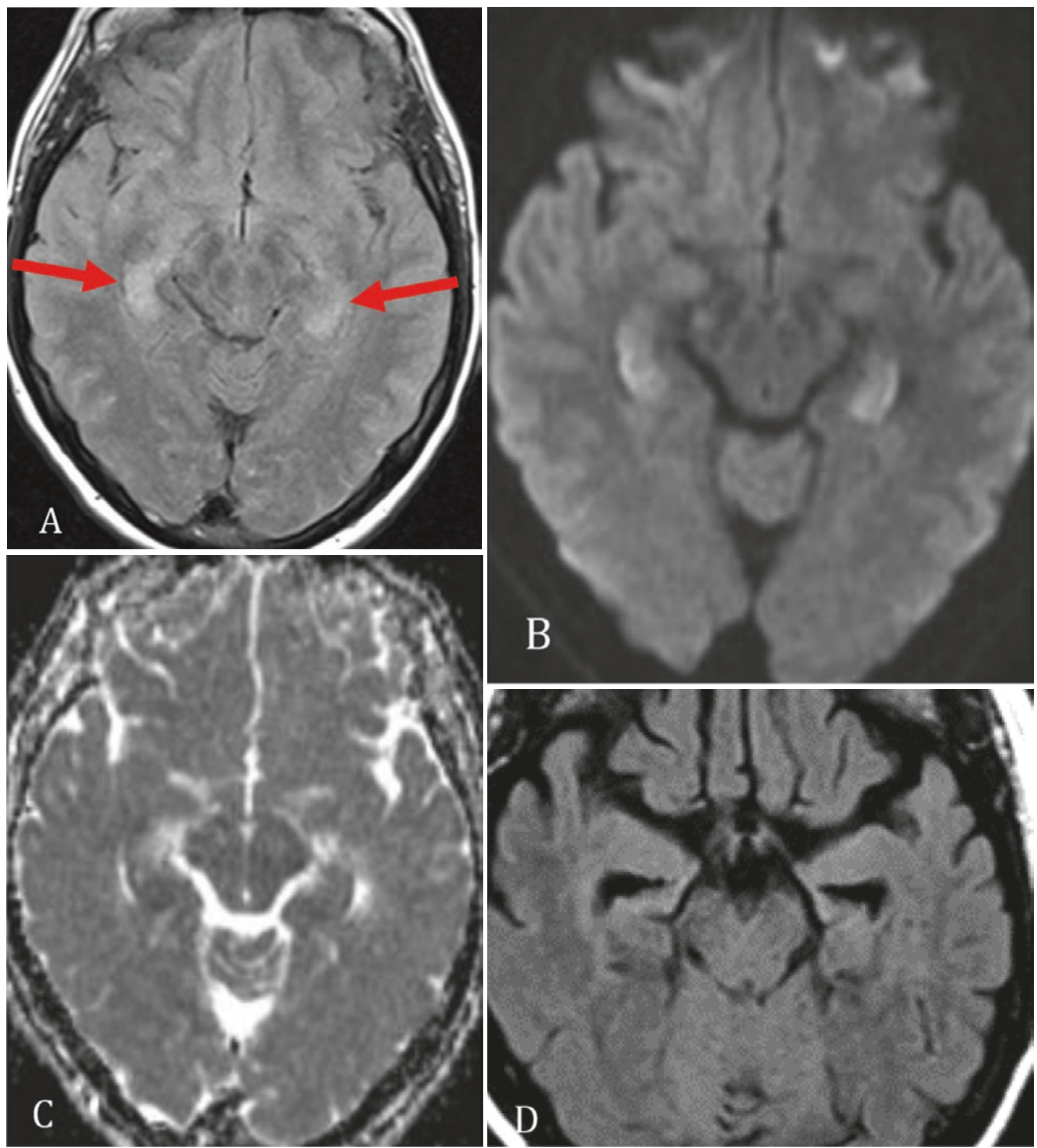

Fig. 6.7 Axial FLAIR (a) shows bilateral high signal in the hippocampus. On trace DWI (b), high signal is detected with a subtle decrease in ADC values (c). On follow-up MR, bilateral hippocampal atrophy is observed (d) 

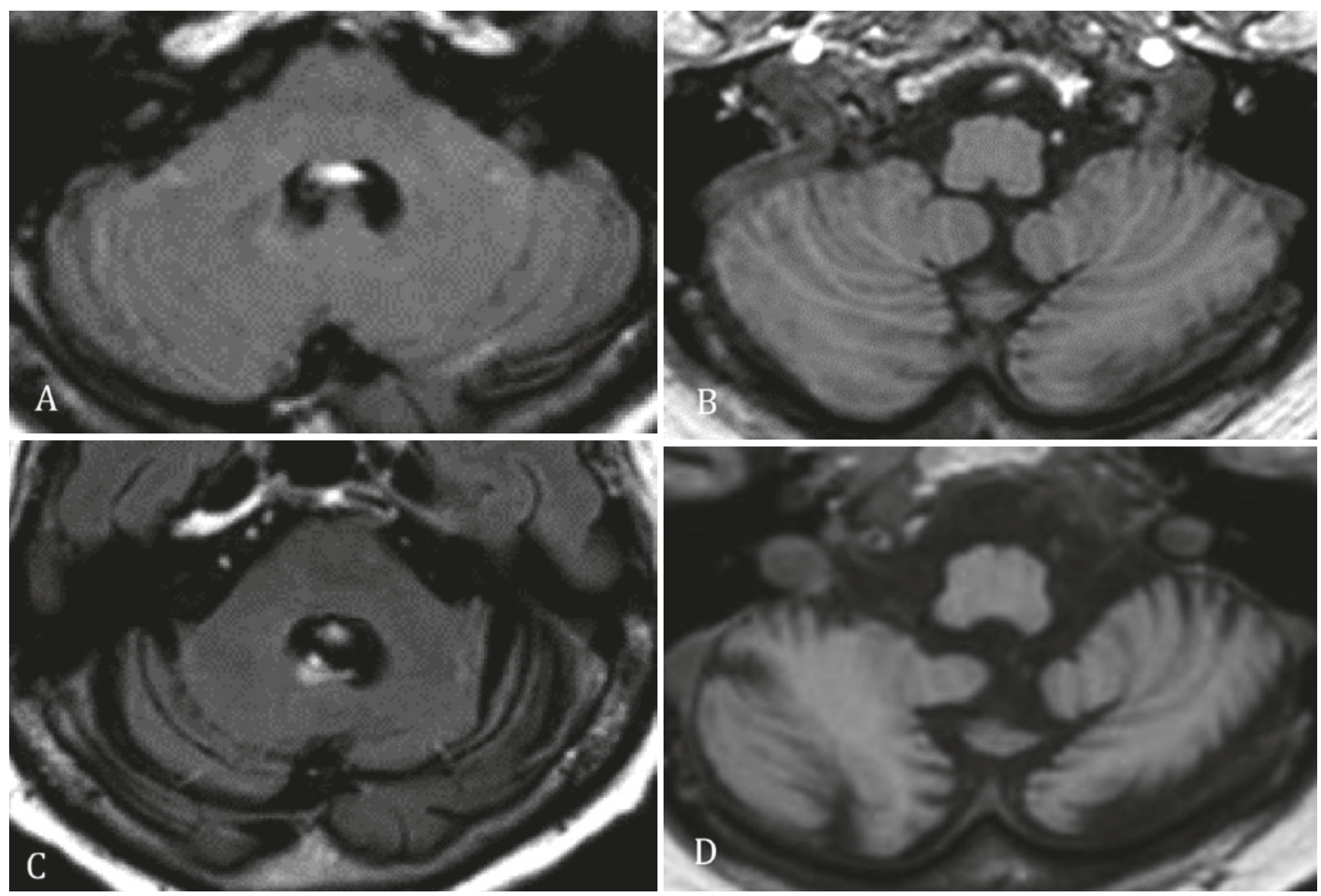

Fig. 6.8 Initial MR exam in a patient with proven anti-Yo encephalitis $(\mathbf{a}, \mathbf{b})$. Follow-up MR 2 years later shows marked cerebellar atrophy $(\mathbf{c}, \mathbf{d})$

frontal lobe, subcortical WM, and periventricular region have been described. Occasionally, leptomeningeal and cortical contrast enhancement will be detected.

Anti-neuronal nuclear antibody $1($ Anti-Hu) encephalitis is associated with lung cancer and is usually associated with a bad prognosis. It will less frequently be recognized as limbic encephalitis, and usually affects the brainstem.

Anti-Yo is associated with ovarian and breast cancer, and it typically presents with paraneoplastic cerebellar degeneration (Fig. 6.8).

\subsection{Neurosarcoidosis}

Neurosarcoidosis is a multisystem granulomatous disease with sarcoid-type granulomas and epithelial cells and its diagnosis is based on the clinical determination of multisystem diseases in combination with histologic confirmation.

\section{Key Points}

- Perform complete MRI including T1 after contrast administration, FLAIR, T2, DWI, and SWI

- Be aware of possible spine lesions

- Think of neurosarcoidosis when several different brain compartments are involved

- Differential diagnosis: meningitis, tuberculosis, leukemia, lymphoma, and vasculitis, among others

Neurosarcoidosis has been described in $5 \%$ of patients with sarcoidosis. The disease might present with a variety of clinical presentations, such as cranial neuropathy, encephalopathy, meningitis, hydrocephalus, seizures, as well as spinal cord abnormalities and peripheral neuropathy and myopathy.

Typical imaging findings, although nonspecific, include dural thickening or dural masses, leptomeningeal involvement (Fig. 6.9), enhancing and non-enhancing parenchymal lesions (Figs. 6.10 and 6.11), and cranial nerve involvement (Fig. 6.12). The latter is the most common finding. Spine 

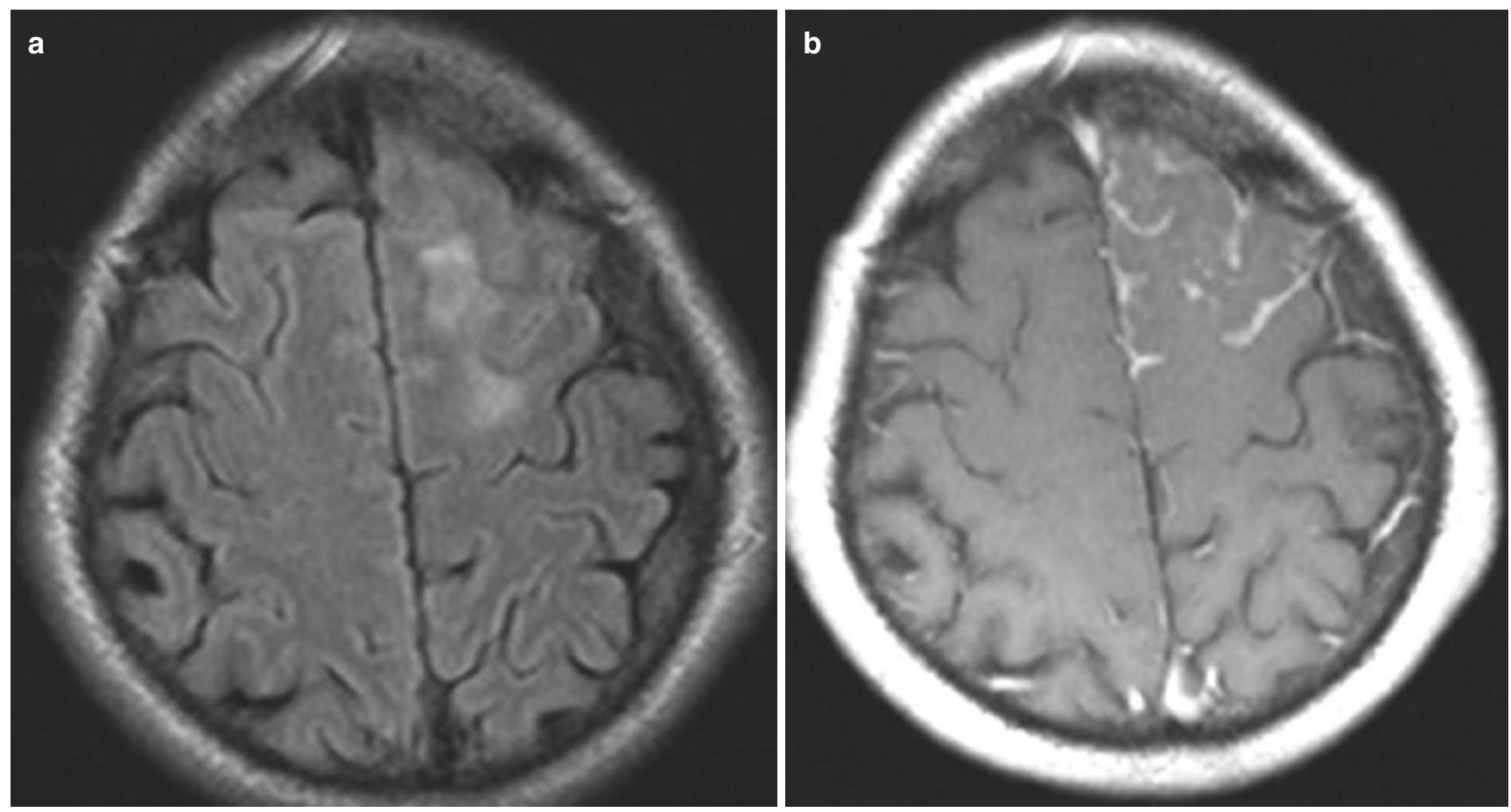

Fig. 6.9 Axial FLAIR (a) and T1-weighted post-contrast administration (b) demonstrate diffuse, focal, increased FLAIR signal in the underlying brain parenchyma and focal left-sided leptomeningeal enhancement in a patient with neurosarcoidosis
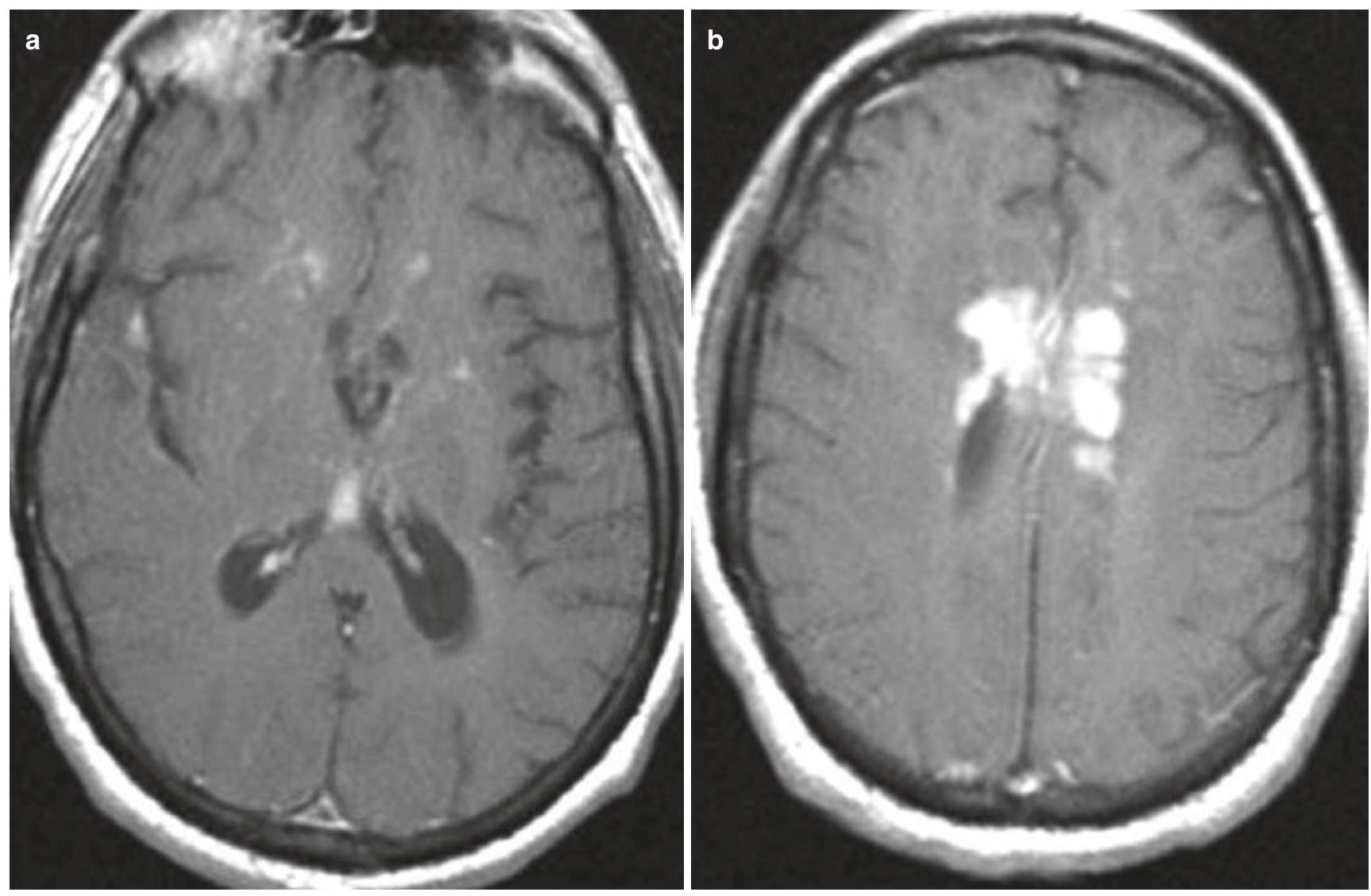

Fig. 6.10 Axial post-contrast-enhancing T1-weighted images $(\mathbf{a}, \mathbf{b})$ demonstrating focal periventricular granulomatous contrast-enhancing masses 

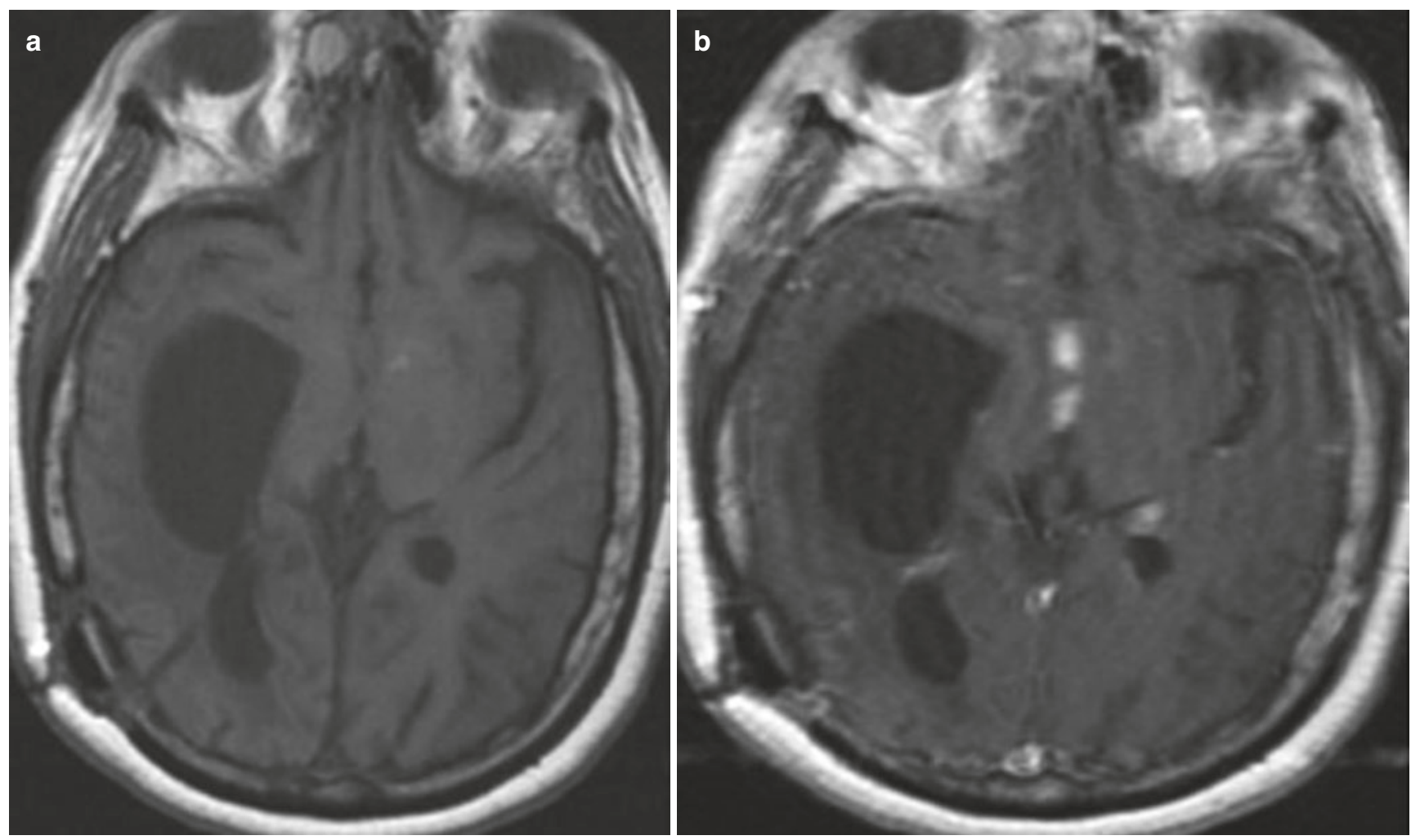

Fig. 6.11 Axial pre- (a) and post-contrast T1-weighted (b) images demonstrating basal granulomatous contrast-enhancing mass lesions obstructing the CSF circulation and causing secondary hydrocephalus
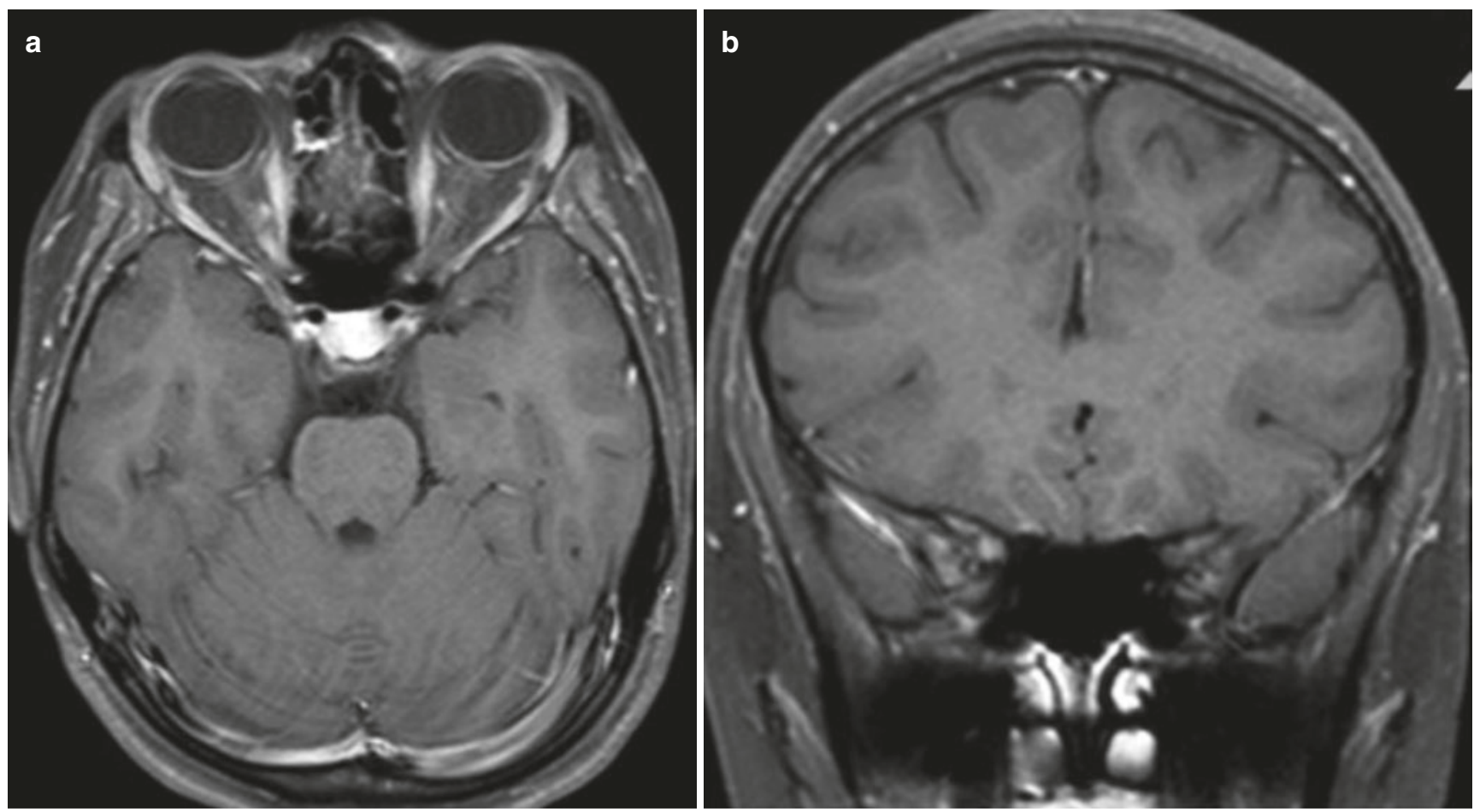

Fig. 6.12 Axial (a) and coronal (b) post-contrast T1-weighted images showing thickening and pathological contrast enhancement in the posterior pre-chiasmatic portion of the right optic nerve 

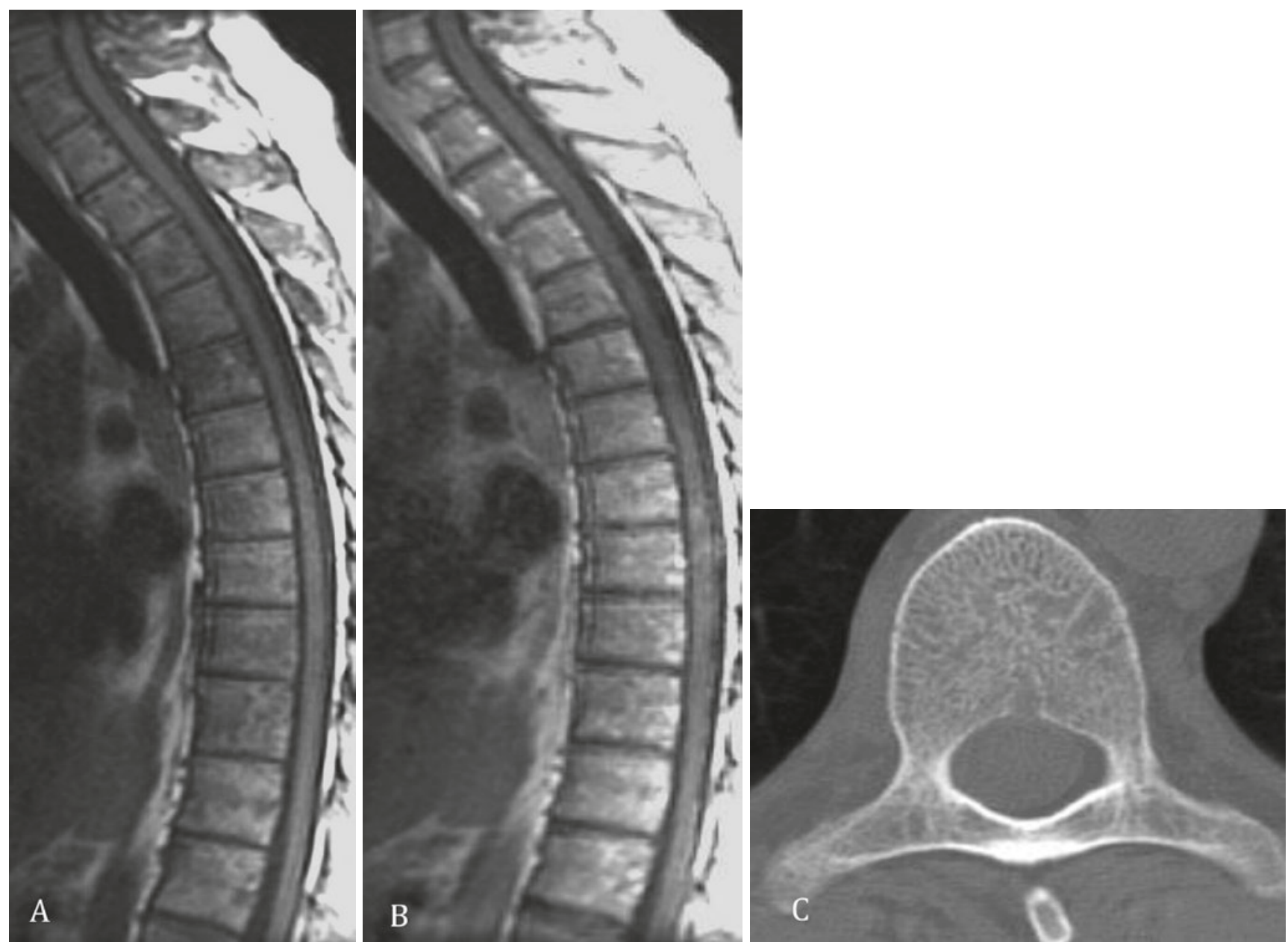

Fig. 6.13 Sagittal pre- (a) and post-contrast-enhancing T1-weighted (b) images over the thoracic spine demonstrating lesions in the vertebral bodies and discrete contrast enhancement in the spinal cord at the level of the mid-thoracic region. Axial CT (c) demonstrates the vertebral lesions

involvement may exist, even less frequently, such as lesions in the vertebral body, spinal cord lesions, and spinal and nerve root enhancement (Fig. 6.13).

Common radiological imaging methods include CT and MRI. Imaging findings on CT are, in general, nonspecific and might include hydrocephalus, periventricular hypoattenuation and contrast enhancement, calcification, meningeal contrast enhancement, focal white matter lesions, and lesions in the optic nerves or chiasm. The imaging modality of choice for the evaluation of suspected neurosarcoidosis is MRI. The protocol should include FLAIR/T2-weighted, T1-weighted preand post-contrast administration, DWI and T2*, or SWI. MRI can better depict leptomeningeal abnormalities and lesions in the brain parenchyma, as well as demonstrate the cranial nerve involvement, which often presents as cranial nerve thickening and enhancement. The most common nerves involved are the optic nerve and the facial nerves. There may also be involvement of the pituitary gland. Spinal involvement, both of the vertebra and the spinal cord, is better seen with MRI. It should be noted that MRI can be normal in patients with neurosarcoidosis, especially in patients on corticosteroids or if the only presenting symptom is cranial neuropathy.

\subsubsection{Leptomeningeal Involvement}

Leptomeningeal involvement occurs in up to $40 \%$ of patients with neurosarcoidosis. It might be focal, multifocal, or diffuse, or even mass-like, and present as linear or nodular enhancement along the brain surface and basal regions. It is best depicted after contrast enhancement (Fig. 6.9). Differential diagnosis for leptomeningeal enhancement should include meningeal carcinomatosis, lymphoma, leukemia, and fungal and bacterial infections.

\subsubsection{Dural or Focal Mass Lesions}

The granulomatous lesions might, if located in the basal regions or adjacent to the ventricles, cause obstruction of the CSF (cerebral spinal fluid) circulation and lead to hydrocephalus (Fig. 6.10). The dural lesions are usually isointense, appearing as a gray mass on T1 sequences of MRI and hypointense on T2 sequences of MRI. Potential differential diagnosis includes meningiomas, metastases, plasmacytoma, and granulomatous infections. 


\subsubsection{Cranial Nerve Involvement}

The involvement of the cranial nerves, most commonly the optic nerve and the fascial nerves, might be demonstrated as thickening and enhancement of the involved nerves, best seen on T1-weighted post-contrast administration and on T2-weighted images (Fig. 6.12a, b). Here, the 3D-CISS sequences might be of value for better depiction of the nerves. Note that several nerves might be involved.

\subsubsection{Spine, Spinal Cord, and Spinal Nerve Involvement}

Spine and spinal cord involvement are generally uncommon in neurosarcoidosis, but do occur both in combination with other CNS involvement or as an isolated finding. Such involvement may present as leptomeningeal enhancement, pachymeningeal, or intramedullary lesions and rarely involves the vertebral bodies. Typical vertebra lesions can present as focal or diffuse lesions in the vertebral bodies and be misdiagnosed as myeloma, lymphoma, or metastasis. Involvement of the spinal cord and spinal nerve roots might present as focal lesions with low signal intensity on $\mathrm{T} 1$, high signal intensity on $\mathrm{T} 2$, and nodular enhancement on contrast administration. The increased signal on $\mathrm{T} 2$-weighted images compared to that of the gray matter is suspected to be related to inflammation and edema. In addition, leptomeningeal or focal contrast enhancement can be seen along or in the spinal cord or along the nerve roots on T1-weighted images after contrast administration. The intramedullary lesions will normally demonstrate slightly increased diffusivity with correspondingly increased values at the ADC map image, reflecting vasogenic edema and inflammation, even if iso- to high signal intensity with normal to slightly increased diffusion on DWI have been reported in a limited number of patients. Cervical or thoracic involvement is more common than lumbosacral. There is a female predominance for spinal involvement. Potential differential diagnosis for vertebral body involvement in neurosarcoidosis includes, for example, myeloma, lymphoma, and metastasis.

\subsection{IgG-Related Diseases}

IgG-related diseases encompass a spectrum of fibroinflammatory disorders characterized by IgG4-positive plasma cell infiltration that can affect almost every organ system. They respond well to corticosteroid therapy.

CNS involvement is rare.

\section{Key Points}

- Thick, dural enhancement-THINK IgG-related pachymeningitis

- Brain edema due to compression may occur

- Rarely may present with leptomeningeal enhancement

IgG-related pachymeningitis is a rare manifestation of the IgG4-related fibroinflammatory disease spectrum. Inflammation leads to a localized or diffuse thickening of the meninges overlying the supratentorial hemispheres, skull base, or spinal cord. The symptoms are related to mass effect, or focal deficits caused by the compression of blood vessels or nerves. This was previously described as idiopathic, hypertrophic, cranial pachymeningitis (IHCPM). Tissue biopsy is the gold standard for diagnosis in most settings (Fig. 6.14).

\subsection{Vasculitis}

Vasculitis is defined as an inflammation of the vessel wall. The classification of CNS vasculitis can be divided into primary systemic vasculitides and CNS vasculitis associated with other underlying disorders. The inflammation of the vessel wall can affect vessels of various sizes, i.e., small, medium, and large vessels or can affect all the vessels, regardless of size.

\section{Key Points}

- Vasculitis presents with various imaging findings, some quite nonspecific

- The imaging protocol preferably should include vessel wall imaging (VWI), DWI, and pre- and post-contrast T1-weighted images

- MRA, CTA, or conventional digital subtraction angiography demonstrate a "string-of-bead" appearance of the intracranial vessels

Giant cell arteritis, Takayasu's arteritis, granulomatosis with polyangiitis (GPA), Churg-Strauss syndrome, Kawasaki disease, and primary angiitis of the CNS (PACNS) are considered primary vasculitides. Nervous system vasculitis can also be associated with connective tissue disorders, like systemic lupus erythematosus (SLE), Sjögren's disease, rheumatoid arthritis (RA), lymphoproliferative diseases, malignancies, drug and substance abuse, or infections. The diagnosis of vasculitis is a combination of symptoms, as well as clinical and laboratory findings, and imaging. Still, today, brain biopsy is considered the gold standard for the final diagnosis of vasculitis. 

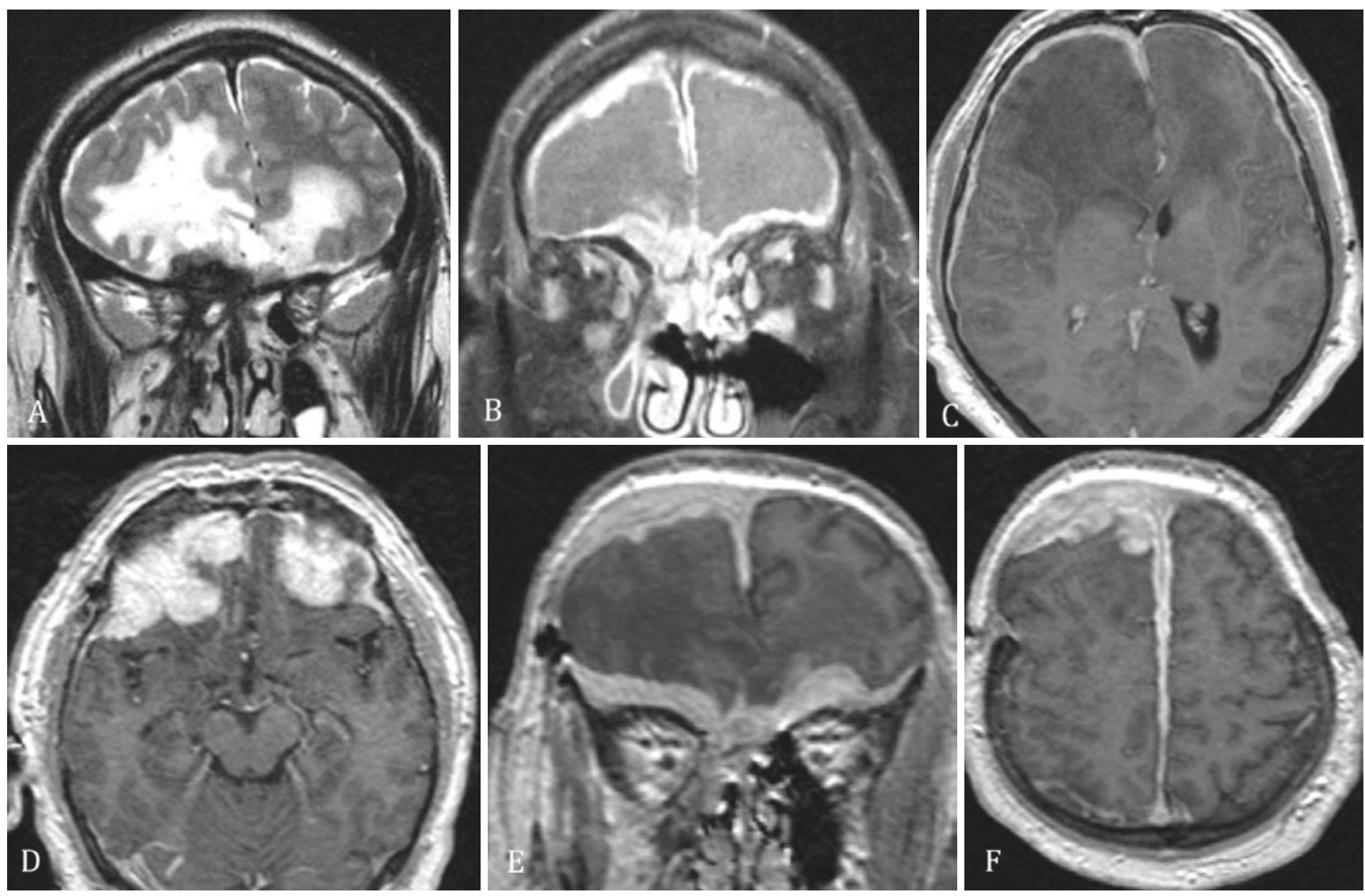

Fig. 6.14 Thick, dural enhancement is observed bilaterally frontally and along the falx (b-f). T2-high signal intensity is also seen in the parenchyma of both frontal lobes, reflecting compression of the brain parenchyma (a)

\subsubsection{Imaging in Vasculitis}

The MR imaging protocol shall include T1-weighted images pre- and post-contrast, FLAIR/T2-weighted, DWI, and, for the evaluation of microbleeds, SWI or T2*-w, MRA-3D TOF, and, if possible, vessel wall imaging (VWI) [9-11].

If MRI is inconclusive, digital subtraction angiography can be added to the radiological work-up.

The MR imaging findings in patients with vasculitis may vary depending on the vessels involved. The examination can be normal and include different findings, such as acute ischemic changes, old infarcts, nonspecific focal or multiple hyperintense white matter lesions, confluent white matter lesions, patchy or linear contrast enhancement, leptomeningeal contrast enhancement, and brain atrophy (Fig. 6.15). Microbleeds are rare in contrast to, for example, amyloid angiopathy, a common differential diagnosis. On MR angiography (3D TOF) shows multiple, focal, irregular stenosis of more than one of the medium or large vessels (small vessels are not visualized on MRA) (Figs. 6.16 and 6.19b). The digital subtraction angiography might demonstrate focal stenosis and the string-of-bead sign in various intracranial vessels (Figs. 6.17, 6.18 , and $6.19 \mathrm{c}$ ).

\subsubsection{Primary Angiitis of the CNS (PACNS)}

Primary angiitis of the CNS (PACNS) is a rare disease of unknown etiology with a predominance in males compared to females (7:3). Imaging will demonstrate irregularities in the peripheral vessels that results in acute ischemic changes that are secondary to the inflammation of the vessel wall, stenosis, or obstruction of the vessel lumen, with increased local coagulation and alterations in vasomotor tone. A differential diagnosis for PACNS includes the reversible cerebral vasoconstriction syndrome (RCVS). RCVS more commonly affects women and is characterized by severe headaches (thunderclap headache) with or without focal neurologic deficits and/or seizures. In RCVS, the segmental constriction of cerebral arteries resolves within 3 months.

\subsubsection{Granulomatosis with Polyangiitis (GPA)}

Granulomatosis with polyangiitis (GPA) (previously called Wegener's granulomatosis) is a systemic disease of small and medium vessels with an unclear pathogenesis. The disease primarily affects the upper and lower respiratory airways, joints, eyes, skin, heart, liver, glands, and, in $25-50 \%$, the peripheral 

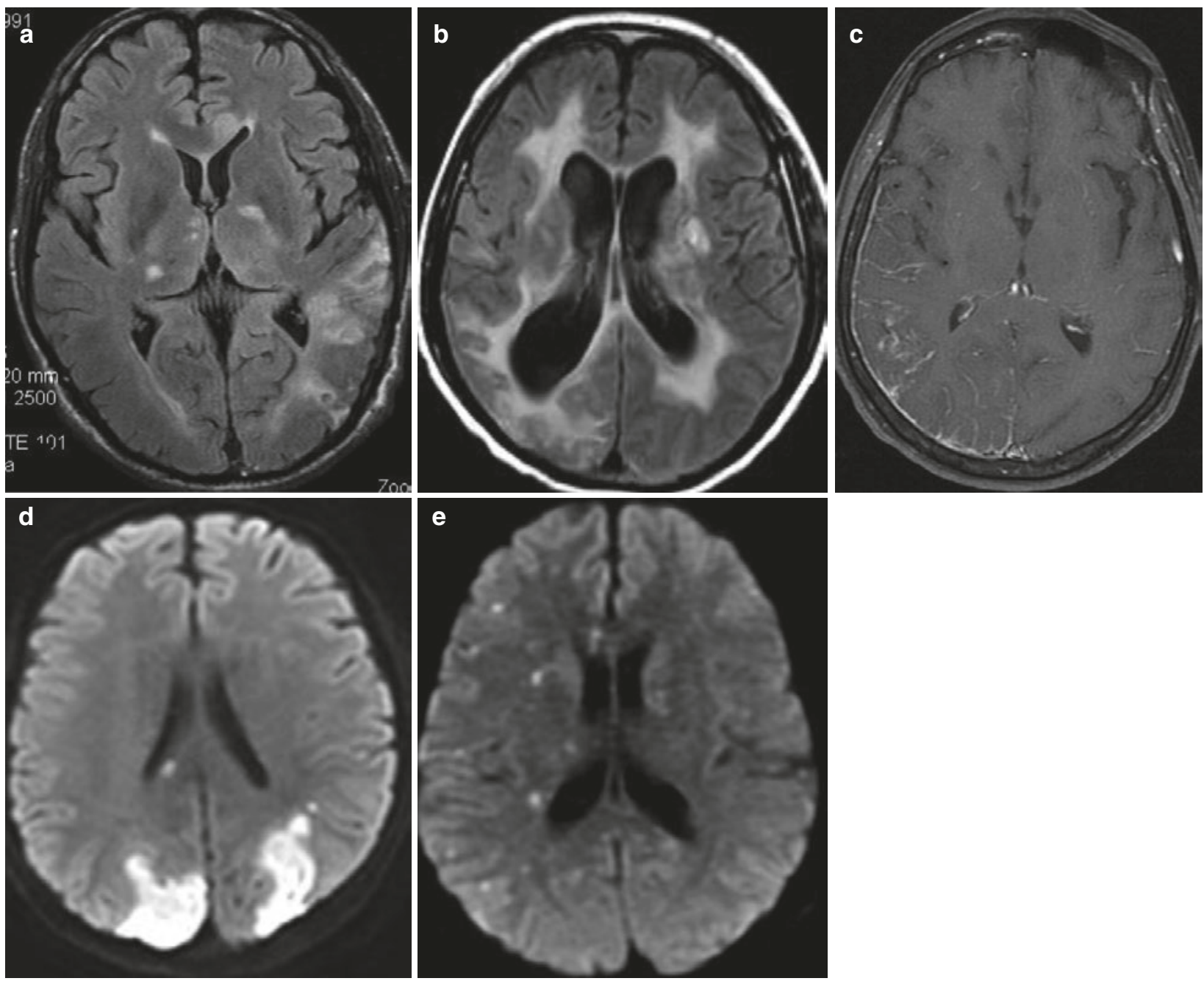

Fig. 6.15 Different imaging findings in patients with known vasculitis; (a) hyperintense FLAIR lesions, (b) confluent white matter signal abnormalities, (c) leptomeningeal contrast enhancement, (d) cortical infracts, (e) multiple acute lacunar infarcts

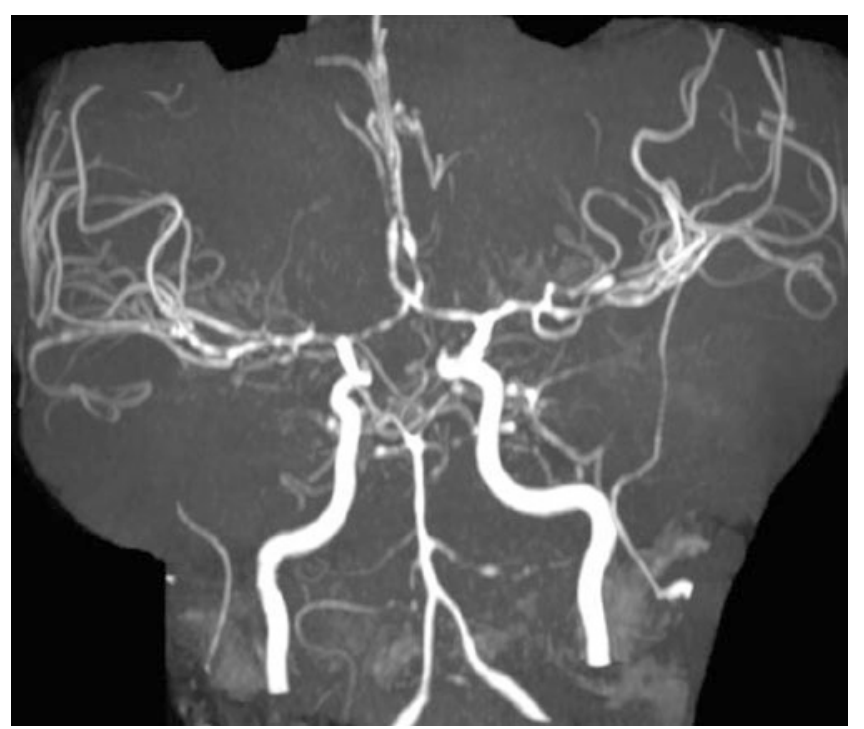

Fig. 6.16 MRA 3D TOF demonstrating bilateral multiple focal stenosis of the anterior and middle cerebral arteries

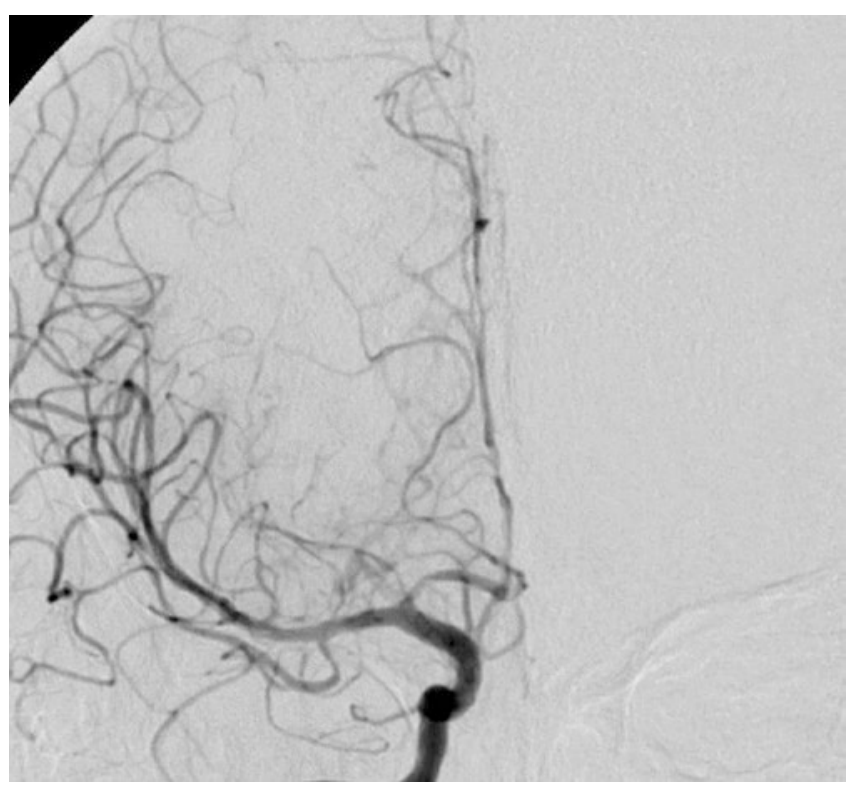

Fig. 6.17 Coronal view after right, selective, internal carotid artery injection, demonstrating focal stenosis in the right pericallosal artery 

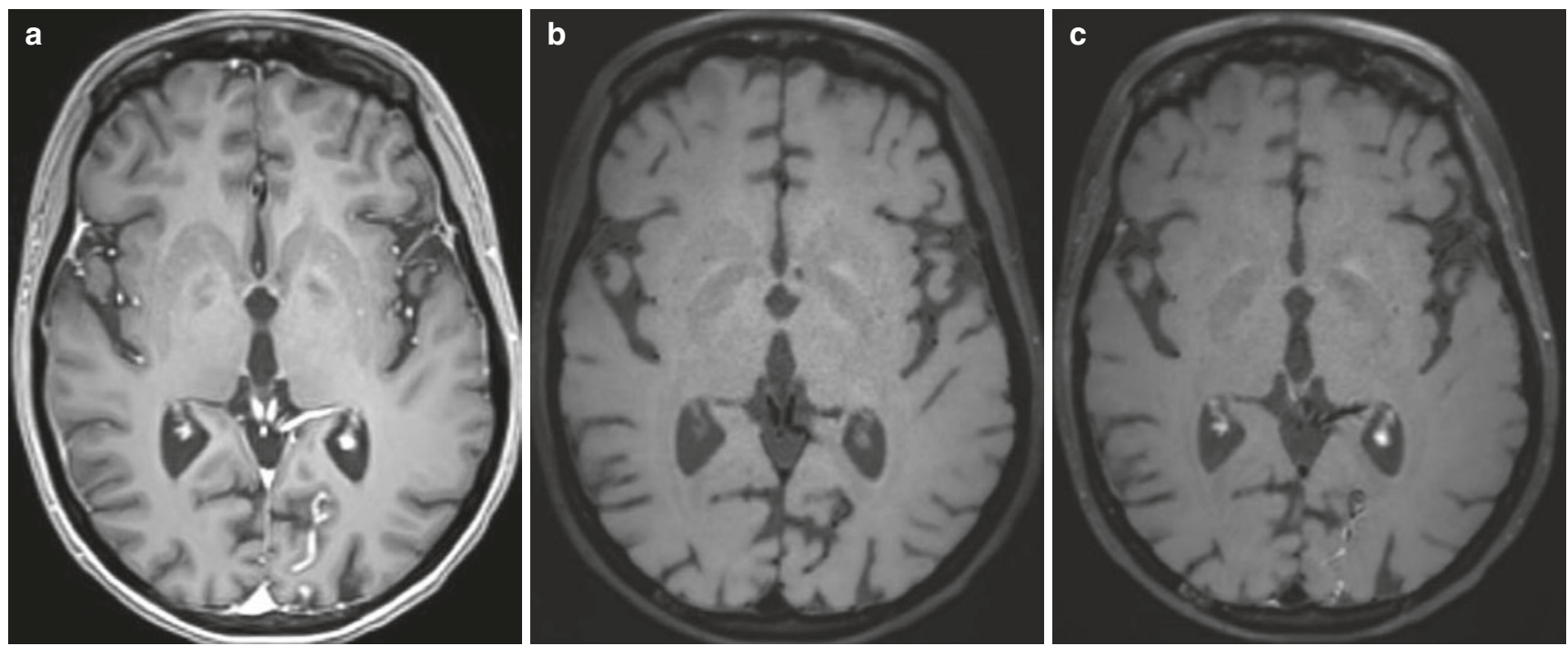

Fig. 6.18 On post-contrast T1wi, enhancement of the branches of the left posterior cerebral artery is noted (a). Intense enhancement of the vessel wall on black-blood sequence $(\mathbf{b}, \mathbf{c})$ suggests vasculitis

Fig. 6.19 Axial FLAIR (a) demonstrates focal and confluent areas of increased signal in the periventricular and deep white matter, (b) focal stenosis and lumen obstruction of the middle and anterior cerebral arteries, and (c) multiple focal stenosis of several intracranial vessels peripherally, on sagittal DSA, in a patient with PACNS
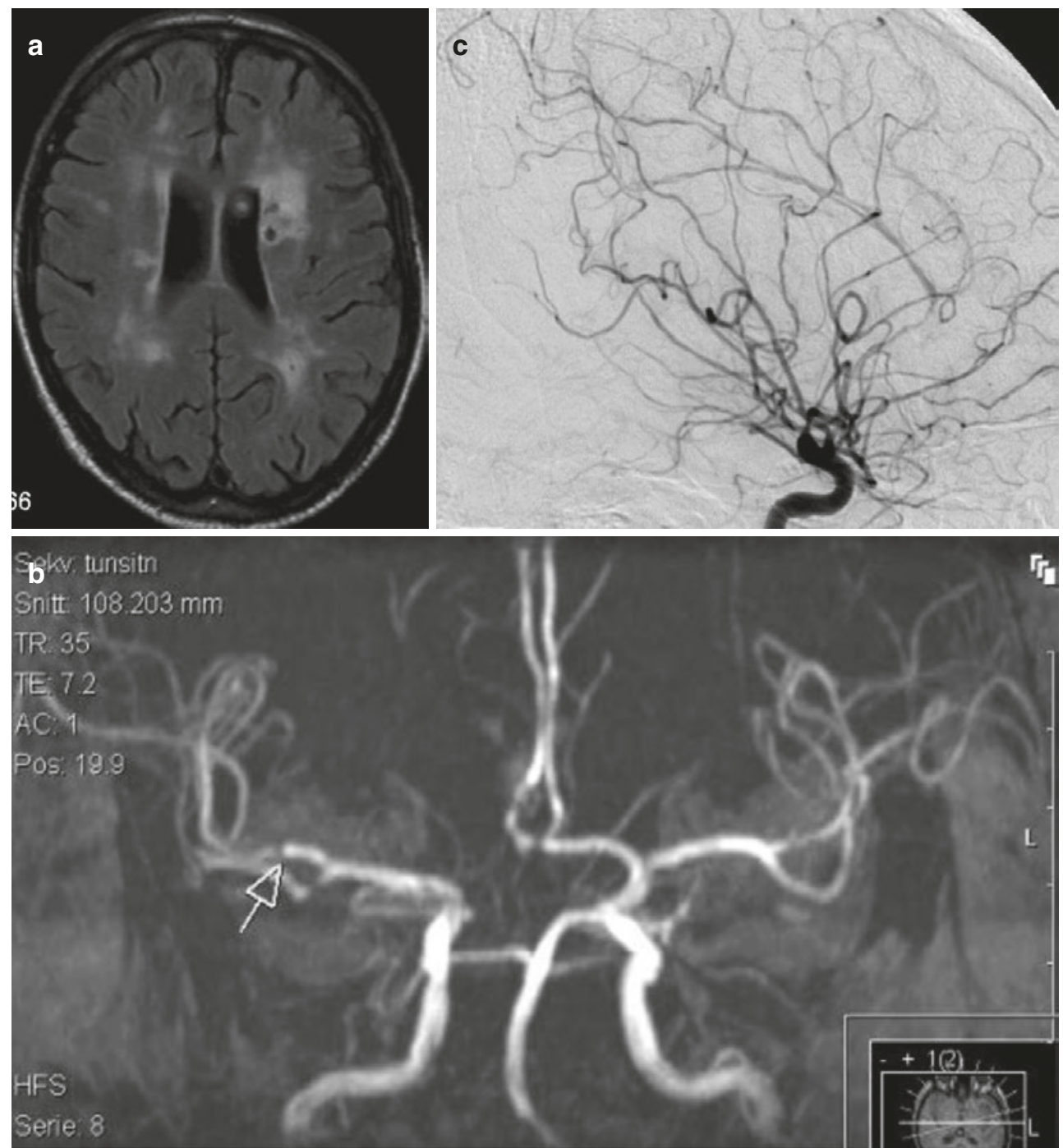
nervous system in the form of necrotizing vasculitis, peripheral neuropathies, and cranial nerve palsy. The involvement of the CNS is far less frequent, with only $2-8 \%$ in the form of CNS vasculitis with intracranial granulomas directly or due to spread from the sinus and orbits. CNS involvement in this form is associated with a very high mortality rate $(>90 \%)$ if untreated. This may present with focal granulomas that have spread from the frontal sinus (Fig. 6.20), or focal white matter hyperintesies and/or leptomeningeal contrast henhancement (Fig. 6.21).

\subsubsection{Neurosyphilis}

Neurosyphilis (CNS lues) is a classic infectious cause of cerebral vasculitis. The patient often presents with an ischemic event predominantly related to the middle cerebral arteries or branches of the basilar artery. Typical imaging findings are multiple lacunar infarcts, arteritis changes with focal stenosis, ectasia of the basilar artery, focal areas of increased T2 signal in the white matter, and the presence of patchy enhancement (Fig. 6.22).
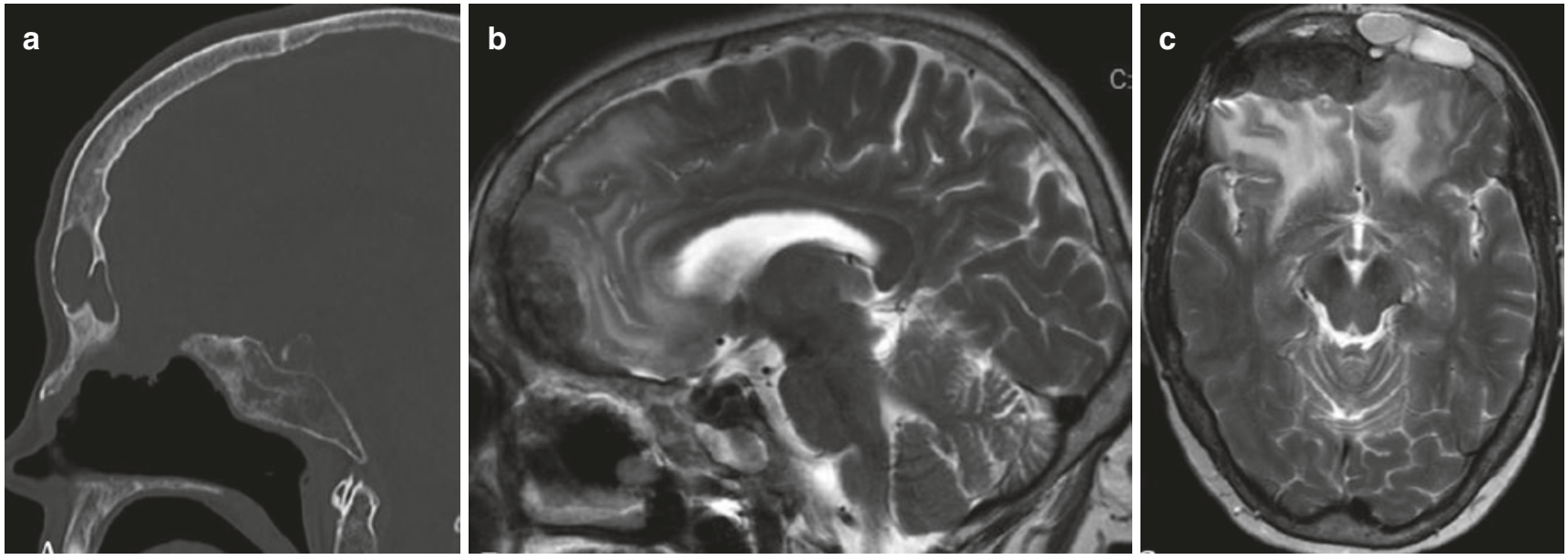

Fig. 6.20 (a) Sagittal CT (bone window) demonstrates opacification of the frontal sinus. (b, c) Granuloma extension into the frontal lobes bilaterally with surrounding edema and gliosis
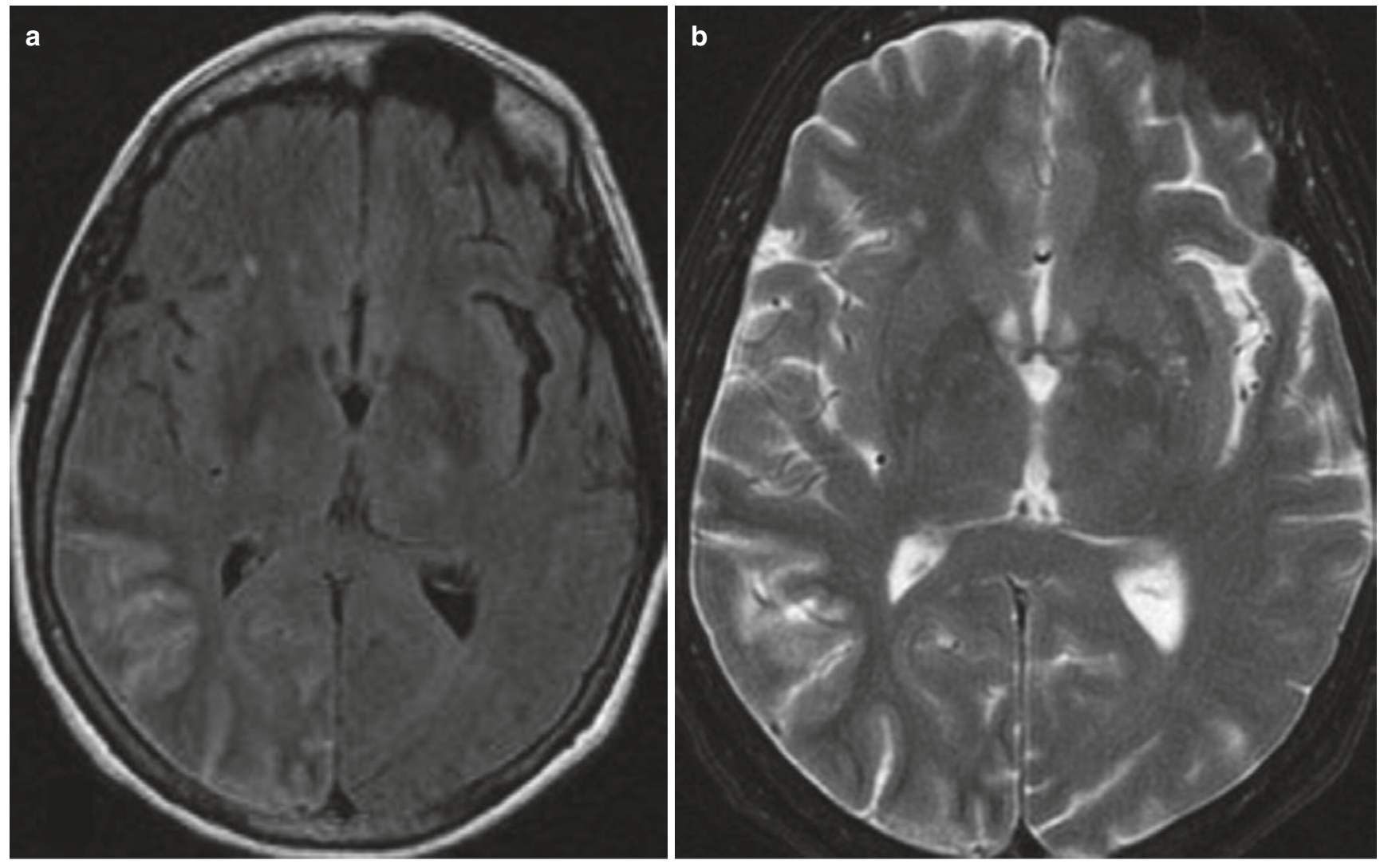

Fig. 6.21 Axial (a) FLAIR, (b) T2-weighted, and (c, d) T1-weighted image after contrast administration demonstrating focal brain swelling and leptomeningeal contrast enhancment 

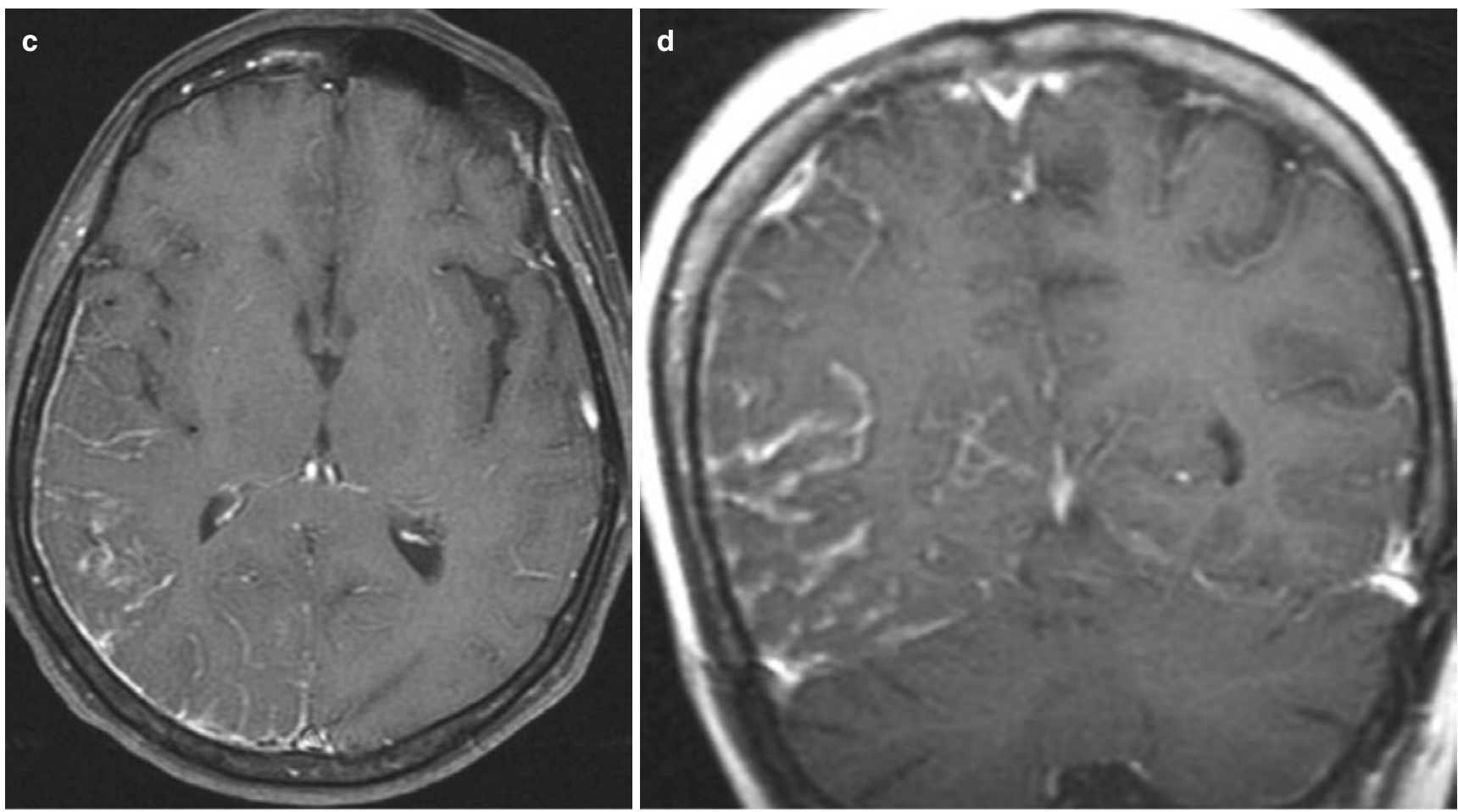

Fig. 6.21 (continued)
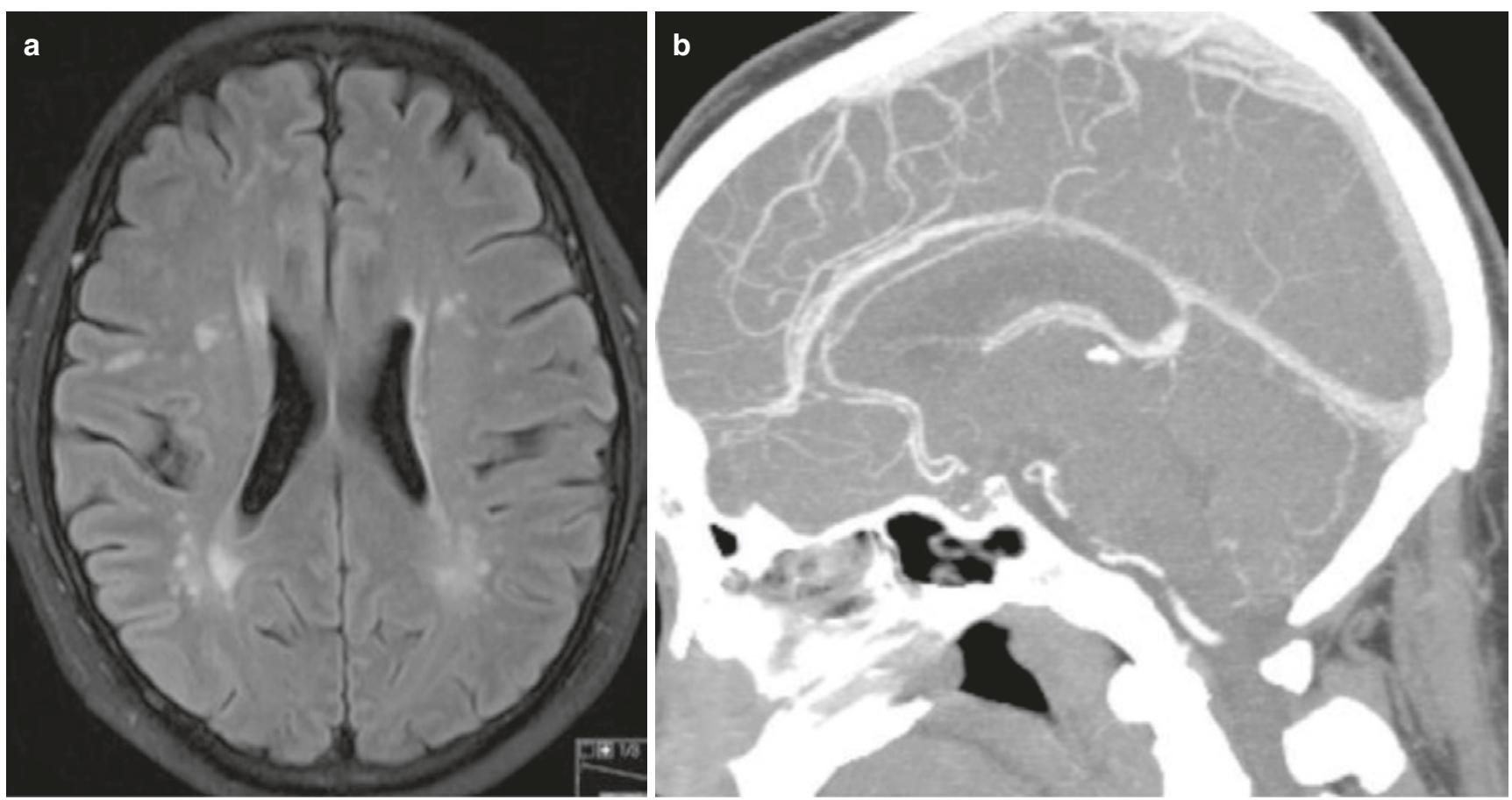

Fig. 6.22 (a) Axial FLAIR demonstrating white matter lesions, (b) CT angiography demonstrating stenosis and irregularities in the basialry artery, and (c) stenosis in both middle cerbral arteries and stenosis of the basilar artery in a 67-year-old male with diagnosed neurosyphilis 


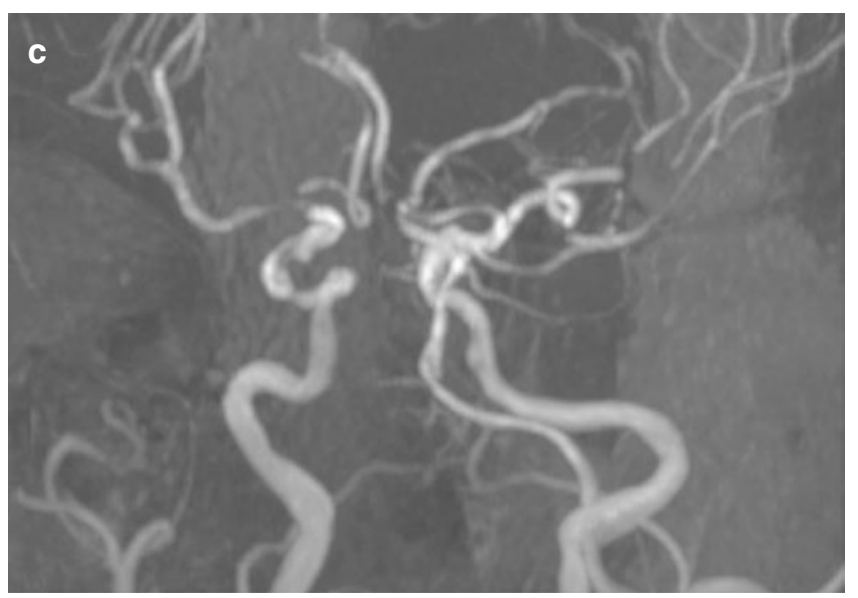

Fig. 6.22 (continued)

\subsection{Concluding Remarks}

Infectious and inflammatory CNS disorders are difficult clinical diagnoses due to the similarities in clinical symptoms and laboratory findings. The role of imaging remains critical and the use of advanced techniques and state-of-the-art MRI of the brain and spine is mandatory for early detection, differentiation, and follow-up.

\section{Take Home Messages}

- Use FLAIR and post-contrast 3D T2-FLAIR for detection of meningeal pathology

- Use multimodal MRI approach in ring-enhancing brain lesions

- Use vessel wall MR imaging for evaluation of CNS vasculitis

- In autoimmune encephalitis MRI may show no abnormality

\section{References}

1. Thurnher MM. Infections in immunocompromised patients. In: Barkhof F, Jäger R, Thurnher M, Rovira A, editors. Clinical neuroradiology. Berlin: Springer; 2019.

2. Fukuoka H, Hirai T, Okuda T, et al. Comparison of the added value of contrast-enhanced 3D fluid-attenuated inversion recovery and magnetization-prepared rapid acquisition of gradient echo sequences in relation to conventional postcontrast T1-weighted images for the evaluation of leptomeningeal diseases at 3T. AJNR Am J Neuroradiol. 2010;31:868-73.

3. Toh $\mathrm{CH}$, Wei KC, Chang CN, et al. Differentiation of pyogenic brain abscesses from necrotic glioblastomas with use of susceptibilityweighted imaging. AJNR Am J Neuroradiol. 2012;33:1534-8.

4. Antulov R, Dolic K, Fruehwald-Pallamar J, Miletic D, Thurnher MM. Differentiation of pyogenic and fungal brain abscesses with susceptibility-weighted MR sequences. Neuroradiology. 2014;56(11):937-45.

5. Thurnher MM. Bacterial infections. In: Barkhof F, Jäger R, Thurnher M, Rovira A, editors. Clinical neuroradiology. Berlin: Springer; 2019.

6. Rey C, Koric L, Guedj E, et al. Striatal hypermetabolism in limbic encephalitis. J Neurol. 2012;259(6):1106-10.

7. Kelley BP, Patel SC, Marin HL, Corrigan JJ, Mitsias PD, Griffith B. Autoimmune encephalitis: pathophysiology and imaging review of an overlooked diagnosis. AJNR Am J Neuroradiol. 2017;38(6):1070-8.

8. Graus F, Titulaer MJ, Balu R, et al. A clinical approach to diagnosis of autoimmune encephalitis. Lancet Neurol. 2016;15:391-404.

9. Razek AAKA, Alvarez H, Bagg S, Refaat S, Castillo M. Imaging spectrum of CNS vasculitis. Radiographics. 2014;34:873-94.

10. Mandell DM, Mossa-Basha M, Qjao Y, et al. Intracranial vessel wall MRI: principles and expert consensus recommendations of the American Society of Neuroradiology. AJNR Am J Neuroradiol. 2017;38(2):218-29.

11. Cannerfelt B, Nystedt J, Jönsen A, Lätt J, van Westen D, Lilja A, Bengtsson A, Nilsson P, Mårtensson J, Sundgren PC. White matter lesions and brain atrophy in systemic lupus erythematosus patients: correlation to cognitive dysfunction in a cohort of systemic lupus erythematosus patients using different definition models for neuropsychiatric systemic lupus erythematosus. Lupus. 2018;27(7):1140-9.

Open Access This chapter is licensed under the terms of the Creative Commons Attribution 4.0 International License (http://creativecommons. $\mathrm{org} /$ licenses/by/4.0/), which permits use, sharing, adaptation, distribution and reproduction in any medium or format, as long as you give appropriate credit to the original author(s) and the source, provide a link to the Creative Commons license and indicate if changes were made.

The images or other third party material in this chapter are included in the chapter's Creative Commons license, unless indicated otherwise in a credit line to the material. If material is not included in the chapter's Creative Commons license and your intended use is not permitted by statutory regulation or exceeds the permitted use, you will need to obtain permission directly from the copyright holder. 


\title{
Traumatic Neuroemergency: Imaging Patients with Traumatic Brain Injury-An Introduction
}

\author{
Paul M. Parizel and C. Douglas Philips
}

\section{Learning Objectives}

- Understand the role of imaging in the urgent evaluation of patients with CNS trauma

- Review common surgical vs. non-surgical CNS abnormalities in trauma patients

- Compare MR and CT for evaluation of brain trauma

\section{Key Points}

- CT has become the most important diagnostic test in patients with CNS trauma in the urgent setting.

- CT has the ability to depict traumatic lesions that will require urgent surgical management and to also assess other traumatic lesions that are notable but not surgical indications.

- MR can accurately detect a range of CNS injuries that are ultimately critical in the prognosis of major CNS trauma.

\subsection{Introduction}

Traumatic brain injury (TBI) remains a leading cause of mortality and disability among young individuals, especially in high-income countries [1]. According to a meta-analysis, combining reports from 23 European countries between

\section{P. M. Parizel}

Department of Radiology, Antwerp University Hospital, Faculty of Medicine and Health Sciences, University of Antwerp,

Antwerp, Belgium

C. D. Philips $(\square)$

Weill Cornell Medical Center, NewYork-Presbyterian Hospital,

New York, NY, USA

e-mail: dphillips@med.cornell.edu
1980 and 2003, the incidence rate is about 235 per 100,000 [2]. Increasingly, imaging techniques play a crucial role in the diagnosis and management of patients with craniocerebral trauma, and may influence life or death decisions. Additionally, the role of imaging in the prediction of prognosis is well established and of growing importance in the research arena.

\subsection{Imaging Techniques}

We have moved well beyond the era of projectional radiography, as the diagnostic efficacy of plain skull films in TBI is poor. At present, for the management of patients with traumatic brain injury, plain X-ray films play no role; they have entirely been replaced by computed tomography (CT) imaging [3].

$\mathrm{CT}$ is the initial imaging technique for evaluation of craniocerebral trauma. In most trauma centers and emergency rooms, CT is readily available and available within a short distance from the trauma bay. Many emergency departments routinely position one or several CT scanners in the receiving area for triage. Moreover, a CT examination can be performed in a few seconds with a modern, state-ofthe-art multi-detector CT (MDCT) scanner. MDCT allows acquisition of multiple thin sections in a much shorter scan time than a single-detector system [4] and has essentially replaced them. Image data can be reconstructed in every plane as well as in 3D views (Fig. 7.1). Routine utilization of these multiplanar reconstructed (MPR) images also appears to improve diagnosis of traumatic injuries and has been supported in the literature to increase CT accuracy [5]. Cranial CT is used for evaluation of all forms of intracranial hemorrhage, fractures, brain edema, herniation and other associated injuries, as well as to evaluate foreign bodies. CT angiography (dynamic contrast-enhanced CT to visualize vascular structures) may be indicated whenever vascular injury is suspected, and also patients with a highrisk mechanism trauma, such as, for example, intra-oral 


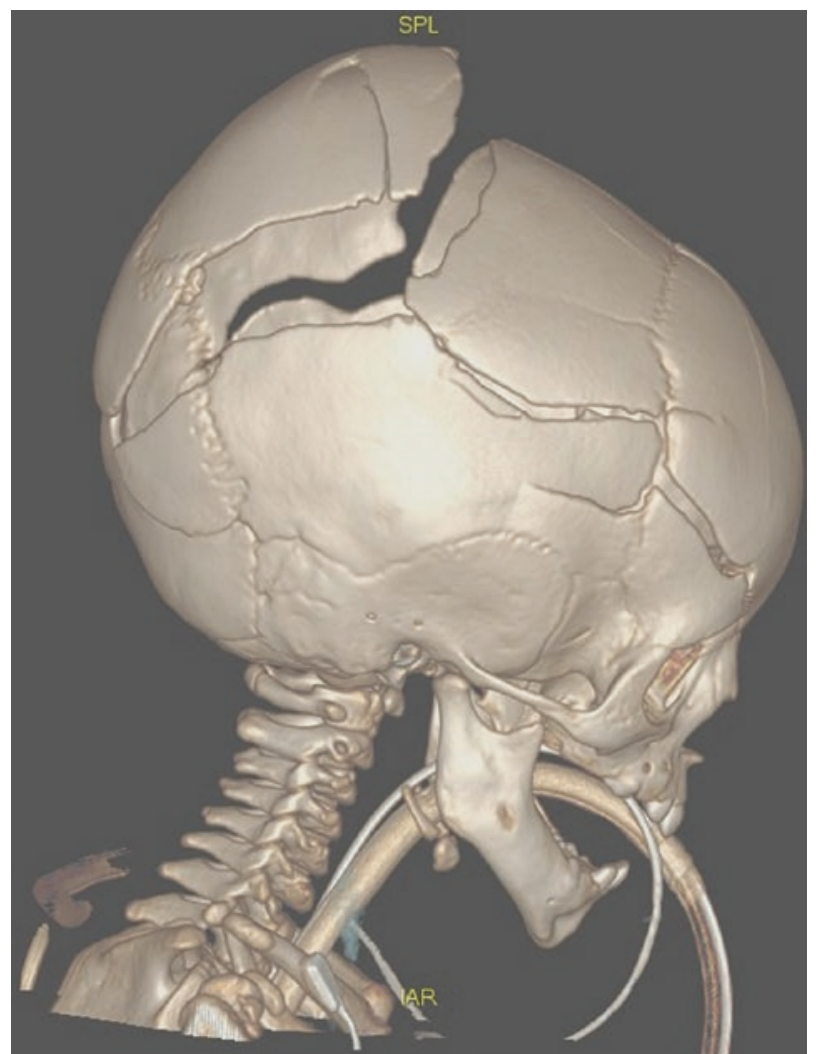

Fig. 7.1 Volume rendering technique (VRT), obtained from a 3D CT-scan data set, reveals multiple skull fractures and displaced bone fragments. The sharp outline of the fracture lines is clearly distinct from the jagged and serrated edges of the cranial sutures in this young child with severe craniocerebral trauma

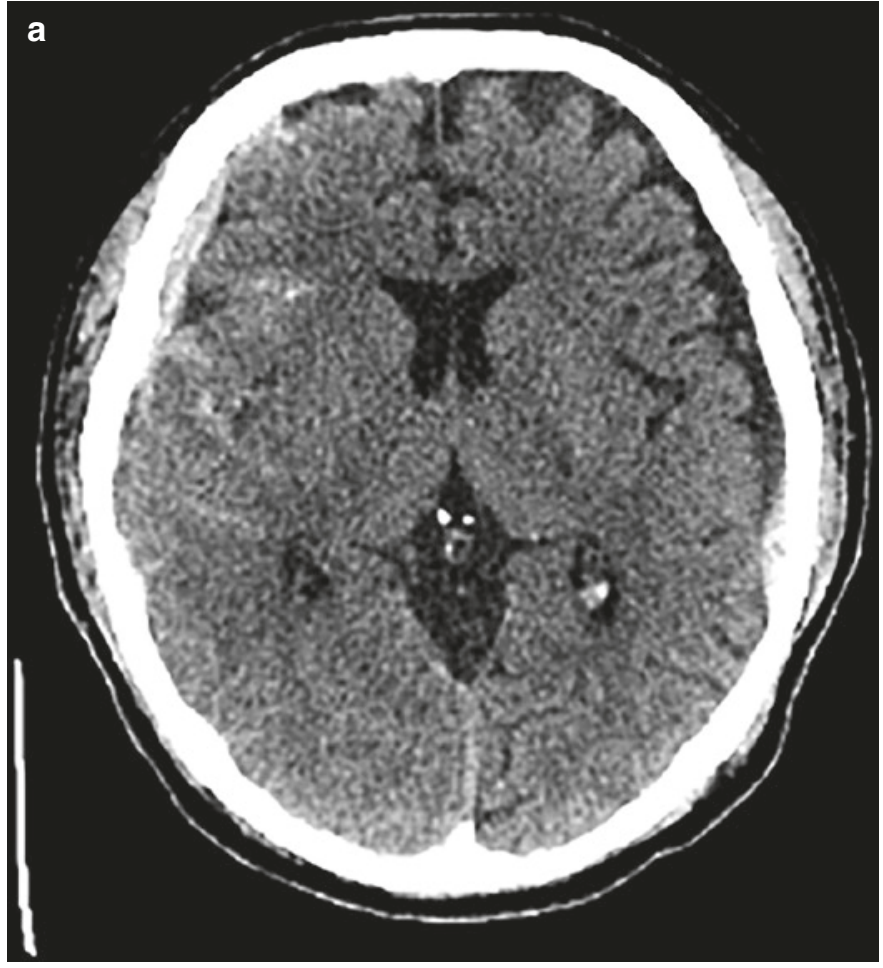

Fig. 7.2 (a, b) Axial non-contrast CT-scan, revealing a left parietal epidural hematoma ("coup" side) with accompanying subarachnoid hemorrhage. A fracture was evident on bone images. On the "contre- trauma, high energy crashes, near-hanging, and fractures of skull base and midface [6] or for suspicious hemorrhage patterns.

MRI is more sensitive than $\mathrm{CT}$ for the detection of parenchymal lesions [7, 8]. It is also indicated whenever there is a discrepancy between the clinical neurological status of the patient and the CT findings.

In this review paper, we aim to present a systematic guide towards interpretation of imaging studies in the patient with TBI.

\subsection{Scalp Lesions}

Scalp lesions are common in head trauma victims (Figs. 7.2 and 7.3). Although they are of little clinical importance, identification of scalp lesions may direct the attention of the radiologist towards the point of impact [9]. CT is rarely performed for isolated scalp injury, but to detect the suspected intracranial injuries. CT not only visualizes the soft tissue swelling, but through appropriate window (W) and level (L) settings will also illustrate intracranial abnormalities [8]. One important factor influencing the detection rate of soft tissue lesions in CT is the perception of density differences within the lesions and the surrounding tissues such as blood, subcutaneous fatty tissue, or cerebrospinal fluid [10].

Table 7.1 provides an overview of some of the most commonly encountered scalp lesions.

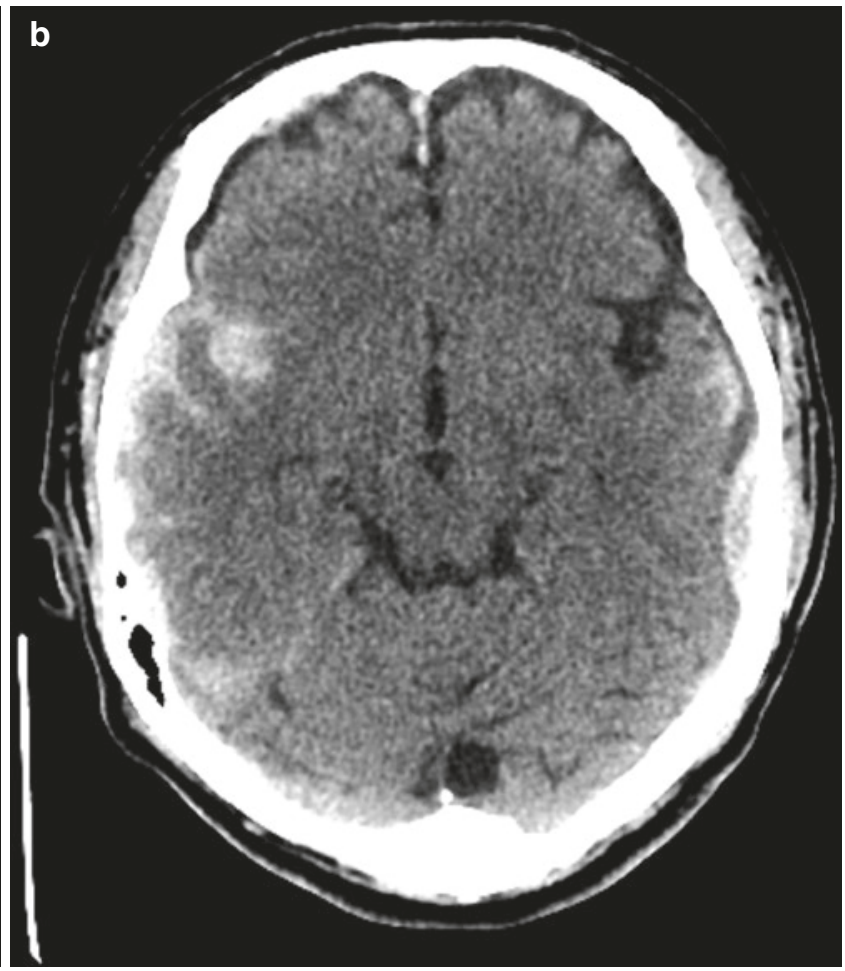

coup" side, there is a hyperdense subdural hematoma overlying the right cerebral hemisphere, as well as a basifrontal hemorrhagic contusion 

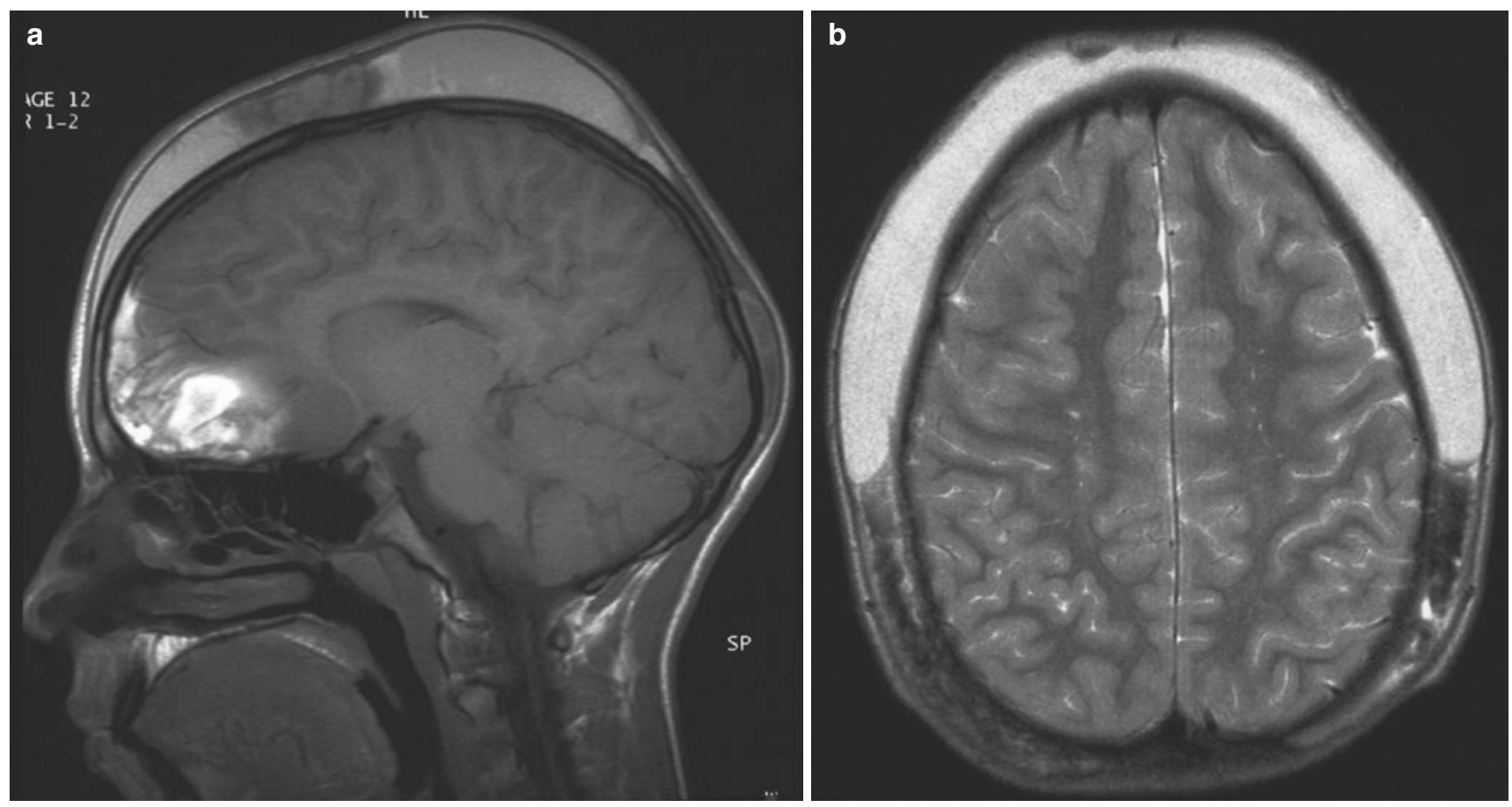

Fig. 7.3 (a, b) Hemorrhagic cerebral contusions and subgaleal hematoma. MRI examination performed with (a) sagittal SE T1-weighted images and (b) axial TSE T2-weighted images. Extracranially, there is marked swelling of the subcutaneous soft tissues, due to a massive sub- galeal hematoma, which extends from the frontal to the posterior parietal region. Intracranially, there are hemorrhagic cerebral contusions in the right frontal and anterior temporal lobes

Table 7.1 Overview of scalp lesions

\begin{tabular}{|c|c|c|c|c|}
\hline Lesion type & Caput succedaneum & $\begin{array}{l}\text { Subgaleal hematoma (extracranial } \\
\text { subdural hematoma) }\end{array}$ & Subgaleal hygroma & $\begin{array}{l}\text { Cephalohematoma (extracranial } \\
\text { epidural hematoma) }\end{array}$ \\
\hline Occurrence & $\begin{array}{l}\text { After normal vaginal } \\
\text { delivery }\end{array}$ & After head trauma (or after birth) & $\begin{array}{l}\text { Birth trauma } \\
\text { (forceps delivery) }\end{array}$ & $\begin{array}{l}\text { Birth trauma (skull fracture during } \\
\text { birth) }\end{array}$ \\
\hline Location & $\begin{array}{l}\text { Superficial of the galea } \\
\text { aponeurotica }\end{array}$ & Beneath the galea aponeurotica & $\begin{array}{l}\text { Beneath the galea } \\
\text { aponeurotica }\end{array}$ & Subperiosteal (flat skull bones) \\
\hline Composition & $\begin{array}{l}\text { Edema (with microscopic } \\
\text { hemorrhages) }\end{array}$ & Venous blood & Cerebrospinal fluid & Subperiosteal hemorrhage \\
\hline Clinical presentation & Pitting edema & $\begin{array}{l}\text { Diffusely spreading, firm } \\
\text { fluctuating mass }\end{array}$ & - & Well defined, focal, firm mass \\
\hline Skull fracture & & Yes or no & Yes & Yes \\
\hline Crosses suture lines? & Yes & Yes & Yes & No \\
\hline
\end{tabular}

\subsection{Skull Fractures}

Skull fractures constitute a diverse group of traumatic bone injuries with different mechanism of occurrence, topography, complications, and required management. They can be classified according to various parameters:

- Shape of the fracture: linear, comminuted, stellate

- Depressed, non-depressed, open

- Anatomic location and extent of the fracture: skull vault, skull base, craniofacial junction

Simple linear fractures are most common type, especially in children younger than 5 years. Temporal bone fractures represent $15-48 \%$ of all skull fractures and are considered as a separate category of skull injury. Skull base and temporal bone fractures have their own clinical significance; a detailed discussion of these injuries is beyond the scope of this text.

CSF leaks, cranial nerve injuries, orbital injuries, and craniocervical junction injuries are common accompaniments of fractures, especially near the skull base.

Depressed skull fractures (with displacement of the outer table of the skull to at least the level of the inner table) occur in $75 \%$ of cases in the frontoparietal region and are often open (75-90\%).

Patients with skull fractures have a fivefold increased risk of having an intracranial hemorrhage (ICH) and CT is mandatory [11]. Linear skull fractures may be occult on skull radiographs because of the double-layered skull along fractures, when the cross-section is oblique to the direction of the X-rays [12]. Similarly, skull fractures may be difficult to see on CT, particu- 
Table 7.2 Epidural versus subdural hematoma

Epidural hematoma (EDH)
'Coup' side
Associated with skull fracture in $\pm 90 \%$ of cases
Does not cross suture lines
Not limited by falx or tentorium (may extend from supra- to
infratentorial or across midline)
Origin
- Arterial (majority, due to tearing of one or more branches of the
meningeal arteries, most commonly the middle meningeal artery),
- Venous (minority, due to laceration of a dural venous sinus,
e.g., along the sphenoparietal sinus)
Medical emergency
Magnitude of the mass effect caused by EDH is directly related
to the size of the extracerebral collection
CT is preferred imaging technique because
- Rapid accessibility
- Shows both the hemorrhage and the skull fracture
MR can be useful for
- Detection of parenchymal repercussions (edema, mass effect,
herniations)

larly when parallel to the display plane. The interpreting physician may need to correlate MPR images as well as occasionally rely on the scout image to depict these linear fractures.

In patients with significant TBI, skull fractures are common and are often overlooked on clinical examination. However, in some cases, there is a poor correlation between skull fractures and intracranial injury; severe brain injuries can occur, even without the presence of a skull fracture [8].

\subsection{Extra-Axial Lesions}

There are four types of extra-axial hemorrhage to be considered: epidural, subdural, subarachnoid, and intraventricular.

\subsubsection{Epidural Hematoma}

Epidural hematoma (EDH) is an extracerebral collection of blood between the inner table of the skull and the dura, essentially underlying a bare bone surface. EDH occurs in $<1 \%$ of patients with head injuries [13]. EDH is typically found on the "coup" injury side, and is commonly associated with a skull fracture and laceration of the underlying blood vessel(s), notably the dural vasculature [14]. The recommended imaging method is CT, which depicts both the fracture and resulting hemorrhage. EDHs are commonly biconvex in appearance and typically do not cross suture lines but may cross the tentorium or falx; their mass effect is mostly caused by the size of extracerebral collection [14]. In the early stage, an epidural hematoma may appear heterogeneous on CT with hyperdense areas of clotted blood and hypodense whirls of non-coagulated blood that in time becomes more homogeneous [14]. This "swirl sign" is an ominous feature, and should be carefully noted and communicated to the treating physicians, as it represents active hemorrhage into the collection [15]. Rapid progression has been noted.

\section{Subdural hematoma (SDH)}

\section{'Contre-coup' side}

No consistent relationship with skull fractures

Does cross suture lines

Limited by falx and tentorium (confined to supra- or infratentorial compartment, does not cross midline)

Origin

- Venous, due to laceration of superficial bridging cortical veins

May be chronic

Magnitude of the mass effect caused by SDH is more often associated with underlying parenchymal injury MRI is preferred imaging technique because

- MRI is more sensitive than CT, especially in the detection of so-called isodense SDH's which may be difficult to see on CT

- Multiplanar imaging capability

- Better definition of multi-compartmental nature of SDH

EDH may be arterial or venous in origin (Table 7.2). Arterial EDH is caused by injury of a meningeal artery, commonly the middle meningeal artery. The typical location is the temporoparietal region, with $75 \%$ of EDH occurring adjacent to the temporal bone (Fig. 7.4). When the blood vessel is lacerated, arterial bleeding results in a rapidly expanding hematoma that exerts mass effect on the brain, focal deformity of the brain and an increase in intracranial pressure (ICP). The size of the EDH may continue to increase until the arterial perfusion pressure is equilibrated with the elevated ICP. Emergency decompressive surgery is indicated to remove the $\mathrm{EDH}$, stop hemorrhage, and reduce compression on the brain.

Venous EDH results from rupture/laceration of a dural venous sinus, or bleeding from diploic veins, again often associated with a skull fracture [16]. Venous EDH is usually located near the anterior wall of the middle cranial fossa (injury of sphenoparietal sinus and/or fracture of the greater wing of the sphenoid bone), the vertex (injury of the superior sagittal sinus and/or fracture of the frontal or parietal bone), or against the occiput near the tentorium (injury of the transverse sinus and/or occipital fracture) [8] (Fig. 7.5).

Few EDH patients will not undergo craniotomy and evacuation, often on an emergent basis. One unusual and typically indolent EDH is known; anterior temporal EDH is often a lowpressure hemorrhage and typically do not expand. There is no usual requirement for surgical intervention [16]. Conversely, posterior fossa EDH may urgently require surgical evacuation because of early brainstem compression [16].

\subsubsection{Subdural Hematoma}

A subdural hematoma (SDH) represents an extracerebral collection of blood in the potential space between the dura mater and the arachnoid membrane, or "epiarachnoid." 


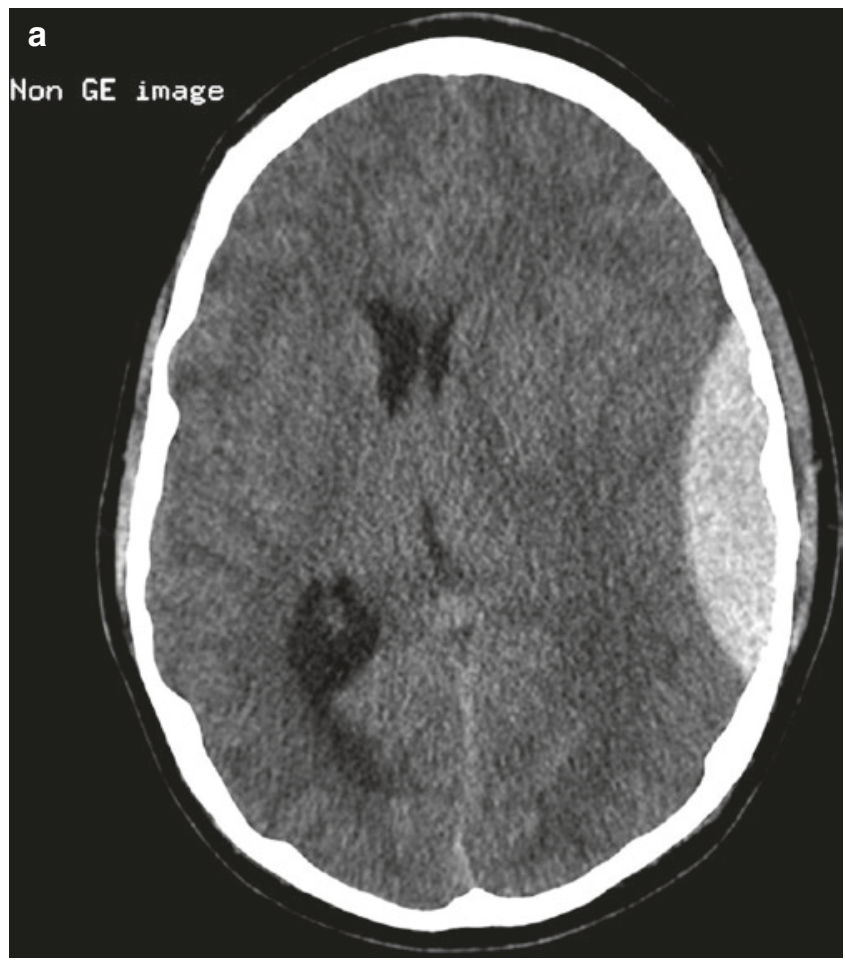

Fig. 7.4 (a, b) Acute left arterial epidural hematoma. Non-contrast CT-scan upon admission. The left-sided hematoma is biconvex, and is limited by the coronal suture anteriorly and by the lambdoid suture pos-

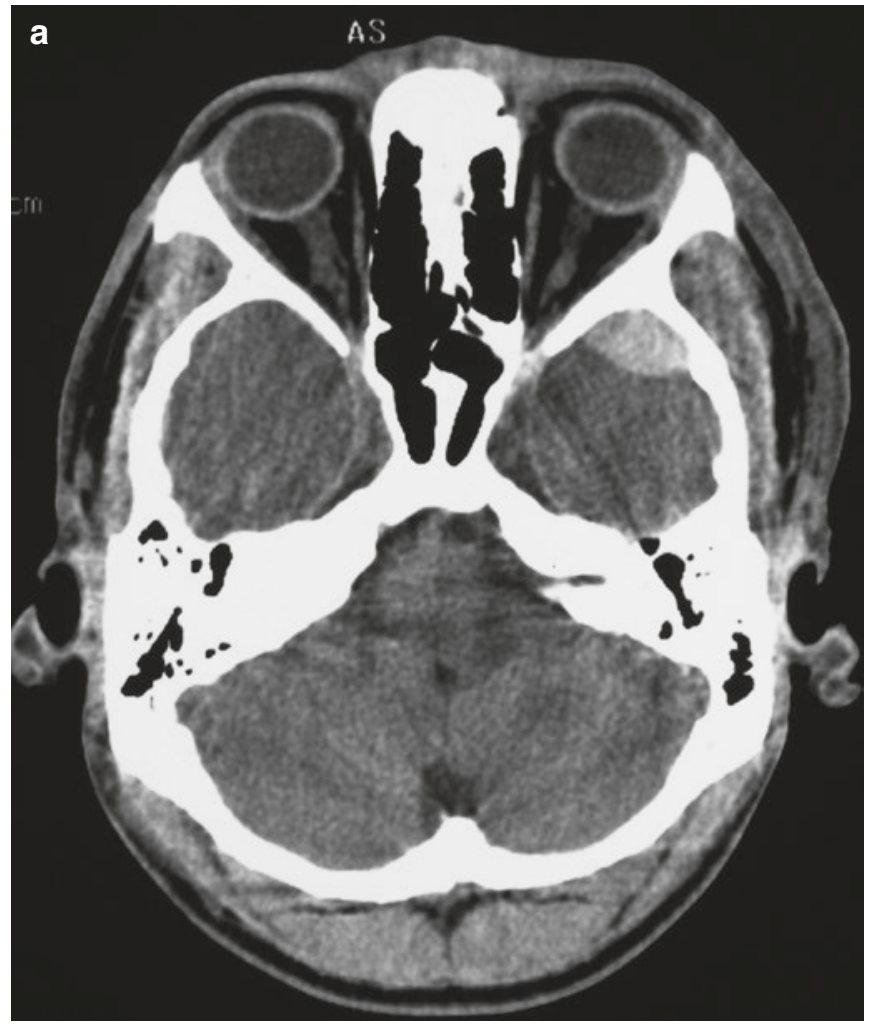

Fig. 7.5 (a, b) Venous epidural hematomas, shown in two different patients. Venous epidural hematomas typically are found (a) anteriorly in the middle cranial fossa adjacent to the sphenoparietal sinus and

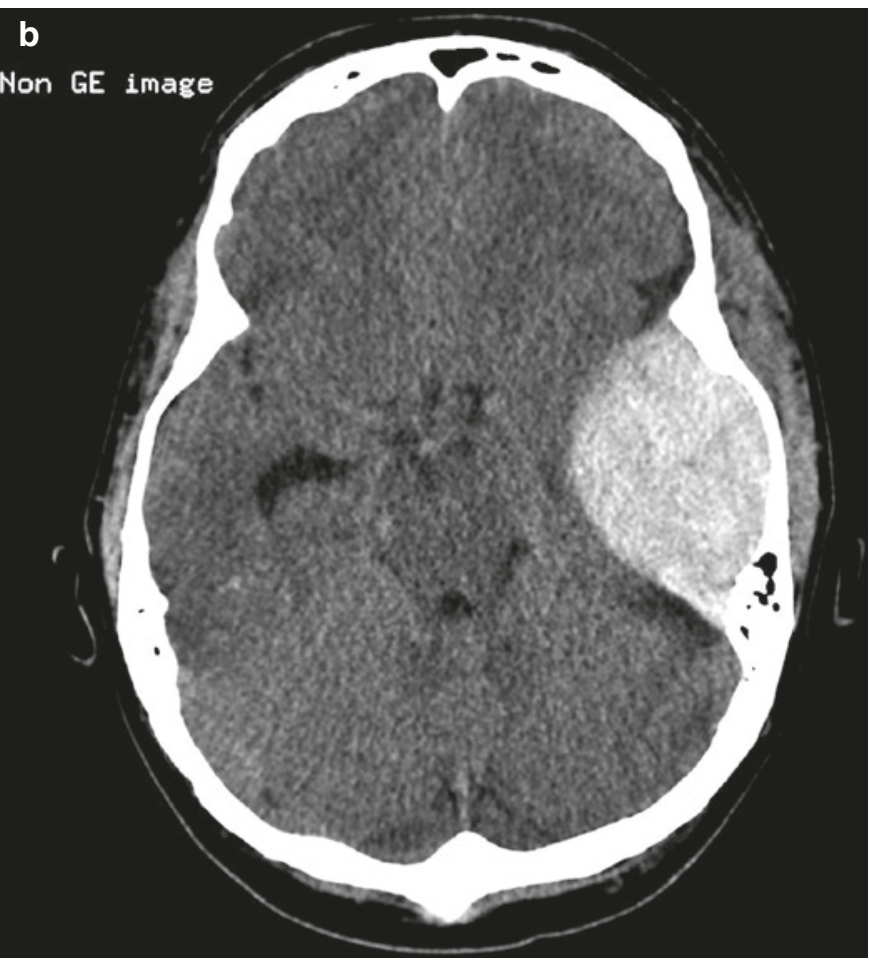

teriorly. The mass effect causes a shift of the midline structures with compression of the left lateral ventricle and dilatation of the right lateral ventricle

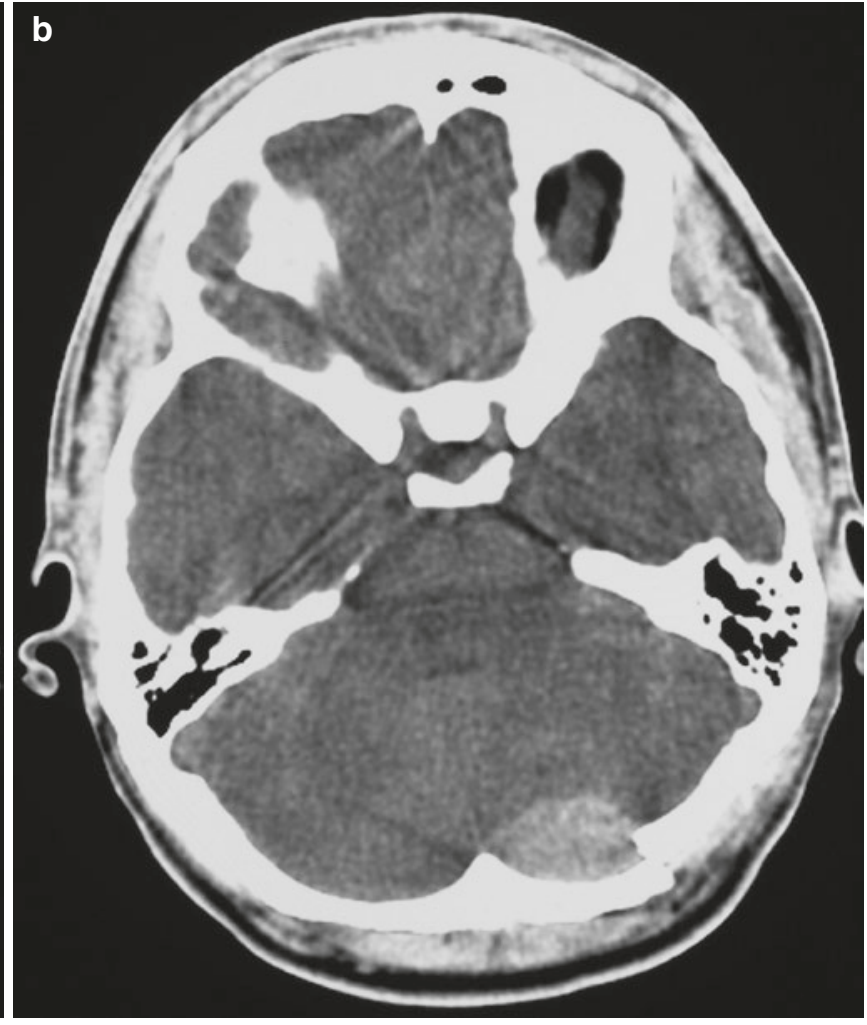

greater wing of the sphenoid bone, or (b) adjacent to the transverse sinus. They present a typical, biconvex lens-shaped configuration 
SDHs are relatively common and occur in about $30 \%$ cases of severe head trauma [13]. They are more commonly found on the contre-coup injury side and are not as strongly associated with fractures as EDH [14]. The origin is almost invariably venous, caused by injury of superficial bridging cortical veins. They do cross sutures and may be holohemispheric, and are typically limited by the falx and tentorium [14].

On CT, an acute SDH is seen as crescentic hyperdense structure between brain and the inner border of skull (Fig. 7.6). On modern CT scanners, the arachnoid space may
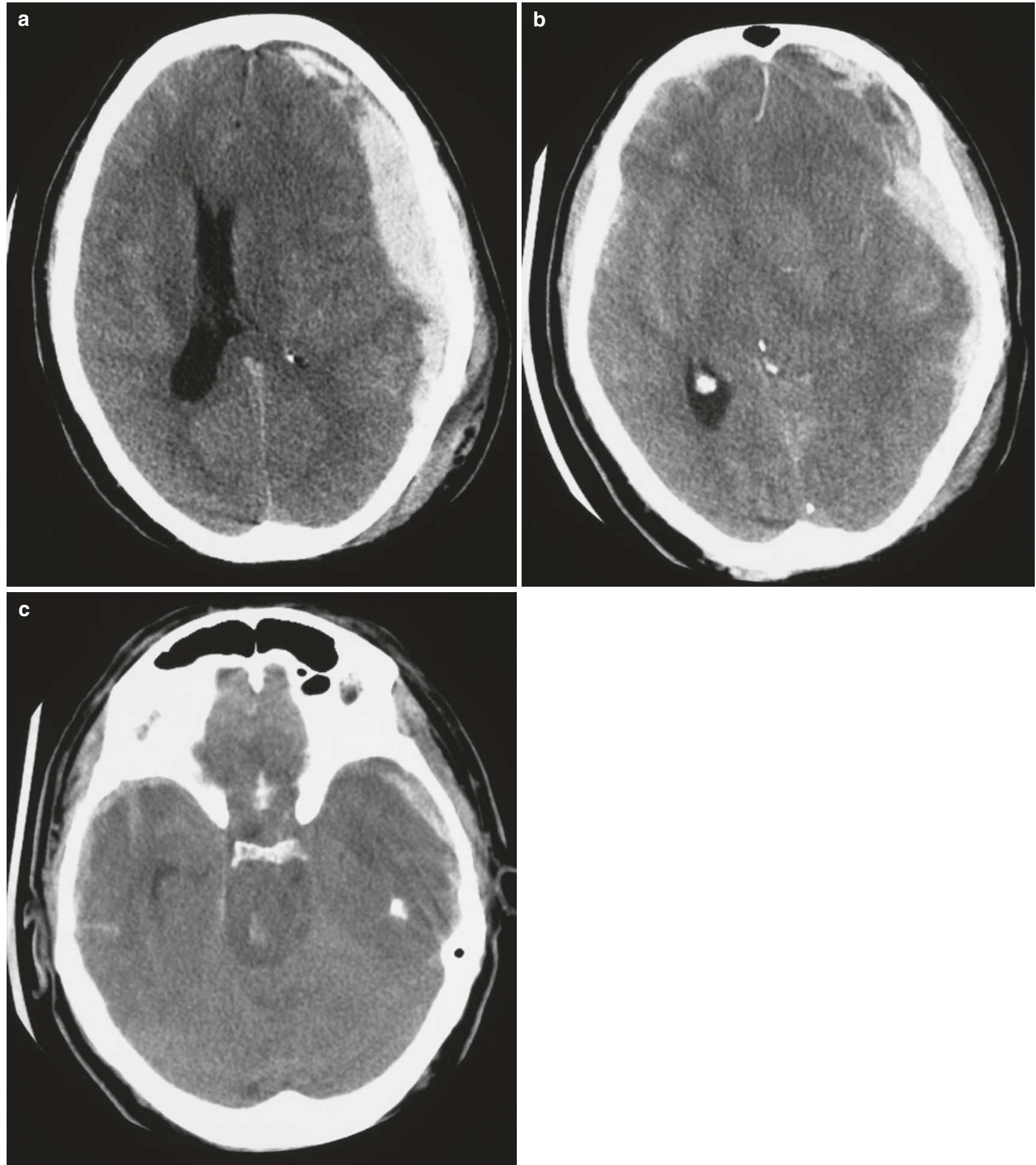

Fig. 7.6 (a-c) Acute left subdural hematoma. The CT-scan shows hyperdense areas of clotted blood and some hypodense components indicating active bleeding. There is mass effect on the left cerebral hemisphere, with transtentorial herniation. The high-density area in the

brainstem indicates a secondary brainstem hemorrhage or so-called Duret hemorrhage. There are also hemorrhagic cerebral contusions in both cerebral hemispheres 

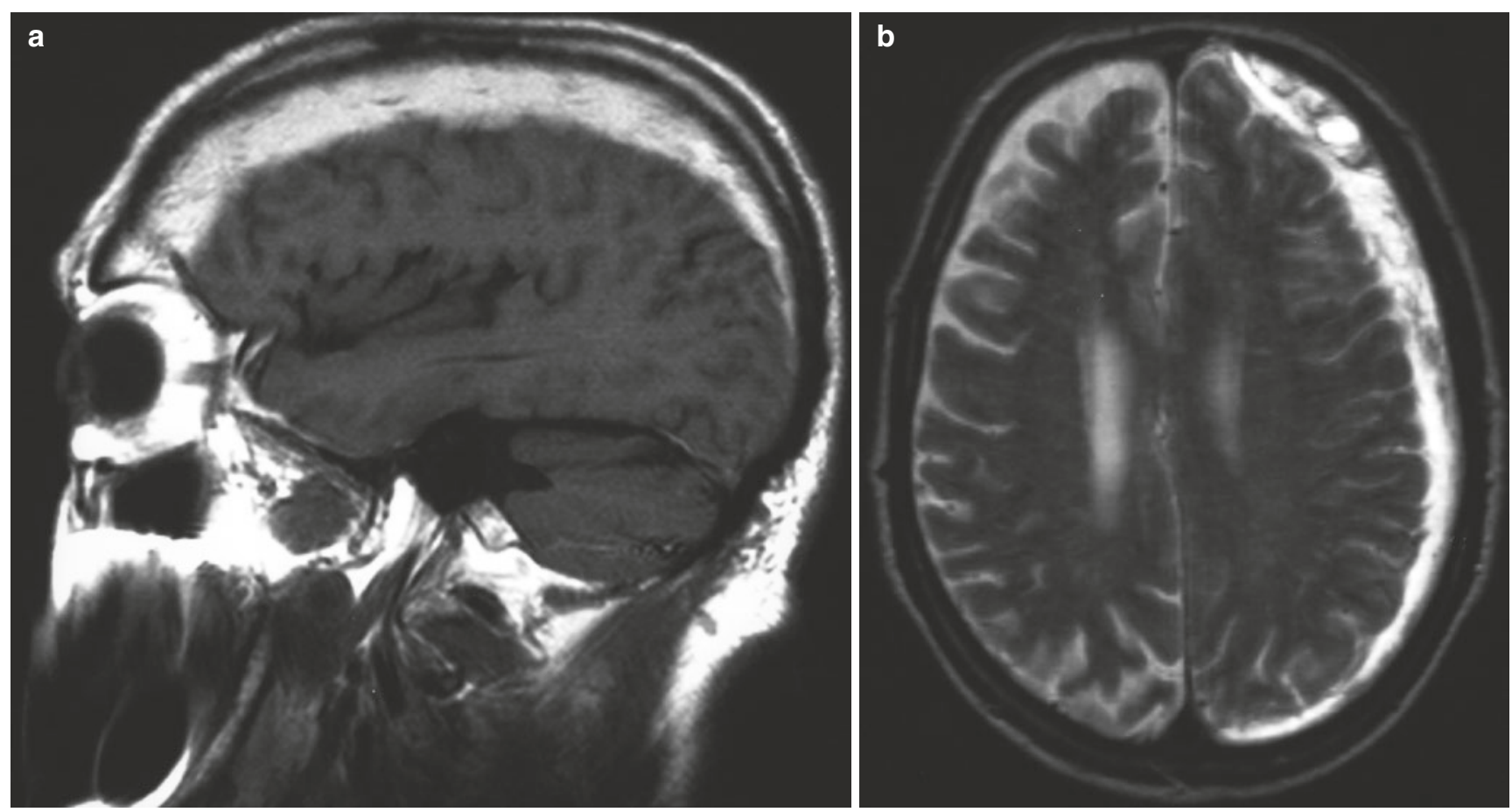

Fig. 7.7 (a, b) Subacute left subdural hematoma. MRI examination performed with (a) sagittal SE T1-weighted image and (b) axial TSE T2-weighted image. The MR images show the typical appearance of a crescent-shaped left subdural hematoma. There are multiple internal septations, representing strands of fibrovascular granulation tissue, derived from the inner (meningeal) layer of the dura. The hematoma extends over the surface of the left cerebral hemisphere from the frontal to the occipital region. The blood is hyperintense on T1-WI and on T2-WI, indicating the presence of extracellular methemoglobin be seen adjacent to the brain opposing the SDH. SDHs do not penetrate into sulci. Over time, the density decreases and they may become isodense to the adjacent brain. Therefore, MRI may be a preferred imaging method in later stages.

Subacute SDH occurs as hyperintense lesion on T1 and T2-weighed images due to extracellular methemoglobin [17] (Fig. 7.7). Chronic subdural hematoma is also well evaluated by MRI, which can clearly distinguish the size and extent of the collection as well as the development of the enhancing, capillary-rich membrane associated with resorbing SDH [17]. Chronic subdural hematomas are variable in signal but may retain T1 or FLAIR hyperintensity (Fig. 7.8).

The development of the vascular membranes, that are common in chronic SDH, is an important feature in the subsequent development of rehemorrhage into these collections, representing the "acute on chronic" form of $\mathrm{SDH}$. These collections may appear quite complex in nature, with mixed and layered signal intensities, and are difficult to manage [18].

Finally, the occasional occurrence of an acute low-density subdural collection in the trauma patient is a known phenomenon. A tear in the arachnoid may allow CSF to enter the potential subdural space and produce an enlarging fluid collec- tion known as a subdural hygroma. These may spontaneously resolve but also are monitored for mass effect.

\subsubsection{Traumatic Subarachnoid Hemorrhage}

Traumatic subarachnoid hemorrhage (SAH) is common and often focal. It can be due to superficial cerebral abrasions, direct leptomeningeal vascular injury, or intraventricular hemorrhage with subarachnoid reflux [8]. The estimated incidence rates for SAH are 10-25 per 100,000 per year [19]. Acute SAH is easy recognizable on non-contrast CT as gyriform high attenuation in the subarachnoid space. It will penetrate sulci. The attenuation of blood on CT decreases as the hemoglobin concentration decreases [20]. The combination of SWI and FLAIR yields a distinctly higher detection rate for SAH due to their complementary detection capabilities [21]. FLAIR is sensitive for superficial/convexity SAH, while SWI adds value for the centrally located SAH, e.g., the interhemispheric fissure, interpeduncular fossa, supracerebellar cistern, and intraventricular subarachnoid blood [21]. The recognition of subtle SAH is important in the trauma setting, as this finding alone will typically result in an extended in-hospital evaluation of the patient. 


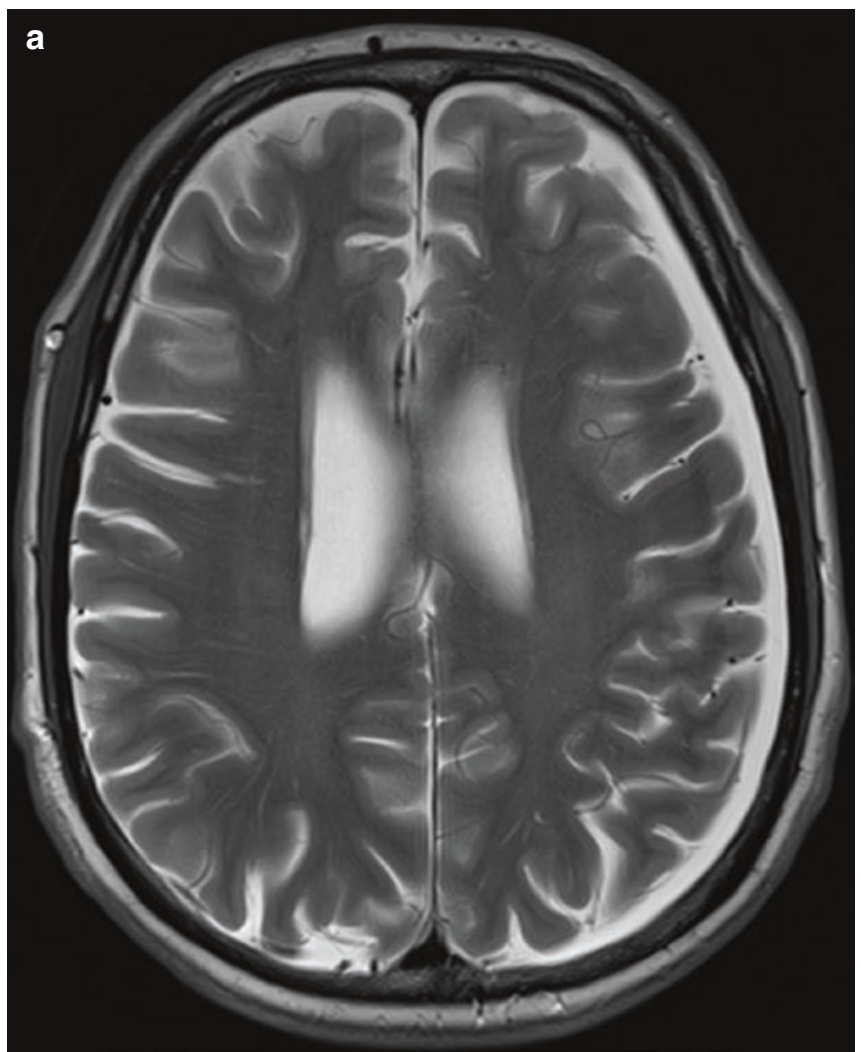

Fig. 7.8 (a, b) Chronic left subdural hematoma. MRI examination, including (a) axial TSE T2-weighted image and (b) axial turbo-FLAIR image with fat saturation. On the T2-weighted scan, the chronic subdural hematoma overlying the left cerebral hemisphere is hyperintense,

\subsubsection{Traumatic Intraventricular Hemorrhage}

Traumatic intraventricular hemorrhage (IVH) occurs as a result of the tearing of subependymal or choroidal veins or reflux of SAH into the ventricular system. It may also be seen in association with diffuse axonal injury of the corpus callosum. The incidence of IVH in nonpenetrating head injury is $1.5-3 \%$ and $10-25 \%$ of patients with severe head injury [22].

On CT IVH may appear as a hyperdense collection in the ventricular system. MRI is more sensitive to IVH and DWI and FLAIR are the preferred sequences for identifying IVH (Fig. 7.9). During the first $48 \mathrm{~h} \mathrm{IVH} \mathrm{appears} \mathrm{hyperintense} \mathrm{on}$ FLAIR, but subsequently the signal intensity may be variable [23]. Pulsatile flow artifacts in the third and fourth ventricle may compromise the usefulness of the FLAIR technique [8]. 3D-FLAIR techniques are more useful in this regard. SWI is extremely sensitive in detecting intraventricular hemorrhage. IVH appears as a typical hypointense area of susceptibility [24].

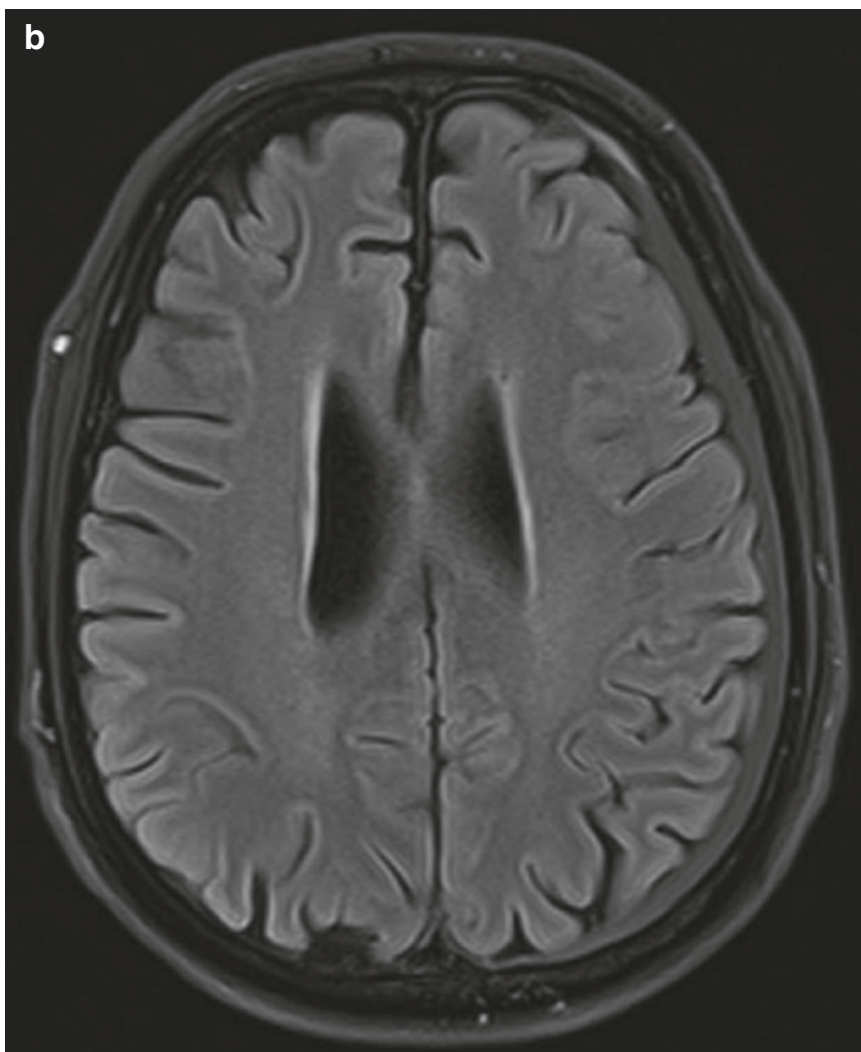

and appears isointense to cerebrospinal fluid (CSF); however, on the FLAIR image, the signal intensity is higher than CSF, indicating a different protein content

\subsection{Intra-Axial Lesions}

\subsubsection{Cerebral Contusion, Hemorrhagic Vs Non-hemorrhagic, Coup Versus Contre-Coup}

Cerebral contusions are focal injuries of the brain surface due to direct impact with the skull and are most common at sides of bony protuberances or overlying irregular areas of the floor of the anterior or middle fossa. They are, in essence, to be considered as "a bruise of the brain." The consequent lesions are hemorrhagic or edematous and may progress to necrosis [25].

All contusions are hemorrhagic, but the extent of edema versus hemorrhage in an individual contusion will determine its appearance on CT. MR will consistently depict hemorrhage, notably on SWI or GRE sequences. Hemorrhagic contusions are superficial and have either a "salt-and-pepper" appearance or may appear more solid [26]. When a traumatic brain injury results in a cerebral contusion, they may prog- 


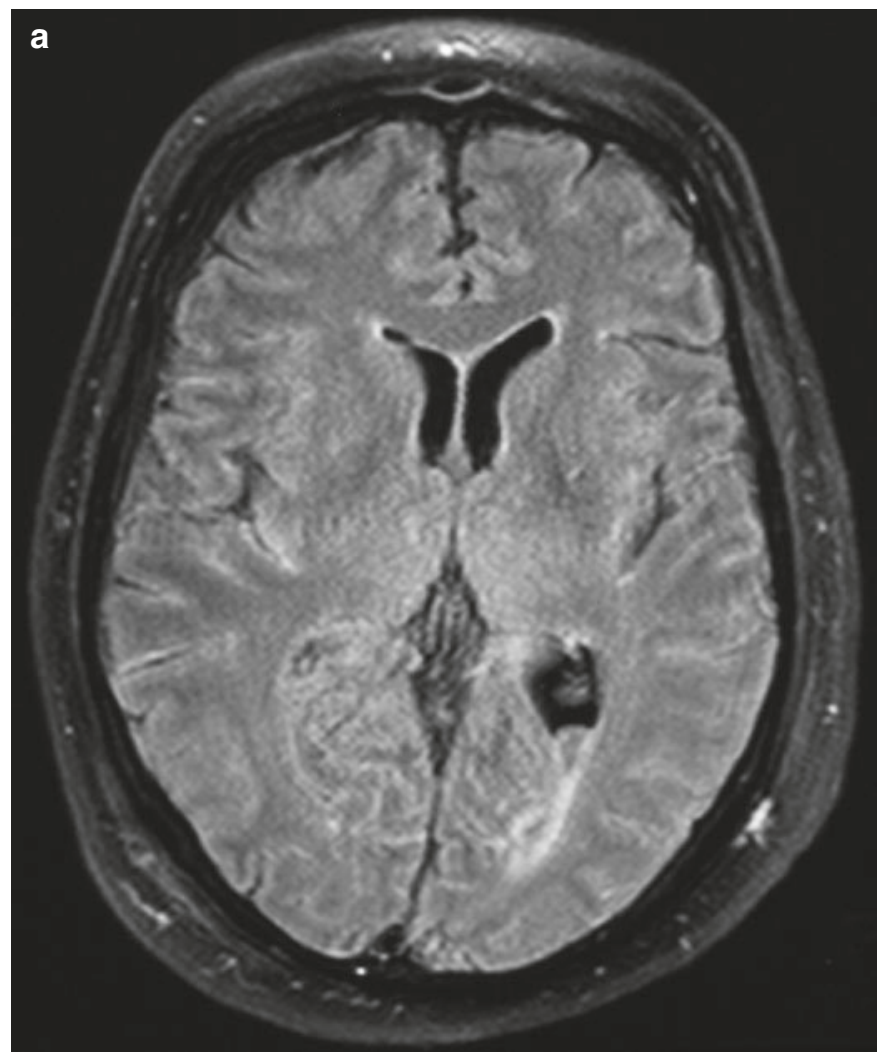

Fig. 7.9 (a, b) Posttraumatic intraventricular and subarachnoid hemorrhage. MRI examination, including (a) axial turbo-FLAIR image with fat saturation and (b) axial diffusion-weighted trace image $(b=1000)$. In the left occipital horn, there is intraventricular blood, with a hemor-

ress during the first several hours and can be associated with SAH [27]. Predominantly affected areas are located in the supratentorial region-anterior frontal and temporal lobes and the gyri around the Sylvian fissure.

On CT, hemorrhagic contusions appear as heterogeneous, hyperdense cortical lesions surrounded by an irregularly marginated hypodense (edematous) component. In the acute stage, CT may be more sensitive than MRI, as acute hemorrhage can be nearly indistinguishable from brain parenchyma on MRI. Subsequent CT performed between 24 and $48 \mathrm{~h}$ after traumatic brain injury often demonstrates previously undetected contusions or more extensive hemorrhage and increased edema. MRI is more sensitive than CT to depict cerebral contusions after the first $24 \mathrm{~h}$ because of the visualization of "non-hemorrhagic" contusion, or contusions with predominant edema. This is particularly true of FLAIR $[8,25]$. The ability of MRI to detect hemorrhagic brain lesions increases proportionally to the evolution of blood products in these lesions. Hemosiderin and also deoxyhemoglobin have increased magnetic susceptibility that make SWI or T2*

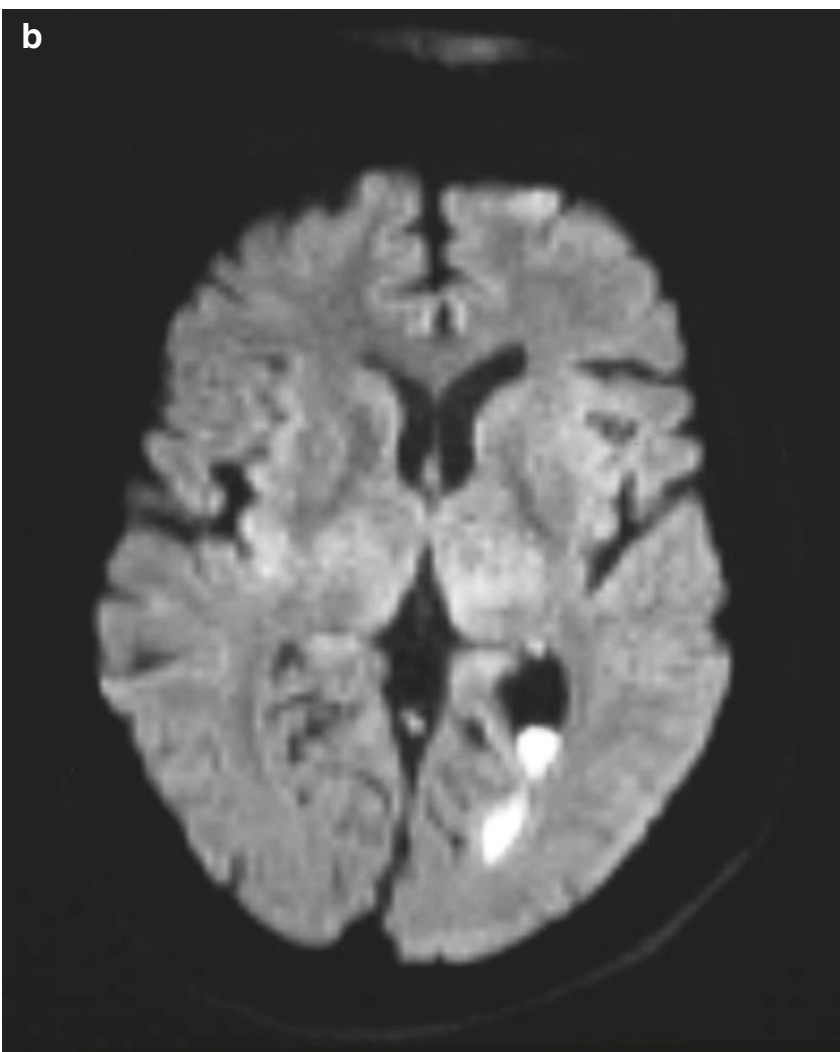

rhagic sedimentation level, and markedly restricted diffusion. In addition, there is evidence of subarachnoid hemorrhage, best seen on the FLAIR images

gradient-echo sequences very sensitive for detecting hemorrhagic lesions [28, 29]. Four to six months after injury the lesions become cystic and lose volume and therefore hypodense on CT [30], although MR findings of hemorrhage persist.

Table 7.3 provides an overview of sequential signal intensity changes of intracranial hemorrhage on MRI.

Contusions may be bihemispheric and occur along the vector of the force applied to the skull on the site of impact and the opposite region of brain. These injuries are referred to as "coup" (side of injury) or "contre-coup" (opposite the injury). In closed-head injury, for example, after an abrupt stop, the force translation can result in this pattern [30]. The coup-injury is on the side of primary impact, often identified by scalp lesions or skull fracture. "Contre-coup" lesions are the mechanical consequence of an acceleration/deceleration trauma and occur on the opposite side of impact. They are often larger and more extensive than the coup lesions, perhaps because of the initial displacement of the brain toward the contre-coup location $[8,30,31]$. 
Table 7.3 Sequential signal intensity changes of intracranial hemorrhage on MRI (1,5 T)

\begin{tabular}{|c|c|c|c|c|c|}
\hline & Hyperacute hemorrhage & Acute hemorrhage & $\begin{array}{l}\text { Early subacute } \\
\text { hemorrhage }\end{array}$ & $\begin{array}{l}\text { Late subacute } \\
\text { hemorrhage }\end{array}$ & Chronic hemorrhage \\
\hline $\begin{array}{l}\text { What } \\
\text { happens }\end{array}$ & $\begin{array}{l}\text { Blood leaves the } \\
\text { vascular system } \\
\text { (extravasation) }\end{array}$ & $\begin{array}{l}\text { Deoxygenation with } \\
\text { formation of deoxy-Hb }\end{array}$ & $\begin{array}{l}\text { Clot retraction and } \\
\text { deoxy-Hb is oxidized to } \\
\text { met-Hb }\end{array}$ & $\begin{array}{l}\text { Cell lysis (membrane } \\
\text { disruption) }\end{array}$ & $\begin{array}{l}\text { Macrophages digest the } \\
\text { clot }\end{array}$ \\
\hline Time frame & $<12 \mathrm{~h}$ & $\begin{array}{l}\text { Hours-days (weeks in } \\
\text { center of hematoma) }\end{array}$ & A few days & 4-7 days -1 month & Weeks-years \\
\hline $\begin{array}{l}\text { Red blood } \\
\text { cells }\end{array}$ & Intact erythrocytes & $\begin{array}{l}\text { Intact, but hypoxic } \\
\text { erythrocytes }\end{array}$ & $\begin{array}{l}\text { Still intact, severely } \\
\text { hypoxic }\end{array}$ & $\begin{array}{l}\text { Lysis (solution of } \\
\text { lysed cells) }\end{array}$ & $\begin{array}{l}\text { Gone; encephalomalacia } \\
\text { with proteinaceous fluid }\end{array}$ \\
\hline State of $\mathrm{Hb}$ & $\begin{array}{l}\text { Intracellular oxy-Hb } \\
(\mathrm{HbO} 2)\end{array}$ & $\begin{array}{l}\text { Intracellular deoxy-Hb } \\
(\mathrm{Hb})\end{array}$ & $\begin{array}{l}\text { Intracell. met-Hb } \\
(\mathrm{HbOH}) \text { (first at } \\
\text { periphery of clot) }\end{array}$ & $\begin{array}{l}\text { Extracell. met-Hb } \\
(\mathrm{HbOH})\end{array}$ & $\begin{array}{l}\text { Hemosiderin (insoluble) } \\
\text { and ferritin (water soluble) }\end{array}$ \\
\hline $\begin{array}{l}\text { Oxidation } \\
\text { state }\end{array}$ & $\begin{array}{l}\text { Ferrous }(\mathrm{Fe} 2+) \\
\text { No unpaired e- }\end{array}$ & $\begin{array}{l}\text { Ferrous }(\mathrm{Fe} 2+) \\
4 \text { unpaired e- }\end{array}$ & $\begin{array}{l}\text { Ferric }(\mathrm{Fe} 3+) \\
5 \text { unpaired e- }\end{array}$ & $\begin{array}{l}\text { Ferric }(\mathrm{Fe} 3+) \\
5 \text { unpaired } \mathrm{e}-\end{array}$ & $\begin{array}{l}\text { Ferric }(\mathrm{Fe} 3+) \\
2000 \times 5 \text { unpaired }--\end{array}$ \\
\hline $\begin{array}{l}\text { Magnetic } \\
\text { properties }\end{array}$ & Diamagnetic $(c<0)$ & Paramagnetic $(c>0)$ & Paramagnetic $(c>0)$ & Paramagnetic $(c>0)$ & $\begin{array}{l}\text { FeOOH is } \\
\text { superparamagnetic }\end{array}$ \\
\hline SI on T1-wi & $\approx$ or $\downarrow$ & $\begin{array}{l}\approx(\text { or } \downarrow) \text { (no PEDD } \\
\text { interaction) }\end{array}$ & $\uparrow \uparrow$ (PEDD interaction) & $\begin{array}{l}\uparrow \uparrow(\mathrm{PEDD} \\
\text { interaction) }\end{array}$ & $\begin{array}{l}\approx(\text { or } \downarrow) \text { (no PEDD } \\
\text { interaction) }\end{array}$ \\
\hline SI on T2-wi & $\uparrow$ (high water content) & $\begin{array}{l}\downarrow \mathrm{T} 2 \text { PRE } \\
\text { (susceptibility effect) }\end{array}$ & $\begin{array}{l}\downarrow \downarrow \text { T2 PRE } \\
\text { (susceptibility effect) }\end{array}$ & $\uparrow \uparrow$ No T2 PRE & $\begin{array}{l}\downarrow \downarrow \text { T2 PRE (susceptibility } \\
\text { effect) }\end{array}$ \\
\hline
\end{tabular}

Notes: $\mathrm{Hb}$ hemoglobin, $e$ - electrons, $\mathrm{FeOOH}$ ferric oxyhydroxide, $\uparrow=$ increased SI relative to normal gray matter, $\downarrow=$ decreased SI relative to normal gray matter

\subsubsection{Diffuse Axonal Injury (DAI)}

Diffuse axonal injury is a common type of primary neuronal injury in patients with severe head trauma with an incidence up to $50 \%$ of trauma victims [30,32]. DAI occurs when the brain is exposed to shear-strain forces that leads to lesions at interfaces between two adjacent tissues with different densities or rigidities, such as gray and white matter [33] or areas where the brain is relatively anchored to an adjacent structure, such as the cerebral or cerebellar peduncles or corpus callosum. Predominantly affected areas in decreasing order of frequency are: gray-white matter junction, corpus callosum, basal ganglia, brainstem, and mesencephalon. The severity of the lesions increases towards the cerebellum, whereas axonal injury in the brainstem is considered to be an indicator for degeneration to coma in DAI $[8,33]$.

Its' important clinical relevance and various clinical manifestations have contributed to the development of radiological imaging techniques for detection of DAI. As mentioned, DAI is a common primary injury in brain trauma and CT examination is still the mode of choice in severe trauma patients in the acute phase because of its great value in detecting petechial hemorrhagic lesions, fractures and because of its rapid and convenient acquisition. Unfortunately, CT significantly underestimates the extent and number of DAI lesions, especially of non-hemorrhagic lesions [34] (Fig. 7.10). Therefore, whenever there is a discrepancy between the clinical status of the patient and the CT findings, MRI can provide very important information. In the early stage, diffusion-weighted images are of great importance to detect DAI. In addition, FLAIR sequences are useful for the detection of areas of gliosis. Gradient-echo sequences (GRE) and more recently susceptibility-weighted imaging (SWI) are also superior to $\mathrm{CT}$ in subacute or chronic hemorrhagic head injuries because of paramagnetic blood degradation products [33, 34].

New advanced MRI techniques like diffusion tensor imaging (DTI) offer more sensitive detection and differentiation of DAI. In DTI, the specific water diffusion direction in the white matter is used to detect microstructural conditions that result in restriction in the diffusion of the molecules and alter the values seen on FA (fractional anisotropy) maps [35, 36]. A recent study showed that DTI is a very qualitative and useful way for clinical evaluation of DAI. The authors found decreased anisotropy in the corpus callosum even in patients with normal results on FLAIR and GRE [37]. Multiple additional studies demonstrate the utility of DTI, but application to individual patients remains challenging because of a wide number of variables [38]. DTI and the derived FA values remain an important research tool, and are being widely investigated to determine what value they may provide in the individual patient. For the present time, these remain largely research tools.

\subsection{Vessel Injury}

\subsubsection{Carotid Artery-Cavernous Sinus Fistula (CCF)}

In the traumatic carotid artery-cavernous sinus fistula $(\mathrm{CCF})$, there occurs a direct communication between the cavernous segment of the internal carotid artery (ICA) and the cavernous sinus. The underlying cause is often a laceration of the ICA due to blunt or penetration head trauma. The fistula leads to engorgement and dilatation of the cavernous sinus with successive congestion of the superior ophthalmic vein (SOV) and inferior petrosal sinus [28]. 

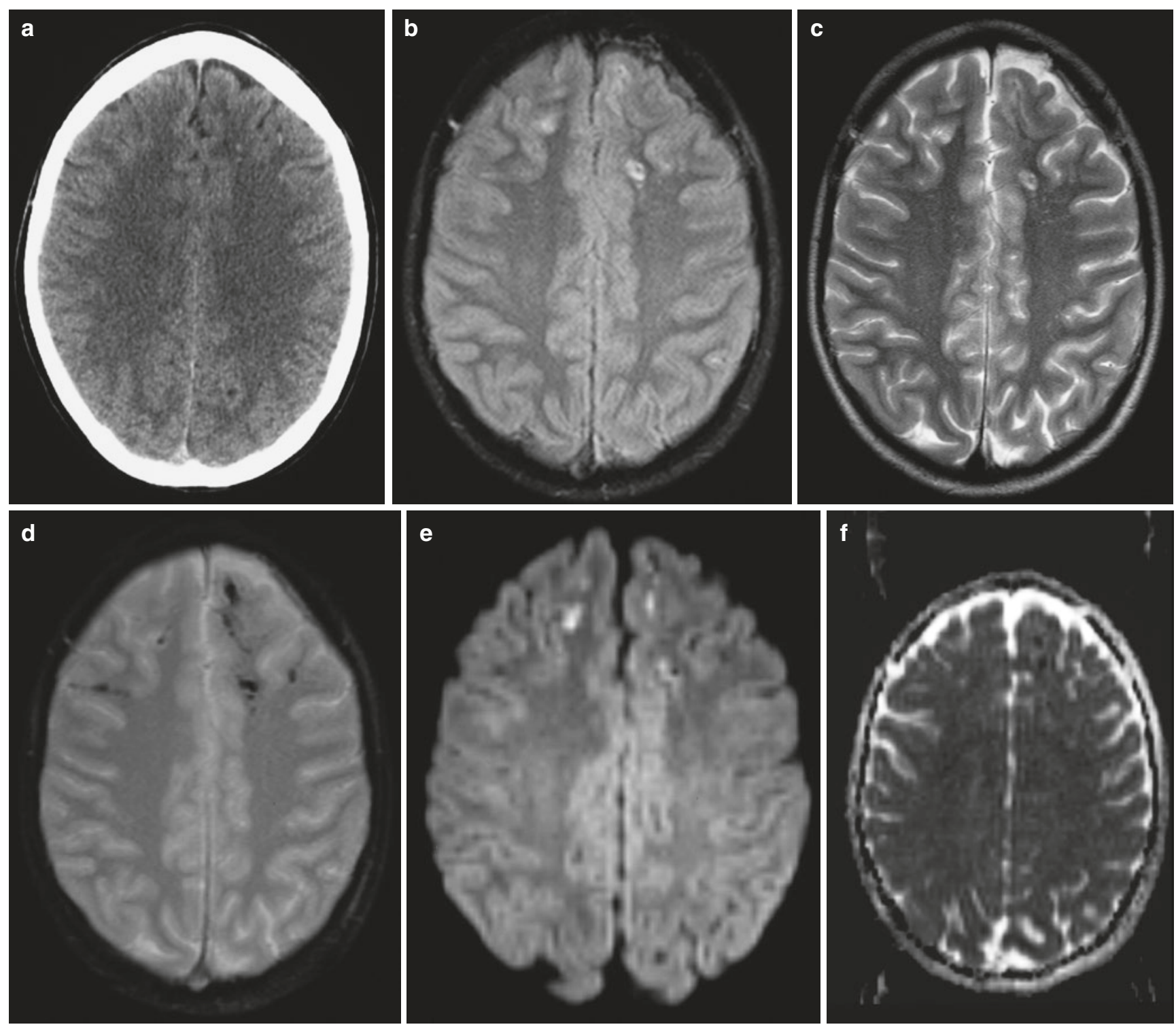

Fig. 7.10 (a-f) Diffuse axonal injuries in a 14-year-old boy, 4 days after a motor vehicle accident: (a) axial non-contrast CT-scan; (b) axial turbo-FLAIR image; (c) axial TSE T2-weighted image; (d) axial gradient-echo FLASH T2*-weighted image $(\mathrm{TE}=25 \mathrm{~ms})$; (e) axial diffusion-weighted image (DWI) with $b=1000$; (f) axial apparent diffusion coefficient (ADC) map. The non-contrast CT-scan shows several punctate petechial hemorrhages at the gray-white matter junction

Specific imaging signs of CCF are enlargement of the cavernous sinus or ipsilateral SOV on CT or MR and multiple flow-voids on MRI. MRA may demonstrate arterialized flow in the cavernous sinus or SOV.

\subsubsection{Traumatic Aneurysms}

Traumatic intracranial aneurysms are rare and represent $<1 \%$ of all aneurysms. They are most common in children [39]. Predominant locations are the cavernous and infraclinoid segment of ICA, as well as along the anterior cerebral artery.

of the frontal lobes. These lesions are hyperintense on turbo-FLAIR and T2-weighted images. On the T2*-weighted image, multiple hypointense hemosiderin deposits are seen at the gray-white matter junction and in the corpus callosum. The appearance, multiplicity, and topographical distribution are typical of hemorrhagic shearing injuries. On the DWI scan, the lesions are hyperintense, and on the ADC maps, the lesions are hypointense, indicating restricted diffusion

The gold-standard in imaging of traumatic aneurysms is cerebral angiography, but CT angiography (CTA) is widely used because of its speed and ease of acquisition [40].

\subsubsection{Traumatic Vascular Dissection}

Traumatic vascular dissections are unusual complications of significant head injury. The injury is caused by a tear in the intimal layer of an artery, resulting in several potential findings, notably diversion of blood flow into a potential "false" lumen within the media. Dissection occurs as a consequence of con- 
tiguous bony injury (fractures), rotational forces and torsion of the vessel in blunt head trauma or less frequently with penetrating trauma [41]. The incidence in blunt trauma victims is $0.86 \%$ for ICA and $0.53 \%$ for traumatic vertebral artery dissections. A common indication for vascular imaging in this setting is fractures of the skull base extending through the carotid canal.

Again, different techniques may provide a diagnosis of vessel dissection. Conventional angiography remains a useful examination. Although the intramural hematoma cannot be visualized, the "string-sign" and a "flame-shaped tapering" are common radiological features [42]. Ultrasound examinations, especially B-mode and Color-flow Doppler, are also increasingly used in detection of traumatic dissections. To detect subtle luminal abnormalities or wall abnormalities in dissection, both CT and MRI, as well as CTA and MRA are superior to catheter angiography.

\subsection{Secondary Lesions}

\subsubsection{Definition}

Secondary brain damage results from the posttraumatic pathophysiologic cascade that follows the initial injury and contributes to delayed tissue injury and neuronal loss.

\subsubsection{Intracranial Hypertension}

Intracranial hypertension may occur after severe cerebral injury and can be a significant finding, resulting in progressive neural dysfunction. After traumatic brain injury, intracranial hypertension may be caused by cumulative edema, hemorrhage, or swelling after contusion. The presence of any of these signs is suspicious: loss of gray-white junction indicating cerebral edema, midline shift, a significant hematoma, herniation, or change in ventricular shape or size [28].

\subsubsection{Brain Herniation (Table 7.4)}

Cerebral herniation is the most common and dangerous secondary effect of increased ICP [8]. Cerebral herniation is the most common secondary effect of an expanding intracranial mass. Due to the herniation the subarachnoid spaces and basal cisterns become obliterated, hydrocephalus develops, vascular compression may result in brain ischemia, and compression on vital brain tissue may cause profound neurological deficits.

\section{8.2.2 Secondary Brainstem Hemorrhage (Duret Hemorrhage)}

Secondary brainstem hemorrhage (also known as "Duret hemorrhage"), can occur in craniocerebral trauma

Table 7.4 Cerebral herniation types

\begin{tabular}{|c|c|c|c|c|c|c|}
\hline $\begin{array}{l}\text { Type of } \\
\text { cerebral } \\
\text { herniation }\end{array}$ & $\begin{array}{l}\text { Subfalcial h. } \\
\text { (cingulate h.) }\end{array}$ & Tonsillar h. & $\begin{array}{l}\text { Descending } \\
\text { transtentorial h. }\end{array}$ & $\begin{array}{l}\text { Ascending } \\
\text { transtentorial h. }\end{array}$ & Uncal h. & External h. \\
\hline Definition & $\begin{array}{l}\text { Medial } \\
\text { displacement of } \\
\text { cingulate gyrus } \\
\text { under inferior free } \\
\text { margin of the falx }\end{array}$ & $\begin{array}{l}\text { Inferior } \\
\text { displacement of } \\
\text { cerebellar } \\
\text { tonsil(s) through } \\
\text { foramen magnum }\end{array}$ & $\begin{array}{l}\text { Downward shift of } \\
\text { diencephalon, } \\
\text { mesencephalon, and } \\
\text { upper brainstem }\end{array}$ & $\begin{array}{l}\text { Superior } \\
\text { displacement of } \\
\text { the vermis } \\
\text { through the } \\
\text { tentorial incisura }\end{array}$ & $\begin{array}{l}\text { Herniation of medial } \\
\text { temporal lobe through } \\
\text { tentorial notch }\end{array}$ & $\begin{array}{l}\text { Brain tissue } \\
\text { extrudes externally } \\
\text { through a skull } \\
\text { defect }\end{array}$ \\
\hline Cause & $\begin{array}{l}\text { Supratentorial } \\
\text { mass (e.g., EDH, } \\
\text { SDH, } \\
\text { hemispheric mass } \\
\text { lesion, ...) }\end{array}$ & $\begin{array}{l}\text { Posterior fossa } \\
\text { mass lesions or } \\
\text { supratentorial } \\
\text { mass effect }\end{array}$ & $\begin{array}{l}\text { Increasing } \\
\text { supratentorial mass } \\
\text { effect }\end{array}$ & $\begin{array}{l}\text { Posterior fossa } \\
\text { mass lesion } \\
\text { (tumor) }\end{array}$ & $\begin{array}{l}\text { Temporal lobe mass } \\
\text { lesion, e.g., focal } \\
\text { hematoma }\end{array}$ & $\begin{array}{l}\text { Increased ICP in } \\
\text { association with a } \\
\text { traumatic or } \\
\text { surgical skull } \\
\text { defect }\end{array}$ \\
\hline Imaging & $\begin{array}{l}\text { - Mass lesion } \\
\text { - Bowing of falx } \\
\text { - Compression of } \\
\text { ipsilateral } \\
\text { lateral ventricle } \\
\text { - Contralateral } \\
\text { ventricle } \\
\text { enlarges due to } \\
\text { obstruction of } \\
\text { the foramen of } \\
\text { Monro }\end{array}$ & $\begin{array}{l}\text { - Downward } \\
\text { displacement } \\
\text { of cerebellar } \\
\text { tonsils below } \\
\text { level of } \\
\text { foramen } \\
\text { magnum } \\
\text { - Obliteration of } \\
\text { cisterna magna }\end{array}$ & $\begin{array}{l}\text { - Obliteration of } \\
\text { peri-mesencephalic } \\
\text { cisterns with } \\
\text { complete plugging } \\
\text { of tentorial incisura } \\
\text { - Downward shift of } \\
\text { pineal calcification } \\
\text { - Brainstem is } \\
\text { foreshortened } \\
\text { (sagittal plane) and } \\
\text { compressed (axial } \\
\text { plane) }\end{array}$ & $\begin{array}{l}\text { - Fourth ventricle } \\
\text { becomes } \\
\text { obliterated } \\
\text { - Effacement of } \\
\text { the superior } \\
\text { cerebellar and } \\
\text { quadrigeminal } \\
\text { cisterns }\end{array}$ & $\begin{array}{l}\text { - Obliteration of the } \\
\text { suprasellar cistern } \\
\text { - Shift of } \\
\text { mesencephalon to } \\
\text { opposite side } \\
\text { - Widening of } \\
\text { ipsilateral CPA } \\
\text { cistern } \\
\text { - Obstructive } \\
\text { hydrocephalus (due } \\
\text { to aqueductal } \\
\text { obstruction) }\end{array}$ & $\begin{array}{l}\text { - Extracranial } \\
\text { displacement of } \\
\text { brain tissue } \\
\text { - Bone defect can } \\
\text { be }\end{array}$ \\
\hline $\begin{array}{l}\text { Compression } \\
\text { of vascular } \\
\text { structures }\end{array}$ & $\begin{array}{l}\text { ACA infarction } \\
\text { (pericallosal and } \\
\text { callosomarginal } \\
\text { arteries) }\end{array}$ & PICA infarction & $\begin{array}{l}\text { PCA compression } \\
\text { leads to occipital lobe } \\
\text { ischemia or infarction }\end{array}$ & $\begin{array}{l}\text { SCA compression } \\
\text { may result in } \\
\text { cerebellar } \\
\text { infarction }\end{array}$ & $\begin{array}{l}\text { Compression of the } \\
\text { contralateral PCA } \\
\text { against the tentorial } \\
\text { edge }\end{array}$ & $\begin{array}{l}\text { Venous obstruction } \\
\text { may result in } \\
\text { venous infarction } \\
\text { (propensity to } \\
\text { hemorrhage) }\end{array}$ \\
\hline $\begin{array}{l}\text { Other } \\
\text { complications }\end{array}$ & $\begin{array}{l}\text { Intracranial } \\
\text { hypertension may } \\
\text { cause descending } \\
\text { transtentorial h }\end{array}$ & $\begin{array}{l}\text { Hydrocephalus } \\
\text { and } \\
\text { syringomyelia }\end{array}$ & $\begin{array}{l}\text { "Duret" brainstem } \\
\text { hemorrhage } \\
\text { (disruption of } \\
\text { perforating arteries to } \\
\text { brainstem) }\end{array}$ & $\begin{array}{l}\text { Hydrocephalus } \\
\text { due to } \\
\text { compression of } \\
\text { the aqueduct }\end{array}$ & $\begin{array}{l}\text { - Can progress to } \\
\text { descending } \\
\text { transtentorial h. } \\
\text { - Obstructive } \\
\text { hydrocephalus }\end{array}$ & $\begin{array}{l}\text { Pressure necrosis } \\
\text { with swelling of } \\
\text { the adjacent brain } \\
\text { at the margins of } \\
\text { the defect }\end{array}$ \\
\hline
\end{tabular}


patients with rapidly evolving descending cerebral herniation [43]. It is evident on CT as a hyperdense brainstem lesion, typically found in lower mesencephalon and ventral pons (Fig. 7.6). It is not clear whether the origin is arterial or venous, but in the majority of cases the outcome is fatal $[8,43]$.

\subsubsection{Ischemic Lesions and Infarction}

Posttraumatic ischemic lesions are common craniocerebral trauma complications with poor clinical outcome. MRI plays an important role in diagnosing this condition by demonstrating multiple areas of abnormal signal intensity on T2-weighted and FLAIR images [8]. On diffusion-weighted scans, these lesions are seen in the acute phase as hyperintense signal with corresponding hypointense signal on ADC [44]. Of all posttraumatic ischemic lesions, the classic lesion is an ipsilateral posterior cerebral artery infarction in the setting of significant supratentorial mass effect, secondary to any traumatic lesion with mass effect. The causative lesion may be an extra-axial hemorrhage or intracranial hemorrhage associated with edema and mass effect. The posterior cerebral artery may be compressed and transiently occluded between the brain and the free edge of the tentorium. Other intracranial arteries may be occluded or compromised secondary to herniation, including the middle and anterior cerebral arteries [45].

\subsubsection{Infections}

The risk of local wound infections, meningitis, ventriculitis, or cerebral abscess are particularly high among penetrating head trauma because of the presence of contaminated foreign objects, skin, hair, and bone fragments driven into the brain tissue along the projectile track [46].

\subsection{Posttraumatic Sequelae}

\subsubsection{Posttraumatic Encephalomalacia}

The term "posttraumatic encephalomalacia" refers to loss of brain tissue after craniocerebral trauma. Gross pathology shows weakening or cystic cavitation of brain tissue, with blurred cortical margins and surrounded by gliotic scar tissue. The basal part of the temporal lobes and anterior pole of the temporal lobes are most frequently affected. CT scans show hypodense areas of tissue loss, particularly in these parts of the brain. On MRI, encephalomalacia is identified as (multi-) cystic areas of tissue loss, surrounded by an irregular rim of gliosis, best seen on FLAIR images as a hyperintense rim (Fig. 7.11). Posttraumatic encephalomalacia is a very serious condition, resulting in life-long disability, neurological dysfunction, epilepsy, or even death in some cases. Another common form of posttraumatic encephalomalacia involves the olfactory nerve, which is frequently injured in shear injury.
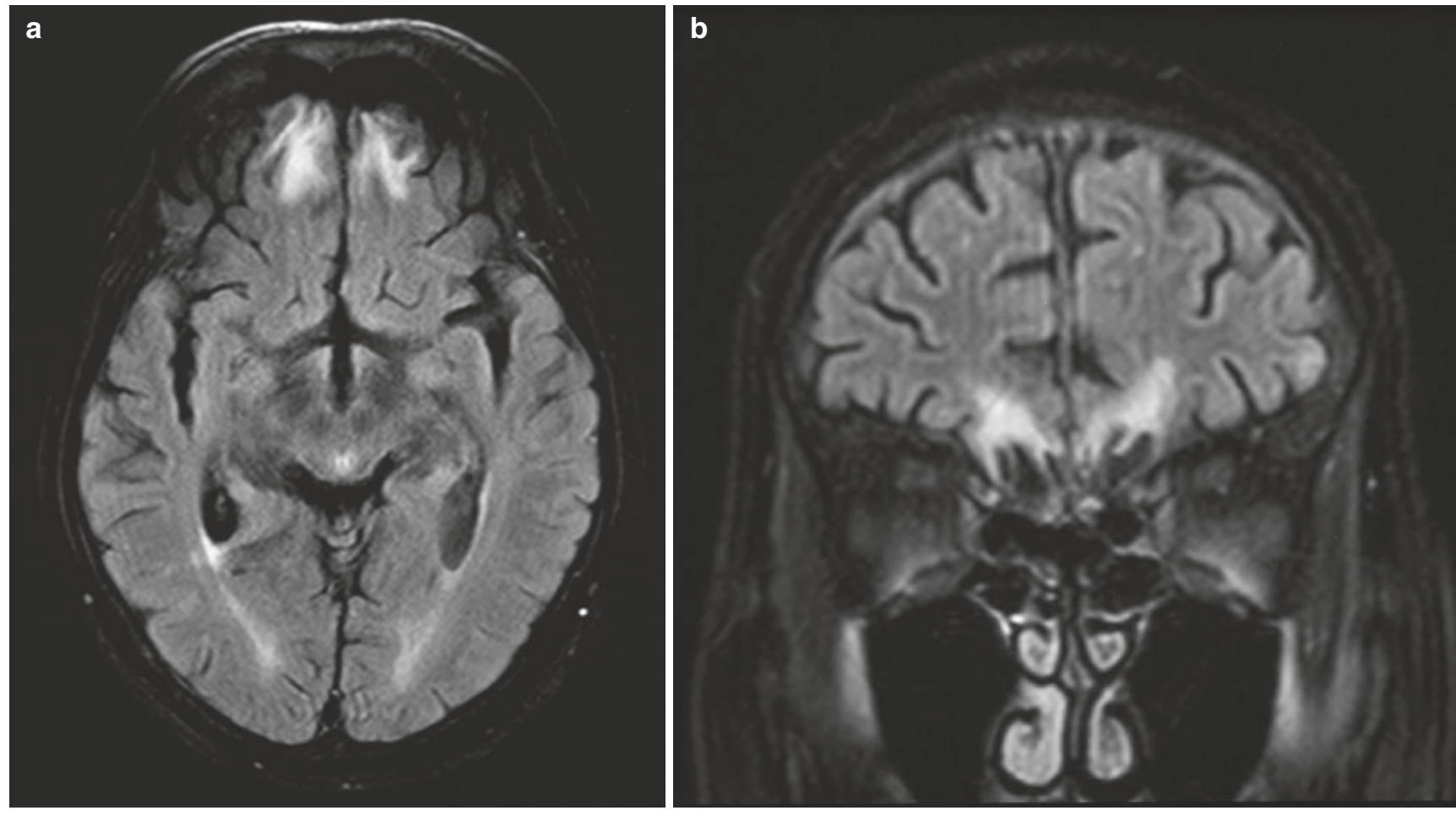

Fig. 7.11 (a, b) Posttraumatic encephalomalacia and gliosis. Axial (a) and coronal (b) turbo-FLAIR images with spectral fat saturation. MRI performed 4 years after severe head injury. There are areas of posttraumatic tissue loss in the basal part of the frontal lobes bilaterally. The areas of tissue loss are surrounded by gliosis, which is hyperintense on the FLAIR images. In addition, there are old diffuse axonal injuries, seen as gliotic foci at the gray-white matter interface, e.g., in the upper part of the left frontal lobe 


\subsubsection{Growing Skull Fracture}

A growing skull fracture is a rare but well-known complication of pediatric head trauma [47]. Its incidence ranges from $<0.05 \%$ to $1.6 \%$ [48]. CT and MRI findings include a diastatic fracture and brain herniation through the skull defect; it can also be accompanied by cystic lesions (meningocele) within the fracture and encephalomalacia [48].

\subsubsection{Traumatic CSF Leaks}

Traumatic cerebrospinal leakage is a complication seen in $2 \%$ of all head-injured patients and in $12-30 \%$ of cases of basilar skull fractures [49]. The most common locations are the cribriform plate (forming the roof of the ethmoid) and the walls of the frontal sinus [50]. Intracranial air may be an important sign. Careful evaluation of fractures that are contiguous to sinuses is key to making a correct diagnosis. Persistent opacification of sinuses contiguous to a fracture are of particular suspicion. Suggesting the presence of a CSF leak will usually result in testing of fluid in the contiguous sinus for the presence of beta- 2 transferrin, a dependable marker of CSF.

\subsubsection{Diabetes Insipidus}

The prevalence of central diabetes insipidus (DI) after TBI is $1.7-26 \%$, often resulting from injury or transection of the infundibulum. MRI is the technique of choice for pituitary imaging. A sagittal T1-weighted image demonstrates a posterior pituitary "bright spot" in healthy individuals. The absence of this "bright spot" in diabetes insipidus is a nonspecific sign of DI [51]. In addition to the absence of this sign, an ectopic "bright spot" in the proximal stump of the transected, retracted proximal stalk or hypothalamus may be identified. If there is clinical suspicion of trauma-related DI, a dedicated pituitary examination is the appropriate examination.

\subsection{Quantitative Imaging in Patients with Traumatic Brain Injury}

\subsubsection{Diffusion Weighted Imaging (DWI) with ADC Maps}

Diffusion-weighted imaging measures the motion of water molecules and thus helps visualize the physiologic state in the brain [8]. There are physiologic phase-shifts or signal loss due to this normal semi-random motion of water molecules. In distinction, areas with abnormal diffusion reflect alterations in the normal water diffusion and appear bright on DWI [29]. The apparent diffusion coefficient (ADC) values indicate the decrease in the diffusion with corresponding hypointense signal on ADC maps [29]. DWI has become an important tool in diagnosing TBI, acute stroke, and white matter diseases [8].

\subsubsection{Diffusion Tensor Imaging (DTI), Diffusion Kurtosis Imaging (DKI)}

Diffusion tensor Imaging (DTI) is derived from specialized DWI with multiple directions, and can measure not only the degree, but also the direction of water diffusion [52]. It allows in-vivo visualization of the integrity of white matter tracts. The main application is in diffuse axonal injury.

Diffusion kurtosis Imaging (DKI) is an extended technique, derived from DTI, and allows diffusional kurtosis to be evaluated using diffusion-weighted imaging techniques [53]. With DKI, both gray and white matter can be evaluated.

\subsubsection{Outcome Prediction with Imaging Parameters}

Imaging is a very important clinical tool for diagnosis, prognosis, and visualizing therapy outcomes following TBI. Although structural imaging techniques such as CT or MRI offer a rapid evaluation of the brain status and may help predict survival, more information is necessary. Detailed information of cerebral perfusion has also proven useful in management and in assessing outcomes [29].

Several imaging techniques are available to visualize the brain physiology: CT perfusion, MR perfusion, SPECT, fMRI (functional MRI), PET, and MRS (MR Spectroscopy). While obviously important research tools, these techniques are occasionally finding their way into clinical imaging to answer clinical questions.

MRI sequences like DTI, FLAIR, GRE/SWI, and DWI are increasingly used in many research models predicting outcome [54].

\subsection{Conclusion}

Imaging studies represent an essential element in the management of patients with significant neurologic trauma. Diagnosis of the extent of the initial injury is critical to triage patients to surgical or non-surgical management. Repeated follow up to determine potential secondary injury patterns and also determine the success of management schemes are of significant value and improve patient outcomes. Imaging techniques have shown considerable promise in improving 
our knowledge of the patterns and significance of intracranial injury and in suggesting and monitoring therapies to treat these patients and determine their prognosis.

\section{Take Home Messages}

- A rapid and thorough CT examination of the acutely traumatized patient is standard of care in major trauma centers.

- Develop a protocol for urgent CT screening and reporting of major trauma patients.

- Rapid access to imaging technologies is critical to good outcomes in patients who have been significantly injured.

- If the CT study is not adequate to explain the patient's mental status, MR is the next most important examination to be performed.

\section{References}

1. Maas AI, Stocchetti N, Bullock R. Moderate and severe traumatic brain injury in adults. Lancet Neurol. 2008;7(8):728-41.

2. Tagliaferri F, Compagnone C, Korsic M, Servadei F, Kraus J. A systematic review of brain injury epidemiology in Europe. Acta Neurochir. 2006;148(3):255-68.

3. Le TH, Gean AD. Neuroimaging of traumatic brain injury. Mt Sinai J Med. 2009;76(2):145-62.

4. Jones TR, Kaplan RT, Lane B, Atlas SW, Rubin GD. Single- versus multi-detector row $\mathrm{CT}$ of the brain: quality assessment. Radiology. 2001;219(3):750-5.

5. Langford S, Panigrahy A, Narayanan S, Hwang M, Fitz C, Flom L, Lee VK, Zuccoli G. Multiplanar reconstructed CT images increased depiction of intracranial hemorrhages in pediatric head trauma. Neuroradiology. 2015;57(12):1263-8.

6. Bykowski J, Wong W. Angiographic evaluation and treatment for head and neck vascular injury. Appl Radiol. 2012;41(3):10-6.

7. Provenzale J. Imaging of traumatic brain injury: a review of the recent medical literature. AJR Am J Roentgenol. 2010;194(1):16-9.

8. Parizel PM, Van Goethem JW, Ozsarlak O, Maes M, Phillips $\mathrm{CD}$. New developments in the neuroradiological diagnosis of craniocerebral trauma. Eur Radiol. 2005;15(3):569-81.

9. Yen K, Vock P, Tiefenthaler B, Ranner G, Scheurer E, Thali MJ, Zwygart K, Sonnenschein M, Wiltgen M, Dirnhofer R. Virtopsy: forensic traumatology of the subcutaneous fatty tissue; multislice computed tomography (MSCT) and magnetic resonance imaging (MRI) as diagnostic tools. J Forensic Sci. 2004;49(4):799-806.

10. Malli N, Ehammer T, Yen K, Scheurer E. Detection and characterization of traumatic scalp injuries for forensic evaluation using computed tomography. Int J Legal Med. 2013;127(1):195-200.

11. Hofman PA, Nelemans P, Kemerink GJ, Wilmink JT. Value of radiological diagnosis of skull fracture in the management of mild head injury: meta-analysis. J Neurol Neurosurg Psychiatry. 2000;68(4):416-22.

12. Nakahara K, Shimizu S, Utsuki S, Oka H, Kitahara T, Kan S, Fujii $\mathrm{K}$. Linear fractures occult on skull radiographs: a pitfall at radiological screening for mild head injury. J Trauma. 2011;70(1):180-2.

13. Tallon JM, Ackroyd-Stolarz S, Karim SA, Clarke DB. The epidemiology of surgically treated acute subdural and epidural hemato- mas in patients with head injuries: a population-based study. Can J Surg. 2008;51(5):339-45.

14. Parizel PM, Phillips CD. Neuroradiological diagnosis of craniocerebral trauma: current concepts. In: Hodler J, von Schulthess GK, Zollikofer CL, editors. Diseases of the brain, head \& neck, spine 2012-2015. Basel: Springer; 2012.

15. Al-Nakshabandi NA. The swirl sign. Radiology. 2001;218(2):433.

16. Gean AD, Fischbein NJ, Purcell DD, Aiken AH, Manley GT, Stiver SI. Benign anterior temporal epidural hematoma: indolent lesion with a characteristic CT imaging appearance after blunt head trauma. Radiology. 2010;257(1):212-8.

17. Senturk S, Guzel A, Bilici A, Takmaz I, Guzel E, Aluclu MU, Ceviz A. CT and MR imaging of chronic subdural hematomas: a comparative study. Swiss Med Wkly. 2010;140(23-24):335-40.

18. Honda Y, Sorimachi T, Momose H, Takizawa K, Inokuchi S, Matsumae M. Chronic subdural haematoma associated with disturbance of consciousness: significance of acute-on-chronic subdural haematoma. Neurol Res. 2015;37(11):985-92.

19. León-Carrión J, Domínguez-Morales Mdel R, Barroso y Martín JM, Murillo-Cabezas F. Epidemiology of traumatic brain injury and subarachnoid hemorrhage. Pituitary. 2005;8(3-4):197-202.

20. Fainardi E, Chieregato A, Antonelli V, Fagioli L, Servadei F. Time course of CT evolution in traumatic subarachnoid haemorrhage: a study of 141 patients. Acta Neurochir. 2004;146(3):257-63.

21. Verma RK, Kottke R, Andereggen L, Weisstanner C, Zubler C, Gralla J, Kiefer C, Slotboom J, Wiest R, Schroth G, Ozdoba C, El-Koussy M. Detecting subarachnoid hemorrhage: comparison of combined FLAIR/SWI versus CT. Eur J Radiol. 2013;82(9): $1539-45$.

22. LeRoux PD, Haglund MM, Newell DW, Grady MS, Winn HR. Intraventricular hemorrhage in blunt head trauma: an analysis of 43 cases. Neurosurgery. 1992;31(4):678-84.

23. Sohn CH, Baik SK, Lee HJ, Lee SM, Kim IM, Yim MB, Hwang JS, Lauzon ML, Sevick RJ. MR imaging of hyperacute subarachnoid and intraventricular hemorrhage at 3T: a preliminary report of gradient echo T $2 *$-weighted sequences. AJNR Am J Neuroradiol. 2005;26(3):662-5.

24. Wu Z, Li S, Lei J, An D, Haacke EM. Evaluation of traumatic subarachnoid hemorrhage using susceptibility-weighted imaging. AJNR Am J Neuroradiol. 2010;31(7):1302-10.

25. Toyama Y, Kobayashi T, Nishiyama Y, Satoh K, Ohkawa M, Seki K. CT for acute stage of closed head injury. Radiat Med. 2005;23(5):309-16.

26. Alahmadi H, Vachhrajani S, Cusimano MD. The natural history of brain contusion: an analysis of radiological and clinical progression. J Neurosurg. 2010;112(5):1139-45.

27. Kurland D, Hong C, Aarabi B, Gerzanich V, Simard JM. Hemorrhagic progression of a contusion after traumatic brain injury: a review. J Neurotrauma. 2012;29(1):19-31.

28. Lee B, Newberg A. Neuroimaging in traumatic brain imaging. NeuroRx. 2005;2(2):372-83.

29. Coles JP. Imaging after brain injury. Br J Anaesth. 2007;99(1):49-60.

30. Besenski N. Traumatic injuries: imaging of head injuries. Eur Radiol. 2002;12(6):1237-52.

31. Drew LB, Drew WE. The contrecoup-coup phenomenon: a new understanding of the mechanism of closed head injury. Neurocrit Care. 2004;1(3):385-90.

32. Hammoud DA, Wasserman BA. Diffuse axonal injuries: pathophysiology and imaging. Neuroimaging Clin N Am. 2002; 12(2):205-16.

33. Li XY, Feng DF. Diffuse axonal injury: novel insights into detection and treatment. J Clin Neurosci. 2009;16(5):614-9.

34. Parizel PM, Ozsarlak, Van Goethem JW, van den Hauwe L, Dillen C, Verlooy J, Cosyns P, De Schepper AM. Imaging findings in diffuse axonal injury after closed head trauma. Eur Radiol. 1998;8(6):960-5. 
35. Niogi SN, Mukherjee P, Ghajar J, Johnson C, Kolster RA, Sarkar R, Lee H, Meeker M, Zimmerman RD, Manley GT, McCandliss BD. Extent of microstructural white matter injury in postconcussive syndrome correlates with impaired cognitive reaction time: a 3T diffusion tensor imaging study of mild traumatic brain injury. AJNR Am J Neuroradiol. 2008;29(5):967-73.

36. Hurley RA, McGowan JC, Arfanakis K, Taber KH. Traumatic axonal injury: novel insights into evolution and identification. J Neuropsychiatry Clin Neurosci. 2004;16(1):1-7.

37. Chan JH, Tsui EY, Peh WC, Fong D, Fok KF, Leung KM, Yuen MK, Fung KK. Diffuse axonal injury: detection of changes in anisotropy of water diffusion by diffusion-weighted imaging. Neuroradiology. 2003;45(1):34-8.

38. Hulkower MB, Poliak DB, Rosenbaum SB, Zimmerman ME, Lipton ML. A decade of DTI in traumatic brain injury: 10 years and 100 articles later. AJNR Am J Neuroradiol. 2013;34(11):2064-74.

39. Larson PS, Reisner A, Morassutti DJ, Abdulhadi B, Harpring JE. Traumatic intracranial aneurysms. Neurosurg Focus. 2000;8(1):e4.

40. Mao Z, Wang N, Hussain M, Li M, Zhang H, Zhang Q, Zhang P, Zhi X, Ling F. Traumatic intracranial aneurysms due to blunt brain injury - a single center experience. Acta Neurochir. 2012;154(12):2187-93.

41. Schievink WI. Spontaneous dissection of the carotid and vertebral arteries. N Engl J Med. 2001;344(12):898-906.

42. Caplan LR. Dissections of brain-supplying arteries. Nat Clin Pract Neurol. 2008;4(1):34-42.

43. Parizel PM, Makkat S, Jorens PG, Ozsarlak O, Cras P, Van Goethem JW, van den Hauwe L, Verlooy J, De Schepper AM. Brainstem hemorrhage in descending transtentorial herniation (Duret hemorrhage). Intensive Care Med. 2002;28(1):85-8.
44. Parizel PM, Demey HE, Veeckmans G, Verstreken F, Cras P, Jorens PG, De Schepper AM. Early diagnosis of cerebral fat embolism syndrome by diffusion-weighted MRI (starfield pattern). Stroke. 2001;32(12):2942-4.

45. Server A, Dullerud R, Haakonsen M, Nakstad PH, Johnsen UL, Magnaes B. Post-traumatic cerebral infarction. Neuroimaging findings, etiology and outcome. Acta Radiol. 2001;42(3):254-60.

46. Kazim SF, Shamim MS, Tahir MZ, Enam SA, Waheed S. Management of penetrating brain injury. J Emerg Trauma Shock. 2011;4(3):395-402.

47. Drapkin AJ. Growing skull fracture: a posttraumatic neosuture. Childs Nerv Syst. 2006;22(4):394-7.

48. Ersahin Y, Gülmen V, Palali I, Mutluer S. Growing skull fractures (craniocerebral erosion). Neurosurg Rev. 2000;23(3):139-44.

49. Friedman JA, Ebersold MJ, Quast LM. Post-traumatic cerebrospinal fluid leakage. World J Surg. 2001;25(8):1062-6.

50. Hofmann E, Behr R, Schwager K. Imaging of cerebrospinal fluid leaks. Klin Neuroradiol. 2009;19(2):111-21.

51. Makulski DD, Taber KH, Chiou-Tan FY. Neuroimaging in posttraumatic hypopituitarism. J Comput Assist Tomogr. 2008; 32(2):324-8.

52. Xiong KL, Zhu YS, Zhang WG. Diffusion tensor imaging and magnetic resonance spectroscopy in traumatic brain injury: a review of recent literature. Brain Imaging Behav. 2014;8(4):487-96.

53. Jensen JH, Helpern JA. MRI quantification of non-Gaussian water diffusion by kurtosis analysis. NMR Biomed. 2010;23(7):698-710.

54. Huisman TA, Schwamm LH, Schaefer PW, Koroshetz WJ, ShettyAlva N, Ozsunar Y, Wu O, Sorensen AG. Diffusion tensor imaging as potential biomarker of white matter injury in diffuse axonal injury. AJNR Am J Neuroradiol. 2004;25(3):370-6.

Open Access This chapter is licensed under the terms of the Creative Commons Attribution 4.0 International License (http://creativecommons. org/licenses/by/4.0/), which permits use, sharing, adaptation, distribution and reproduction in any medium or format, as long as you give appropriate credit to the original author(s) and the source, provide a link to the Creative Commons license and indicate if changes were made.

The images or other third party material in this chapter are included in the chapter's Creative Commons license, unless indicated otherwise in a credit line to the material. If material is not included in the chapter's Creative Commons license and your intended use is not permitted by statutory regulation or exceeds the permitted use, you will need to obtain permission directly from the copyright holder. 


\title{
Differential Diagnosis of Intracranial Masses
}

\author{
James G. Smirniotopoulos and Hans Rolf Jäger
}

\section{Learning Objectives}

- Distinguish intraaxial from extraaxial and intraventricular masses

- Describe secondary effects: "volume effect" and perilesional vasogenic edema

- Distinguish metastatic disease at gray-white junction from deeper infiltrating gliomas

\subsection{Introduction}

Accurate image interpretation requires a knowledge base, understanding the patient's clinical features, and experience. Radiology is often taught as an apprenticeship of pattern recognition. However, the best interpretations use pattern analysis instead of pattern recognition: progression of clinical course (acute-rapid, subacute-smoldering, chronicprolonged); location of lesion (intra- vs. extraaxial); secondary effects (volume, edema, herniation); enhancement (solid, ring-like or leading edge, non-enhancing); blood-products; MR Spectroscopy (MRS); Diffusion-weighted Imaging (DWI and ADC values); response to treatment.

\subsubsection{Time Course of Disease}

Cerebrovascular events and traumatic lesions usually present acutely: skull impact and inertial trauma cause epidural, subdural, subarachnoid, and parenchymal hemorrhage; aneurys-

J. G. Smirniotopoulos $(\bowtie)$

Department of Radiology, Sigma Healthcare Consulting,

Silver Spring, MD, USA

\section{H. R. Jäger}

Neuroradiological Academic Unit, UCL Institute of Neurology,

London, UK

e-mail: r.jager@ucl.ac.uk mal bleeding ("thunderclap headache") into the subarachnoid space (SAH); deep hypertensive bleeds (basal ganglia and thalamus); acute neurologic deficit from ischemia-infarction (aphasia, paralysis, etc.). At the other end of the time spectrum, degenerative diseases and dementia may progress over months to years. In between, with symptom duration of weeks to months to years, are intracranial neoplasms. More rapid onset of symptoms occurs with high-grade glioma (glioblastoma) and metastatic disease.

\section{Key Point}

- Every image should be interpreted in the clinical context for each patient. When things are inconsistent, expand your differential diagnosis.

\subsection{Intraaxial Vs. Extraaxial}

Intraaxial lesions involve the parenchyma of the brain (including brainstem and cerebellum). They may follow an anatomic distribution (e.g., vascular territory for ischemia/infarction) or non-anatomic distribution (abscess). Diffuse gliomas will expand the infiltrated white-matter and tracts, causing effacement of ventricles and sulci. Extraaxial lesions will displace the brain away from the skull—often enlarging the subarachnoid cisternal spaces at the edges adjacent to the mass (Fig. 8.1).

\subsection{Pattern Analysis}

Radiology (also pathology and dermatology) are often taught using "pattern recognition." This works well once you have accumulated-through experience-a built-in library of "patterns" that you may match with an unknown case. Another approach is a break down of the imaging features, analyze them separately, and then synthesize a differential diagnosis. Patterns of contrast enhancement may suggest 

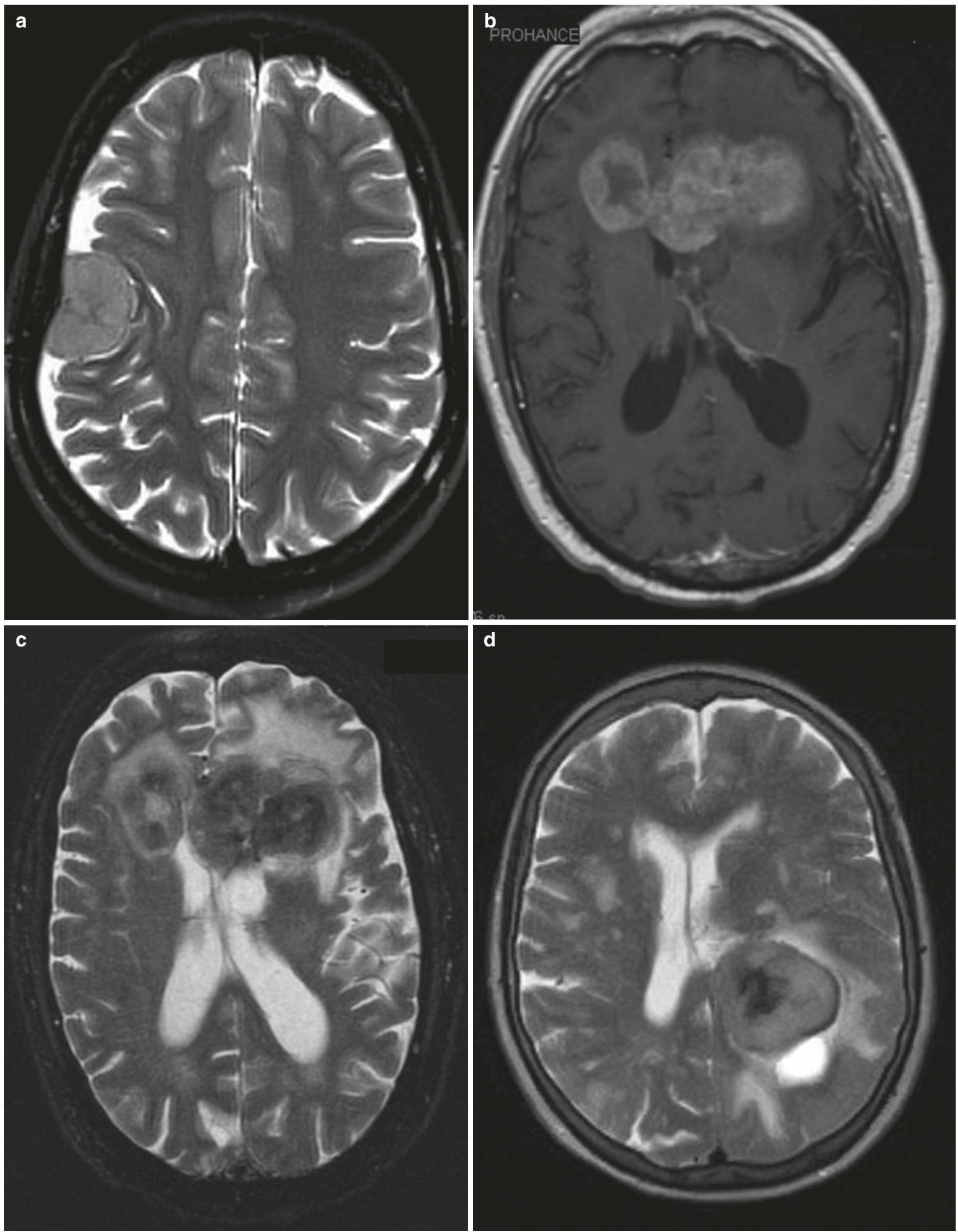

Fig. 8.1 (a) Extraaxial mass (meningioma). Axial T2-weighted MR shows a right-side gray-matter signal-intensity hemispheric-shape mass with enlargement of the SAS at its margins. There is underlying hyperostosis. (b, c) Intraaxial primary CNS lymphoma. Axial T1-weighted with gadolinium (b) shows an enhancing intraaxial mass in the genu of the corpus callosum. On the T2-weighted image (c), the lesion shows lower signal (restricted diffusion) and perilesional vasogenic edema in

the white-matter and sparing the cortical gray-matter. Infiltrating expansile lesions of the corpus callosum are usually diffuse glioma or primary CNS lymphoma. (d) Intraventricular meningioma. Axial T1-weighted image shows a rounded lesion in the trigone of the left lateral ventricle. Ventricular meningiomas arise from arachnoid cells within the choroid plexus, and the trigone is the most common location 
more specific diagnoses: gyral gray-matter enhancement occurs with reperfusion after ischemia, post-ictal or seizures, and meningoencephalitis; ring enhancement implies "central necrosis"- but may be seen with an organized abscess as well as neoplasms [1].

\subsection{Neoplastic Vs. Non-neoplastic Disease}

Neoplastic diseases are usually "tumefactive" - they take up space ("positive volume effect") and displace adjacent structures causing secondary damage and herniation. Both primary and secondary (metastatic) lesions may have added volume from accumulation of interstitial fluid-brain edema-most often due to abnormal increased permeability from breakdown of the blood-brain-barrier (BBB): vasogenic edema. In contrast, inflammatory demyelination often has minimal mass effect - it's "volume neutral". Chronic destructive processes (demyelination, healed viral infections, old infarcts) have "negative volume effect" (Fig. 8.2).

\section{Key Point}

- Chronic destructive lesions cause a loss of brain volume or "negative volume effect," with compensatory enlargement of ventricles and sulci. Adding cells (blood, pus, neoplasm) usually cause proportionate "positive volume effect" with compression of ventricles and effacement of sulci.
Tumefactive demyelinating lesions (TDL) are a frequent differential dilemma. If correctly identified, they should not be biopsied. Instead, a trial of immune-modulating therapy should be given. Features suggesting a TDL (instead of a neoplasm) include: lesion centered in white-matter (including corpus callosum); central-vein sign; "garland-like" or incomplete rim-enhancement ("open ring" or "horseshoe"); less than expected volume effect; rCBV lower than neoplasm; ADC values higher than neoplasm (GBM or lymphoma); and non-contrast CT showing low attenuation (compared to solid portions of GBM and hyper-attenuation of lymphoma [2-4] (Fig. 8.3).

\section{Key Point}

- Tumefactive demyelination may be distinguished from a neoplasm by paucity of mass-effect; mini$\mathrm{mal} / \mathrm{no}$ perilesional edema; "open ring" enhancement; increased ADC; and low perfusion values.

\subsection{Single Vs. Many Lesions}

The vast majority of intracranial lesions may present as a single/solitary mass: primary neoplasm; hematoma; abscess; infarction. Multiple lesions primarily occur as part of a systemic disease: inflammatory; toxic; metabolic; genetic; or hematogenous dissemination (Fig. 8.4). Hematogenous dissemination of infection and metastatic neoplasms can pres-
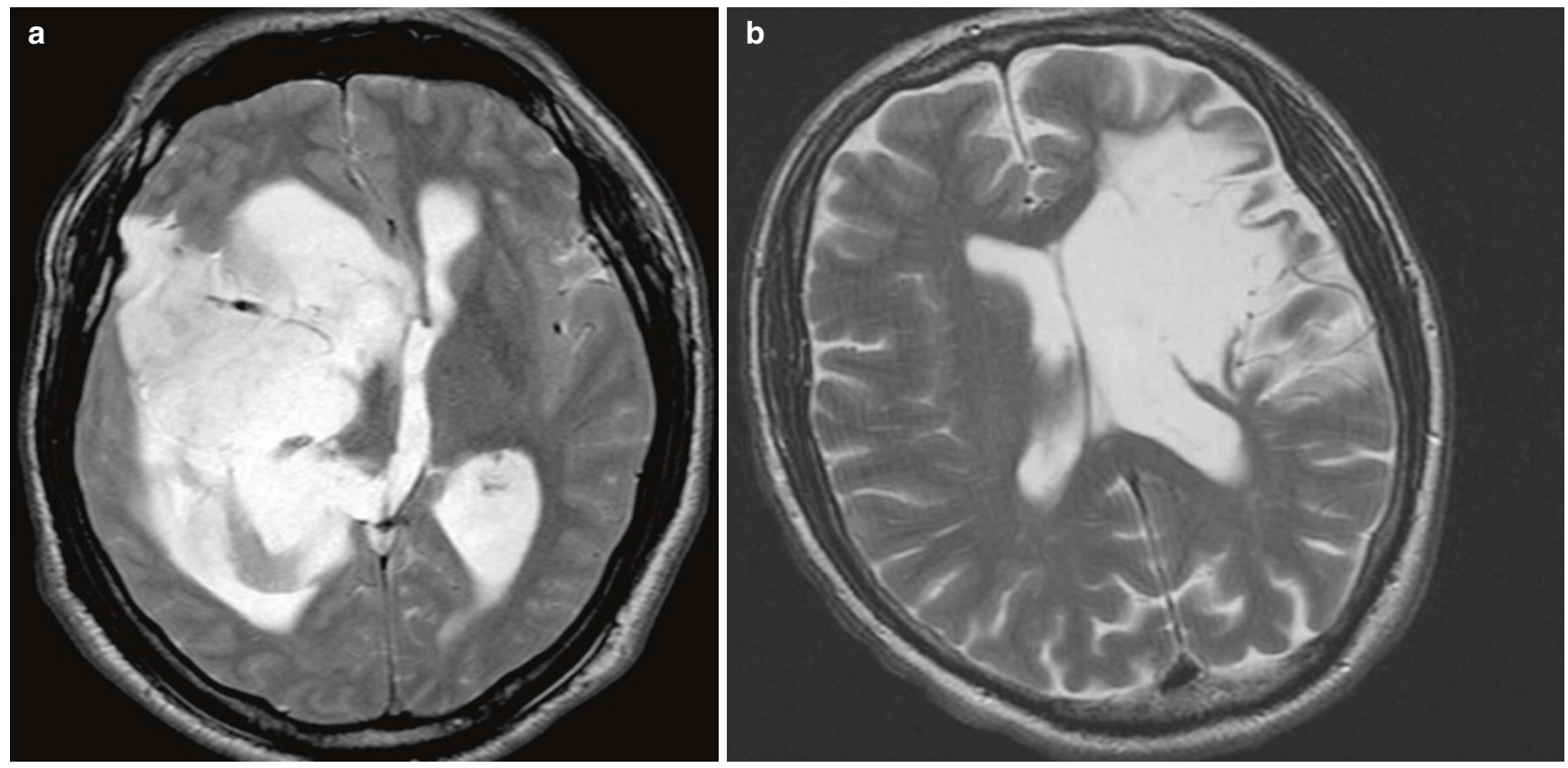

Fig. 8.2 (a) Oligodendroglioma. Axial T2-weighted image shows a single, large, deep, expansile lesion effacing both the cortical sulci and the adjacent ventricle. A lesion of this size, to present so late in its natural history, must be both slowly growing and non-destructively infiltrating. Positive "volume effect." (b) Chronic cerebral infarction. Axial T1-weighted image shows a large region of hyperintensity in the territory of the left middle cerebral artery. There is compensatory enlargement of the adjacent ventricle, overlying sulci, and subarachnoid space. Negative "volume effect" 


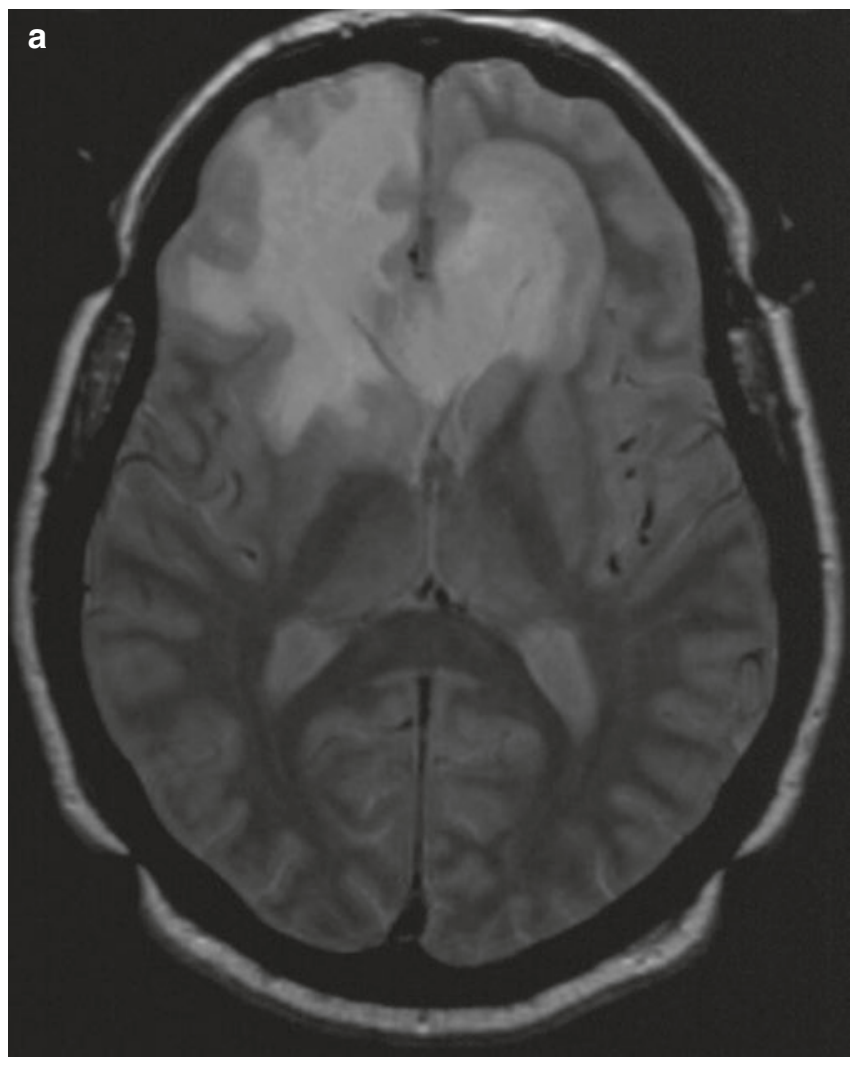

Fig. 8.3 (a, b) Tumefactive Demyelination (path-proven). Axial proton-density (a) and T1-weighted MR with gadolinium (b) show a large expansile lesion of the corpus callosum, extending into both frontal lobes. This is strongly suggestive of a diffuse high-grade astrocy-

ent with solid nodular as well as ring-enhancing lesions. Approximately $40-60 \%$ of patients with hematogenous metastasis will present initially with a single or solitary metastasis [5]. Inflammatory and auto-immune diseases (without infection) typically show multiple lesions: multiple sclerosis-MS, acute disseminated encephalomyelitisADEM (Fig. 8.4e).

\section{Key Point}

- Multiple lesions have many possible causes-some neoplastic, others toxic, metabolic, inflammatory, or genetic.

\subsection{Metastatic Disease}

Solid tumors require angiogenesis to enlarge beyond a couple of millimeters. The bio-machinery to grow new vessels can remodel existing vessels and allow embolization of tumor cells. Once in circulation, the tumor emboli are distributed by blood flow: hematogenous dissemination. The

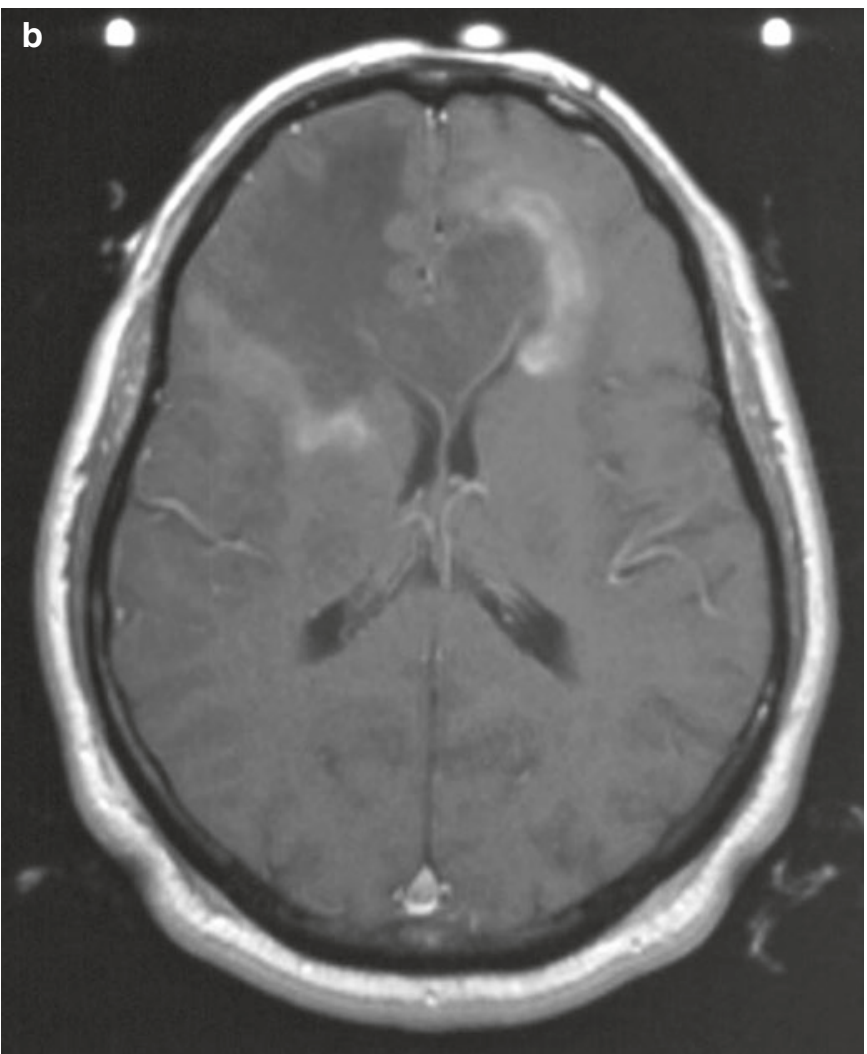

toma (glioblastoma). However, there is discontinuous peripheral "garland-like" enhancement-more characteristic of inflammatory demyelination. The appearance was so suggestive of neoplasm that a biopsy was performed

brain requires about $20 \%$ of our cardiac output. It is a vast "filtration bed" where platelet thrombi, clots, and tumor emboli may lodge. The majority of these "particulates" will lodge in two places: in the subcortical gray-white junction, and in the penetrating vessels that supply the deep graymatter (Fig. 8.4b, c) [6] Within the brain parenchyma, metastases are remarkably well localized, round solid or ring enhancing, often with a rim of reactive gliosis, and surrounded by perilesional vasogenic edema (Fig. 8.4d). Metastases from highly cellular primary tumors (such as small cell carcinoma) may show restricted diffusion. Survival after presentation with parenchymal brain metastasis may be as short as 6 months.

\section{Key Point}

- Metastatic lesions are usually peripheral cortical or subcortical, round, and well-demarcated with enhancement and perilesional vasogenic edema. At initial presentation with metastatic disease, $40-60 \%$ of patients will have a single or solitary (only brain) metastatic lesion. 

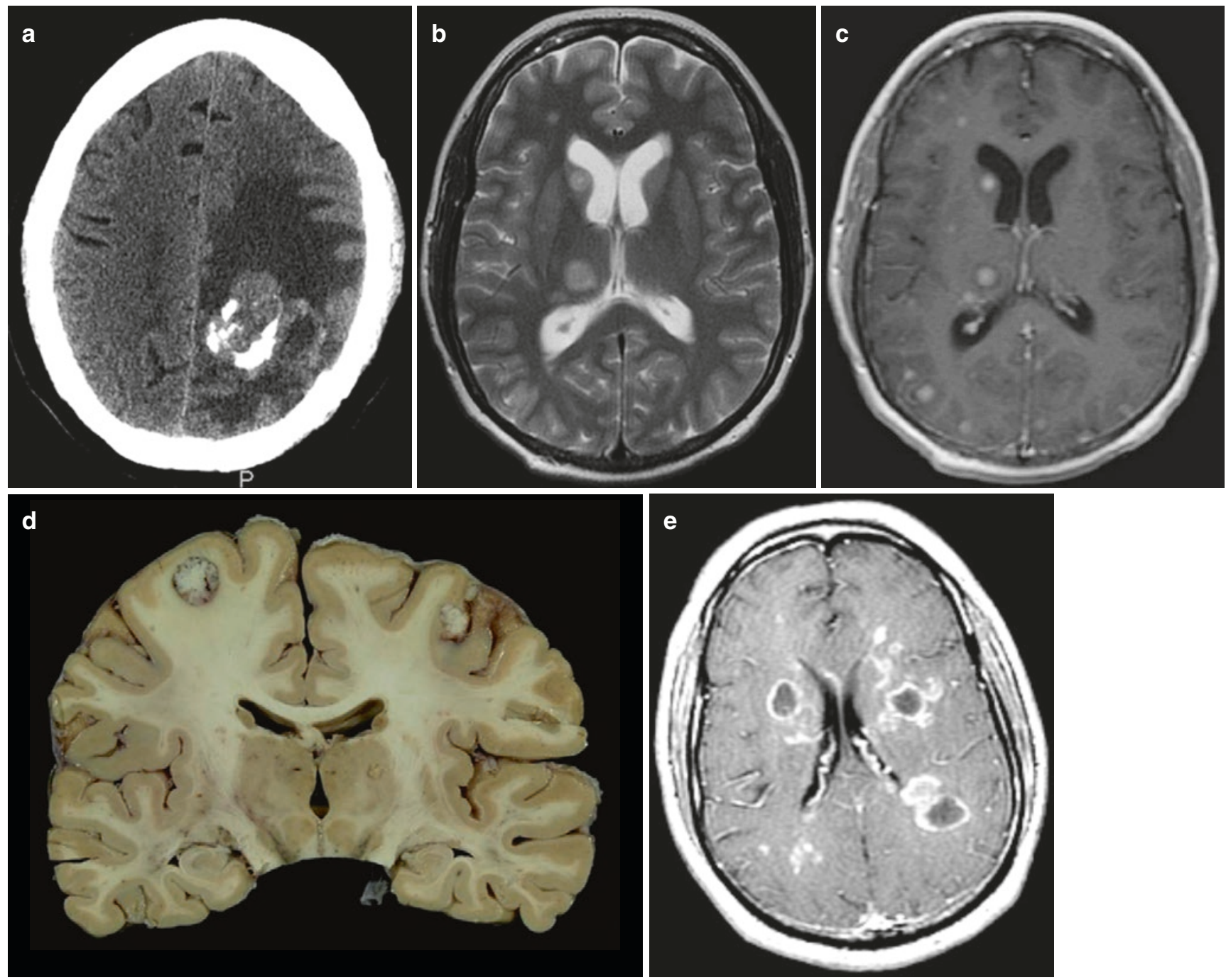

Fig. 8.4 (a) Hemorrhagic glioblastoma (WHO Gr4). Axial noncontrast CT shows a heterogenous lesion with multi-focal high attenuation (clots), surrounded by vasogenic edema in the white-matter and preservation of normal cortical gray-matter. Vasogenic edema spares the gray-matter. Single lesion. (b, c) Metastatic breast carcinoma. Axial MR (b) T2-weighted and (c) T1-weighted with gadolinium show multiple solid nodular lesions in the deep gray-matter (caudate, putamen, and thalamus) as well as multiple cortical/subcortical enhancing lesions. Multiple lesions, bilateral, but asymmetric. (d) Metastatic breast cancer (different patient). Coronal gross brain. There is a solitary, sharply demarcated and round lesion in the subcortical white-matter near the gray-white junction of the high convexity of the left hemisphere. Metastasis in the brain is usually round and focal. (e) ADEMAcute disseminated encephalomyelitis. Axial T1-weighted after gadolinium. There are multiple ring-enhancing lesions. Notice they are centered in the white-matter, and they are associated with smaller nodular enhancing lesions. ADEM is usually a monophasic process associated with an immune stimulus (infection or vaccination) primarily in children and young adults. In many cases - but not this one-we would see many "open ring" lesions. This appearance is concerning for abscesses-like toxoplasmosis. However, the patient was not immune suppressed

\subsection{Primary Neoplasms}

\subsubsection{Extraaxial Neoplasms}

Extraaxial neoplasms arise from the supporting tissues of the meninges (meningioma) and the nerves passing from central to peripheral (schwannoma). These are the two most common extraaxial and most common non-glial tumors. Meningiomas occur anywhere around the cerebral convexi- ties, along the falx and tentorium, and on the skull base. Meningiomas arise from the arachnoid and usually have a broad base of attachment to the adjacent dura — giving them a hemispheric or "globose" shape (Fig. 8.5a). A second morphology is the "en plaque" meningioma-a thickening growing along the inner table of the skull like a flat bread (pita bread) (Fig. 8.5b). Meningiomas usually maintain a homogenous CT high attenuation, and MR gray-matter-like signal-intensity, and enhancement as they grow larger (Fig. 8.5). They are commonly associated with adjacent 

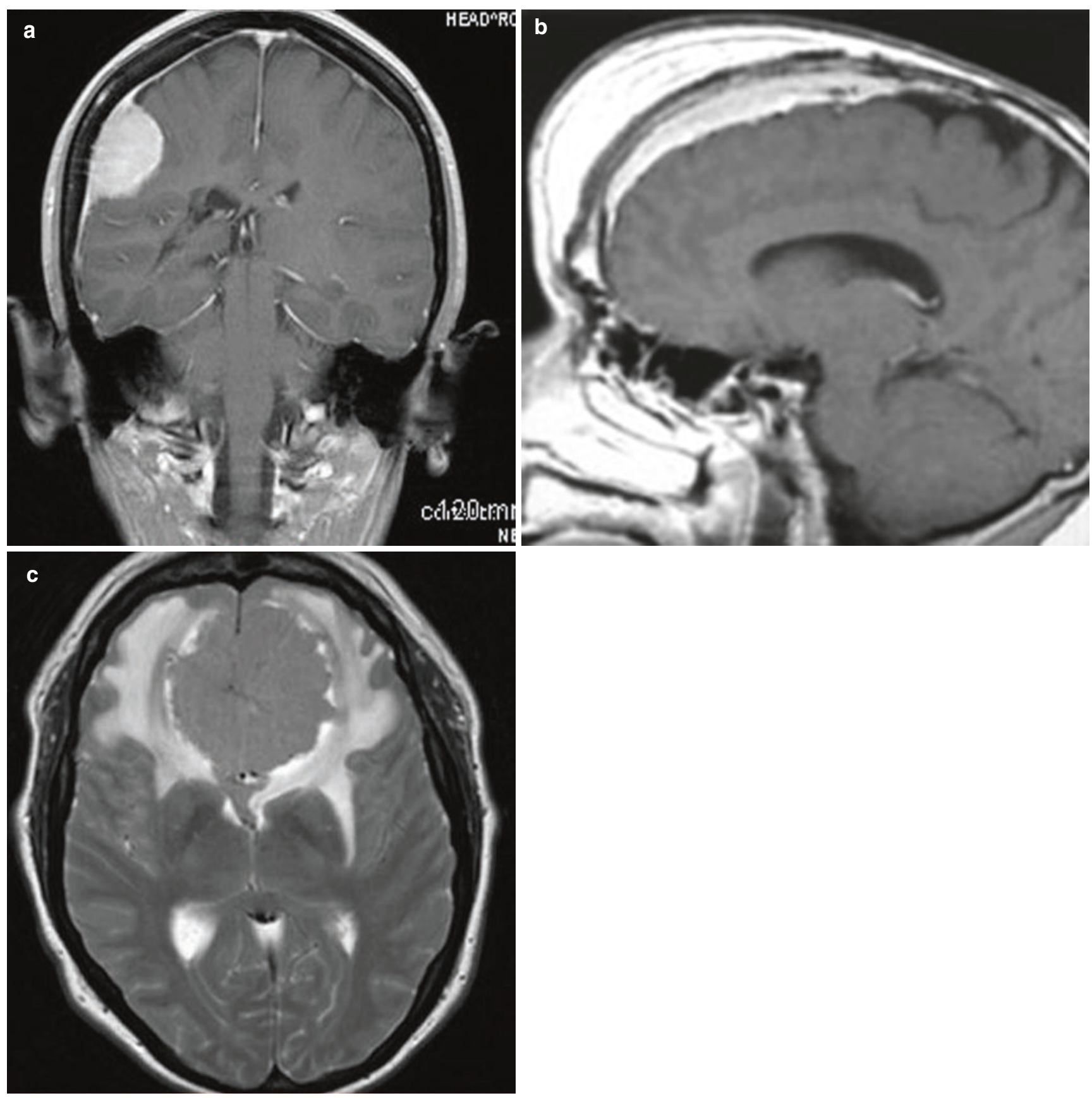

Fig. 8.5 (a, b) Meningioma. (a) Coronal T1-weighted MR after gadolinium This globose meningioma shows a sharply demarcated, hemispheric, extraaxial lesion with overlying calvarial thickening (hyperostosis). The nearby adjacent dura is slightly thickened with avid enhancement - the "dural tail." This represents a reactive process, not neoplastic infiltration. (b) Sagittal T1-weighted MR after gadolinium. This en plaque meningioma has invaded through the skull, forming a large scalp mass. These disturbing imaging features do not correlate

hyperostosis and an enhancing "dural tail" [7]. Intraaxial vasogenic edema occurs in the majority of meningiomas and is only loosely correlated with histology [8]. It is more often seen when the tumor does not have a clear visible CSF-cleft; has high signal-intensity on T2; and, a pial blood

with up-grade in histology - this was a WHO grade 1 tumor. (c) Meningioma. (c) Axial T2-weighted MR shows a bi-frontal soft-tissue mass centered on the falx, with non-specific gray-matter signalintensity. There is bi-frontal intraaxial vasogenic edema that skirts the gray-matter of the lenticular nuclei. Meningiomas easily infiltrate through the dura (not a sign of malignancy)—while other lesions (metastasis) usually do not

supply (rather than dural-only) [9]. On perfusion imaging, they usually have increased $\mathrm{rCBV}$ and $\mathrm{rCBF}$ - while showing prolonged MTT (Meant Transit Time). This is the MR equivalent of a prolonged tumor blush and delayed venous filling. On routine MRS about $1 / 2$ of well-differentiated 
meningioma will show an alanine peak (1.35-1.55 ppm), and, at 3T, there is a "characteristic" glutamine/glutamate MRS peak at $3.8 \mathrm{ppm}[10]$.

An important differential diagnosis of "en plaque" meningiomas are dural metastases in the appropriate clinical setting (e.g., in a patient with CA breast).

Schwannomas arise from the peripheral portions of the cranial nerves [3-12] - so they are ventral to the brainstem, most commonly in the posterior fossa. The most common site of origin is the inferior division of the vestibular nerve, at the apex of the internal auditory canal (IAC)_VS (vestibular Schwannoma). The tumors will compress the adjacent cochlear nerve-causing high-frequency hearing loss, but vestibular dysfunction may be retained. They grow out of the IAC into the cerebellopontine angle (CPA) cistern-where they present the bulk of the mass (Fig. 8.6). Like meningiomas VS will show avid enhancement. However, unlike meningioma, large Schwannoma become heterogenous from benign cystic degeneration. There are no imaging signs that reliably predict tumor growth, but larger tumors at presentation tend to grow faster [11].

\section{Key Point}

- Meningiomas usually remain homogeneous, even when large. Vestibular schwannomas are distinguished by location at a nerve (e.g., arising within the internal auditory canal), and they often become heterogeneous as they grow and enlarge. Almost all extraaxial and non-glial neoplasms show contrast enhancement.

\subsubsection{Intraaxial Neoplasms}

The overwhelming majority of primary intraaxial neoplasms arise from the glia—or glial cell precursors.

After glial tumors, primary CNS lymphoma (PCNSL) represents an important intrinsic neoplasm.

There are several types of glioma-usually categorized as circumscribed or diffuse (Table 8.1). The most frequent glioma in adults is, unfortunately, glioblastoma multiforme (GBM) which has the worst prognosis-median survival 12-15 months. With the exception of intraventricular neoplasms (ependymoma, choroid plexus tumors), most gliomas
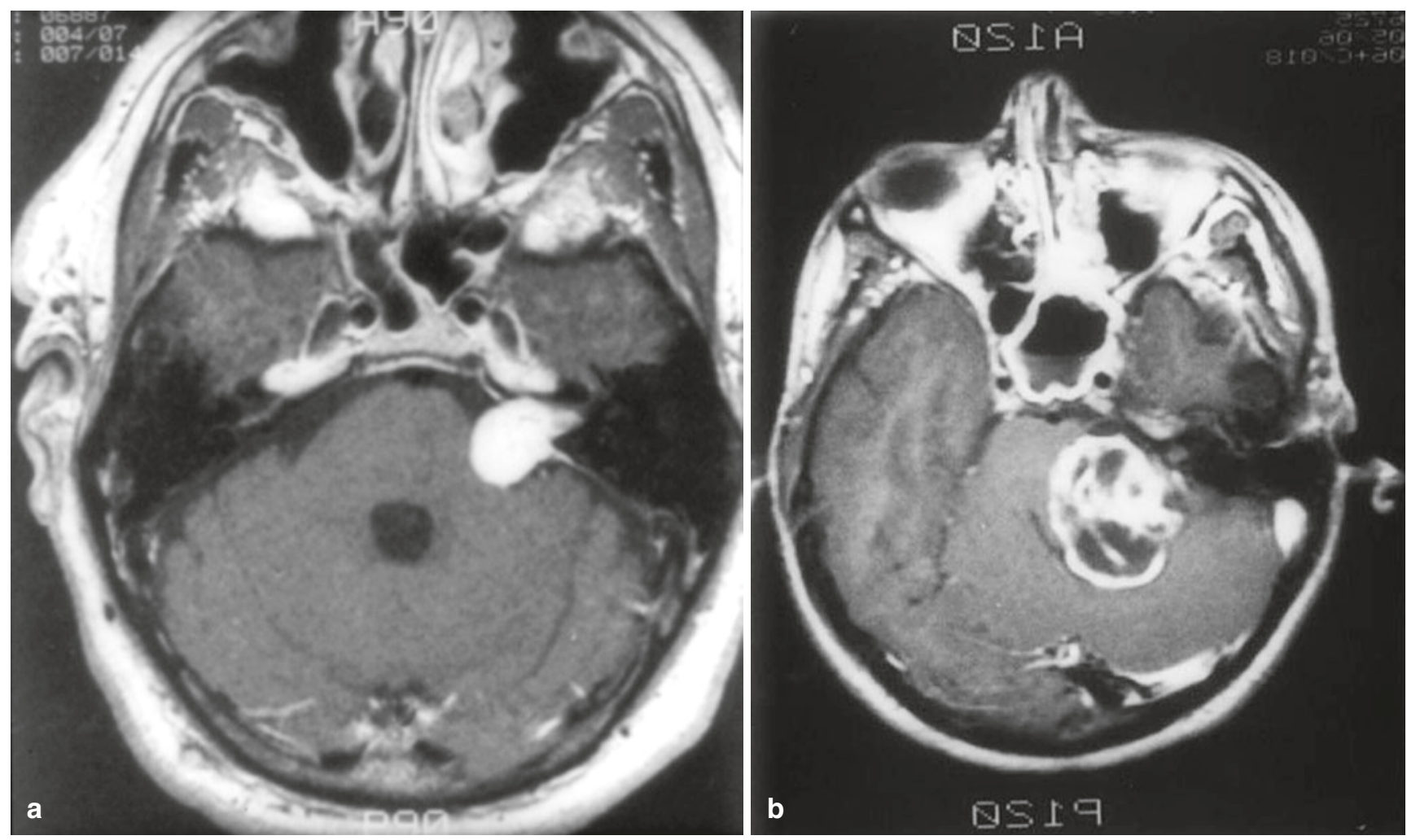

Fig. 8.6 (a) Vestibular Schwannoma. Axial T1-weighted MR after gadolinium shows a homogeneously enhancing left-sided mass in the cerebello-pontine angle cistern with a conical component inside an enlarged internal auditory canal. Note: there is a small "dural tail" along the posterior margin of the mass. The "dural tail" is a reactive process that may occur with any lesion abutting the dura. (b) Vestibular schwan- noma. Axial T1-weighted MR after gadolinium. Large extraaxial mass in the cerebello-pontine angle cistern. This is an "older" schwannoma that has become heterogeneous from benign cystic degeneration. If this lesion were incorrectly localized as intraaxial, the complex morphology would suggest a glioblastoma 
Table 8.1 Glial and mixed neoplasms

\begin{tabular}{|c|c|c|}
\hline \multirow[b]{2}{*}{ Circumscribed glioma } & \multicolumn{2}{|c|}{ Diffusely infiltrating gliomas } \\
\hline & Lower-grade diffuse & High-grade diffuse \\
\hline $\begin{array}{l}\text { Pilocytic astrocytoma } \\
\text { Gr1 }\end{array}$ & $\begin{array}{l}\text { Astrocytoma IDH-wt } \\
\text { Gr2 }\end{array}$ & $\begin{array}{l}1 \text { Glioblastoma } \\
\text { IDH-wt Gr4 }\end{array}$ \\
\hline $\begin{array}{l}\text { Subependymal giant-cell } \\
\text { astrocytoma Gr1 }\end{array}$ & $\begin{array}{l}\text { Astrocytoma } \\
\text { IDH-mut Gr2 }\end{array}$ & $\begin{array}{l}2 \text { Glioblastoma } \\
\text { IDH-mut Gr4 }\end{array}$ \\
\hline $\begin{array}{l}\text { Pleomorphic } \\
\text { xanthoastrocytoma Gr2,3 }\end{array}$ & $\begin{array}{l}\text { Anaplastic Astro } \\
\text { IDH-wt Gr3 }\end{array}$ & \\
\hline $\begin{array}{l}\text { Pilomyxoid } \\
\text { astrocytoma Gr2 }\end{array}$ & $\begin{array}{l}\text { Anaplastic Astro } \\
\text { IDH-mut Gr3 }\end{array}$ & \\
\hline Ganglioglioma Gr1 & & \\
\hline Ependymoma Gr1,2,3 & $\begin{array}{l}\text { Oligodendroglioma } \\
\text { Gr2 }\end{array}$ & \\
\hline $\begin{array}{l}\text { Choroid plexus } \\
\text { neoplasms (papilloma, } \\
\text { carcinoma, etc.) Gr1,2,3 }\end{array}$ & $\begin{array}{l}\text { Anaplastic } \\
\text { oligodendroglioma } \\
\text { Gr3 }\end{array}$ & \\
\hline
\end{tabular}

$G r$ WHO grade, mut mutant, $w t$ wild-type

Note: Oligodendrogliomas are invariably IDH-mut and 1p19q co-deleted

are large lesions that arise in the deeper periventricular white-matter-only rarely in the gray-matter (most often in the thalamus) (Fig. 8.7) Diffuse gliomas follow the whitematter tracts-using them as a scaffolding for spreadextending horizontally through the corpus callosum, vertically via cortico-spinal tracts, and through the association tracts within a single hemisphere (Fig. 8.7). Diffuse gliomas may also extend toward the cortical surface and infiltrate the gray-matter. The perilesional signal abnormality - seen best on FLAIR and T2-weighted images-is a mixture of vasogenic edema with infiltrating tumor cells. However, since vasogenic edema will not involve the cortex, cortical signal changes represent neoplastic infiltration.

\section{Key Point}

- Cortical gray-matter signal changes with a large white-matter lesion suggest neoplastic infiltration-a differential feature favoring diffuse gliomas, not seen with metastasis nor tumefactive demyelination.

Gliomagenesis for diffuse gliomas follows several different pathways. Up to $80 \%$ of lower-grade (WHO Gr 2-3) diffuse glioma have a gain-of-function "priming mutation" in IDH (isocitrate dehydrogenase-90\% IDH1 and $10 \%$ IDH2) that produces a 10,000 -fold increase in dextro-2-hydroxygutarate (D2HG) - that can be used as a tumor marker on 3T MRS [12]. Astrocytoma adds mutations in TP53 and ATRX. Oligodendrogliomas begin with the same IDH mutation, but add other mutations (TERT) and a codeletion of $1 \mathrm{p} 19 \mathrm{q}$, which is now the de facto standard marker [13]. Glioblastoma (GBM) arises from a more complex series of mutations: $90 \%$ are IDH-wild type (primary GBM, usually older patients. However, about $10 \%$ of GBM are secondary, arising from a prior lower-grade lesion that has transformed. These are usu- ally IDH-mutated and present in younger patients, often $<40$ years old.

Lower-grade diffuse gliomas (WHO Gr2,3) are deeply infiltrating masses without necrosis. They expand and nondestructively spread through the brain-often delaying the onset of a neurologic presentation. Diffuse lower-grade astrocytoma (WHO Gr 2,3) is usually homogeneous low attenuation on CT with water-like signal-intensity on MR. WHO Gr 2 astrocytomas do usually do not enhance, because they do not produce neovascularity. Instead, the tumor cells disperse and infiltrate into the surrounding brain and are supplied by normal pre-existing capillaries. Should enhancement appear on serial imaging of a known low-grade astrocytoma, it usually indicates transformation to a higher grade tumor.

IDH-mutant astrocytoma is usually in the frontal lobe, sharply demarcated, and often display the "T2-FLAIR mismatch" sign [14]. IDH-wild type astrocytoma often has blurry margins-without the mismatch sign (Fig. 8.7e, f).

IDH-mutant gliomas have a much better prognosis than IDH-wild type. Virtually all (probably all) oligodendrogliomas have IDH mutations - and they enjoy the best prognosis of all diffuse gliomas. Next in survival is the IDH-mutant astrocytoma-about $70 \%$ of lower-grade WHO Gr 2,3 tumors. IDH-wild type astrocytoma (30\% of WHO Gr 2,3) tends to have the same poor prognosis as IDH-wild glioblastomas. In some centers, they are monitored closely for progression and may then be treated like glioblastoma [15].

Oligodendrogliomas present at all ages-with the majority seen in the 4th-6th decades. They are more slowly growing than astrocytoma with a prolonged clinical history that often spans a decade. The long clinical course is often (30$90 \%$ ) associated with dense nodular calcifications (Fig. 8.8). Somewhat different from astrocytoma, they tend to grow toward the cortical surface of the cerebral hemispheres-and they may produce remodeling of the inner table of the skull. Oligodendrogliomas usually have a fine network of capillary vessels, and they may show contrast enhancement, even if they are low grade. Their vascularity may be associated with symptomatic hemorrhage.

Circumscribed gliomas may be intraventricular (ependymoma and choroid plexus neoplasms) or parenchymal. Circumscribed parenchymal gliomas are astrocytic: pilocytic (PA) WHO Gr1; pleomorphic xanthoastrocytoma (PXA); WHO Gr2,3 pilomyxoid astrocytoma (PMA) WHO Gr2; subependymal giant-cell astrocytoma (SEGA) WHO Gr1; or ganglioglioma (GG) WHO Gr1-a mixed glioneuronal neoplasm. Circumscribed astrocytoma usually has mutations in BRAF: BRAF-fusion for infratentorial pilocytic tumors; and BRAF V600E (substitution) for supratentorial PXA, PMA, and GG [16]. SEGA has one of the two mutations associated with tuberous sclerosis: TSC 1 on chromosome 9 or TSC 2 on chromosome 16. Most circumscribed gliomas show avid contrast enhancement. Yet, these neoplasms enjoy a much better prognosis compared to non-enhancing diffuse glio- 


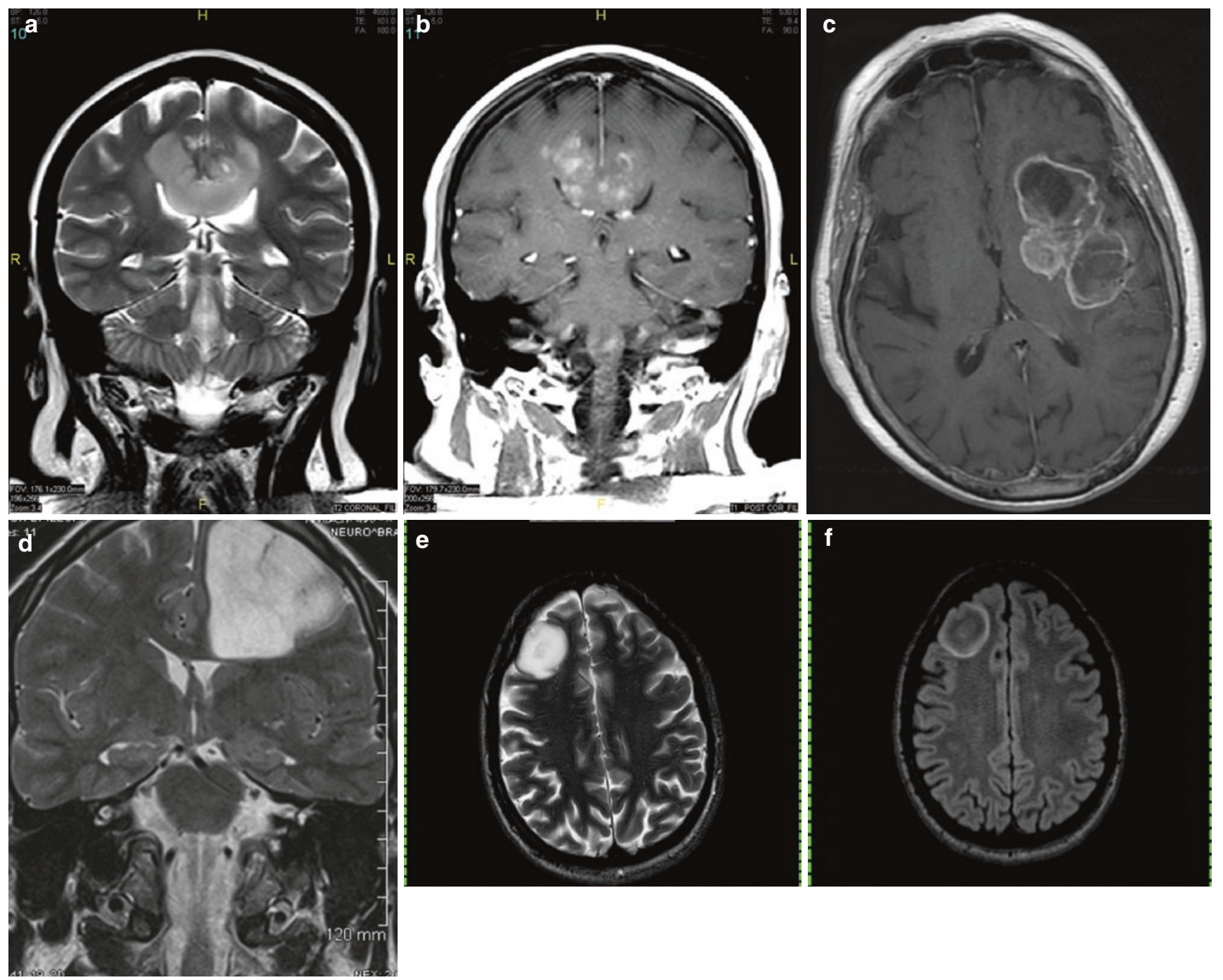

Fig. 8.7 (a, b) Diffuse Glioma a,b Glioblastoma. Coronal T2-weighted MR (a) and T1-weighted after gadolinium (b) demonstrates expansion of the mid-body of the corpus callosum with abnormal hyperintensity. The lesion shows heterogenous patchy enhancement, but without macroscopic necrosis. (c) Glioblastoma WHO Gr4. Axial T1-weighted MR with gadolinium shows a large and heterogenous left insular mass with heterogeneous enhancement. This appearance is "classic" for glioblastoma multiforme with macroscopic areas of necrosis. (d). Astrocytoma WHO Gr2,3. Coronal T2-weighted MR clearly shows abnormal hyper-

mas. The other curiosity is that most BRAF-mutant neoplasms are "fluid-secreting"-producing the characteristic appearance of a solid nodule of enhancement and a homogenous fluid-pool without peripheral enhancement (Fig. 8.9).

Pilocytic astrocytoma is most common in young patients (9-15 years of age) in the cerebellar hemispheres. Pleomorphic xanthoastrocytoma is present in children and mostly younger adults, typically in a superficial location of the cerebral hemispheres. Ganglioglioma usually present with seizures in young adults and most commonly located in the temporal lobe. Pilomyxoid astrocytoma is usually around the third ventricle (Fig. 8.9). intense signal extending toward the surface. This neoplasm has infiltrated and expanded the cerebral cortex, effacing the sulci. This is not vasogenic edema, which usually spares the gray-matter. (e, f) Diffuse astrocytoma WHO Gr 2 (c, d). Axial T2-weighted (c) and FLAIR (d) IDH-mutated diffuse astrocytomas are usually well-demarcated and homogeneous non-enhancing areas of water-like signal-intensity. They often display the "T2-FLAIR mismatch" sign-as shown here, where the center is dark on FLAIR despite hyperintensity on T2

\subsection{Primary CNS Lymphoma}

Whereas the incidence of PCNL in immunocompromised patients has declined following the introduction of effective antiviral therapy, there has been a steady increase of PCNSL in immunocompetent patients.

PCNSL are mostly large B-cell lymphomas and commonly found in the periventricular regions or deep gray nuclei. Owing to their high cellularity, they demonstrate typically restricted diffusion. They enhance strongly with a homogenous enhancement pattern in immunocompetent 

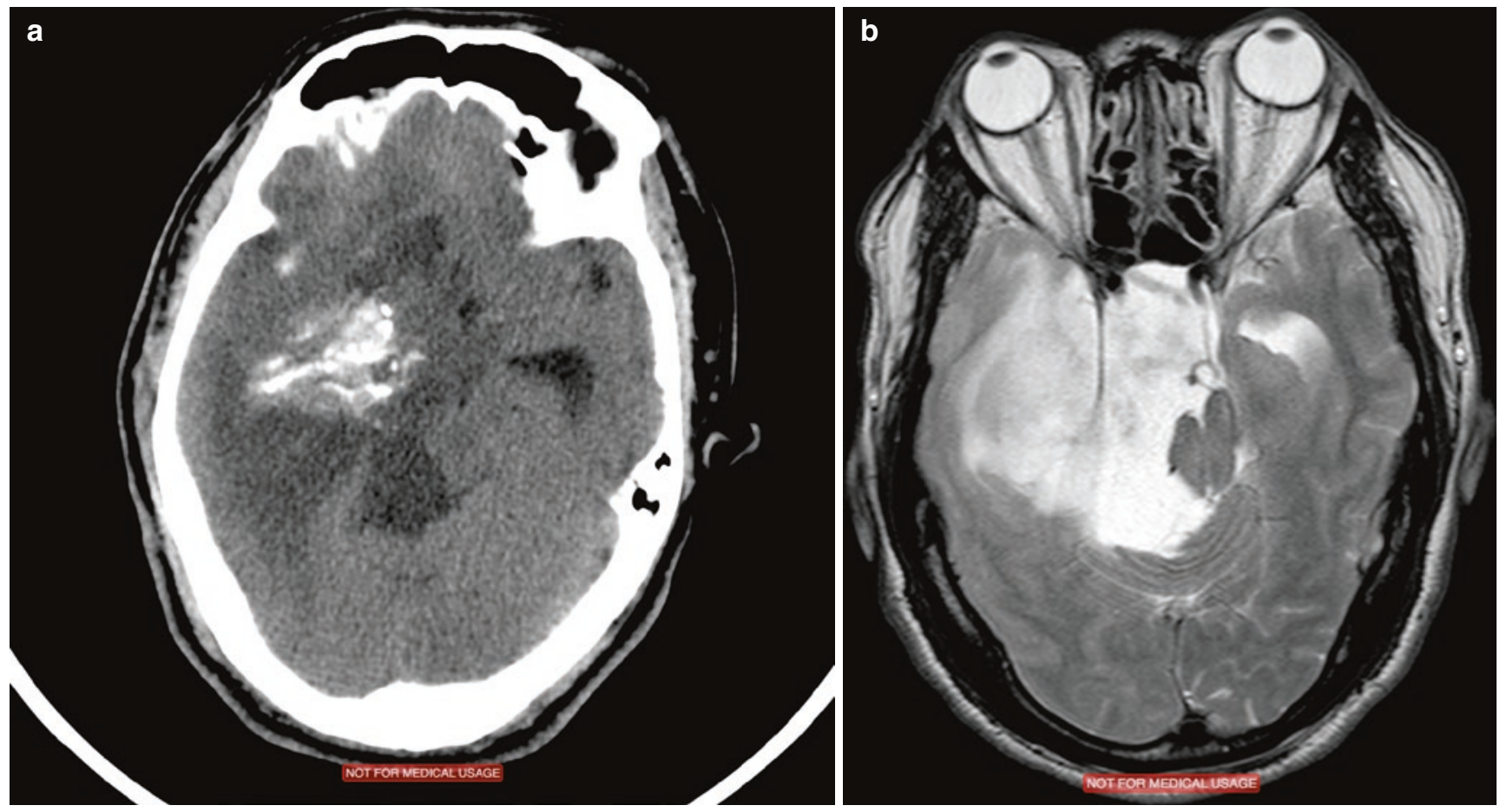

Fig. 8.8 (a, b) Oligodendroglioma WHO Gr2 (same patient as Fig 8.2a). Axial non-contrast CT (a) and T2-weighted MR (b) show a very large area of low attenuation and corresponding high signal-intensity. Surprisingly, the conglomerate hyper-attenuating calcifications do not cause corresponding signal voids on the MR. This tumor was proven to have $1 \mathrm{p} 19 \mathrm{q}$ codeletion by FISH (fluorescent in-situ hybridization) analysis. It was also IDH-mutant, like the majority of diffuse lower-grade gliomas patients and ring enhancement in the immunocompromised. On perfusion imaging, the $\mathrm{rCBV}$ is only minimally increased (much less than, e.g., in glioblastomas), as lymphoma grows angiocentrically and does not stimulate neoangiogenesis.

\section{Key Point}

- Glioblastomas are usually (90\%) IDH-wild type with enhancement and necrosis. Diffuse lower-grade gliomas (WHO Gr2,3) overwhelmingly harbor IDH mutations and do not enhance. The T2-FLAIR mismatch sign indicates an IDH-mutant astrocytoma, distinguishing from oligodendroglioma and IDHwild type astrocytoma. Dense calcifications suggest oligodendroglioma. Circumscribed gliomas usually show prominent contrast enhancement, a fluid component, and have BRAF mutations.

\subsection{Concluding Remarks}

There are multiple tools for triangulating a short differential diagnosis list, and it is important to use "pattern analysis" rather than simple "pattern recognition." Key features include lesion location, relative "volume effect"; secondary vasogenic edema; homogeneity or heterogeneity of the lesion; and patterns of contrast enhancement (homogeneous, patchy, closed or open ring). Multiple lesions usually represent a systemic process: inflammatory; toxic; metabolic; genetic; or hematogenous dissemination. Extraaxial masses are nonglial, while intraaxial neoplasms may be metastatic from extra-CNS sources, or primary gliomas.

\section{Take Home Messages}

- Lesions with vasogenic edema should also demonstrate contrast enhancement

- Solitary deep lesions are more likely to be primary gliomas

- Round nodular lesions at the gray-white junction are more likely to be metastatic

- Diffuse gliomas affect the cortex, vasogenic edema does not

- IDH-mutant diffuse astrocytoma shows T2-FLAIR mismatch sign

- Extraaxial lesions are meningioma, schwannoma, and congenital cysts 

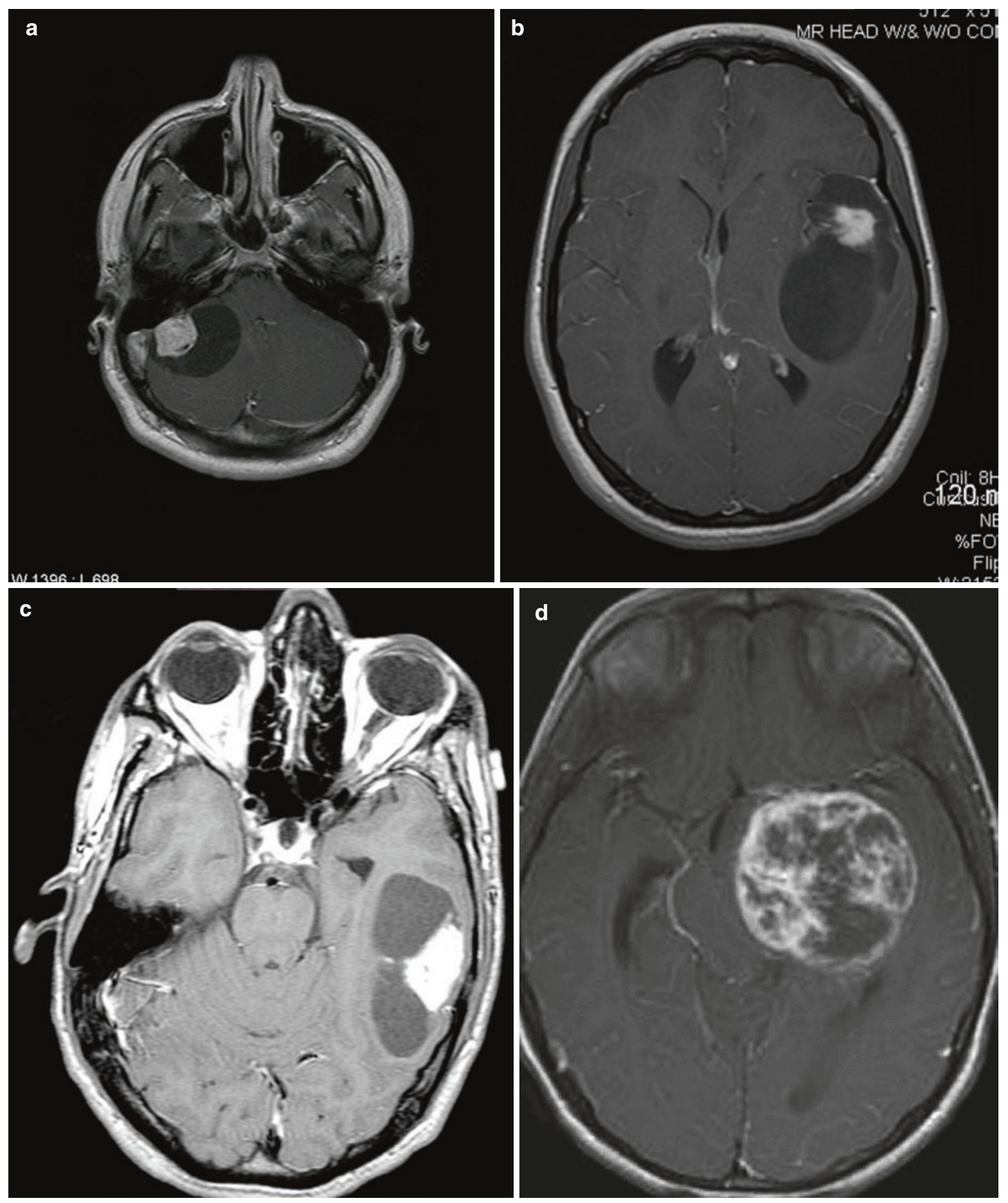

Fig. 8.9 (a, b, c, d) BRAF-mutated circumscribed astrocytoma. Axial T1-weighted MR after gadolinium: (a) pilocytic astrocytoma; (b) pleomorphic xanthoastrocytoma; (c) ganglioglioma; and (d) pilomyxoid astrocytoma. Fluidsecreting neoplasms usually show contrast enhancement limited to a nodule with a peripheral puddle of fluid $(\mathbf{a}-\mathbf{c})$. The absence of a complete rim of enhancement is strongly suggestive of a "fluid-secreting" circumscribed glioma - rather than necrosis in a highgrade tumor. However, in some cases (d), fluid producing tumors can show extremely heterogeneous patterns of enhancement- and may mimic an aggressive and necrotic tumor. The fluid attenuation and signal-intensity may deviate from CSF usually due to protein and rarely from hemorrhage 


\section{References}

1. Smirniotopoulos JG, Murphy FM, Rushing EJ, Schroeder JW. Patterns of contrast enhancement in the brain and meninges. Radiographics. 2007;27(2):525-51.

2. Kim DS, Na DG, Kim KH, Kim JH, Kim E, Yun BL, et al. Distinguishing tumefactive demyelinating lesions from glioma or central nervous system lymphoma: added value of unenhanced CT compared with conventional contrast-enhanced MR imaging. Radiology. 2009;251(2):467-75.

3. Suh CH, Kim HS, Jung SC, Choi CG, Kim SJ, Kim SJ. MRI findings in tumefactive demyelinating lesions: a systematic review and meta-analysis. AJNR Am J Neuroradiol. 2018;39(9):1643-9.

4. Suthiphosuwan S, Sati P, Guenette M, Montalban X, Reich DM, Bharatha A, et al. The central vein sign in radiologically isolated syndrome. AJNR Am J Neuroradiol. 2019;40(5):776-83.

5. Nussbaum ES, Djalilian HR, Cho KH, Hall WA. Brain metastases. Histology, multiplicity, surgery, and survival. Cancer. 1996;78(8):1781-8.

6. Hwang TL, Close TP, Grego JM, Brannon WL, Gonzales F. Predilection of brain metastasis in gray and white matter junction and vascular border zones. Cancer. 1996;77(8):1551-5.

7. Lyndon D, Lansley JA, Evanson J, Krishnan AS. Dural masses: meningiomas and their mimics. Insights Imaging. 2019;10(1):11.

8. Lee KJ, Joo WI, Rha HK, Park HK, Chough JK, Hong YK, et al. Peritumoral brain edema in meningiomas: correlations between magnetic resonance imaging, angiography, and pathology. Surg Neurol. 2008;69(4):350-5
9. Osawa T, Tosaka M, Nagaishi M, Yoshimoto Y. Factors affecting peritumoral brain edema in meningioma: special histological subtypes with prominently extensive edema. J Neuro-Oncol. 2013;111(1):49-57.

10. Crisi G. H MR spectroscopy of meningiomas at 3.0T: the role of glutamate-glutamine complex and glutathione. Neuroradiol J. 2011;24(6):846-53.

11. D'Haese S, Parmentier H, Keppler H, Van Vooren S, Van Driessche $\mathrm{V}$, Bauters W, et al. Vestibular schwannoma: natural growth and possible predictive factors. Acta Otolaryngol. 2019;139(9):753-8.

12. Andronesi OC, Rapilino O, Gerstner E, Chi A, Batchelor T. Detection of oncogenic IDH1 mutations using magnetic resonance spectroscopy of 2-hydroxyglutarate. J Clin Invest. 2013;123(9):3659-63.

13. Wen PY, Reardon DA. Neuro-oncology in 2015: progress in glioma diagnosis, classification and treatment. Nat Rev Neurol. 2016;12(2):69-70.

14. Suh CH, Kim HS, Jung SC, Choi CG, Kim SJ. Imaging prediction of isocitrate dehydrogenase (IDH) mutation in patients with glioma: a systemic review and meta-analysis. Eur Radiol. 2019;29(2):745-58.

15. Eckel-Passow JE, Lachance DH, Molinaro AM, Walsh KM, et al. Glioma groups based on $1 \mathrm{p} / 19 \mathrm{q}$, IDH, and TERT promoter mutations in tumors. N Engl J Med. 2015;372(26):2499-508.

16. Maraka S, Janku F. BRAF alterations in primary brain tumors. Discov Med. 2018;26(141):51-60.

Open Access This chapter is licensed under the terms of the Creative Commons Attribution 4.0 International License (http://creativecommons. org/licenses/by/4.0/), which permits use, sharing, adaptation, distribution and reproduction in any medium or format, as long as you give appropriate credit to the original author(s) and the source, provide a link to the Creative Commons license and indicate if changes were made.

The images or other third party material in this chapter are included in the chapter's Creative Commons license, unless indicated otherwise in a credit line to the material. If material is not included in the chapter's Creative Commons license and your intended use is not permitted by statutory regulation or exceeds the permitted use, you will need to obtain permission directly from the copyright holder. 


\title{
Toxic and Metabolic Disorders: Metabolic Diseases
}

\author{
Marco Essig
}

\section{Learning Objectives}

- To be able to detect typical imaging findings in congenital metabolic diseases

- To be able to detect typical imaging findings in acquired metabolic diseases

- To understand the use of the different imaging modalities, mainly CT and MRI in metabolic diseases

\section{Key Points}

- Metabolic diseases lead to changes in the imaging studies that we need to be aware of to trigger further investigation as the exact differential diagnosis might be challenging

- Toxic changes to the brain and spine are common and need to be identified in both an emergency and non-emergent situation

- MRI is the imaging method of choice and functional imaging techniques like MR spectroscopy and perfusion methods may add important additional information

\subsection{Most Common Congenital Metabolic Disorders Presenting or Persisting into Adulthood}

\subsubsection{X-Linked Adrenoleukodystrophy}

This is a disorder of peroxisomal fatty acid beta oxidation leading to accumulation of long chain fatty acids, which can

\footnotetext{
M. Essig ( ()

Department of Radiology, University of Manitoba,

Winnipeg, MB, Canada

e-mail: messig@exchange.hsc.mb.ca
}

manifest itself in childhood (typically around 7 years of age) or in adult life. Adult onset cerebral ALD accounts for about $5 \%$ and usually presents with psychiatric features followed by dementia, ataxia, and seizures. As an X-linked disorder ALD is more frequent in males but around $15-20 \%$ of heterozygote females carriers become symptomatic.

The typical MRI appearance are T2 and FLAIR hyperintense white matter abnormalities staring in the parietooccipital regions with early involvement of the splenium of the corpus callosum and corticospinal tracts. These signal abnormalities correspond to areas of demyelination. Active demyelination at the edge of the lesions is associated with contrast enhancement and restricted diffusion on DWI.

Adrenomyeloneuropathy (AMN) is a later onset adult phenotype presenting with slowly progressive spastic paraparesis, bladder and bowel dysfunction, sexual dysfunction, and peripheral neuropathy. Typical imaging appearances include increased T2/FLAIR signal in the posterior limbs of the internal capsules, brainstem and cerebellar white matter which may be followed by spinal cord atrophy [1].

\subsubsection{Globoid Cell Leukodystrophy (Krabbe Disease)}

Globoid cell leukodystrophy (GLD) has been linked to a mutation in the GALC gene on chromosome 14, leading to a deficiency of galactosylceramide $\beta$-galactosidase that causes accumulation of sphingolipids in the lysosomes.

Late adolescent and adult forms (10\%) present with slowly progressive gait abnormalities or spastic paresis. Other features include cognitive decline, seizures, and cortical blindness.

MRI shows predominantly posterior T2/FLAIR hyperintense white matter changes with sparing of the $U$ fibres and involvement of the splenium of the corpus callosum, extending along the corticospinal tracts into the posterior limbs of the internal capsules and the pyramidal tracts. 


\subsubsection{Metachromatic Leukodystrophy (MLD)}

Several variants have been described, all of which have deficient activity of arylsulfatase A, which results in defective degradation of sulfatides in the lysosomes.

Approximately 20\% of MLD present in adulthood often with psychiatric symptoms, followed by spastic paraparesis, cerebellar ataxia, and cognitive decline.

MRI demonstrates symmetrical areas of T2 prolongation in the periventricular white matter with sparing of the subcortical U fibres. Early involvement of the peritrigonal white matter, corpus callosum, and cerebellar white matter is common, showing typically a "tigroid" pattern of radiating stripes. As opposed to ALD, there is no contrast enhancement of the lesion edge.

\subsubsection{Mitochondrial Encephalomyopathy with Lactic Acidosis and Stroke-like Episodes (MELAS)}

Mitochondrial encephalomyopathy with lactic acidosis and stroke-like episodes (MELAS) syndrome is a typical example of a mitochondrial disorder. About $40 \%$ of patients present in late childhood or early adulthood.

Clinical features are led by encephalomyopathy, lactic acidosis, and stroke-like episodes; other features include seizures, hearing loss, and neuropsychiatric dysfunction.

MRI demonstrates T2/FLAIR hyperintense areas involving cortical and deep grey matter, not confined to any of the major vascular territories. On diffusion-weighted imaging, $\mathrm{ADC}$ values can be elevated or decreased, depending on the acuity of symptoms.

\subsubsection{Fabry Disease (Galactocerebrosidase Deficiency)}

Fabry disease is a lysosomal storage disease related to a deficiency of a-galactosidase. It is an X-linked inherited disorder with varied clinical presentations and an estimated prevalence of one in 50,000 persons.

The deposition of globotriaosylceramide- 3 in the endothelium and smooth muscles leads to involvement of multiple organ systems, including the blood vessels, heart, and kidneys.

Macro and microvascular complications are the leading CNS manifestations, with the first cerebrovascular event usually occurring around 40 years of age.

$\mathrm{CT}$ and MRI features are those of macrovascular disease (acute or chronic infarcts and parenchymal haemorrhage), and signs of microvascular disease (hyperintense foci in the periventricular and subcortical white matter and cerebral microhaemorrhages).
Characteristic MRI features of Fabry's disease are T1 hyperintensity within the pulvinar nuclei of the thalamus (the "pulvinar sign") and ectasia of the basilar artery on MRA, which has been found to be one of the best indicators of the presence of the disease in adults.

\subsubsection{Alexander Disease}

Defects in the glial fibrillary acidic protein gene have been identified as the underlying cause of this disease, in which approximately $25 \%$ of patients present in adulthood, most commonly with bulbar dysfunction, pyramidal involvement, cerebellar ataxia, and sleep abnormalities.

The MRI findings of the infantile and juvenile form include extensive white matter change with frontal predominance, a periventricular rim with high signal on T1-weighted images and low signal on T2-weighted images, abnormalities of the basal ganglia and thalami, brainstem abnormalities and contrast enhancement.

In adult onset disease, MRI findings consist of atrophy and abnormal signal within the medulla and upper cord, which has been termed a "tadpole" appearance and may be accompanied by periventricular white matter change.

\subsubsection{Primary Familial Brain Calcification}

Primary familial brain calcification, idiopathic basal ganglia calcification, and bilateral strioapallidodentate calcinosis are terms used for what was formerly called Fahr disease. This is a group of genetic disorders with currently four known mutations that are inherited in an autosomal dominant pattern. Symptomatic patients present in the late 40s with a mostly Parkinsonian movement disorder, followed by cognitive decline, cerebellar features, and speech disorders. Symmetrical calcification involving the lentiform nuclei, caudate nuclei, thalami and dentate nuclei is mostly readily detected by CT. On MRI, the structures involved may show high signal on T1-weighted spin echo sequences and signal loss on susceptibility-weighted imaging.

\subsection{Acquired Metabolic Disorders}

These are mostly seen in young adults or adults and present with typical imaging findings.

\subsubsection{Wernicke's Encephalopathy}

Wernicke's encephalopathy (WE) is caused by a deficiency of vitamin B1 (thiamine), which may be due to alcohol 
abuse, malabsorption, poor nutrition, increased metabolism, or iatrogenic elimination (haemodialysis).

Thiamine depletion leads to failure of conversion of pyruvate to acetyl-CoA and $\alpha$-ketoglutarate to succinate and the lack of Krebs cycle resulting in cerebral lactic acidosis with intra- and extracellular oedema, swelling of astrocytes, oligodendrocytes, myelin fibres, and neuronal dendrites.

The classic clinical triad of ocular dysfunctions (nystagmus, conjugate gaze palsy, ophthalmoplegia), ataxia, and confusion is observed only in $30 \%$ of cases. Treatment consists of thiamine infusion, which can prevent progression to Korsakoff's dementia or death.

MRI shows T2/FLAIR hyper intensities in the periaqueductal and medial thalamic regions, mammillary bodies, hypothalamus, tectum, and cerebellum (Fig. 9.1).

Contrast enhancement occurs in $80 \%$ in the mammillary bodies (often prior to $\mathrm{T} 2$ hyperintensities) and in $50 \%$ in the peri-acqueductal regions.

Imaging abnormalities can regress with treatment, but the prognosis is usually poor once there is cortical involvement or T1 hyperintensity in the thalami and mammillary bodies indicating haemorrhagic change.

Chronic cases show atrophy of the mammillary bodies.

\subsubsection{Subacute Combined Degeneration}

This is a disorder of the spinal cord secondary to vitamin B12 deficiency, characterised by gliosis and spongiform degeneration of the posterior and lateral columns. Clinical presentations are in spastic paraparesis and spinal ataxia.

MRI demonstrates T2 hyperintensities in the posterior and lateral columns of the spinal cord, which can reverse after adequate B12 administration. Chronic non- or undertreated cases show spinal cord atrophy.
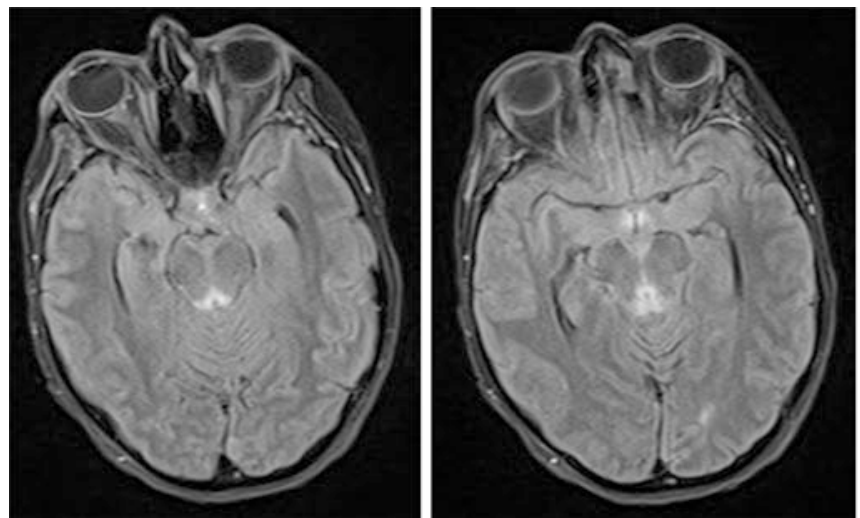

Fig. 9.1 Typical FLAIR hyperintense tissue changes in the medial thalamus, the periaqueductal area, mammillary bodies, and tectal plate
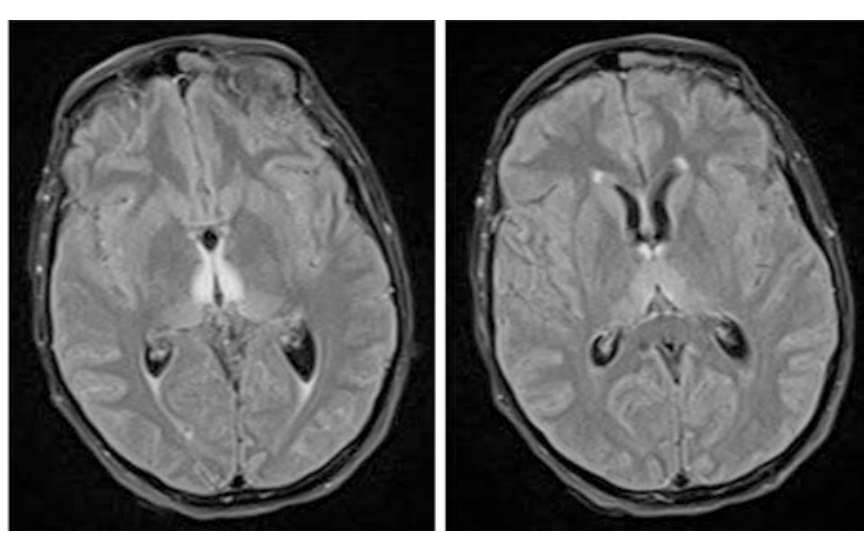

\subsubsection{Osmotic Myelinolysis Syndrome}

This entity includes central pontine myelinolysis (CPM) and extrapontine myelinolysis and occurs usually in patients with hyponatremia that has been corrected too quickly.

The traditionally proposed pathophysiological mechanism is a disruption of the blood-brain-barrier resulting in vasogenic oedema, fibre tract compression, and myelinolysis. Additional implicated mechanisms are cerebral dehydration, intramyelinic oedema, and oligodendrocyte degeneration. The most common damage is in the central pontine fibres (CPM). Extrapontine demyelination (in sites such as basal ganglia, thalami, lateral geniculate body, cerebellum and cerebral cortex) may occur together with CPM or in isolation (in approximately 10\%).

Clinical symptoms of CPM include paralysis, dysphagia, dysarthria, and pseudobulbar palsy.

Typical MRI features of central pontine myelinolysis are T2/FLAIR hyperintensity in the central pons showing a symmetric "trident" or "bat-wing"-pattern, due to sparing of the peripheral fibres and the axons of the corticospinal tracts.

In the acute phase DWI shows restricted diffusion with a decreased ADC. This may occur within $24 \mathrm{~h}$ of symptom onset and precede the signal abnormalities seen on $\mathrm{T} 2$ or FLAIR images. The ADC values usually return to baseline within 3-4 weeks.

CPM can appear moderately hypointense in $\mathrm{T}_{1}$ and may infrequently show contrast enhancement. If the patient survives the acute phase, the pontine lesions can cavitate and appear markedly hypointense on $\mathrm{T} 1$ weighted images.

\subsubsection{Hepatic Encephalopathy}

The term hepatic encephalopathy includes a spectrum of neuropsychiatric abnormalities in patients with liver dys- 

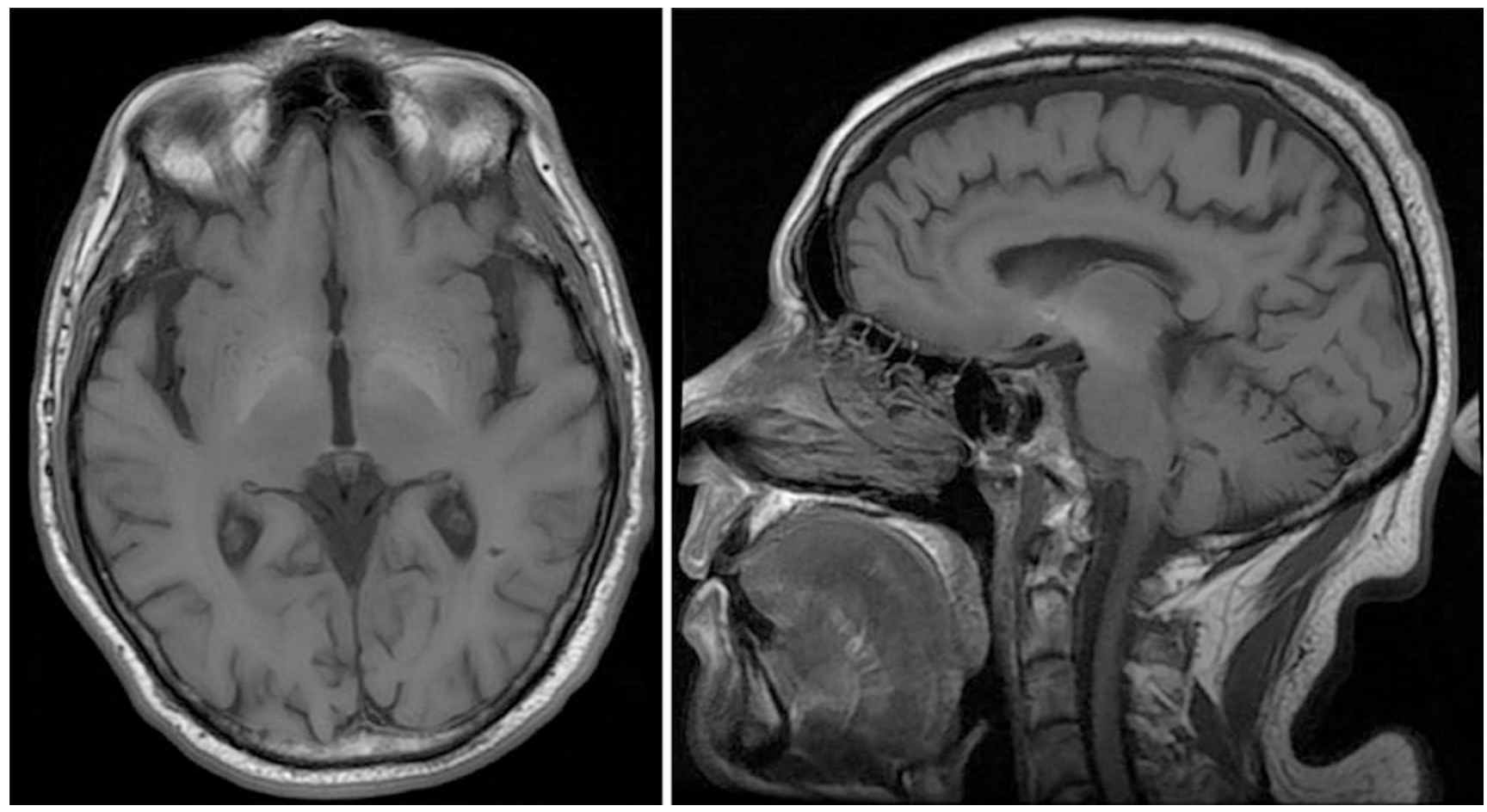

Fig. 9.2 Hepatic Encephalopathy-typical T1 hyperintense tissue changes in the basal ganglia based on manganese deposition

function. Most cases are associated with cirrhosis and portal hypertension or portal-systemic shunts, but the condition can also occur in acute liver failure.

Classical MR abnormalities in chronic hepatic encephalopathy include high signal intensity in the globus pallidus on T1-weighted images, and less frequently, in the substantia nigra and the tegmentum of the midbrain, secondary to increased concentrations of manganese (Fig. 9.2) [2].

Magnetisation transfer imaging, fast FLAIR, and DWI sequences may demonstrate white matter abnormalities secondary to increased CNS ammonia concentration. These return to normal with restoration of liver function, and are thought to reflect mild diffuse interstitial brain oedema, which appears to play an essential role in the pathogenesis of hepatic encephalopathy.

In acute hepatic encephalopathy, bilateral symmetric T2-hyperintensities of the cortical grey matter are often associated with restricted diffusion. There may be additional involvement of the subcortical white matter, basal ganglia, thalami, and midbrain. These imaging abnormalities are thought to reflect cytotoxic oedema secondary to acute hyperammonemia.

\subsubsection{Hypoglycemic Encephalopathy}

An acute decrease in serum glucose levels can arise from an excess of exogenous or endogenous insulin and hypoglycaemia inducing drugs. This causes a decline of the cell membrane ATPase pump activity and release of excitatory neurotransmitters such as aspartate.

CT can demonstrate enhancing hypodensities in the basal ganglia, cerebral cortex, hippocampus, and substantia nigra.

MRI is more sensitive and shows $\mathrm{T} 2$ hyperintensities as well as restricted diffusion in the posterior limb of the internal capsules, hippocampi, the basal ganglia and cortical areas and splenium of the corpus callosum. These changes are likely to reflect cytotoxic oedema and extensive DWI changes in the basal ganglia and deep white matter are associated with poor clinical outcome.

\subsubsection{Hyperglycaemic Encephalopathy}

Hyperglycaemia occurs in uncontrolled diabetes mellitus and can lead to osmotic derangements the basal ganglia and subthalamic region. 
Imaging findings are hyperdense changes on $\mathrm{CT}$ and $\mathrm{T} 1$ hyperintensities on MRI within the putamen and caudate nuclei, which may be uni- or bilateral. SPECT has been reported to show hypoperfusion in the basal ganglia. After correction of blood glucose, the imaging abnormalities usually regress [3].

\section{Take Home Messages}

- Both congenital and acquired metabolic diseases present with typical imaging findings.

- Even sometimes subtle, the course will enable to identify patterns that help in the differential process.

- Only the combination between clinical information, laboratory findings, and imaging will allow to make a solid differential diagnosis.

\section{References}

1. Ahmed RM, Murphy E, Davagnanam I, et al. A practical approach to diagnosing adult onset leukodystrophies. J Neurol Neurosurg Psychiatry. 2014;85:770-81.

2. Alonso J, Córdoba J, Rovira A. Brain magnetic resonance in hepatic encephalopathy. Semin Ultrasound CT MR. 2014;35(2):136-52.

3. Alleman AM. Osmotic demyelination syndrome: central pontine myelinolysis and extrapontine myelinolysis. Semin Ultrasound CT MR. 2014;35(2):153-9.

Open Access This chapter is licensed under the terms of the Creative Commons Attribution 4.0 International License (http://creativecommons. $\mathrm{org} /$ licenses/by/4.0/), which permits use, sharing, adaptation, distribution and reproduction in any medium or format, as long as you give appropriate credit to the original author(s) and the source, provide a link to the Creative Commons license and indicate if changes were made.

The images or other third party material in this chapter are included in the chapter's Creative Commons license, unless indicated otherwise in a credit line to the material. If material is not included in the chapter's Creative Commons license and your intended use is not permitted by statutory regulation or exceeds the permitted use, you will need to obtain permission directly from the copyright holder. 


\title{
Imaging the Patient with Epilepsy
}

\author{
Charlie Chia-Tsong Hsu, Lars Stenberg, and Timo Krings
}

\section{Learning Objectives}

- To understand typical imaging criteria to identify and differentiate causes of medication refractory epilepsy

- To understand the limitations of imaging for patients with medication refractory epilepsy if no dedicated Epilepsy MR protocol is used.

- To appreciate the importance of additional clinical information when evaluating the patient with medication refractory epilepsy.

C. C.-T. Hsu

Division of Neuroradiology, Department of Medical Imaging, Gold Coast University Hospital, Southport, QLD, Australia

Division of Neuroradiology, Queensland Diagnostic Imaging, Healius Health Care, Varsity Lakes, QLD, Australia

\section{Stenberg}

Section of Neuroradiology, Department of Medical Imaging and Physiology, Skåne University Hospital, Lund, Sweden

Department of Diagnostic Radiology, Lund University, Lund, Sweden

e-mail: Lars.Stenberg@skane.se

T. Krings $(\bowtie)$

Department of Radiology and Surgery, University of Toronto, Toronto, ON, Canada

The David Braley and Nancy Gordon Chair of Interventional Neuroradiology, University of Toronto, Toronto, ON, Canada

Division of Neuroradiology, Toronto Western Hospital, Toronto, ON, Canada

Joint Department of Medical Imaging at the University Health Network, Toronto, ON, Canada

Women's College Hospital, Toronto, ON, Canada

Mount Sinai Hospital, Toronto, ON, Canada

Medical Imaging, Toronto Western Hospital, Toronto, ON, Canada e-mail: timo.krings@uhn.ca

\subsection{Introduction}

Approximately 4\% of the general population will experience a seizure during their lifetime. Imaging findings of "firstever" seizure patients are in most cases normal, and abnormalities are only present in approximately $15 \%$ of patients as seizures can be provoked by fever, sleep deprivation, stroboscopic lights, or drugs. However, an underlying lesion will lower the seizure threshold and thus make a patient more susceptible to experience a seizure. As "first-ever" seizures are a medical emergency the initial imaging modality of choice in these cases is an unenhanced CT head scan to exclude acute medical emergencies that may go along with seizures prior to a more extensive workup depending on clinical history and presentation. Imaging abnormalities encountered in patients experiencing their first-ever seizures include (but are not restricted to) virtually all diseases affecting the brain. As such you may find vascular abnormalities (such as microangiopathy, arteriovenous malformations (AVM), sinus thrombosis, hemorrhage, cavernomas, or stroke), tumors (metastases, primary tumors), infections (encephalitis, meningitis, abscess), sequelae of previous head injury, and toxic or metabolic conditions (e.g., PRES) in these patients.

In contrast to the "first-ever" seizure, patients diagnosed with "epilepsy" have recurrent and unprovoked seizures. Approximately $1 \%$ of the general population will be diagnosed with this condition and as seizures are recurrent and unprovoked, an underlying lesion is far more common as compared to patients with their first-ever seizure. Being "unprovoked," lesions that can irritate the brain (i.e., are "epileptogenic") may be present. On brain imaging, lesions will be seen in nearly $50 \%$ of patients; however, these are non-specific and can encompass a wide variety of underlying conditions that can provoke the recurrent seizure attacks. Imaging findings in patients with seizures include but are not restricted to: vascular conditions such as microangiopathy, previous ischemia, vascular malformations, previous hemor- 
rhage or cavernomas, tumors (metastases, primary tumors), remote infections (encephalitis, abscess), previous head injury, congenital malformations, or toxic metabolic conditions. Imaging of choice in patients with epilepsy is MRI given the larger variety of potential underlying diseases.

The vast majority of patients with epilepsy can be treated satisfactorily with antiepileptic drugs. However, $0.4 \%$ of the general population will have recurrent and unprovoked seizures that do not respond to medication. These patients are potentially treatable with surgery, and surgical intervention is an appropriate consideration for $3 \%$ of people who develop epilepsy [1]. The major focus of this chapter will be on the imaging findings in those patients who are diagnosed with "medication refractory" epilepsy, i.e., patients where the seizure focus is too strong to be controlled by medication which indicates that the underlying lesion has to have a strong epileptogenic potential. In these patients, structural imaging will find abnormalities in up to $85 \%$ of patients. Lesions with a strong epileptogenic potential are either close to epileptogenic structures or consist of abnormal neurons. Lesions that are often involved in medication refractory epilepsy are: mesial temporal lobe sclerosis (MTS) (primary or secondary to a long standing seizure disorder), malformations of cortical development, certain epileptogenic tumors (e.g., dysembryoplastic neuroepithelial tumors (DNET), temporal lobe gliomas gangliogliomas), vascular malformations, trauma, remote infection, and certain phakomatoses. Imaging findings in some of these conditions will be subtle which necessitates both a dedicated imaging protocol (as compared to a standard MR) and an "expert" experience in reading these types of scans. In a landmark study of von Oerzten et al. [2], the sensitivity of "nonexpert" reports of standard MRI reports for focal lesions was $39 \%$, while sensitivity of "expert" reports of standard MRI increased to 50\%. "Expert" reports of epilepsy dedicated MRI protocols further increased the sensitivity in detecting subtle lesions to $91 \%$. Dedicated MRI showed focal lesions in $85 \%$ of patients with "non-lesional" standard MRI. Neuropathological diagnoses were predicted correctly in $22 \%$ of "non-expert" standard MRI reports but by $89 \%$ of dedicated MRI reports. Thus, the combination of dedicated MRI protocols and dedicated radiologists trained in evaluating patients with medication refractory seizures increases significantly the sensitivity of MRI in this subgroup of patients. A multidisciplinary approach that involves close communication between epilepsy neurologists, neuroradiology, EEG, nuclear medicine, neuropsychology, and neurosurgery is an important feature of modern epilepsy management.

The necessity of expert MR reading with a dedicated imaging protocol is further highlighted by the fact that postsurgical seizure freedom is achieved significantly more often when a circumscribed, resectable epileptogenic lesion can be identified on MRI preoperatively compared to patients that are rated non-lesional [3]. As pointed out by Wellmer et al. in
2013 [4, 5], the possible reasons for undetected epileptic lesions in standard outpatient MRI are insufficient clinical information from the referring clinician, routine MR protocols not optimized for the spectrum of epileptogenic lesions, and unfamiliarity with the spectrum of epileptogenic lesions. Wellmer pointed out that "because even the best focus hypothesis and most profound knowledge of epileptogenic lesions do not permit the detection of lesions when they are invisible on the MRI scan, the starting point for any improvement of outpatient MRI diagnostics should be defining an MRI protocol that is adjusted to common epileptogenic lesions."

This indicates that a specific imaging protocol to identify these lesions is necessary. This protocol should take into account that-as small epileptogenic lesions are usually those that provide the best chance for postoperative seizure freedom-slice thickness should be adjusted to detect small lesions and multiple (coronal, axial, sagittal) cut planes are acquired to ensure that physiologic structures or partial volume effects within the folded cortex are not taken for pathology and vice versa. Coronal sequences have to be angulated perpendicular to the hippocampal axis to allow hippocampal volume estimation and caution has to be taken that they are oriented in a plane that ensures direct comparison with the contralateral hemisphere. In our practice, we employ this coronal angulation for both T2FLAIR and T1 IR sequences. High resolution T1-weighted sequences with isotropic voxel sizes allow for multiplanar reformation and further evaluation (including 3D reformats, "pancake" views, surface rendering, and volumetric assessments). T2 gradient echo or susceptibility weighted sequences are highly sensitive to detect blood products or calcifications and should therefore be part of a seizure imaging protocol (Fig. 10.1).

In a recent analysis performed by Wellmer et al. on the prevalence of epileptogenic lesions among 2740 patients the following pathologies were found: mesial temporal lobe sclerosis $(32 \%)$, tumors (including low and high grade tumors as well as malformative tumors and benign epilepsy associated tumors) in approximately $17 \%$ of patients, cortical dysplasias in $11 \%$, glial scars (including posttraumatic, post-ischemic, post-hemorrhagic, postinfectious/abscess, ulegyria, and postsurgical scars) in $11 \%$, vascular diseases (cavernoma AVM, pial angiomatosis) in 5\%, malformations of cortical development including nodular heterotopia, subcortical band heterotopia, polymicrogyria, lissencephaly, pachygyria, agenesis of corpus callosum, craniocephalic malformations, hemiatrophy, lobar dysgenesis, hemimegalencephaly, or hamartomas in $3 \%$, and sequelae of encephalitis in $1 \%$ while in approximately $20 \%$ no lesion could be detected.

Lesion location - presumably related to the different epileptogenic potential in different brain regions - demonstrates preponderance for the temporal lobes $(60 \%)$ followed by the frontal lobe $(20 \%)$, the parietal lobe $(10 \%)$, the periventricu- 

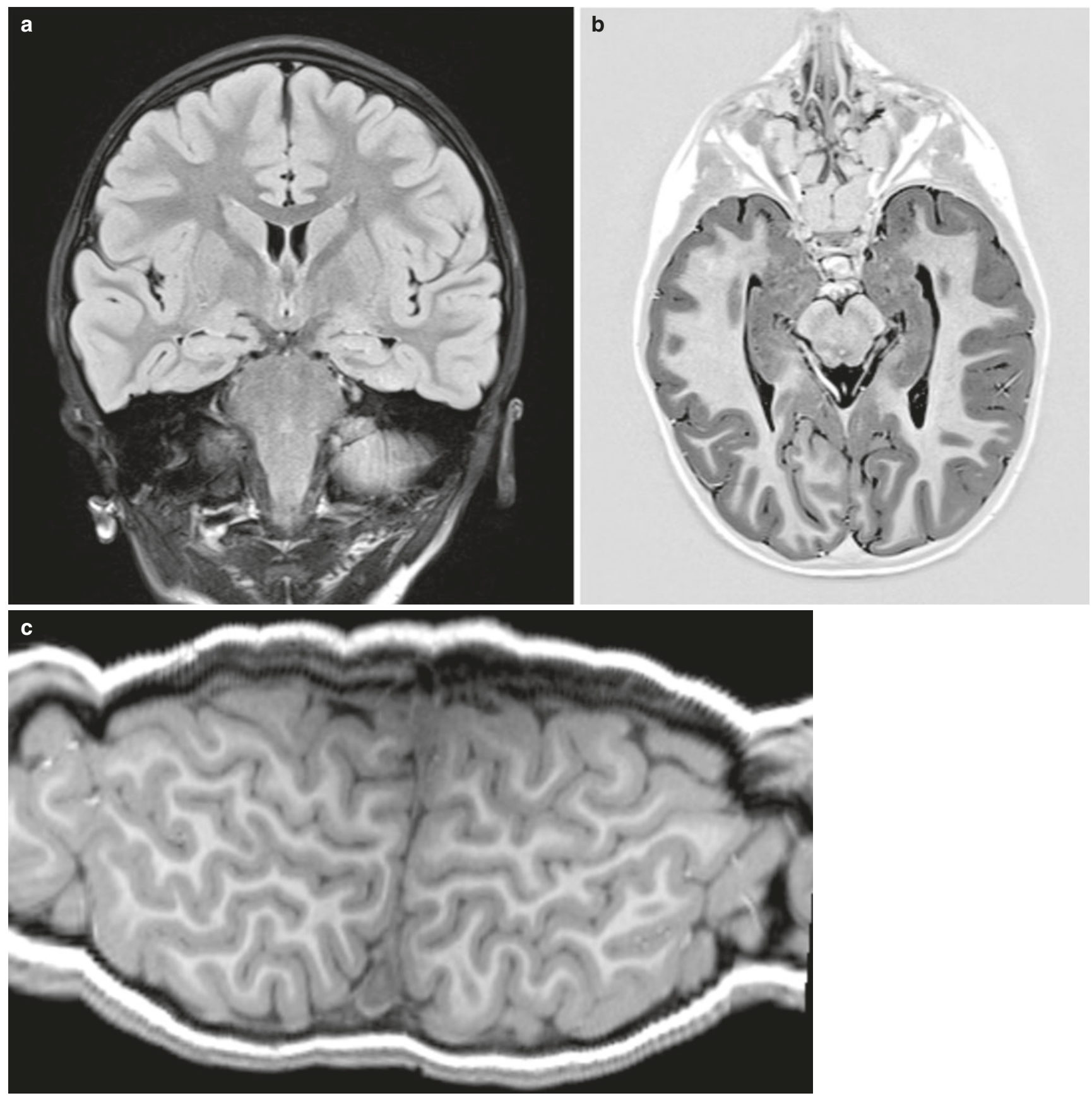

Fig. 10.1 (a-c) Some examples of sequences employed in our institutional dedicated epilepsy protocol: (a) coronal T2 FLAIR, perpendicular to the hippocampal axis; (b) axial T1 inversion recovery (IR) parallel

to the hippocampal axis; (c) T1 3D image set presented as "pancake view" for a better overview of the gyral pattern

higher field strengths (7T) (Fig. 10.2) will probably further increase the detection rate of epileptogenic substrates such as mesial temporal lobe sclerosis (MTS), focal cortical dysplasia (FCD), and polymicrogyria [8-11]. Epilepsy imaging at $1.5 \mathrm{~T}$ is in the opinion of the authors no longer acceptable. There are multiple adjunct imaging techniques: mended that for an epilepsy-specific protocol T2FLAIR, T2/ STIR in two parallel planes, T2 gradient echo/SWI sequences, and an isotropic 3D-T1 are necessary. We strongly recommend these imaging sequences to be done on a 3T scanner given the higher spatial resolution $[6,7]$. The use of even
- Functional MRI (fMRI) can map eloquent cortex and provide information regarding language lateralization [12] (Fig. 10.3), and the use of diffusion tensor imaging (DTI) 


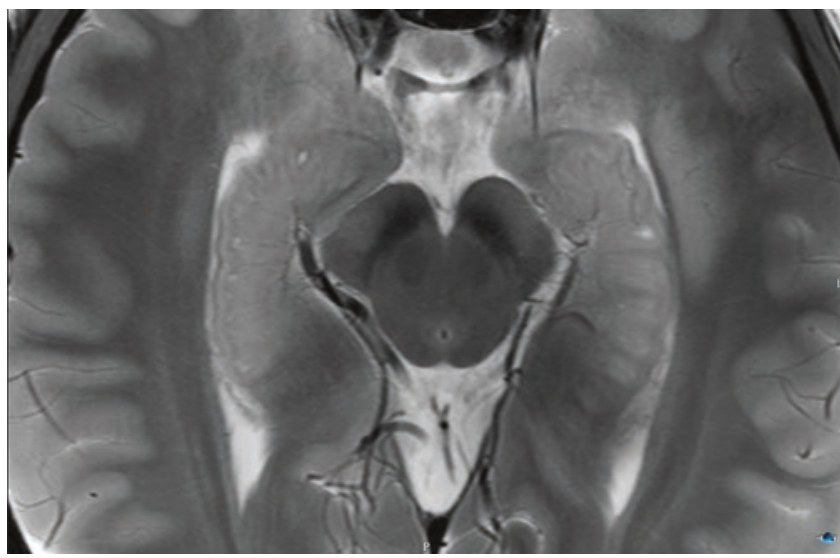

Fig. 10.2 axial T2 at 7T parallel to the hippocampal axis. Excellent in plane resolution provides detailed imaging of hippocampus

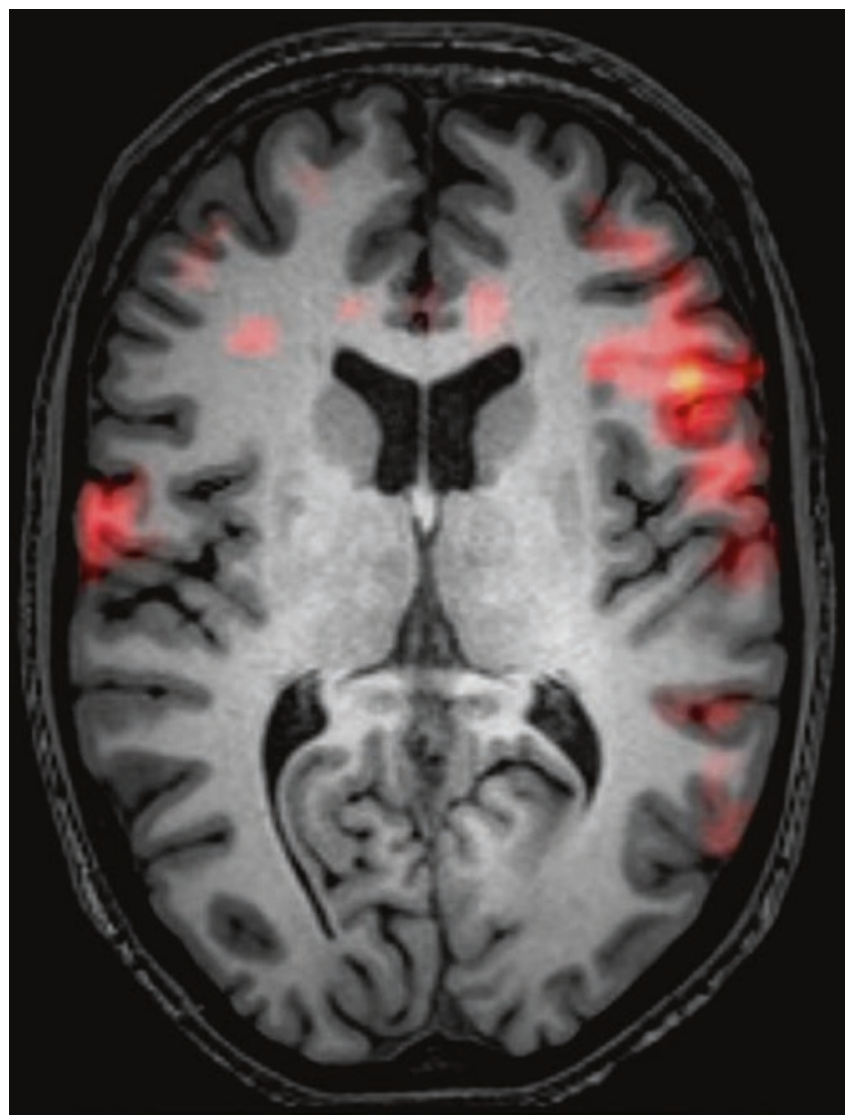

Fig. 10.3 Functional MRI. Red areas indicate activation during a simple word generation task. Activation is seen predominantly in the left hemisphere in the frontal language region; typical language lateralization

and tractography may help to avoid injury to the optic radiation during temporal lobe resection [13].

- Radionuclide imaging can add useful information in selected cases [14]. Subtraction of ictal and interictal SPECT co-registered to MRI (SISCOM) can show a seizure-induced hyperperfusion (Fig. 10.4), whereas ${ }^{18 F}$ FDG-PET may show hypometabolism in the seizure onset zone. This is particularly useful in later- alization of temporal lobe epilepsy in the MR-negative patient.

- Dipole source analysis using either EEG or MEG data can also be added for the localization of seizure activity.

Imaging evaluation should be standardized using a stepwise approach to evaluate the hippocampus and mesial temporal lobe structures, the ventricular outline, the gyral, and the sulcal anatomy. Particular emphasis should be paid upon the T2/FLAIR signal within the cortex and hippocampus, its similarity to other regions of neo- and archicortex, the internal architecture of the hippocampus, the indentations of the pes hippocampi, the fornix and mammillary bodies, and the gray-white matter interface of the neocortex (blurring, gray matter thinning or thickening). Selection of narrow window width better optimizes contrast between the gray and white matter which can assist in lesion detection (Fig. 10.5).

\section{Key Point}

- In selected patients, i.e., those with medication refractory epilepsy, abnormalities can be found in a high percentage if images are performed with a dedicated imaging protocol, and expert read-out. A variety of non-radiological adjunct tests are available that may help in the localization of the seizure focus and preferably these challenging cases are therefore discussed in multidisciplinary conferences.

In the following we will discuss the imaging features of epileptogenic lesions highlighting imaging pearls and pitfalls.

\subsubsection{Mesial Temporal Lobe/Hippocampal Sclerosis}

Patients with mesial temporal sclerosis (MTS) often harbor complex partial seizures with a seizure semiology (given its temporal lobe origin) that is characterized by déjà vu (or jamais vu) sensations, epigastric auras, lip smacking, or other oral automatisms and often have in their past medical history febrile seizures as a child with progressive worsening of seizure frequency and severity over time. Understanding the normal hippocampal internal architecture is critical to make the correct diagnosis. The hippocampus is composed of four distinct cellular layers with stratum oriens as the most superficial layer followed by stratum pyramidale, stratum radiatum, and stratum lacunosum as the inner most layer. Histopathologic correlation with 3T MRI of resected specimen of hippocampi revealed the inner hypointense band on the coronal T2-weighted sequence as the stratum lacunosum (Fig. 10.6). MTS is characterized with extensive gliosis 

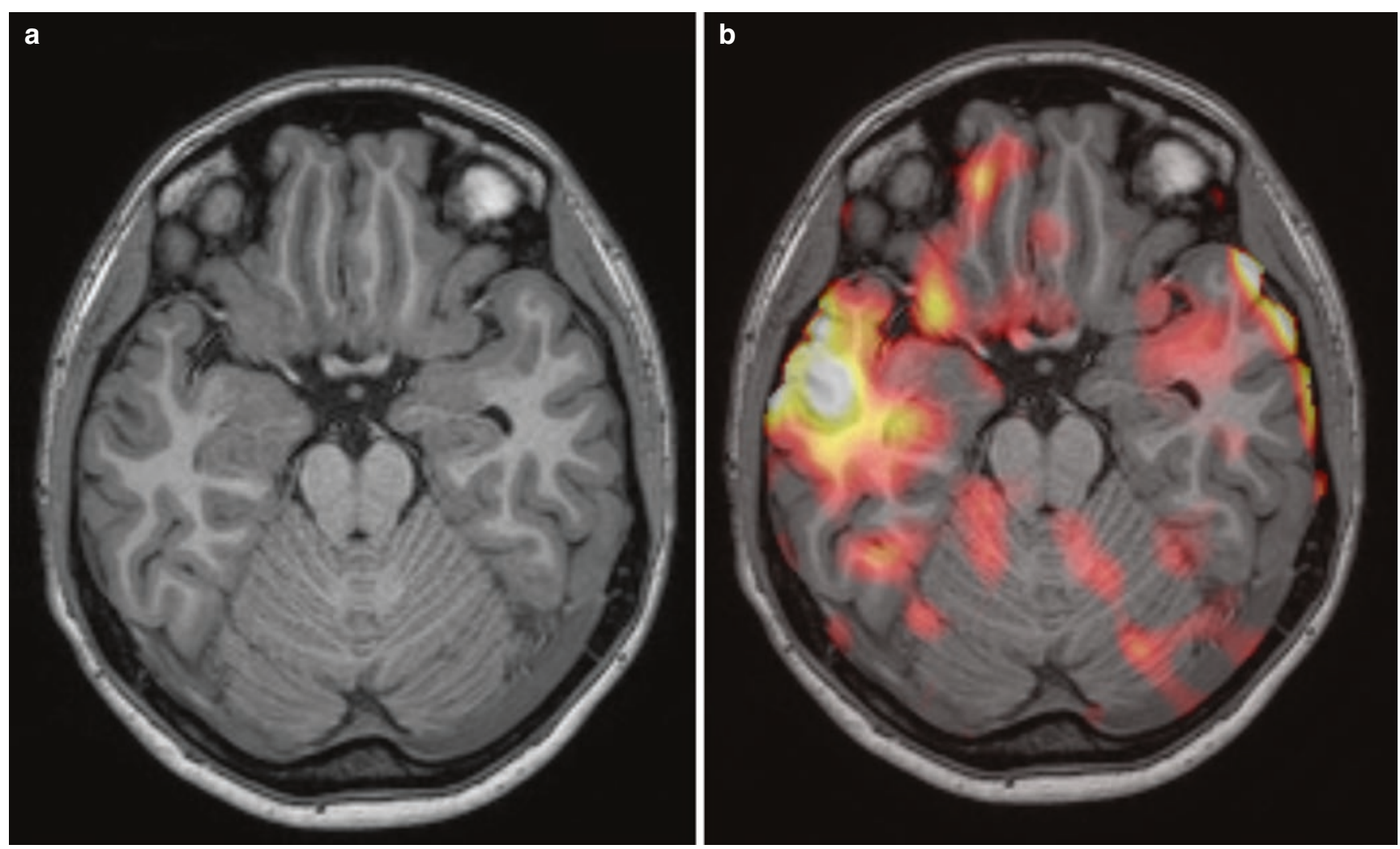

Fig. 10.4 (a, b) SISCOM: (a) axial T1 with small subcortical area in the right temporal lobe with prolonged T1; (b) co-registration of SPECT on MR-images shows ictal hyperperfused area exactly in the same spot as the suspected lesion

throughout the cornu ammonis (CA) regions, but early in the course of the disease there is loss of tissue in the stratum pyramidale most pronounced in CA1 region [15]. Identification of the stratum lacunosum as a landmark provides an imaging reference point for qualitative and quantitative analysis of tissue loss in MTS. Atrophy of the hippocampus and gliosis within the hippocampus will manifest itself as increased signal intensity on T2/FLAIR sequences (Fig. 10.7). The atrophy will lead to loss of the pes hippocampi interdigitations, widening of the temporal horn and atrophy of the white matter of the temporal lobe. As a consequence of Wallerian degeneration, there will be atrophy of the projecting pathways of the hippocampus, i.e., the Papez circuit, with atrophy of the ipsilateral fornix and the mammillary body. In the early stages of MTS the imaging findings are subtle with loss of the internal architecture of the hippocampus with or without associated FLAIR signal changes. Hard windowing of the FLAIR weighted sequences will make identification of the diseased hippocampus easier. In nearly $20 \%$ of patients with MTS, dual pathology is present with a second epileptogenic focus. It is believed that in these cases the other epileptogenic lesion triggered the mesial temporal lobe sclerosis (similar to febrile seizures as a child can trigger or "kindle" a mesial temporal lobe sclerosis). Identification of the second focus is of great importance as failure to do so may result in surgical failure if only a selective amygdalohippocampectomy is performed thus leaving the "primary" focus behind. On the other hand, failure to identify MTS in patients with other lesions may also lead to surgical failure following lesionectomy. Dual pathology may consist also of bilateral mesiotemporal lobe sclerosis as one hemisphere may trigger the other hippocampus to become sclerotic thus constituting bilateral abnormalities. As the internal reference (i.e., the contralateral hippocampus) is similarly affected, comparison of the signal with other regions of archicortex (3-4 layered cortex) can identify whether a mesial temporal lobe sclerosis is present bilaterally. Thus, if the T2/FLAIR signal of the hippocampus is bilateral symmetrical but higher as compared to the cingulum or insula, you have to consider bilateral mesial temporal lobe sclerosis.

\section{Key Point}

- Mesial temporal lobe sclerosis is the most commonly seen cause for medication refractory epilepsy and is characterized by an indistinct gray-white matter differentiation, abnormal high signal on T2/ Flair sequences, and atrophy. In up to $20 \%$ of cases additional epileptogenic pathology is found in patients with mesial temporal lobe sclerosis. 

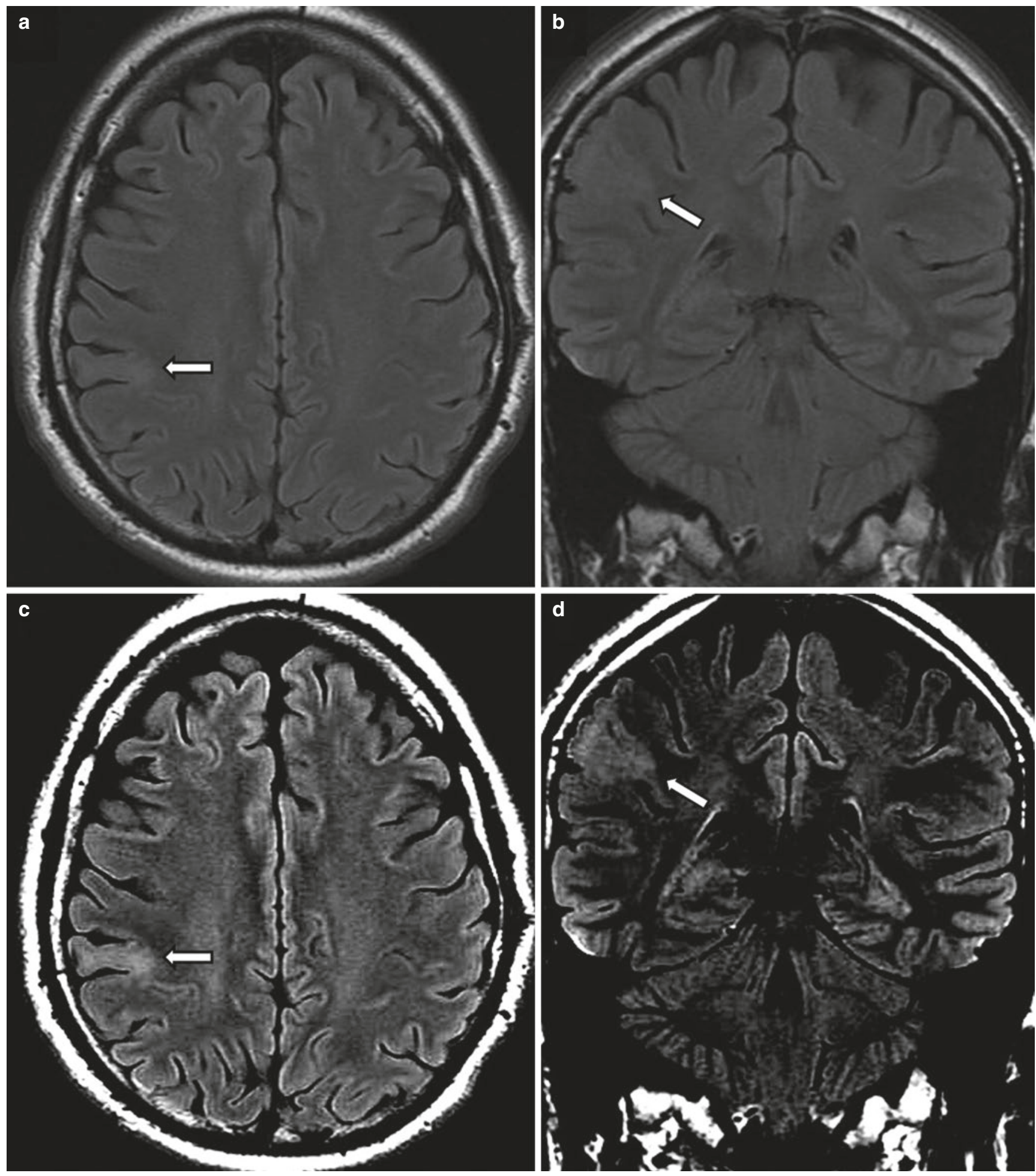

Fig. 10.5 (a, b) Right lateral precentral gyrus type II FCD. (a) axial and (b) coronal FLAIR images at standard window level setting as compared to narrowed window width setting of the same images in $(\mathbf{c}, \mathbf{d})$ which makes the lesion more conspicuous

\subsubsection{Malformations of Cortical Development}

In order to understand the different types of malformations of cortical development it is important to briefly review the embryology of normal brain development: During the seventh week of gestation, neuronal proliferation in the sub- ependymal germinal matrix occurs. After the eighth week these cells migrate outward in multiple waves of radial outward migration aided by radial glial cell guidance in a process coined chemotaxis. The third and last part of the cortical development, the lamination, is the organization of the cells within different cortical layers, a process that is orchestrated 

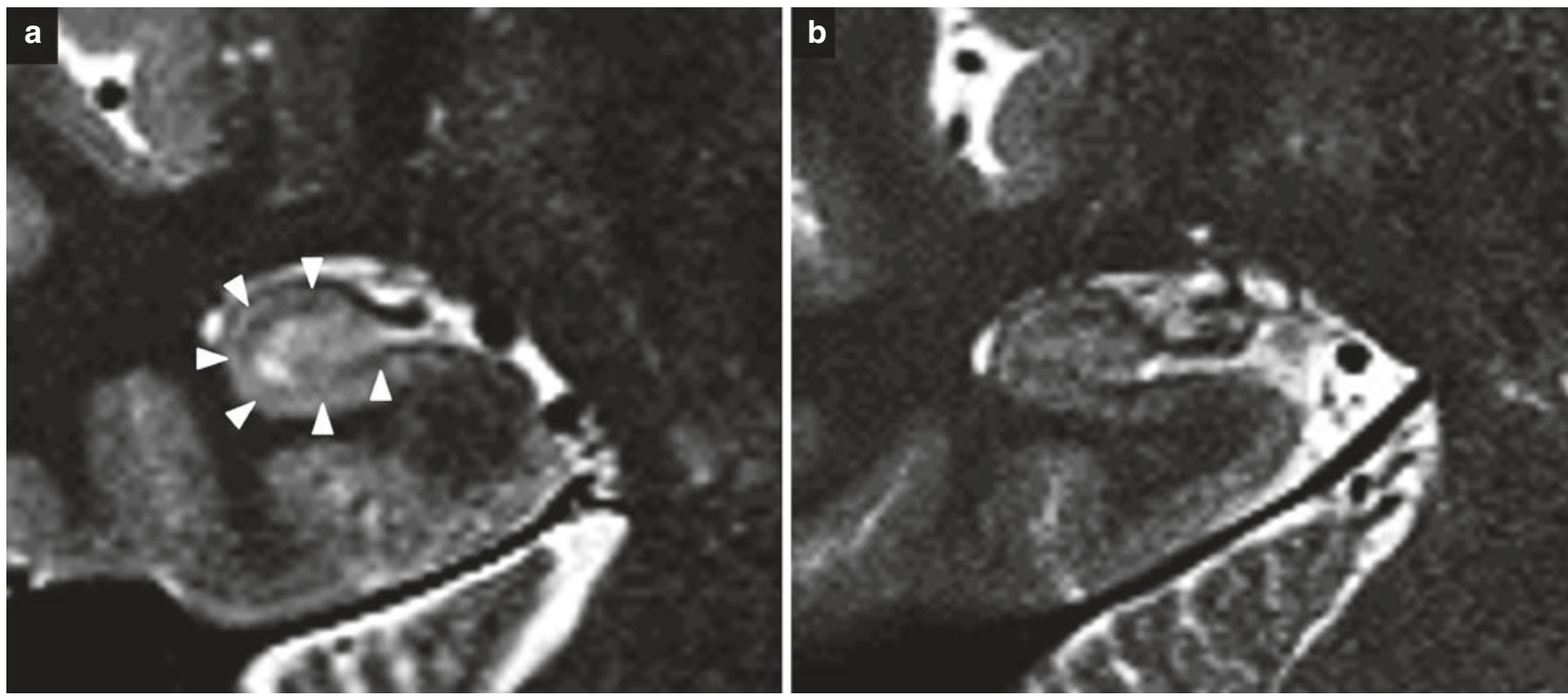

Fig. 10.6 (a, b) Right hippocampus internal architecture: (a) coronal T2 at 3T showing an inner hypointense layer of the hippocampus representing the stratum lacunosum; (b) at 1.5T the ultrastructural detail of the hippocampus is obscured due to the decreased spatial resolution and SNR

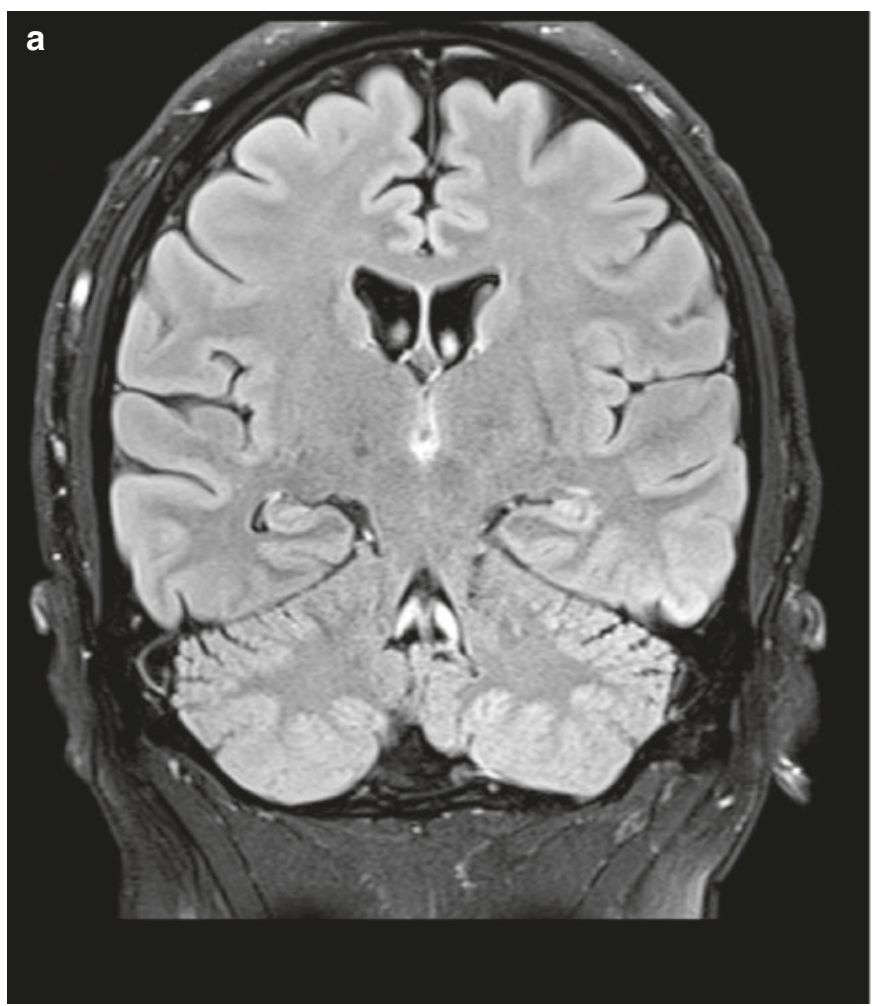

Fig. 10.7 (a, b) Mesial temporal sclerosis (MTS): (a) coronal T2FLAIR shows increased signal in the left hippocampus; (b) axial T1IR demonstrates volume loss in the left hippocampus. The findings

by the subplate (the lowest layer of cortex). Chromosomal mutations, destructive events (ischemia/infections), or toxins may inhibit either of these three processes (proliferation, chemotaxis, or cortical organization) which will lead to abnormalities in stem cell development, migration, or lamination.

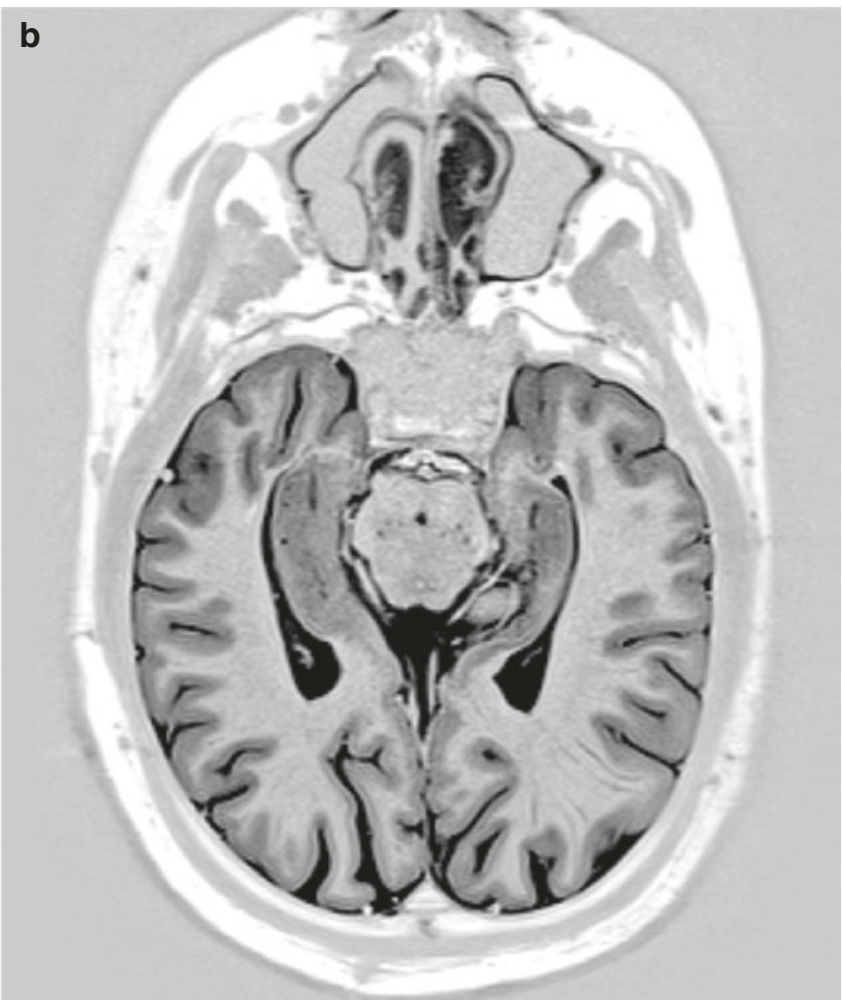

shown are relatively subtle. MRI fails to recognize pathologically detected mesial temporal lobe sclerosis in up to $20 \%$ of the cases

Malformations of cortical development are present in up to $25 \%$ of patients with intractable childhood epilepsy. They are associated with chromosomal alterations, congenital infections, or in utero ischemia. In addition to epilepsy these patients may have developmental delay and focal neurological deficits. 
Malformations related to abnormal stem cell development include the focal or transmantle cortical dysplasias (balloon cell or type II FCDs) and the hemimegalencephalies.

Type II FCD is characterized on histology by dysmorphic neurons with or without balloon cells in addition to cortical dyslamination and are identical to cortical hamartomas in tuberous sclerosis. The transmantle sign is a specific radiologic feature of FCD type II, which is more frequently detected in patients with FCD type IIb than FCD type IIa. Histologically the transmantle sign reflects abnormal cells extending from the ventricle to the cortex manifesting as a linear T2-weighted or FLAIR hyperintensity from ventricle towards the cortex (the radial band or foot) and can be seen in association with a subcortical FLAIR hyperintensity. The abnormal FLAIR hypersignal is again better seen with a narrowed window width. The junction between cortex and white matter is indistinct and the cortical gray matter may be focally thickened (Fig. 10.8).

Signal intensity of the transmantle sign on the T1-weighted sequence could reveal the histologic composition of the type II FCD. Traditionally transmantle sign appears T2-weighted and FLAIR hyperintense and T1-weighted hypointense. More recently a study by Kumura et al. revealed that T1-weighted hyperintense transmantle sign is associated with higher density of balloon cells without significant differences in the number of dysmorphic neurons or the severity of gliosis (Fig. 10.9) [16]. Identifying a T1 hyperintense transmantle sign may have clinical relevance. Although the exact role of balloon cells remains uncertain, some studies have suggested a protective role in reducing spread of the epileptogenic activity.

In hemimegalencephaly a diffuse hamartomatous overgrowth as a result of abnormal stem cell proliferation is present resulting in broad gyri, shallow sulci, and a blurred gray-white matter junction. The ipsilateral ventricle is often enlarged and demonstrates an abnormal straight course of the frontal horn (Fig. 10.10). Clinically, patients present with macrocephaly, hemiplegia, developmental delay, and seizures. The affected hemisphere has no function and thus hemispherectomy can be proposed to these patients. Pre-operative detailed clinical and radiologic assessment is required to determine if there are co-existing abnormalities in the contralateral hemisphere. In addition, diffusion tensor imaging has shown a subset of patients with hemimegalencephaly harbor aberrant midsagittal fiber tracts with intra- or interhemispherically connection which may need to be surgically addressed to improve surgical outcome [17].

Malformations related to abnormal migration are the lissencephalies, the agyria-pachygyrias, and the heterotopias.

In the lissencephalies, there has been a global halt in the migration due to an impaired last phase of neural migration leading to paucity of the gyral and sulcal development with a smooth brain surface and diminished white matter. Patients present with global developmental delay and seizures. Two different types of lissencephaly can be distinguished: the posterior agyria (related to an alteration on Chromosome 17) and the anterior agyria which is an $\mathrm{x}$-linked disease (Fig. 10.11).

Female carriers of the affected X-chromosome present with band heterotopias that is more present in the frontal lobes compared to the parietal lobes. Thus, if females present with band heterotopias, genetic counseling may be indicated as half of their male offspring may be affected by anterior agyria. The band may be thin or thick depending on the amount of arrested neurons during migration. Patients with a thick band have less normal cortex (that can be thinned) and thus present with a more severe developmental delay.

In addition to the "band heterotopia," focal subcortical heterotopia can be present, on imaging, swirling, curvilinear bands of gray matter as well as thinned cortex, and paucity of the white matter are seen. The ipsilateral ventricle may be distorted and there can be an associated callosal hypogenesis.

The third type of heterotopia is called periventricular nodular heterotopia or subependymal heterotopia. On imaging an exophytic smooth ovoid mass in the residual germinal matrix, i.e., along the ventricle is seen (Fig. 10.12). Again, you may have associated anomalies including Chiari malformations, cephaloceles, corpus callosum agenesis, or a Dandy Walker syndrome. In contrast to the other malformations of abnormal migration, the periventricular nodular heterotopia may exhibit quite mild symptoms with normal development and late onset of seizures. If the periventricular heterotopia completely lined the walls of both ventricles, a familiar form has to be considered. Periventricular nodular heterotopia can have different inheritance patterns. Specifically, mutation in the FLNA gene is inherited in an X-linked dominant pattern with male offspring experiencing more severe symptoms of the disorder than females, and in most cases die before birth. Hence genetic counseling for female patient of reproductive age with extensive periventricular heterotopia may be considered.

Malformations related to abnormal cortical organization encompass polymicrogyria, schizencephaly, and FCD type I (non-balloon cell). In polymicrogyria, neurons reach the cortex but distribute abnormally, thus multiple small gyri are formed (Fig. 10.13). Polymicrogyria is most commonly found around the posterior Sylvian fissures, when bilaterally present in the perisylvian region patients present with pseudobulbar palsy.

In open-lip schizencephaly a cleft that is lined by gray matter reaches from the periphery to the ventricle, while in the closed-lip schizencephaly gray matter is reaching from the periphery to the ventricle and a dimple is seen in the ventricular wall. Schizencephaly can be 
multifocal and bilateral. The cortex lining the defect is polymicrogyric with ill-defined margins to the white matter. Finally, FCD type I (non-balloon cell) is a disorder of lamination. Imaging features are very subtle and only mild focal blurring of the gray-white matter junction may be present. This type of dysplasia is often undetectable on MRI.

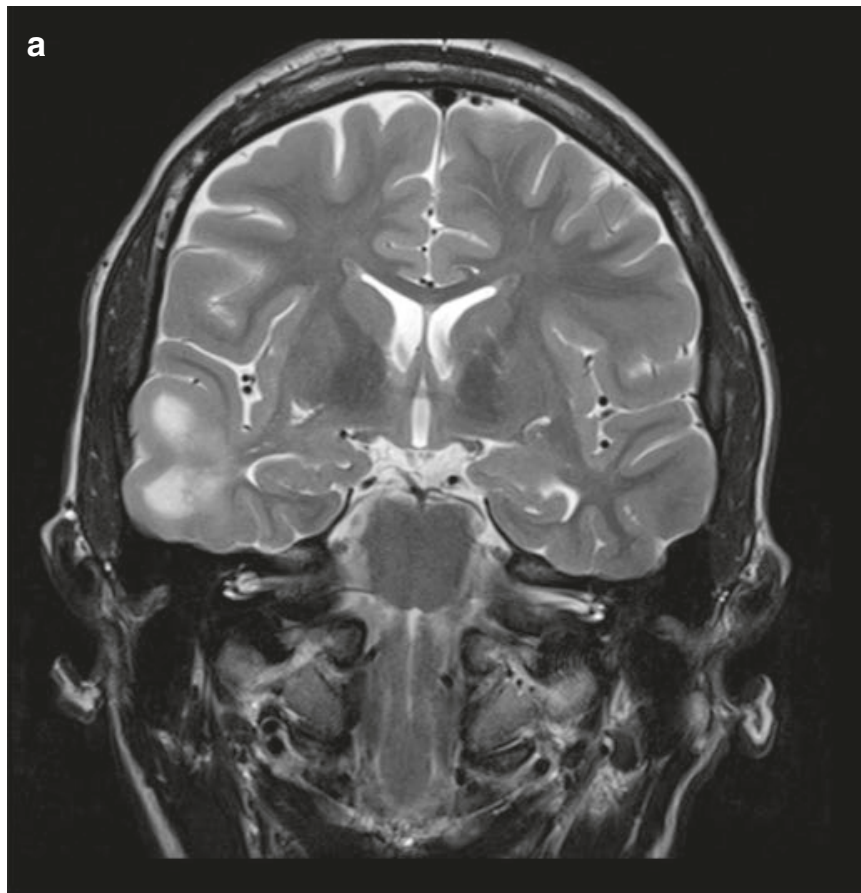

C

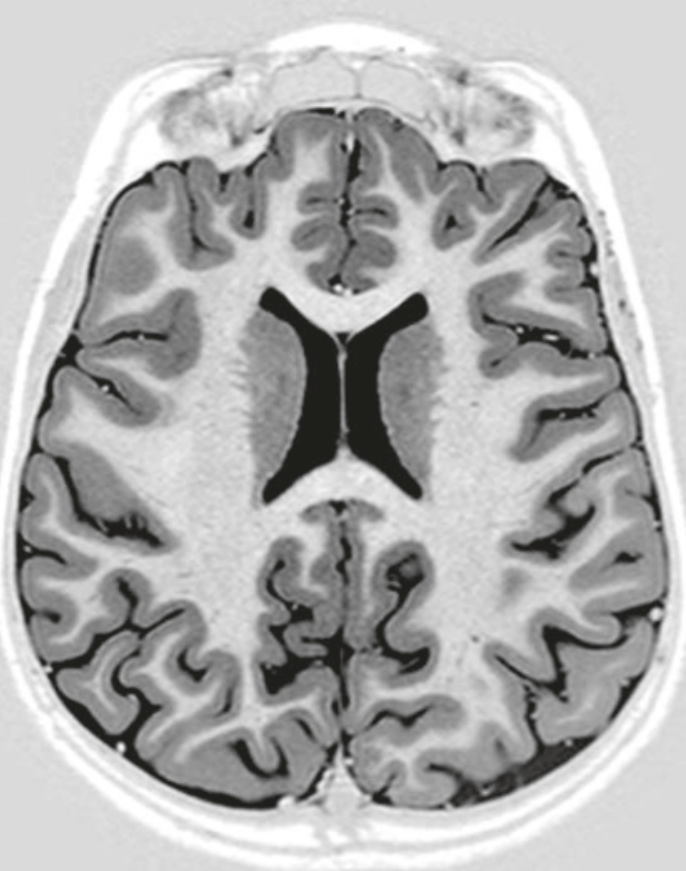

Fig. 10.8 Three cases of FCD type II. (a, b) patient 1; (a) coronal T2 with large subcortical area in the right temporal lobe with prolonged T2. The increased signal stretches in to the temporal horn of the right ventricle; (b) coronal T1 with gadolinium. The corresponding area has decreased signal on T1-weighted image. No enhancement. (c, d) patient 2; (c) axial T1IR with very subtle signal changes at the bottom of a

\section{Key Point}

- Malformations of cortical development are commonly seen in pediatric patients with medication refractory epilepsy. Often these patients have some form of cognitive impairment or developmental delay.
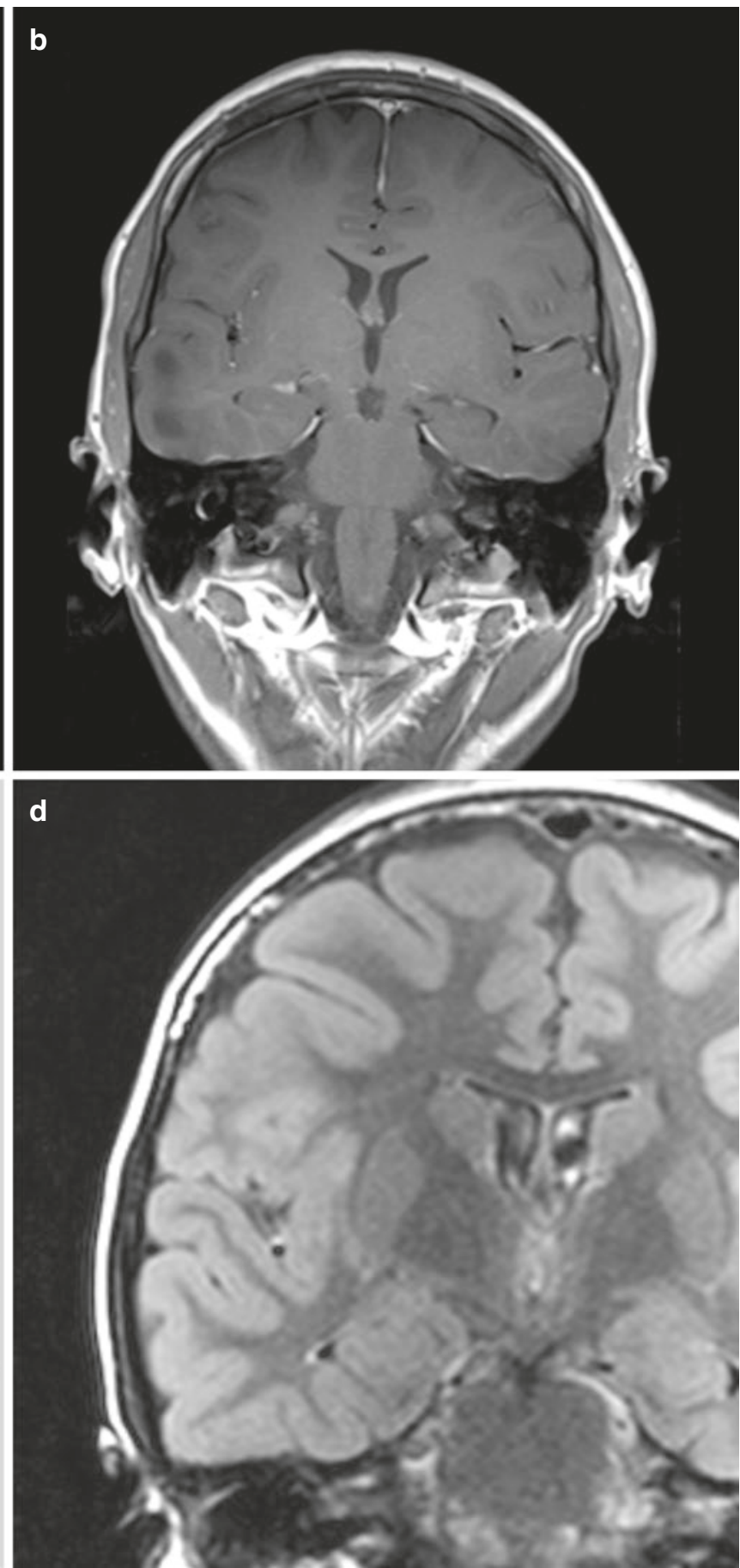

sulcus lateral in the frontal lobe; (d) coronal T2FLAIR shows increased signal in the same are with a faint band stretching towards the lateral ventricle. (e) patient 3, boy, 3 months old, axial T1IR shows a region with thickened cortex in the right frontal lobe. Notice the premature myelinization of the white matter tracts involved in the seizures 
e

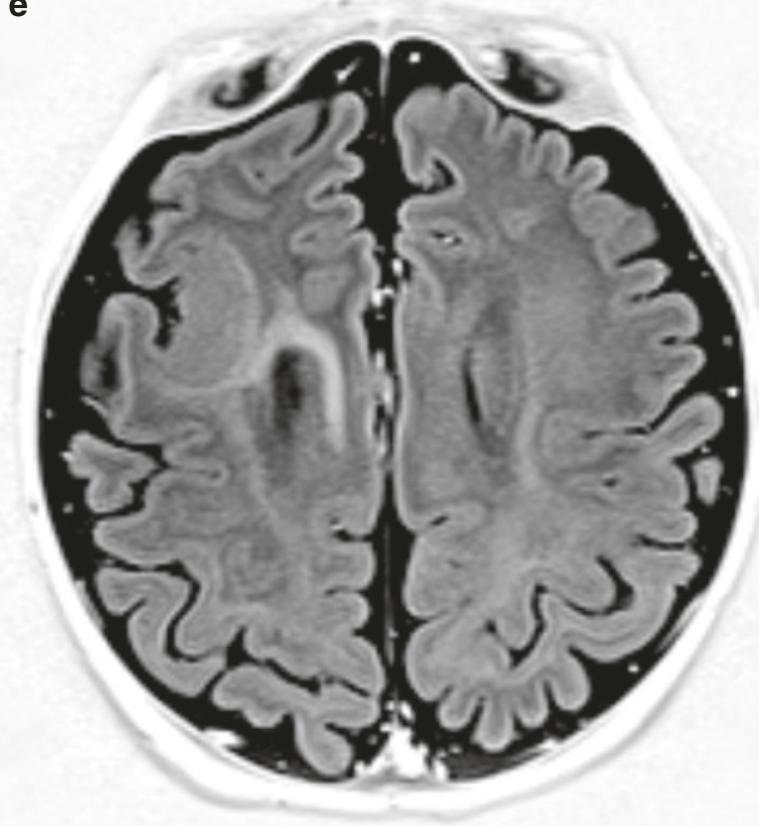

Fig. 10.8 (continued)

\subsubsection{Epileptogenic Tumors}

While virtually all tumors may cause epilepsy, there are certain tumors that have a very high propensity of eliciting medication refractory seizures. As most of these are benign and just by means of location (i.e., within the cortical-white matter interface and with temporal lobe predilection) cause the seizures, these are often very good candidates for surgery. As a general discussion of all tumors is beyond the scope of this chapter, we will focus only on three tumors that are commonly associated with seizures: the gangliogliomas, the DNETs, and the tuber cinereum hamartomas.

Gangliogliomas are cortically based, partly cystic tumors that may calcify and that harbor an enhancing nodule (Fig. 10.14). Gangliogliomas occur in young adults and older children, when present under the age of 10 they are often larger with more cystic components. They are mainly located in the temporal lobes but can also occur in parietal and frontal lobes. Cortical dysplasias (coined type III) can be associated with a ganglioglioma. Differential diagnoses for gangliogliomas are DNETs, pilocytic astrocytomas, pleomorphic xanthoastrocytomas, gliomas, and neurocysticerco-

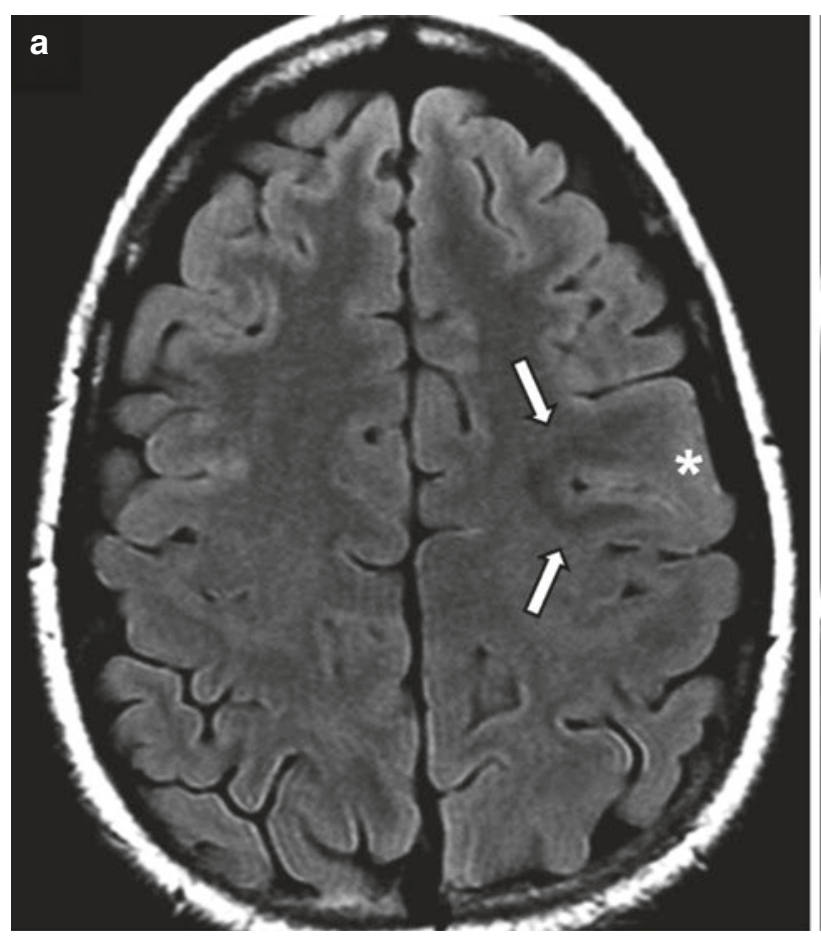

Fig. 10.9 Left frontal type II FCD. (a, b) axial and coronal FLAIR images demonstrate focal gyral thickening posteriorly in the left frontal gyrus with an associated curvilinear hypointense band following the

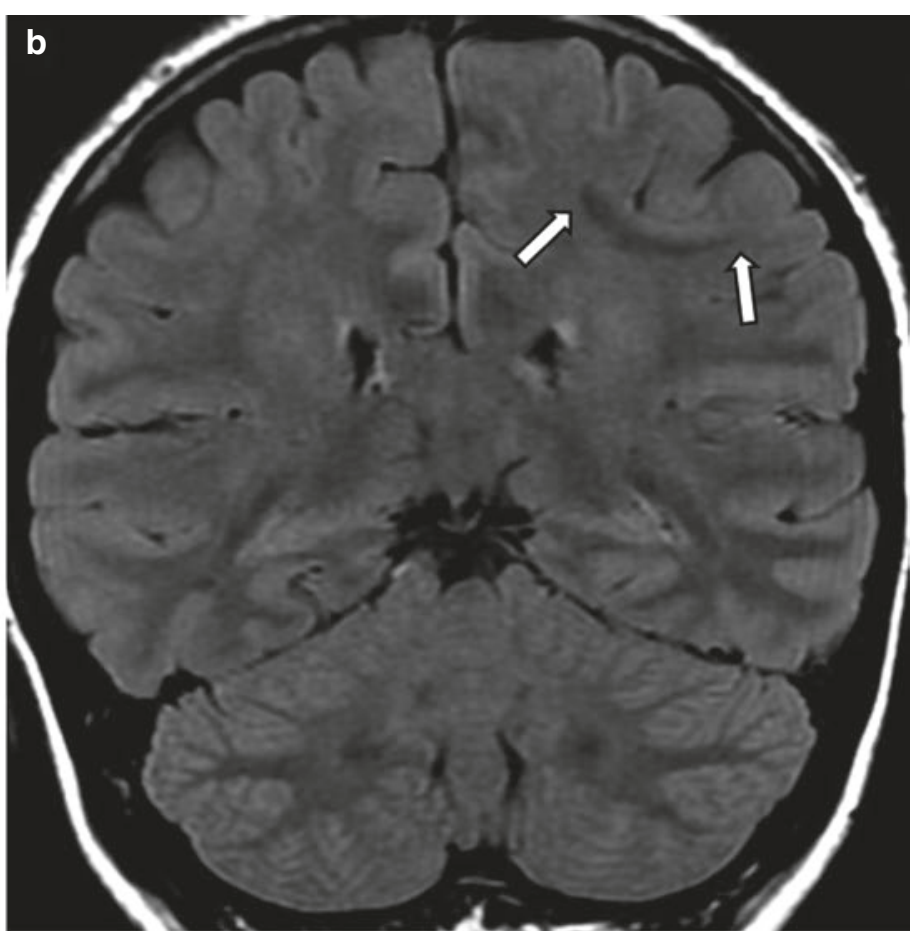

bottom of the sulcus. (c) T1 inversion recovery reveal hyperintensity in this area 


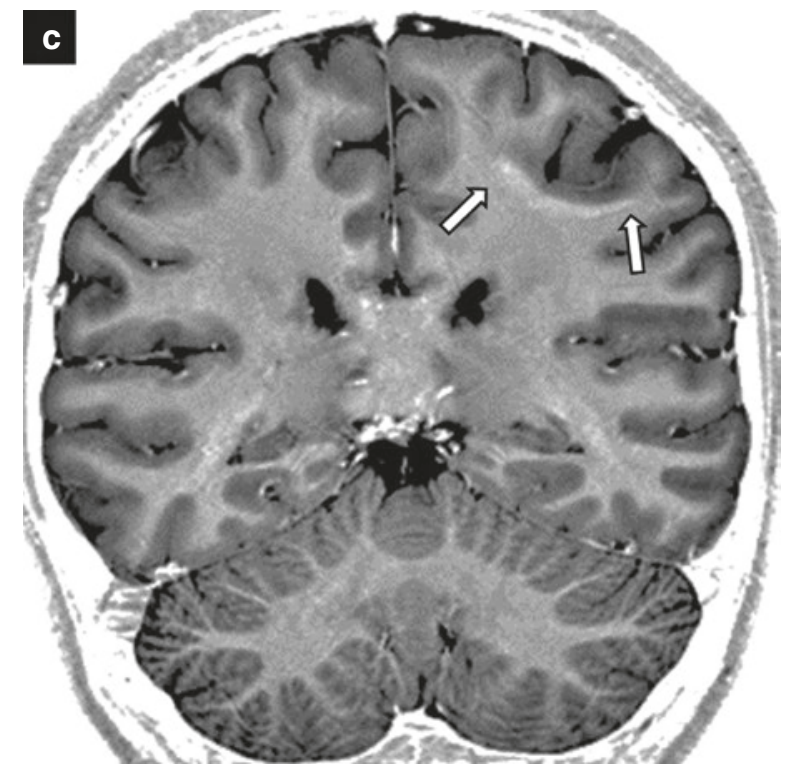

Fig. 10.9 (continued) sis. In adults, solid appearing ganglioglioma may mimic high grade glioma and advanced imaging techniques such as MR perfusion maybe a useful tool for pre-operative evaluation (Fig. 10.15).

DNETs are well demarcated, bubbly, intracortical masses that also are most common in the temporal, parietal, and frontal lobes (Fig. 10.16). They may calcify and enhancement is very rare and if present should lead to more intensive follow-up as the enhancing portion of a DNET may recur following surgery. Top differential diagnoses for DNETs are cortical dysplasia, ganglioglioma, pilocytic astrocytoma, glioma, neuroepithelial cysts, and dilated VR spaces.

Tuber cinereum hamartomas present with the combination of gelastic seizures and precocious puberty. They are located at the floor of the third ventricle (i.e., the tuber cinereum) do not enhance and are isointense to cortex (Fig. 10.17). They are non-neoplastic tumors with disorganized collection of neurons and glia.
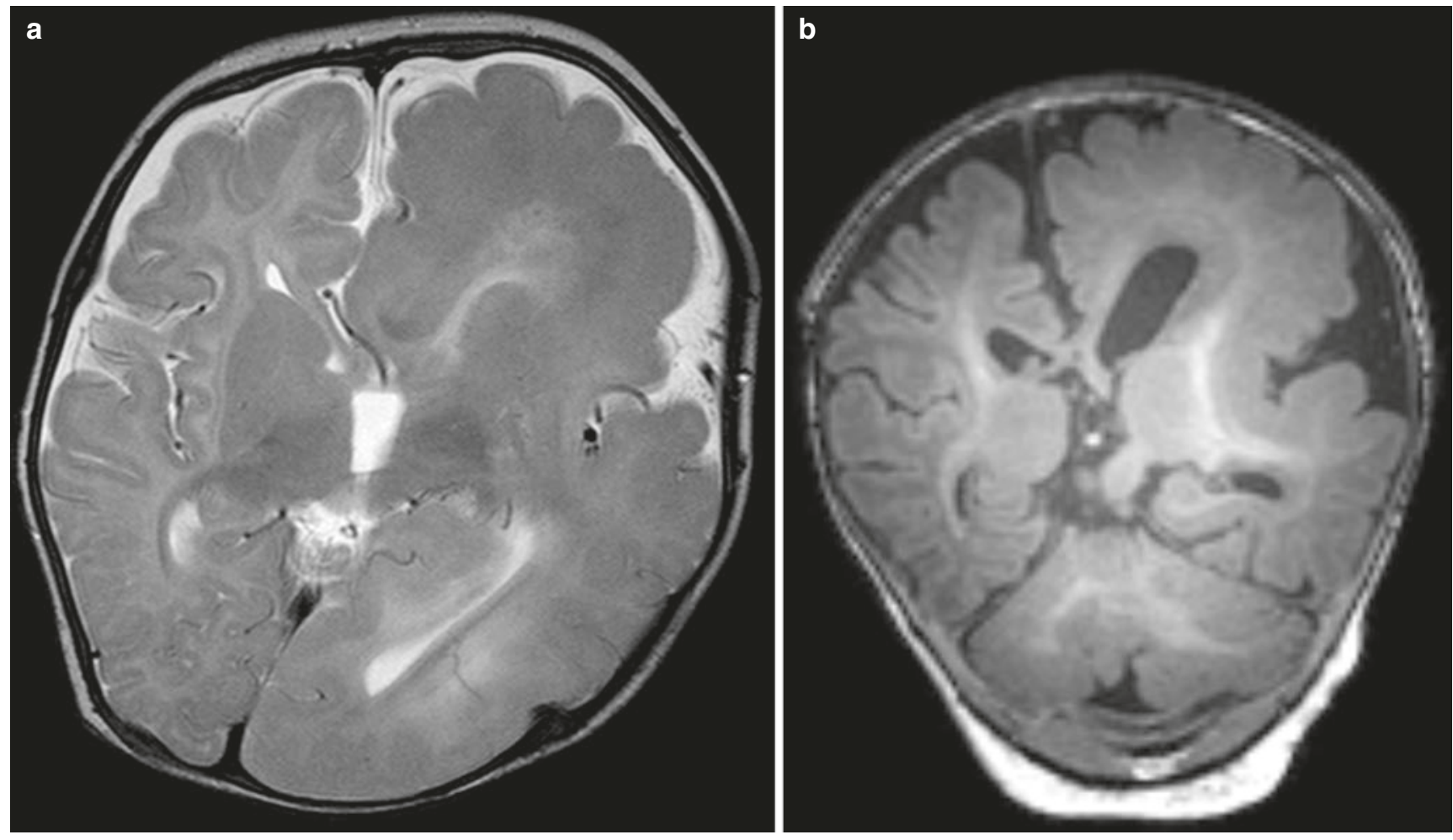

Fig. 10.10 (a, b) Boy, 6 months. Hemimegalencephaly. (a) axial T2; (b) coronal T1. The left hemisphere is enlarged with broad gyri and shallow sulci. The ipsilateral ventricle is enlarged with an abnormal

shape of the frontal horn. Indistinct gray/white matter is noted throughout the entire hemisphere 

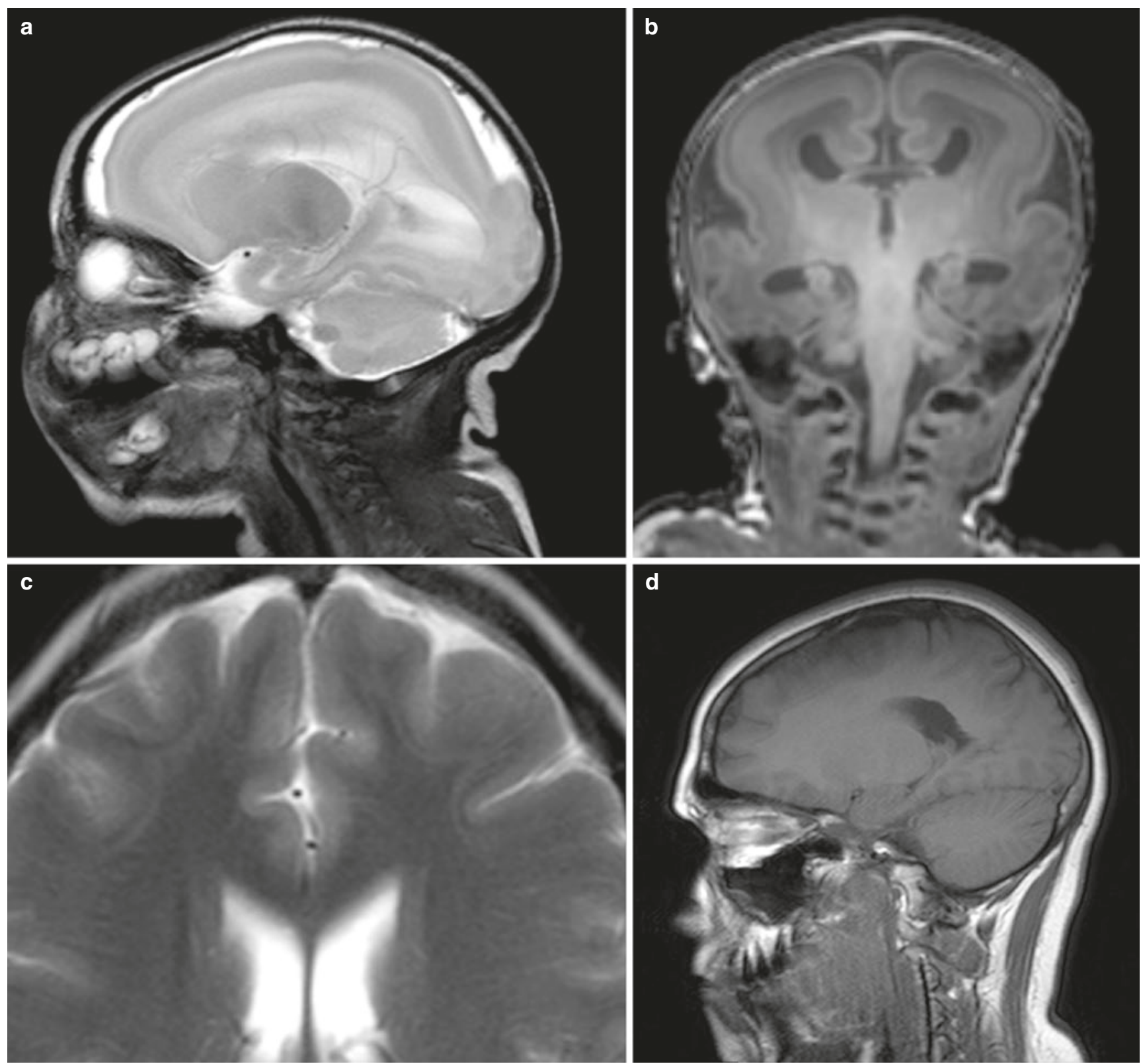

Fig. 10.11 (a, b) X-linked lissencephaly, boy, 2 weeks old. (a) sag t2; (b) coronal $\mathrm{T} 1$. Lissencephaly with agyria more pronounced in the anterior part of the brain. In addition, callosal hypogenesis. (c, d) The mother of the boy in (a, b) female carrier. (c) Axial T2 (detail); (d) coronal T1; Subtle subcortical band heterotopia in both frontal lobes

\subsubsection{Miscellaneous: Vascular Malformations/ Trauma/Infection/Phakomatoses}

Similar to the previous paragraph, it is beyond the scope to in detail describe imaging features of vascular malformations, infections or trauma that can go along with seizures and most of the entities are described in other chapters of this syllabus. We therefore only want to highlight few epilepsy-relevant facts and features of these miscellaneous conditions.

Brain AVMs can cause seizures due to previous hemorrhage and scarring, hemosiderin deposition (especially when close to the cortex), or gliosis. AVMs in the temporal lobe have a higher likelihood of producing seizure due to interference of the normal blood supply and drainage of potentially epileptogenic structures such as the hippocampus.

While cavernomas that are deeply located in the white matter rarely cause seizures, those that are cortically located and have hemosiderin staining reaching the cortex, and in particular the mesial temporal lobe structures, are very often associated with seizures as the hemosiderin stain is believed to have a strong irritative potential for neurons. They are best visualized on T2 gradient echo or SWI sequences where they 

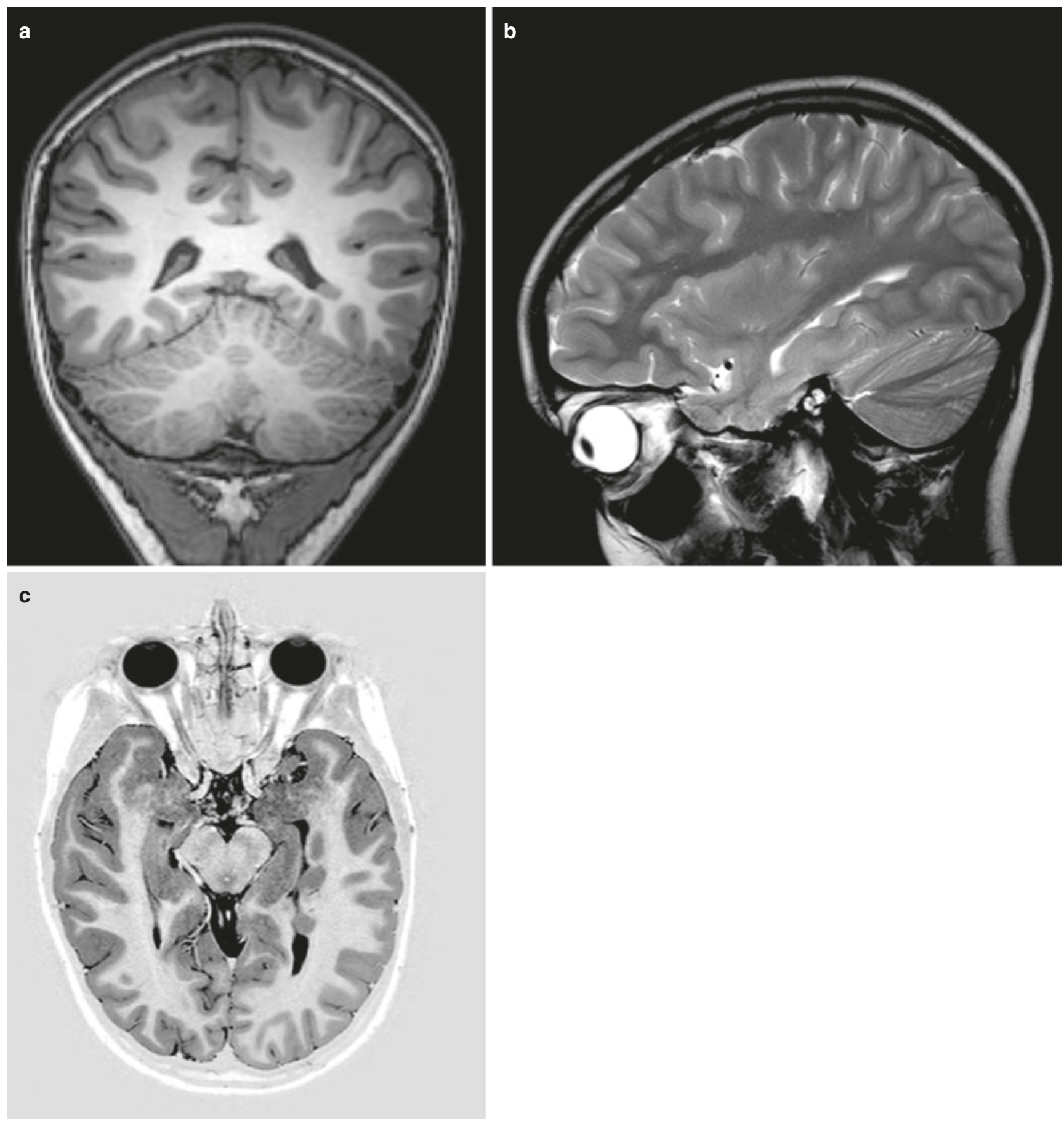

Fig. 10.12 (a-c) Periventricular nodular heterotopia: (a) coronal T1; (b) sagittal T2; (c) axial T1IR. Well delineated smooth ovoid masses lateral to the trigone and temporal horn of the left ventricle. Note that the signal is identical to that of cortex in all sequences

demonstrate with the classical blooming artifact (Fig. 10.18). Cavernomas may be multiple and they can be associated with developmental venous anomalies (DVA). New intracavernomatous thrombosis or hemorrhage may lead to change in seizure frequency.

Patients with previous trauma can experience posttraumatic seizure disorder, especially after having sustained con- tusional hemorrhages of their temporal lobes as gliosis and hemosiderin staining can cause irritation of the surrounding cortex.

Neonatal anoxic ischemia or hypoxemia can cause ulegyria-i.e., a scar/defect of the cerebral cortex that mainly involves the cortex in the depth of the sulcus whereas the cortical crowns remain relatively unaffected. This peculiar pattern can be 

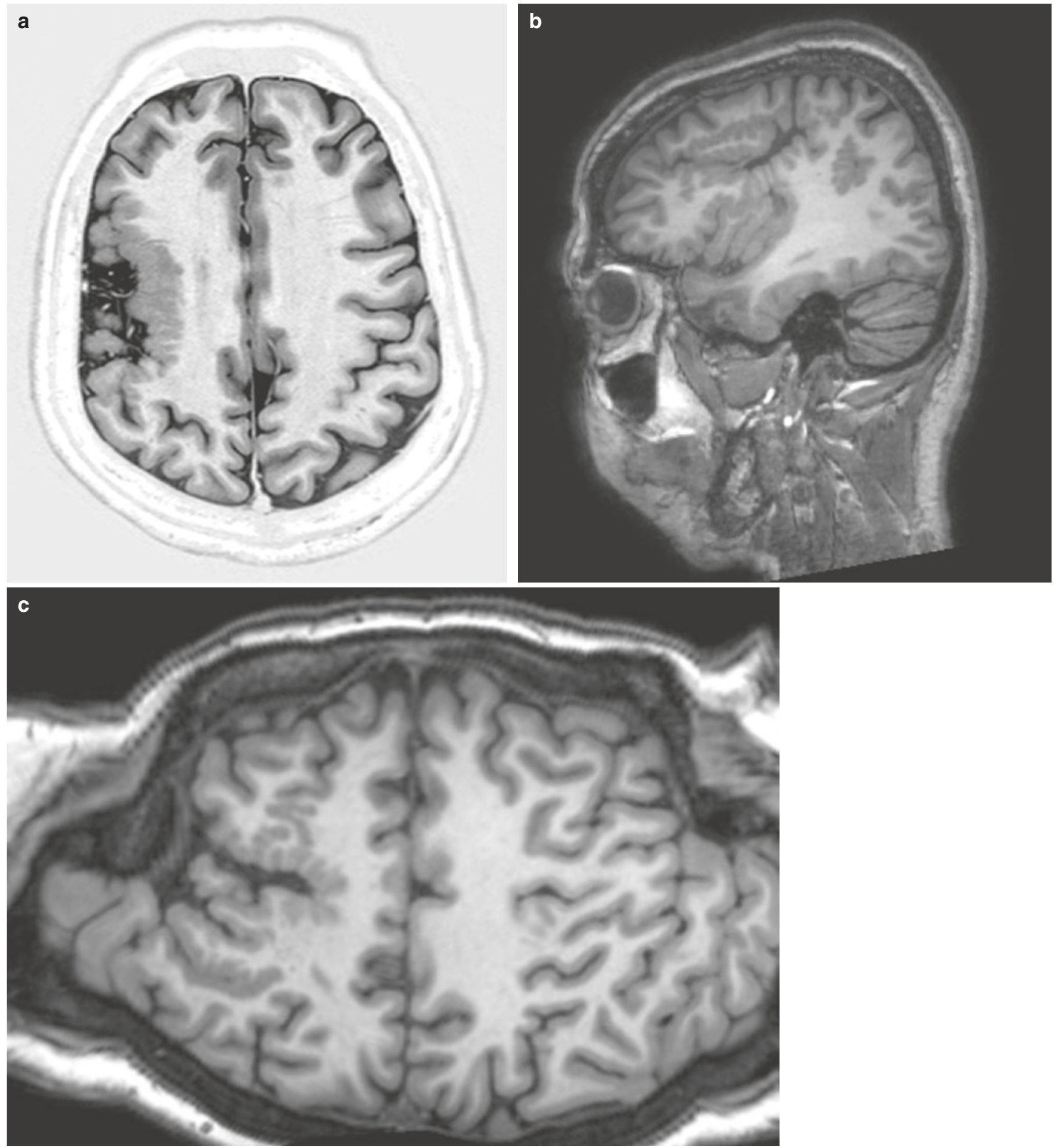

Fig. 10.13 (a-c) Two patients with polymicrogyria. (a) Axial T1IR; (b): sagittal T1. Patient 1. Abnormal gyration in the right hemisphere with a large region with polymicrogyria. (c) Patient 2, "pancake view" from a 3D T1-sequence gives a very good overview of the migration anomalies in the right hemisphere. It also increases the chance to detect subtle changes - see small area with polymicrogyria in the left hemisphere! 

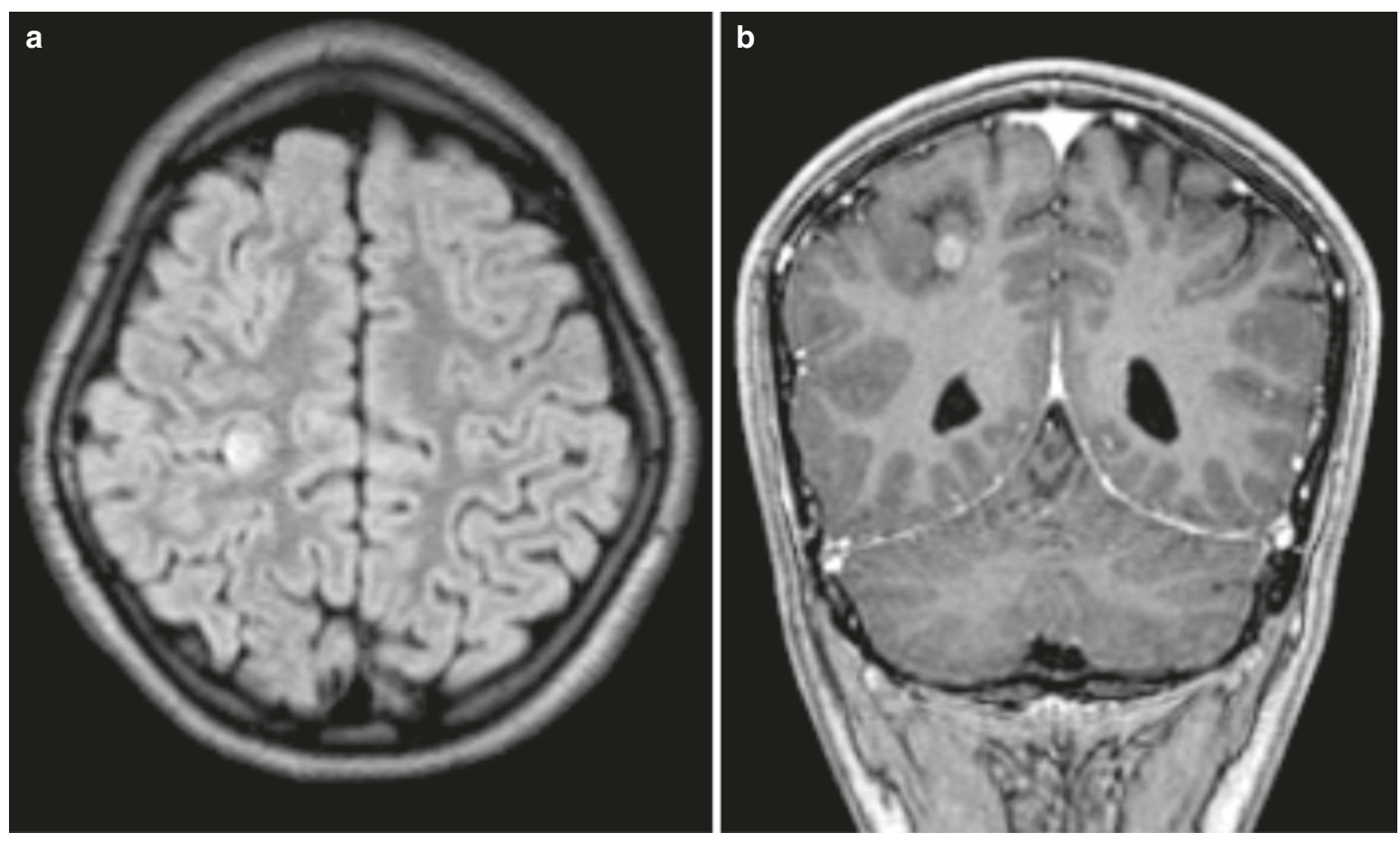

Fig. 10.14 (a, b) Ganglioglioma close to the right postcentral sulcus. (a) axial T2FLAIR with a small, cortical/subcortical, nodular high-signal area in the right parietal lobe close to the postcentral sulcus; (b) coronal T1 with gadolinium shows contrast enhancement in the nodulus
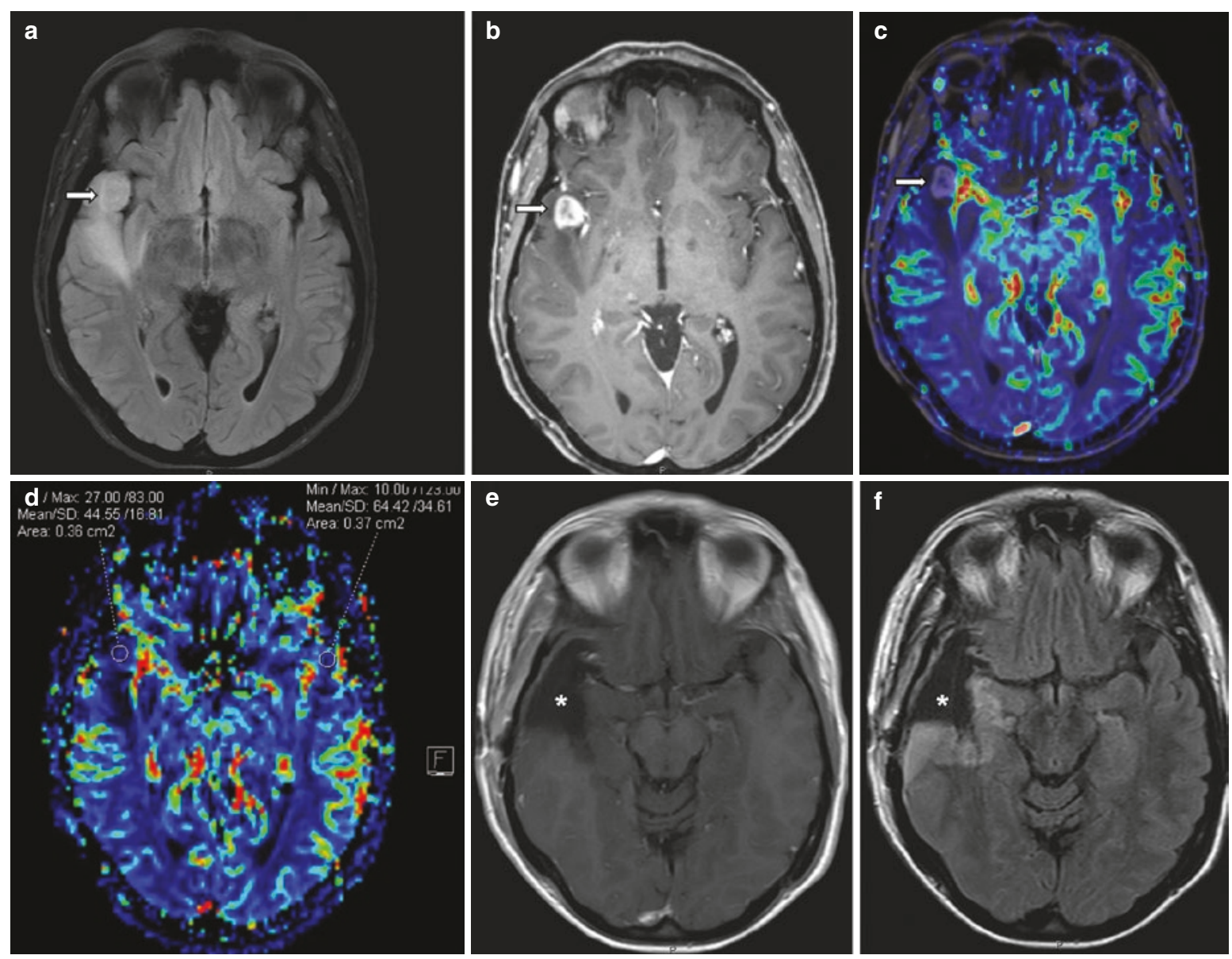

Fig. 10.15 Right superior temporal gyrus ganglioglioma in a middleaged patient. Pre-operative MRI (a, b) axial T2FLAIR and post gadolinium enhanced axial T1 images showed an avidly enhancing cortically based mass with moderate peri-lesional edema. (c, d) Despite the avid lesional enhancement, MR perfusion showed low rCBV value consistent with a low-grade glioma. (e, f) Diagnosis of ganglioglioma was confirmed following mesial sparing temporal lobectomy 

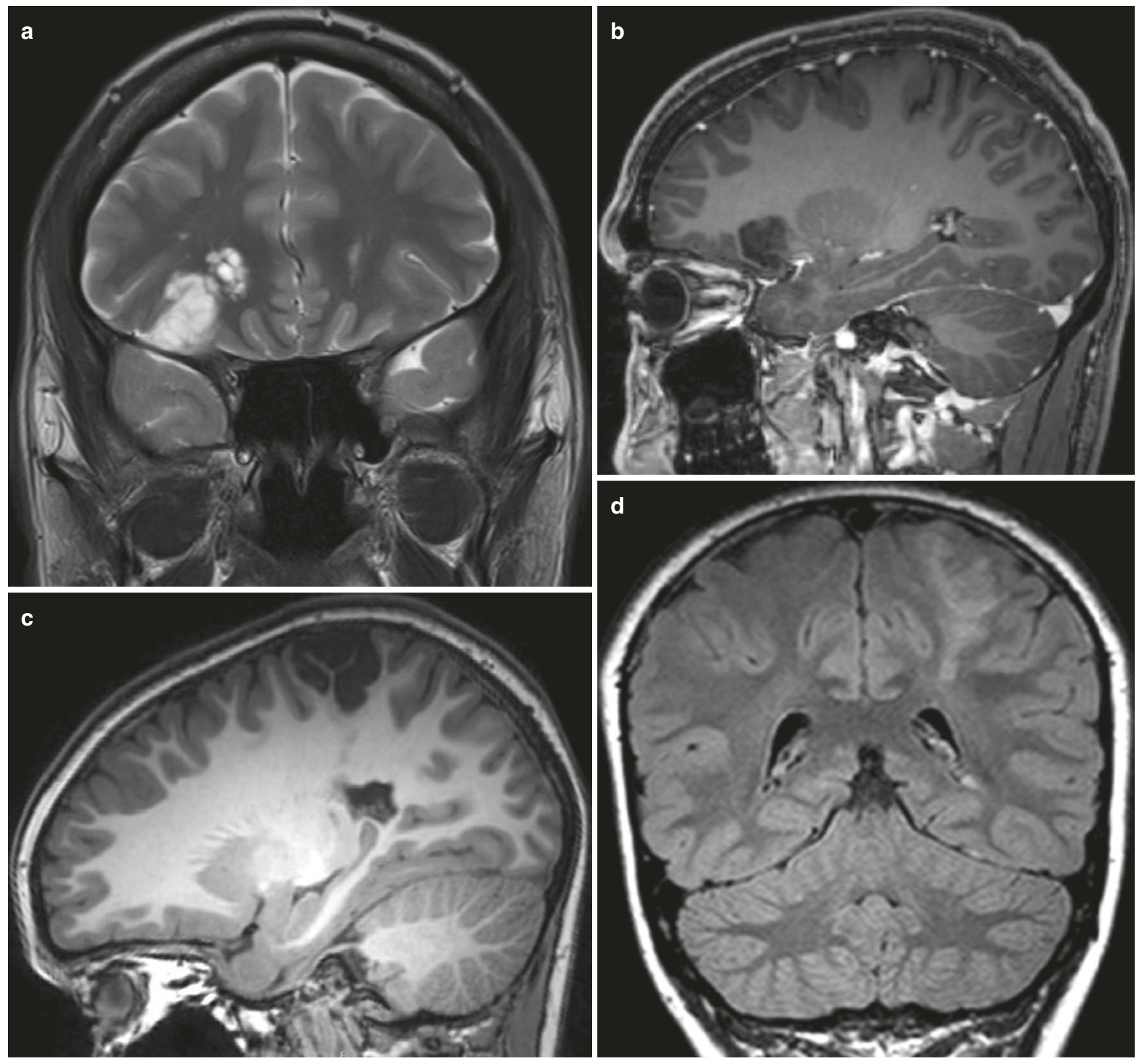

Fig. 10.16 Two patients with DNET. (a, b) Patient 1. (a) coronal T2; (b) sagittal T1 with gadolinium; well delineated cortical/subcortical bubbly mass in the right frontal lobe with prolonged $\mathrm{T} 2$ and no contrast enhancement typical of a DNET. (c, d) Patient 2: (c) sagittal T1; (d)

explained by the vascular supply of the gyri in the newborn that leads to a better perfusion of the apices of the gyri as compared to the depth of the sulci. There will be paucity of the white matter and, as the lesion occurred prior to complete myelination, a relatively mild gliosis. If the perinatal ischemia has only involved one hemisphere (perinatal stroke) a Dyke Davidoff Mason syndrome will ensue where stable hemiatrophy is present with hypertrophy of the skull and the sinuses, paucity of white matter, ventricular enlargement, and mild gliosis.

Virtually any infection (bacterial, fungal, parasitic) can produce epileptogenic lesions, and world-wide, infections are coronal T2 FLAIR; This DNET in the left parietal lobe is associated with a FCD type II. Notice the streak with signal changes which stretches towards the lateral ventricle

the leading cause of epilepsy. A typical example is neurocysticercosis which is a very common cause of focal epilepsy in the developing world. In the early vesicular, colloidal or granular nodular stages, the ictal focus is likely to originate from the cysticercus zone. Recurrent seizures might cause hippocampal damage or dysfunction. In the late nodular calcified stage the cysticercus zone becomes less active and but damages to the mesial temporal structures may lead to acquired MTS which becomes the new ictal focus (Fig. 10.19).

Rasmussen's encephalitis is a presumably autoimmune mediated chronic inflammation of the brain that presents 

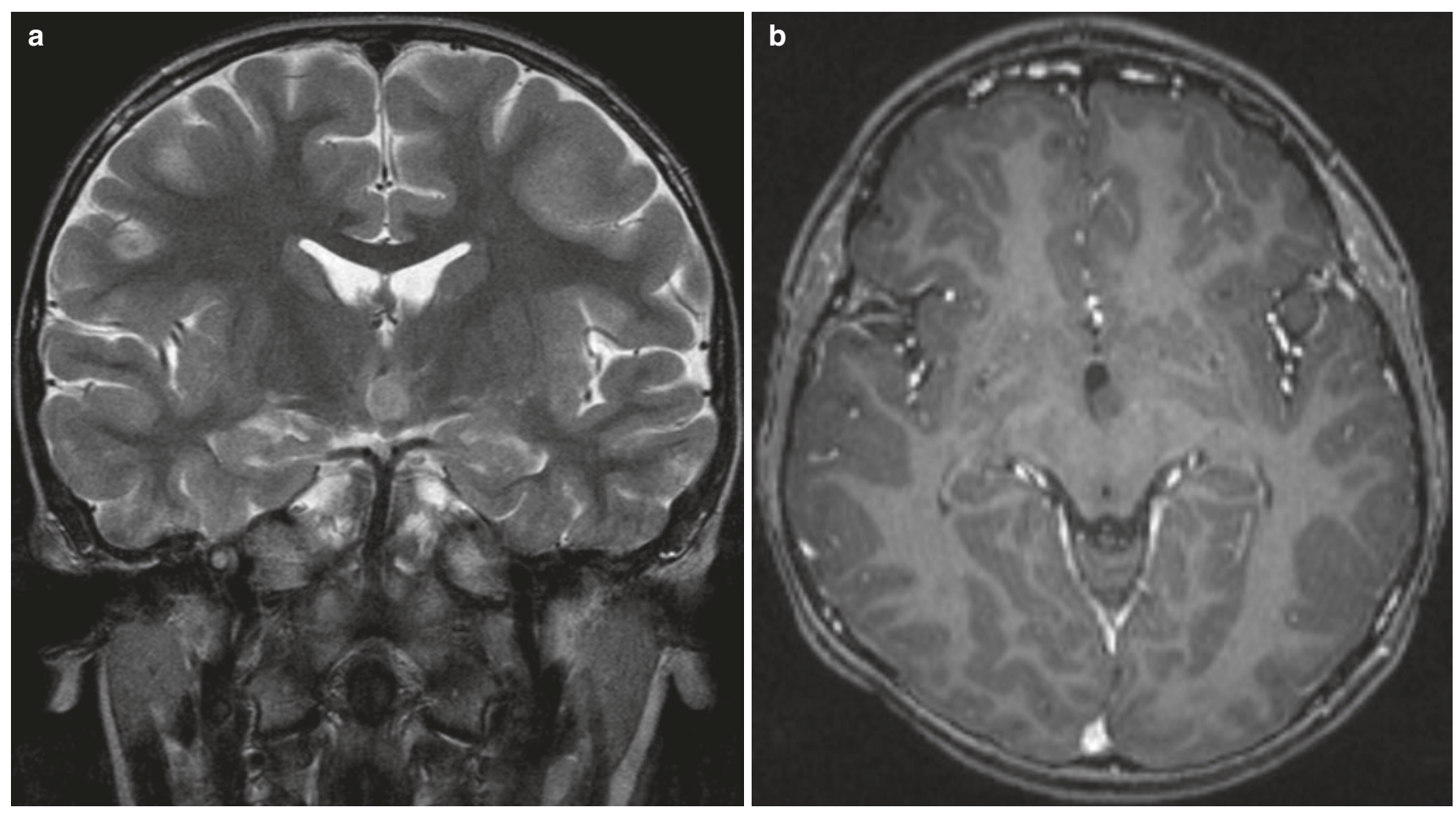

Fig. 10.17 (a, b) Hypothalamic hamartoma. (a) Coronal T2 with a lobular mass close to the left wall of the third ventricle. (b) Axial T1 with gadolinium detects no contrast enhancement in the mass
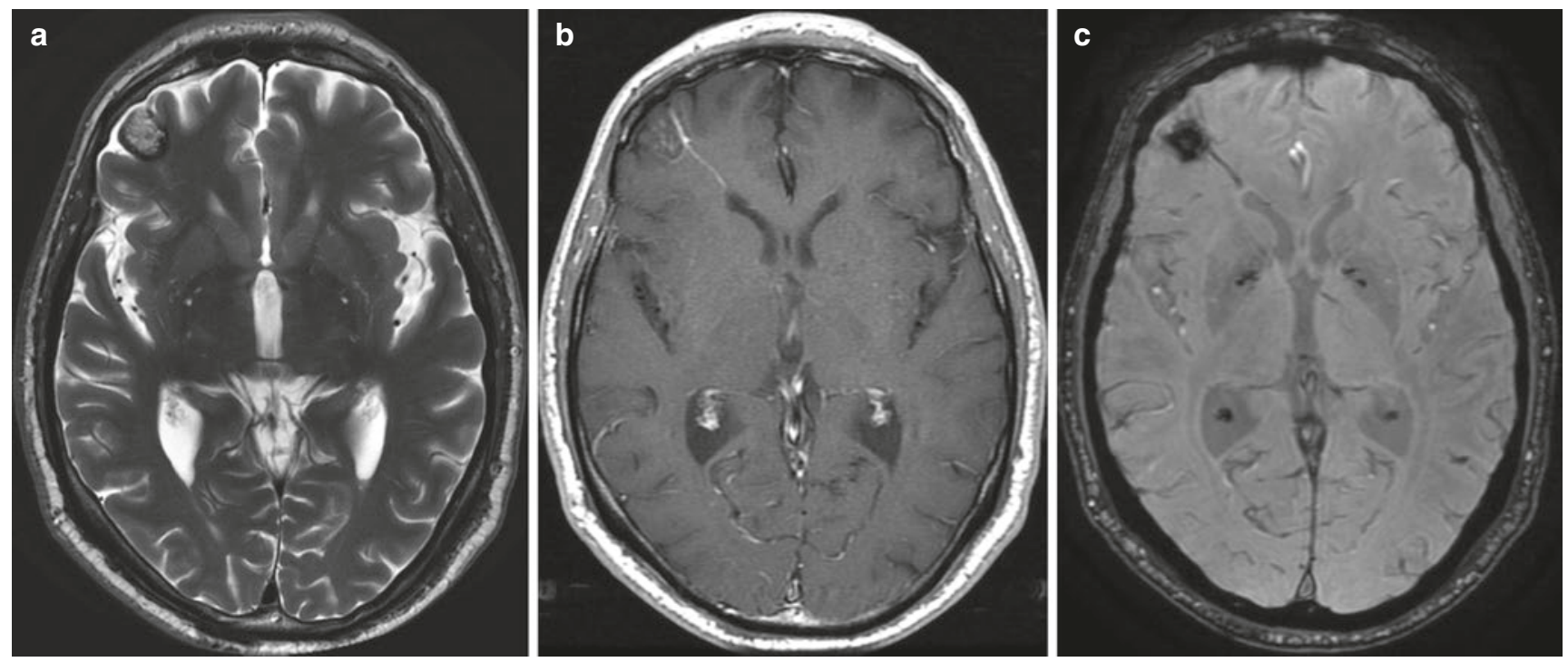

Fig. 10.18 (a-c) Cavernoma in the right frontal lobe with an associated DVA. (a) axial T2 shows the superficial lesion with heterogenous signal; (b) axial T1 with gadolinium shows an associated vascular

with progressive gliosis and volume loss. Patients experience seizures and a progressive hemiparesis.

Antero-basal temporal lobe encephaloceles are lesions that are either related to a congenital defect of the bone or to previous trauma. Brain tissue can extend into the pterygopalatine fossa through the bony defect at the base of the structure, a DVA; (c) axial SWAN-sequence. This susceptibility sensitive sequence shows the classical blooming artifact of the blood products within the cavernoma

greater sphenoid wing in the region of the foramen rotundum and pterygoid process. The herniated brain demonstrates high T2/FLAIR signal and is believed to be the epileptogenic focus. Following resection of the abnormal brain tissue seizure freedom can be obtained in a very large proportion of cases. 

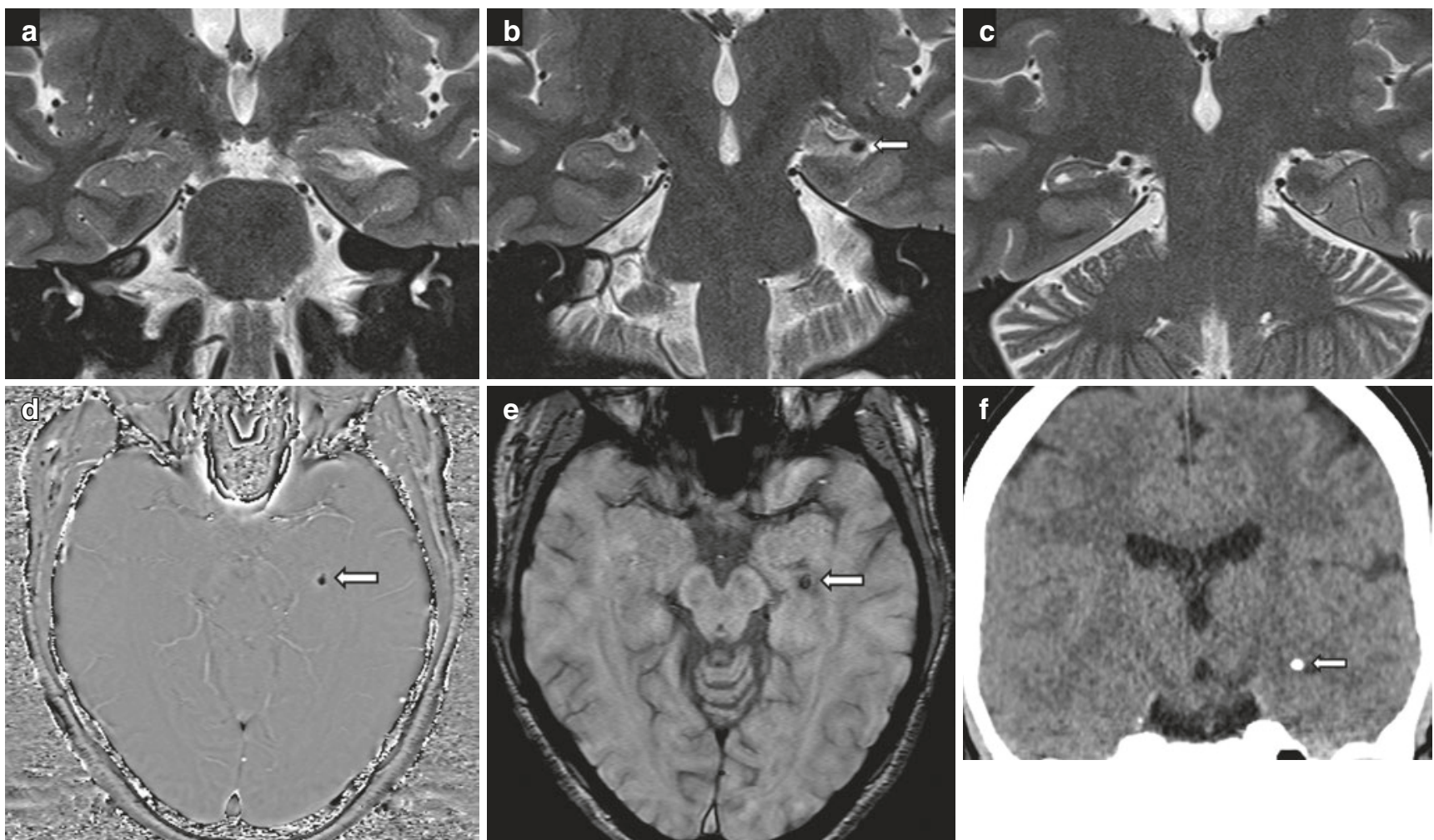

Fig. 10.19 Nodular calcified neurocysticercosis in the left hippocampus leading to acquired MTS. (a-c) Coronal T2 images show a small circumscribed hypointense lesion in the left hippocampal head with associated significant volume of the left hippocampus and obscuration of the internal hippocampal architecture. (d, e) SWI and phase image show positive phase shift suggestive presence of calcification. (f) CT scan confirms the calcification
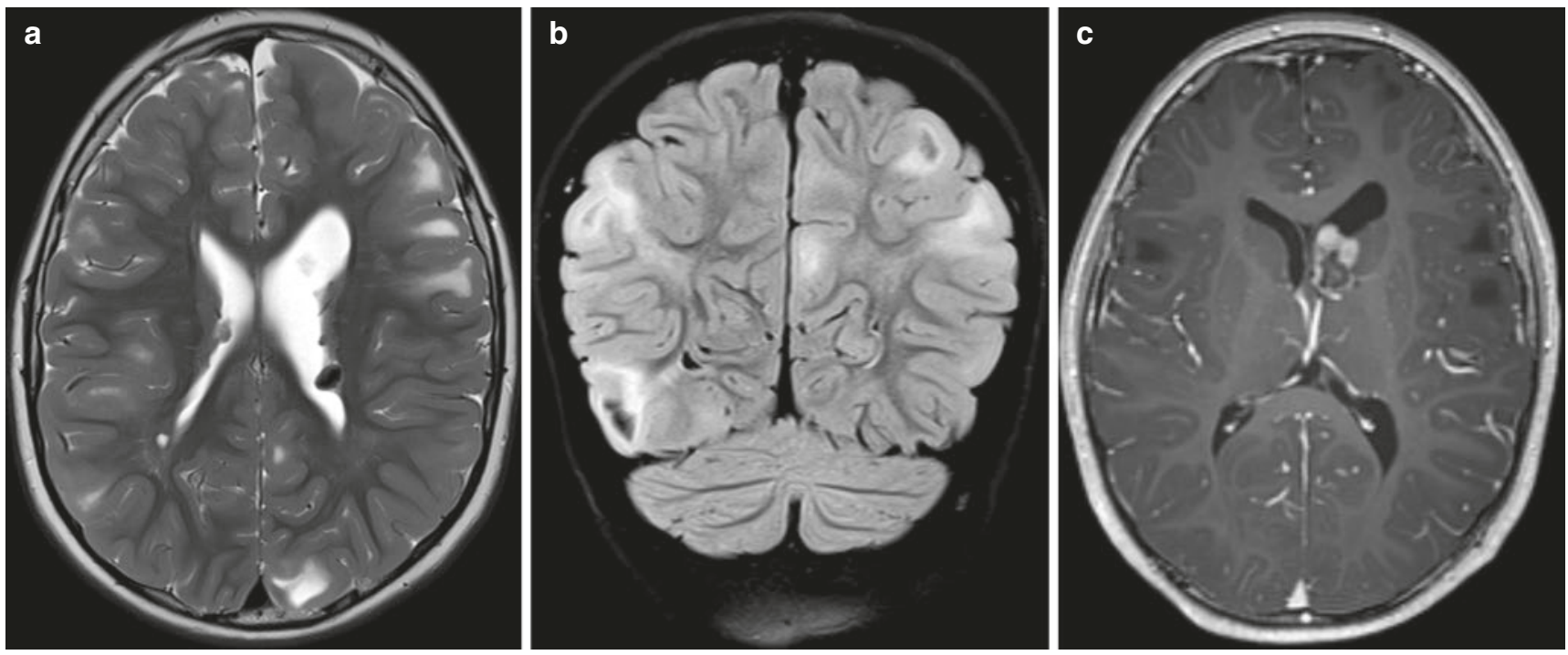

Fig. $10.20(\mathbf{a}-\mathbf{c})$ Tuberous sclerosis; (a) axial T2 and (b) coronal T2FLAIR show subependymal hamartomas and widespread cortical and subcortical signal changes; (c) axial T1 with gadolinium with a large giant cell astrocytoma in a classical position, close to the foramen of Monroe in the left lateral ventricle 

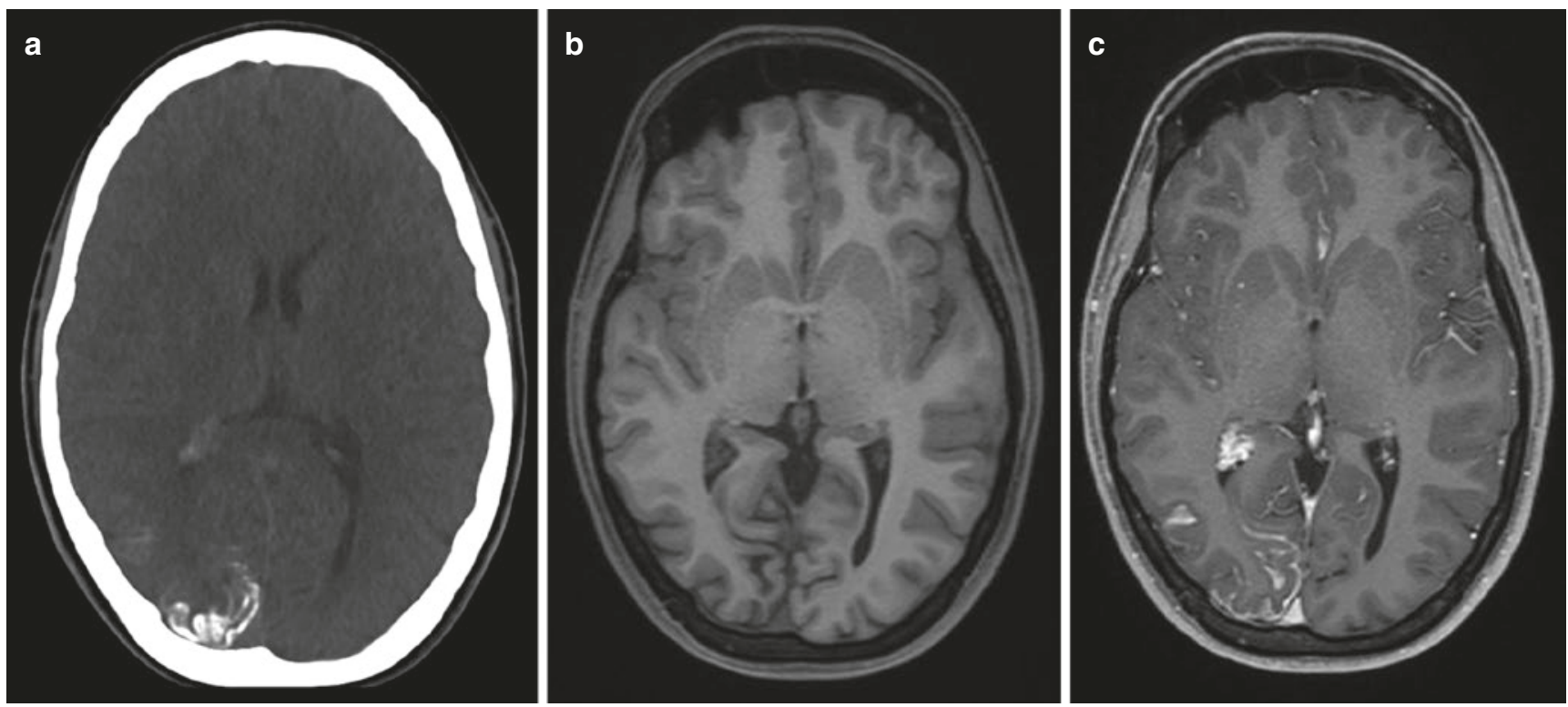

Fig. 10.21 (a-c) Sturge-Weber syndrome; (a) axial CT shows curvilinear cortical calcifications in the right occipital lobe; (b) axial T1 without and (c) with gadolinium shows contrast enhancement caused

The two phakomatoses commonly associated with seizures are tuberous sclerosis and Sturge-Weber syndrome. In tuberous sclerosis, multiple hamartomas are present within the cortical/subcortical region (Fig. 10.20). These are similar in histology to the FCD type II and are therefore believed to be epileptogenic. In addition, patients may develop subependymal calcification as well as a subependymal giant cell astrocytoma; however, the latter two lesions are not believed to be epileptogenic. In Sturge-Weber syndrome the cortical calcification as well as the pial angiomatosis along the cortex is presumably related to the seizures. In addition, patients may present with choroid plexus hypertrophy and brain hemiatrophy as well as a facial port-wine stain (Fig. 10.21).

\section{Key Point}

- Many other pathologies including tumors, vascular malformations, phakomatoses, or remote infections can cause medication refractory epilepsy especially if the structures involved are close to the mesial temporal lobe structures.

\subsection{Conclusion}

Neuroimaging in patients with refractory epilepsy will find abnormalities in as high as $85 \%$ of cases and therefore plays a crucial role in the identification of epileptogenic lesions and their possible surgical removal. A dedicated epilepsy protocol is necessary to identify these lesions and the MR

by pial angiomatosis. Note also hypertrophy of the ipsilateral choroidal plexus, typical for this phakomatosis

should be interpreted in conjunction with EEG, MEG, neuropsychological testing, and clinical semiological data to increase the likelihood of identifying these often very subtle lesions.

\section{Take Home Messages}

- When evaluating a dedicated seizure protocol MR, a structured approach is helpful that includes a detailed assessment of (a) the hippocampus and mesial temporal lobe structures, (b) the ventricular outline, and (c) the gyral and the sulcal anatomy.

- Particular emphasis should be paid upon the T2/ FLAIR signal within the cortex and hippocampus, its similarity to other regions of neo- and archicortex, the internal architecture of the hippocampus, the indentations of the pes hippocampi, the fornix and mammillary bodies, and the grey-white matter interface of the neocortex (blurring, gray matter thinning or thickening).

- The malformations of cortical development can be differentiated into disorders of neuronal proliferation, migration and cortical organization.

\section{References}

1. Duncan JS. Imaging in the surgical treatment of epilepsy. Nat Rev Neurol. 2010;6:537-50.

2. von Oertzen J, Urbach H, Jungbluth S, et al. Standard magnetic resonance imaging is inadequate for patients with refractory focal epilepsy. J Neurol Neurosurg Psychiatry. 2002;73:643-7. 
3. Téllez-Zentenoa JF, Hernández Ronquilloa L, Moien-Afshari F, et al. Surgical outcomes in lesional and non-lesional epilepsy: a systematic review and meta-analysis. Epilepsy Res. 2010;89:310-8.

4. Wellmer J, Quesada CM, Rothe L, et al. Proposal for a magnetic resonance imaging protocol for the detection of epileptogenic lesions at early outpatient stages. Epilepsia. 2013;54:1977-87.

5. Strandberg M, Larsson EM, Backman S, et al. Pre-surgical epilepsy evaluation using 3T MRI. Do surface coils provide additional information? Epileptic Disord. 2008;10:83-92.

6. Winstona GP, Micallef C, Brian E, Kendell BE, et al. The value of repeat neuroimaging for epilepsy at a tertiary referral centre: 16 years of experience. Epilepsy Res. 2013;105:349-55.

7. Balchandani P, Naidich TP. Ultra-high-field MR neuroimaging. Am J Neuroradiol. 2015;36:1204-15.

8. De Ciantis A, Barkovich AJ, Cosottini M, et al. Ultra-high-field MR imaging in polymicrogyria and epilepsy. Am J Neuroradiol. 2015;36:309-16.

9. Breyer T, Wanke I, Maderwald S, et al. Imaging of patients with hippocampal sclerosis at 7 Tesla: initial results. Acad Radiol. 2010;17:421-6.

10. Neel Madan N, Grant PE. New directions in clinical imaging of cortical dysplasias. Epilepsia. 2009;50:9-18.

11. Bauer PR, Reitsma JB, Bernard M, Houweling BM, et al. Can fMRI safely replace the Wada test for preoperative assessment of language lateralisation? A meta-analysis and systematic review. J Neurol Neurosurg Psychiatry. 2014;85:581-8.

12. Piper RJ, Yoong MM, Kandasamy J, et al. Application of diffusion tensor imaging and tractography of the optic radiation in anterior temporal lobe resection for epilepsy: a systematic review. Clin Neurol Neurosurg. 2014;124:59-65.

13. Desai A, Bekelis K, Thadani VM, et al. Interictal PET and ictal subtraction SPECT: sensitivity in the detection of seizure foci in patients with medically intractable epilepsy. Epilepsia. 2013;54:341-50.

14. Barkovich AJ, Guerrini R, Kuzniecky RI, et al. A developmental and genetic classification for malformations of cortical development: update. Brain. 2012;135:1348-69.

15. Howe KL, Dimitri D, Heyn C, et al. Histologically confirmed hippocampal structural features revealed by $3 \mathrm{~T}$ MR imaging: potential to increase diagnostic specificity of mesial temporal sclerosis. Am J Neuroradiol. 2010;31:1682-9.

16. Kimura Y, Shioya A, Saito Y, et al. Radiologic and pathologic features of the transmantle sign in focal cortical dysplasia: the T1 signal is useful for differentiating subtypes. Am J Neuroradiol. 2019;40:1060-6.

17. Sato N, Ota M, Yagishita A, Miki Y, et al. Aberrant midsagittal fiber tracts in patients with hemimegalencephaly. Am J Neuroradiol. 2008;29:823-7.

\section{Suggested Readings}

Gaitanis JN, Donahue J. Focal cortical dysplasia. Pediatr Neurol. 2013;49:79-87.

Guerrini R, Duchowny M, Jayakar P, et al. Diagnostic methods and treatment options for focal cortical dysplasia. Epilepsia. 2015;56:1669-86.

Pohlmann-Eden B, Crocker CE, Matthias H, Schmidt MH. A conceptual framework for the use of neuroimaging to study and predict pharmacoresistance in epilepsy. Epilepsia. 2013;54:75-9.

Ryvlin P, Cross JH, Rheims S. Epilepsy surgery in children and adults. Lancet Neurol. 2014;13:1114-26.

Vattoth S, Manzil FFP, Singhal A, et al. State of the art epilepsy imaging an update. Clin Nucl Med. 2014;39:511-26.

Open Access This chapter is licensed under the terms of the Creative Commons Attribution 4.0 International License (http://creativecommons. org/licenses/by/4.0/), which permits use, sharing, adaptation, distribution and reproduction in any medium or format, as long as you give appropriate credit to the original author(s) and the source, provide a link to the Creative Commons license and indicate if changes were made.

The images or other third party material in this chapter are included in the chapter's Creative Commons license, unless indicated otherwise in a credit line to the material. If material is not included in the chapter's Creative Commons license and your intended use is not permitted by statutory regulation or exceeds the permitted use, you will need to obtain permission directly from the copyright holder. 


\title{
Neuroimaging in Dementia
}

\author{
Meike W. Vernooij and Mark A. van Buchem
}

\section{Learning Objectives}

- Understanding that dementia is a clinical syndrome with a variety of underlying pathologies or subtypes (varying by age of presentation of the patient); and that neuroimaging plays an important role in guiding clinical diagnostic assessment.

- Gaining insight into the distinction between imaging findings in "normal aging" versus those in neurodegeneration.

- Being able to list the most typical imaging findings of the most common subtypes of dementia.

- Acquiring knowledge on the recommended imaging protocol in the memory clinic setting as well as the elements essential for the radiological report.

\subsection{Dementia: A Syndrome, Not a Disease}

Dementia is an acquired syndrome in which a person suffers from progressive impairment in one of the following six cognitive domains: complex attention, executive function, learning and memory, language, perceptual-motor function, and/ or social cognition. These cognitive deficits cannot be attributed to another mental disorder (e.g., depression or schizophrenia) and must be severe enough to interfere with independent daily functioning to be classified as a "major neurocognitive disorder" (major NCD) according to the

M. W. Vernooij $(\bowtie)$

Department of Radiology and Nuclear Medicine, Erasmus MC University Medical Center, Rotterdam, The Netherlands

Department of Epidemiology, Erasmus MC University Medical Center, Rotterdam, The Netherlands

e-mail: m.vernooij@erasmusmc.nl

M. A. van Buchem

Department of Radiology, Leiden University Medical Centre,

Leiden, The Netherlands

e-mail: m.a.van_buchem@lumc.nl
DSM-V 2013 edition [1]. This new choice in terminology in the DSM-V was chosen in order to be able to also define a "mild neurocognitive disorder" (equivalent to mild cognitive impairment (MCI) and/or prodromal dementia), with the key distinction that persons with mild NCD remain independent in daily functioning. This shift to diagnose NCD as early as possible stems from the understanding that these diseases have a long prodromal phase, in which early diagnosis and early treatment may have beneficial effects. Importantly, mild NCD is not always a precursor to major NCD (dementia), and the diagnosis does not require further decline.

The syndrome of dementia has a multitude of underlying causes and the diagnostic work-up is primarily aimed at identifying these and to assign a "subtype." The clinical work-up relies heavily on history taking, clinical and neuropsychological (cognitive) assessment and can be complemented by a number of ancillary tests, of which neuroimaging is one of the most important.

\subsection{Dementia: Population Prevalence and Subtype Proportions}

Due to the aging of populations worldwide, the absolute number of cases of dementia is estimated to double worldwide between 2020 and 2040, to a staggering 81 million over the age of 60 living with dementia in the year 2040, with the highest rise in developing countries [2]. Pooled prevalence data of 11 European population-based studies published in 2000 showed the following estimates of prevalence of dementia in the elderly ( $>65$ years): age-standardized prevalence was $6.4 \%$ for dementia (all causes), $4.4 \%$ for Alzheimer's disease (AD), and $1.6 \%$ for vascular dementia (VaD). Prevalence of dementia was higher in women than in men and nearly doubled with every 5 years increase of age: from $0.8 \%$ in the group age 65-69 years to $28.5 \%$ over the age of 90 years [3].

In the elderly, the most common underlying cause (or subtype) of dementia is Alzheimer's disease (45-54\%), followed by vascular dementia (14-16\%), Lewy body (5\%), 


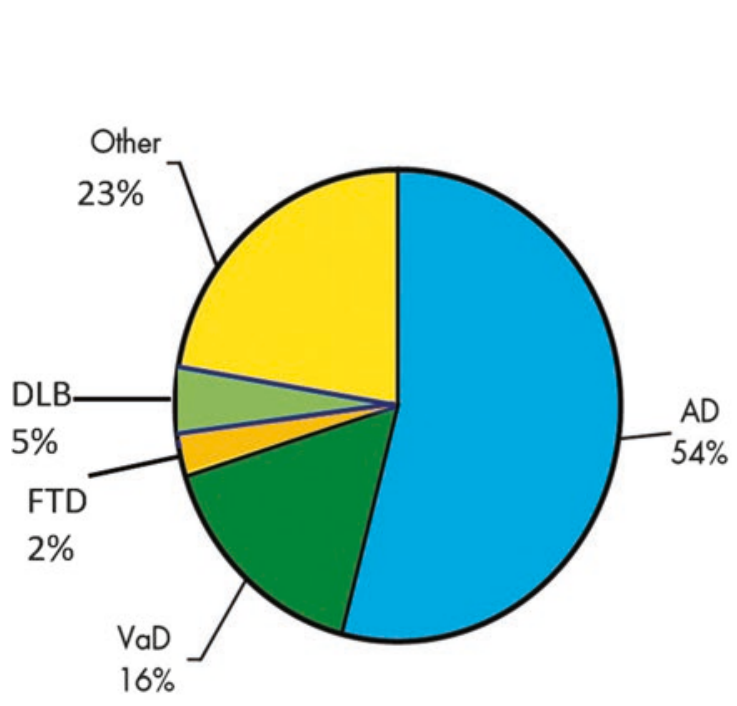

Age $\geq 65$ years

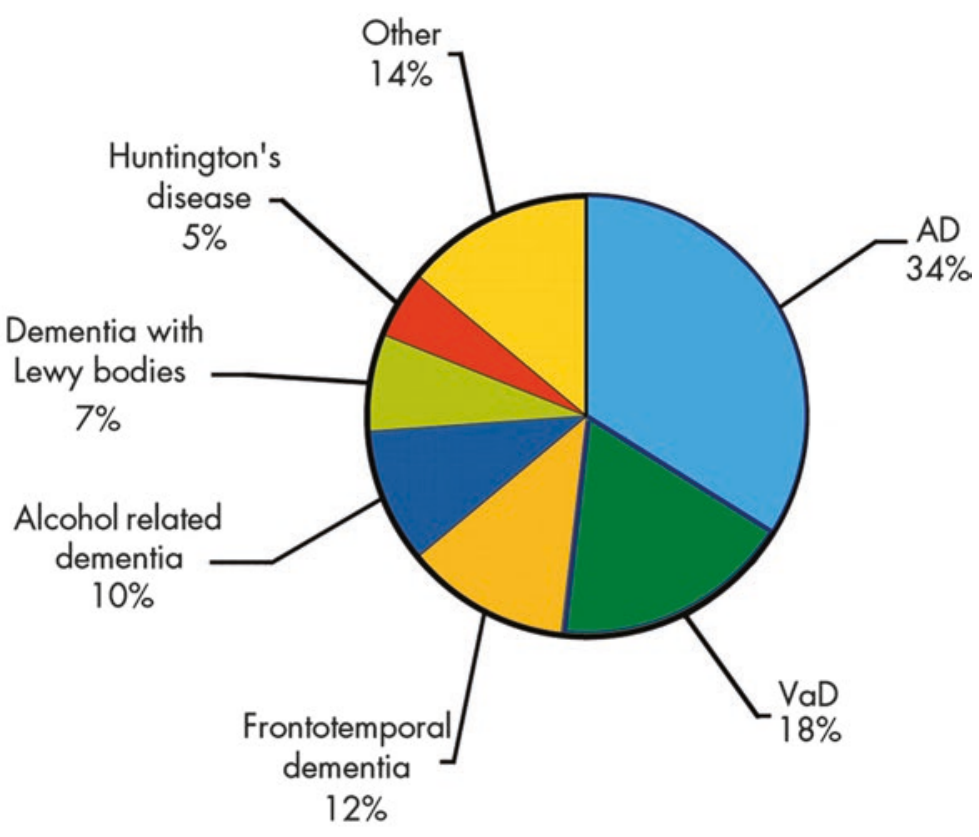

Age $<65$ years

Fig. 11.1 Subtype proportions of dementia according to age of onset. Adapted from van der Flier and Scheltens [5]

frontotemporal dementia (2\%), and alcohol induced dementia $(0.7 \%)[4,5]$ (Fig. 11.1).

It is important to realize that in early onset dementia ( $<65$ years), these subtype proportions differ, with the primary clinical subtypes still being Alzheimer's disease (18-34\%) and vascular dementia (13-18\%), but now with a much higher proportion of frontotemporal dementia (11-12\%) [6, 7]. Also other causes such as alcohol-related dementia (9-18\%), traumatic brain injury, Huntington's disease, and HIV-related dementia are more prevalent in early onset dementia versus late onset (Fig. 11.1). Due to the fact that younger patients ( $<65$ years) more often have alternative diagnoses or a genetic cause of dementia, imaging plays an even more important role in this group to aid clinical diagnosis, in particular since different subtypes bring about different prognostic consequences.

It is crucial to understand that in many cases, especially in the elderly, there are multiple causative factors, of which a combination of Alzheimer pathology and vascular pathology is one of the most prominent ("mixed pathology," not yet indicated in Fig. 11.1 but in late onset dementia responsible for up to $30-40 \%$ of dementias) [8]. This should be recognized in the diagnosis and should be kept in mind when reading imaging exams of memory clinic patients.

Furthermore, the proportion of subtypes differ according to autopsy versus clinical diagnosis [9] indicating that current clinical criteria for subtyping dementia may not adequately capture the true underlying pathology. This is also the reason why in the field of research, biomarkers have become an essential component which have also been incor- porated in new, unbiased descriptive classification schemes that do not specify disease labels. One of these is the "A/T/N" system [10], in which seven major AD biomarkers are used to define three binary categories for presence/absence of these markers (amyloid in PET or CSF ("A"); tau on PET or in CSF ("T") or neurodegeneration on FDG PET, MRI, or CSF total tau ("N")). Each biomarker category is rated as positive or negative. An individual score might, for example, appear as $\mathrm{A}+/ \mathrm{T}+/ \mathrm{N}-$, or $\mathrm{A}+/ \mathrm{T}-/ \mathrm{N}-$, etc. Given the present lack of consensus among $\mathrm{AD}$ specialists on terminology across the clinically normal to dementia spectrum, a biomarker classification scheme will have broadest acceptance if it is independent from any clinically defined diagnostic scheme. Though such a biomarker-based classification will not be implemented in clinical practice in the short term, it is important for radiologists to be aware of the shifting views in the field on traditional disease labels.

\section{Key Points}

- Dementia is a syndrome with a multitude of underlying causes, the proportions of these differ for early onset ( $<65$ years) versus late onset dementia.

- Alzheimer's disease and vascular dementia are at all ages the most common subtypes according to clinical diagnosis, but neuropathology studies show a very high proportion of mixed pathology (AD pathology + vascular pathology), up to $30-40 \%$ in old age. 


\subsection{Dementia: Role of Neuroimaging}

Neuroimaging is one of the most important ancillary tests in the clinical work-up of a patient suspected of dementia. The primary aim of imaging used to be the exclusion of treatable causes of cognitive impairment (such as subdural hematomas, brain tumors, or normal pressure hydrocephalus), but imaging is increasingly helpful in the identification of underlying causes of dementia. Though for the majority of diseases causing dementia, no specific imaging criteria have been formulated in the clinical diagnostic criteria, it is notable that more recent revisions of criteria are increasingly including imaging (for positive as well as negative predictive value) [11].

Though Alzheimer's disease is at any age the most prevalent dementia subtype, the most important differential diagnoses of $\mathrm{AD}$, depending on age of onset, are vascular dementia (VaD), FTD, non-fluent and semantic subtypes of primary progressive aphasia (PPA), dementia with Lewy bodies (DLB), and Creutzfeldt-Jakob disease (CJD). Especially in the early stages of the disease, when symptoms are often unspecific, differential diagnosis may be challenging. Accurate differentiation between the several types of dementia, however, is of especially great relevance in the early stages, when most can be gained in terms of symptom reduction, informing the patient on the prognosis and increased quality of life. Also, early and accurate differentiation is important for inclusion into clinical drug trials and therapy evaluation in a trial setting. Neuroimaging may play an important role in this regard.

\subsection{Dementia Versus Normal Aging}

This section contains text extracts that are reprinted with permission from Vernooij and Barkhof [12].

In the evaluation of brain imaging in a memory clinic setting, knowledge on the background of "normal" brain aging and its associated changes is important but often overlooked. Autopsy studies in brains of aged people without neurological disease invariably show the presence of plaques, tangles, Lewy bodies, and neuronal loss. These are pathological findings that are also considered hallmarks of neurodegenerative diseases, but are more abundantly present and regionally clustered in those diseases. This observation that almost all aged brains show characteristic changes that are strongly linked to neurodegeneration has led to the hypothesis that normal brain aging and neurodegenerative diseases form a continuum, and that pathological neurodegeneration perhaps is an expression of accelerated aging. Though this is likely a too simplified view, it is a fact that there is considerable overlap between patterns of brain pathology in aging and neurodegenerative disease and that knowledge on changes in brain aging is indispensable as a background against which to evaluate neurodegenerative disease.

The most important imaging findings in aging which overlap with those found in neurodegenerative diseases are brain atrophy and imaging markers of small vessel disease. Below we describe shortly those findings in the age-related setting, and when these can still be considered normal for aging or when they can provide an explanation for dementia.

\subsubsection{Brain Atrophy in Aging}

Regarding brain atrophy, there is consistent evidence that brain volume declines with aging, and that age-related volume loss appears as early as the age of $30(0.2 \%$ loss of total brain volume per year), and accelerates after the age of 70 up to $0.5 \%$ total brain volume loss per year (5\% per decade) in cognitively healthy elderly [13]. At the age of 75 , this roughly equates to a $10 \%$ brain volume loss, which is readily appreciable to the naked eye (Fig. 11.2).

The most important rules of thumb to distinguish atrophy in aging from neurodegeneration are that age-related brain atrophy is generally symmetric and generalized, whereas in neurodegeneration we see specific lobar patterns (further described below) and can see asymmetries. Semi-quantitative visual rating scales such as the global cortical atrophy scale (GCA) and medial temporal atrophy scale (MTA) (Tables 11.1 and 11.2, Figs. 11.3 and 11.4) are be helpful to distinguish "normal" from "abnormal" based on a visual assessment. Though these scales are quite crude and not sensitive to subtle or longitudinal changes, they have consistently been shown to correlate well with pathology data and with volumetric assessments and are easy to use in everyday clinical practice.

Yet, without absolute quantification it is very hard to assess what "normal" or even "expected" is for a specific age and sex, in particular when assessing atrophy on a regional or substructure level. Advances are being made in image analysis algorithms to perform fully automated tissue and structure quantification on individual level scans and compare these to normative databases, in order to aid everyday radiology assessments (Fig. 11.2).

On visual rating, asymmetry and focal brain atrophy should be considered abnormal, as is a GCA score of 3 ("knife-blading") in all persons or a score of 2 (clear gyral shrinking) in persons aged $<75$ years.

In individuals until age 75 years, the cutoff for abnormal MTA lies at an average of $\geq 1.5$, i.e., having a score of 2 (or higher) on one side is considered abnormal. In those over age 75 years, having an MTA score of 2 (or higher) on both sides (i.e., an average of $\geq 2.0$ ) is considered abnormal [17]. 


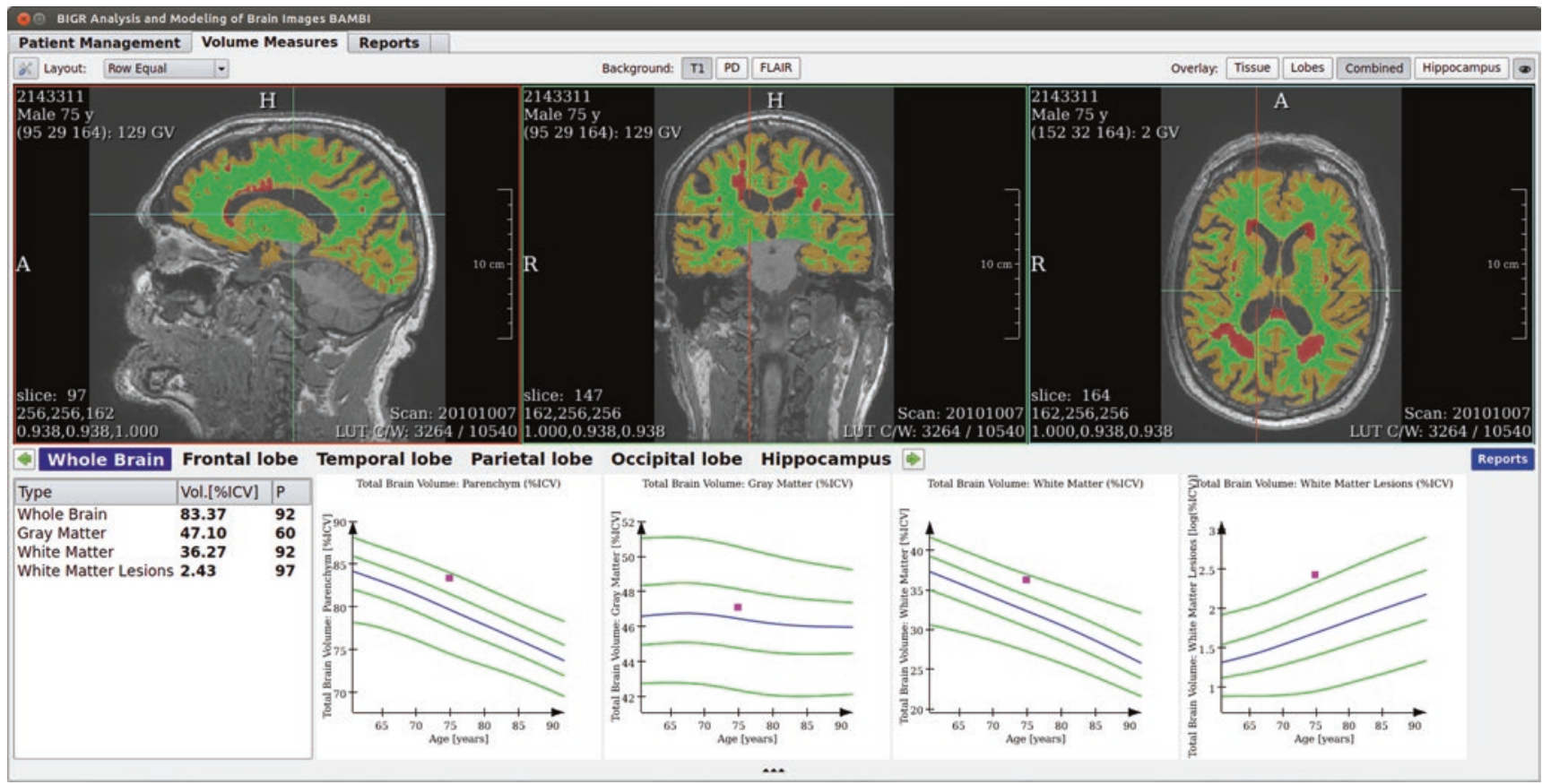

Fig. 11.2 Automated volumetry in the memory clinic. Screenshot example showing brain tissue volumetric results and application of reference curves to assess individual brain atrophy. (Reprinted with permission from Vernooij and Barkhof [12])

Table 11.1 Visual rating scale of global cortical atrophy (GCA)

\begin{tabular}{|c|c|c|c|}
\hline \multicolumn{2}{|c|}{ Degree of atrophy } & Gyri & Sulci \\
\hline $\mathrm{GCA}=0$ & No & Normal volume & $\begin{array}{l}\text { Normal } \\
\text { width }\end{array}$ \\
\hline $\mathrm{GCA}=1$ & $\begin{array}{l}\text { Mild (may be } \\
\text { considered normal in } \\
\text { the elderly) }\end{array}$ & Normal & $\begin{array}{l}\text { Some } \\
\text { opening of } \\
\text { sulci }\end{array}$ \\
\hline $\mathrm{GCA}=2$ & Moderate & Reduced & Enlarged \\
\hline $\mathrm{GCA}=3$ & Severe & $\begin{array}{l}\text { Severely reduced } \\
\text { ("knife blade") }\end{array}$ & $\begin{array}{l}\text { Severely } \\
\text { enlarged }\end{array}$ \\
\hline
\end{tabular}

Data from Pasquier et al. [14]
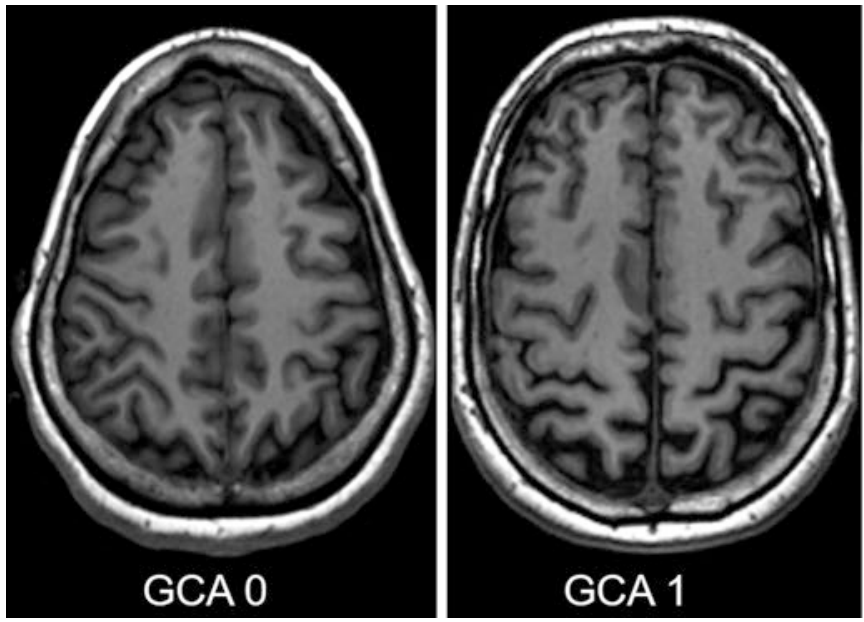

Fig. 11.3 Global cortical atrophy scale. Illustration showing the different categories on the GCA scale (see also Table 11.1): no atrophy in the parietal lobe (GCA 0), some opening of sulci (GCA 1), reduced volume
Table 11.2 Visual rating scale of medial temporal lobe atrophy (MTA)

\begin{tabular}{|l|l|l|l|} 
& Choroid fissure & Temporal horn & $\begin{array}{l}\text { Hippocampal } \\
\text { height }\end{array}$ \\
\hline MTA $=0$ & Normal & Normal & Normal \\
\hline MTA $=1$ & Widened & Normal & $\begin{array}{l}\text { Normal } \\
\text { Reduced }\end{array}$ \\
\hline MTA $=3$ & $\begin{array}{l}\text { Soderately } \\
\text { widened }\end{array}$ & Widened & $\begin{array}{l}\text { Moderately } \\
\text { reduced }\end{array}$ \\
\hline
\end{tabular}

MTA = 4 Severely widened Severely widened Severely reduced Data from Scheltens et al. [15]
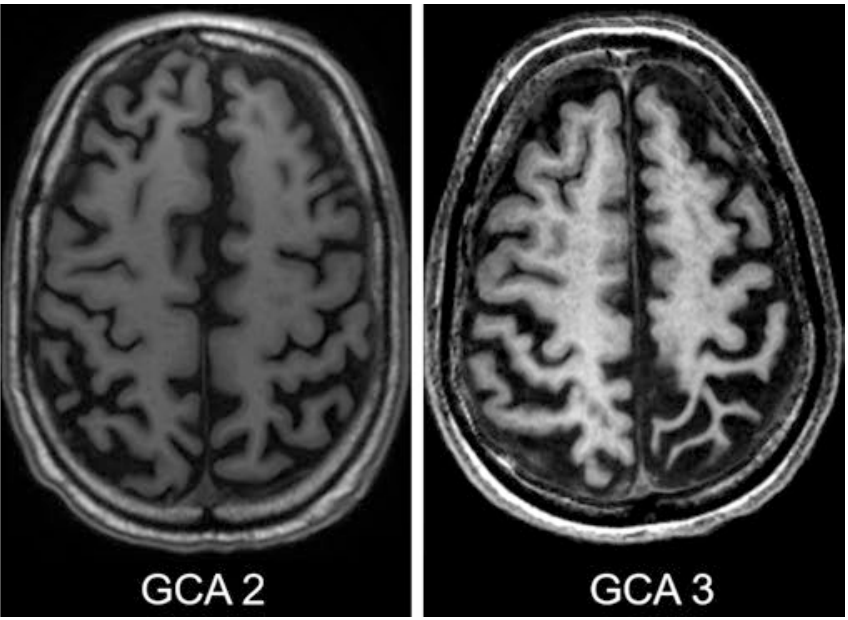

of gyri and further widening of sulci (GCA 2), and severe volume reduction (knife-blading) in the left parietal lobe (GCA 3) 


\subsubsection{Vascular pathology in aging}

Vascular pathology commonly seen in aging and which may overlap with vascular pathology in mild and major NCD, are white matter hyperintensities $(\mathrm{MH})$, silent brain infarcts (usually lacunes), and cerebral microbleeds.

Studies in community-dwelling subjects have shown that after the age of 45 years, only $5-10 \%$ of persons are completely free of $\mathrm{WMH}$, and that their presence and volume increase steeply with age. Large observational studies have provided compelling evidence of links of WMH with risk of stroke, dementia, cognitive dysfunction (in particular executive function and information processing speed), gait disturbance, and falls as well as depression. All of these observations have led to WMH being considered an expression of cerebral small vessel disease and not an innocuous finding, especially when larger in volume. In everyday clinical practice, standardized visual rating scales such as the Fazekas scale as described in Fig. 11.5 can be applied. Due to ease of use, this scale shows very high intra- and interrater reliability. In general, a Fazekas score of 1 can be con- sidered normal, whereas scores 2 and 3 indicate the presence of small vessel disease. A score of 3 is abnormal at any age, and found both in frontal and parietal lobes, is indicative of a large $\%(>25 \%)$ of white matter being pathologic and contributes to the clinical criteria for vascular dementia.

Silent brain infarctions are highly frequent in asymptomatic aging individuals, with prevalence estimates ranging from $8 \%$ to as high as $28 \%$ and increase with age [18]. Most of these infarcts are lacunes in the basal ganglia or deep white matter, presumed to be caused by underlying hypertensive small-vessel disease or small emboli. Although by definition silent infarcts go without overt clinical symptoms, there is compelling evidence that they are associated with subtle deficits in physical and cognitive function [18]. Moreover, the presence of silent infarcts, both lacunes and microinfarcts, more than doubles the risk of stroke and dementia, especially when multiple lesions are present.

Microbleeds, visible as hypointense focal lesions on T2*weighted sequences susceptible to the paramagnetic properties of blood-break down products, are thought to represent small chronic hemorrhages caused by damaged small vessels.
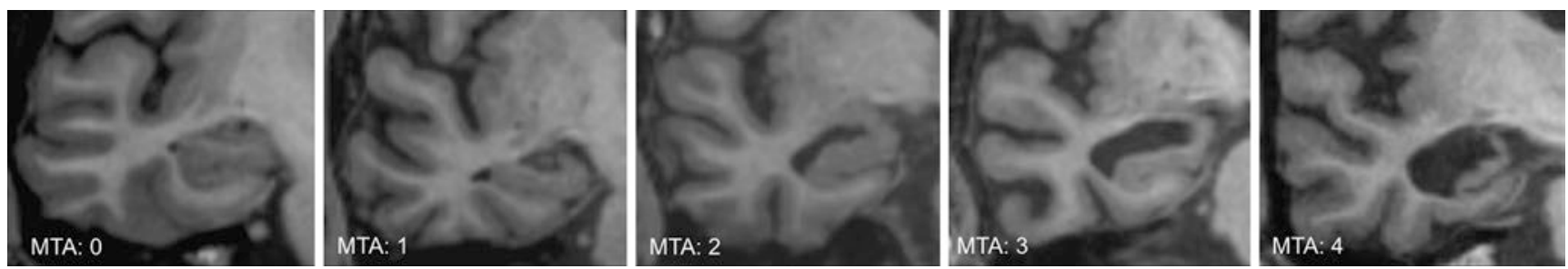

Fig. 11.4 Medial temporal atrophy scale. Coronal T1-weighted images of the hippocampus for stages $0-4$ of the MTA rating scale (see Table 11.2). Note progressive widening of the choroid fissure (from stage 1 onwards) and temporal horn (from stage 2 onwards), as well as hippocampal body volume loss (stages 3 and 4). (Reprinted with permission from Vernooij and Smits [16]. Copyright Elsevier (2012))
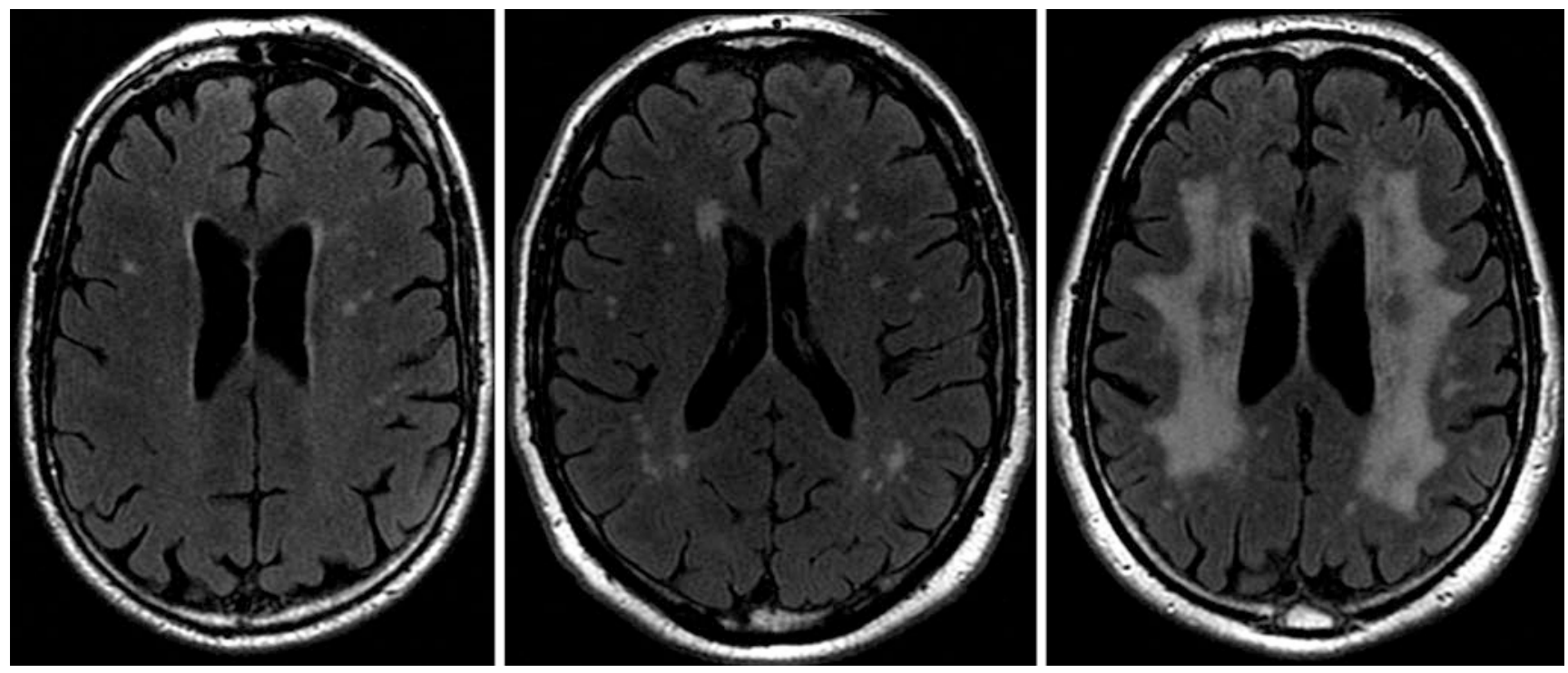

Fig. 11.5 Fazekas scale for white matter lesions. From left to right, Fazekas scale 1 (punctiform lesions), 2 (early confluent lesions), and 3 (confluent lesions) (not shown: score $0=$ no lesions). (Reprinted with permission from Vernooij and Smits [16]. Copyright Elsevier (2012)) 
Population cohorts in healthy elderly describe prevalence estimates ranging from 5 to $30 \%$, with increasing prevalence with age, but also with magnetic field strength, hindering easy comparison between populations or pooling of results. The topographic distribution of microbleeds is thought to reflect the type of underlying vasculopathy. Lobar microbleeds, especially those that are multiple and strictly lobar (cortico-subcortical junction of cerebral lobes) in location, are presumed to be caused by amyloid angiopathy, whereas deep microbleeds (basal ganglia, thalamus, brainstem, cerebellum) reflect hypertensive arteriopathy. Generally, a single microbleed in any brain location can be considered to be not of clinical relevance. However, having multiple (two or more) microbleeds in strictly lobar brain locations in a person aged over 55 years without other causes for microbleeds (e.g., previous radiotherapy, head trauma) classifies as "probable CAA" according to the Boston criteria. Due to the frequent co-occurrence of CAA and $\mathrm{AD}$ pathology, multiple lobar microbleeds can often be observed in patients fulfilling the clinical criteria of AD. The clinical diagnosis of CAA is further supported if superficial siderosis is also present (see Fig. 11.6). Similarly, having multiple microbleeds in deep brain locations or in the brainstem should trigger the presence of hypertensive arteriolosclerosis. Cerebellar microbleeds can occur both in the context of CAA or hypertension and thus are not contributing meaningfully to differential diagnosis.

\section{Key Points}

- The aging brain shows characteristic changes that are strongly linked to neurodegeneration.

- On visual rating, asymmetry and focal brain atrophy should be considered abnormal, as is a GCA score of 3 ("knife-blading") in all persons or a score of 2 (clear gyral shrinking) in persons aged $<75$ years.
- In individuals up to 75 years, an MTA score of 2 or higher on one side is abnormal, whereas in persons over the age of 75, a score of 2 or higher on both sides is considered abnormal.

- A Fazekas score of 2 or 3 is indicative of underlying small vessel disease.

- Silent brain infarcts, even though very frequent in aging subjects, more than double the risk of stroke and dementia and should be considered as indicative of small vessel disease.

- Microbleeds in lobar, deep, or cerebellar locations occur frequently in old age (up to $30 \%$ ). Generally, a single microbleed is considered not relevant, whereas the presence of multiple microbleeds raises suspicion of underlying cerebral amyloid angiopathy (when strictly lobar in location) or hypertensive arteriopathy (when deep or in brainstem).

\subsection{Imaging Characteristics of Most Common Subtypes of Dementia}

This section contains text extracts from Vernooij and Smits [16]. Copyright Elsevier (2012); reprinted with permission.

\subsubsection{Typical Imaging Presentation of Alzheimer's Disease}

The radiological hallmark finding of AD is cortical atrophy due to neuronal degeneration and loss. Atrophy is diffuse, but more prominent in the temporal and parietal lobes with the hippocampus most severely, and disproportionately
Fig. 11.6 Superficial siderosis and lobar microbleeds. T $2 *$ gradientecho images (or susceptibility-weighted images) showing linear hypointensity on the cortical surface (left), compatible with superficial siderosis, and multiple focal hypointensities in the brain parenchyma in a lobar location (right), reflecting cerebral microbleeds
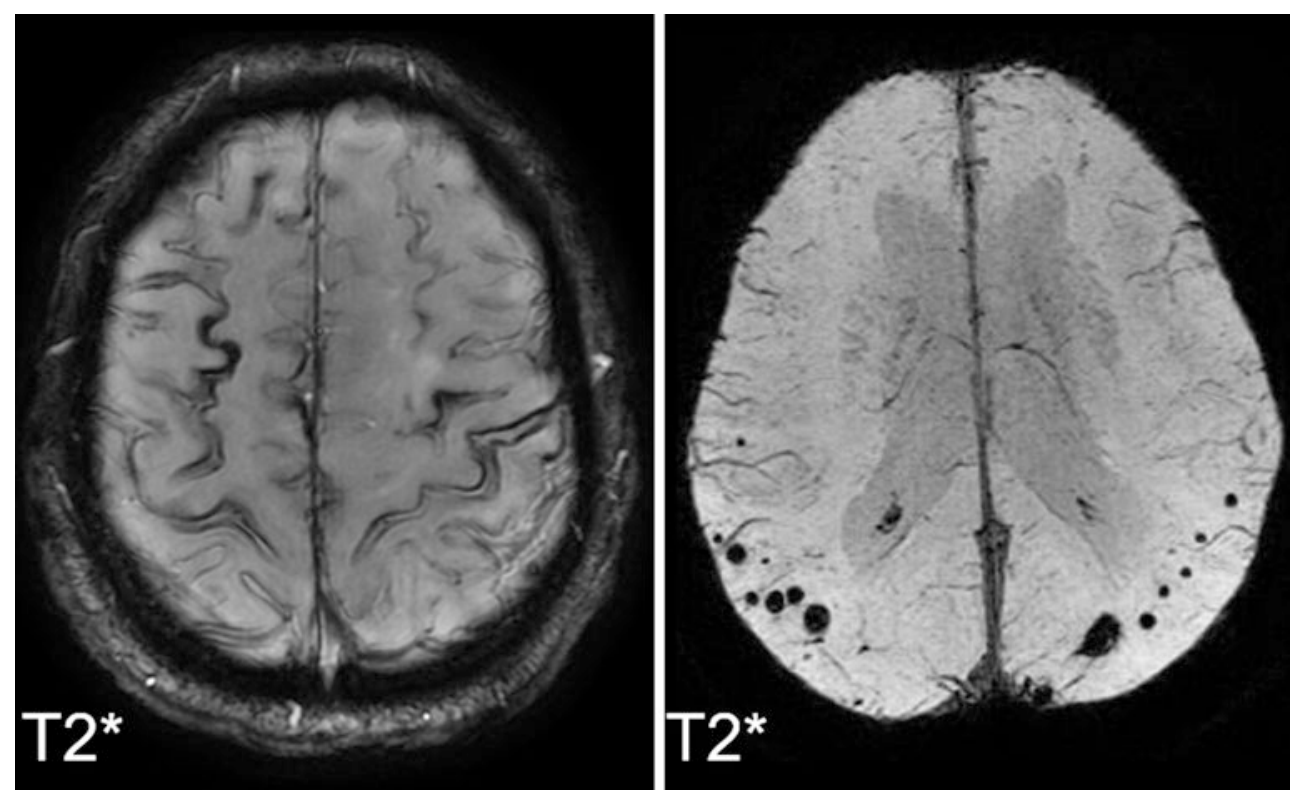
affected (Fig. 11.7). The primary motor and sensory cortices are relatively spared until late in the disease. Findings are bilateral and generally symmetrical, but a certain degree of asymmetry may occur.

Sensitivity and specificity to use MTA in distinguishing patients with $\mathrm{AD}$ from healthy controls are in the range of 85 and $88 \%$, respectively. Note that hippocampal atrophy is also common in other types of dementia, such as dementia with Lewy bodies, frontotemporal dementia, and the Heidenhain variant of Creutzfeldt-Jakob disease. Consequently, diagnostic accuracy to distinguish AD from other dementia is considerably lower than from healthy controls.

Asymmetry of the hippocampus does not exclude AD, although marked asymmetry suggests alternative diagnosis, most notably frontotemporal dementia (FTD, see further).

Fig. 11.7 Typical atrophy pattern in AD. Seventy-yearold female with clinical dementia. Coronal T1-weighted images show bilateral hippocampal atrophy (MTA 3 both sides, panels 1 image shows bilateral symmetric cortical atrophy, most prominent in parietal lobes. This pattern is typical for atrophy in (late onset) Alzheimer's disease and 2) and axial T1-weighted
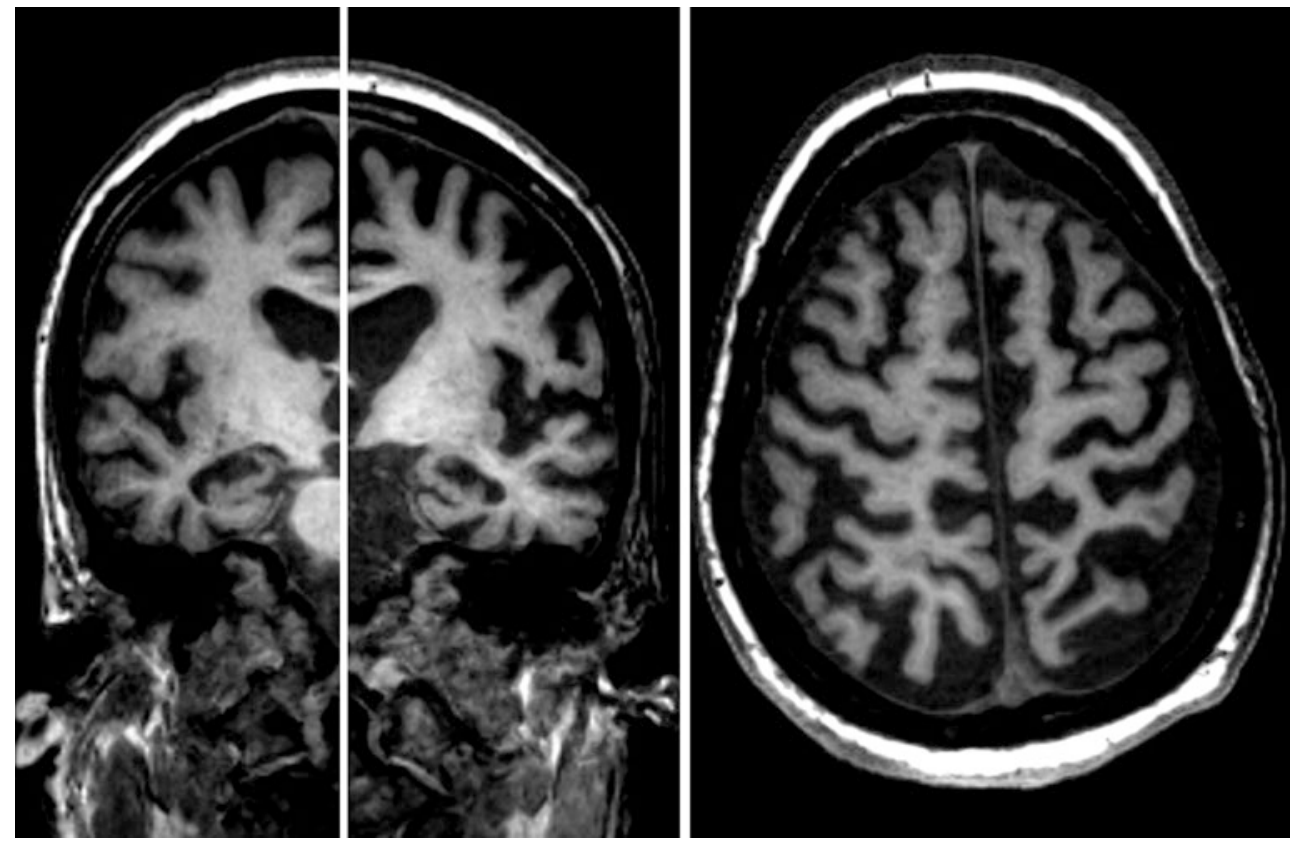

Fig. 11.8 Posterior cortical atrophy (precuneus atrophy) in atypical AD. Fifty-six-yearold female with clinical dementia. Sagittal T1-weighted image shows precuneus atrophy (and widening of the parietooccipital sulcus), whereas there is a normal hippocampal volume on coronal

T1-weighted image (MTA 1). This pattern is typical for posterior cortical atrophy in (early onset) Alzheimer's disease
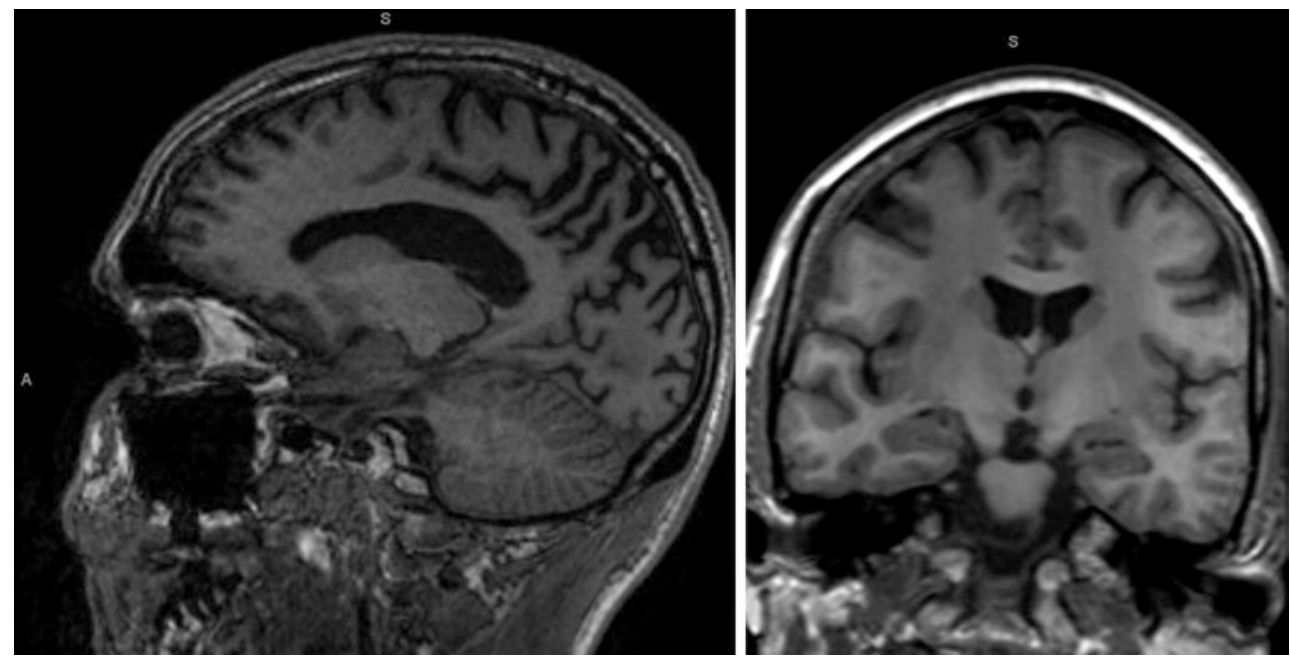
less prominent memory loss and show associated atrophy of the parieto-occipital and posterior temporal cortices. Atrophy is generally asymmetrical, the right hemisphere being more affected than the left. Though the underlying pathological substrate may differ, Alzheimer pathology was found to be most prevalent in these patients.

Logopenic progressive aphasia has been described as a clinically, neuropathologically, and radiologically distinct subtype of primary progressive aphasia (PPA, see more information in Sect. 11.5.4). The syndrome is characterized by language disorders rather than memory impairment, which consists of slow speech and deficits in sentence repetition. On brain imaging there is marked asymmetrical atrophy of the posterior temporal cortex, including the posterior superior and middle temporal gyri, and the inferior parietal lobule, with the left hemisphere more affected than right. Clinically, LPA is difficult to distinguish from the other PPA subtypes, which have predominantly underlying FTD pathology. Structural brain imaging plays an important role in differential diagnosis.

\subsubsection{Frontotemporal Dementia (FTD)}

FTD is in particular in patients under the age of 65 years a frequent occurring cause of dementia, occurring in up to $12-15 \%$ of early onset dementia. While also characterized by neuronal loss, FTD is histopathologically distinct from AD due to the presence of tau-positive or ubiquitin-positive inclusions. The three main variants are the behavioral or frontal variant (bvFTD; 40\%), progressive non-fluent aphasia (PNFA; 20\%), and semantic dementia (SD; 40\%). The latter two are clinically characterized by a predominance of language impairment and considered subtypes of primary progressive aphasia, which is discussed separately below (Fig. 11.9).

Brain imaging in bv-FTD shows frontal and temporal cortical atrophy with an anterior to posterior gradient, which is bilateral and may be asymmetrical. There may be associated white matter hyperintensities on T2-weighted/T2-FLAIR imaging. Atrophy of the frontal lobe, especially if there is concurrent atrophy of the caudate head, results in disproportionate widening of the frontal horns of the lateral ventricles. In the early stages widening of the sulci in the orbitofrontal region may be seen as an early manifestation of orbital frontal atrophy. Compared with AD, medial temporal lobe atrophy in FTD is more pronounced anteriorly and more asymmetrical.

\subsubsection{Primary Progressive Aphasia}

PPA is a slowly progressive impairment of language, with three subtypes: logopenic progressive aphasia (LPA) (commonly AD pathology, see Sect. 11.5.2), PNFA, and SD (the latter two mainly FTD pathology).

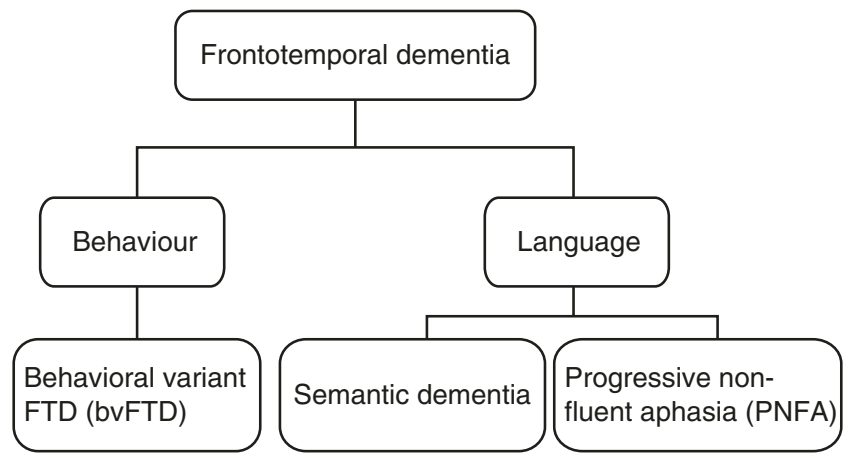

Fig. 11.9 Clinical subtypes of frontotemporal dementia

Patients with PNFA have slow, effortful speech with errors in the production of speech sounds and grammatical deficits. On brain imaging, atrophy is seen in the anterior perisylvian region, involving the inferior, opercular, and insular regions, with a left-hemispheric predominance.

SD is clinically characterized by impairment of semantic performance. Brain imaging shows atrophy of the ventral and lateral regions of the anterior temporal lobes, including the anterior hippocampus and the amygdala. Again, atrophy is often asymmetrical with the left hemisphere more affected than the right, and there is an anterior to posterior gradient.

\subsubsection{Vascular Dementia}

In the elderly, cerebrovascular disease is the second most common pathology underlying dementia after $\mathrm{AD}$. VaD is a heterogeneous entity, including both large and small vessel disease, and involving the gray and/or white matter. By far the most common underlying pathology is small vessel disease, leading to diffuse confluent white matter changes and multiple lacunar infarcts of the deep white and (notably the thalamic) gray matter. Typically, the subcortical U-fibers are spared. When infarction of the deep perforating arteries is complete, lacunar infarcts occur, which are visualized on MRI as small lesions with signal intensity of CSF on all sequences, surrounded by a rim of T2-weighted hyperintensity. A multilacunar state is also known as "état lacunaire," not to be confused with "état criblé," which is the term used for multiple enlarged perivascular spaces in the basal ganglia. Both states are considered pathological and manifestations of small vessel disease. Large vessel disease manifests as multiple (strategic infarcts.

Since cerebrovascular changes are common in healthy elderly, as outlined earlier in this chapter, there are strict criteria to diagnose $\mathrm{VaD}$ set out by the National Institute of Neurological Disorders and Stroke and Association Internationale pour la Recherché et l'Enseignement en Neurosciences (NINDS-AIREN) [19]. A patient must meet the criteria of a dementia syndrome, have evidence of cere- 
brovascular disease on clinical examination and on imaging, and there has to be a temporal relationship between the onset of dementia and cerebrovascular disease.

Radiological criteria are crucial to diagnosis and specified in detail:

- Confluent white matter lesions need to involve at least $25 \%$ of the total white matter to reach the diagnosis of $\mathrm{VaD}$.

- Lacunar infarcts need to involve multiple basal ganglia and the frontal white matter, and thalamic lesions need to be bilateral.

- Strategic_large vessel-infarcts meet the criteria when they involve the following territories: bilateral anterior cerebral artery, paramedian thalamic, inferior medial temporal lobe, parieto-temporal and temporo-occipital association areas and angular gyrus, superior frontal and parietal watershed areas in the dominant hemisphere.

There seems to be a complex interrelationship between AD and cerebrovascular disease that extends beyond the coexistence of these two disease processes. Cerebrovascular disease and $\mathrm{AD}$ often coexist, while stroke often exacerbates pre-existing, sometimes previously subclinical, pathology. Furthermore, $\mathrm{AD}$ and $\mathrm{VaD}$ share common risk factors, such as diabetes and hypertension, as well as genetic factors for brain tissue vulnerability (presenilins, amyloid precursor protein, APOE genes). Microbleeds, considered to be a manifestation of cerebral amyloid angiopathy (CAA), are seen at high frequency in patients with $\mathrm{AD}$. CAA is a microangiopathy with $\beta$-amyloid deposition in the vessel walls. On brain imaging, the presence of two or more microbleeds in a lobar distribution is suggestive of CAA, while other features include subpial siderosis and evidence of past lobar hemorrhage. Although sporadic CAA is commonly seen in the elderly, being the leading cause of lobar hemorrhage, in its severe form it is also recognized as a risk factor for dementia. This typically constitutes a subcortical vascular dementia, but severe CAA is also considered a feature of $\mathrm{AD}$, sharing the APOE $\varepsilon 4$ allele as a common risk factor.

\subsubsection{Dementia with Lewy Bodies (DLB)}

DLB is clinically distinguished from AD by the presence of visually hallucinations, which occur in $80 \%$ of cases. Visual hallucinations may, however, also occur in AD. On brain MRI, there is medial temporal lobe and global cortical atrophy with relative sparing of the primary motor and sensory cortex, as is seen in AD. Atrophy, however, is less pronounced than in $\mathrm{AD}$ when the severity of disease is taken into account. In the early stages of the disease, medial temporal lobe volume may be preserved. Similar to AD, hyperintensity of the white matter on T2-weighted images is seen at higher frequency in DLB than in healthy controls.

\subsubsection{Creutzfeldt-Jakob Disease}

Sporadic CJD, accounting for $85-90 \%$ of cases, generally presents as a subacute dementia syndrome between the ages of 50 and 70 years. Rapid progression and concomitant focal neurological deficits and myoclonus will clinically distinguish CJD from AD during the course of the disease, but initially cognitive symptoms may be similar. Brain MRI typically shows marked hyperintensity on T2-weighted sequences bilaterally in the caudate nucleus and the putamen, and to a lesser extent in the thalamus and neocortex. Diffusion weighted imaging, showing restricted diffusion in the affected gray matter, seems to be the most sensitive sequence to detect CJD-related abnormalities (Table 11.3).

\section{Key Point}

- Structural neuroimaging can help identify underlying causes of dementia through the identification of patterns of atrophy (including symmetry and lobar patterns) as well as presence and distribution of vascular lesions.

Table 11.3 Summary of structural MR imaging findings for the most common dementia subtypes

Typical Alzheimer's disease

- Disproportionate hippocampal atrophy with temporoparietal atrophy

- Relative sparing of the primary motor and sensory cortex

- Bilateral, more or less symmetrical

- Microbleeds in a lobar, subcortical distribution

Atypical Alzheimer's disease

- No or little hippocampal atrophy

- Focal cortical atrophy

- Precuneus: early onset AD

- Parieto-occipital and posterior temporal lobe: PCA

- Posterior temporal cortex and the inferior parietal lobule: LPA

Frontotemporal dementia

- Bilateral frontal and temporal atrophy with anterior to posterior gradient

- Left more than right anterior perisylvian region: PNFA

- Left more than right ventrolateral temporal region: SD

- (Asymmetrical) hippocampal atrophy, more pronounced anteriorly

- Disproportionate widening of the frontal horns: Bv-FTD

- Relative sparing of the parietal and occipital lobes

Creutzfeldt-Jakob disease

- T2-hyperintensity and diffusion restriction bilaterally in the caudate nucleus and putamen, and to a lesser extent in the thalamus and neocortex

Dementia with Lewy bodies

- Medial temporal lobe and global cortical atrophy

- Relative sparing of the primary motor and sensory cortex

- Less extensive than $\mathrm{AD}$ when accounting for disease severity

From Vernooij and Smits [16]; reprinted with permission 


\subsection{Other Dementia Causes}

\subsubsection{Normal Pressure Hydrocephalus (NPH)}

NPH is still a poorly understood disease which seems to result from a disbalance in CSF production and resorption, though an alternative hypothesis is that periventricular white matter damage is a causative factor. The clinical picture is typically characterized by a triad of gait apraxia, dementia, and urinary incontinence; yet, this full trias is only present in less than $33 \%$ of patients. NPH is uncommon under the age of 60 years, and increases in frequency thereafter. As the name suggests, CSF pressure on lumbar puncture is in the normal range. Regardless of underlying etiology, in a number of patients with NPH the clinical symptoms improve with CSF shunting, making this a potentially treatable cause of dementia.

The imaging diagnosis of NPH is challenging. Most striking is the widening of the central CSF spaces (in particular the lateral and third ventricle), with a marked discrepancy between ventricular width and sulcal width. In other words, the ventricular widening cannot be explained by the amount of brain atrophy. A disproportionate widening of the Sylvian fissure can be seen in comparison to the other sulci which appear rather small (Fig. 11.10). Also, some sulci may focally dilate over the convexity or medial surface ("trans-

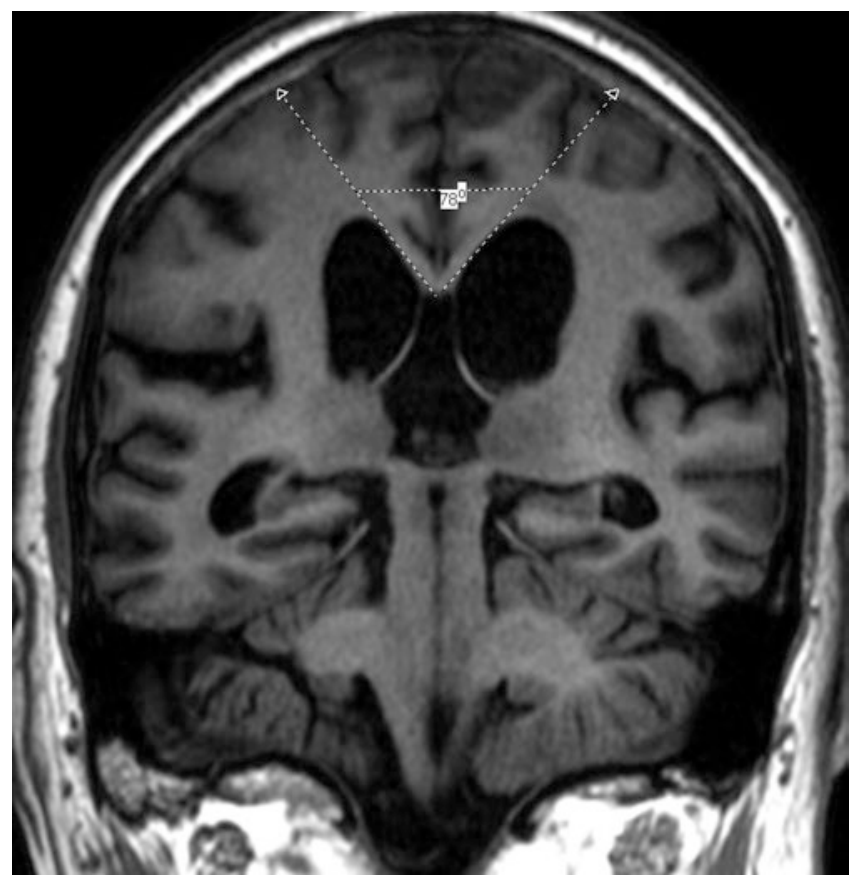

Fig. 11.10 Normal pressure hydrocephalus. Seventy-year-old male with cognitive problems and gait imbalance. MRI shows widening of central CSF spaces, a disproportionate widening of Sylvian fissures relative to other sulci, and crowding of gyri at the vertex/parafalcine region. Corpus callosum angle, measured at the level of the posterior commissure, is $78^{\circ}\left(\right.$ normal $\left.>100^{\circ}\right)$ port sulci"). As the ventricles enlarge, shearing forces are exerted on the white matter, causing in particular the medial lying fibers of the corticospinal tract to be damaged, hence leading to the gait disturbance. This is on imaging exemplified by sharpening of the callosal angle (normal 100-120 $0^{\circ}$, in NPH $50-80^{\circ}$ ) and upward bowing of the corpus callosum, leading to tight high convexity sulci at the vertex and the parafalcine region.

\subsubsection{Corticobasal Syndrome (CBS)}

Corticobasal syndrome is characterized by asymmetric involuntary movements (rigidity, tremor, myoclonus) and apraxia and alien limb phenomena. Additionally, many patients show cognitive deficits or dementia. CBS has several distinct histopathologies, including corticobasal degeneration (tauopathy, related to FTD), and Alzheimer's disease.

Progressive supranuclear palsy, and DLB. While there are no specific imaging findings, CBS with underlying AD pathology usually shows posterior temporal and inferior parietal atrophy, whereas focal atrophy in the premotor cortex or supplemental motor area (often asymmetric) may suggest CBD [20].

\subsubsection{Overlap Between Dementia and Movement Disorders}

There is an overlap between dementia and movement disorders. Parkinson's disease, as the most common neurodegenerative movement disorder, has a cumulative prevalence of dementia of 75-90\% (PD-dementia or PDD). Cognitive dysfunction or dementia is also common in several other movement disorders such as atypical parkinsonism: PSP, DLB, and CBS (see Sect. 11.6.2). Among hyperkinetic disorders, Huntington's disease, spinocerebellar ataxia, and Wilson's disease are best known for their cognitive correlates. Imaging findings are usually dominated by the typical pattern of imaging findings associated with the movement disorder. In some patients cognitive decline may be the dominant clinical manifestation, notably during early stages of the disease, putting a larger emphasis on neuroimaging to aid in the clinical diagnosis.

\subsection{Recommended MR Imaging Protocol in Patients Suspected for Dementia}

The description of imaging findings for the various subtypes above means that the first aims in the imaging evaluation of a patient suspected for dementia should be: 
Table 11.4 Recommended MR imaging protocol for dementia

\begin{tabular}{|c|c|c|}
\hline & Sequence & Findings \\
\hline $\begin{array}{l}\text { 3D } \\
\text { (isotropic) }\end{array}$ & T1-weighted & $\begin{array}{l}\text { Assessment of atrophy (GCA, } \\
\text { MTA) } \\
\text { Coronal plane: hippocampal } \\
\text { atrophy. } \\
\text { Sagittal plane: precuneus atrophy }\end{array}$ \\
\hline $2 \mathrm{D} / 3 \mathrm{D}$ & T2-FLAIR & $\begin{array}{l}\text { Atrophy, white and gray matter } \\
\text { signal abnormalities } \\
\text { Coronal plane: hippocampal signal } \\
\text { abnormalities } \\
\text { WMH and lacunes }\end{array}$ \\
\hline $\begin{array}{l}2 \mathrm{D} \\
\text { transverse }\end{array}$ & $\begin{array}{l}\text { TSE/FSE } \\
\text { T2-weighted }\end{array}$ & $\begin{array}{l}\text { Lesions in thalamus and posterior } \\
\text { fossa }\end{array}$ \\
\hline $\begin{array}{l}2 \mathrm{D} \\
\text { transverse }\end{array}$ & DWI & $\begin{array}{l}\text { Diffusion restriction: recent } \\
\text { ischemia; CJD }\end{array}$ \\
\hline $\begin{array}{l}2 \mathrm{D} \\
\text { transverse }\end{array}$ & T2*-weighted & Microbleeds, superficial siderosis \\
\hline
\end{tabular}

(a) To assess the extent and pattern of brain atrophy, in particular lobar cortical atrophy (with GCA) and medial temporal lobe atrophy (MTA; for evidence of Alzheimer's pathology);

(b) To determine the degree of vascular damage, including the occurrence of strategic vascular lesions.

We recommend that the MRI scanning protocol includes T1-weighted, T2-weighted, T2-FLAIR, diffusion weighted, and T2*-weighted sequences (Table 11.4). Routine application of contrast is not indicated, although it may be needed in atypical cases when granulomatous disease, vasculitis, and infection are considered as differential diagnoses. 3D T1-weighted images should be reconstructed in a coronal plane perpendicular to the long axis of the hippocampus for adequate assessment of medial temporal atrophy.

\subsection{Reporting Findings in Dementia}

Reporting MRI findings in dementia includes both those pointing towards the pathology underlying the dementia syndrome and those suggesting an alternative cause of neurocognitive degeneration. The latter would include focal abnormalities such as brain tumor or subdural hematoma. The hippocampus needs to be scrutinized for signal abnormality on T2-weighted sequences to diagnose ischemia or sclerosis. Areas of diffusion restriction may indicate pathologies such as acute ischemia, herpes simplex encephalitis, alcoholic encephalopathy, or CJD. T2-weighted hyperintensity in certain brain regions may point to a specific diagnosis, such as in the periaqueductal gray or mammillary bodies in case of alcoholic encephalopathy.

The radiological report further needs to include a structured and standardized assessment of the global cortical atrophy, the medial temporal lobe and white matter lesion load, according to, for instance, the GCA, MTA, and Fazekas visual rating scales, respectively (see Tables 11.1 and 11.2, Figs. 11.3, 11.4, and 11.5). Atrophy should always be rated on the same imaging sequence, preferably the FLAIR or T1-weighted sequence. The degree of asymmetry, as well as focal regions of atrophy should be reported separately. Both the number and distribution of microbleeds need to be reported. Large and small vessel cerebrovascular changes need to be specified according to the topographic and severity operational NINDS-AIREN criteria to enable diagnosis of $\mathrm{VaD}$.

\subsection{Concluding Remarks}

The dementia syndrome encompasses a wide variety of underlying causal pathologies, for many of which neuroimaging can give additional information in the diagnostic workup. It is important for (neuro)radiologists to read imaging exams from memory clinic patients against a background of normal aging brain pathology. Patterns of atrophy and vascular lesions can direct towards specific underlying diagnoses. Standardized image acquisition and structured reporting (including the use of semi-quantitative visual rating scales) will increase diagnostic yield and facilitate communication with referring clinicians.

\section{Take Home Messages}

- Dementia is a complex clinical syndrome with multiple underlying causes, of which presentation and prevalence differ according to patient demographics.

- Knowledge on typical and less typical imaging presentations of the various causes of dementia is essential in order to exploit the value of neuroimaging in the memory clinic to its full potential.

- Radiologists should perform a standardized imaging protocol, which enables both the assessment of patterns of atrophy and vascular lesions, and employ a systematic assessment (using semi-quantitative visual rating scales) in order to improve diagnostic yield.

\section{References}

1. Sachdev PS, Blacker D, Blazer DG, Ganguli M, Jeste DV, Paulsen JS, et al. Classifying neurocognitive disorders: the DSM-5 approach. Nat Rev Neurol. 2014;10(11):634-42.

2. Rizzi L, Rosset I, Roriz-Cruz M. Global epidemiology of dementia: Alzheimer's and vascular types. Biomed Res Int. 2014;2014:908915.

3. Lobo A, Launer LJ, Fratiglioni L, Andersen K, Di Carlo A, Breteler MM, et al. Prevalence of dementia and major subtypes in Europe: 
a collaborative study of population-based cohorts. Neurologic Diseases in the Elderly Research Group. Neurology. 2000;54(11 Suppl 5):S4-9.

4. Goodman RA, Lochner KA, Thambisetty M, Wingo TS, Posner SF, Ling SM. Prevalence of dementia subtypes in United States Medicare fee-for-service beneficiaries, 2011-2013. Alzheimer's Dement. 2017;13(1):28-37.

5. van der Flier WM, Scheltens P. Epidemiology and risk factors of dementia. J Neurol Neurosurg Psychiatry. 2005;76(Suppl 5):v2-7.

6. McMurtray A, Clark DG, Christine D, Mendez MF. Early-onset dementia: frequency and causes compared to late-onset dementia. Dement Geriatr Cogn Disord. 2006;21(2):59-64.

7. Picard C, Pasquier F, Martinaud O, Hannequin D, Godefroy O. Early onset dementia: characteristics in a large cohort from academic memory clinics. Alzheimer Dis Assoc Disord. 2011; 25(3):203-5.

8. Schneider JA, Arvanitakis Z, Bang W, Bennett DA. Mixed brain pathologies account for most dementia cases in communitydwelling older persons. Neurology. 2007;69(24):2197-204.

9. Brunnstrom H, Gustafson L, Passant U, Englund E. Prevalence of dementia subtypes: a 30-year retrospective survey of neuropathological reports. Arch Gerontol Geriatr. 2009;49(1):146-9.

10. Jack CR Jr, Bennett DA, Blennow K, Carrillo MC, Feldman HH, Frisoni GB, et al. A/T/N: an unbiased descriptive classification scheme for Alzheimer disease biomarkers. Neurology. 2016;87(5):539-47.

11. McKhann GM, Knopman DS, Chertkow H, Hyman BT, Jack $\mathrm{CR}$ Jr, Kawas $\mathrm{CH}$, et al. The diagnosis of dementia due to Alzheimer's disease: recommendations from the National Institute on Aging-Alzheimer's Association workgroups on diagnostic guidelines for Alzheimer's disease. Alzheimer's Dement. 2011;7(3):263-9.
12. Vernooij MW, Barkhof F. Neuroimaging in normal brain aging. In: Barkhof F, Jäger H, Thurnher M, Rovira À, editors. Clinical neuroradiology. Cham: Springer; 2019.

13. Ikram MA, Vrooman HA, Vernooij MW, van der Lijn F, Hofman $A$, van der Lugt A, et al. Brain tissue volumes in the general elderly population. The Rotterdam Scan Study. Neurobiol Aging. 2008;29(6):882-90.

14. Pasquier F, Leys D, Weerts JG, Mounier-Vehier F, Barkhof F, Scheltens P. Inter- and intraobserver reproducibility of cerebral atrophy assessment on MRI scans with hemispheric infarcts. Eur Neurol. 1996;36(5):268-72.

15. Scheltens P, Leys D, Barkhof F, Huglo D, Weinstein HC, Vermersch $\mathrm{P}$, et al. Atrophy of medial temporal lobes on MRI in "probable" Alzheimer's disease and normal ageing: diagnostic value and neuropsychological correlates. J Neurol Neurosurg Psychiatry. 1992;55(10):967-72.

16. Vernooij MW, Smits M. Structural neuroimaging in aging and Alzheimer's disease. Neuroimaging Clin N Am. 2012;22(1):33-55, vii-viii.

17. Pereira JB, Cavallin L, Spulber G, Aguilar C, Mecocci P, Vellas B, et al. Influence of age, disease onset and ApoE4 on visual medial temporal lobe atrophy cut-offs. J Intern Med. 2014;275(3):317-30.

18. Vermeer SE, Longstreth WT Jr, Koudstaal PJ. Silent brain infarcts: a systematic review. Lancet Neurol. 2007;6(7):611-9.

19. Roman GC, Tatemichi TK, Erkinjuntti T, Cummings JL, Masdeu $\mathrm{JC}$, Garcia JH, et al. Vascular dementia: diagnostic criteria for research studies. Report of the NINDS-AIREN International Workshop. Neurology. 1993;43(2):250-60.

20. Chahine LM, Rebeiz T, Rebeiz JJ, Grossman M, Gross RG. Corticobasal syndrome: five new things. Neurol Clin Pract. 2014;4(4):304-12.

Open Access This chapter is licensed under the terms of the Creative Commons Attribution 4.0 International License (http://creativecommons. org/licenses/by/4.0/), which permits use, sharing, adaptation, distribution and reproduction in any medium or format, as long as you give appropriate credit to the original author(s) and the source, provide a link to the Creative Commons license and indicate if changes were made.

The images or other third party material in this chapter are included in the chapter's Creative Commons license, unless indicated otherwise in a credit line to the material. If material is not included in the chapter's Creative Commons license and your intended use is not permitted by statutory regulation or exceeds the permitted use, you will need to obtain permission directly from the copyright holder. 


\title{
Imaging Evaluation of Patients with Cranial Nerve Disorders
}

\author{
Alexandre Krainik and Jan W. Casselman
}

\section{Abbreviations}

$\begin{array}{ll}\text { ADC } & \text { Apparent diffusion coefficient } \\ \text { CN } & \text { Cranial nerves } \\ \text { CNS } & \text { Central nervous system } \\ \text { CT } & \text { Computed tomography } \\ \text { CTA } & \text { Computed tomography angiography } \\ \text { DWI } & \text { Diffusion weighted images } \\ \text { FS } & \text { Fat-saturated } \\ \text { MRA } & \text { Magnetic resonance angiography } \\ \text { MRI } & \text { Magnetic resonance imaging } \\ \text { MS } & \text { Multiple sclerosis } \\ \text { NF } & \text { Neurofibromatosis } \\ \text { NMOsd } & \text { Neuromyelitis optica spectrum disorders } \\ \text { NVC } & \text { Neurovascular conflict } \\ \text { SWI } & \text { Susceptibility weighted images } \\ \text { TOF } & \text { Time of flight } \\ \text { TZ } & \text { Transition zone } \\ \text { WI } & \text { Weighted images }\end{array}$

\section{Learning Objectives}

- To know the imaging appearance of the most frequent cranial nerve lesions and be aware of associated $\mathrm{CN}$ disorders

- To understand that different lesions can be found on the different anatomical segments of the cranial nerves (intraaxial, extraaxial intracranial, extracranial) and that dedicated imaging techniques are needed to investigate these different segments and various pathologies

\section{A. Krainik $(\bowtie)$}

Department of Neuroradiology, University Hospital of Grenoble, Grenoble, France

J. W. Casselman

AZ Sint-Jan Brugge-Oostende AV, Brugge, Belgium

\section{Key Points}

- Intraaxial lesions usually cause multiple $\mathrm{CN}$ disorders in association with CNS dysfunctions

- Extraaxial intracranial lesions may cause unique or multiple $\mathrm{CN}$ disorders with meningeal syndrome and intracranial hypertension

- Skull base lesions usually cause unilateral unique or multiple $\mathrm{CN}$ disorders.

- Extracranial lesions may cause unilateral unique or multiple $\mathrm{CN}$ disorders with craniocervical objective lesions.

- Painful CN disorder may reveal aneurysm and dissection

\section{Key Points}

- Be aware of clinical history, presentation, and biological tests for imaging interpretation

- Master CN anatomy including nucleus, apparent origin, route, and target

- MRI is the modality of choice to investigate $\mathrm{CN}$ disorder

- CT is useful to depict calcification and skull lesion

- Add MRA and CTA when painful CN disorder

\subsection{Introduction}

Cranial nerves $(\mathrm{CN})$ are 12 pairs of symmetrical nerves that apparently originate from the cerebrum (I, II) and the brainstem (III-XII). Beyond this regional common thread, $\mathrm{CN}$ imaging encompasses complex anatomical and technical considerations that need to be known for clinical practice.

$\mathrm{CN}$ disorders are caused by a wide spectrum of diseases and imaging plays a major role in the etiological inquiry and 
the therapeutic planning. Guided by medical history and clinical examination, and completed by neurophysiological and biological tests, radiologists have to select the appropriate modalities, to adapt the spatial coverage, and to choose the most relevant protocol.

$\mathrm{CN}$ imaging has to demonstrate the location of the disease: into the brain (intraaxial), out of the brain and into the skull (extraaxial intracranial), out of the skull (extracranial). According to the location, $\mathrm{CN}$ imaging allows to suggest pathophysiological hypotheses and etiologies including vascular, inflammatory, infectious, tumoral, traumatic, or dysfunctional disorders.
This chapter briefly reviews most common diseases that cause $\mathrm{CN}$ disorders. Additional details can be found in [1-8].

\subsection{Anatomical and Technical Challenges}

\subsubsection{Anatomical Challenge}

The $\mathrm{CN}$ anatomy is complex regarding their number, brain nuclei and connections, apparent origins, cisternal and foraminal courses, end organs. Mastering the $\mathrm{CN}$ anatomy and function is necessary to investigate $\mathrm{CN}$ disorders appropriately (Table 12.1).

Table 12.1 Cranial nerves disorders and apparent routes

\begin{tabular}{|c|c|c|c|c|}
\hline $\mathrm{CN}$ name & Main dysfunctions & CNS coverage & Skull base coverage & Face and neck coverage \\
\hline I: Olfactory & Anosmia & $\begin{array}{l}\text { Basal forebrain: olfactory } \\
\text { tracts, bulbs, and striae. Uncus, } \\
\text { parahippocampal and cingular } \\
\text { gyri }\end{array}$ & $\begin{array}{l}\text { Anterior skull base: } \\
\text { cribriform plate }\end{array}$ & Nasal mucosa \\
\hline II: Optic & $\begin{array}{l}\text { Vision loss } \\
\text { Prechiasmatic: onilateral } \\
\text { anopia } \\
\text { Chiasmatic: bitemporal } \\
\text { hemianopia } \\
\text { Retrochiasmatic: } \\
\text { homonymous hemianopia }\end{array}$ & $\begin{array}{l}\text { Basal forebrain: optic } \\
\text { radiation, chiasma, lateral } \\
\text { geniculate bodies, occipital } \\
\text { calcarine sulci }\end{array}$ & $\begin{array}{l}\text { Anterior skull base: } \\
\text { optic canal }\end{array}$ & Orbit: eye ball retina \\
\hline III: Oculomotor & $\begin{array}{l}\text { Oculomotor palsy, ptosis, } \\
\text { mydriasis }\end{array}$ & $\begin{array}{l}\text { Midbrain: interpedoncular } \\
\text { fossa }\end{array}$ & $\begin{array}{l}\text { Middle skull base: } \\
\text { cavernous sinus, } \\
\text { superior orbital } \\
\text { fissure }\end{array}$ & $\begin{array}{l}\text { Orbit: oculomotor m. (superior, } \\
\text { medial, inferior recti, inferior } \\
\text { oblique), levator palpebrae m., } \\
\text { ciliary m. }\end{array}$ \\
\hline IV: Trochlear & Trochlear palsy & Midbrain: tectum & $\begin{array}{l}\text { Middle skull base: } \\
\text { cavernous sinus, } \\
\text { superior orbital } \\
\text { fissure }\end{array}$ & Orbit: trochlear m \\
\hline V: Trigeminal & $\begin{array}{l}\text { Facial anesthesia } \\
\text { Trigeminal neuralgia }\end{array}$ & Pons: anterolateral & $\begin{array}{l}\text { Middle skull base: } \\
\text { cavernous sinus; } \mathrm{V}_{1} \text { : } \\
\text { superior orbital } \\
\text { fissure } \\
\mathrm{V}_{2} \text { : f. rotundum } \\
\mathrm{V}_{3} \text { : f. ovale }\end{array}$ & $\begin{array}{l}\text { Face } \\
V_{1} \text { : forebrain } \\
V_{2} \text { : chick } \\
V_{3} \text { : Jaw, sensory tongue ( } 2 / 3 \text { ant) }\end{array}$ \\
\hline VI: Abducens & Abducens palsy & Medullopontine s. (ant.) & $\begin{array}{l}\text { Middle skull base: } \\
\text { cavernous sinus, } \\
\text { superior orbital } \\
\text { fissure }\end{array}$ & Orbit: abducens m. \\
\hline VII: Facial & $\begin{array}{l}\text { Facial palsy. Hemifacial } \\
\text { spasm }\end{array}$ & Medullopontine s. (lat.) & $\begin{array}{l}\text { Posterior skull base: } \\
\text { internal auditory } \\
\text { canal, stylomastoid f. }\end{array}$ & $\begin{array}{l}\text { Face m., lacrimal and salivary } \\
\text { glands }\end{array}$ \\
\hline $\begin{array}{l}\text { VIII: } \\
\text { Cochleovestibular }\end{array}$ & $\begin{array}{l}\text { Hearing loss, tinnitus, } \\
\text { dizziness }\end{array}$ & Medullopontine s. (lat.) & $\begin{array}{l}\text { Posterior skull base: } \\
\text { internal auditory } \\
\text { canal }\end{array}$ & $\begin{array}{l}\text { Inner ear: cochlea and semicircular } \\
\text { canals }\end{array}$ \\
\hline $\begin{array}{l}\text { IX: } \\
\text { Glossopharyngeal }\end{array}$ & $\begin{array}{l}\text { Ageusia, dysphagia, throat } \\
\text { anesthesia. } \\
\text { Glossopharyngeal } \\
\text { neuralgia }\end{array}$ & Medulla: retroolivary s. & $\begin{array}{l}\text { Posterior skull base: } \\
\text { jugular foramen }\end{array}$ & $\begin{array}{l}\text { Stylopharyngeus } m \text {; sensory tongue } \\
\text { (1/3 post) }\end{array}$ \\
\hline $\mathrm{X}$ : Vagus & Dysphagia, dysphonia & Medulla: retroolivary s. & $\begin{array}{l}\text { Posterior skull base: } \\
\text { jugular foramen }\end{array}$ & Pharyngeal m. \\
\hline XI: Accessory & $\begin{array}{l}\text { Impairment of head } \\
\text { rotation, scapula elevation }\end{array}$ & $\begin{array}{l}\text { Medulla: retroolivary s., lateral } \\
\text { cervical cord }\end{array}$ & $\begin{array}{l}\text { Posterior skull base: } \\
\text { jugular foramen }\end{array}$ & Sternocleidomastoid, trapezius m. \\
\hline XII: Hypoglossal & Palsy of the tongue & Medulla: preolivary s. & $\begin{array}{l}\text { Posterior skull base: } \\
\text { hypoglossal canal }\end{array}$ & Tongue muscles \\
\hline
\end{tabular}

$m$. muscle, s. sulcus, ant. anterior, lat. lateral, post posterior 
Olfactory (I) and optic (II) nerves have both an apparent origin from the basal forebrain, passing through the foramina of the anterior cranial fossa. Both olfactory and optic nerves are tracts of the central nervous system (CNS).

All other $\mathrm{CN}$ originate apparently from the brainstem: oculomotor (III) and trochlear (IV) nerves from the midbrain; trigeminal (V) from the pons; abducens (VI), facial (VII), and cochleovestibular (VIII) nerves from the medullopontine sulcus; glossopharyngeal (IX), vagus (X), accessory (XI), and hypoglossal (XIII) nerves from the medulla oblongata. CN III-VI merge into the cavernous sinuses and pass through the foramina of the middle cranial fossa. CN VIIXII pass through the foramina of the posterior cranial fossa.

Because of this anatomical distribution of the $\mathrm{CN}$ along the basal brain, the brainstem, and the skull base, there is a close relationship between the lesional location and the symptoms. Indeed, CN impairment depends on the location of the macroscopic lesion. When unique, distal extracranial lesions are associated with specific $\mathrm{CN}$ deficit while proximal intraaxial lesions may cause multiple $\mathrm{CN}$ impairment with additional central symptoms.

In clinical practice, imaging is used to explain $\mathrm{CN}$ impairments related to: (1) intraaxial lesions that compromise supranuclear connecting fibers, $\mathrm{CN}$ nuclei, and intramedullary fibers; (2) extraaxial intracranial lesions that compromise cisternal and intraforaminal CN fibers; (3) extracranial lesions that compromise facial and cervical portions of $\mathrm{CN}$.

Further anatomical and functional details can be found in textbooks [3-6].

\subsection{Technical Challenge}

\subsubsection{Conventional Imaging}

MRI is the best imaging modality to investigate $\mathrm{CN}$ disorders. However, computed tomography (CT) is useful to better visualize bone lesion, especially in case of trauma, inflammation (abscess) or in patients with compromised airways. CT still offers an immediate availability, a short acquisition time, and a non-magnetic environment.

Based first on the clinical data, MRI protocols have to be adjusted on $\mathrm{CN}$ regional anatomy by optimizing spatial coverage and selecting most appropriate sequences [2, 8]. Complementary contrast media injection has to be justified in accordance with the clinical presentation, lesional hypotheses, and initial imaging findings (Table 12.2).

The best way to detect cranial nerve pathology is to compare the size and signal intensity of the paired nerves. Due to the course of the cranial nerves this comparison is best achieved in the coronal plane for nerves I-VI and in the axial plane for nerves VII-XII. However, imaging in both axial and coronal planes decreases the chance to overlook pathology.
Frequently nerve enhancement is the only sign indicating cranial nerve involvement and therefore the use of gadolinium is recommended in most cases.

A phased array head coil is best suited to image all cranial nerves down to the level of the mandible but an additional neck coil is needed to visualize the lower cranial nerves in the neck and upper mediastinum (left CN X).

Pathology at the level of the $\mathrm{CN}$ nuclei and intramedullary fibers is best seen on selective proton density, T2-weighted, and multi-echo (m-FFE, Medic, Merge) images. 3D-FLAIR are more reliable than 2D because of better flow saturation. It decreases repetition artifacts and allow a better visualization of the brain stem. Post-contrast 3D-FLAIR is also useful to demonstrate abnormal meningeal and neural enhancements. Additional diffusionweighted images (DWI) are often needed to rule out stroke and to characterize tissular and cystic masses.

The cisternal segment of CN is surrounded by CSF and therefore submillimetric heavily T2-weighted images (WI) are best suited. All these sequences perform well in the center of the image and region around the brainstem and some of the steady state sequences can even cover all 12 cranial nerves. However, the steady state sequences (e.g., CISS, balanced-FFE, etc.) are prone to susceptibility artifacts at the level of the more peripheral located cisternal segments such as the olfactory bulbs and tracts, cranial nerve VII and VIII inside the internal auditory canal. Therefore these peripheral segments are better imaged with TSE sequences (e.g., DRIVE, 3D-TSE, etc.) that unfortunately have a smaller coverage.

Once the nerves are surrounded by a venous plexus (cavernous sinus, basilar plexus behind the clivus, jugular foramen, and hypoglossal canal) they are best depicted as black structures surrounded by high signal intensity enhancing venous structures on 2DSE or TSE, 3DTSE or FFE postcontrast T1-WI. The extracranial segments of the cranial nerves and surrounding lesions can best be depicted on highresolution axial and coronal $\mathrm{T} 1$ and $\mathrm{T} 2-\mathrm{WI}$.

Fat around the cranial nerves will disappear when fat saturation is used and renders visualization of the normal nerves more difficult. On the other hand abnormal enhancement of the nerves will be recognized more easily on fat-saturated images. The use of DIXON sequences avoids this sometimes difficult choice and provides both fat and non-fat-saturated (FS) images in one sequence.

New isotropic 3D unenhanced MR neurography sequences allow detailed visualization of the normal and abnormal extracranial branches and course of nerves V, VII, IX-XII. The sequences are based on a 3D TSE sequence with fat saturation and a motion sensitized driven equilibrium function (black blood function parallel to the nerves) [8]. To acquire all the above mentioned high-resolution images or isotropic submillimetric images in an acceptable 
Table 12.2 Technical recommendations to investigate $\mathrm{CN}$ disorders

\begin{tabular}{|c|c|c|c|c|}
\hline $\begin{array}{l}\mathrm{CN} \text { nuclei and } \\
\text { segments }\end{array}$ & $\begin{array}{l}\text { Brainstem: nuclei and } \\
\text { intramedullary fibers }\end{array}$ & Cisternal segment & $\begin{array}{l}\text { Segments surrounded by } \\
\text { venous plexus-intraforaminal } \\
\text { fibers }\end{array}$ & $\begin{array}{l}\text { Extracranial nerves-face, } \\
\text { neck, mediastinum }\end{array}$ \\
\hline Sequences & $\begin{array}{l}\text { T2-weighted/PD (2D) } \\
\text { DWI (2D) } \\
\text { m-FFE/medic/ } \\
\text { merge(2D)-nuclei }\end{array}$ & $\begin{array}{l}\text { b-FFE XD (3D) } \\
\text { DRIVE TSE T2 (3D) } \\
\text { MRA (3D) unenhanced }\end{array}$ & $\begin{array}{l}\text { T1-weighted TSE HR (2D) } \\
\text { FFE (3D) } \\
\text { Black blood TSE T1 (3D) }\end{array}$ & $\begin{array}{l}\text { T1-weighted TSE HR (2D) } \\
\text { T2-weighted TSE HR (2D) } \\
\text { Neurography (3D) }\end{array}$ \\
\hline Plane & Axial & $\begin{array}{l}\text { 3D sequences, isotropic. Measured } \\
\text { in the axial plane, reformatted in any } \\
\text { other plane }\end{array}$ & $\begin{array}{l}\text { Cavernous sinus: coronal } \\
\text { Basilar plexus: axial } \\
\text { Jugular foramen: } \\
\text { axial > coronal } \\
\text { Hypoglossal canal: } \\
\text { axial > coronal } \\
\text { 3D sequences: reformatted any } \\
\text { plane }\end{array}$ & $\begin{array}{l}\text { Axial + coronal } \\
\text { Neurography: axial 3D } \\
\text { isotropic }\end{array}$ \\
\hline $\begin{array}{l}\text { Resolution } \\
(X \times Y \times Z \text { in } \\
\mathrm{mm})\end{array}$ & $\begin{array}{l}\text { T2-weighted/PD: } \\
0.70 \times 0.88 \times 3.00 \\
\text { DWI: } \\
1.40 \times 1.42 \times 3.00 \\
\text { m-FFE: } \\
0.65 \times 0.87 \times 2.00\end{array}$ & $\begin{array}{l}\text { b-FFE XD: } 0.5 \times 0.5 \times 0.5 \mathrm{~mm} \\
\text { DRIVE T2: } 0.46 \times 0.46 \times 0.50 \mathrm{~mm} \\
\text { MRA: } 0.69 \times 0.70 \times 0.70 \mathrm{~mm}\end{array}$ & $\begin{array}{l}\text { T1-weighted TSE HR: } \\
0.40 \times 0.45 \times 2.30 \mathrm{~mm} \\
\text { FFE: } 0.60 \times 0.59 \times 1.20 \mathrm{~mm} \\
\text { Black blood: } \\
0.55 \times 0.55 \times 0.55 \mathrm{~mm}\end{array}$ & $\begin{array}{l}\text { T1-weighted TSE HR: } \\
0.40 \times 0.45 \times 2.3 \mathrm{~mm} \\
\text { T2-weighted TSE HR: } \\
0.60 \times 0.53 \times 3.3 \mathrm{~mm} \\
\text { Neurography: } \\
0.89 \times 0.90 \times 0.90 \mathrm{~mm}\end{array}$ \\
\hline Range & $\begin{array}{l}\text { T2-weighted/PD: } \\
60 \mathrm{~mm} \\
\text { DWI: } 128 \mathrm{~mm} \\
\text { m-FFE: } 48 \mathrm{~mm}\end{array}$ & $\begin{array}{l}\text { b-FFE XD: } 80 \mathrm{~mm} \\
\text { DRIVE T2: } 36 \mathrm{~mm} \\
\text { MRA: } 120 \mathrm{~mm}\end{array}$ & $\begin{array}{l}\text { T1-weighted TSE HR: } 69 \mathrm{~mm} \\
\text { FFE: } 59 \mathrm{~mm} \\
\text { Black blood: } 39 \mathrm{~mm}\end{array}$ & $\begin{array}{l}\text { T1-weighted TSE HR: } \\
69 \text { mm } \\
\text { T2-weighted TSE HR: } \\
106 \mathrm{~mm} \\
\text { Neurography: } 90 \mathrm{~mm}\end{array}$ \\
\hline $\begin{array}{l}\text { Acquisition } \\
\text { time }\end{array}$ & $\begin{array}{l}\text { T2-weighted/PD: } 2 \mathrm{~min} \\
31 \mathrm{~s} \\
\text { DWI: } 2 \min 21 \mathrm{~s} \\
\text { m-FFE: } 14 \min 46 \mathrm{~s}\end{array}$ & $\begin{array}{l}\text { b-FFE XD: } 4 \min 43 \mathrm{~s}(\mathrm{CS} 10) \\
\text { DRIVE T2: } 2 \min 47 \mathrm{~s}(\mathrm{CS} 3.3) \\
\text { MRA: } 6 \min 50 \mathrm{~s}(\mathrm{CS} 3)\end{array}$ & $\begin{array}{l}\text { T1-weighted TSE HR: } 7 \mathrm{~min} \\
30 \mathrm{~s}(\mathrm{~S} 1.5) \\
\text { FFE: } 4 \text { min } 8 \mathrm{~s}(\mathrm{~S} 1.4) \\
\text { Black blood: } 5 \text { min } 5 \mathrm{~s}(\mathrm{CS} 4)\end{array}$ & $\begin{array}{l}\text { T1-weighted TSE HR: } \\
7 \text { min } 30 \mathrm{~s} \text { (S 1.5) } \\
\text { T2-weighted TSE HR: } \\
3 \text { min } 15 \mathrm{~s}(\mathrm{CS} 2.5) \\
\text { Neurography: } 8 \text { min } 17 \mathrm{~s} \\
\text { (CS 3) }\end{array}$ \\
\hline
\end{tabular}

$2 D$ two dimensional, $3 D$ three dimensional, $b$ - FFE balanced fast field echo, $C N$ cranial nerve, $C S$ compressed sense factor, $D R I V E$ driven equilibrium, $D W I$ diffusion-weighted imaging, $F F E$ fast field echo, medic multi-echo data image combination, merge multiple echo recombine gradient echo, $m$ - $F F E$ merged fast field echo, $H R$ high resolution, $M R A$ magnetic resonance angiography, $P D$ proton density, $S$ sense factor, TSE turbo spin echo

time parallel imaging and if available compressed sense should be used.

\subsubsection{Tractography}

Advances in DWI clinical research have led to the development of cranial nerves tractographie based on diffusion tensor imaging. The visual results depend on the anatomy of the cranial nerve tracked, acquisition parameters, and the postprocessing steps. Currently, cranial nerves tractography techniques only allow to display the thicker nerves, including six isolated cranial nerves (II, III, V, VI, VII/VIII) and IX/X/XI nerves as a group [9] (Fig. 12.1).

The acquisition of high-angular resolution DWI data (at least 32 directions) is required to estimate the local fiber orientation distribution, a prior step to manage voxels with multiple fibers population. Such diffusion signal modeling is required, for example, when tracking nerves with secondary branches or in diseased condition. The other crucial point relies on the susceptibility distribution of the subjects' head, which needs to be corrected by specific postprocessing. It typically uses two b-zero acquisitions with opposing polarities of the phase-encode blips. In addition, the polarity of the diffusion directions has a major impact to pass through skull base foramina. For example, optic nerves tractography would require a postero-anterior acquisition to be feasible along the optic canal, while inner auditory canal nerves would take advantage of an antero-posterior MR acquisition.

It is well-known that probabilistic algorithms are the method of choice to track cranial nerves, including in tumor condition. Global tractographic reconstructions on the whole acquisition volume have also proved efficiency to map cranial nerves ischemia, for example, in anterior optic ischemic neuropathy [10]. Global tractographic approach also allows to obtain cranial nerves reconstructions at a submillimeter scale, regardless to the voxel size acquisition. Technical advances such as faster imaging acquisition and implementation of recent postprocessing developments in clinical software promise new perspectives in neuroradiology, skull base surgery, and neuroanatomy $[9,11,12]$. 

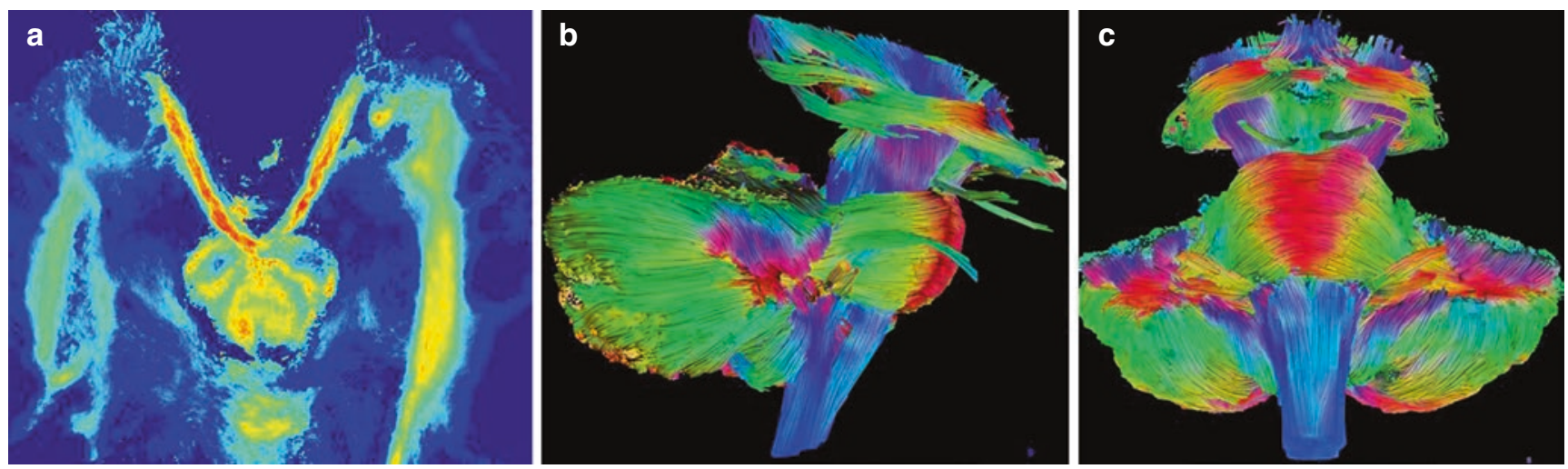

Fig. 12.1 Global tractography of the cranial nerves, brainstem, and cerebellum: (a) axial view of normal oculomotor nerves (III) arising from the interpeduncular fossa; $(\mathbf{b}, \mathbf{c}) 3 \mathrm{D}$ rendering in a healthy volun-

\subsection{Intraaxial Lesions}

Intraaxial lesions such as stroke, meningoencephalitis, central pontine myelinolysis, or glioma might have a spontaneous poor short-term prognosis without specific care. Then, identifying intraaxial lesion in case of $\mathrm{CN}$ impairment remains a diagnostic priority. Despite both olfactory (I) and optic (II) nerves are tracts of the CNS, their lesions are presented in their apparent location.

Because of the complex anatomy of the brainstem, intraaxial lesions are prone to be revealed by a rich clinical semiology, associating multiple $\mathrm{CN}$ impairment and central disorders such as hemiplegia, hemiparesia, extrapyramidal syndrome, dysautonomic syndrome, awareness impairment, nausea, cardiorespiratory dysregulation, etc. Recent imaging details are available in $[1,7]$.

\subsubsection{Vascular Diseases}

\subsubsection{Ischemic Stroke}

Stroke is the major cause of neurologic deficits with acute onset. Ischemic stroke is more frequent than hemorrhage. Except for acute ischemic optic neuropathy, $\mathrm{CN}$ disorders secondary to ischemic stroke is usually due to an occlusion of the basilar artery and its perforating arteries. Occlusion of the distal portion of the vertebral artery, the postero-inferior cerebellar artery and its branches is responsible of ischemia of the medulla. Intravenous thrombolysis is recommended within $4.5 \mathrm{~h}$ after onset. Recommendations for mechanical thrombectomy in the posterior circulation are under investigation, even after $6 \mathrm{~h}$.

Imaging in emergency is needed for brainstem stroke: (1) to distinguish ischemic from hemorrhagic stroke; (2) to estimate the duration of stroke in case of unclear history or awake stroke, (3) to identify intravascular thrombus, occlusion, and stenosis. teer. When compared to surgery, reliable tractography is obtained in most CN but IV and VI [9]

MRI is an excellent modality to demonstrate very acute infarction as a DWI hyperintensity due to a low apparent diffusion coefficient (ADC) (Fig. 12.2). T2 FLAIR hyperintensity reveals stroke after $4 \mathrm{~h}$. T2 $*$ or CT are helpful to rule out hemorrhage. CT angiography (CTA) or MR angiography (MRA) using time of flight (TOF) is useful to identify vascular occlusion or stenosis. Perfusion CT and MR are also useful to show penumbra, this reversible ischemia after reperfusion [13].

\subsubsection{Intraparenchymal Hemorrhage}

Spontaneous hemorrhage of the brainstem is usually due to vascular malformation, such as cavernoma. Arteriovenous malformation and fistula are less frequent. Cavernoma or cavernous hemangioma is a sporadic or familial vascular malformation, characterized by blood cavities due to dilated blood vessels. Abnormal structural support of the vessels may cause leakage and hemorrhage in the surrounding tissue. CT may show hyperdensities due to calcifications and hemorrhage. MRI may show a "pop-corn" like lesion with caverns of varied intensities. T2 may show a hypointense rim. Hemosiderin depositions are best seen using T2* and SWI. Post-contrast T1 images may show subtle enhancement and additional developmental venous anomaly.

\subsubsection{Infectious Diseases}

\subsubsection{Listeria}

Listerial rhombencephalitis is due to Listeria monocytogenes septicemia, which may occur in pregnancy, infant, elderly, and immunocompromised patients after oral contamination of infected fresh products such as cheese. Antiobiotherapy is required in emergency. MRI is the best modality to show pontine enlargement and T2 hyperintensities (Fig. 12.2). Perilesional enhancement might be associated with 

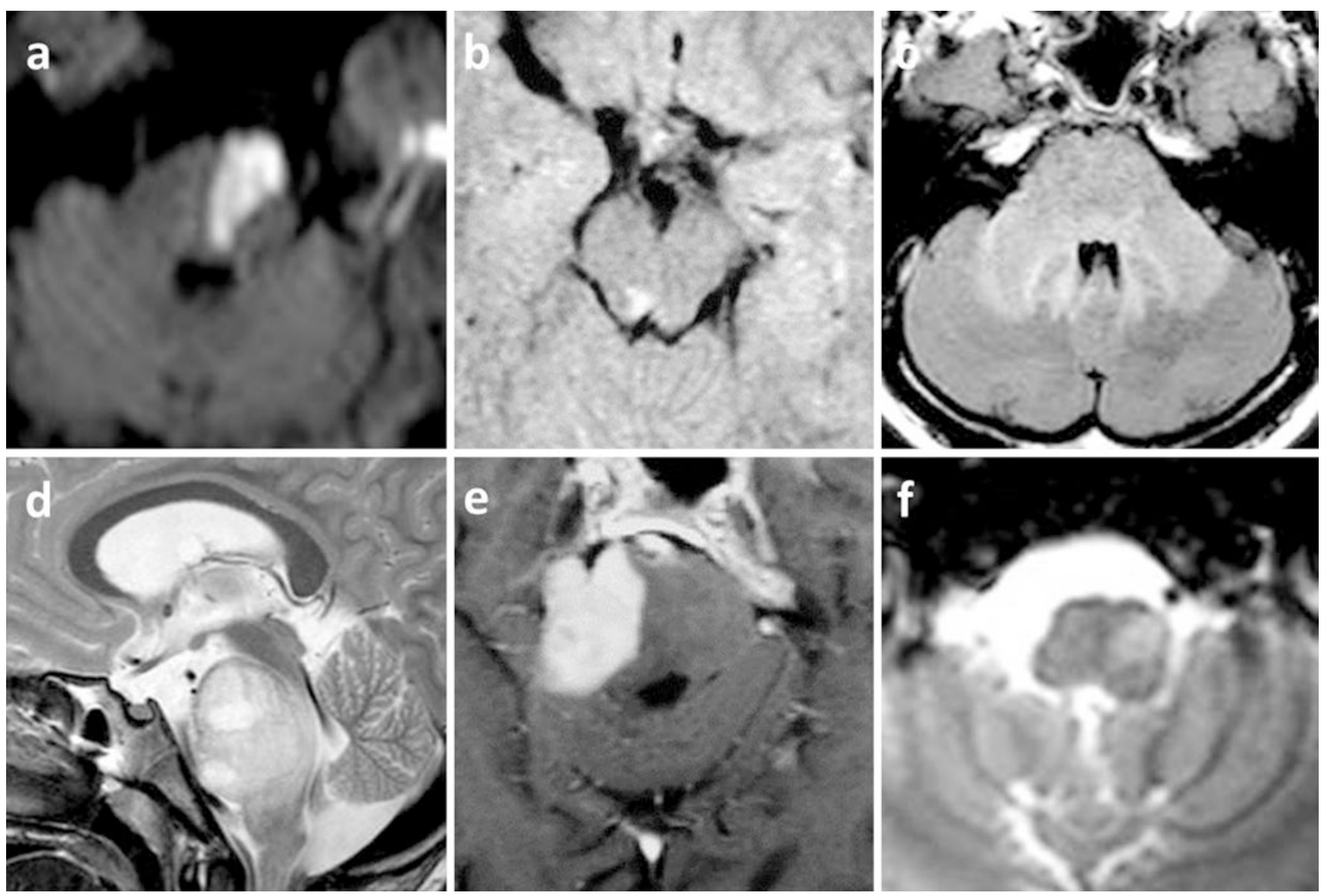

Fig. 12.2 Intraaxial lesions with $\mathrm{CN}$ disorders: (a) ischemic stroke of the left hemi-pons, DWI hyperintense pontine triangle, revealed by right hemiparesia and diplopia; (b) multiple sclerosis with a T2 hyperintense nodule close to $\mathrm{CN}$ III nucleus with a right oculomotor palsy and ptosis; (c) listerial rhombencephalitis as a T2 hyperintense infiltration of pons and cerebellar peduncles in a pregnant woman revealed by febrile confusion with bilateral oculomotor paresis; (d) midline diffuse glioma of the pons as aT2 hyperintense pontine mass, revealed by bilateral CN palsies (oculomotor and facial) and headaches; (e) primary CNS lymphoma, post-contrast T1 enhancing homogeneous right pontine mass, revealed by right trigeminal anesthesia and left hemiparesia; (f) left hypertrophic olivary degeneration (T2* hyperintensity) revealed by left palatal myoclonus, 6 months after ipsilateral mesencephalic cavernoma hemorrhage leptomeningeal and adjacent V4 ependymal enhancements. Foci of low ADC would have a poorer prognosis.

Any septicemia of different origins may also cause intraaxial abscesses.

\subsubsection{CMV}

Cytomegalovirus infection in immunocompromised patients may cause ventriculitis and encephalitis. MRI shows an ependymal enhancement with adjacent $\mathrm{T} 2$ hyperintense foci of inflammation that may insult $\mathrm{CN}$ nuclei and intraaxial fibers.

\subsubsection{Non-infectious Inflammatory Diseases}

\subsubsection{Demyelinating Diseases}

Demyelinating diseases is a heterogeneous group of inflammatory disorders, such as multiple sclerosis, neuromyelitis optica spectrum disorders (NMOsd) with anti-AQP4 or anti-
MOG antibodies, acute disseminated encephalomyelitis, etc. Clinical presentation and biological studies are helpful to discuss among diagnoses. Imaging of the brain and the spinal cord is also critical. All these diseases might be responsible of $\mathrm{CN}$ disorders due to nodular lesions involving the brainstem (Fig. 12.2). NMO with anti-AQP4 antibodies is often associated with periventricular and especially area postrema lesions $[14,15]$. Acute lesions may appear T2 hyperintense, DWI hyperintense due to low ADC within the first days after onset, and open ring perinodular enhancement during the first month. Optic neuritis may have suggestive presentations according to the diseases (see extracranial).

\subsubsection{Behcet's Disease}

Behcet's disease is a multisystem vascular-inflammatory disease of unknown origin that associates recurrent orogenital ulcerations, uveitis, and skin lesions. Males originating from countries along the Silk Road are more affected from 
Mediterranean to Far East. CNS involvement is rare but classical. Besides venous cerebral thrombosis, intraaxial illdefined lesions are often described in the pons, the midbrain, and the diencephalon. Irregular lesional contrast enhancement is possible.

\subsubsection{Tumor}

\subsubsection{Midline Infiltrating Glioma}

Midline infiltrating glioma is rare, usually diagnosed in children and young adults with a poor prognosis. The lesion typically arises in the pons or the thalamus. Rapidly progressive brainstem and cranial nerves disorders develop over few weeks in association with an obstructive hydrocephalus and intracranial hypertension. The lesion is a large expansive mass T1 hypointense, T2 hyperintense (Fig. 12.2). Although considered as a high grade glioma, intralesional necrosis and contrast enhancement are possible but unconstant. Spectroscopy demonstrates a malignant profile, with low NAA, high choline and choline/creatine ratio, and the presence of lactates and lipids.

\subsubsection{Other Intraaxial Tumors}

Other tumors that may infiltrate the brainstem are primary ependymoma, medulloblastoma, CNS lymphoma (Fig. 12.2), metastases. Tumoral hypercellularity appears as a DWI hyperintensity due to low ADC. This feature is common in medulloblastoma and lymphoma. Post-contrast enhancement is usually heterogeneous except in lymphoma which remains homogeneous in immunocompetent patients despite the large size of the tumor.

\subsubsection{Trauma}

\subsubsection{Diffuse Axonal Injuries and Contusions}

Severe traumatic brain injury is defined by Glasgow coma scale $<8$. In case of midbrain lesions, conscientiousness and awareness impairments are predominant. MRI may demonstrate diffuse axonal injuries due to structural neural shearing in case of high velocity trauma. These punctuate lesions, $\mathrm{T} 2 * /$ SWI hypointense, T2 hyperintense may also cause cranial nerve palsies and movements disorders. Additionally, CT and MRI may show post-traumatic contusion of the posterolateral aspect of the midbrain onto the tentorium as an edema and a superficial hemorrhage.

\subsubsection{Toxic/Metabolic}

\subsubsection{Central Pontine Myelinolysis}

Osmotic demyelination syndrome is usually observed after a rapid correction of electrolyte disturbance such as hypona- tremia. Central pontine myelinolysis is T2 hyperintense with a classical trident or piglet shape. Extrapontine myelinolysis may involve basal ganglia and deep white matter.

\subsubsection{Degeneration}

\subsubsection{Progressive Supranuclear Palsy}

Progressive supranuclear palsy is a neurodegenerative disease that is responsible of oculomotor disorders, especially with vertical gaze and convergence impairments. An extrapyramidal syndrome poorly responsive to L-Dopa administration is usually associated. On axial plane, the mesencephalic atrophy is responsible of a concavity of the posterior aspect of the midbrain, the "morning glory" sign. On mid-sagittal plane, the atrophy is responsible of the concavity of the superior aspect of the midbrain, the "hummingbird" sign.

\subsubsection{Hypertrophic Olivary Degeneration}

The hypertrophic olivary degeneration is secondary to a focal lesion, usually a hemorrhage or an ischemia involving the dento-rubro-olivary pathway. The olivary degeneration appears T2 hyperintense and hypertrophic several months after the initial injury (Fig. 12.2). When present, cerebellar changes, including superior cerebellar peduncles are contralateral to the olivary degeneration. Bilateral hypertrophic olivary degeneration is also possible.

\subsection{Extraaxial Intracranial Lesions}

Extraaxial intracranial lesions might insult the cisternal and the transdural portions of CN. These diseases include primary diseases of the nerves, secondary neural inflammation, and compression by vascular, meningeal, and skull bases diseases. $\mathrm{CN}$ disorders reveal the location and the extent of the lesions. Patient's history is critical for diagnosis. CN disorders can be unique or multiple. Additional symptoms such as pain may also suggest particular etiologies such as meningeal and neural inflammation, vascular disorders such as aneurysms and neurovascular conflicts. Headaches with nausea, vomiting, papillary edema, and abducens palsy suggest intracranial hypertension. When neuroimaging is normal, CSF study is needed. In clinical practice, CT is useful in emergency to rule out intracranial hypertension before lumbar puncture and for osseous skull study. MR is usually needed to refine the diagnosis [2, 8, 16-18].

\subsubsection{Vascular Diseases}

The close vicinity of $\mathrm{CN}$ and vessels explains why focal vascular diseases may cause $\mathrm{CN}$ disorders. 


\subsubsection{Aneurysm and Arterial Dissection}

First, life-threatening aneurysm and arterial dissection have to be ruled out in case of unilateral painful $\mathrm{CN}$. Aneurysms of the first portion of the posterior cerebral artery and the cerebral portion of the internal carotid artery may have a mass effect on the superior aspect of the oculomotor nerve (III), responsible of complete extrinsic and intrinsic oculomotor nerve palsy. Because preganglionic parasympathetic pupillomotor fibers to the ciliary ganglion are located on the superomedial surface of the CN III, the loss of pupillary function occurs in about $14 \%$ secondary to the peripheral fibers compression. In most cases, CN III palsy is limited to extrinsic oculomotricity secondary to central fibers ischemia, such as in diabetic patients. Diabetic ophthalmoparesia accounts for $25 \%$ of ocular motor nerve palsies and imaging is still normal in such cases of vasculopathic cranial neuropathy [19].

Painful lower CN disorders may also reveal dissection of the distal part of the vertebral artery or cervical portion of the internal carotid artery. Both CTA and MRA with nonenhanced FS T1 SE images are necessary.

\subsubsection{Arterio-Venous Fistula}

Carotido-cavernous fistulas can eventually cause raised intraocular pressure which can interfere with the blood supply of the optic nerve and can lead to blindness. These fistulas can best be detected on non-enhanced MRA. Abnormal high signal intensity can then be seen inside the cavernous sinus due to the increased speed of the venous flow caused by the fistula with the carotid. A congestive chemosis with a dilation of the superior ophthalmic vein is common.

\subsubsection{Neurovascular Compression Syndromes}

Neurovascular compression syndromes (NVCS) are usually caused by arteries or veins that directly compress and insult the cisternal portion of $\mathrm{CN}$ (Fig. 12.3). Common NVCS involve $\mathrm{CN}$ V with a trigeminal neuralgia $(\mathrm{V}), \mathrm{CN}$ VII with hemifacial spasm, CN VIII with vestibular paroxysmia (VIII), CN IX with glossopharyngeal neuralgia.

Indeed vessels in the posterior fossa elongate and become more tortuous with aging. Among all neurovascular contacts, few are symptomatic. While heavily T2-WI are very efficient in displaying nerve to vessel contact, the relationship with patient symptoms remains debated. In fact, one challenge is to determine which neurovascular contact could be symptomatic. The most common pathophysiological hypothesis relies on the natural weakness of $\mathrm{CN}$ in their transition zone, a short 1-2 $\mathrm{mm}$ segment in which the central myelin that originates from oligodendrocytes and covers the proximal intracisternal portion of $\mathrm{CN}$, turns into peripheral myelin from Schwann cells. Therefore, the correlation between clinical symptoms and the exact location of the neurovascular contact is crucial.
Trigeminal neuralgia (V) secondary to NVC is suspected when neural deformation by vascular contact is located approximately $4 \mathrm{~mm}$ from the apparent $\mathrm{CNV}$ origin from the brainstem, hemifacial spasm (VII) at $2 \mathrm{~mm}$, vestibular paroxysmia (VIII) at $10 \mathrm{~mm}$, and glossopharyngeal neuralgia at $<2 \mathrm{~mm}$ [20].

Venous conflicts are possible but most NVCS are caused by arteries, especially the superior cerebellar artery $(\mathrm{V})$, the anterior inferior cerebellar artery (VII, VIII), the posterior inferior cerebellar, and the vertebral artery (VII, VIII, IX).

Besides the exact location of the contact, reliable signs are a perpendicular neurovascular crossing and the displacement of the nerve. To confirm NVCS, nerves, arteries, and veins are distinguished using heavily T2-WI for nerves, unenhanced TOF MRA for arteries, and post-contrast 3D-submillimetric T1 WI for veins (Fig. 12.3).

Fusion software with selective color coding for each anatomical structure can be helpful, especially after decompressive surgery to recognize the position of the material (e.g., Teflon, etc.).

\subsubsection{Infectious Diseases}

\subsubsection{Viral Neuritis}

Bell's palsy accounts for a peripheral facial palsy secondary to herpes simplex virus related neuritis. Most patients recover spontaneously facial nerve function in $<2$ months without therapy. Imaging is usually performed in case of atypical clinical presentation, including persisting deficit and recurrence. Thin post-contrast FS T1-WI and 3D-FLAIR show a fundal "tuft" and labyrinthic facial nerve (VII) enhancement. Intrameatic and intralabyrinthic enhancements of the CN VII are always abnormal. Nodular enhancement may suggest differential diagnoses such a schwannoma, hemangioma, metastases, etc. Enhancement of the geniculate ganglion, tympanic, and mastoid segment of the nerve has to be interpreted with caution as these segments are surrounded by an arteriovenous plexus that may also enhance in normal subjects. To avoid this problem, it is recommended to use sequences that do not show the surrounding vessels enhancement like millimetric heavily T2-WI and post-contrast black blood 3DT1-WI (Fig. 12.3). Comparison with the contralateral side is useful to distinguish an excessive CN VII enhancement. Main differential diagnoses include facial nerve schwannoma, hemangioma, perineural tumor spread from parotid tumor, and Ramsay Hunt syndrome.

Ramsay Hunt syndrome accounts for a varicella zoster virus infection responsible of both sensory fibers of facial and cochleovestibular nerves. A facial palsy and a sensorineural hearing loss are associated with an external ear vesicular rash. Fever, vertigo, nausea, and burning pain of the ear are common. CN disorders may occur before the rash. Thus, MRI is helpful to identify a unilateral facial and cochleoves- 

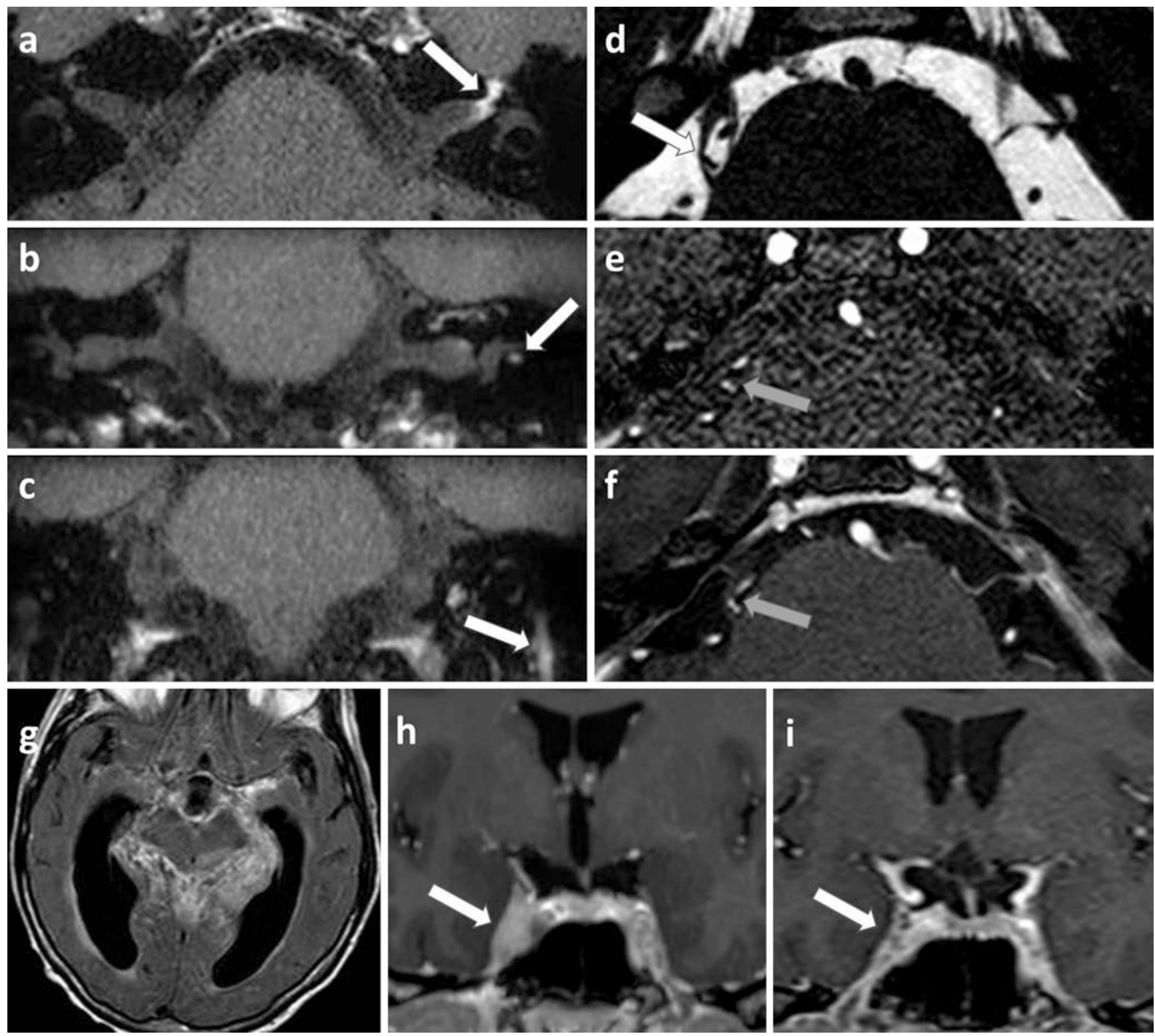

Fig. 12.3 Extraaxial intracranial lesions associated with $\mathrm{CN}$ disorders: $(\mathbf{a}-\mathbf{c})$ left Bell's palsy, a viral neuritis, axial $0.55 \mathrm{~mm}$ thick black blood 3D TSE T1-WI show unilateral left facial nerve enhancement in the fundus of the internal auditory canal and along the labyrinthine segment, and the geniculate ganglion (a arrow). Coronal reformatted images show an enhancement of the tympanic (b arrow) and mastoid segment (c arrow) of the left facial nerve. Note that these black blood images suppress both arterial and vein enhancement, making the diagnosis easier; (d-f) right trigeminal neuralgia in a neurovascular compression syndrome, axial $0.3 \mathrm{~mm}$ thick 3D TSE heavily T2-WI (d) shows the displacement of the transitional zone of the CN V by the right superior cerebellar artery (gray arrows) on TOF MRA (e) and postcontrast $0.5 \mathrm{~mm}$ thick 3D T1-WI (f); (g) tuberculous leptomeningitis, T2 FLAIR hyperintensities of the basal leptomeninges, revealed by bilateral oculomotor and facial palsies and headaches (hydrocephalus); (h, i) Tolosa-Hunt syndrome revealed by a painful right ophthalmoplegia, initial post-contrast T1-WI (e) show a right cavernous thickening without other potential diagnosis (white arrow), high-dose corticoid therapy amended symptoms and 6 months follow-up MRI (f) show normal cavernous sinus tibular enhancement into the fundus and the inner ear on thin post-contrast FS T1-WI and 3D-FLAIR. Additionally, heavily T2-WI shows a thickening of the nerves into the internal auditory canal and the inner ear. Comparison with the contralateral side is very helpful [21]. Other viruses such as HIV may also cause neuritis $[5,22]$.

\subsubsection{Infectious Meningitis}

$\mathrm{CN}$ disorders may reveal meningeal diseases. Indeed, a CSF study is often necessary to set the final diagnosis. In case of meningitis, $\mathrm{CN}$ disorders may consist in a non-specific abducens palsy (VI) to a multiple and bilateral CN palsies associated with a meningeal syndrome. 
Neuroimaging would demonstrate a meningeal enhancement of 3D post-contrast CT, T1, and Flair images. Leptomeningitis is characterized by an enhancement and a thickening of the leptomeninges contiguous to the CNS. Pachymeningitis is characterized by an enhancement and a thickening of the pachymeninges contiguous to the skull. Additional intraparenchymal lesions are common in case of diffuse meningeal diseases, suggestive of meningoencephalitis. Besides pyogenes meningitis, tuberculosis is a common bacterial cause of leptomeningitis involving the skull base and the brainstem (Fig. 12.3). This meningeal inflammation may cause a regional vasculitis and ischemic stroke.

\subsubsection{Skull Base Infections}

Multiple unilateral $\mathrm{CN}$ disorders are suggestive of skull base disease. In addition to clinical and biological data, CT and post-contrast MRI allow to identify symptomatic osteomyelitis usually secondary to naso-sinusal and otomastoid infections.

\subsubsection{Non Infectious Inflammatory Diseases}

\subsubsection{Non-infectious Meningitis}

Among non-infectious leptomeningitis, neurosarcoidosis is the most common cause of $\mathrm{CN}$ disorders. An additional diabetes insipidus is classical. Besides a diffuse leptomeningeal enhancement of post-contrast FS 3D-FLAIR and 3DT1-WI, MRI shows the loss of the bright spot of the posterior pituitary on non-enhanced T1 with thickened infundibulum and stalk.

Non-infectious pachymeningitis is a possible additional feature of granulomatosis and systemic diseases such as Wegener's disease, Langerhans cells, and non-Langerhans cells histiocytosis. Besides pachymeningeal thick enhancement and tumor-like appearance, a possible infundibular and stalk lesion responsible of diabetes insipidus, CT is useful to identify lytic lesions of the skull and the face.

\subsubsection{Tolosa-Hunt Syndrome}

Tolosa-Hunt syndrome is an idiopathic non-neoplastic noninfectious inflammation of the cavernous sinus and the orbital apex. A unilateral painful ophthalmoplegia usually reveals the disease. MR demonstrates an abnormal apical enhancement extended to the cavernous sinus and Meckel's cave that are enlarged with a lateral bulging. After having excluded infections and neoplasms on clinical, biological, and imaging presentation, high dose of steroids is required. Normal MRI at 6-months follow-up is required to set the final diagnosis [23] (Fig. 12.3).

Idiopathic inflammatory infiltration can also be detected in orbital pseudotumor as a T2 hypointense ill-defined infiltration which enhances on post-contrast FS T1-WI. Additional infiltration of the pituitary, infundibulum, lacrimal, and salivary glands is possible in case of IgG4-related disease. A thickening of infraorbitary nerve $\left(\mathrm{V}_{2}\right)$ would very suggestive [24].

\subsubsection{Tumors and Cysts}

\subsubsection{Neural Sheath Tumors}

Schwannoma is the most common neural tumor. This tumor that arises from the nerve sheath after the $\mathrm{TZ}$ may involve any $\mathrm{CN}$ but I and II that do not have Schwann cells. Most common $\mathrm{CN}$ are the vestibular nerve (VIII) within the cerebellopontine angle and the internal auditory meatus, the trigeminal nerve (V) in the Meckel's cave, the mixed nerves (IX, X, XII), and the hypoglossal nerve. The tumor is usually well-defined, homogeneously enhanced on post-contrast T1-WI. Cystic changes may occur in large schwannomas. Multiple schwannomas may occur. Indeed, bilateral vestibular schwannomas is specific of neurofibromatosis type 2 (Fig. 12.4).

In case of single neural mass, main differential diagnoses include facial hemangioma (VII), a small intrameatic heterogeneous lesion, and neuroma that may occur after focal insult [16-18]. Perineural spread of extracranial schwannomas may also extend into the skull.

In case of multiple neural tumors, main differential diagnoses include leptomeningeal metastases and carcinomatosis of extracranial solid tumors, leukemia, and lymphoma. Metastases of intraaxial primary neoplasms such as glioblastoma, medulloblastoma, ependymoma, germinoma are also possible. None of these metastases has a specific appearance. Appropriate diagnosis relies on patient's history, poor general condition, CSF study, and whole body imaging (Fig. 12.4)

\subsubsection{Meningeal Tumors}

Primary meningeal tumors such as meningioma are common. Unique or multiple in case of meningiomatosis, meningioma may displace and shear the $\mathrm{CN}$. Meningioma of the planum sphenoidale and the olfactory groove is a classical cause of anosmia (Fig. 12.4). In the cerebellopontine angle, meningioma is the main differential diagnosis of vestibular schwannoma. On CT, meningioma is often associated with hyperostosis, while schwannoma is responsible of a scalloping of the internal auditory meatus. Meningioma may extent anteriorly to the internal auditory meatus, while vestibular schwannoma usually does not. And perfusion imaging demonstrates the hypervascularization of the meningioma.

Intracranial meningiomas may extent throughout the skull base foramina, along the $\mathrm{CN}$ routes. The optic nerve is the nerve which is most frequently involved in its extracranial 

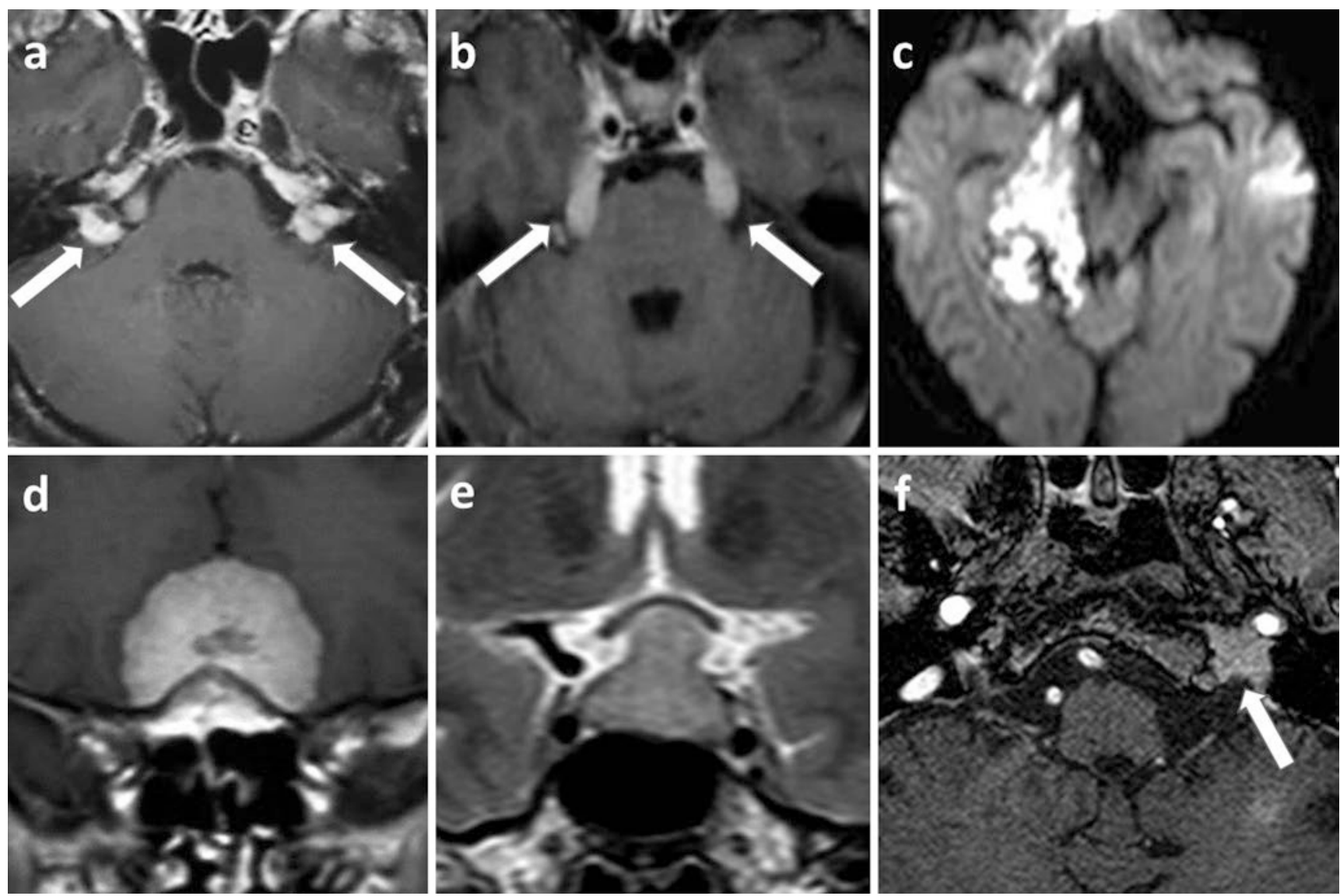

Fig. 12.4 (a) neurofibromatosis type 2, post-contrast T1 bilateral CPA and intrameatic masses, revealed by bilateral sensorineural hearing loss. (b) bilateral trigeminal metastases of leukemia appearing as postcontrast T1 bilateral trigeminal tumoral thickening. (c) epidermoid cyst along the right aspect of the midbrain, DWI hyperintense mass, revealed by right trochlear palsy. (d) meningioma of the cribriform plate, postcontrast $\mathrm{T} 1$ enhancing mass, revealed by anosmia. (e) pituitary mac- roadenoma, intra- and suprasellar mass, that shifts upwards the chiasma was revealed by bitemporal hemianopia. (f) paraganglioma of the left glomus jugular is a contrast-enhanced T1 mass infiltrating the jugular foramen with discrete "salt-and-pepper" appearance on post-contrast FS T1-WI (white arrow) revealed by motor palsies of the left lower nerves (IX-XII) course, followed by the mandibular nerve $\left(\mathrm{V}_{3}\right)$ and the lower cranial nerves IX-XI. Involvement of the later $\mathrm{CN}$ is seen as a mass in the carotid space which must be differentiated from a schwannoma or a paraganglioma. The strong enhancement, dural tail enhancement adjacent to the intracranial component of the meningioma and its iso-intensity with gray matter on all sequences help to recognize them.

Secondary leptomeningeal and pachymeningeal carcinomatosis of CNS tumors and extracranial solid tumors, leukemia, and lymphoma are better revealed on 3D post-contrast T1 and Flair images. Differential diagnosis with granulomatous meningitis can be difficult on imaging only.

\subsubsection{Skull Base Tumors and Tumor-like Lesions}

Multiple unilateral $\mathrm{CN}$ disorders are suggestive of skull base disease. In addition to clinical and biological data, CT and post-contrast MRI allow to identify symptomatic fibrous dysplasia, and bone tumors such as chordoma, chondrocarcinoma, or metastasis.

\subsubsection{Sellar Tumors and Cysts}

\section{Intrasellar and Perisellar Tumors}

Intrasellar and perisellar lesions may cause vision loss, especially bitemporal hemianopia due to chiasmatic compression (Fig. 12.4). Oculomotor disorders may occur in case of extension into the cavernous sinus. Most common lesions include pituitary macroadenoma and craniopharyngioma. The tumor is tissular and cystic-like, respectively. Both enhance on post-contrast images. A common Rathke cleft cyst should not be misdiagnosed.

\section{Rathke Cleft Cyst}

Rathke cleft cyst, a common incidental finding located within the pars intermedia of the pituitary or along the 
pituitary stalk, may rarely cause vision and oculomotor deficit. When symptomatic, the location of the cyst and the presence of small T2 hypointense nodules within the cyst are suggestive. The absence of peripheral and tissular enhancement allows to distinguish the cyst from craniopharyngioma.

\subsubsection{Pineal Tumors and Cysts}

Pineal tumors such as pinealocytoma and germinoma may cause a Parinaud syndrome, an oculomotor disorder associating up-gaze palsy, convergence-retraction nystagmus, and dilated pupils that react to accommodation but not to light. Additional non-specific symptoms are possible, including those related to obstructive hydrocephalus by compression of the aqueduct of the midbrain, and intracranial hypertension. Pineal tumors are suggested in case of nodular enhancement and tissular lesions, even when cystic component is present. Hypercellular germinomas may harbor hyperintense DWI due to restricted diffusion coefficient.

\section{Pineal Cyst}

Pineal cysts are common and usual non-symptomatic, their size is usually below $1 \mathrm{~cm}$. When larger, pineal cyst may cause a Parinaud syndrome and obstructive hydrocephalus that may require surgical CSF derivation in emergency. Pineal cyst contains CSF-like fluid. As protein concentration increases, T1 signal might increase and T2 signal decreases. A thin peripheral enhancement is possible while nodular and tissular enhancements suggest pineal tumors.

\subsubsection{Other Cysts and Cystic-like Lesions}

Cysts and cystic-like lesions are common findings around the $\mathrm{CNS}$ and $\mathrm{CN}$. These lesions are often incidental findings or may be revealed by $\mathrm{CN}$ disorders secondary to compression.

\section{Epidermoid Cyst}

Epidermoid cyst is related to the inclusion of ectodermal epithelial elements. It can be slow growing and revealed by $\mathrm{CN}$ disorders. The insinuating mass has well-defined borders. CT demonstrated a hypodense, similar to CSF. Marginal calcifications are possible. MR shows a CSF-like cyst that may change with protein concentration. The lesion does not enhance. Besides these features that may suggest an arachnoid cyst, a typical incidental finding in adults, MR demonstrates a typical restricted diffusion with hyperintense appearance (Fig. 12.4). Surgery consists in $\mathrm{CN}$ decompression.

\section{Neurenteric Cyst}

Neurenteric cyst is a congenital endodermal cyst that might arise in front of the medulla and the pons. $\mathrm{CN}$ disorders, such as abducens or mixed nerves palsies (VI), are possible. CT usually shows a prepontine lobular hyperdensity. MRI confirms a cyst with high proteins content as T2 hypointensity T1 spontaneous hyperintensity, no enhancement.

\section{Dermoid Cyst}

Dermoid cyst is well-circumscribed unilocular cyst arising from the midline, often close to the sellar and parasellar region. This squamous epithelial cyst contains dermal elements with fatty compounds from sebaceous secretion. Calcifications are common. Rupture of the cyst causes a chemical meningitis and hydrocephalus. CT and MR easily demonstrate the fat density of the cyst content with parietal calcifications. Rupture is suspected in case of diffuse fatty droplets scattered into the subarchnoïd spaces. Surgical treatment is discussed when symptomatic, such as $\mathrm{CN}$ disorders and rupture in association with CSF drainage.

\section{Lipoma}

Lipoma is a non-neoplastic mass of mature adipose tissue. Usually, lipomas do not grow over time and are incidental findings. However, lipomas might be diagnosed in case of $\mathrm{CN}$ disorders when located onto the quadrigeminal plate or into the cerebellopontine angle. CT demonstrates a homogenous low density mass ( -50 to $-100 \mathrm{HU})$ without calcification. MR demonstrates a hyperintense T1 mass which becomes hypointense after fat saturation. The lesion does not enhance. Surgical removal is difficult. When symptomatic, surgery consists in partial removal for $\mathrm{CN}$ decompression.

\subsubsection{Trauma}

Post-traumatic anosmia is usually due to contusion of olfactory bulbs or to olfactory fibers shearing through the cribriform plate that occur after occipital head trauma. High-resolution T2 WI may illustrate olfactory bulbs injuries. Beside this contrecoup anosmia, all other post-traumatic $\mathrm{CN}$ disorders are usually due to shearing or compression secondary to skull base fracture. Intraforaminal portions of $\mathrm{CN}$ are at risk of compression by surrounding hematoma or bone fragment displacement. Optic nerve contusion and shearing can also be observed.

Skull base fracture including petrous bone might be revealed by $\mathrm{CN}$ disorders. When clinically driven, highresolution $\mathrm{CT}$ is very sensitive to demonstrate to symptomatic fracture. MRI allows to depict adjacent edema and focal hemorrhage like in the internal ear. MRI and CTA may also demonstrate vascular injury such as arterial dissection and venous thrombosis.

Parenchymal herniations may also compress CN. Indeed, $\mathrm{CN}$ disorders may reveal slow growing lesion. Transtentorial herniation of the internal temporal gyrus might be revealed by oculomotor (III) and trochlear (IV) palsies. Transoccipital herniation of the cerebellar amygdala might be revealed by mixed nerves (IX-XI) and hypoglossal nerve palsies. 


\subsubsection{Degeneration}

\subsubsection{Superficial Siderosis}

Meningeal superficial siderosis may cause $\mathrm{CN}$ degeneration and disorders, especially sensorineural hearing loss. Chronic meningeal deposition of hemosiderin occurs in repeated trauma, subarachnoid hemorrhage, and slow growing tumor or in cerebral amyloid angiopathy. MRI demonstrated a characteristic hypointense rim coating the CNS and the $\mathrm{CN}$ on $\mathrm{T} 2 *$ and SWI.

\subsection{Extracranial Lesions}

Primary extracranial $\mathrm{CN}$ diseases are rare and include esthesioneuroblastoma of the olfactory nerves (I) and optic nerve (II) glioma, while schwannoma and neurofibroma may arise from all other nerves but CN VIII which has no extracranial segment.

Most extracranial $\mathrm{CN}$ disorders are secondary to a surrounding disease that may insult the nerves. Unilateral symptoms are most common.

Nasal and ethmoidal diseases may cause anosmia due to olfactory nerves (I) impairment.

Orbital diseases may cause partial or complete monocular visual loss (scotoma) because of optic nerve (I) involvement. Diplopia may be due to oculomotor (III), trochlear (IV), and abducens (VI) nerves dysfunctions. Intraorbital apical CN III lesion associates ptosis and mydriasis when the ciliary ganglion that receives afferent parasympathetic fibers is affected.

Superficial and deep facial lesions may cause trigeminal neuropathy when the extracranial branches of $\mathrm{CN} \mathrm{V}$ are injured. Specific signs may suggest particular branches, especially when unilateral: hypoesthesia, numbness, or burning of the forehead $\left(\mathrm{V}_{1}\right)$, the chick $\left(\mathrm{V}_{2}\right)$, the jaw $\left(\mathrm{V}_{3}\right.$. $)$; diminished or absent corneal reflex (ophthalmic branch $\mathrm{V}_{1}$ ); atrophic or weak muscles of mastication (motor branch $\mathrm{V}_{3}$ ). Facial pain is a very unspecific sign but numbness, progressive symptoms and motor weakness should always be taken seriously and are frequently linked to a structural lesion.

Perimastoid and parotid lesion may cause an extracranial CN VII dysfunction with isolated peripheral facial nerve palsy but preserved lacrimal function, stapedius reflex and taste in the anterior two thirds of the tongue.

In the suprahyoid region, CN IX to XII have close relationships explaining why they are often injured simultaneously. Association of CN IX and X disorder is frequent and result in otalgia, dysphagia, absent gag reflex, uvular deviation away from the side of the lesion, endolaryngeal symptoms (hoarseness, dysphagia in cervical esophagus, vocal cord palsy), loss of taste to the posterior $1 / 3$ of the tongue, tachycardia or bradycardia, etc. Isolated disorders of CN XI and XII are common. CN XI palsy is suggested on shoulder drop and inability to raise the arm. CN XII palsy is diagnosed on deviation of the tongue to the side of the lesion, amyotrophy of the intrinsic and extrinsic tongue muscles, and orobuccal dysarthria.

In the infra-hyoid region, lesions would result in an isolated $\mathrm{CN}$ X injury and vagus related oropharyngeal signs will be absent and only the endolaryngeal symptoms will be present. Muscle denervation atrophy is an indirect sign of involvement of the motor cranial nerves and should always be checked [25].

Besides the above mentioned lesions, neural disorders also occur in case of vasculitides, neurotoxin, after radiotherapy.

Because of the large spectrum of extracranial diseases, here are presented most common etiologies.

\subsubsection{Vascular Lesions}

Intraorbital cavernous hemangioma is an ovoid welldelineated enhancing lesion in the orbit and can compress the optic nerve (Fig. 12.5). Venous varix can be recognized on flow void, vascular enhancement, and size increase on procubitus.

Internal carotid artery dissection or (pseudo)aneurysm may cause deficits of the lower cranial nerves IX-XII, due to the compression on the nerves into the carotid space (Fig. 12.6). These nerves can also be damaged during endarterectomy.

Aneurysms of the aortic arch and even cardiomegaly can cause irritation of the left recurrent laryngeal nerve [26].

\subsubsection{Infectious Diseases}

Aggressive bacterial and fungal infections arising from sinuses, temporal bone, and skull base can insult the adjacent cranial nerves. Aspergillus and mucormycosis can be recognized on the very low signal inside the sinuses on T2-WI and the destruction of the bony walls of the sinuses.

All extracranial cranial nerves close to the sinuses and temporal bone can be affected by fungal infection and aggressive bacterial infection: frontal sinus $\left(\mathrm{V}_{1}\right)$, ethmoid and sphenoid (II, III, IV, $\mathrm{V}_{1}, \mathrm{~V}_{2}, \mathrm{VI}-$ orbital apex and superior orbital fissure), temporal bone (VII). Periapical and periodontal dental disease may cause maxillary $\left(\mathrm{V}_{2}\right)$ and mandibular $\left(\mathrm{V}_{3}\right)$ neuropathy which can be seen on postcontrast FS T1-WI or MR neurography [27].

The facial nerve can be involved in malignant otitis externa, a pseudomonas infection in diabetic or immunocompromised patients. Skull base osteomyelitis can also affect the lower cranial nerves IX-XII while mandibular 

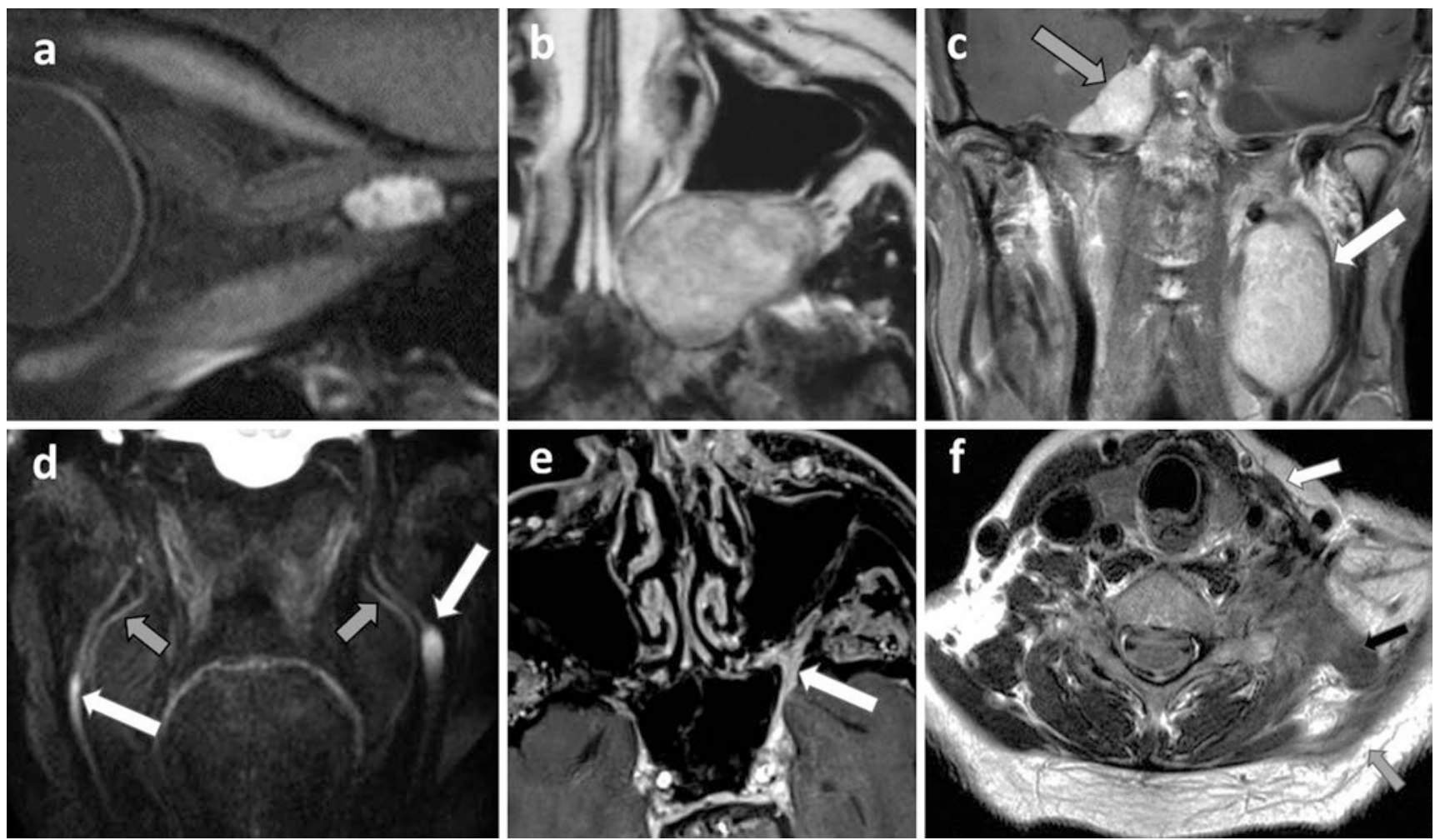

Fig. 12.5 (a) cavernous hemangioma of the orbital apex revealed by unilateral vision loss, the enhancement of the lesion and relation to the optic nerve is clear on this isotropic $0.55 \mathrm{~mm}$ thick post-contrast black blood 3D TSE T1-WI; (b) schwannoma of the left maxillary nerve $\left(\mathrm{V}_{2}\right)$ in the pterygopalatine fossa, as an expansive slightly heterogeneously enhanced on post-contrast T1-WI, scalloping the adjacent bones; (c) neurofibromatosis type 2 with multiple schwannomas of the left vagus nerve (X) (white arrow) and the right Meckel cave (gray arrow) on postcontrast coronal T1-WI; (d) neurofibromas on paracoronal 3D neurog-

raphy with black blood pulse as bilateral thickening and hyperintensity of both inferior alveolar nerves $\left(\mathrm{V}_{3}\right)$ (white arrows), external to lingual nerves (gray arrows). (e) Perineural tumor spread along the left maxillary nerve $\left(\mathrm{V}_{2}\right)$ of an epithelial carcinoma of the nostril (gray arrow), post-contrast FS T1 show a thickened enhancing $V_{2}$ in the pterygopalatine fossa, the rotundum foramen (white arrows); (f) post-traumatic left accessory nerve palsy (XI) with sternocleidomastoid (white arrow), trapezius muscles (gray arrow), and levator scapulae (black arrow) muscles degeneration

osteomyelitis can cause inferior alveolar nerve irritation with pain and numbness. This mandibular osteomyelitis should be differentiated from osteoradionecrosis. Both will result in loss of the high signal inside the mandible and both can enhance. However, osteoradionecrosis can be distinguished when bone sequesters are present, hypointense defects are seen in the cortex and when periosteal bone is formed.

Abscess formation in the carotid space can cause lower cranial nerve IX-XII symptoms and mediastinitis and mediastinal inflammatory adenopathy can cause left sided CN X or recurrent laryngeal nerve deficits.

\subsubsection{Non-infectious Inflammatory Diseases}

\subsubsection{Demyelinating Diseases}

In young adults, spontaneous vision loss is often due to an optic neuritis, which can be a particular feature of demyelin- ating diseases such as multiple sclerosis (MS) and neuromyelitis optica spectrum disorders (NMOsd) with anti-AQP4 or anti-MOG antibodies. Accurate diagnosis assessment is needed because of different treatments with potential deleterious effects when inappropriate. Besides clinical and biological data, whole CNS imaging that includes optic nerves is mandatory. Optic nerve lesions are best identified using post-contrast FS T1-WI and 3D-FLAIR.

In MS, optic neuritis is usually unilateral, short, involving the middle portion of the nerve.

In anti-AQP4+, optic neuritis is usually bilateral, long, involving the posterior portion of the nerves with chiasmatic extension.

In anti-MOG+, optic neuritis is usually bilateral, long, involving the anterior portion of the nerves.

Additional CNS imaging abnormalities are helpful for differential diagnosis.

In MS, MRI demonstrates scattered nodules in the brain and the upper half of spinal cord. Spinal lesions are small 

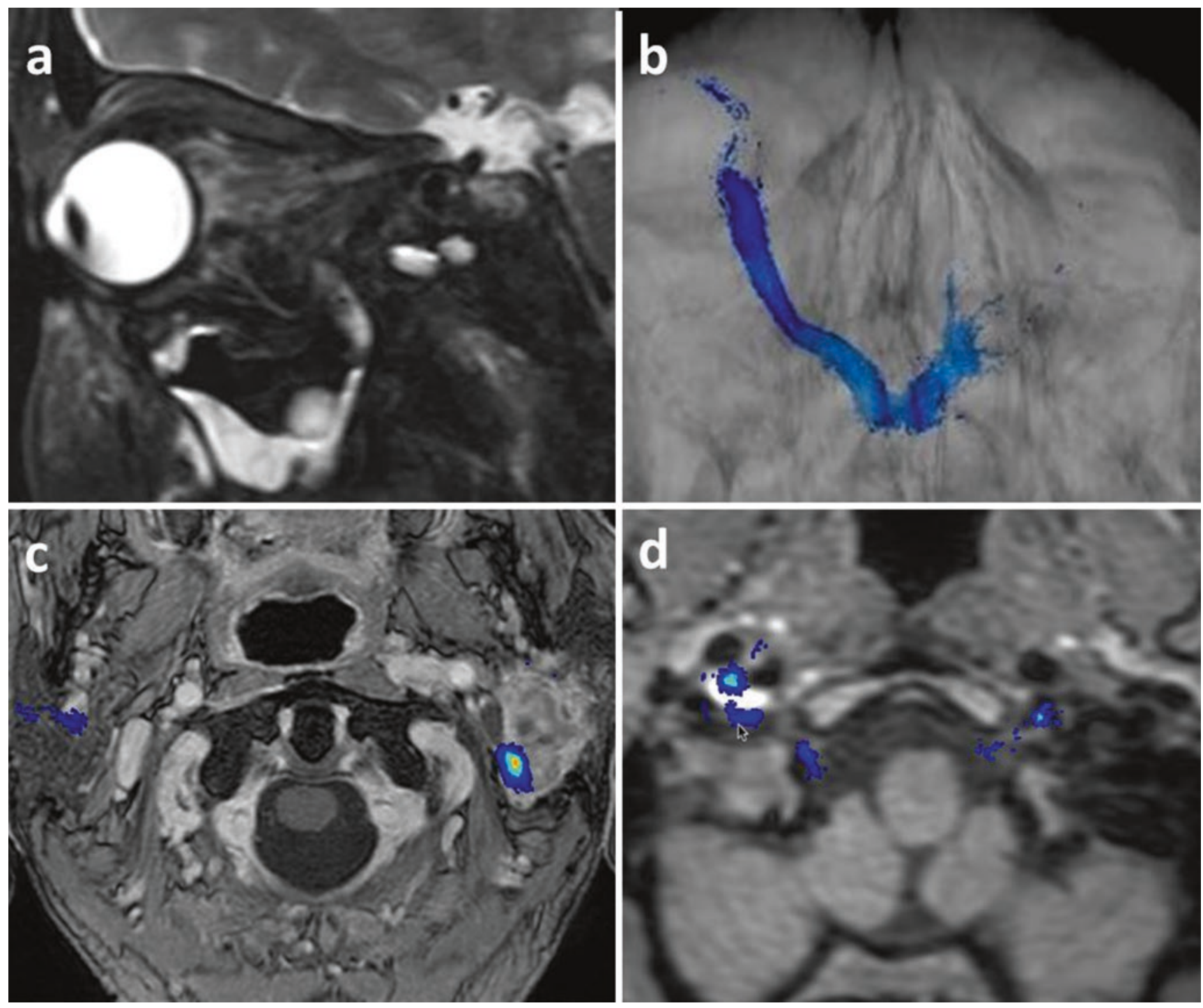

Fig. 12.6 (a, b) post-traumatic left vision loss, sagittal FS T2 (a) shows a retrobulbar edema and an optic nerve hyperintense contusion, optic nerve probabilistic tractography suggests a nerve section of poorer prognosis; (c) facial nerves tractography showing the left posterior dis-

extending over $<2$ vertebral segments on sagittal images, and occupying $<50 \%$ of the cord diameter of axial images. Dissemination in time accounts for plaques of varied contrast uptake.

In anti-AQP4+, brain lesions are mostly periventricular with a suggestive involvement of the area postrema. Spinal cord lesions are usually located in the upper half of the cord, involving three or more vertebral segments on sagittal images, more than $50 \%$ of the cord diameter.

In anti-MOG+, brain lesions are involving more often basal ganglia, thalamus, and the pons. Spinal cord lesions are usually located in the lower half of the cord, involving three or more vertebral segments on sagittal images, more than $50 \%$ of the cord diameter $[14,15]$. placement of the nerve by a parotid cystadenolymphoma; (d) hypoglossal nerves tractography showing the contact with the right internal carotid dissection in a patient referred for a painful hypoglossal palsy

\subsubsection{Granulomatous Diseases}

Extracranial lesions of various granulomatous diseases, such as Wegener's disease, Langerhans cells and non-Langerhans cells histiocystosis, can insult the anterior optic pathways in association with orbital and naso-sinusal lesions. Involvement of the adjacent orbit can occur with potential optic nerve and/ or maxillary nerve involvement. Intracranial extension following the neural routes is possible.

Optic nerves and anterior optic pathways can be affected in $1-5 \%$ of sarcoidosis patients. Sarcoidosis is an inflammatory granulomatosis that can mimic optic nerve meningioma. In these patients careful examination of the systemic signs should lead to the diagnosis. However, bilateral optic nerve involvement, enhancement of the complete optic nerve from 
the globe to the chiasm, bilateral enlargement of lacrimal and parotid glands, leptomeningitis, and infundibular thickening are imaging signs indicative of sarcoidosis $[1,2,5,7]$.

\subsubsection{Orbital Pseudotumor}

Orbital pseudotumor is a benign non-infectious inflammatory condition of the orbit. As a diagnosis of exclusion, systemic causes such as infections and neoplasms should be ruled out. Patients present with sudden painful proptosis, ophthalmoplegia (III, IV, VI), vision loss (II), uveitis and retinal detachment. An ill-defined enhancing pseudo-mass involving any area of the orbit is best detected on postcontrast FS T1-WI. A very fast response to corticosteroid treatment is necessary to confirm the diagnosis.

\subsubsection{Grave's Ophtalmopathy}

Graves' disease is an autoimmune disease affecting the thyroid and Graves' ophtalmopathy is found in more than $25 \%$ of the patients. Extraocular muscle hypertrophy and adipogenesis cause increased ocular pressure and proptosis, possible diplopia (extraocular muscle involvement) and vision loss (II).

\subsubsection{Tumors}

\subsubsection{Primary Nerve Lesions}

\section{Esthesioneuroblastoma}

Esthesioneuroblastoma or olfactory neuroblastoma is a rare tumor arising from the olfactory neurosensory receptor cells in the olfactory epithelium of the high nasal vault. Nasal obstruction, rhinorrhea, epistaxis, anosmia, headaches are common. CT and MRI show a dumbbell tissular mass, enhanced on post-contrast images with peripheral cysts. Bone destruction allows spreading into the ethmoid and the sphenoid sinuses, through the cribriform plate and the anterior cranial fossa.

\section{Optic Nerve Glioma}

As the optic nerve is an extension of the brain, primary tumor is an optic nerve glioma, usually a pilocytic astrocytoma. This glioma occurs under the age of 20 years. The typical appearance is a fusiform lesion on the optic nerve with T2 hyperintensity and a variable enhancement. It causes a kinking of the optic nerve and the lesion may reach the optic chiasm. Optic nerve glioma can be associated with neurofibromatosis type I (NF1), especially when bilateral. NF1associated optic tumors rarely extend beyond the optic pathway and are usually not cyst-like, whereas those not associated with NF1 usually involve the optic chiasm, extend extra-optically, and are frequently cyst-like [28].

\subsubsection{Nerve Sheath Tumors}

As extracranial extension of CNS, intraorbital optic nerves are surrounded by CSF and meningeal sheath. Optic nerve sheath meningioma appears as a fusiform mass surrounding the optic nerve. Post-contrast T1-WI demonstrates a perineural homogeneous enhancement with a "tram track" appearance. CT may show linear calcifications. CSF may be trapped between the tumor and the eyeball.

Optic nerves and olfactory tracts and bulbs have no Schwann cells. However, the olfactory filia which are connecting the olfactory mucosa with the olfactory bulbs do have Schwann cells and hence "olfactory schwannomas" are reported although they do not originate on the olfactory nerve itself.

All other $\mathrm{CN}$ but VIII have an extracranial course with Schwann cells on which nerve sheath tumors can arise, including schwannomas, neurofibromas, and rarely malignant tumors.

They are less often found on the short orbital apex segment of CN III, IV, and VI. The chance to find such tumors on the end branches of the trigeminal nerve (V) is higher (Fig. 12.5) but far less frequent than on its intracranial portion.

Nerve sheath tumors along intraparotid branches of $\mathrm{CN}$ VII do occur but one is most frequently dealing with extensions of a mastoid segment lesion into the parotid gland.

Nerve sheath tumors can be detected on all four lower cranial nerves but schwannomas of $\mathrm{CN} \mathrm{X}$ are most frequent. Extracranial schwannomas of CN XII frequently pass through the hypoglossal canal into subarachnoid space. Extracranial schwannomas of CN IX and XI are rare.

Schwannomas are well-circumscribed, fusiform in shape and have intense enhancement (Fig. 12.5). Cystic, fatty, and hemorrhagic degeneration are possible. Schwannomas are solitary and sporadic in most cases in old adults. However, autosomic dominant neurofibromatosis type 2 (NF2) is likely when multiple schwannomas are present, and bilateral vestibular schwannomas are pathognomonic. In case of hereditary NF2, patients are younger. And the tumoral onset occurs at a younger age than the affected parent.

Schwannomas might be difficult to distinguish them from neurofibromas, discovered at about 20-30 years. Neurofibromas appear as a mild to non-enhancing thickening of a longer segment of the nerve (Fig. 12.5). Similarly, most neurofibromas are solitary and sporadic. However multiple neurofibromas and plexiform neurofibromas almost assure the diagnosis of NF1.

\subsubsection{Secondary Nerve Involvement}

\section{Head and Neck Malignancies}

Squamous cell carcinomas, adenoid cystic carcinomas, adenocarcinomas, lymphomas, melanomas arising in the face, 
sinus, orbit, supra- and infra-hyoid neck can all cause displacement of or invasion of the extracranial $\mathrm{CN}$.

The causative tumors can be different in more specific areas like uveal melanoma and retinoblastoma for the optic nerve, esthesioneuroblastoma for the olfactory bulbs and ophthalmic nerve $\left(\mathrm{V}_{1}\right)$, rhabdomyosarcoma $\left(\mathrm{V}_{3}\right)$, malignant parotid gland tumors such as adenoid cystic carcinoma and mucoepidermoid carcinoma for the facial nerve (VII) and bronchogenic carcinoma and thyroid malignancies for the infra-hyoid vagus nerve (recurrent laryngeal nerve) (X).

Tumoral infiltration of extracranial nerves could be difficult to see but the presence of a tumor at the $\mathrm{CN}$ endpoint or along its extracranial route allows to make the diagnosis. Post-contrast FS T1-WI are helpful to follow thickened enhanced CN. Diffusion tractography is promising for clinical practice, showing nerve displacement and suggesting tumoral infiltration [11] (Fig. 12.6).

It should not be forgotten that the left recurrent laryngeal nerve, branch of the vagus nerve $(\mathrm{X})$, passes through the aortopulmonary window and can therefore be affected by mediastinal adenopathies and malignancies of the aerodigestive tract.

\section{Perineural Tumor Spread}

Adenoid cystic carcinoma, squamous cell carcinoma and lymphoma are the most frequent malignant head and neck lesions with perineural spread, although other malignant tumors also have the potential to follow the cranial nerves [29]. The most frequently involved nerves are the $V_{1}$ (skin and lacrimal malignancies), $\mathrm{V}_{2}$ (skin, oral and nasal cavity and sinus malignancies) (Fig. 12.5), $\mathrm{V}_{3}$ (oral cavity-sublingual and submandibular gland and masticator space malignancies) [30], VII (parotid malignancies), and rarely the CN XII.

The perineural spread can be seen as an abnormal enhancement and thickening of the nerve on post-contrast FS T1-WI (Fig. 12.5). Comparison with the contralateral nerve facilitates the diagnosis. It is crucial to detect intracranial $\mathrm{CN}$ extension as it diminished therapeutic options and result in a poorer prognosis.

CN V and VII have several connections, often involved in perineural tumor spread, which allow tumoral spread along both nerves and their branches. Involvement of the connection between the maxillary nerve $\left(\mathrm{V}_{2}\right)$ and vidian nerve (VII) at the level of the pterygopalatine fossa is the most frequently involved connection. Hence, the pterygopalatine fossa should always be checked in detail as its involvement often heralds intracranial extension along the above mentioned branches.

\section{Paragangliomas}

Paragangliomas are vascular tumors arising from glomus bodies located along carotid bulb and the vagus nerve in the carotid space, the jugular foramen, and the middle ear cavity. They are easy to recognize on MRA as the typical fast blood flow in the arterial feeders can be recognized on these images, setting the final diagnosis. A "salt-andpepper" appearance on post-contrast T1-WI is also classical (Fig. 12.4). These lesions can cause compression on any of the four lower $\mathrm{CN}$ into the carotid space. It is important to detect possible intracranial extension for surgical planning.

\section{Metastases}

Hematogenous metastases from malignant breast, kidney, lung, melanoma, prostate tumors to the head and neck region can involve all extracranial nerves with secondary $\mathrm{CN}$ disorders.

In the orbit, metastases from breast carcinomas are most frequent and compression on the nerve by orbital metastases is far more frequent than direct metastasis to the optic nerve itself.

Other CN III-XII can all be directly involved by the above mentioned metastases or indirectly by metastatic lymph nodes. CN involvement is best seen on post-contrast FS T1-WI in both axial and coronal planes.

\subsubsection{Trauma}

In addition to skull base fractures that threaten foraminal segment of $\mathrm{CN}$, facial bones fractures may also insult extracranial segment of CN (Fig. 12.6).

Branches of the trigeminal nerve (V) are at risk of injury, especially in case of: orbital roof and superior orbital fissure fracture $\left(\mathrm{V}_{1}\right)$; orbital floor and inferior orbital fissure fracture $\left(\mathrm{V}_{2}\right)$, mandible (collum and between the mandibular and the mental foramina) and central skull base fractures $\left(\mathrm{V}_{3}\right)$.

Optic canal fracture threatens $\mathrm{CN}$ II and orbital apex fracture CN III, IV, VI.

$\mathrm{CT}$ is needed to identify fractures and bone fragments displacement which damage $\mathrm{CN}$. $\mathrm{CN}$ contusions or shears are best seen on MR inversion recovery images through the nerves. Post-traumatic neuropathy can be recognized as thickened hyperintense nerves on MR neurography [8].

$\mathrm{CN}$ disorders may also be iatrogenic after sinonasal and periorbital surgery (II, III, IV, VI), tooth implant and mandibular surgery $\left(\mathrm{V}_{3}\right)$, parotid surgery (VII), etc. Neck dissections can result in lower $\mathrm{CN}$ deficits (Fig. 12.5) and surgery in the lower neck can even damage the recurrent laryngeal nerve causing vocal cord palsy. The postsurgical scar formation replaces the fat around the nerves on T1-WI and can enhance in the early postsurgical period. 


\subsection{Conclusion}

Cranial nerves disorders are frequent and reveal a broad variety of diseases. Imaging investigation has to be conducted in agreement with the medical history and presentation. Imaging $\mathrm{CN}$ coverage has to be discussed from nucleus to target. CT and CTA are useful to rule out symptomatic aneurysm and dissection and to better identify skull lesions and calcifications. MRI remains the best imaging modality for $\mathrm{CN}$, and diffusion tractography can illustrate $\mathrm{CN}$ structural changes.

\section{Take Home Messages}

- Master anatomy and function to fully explore $\mathrm{CN}$ from nucleus to target

- Rule out intracranial lesions that require specific treatment.

- Rule out aneurysm and dissection when painful CN disorder using CTA and MRA

- MRI relies on thin slices and 3D, including heavily T2-WI, FLAIR, T1, fat saturation, pre- and postcontrast. Consider optional DWI/DTI and SWI.

Acknowledgments We acknowledge the help of Dr. Arnaud Attyé and Dr. Timothée Jacquesson.

\section{References}

1. Barkhof F, Jäger R, Thurnher M, Rovira A, editors. Clinical neuroradiology: the ESNR textbook. Cham: Springer; 2019. 2272 pp.

2. Casselman J, Mermuys K, Delanote J, Ghekiere J, Coenegrachts K. MRI of the cranial nerves--more than meets the eye: technical considerations and advanced anatomy. Neuroimaging Clin N Am. 2008;18:197-231, preceding $\mathrm{x}$.

3. Chapman $\mathrm{P}$, Harnsberger $\mathrm{H}$, Vattoth $\mathrm{S}$, editors. Imaging anatomy: head and neck. Philadelphia: Elsevier; 2019. 700 pp.

4. Harnsberger H. Cranial nerves. In: Harnsberger H, Osborn A, Macdonald A, Ross J, editors. Diagnostic and surgical imaging anatomy: brain, head and neck, spine. Salt Lake City: Amirsys; 2006. p. 174-259.

5. Koch B, Hamilton B, Hudgins P, Harnsberger H, editors. Diagnostic imaging: head and neck. 3rd ed. Philadelphia: Elsevier; 2017. $1352 \mathrm{pp}$

6. Leblanc A, editor. Encephalo-peripheral nervous system: vascularisation, anatomy, imaging. Berlin: Springer; 2004. 420 pp.

7. Osborn A, Hedlund G, Salzman K, editors. Osborn's brain. 2nd ed. Philadelphia: Elsevier; 2018. 1300 pp.

8. Romano N, Federici M, Castaldi A. Imaging of cranial nerves: a pictorial overview. Insights Imaging. 2019;10:33.
9. Jacquesson T, Cotton F, Attye A, Zaouche S, Tringali S, Bosc J, Robinson P, Jouanneau E, Frindel C. Probabilistic Tractography to predict the position of cranial nerves displaced by skull base tumors: value for surgical strategy through a case series of 62 patients. Neurosurgery. 2019;85:E125-36.

10. Attye A, Jean C, Remond P, et al. Track-weighted imaging for neuroretina: evaluations in healthy volunteers and ischemic optic neuropathy. J Magn Reson Imaging. 2018;48:737.

11. Rouchy RC, Attye A, Medici M, Renard F, Kastler A, Grand S, Tropres I, Righini CA, Krainik A. Facial nerve tractography: a new tool for the detection of perineural spread in parotid cancers. Eur Radiol. 2018;28:3861-71.

12. Attye A, Karkas A, Tropres I, et al. Parotid gland tumours: MR tractography to assess contact with the facial nerve. Eur Radiol. 2016;26:2233-41.

13. van der Zijden T, Mondelaers A, Yperzeele L, Voormolen M, Parizel P. Current concepts in imaging and endovascular treatment of acute ischemic stroke: implications for the clinician. Insights Imaging. 2019;10:64.

14. Dutra BG, Jose da Rocha A, Nunes RH, Martins Maia Junior AC. Neuromyelitis optica spectrum disorders: spectrum of MR imaging findings and their differential diagnosis-erratum. Radiographics. 2018;38:662.

15. Dutra BG, da Rocha AJ, Nunes RH, Maia ACMJ. Neuromyelitis optica spectrum disorders: spectrum of MR imaging findings and their differential diagnosis. Radiographics. 2018;38:169-93.

16. Bonneville F, Savatovsky J, Chiras J. Imaging of cerebellopontine angle lesions: an update. Part 2: intra-axial lesions, skull base lesions that may invade the CPA region, and non-enhancing extraaxial lesions. Eur Radiol. 2007;17:2908-20.

17. Bonneville F, Savatovsky J, Chiras J. Imaging of cerebellopontine angle lesions: an update. Part 1: enhancing extra-axial lesions. Eur Radiol. 2007; 17:2472-82.

18. Krainik A, Cyna-Gorse F, Bouccara D, Cazals-Hatem D, Vilgrain V, Denys A, Rey A, Sterkers O, Menu Y. MRI of unusual lesions in the internal auditory canal. Neuroradiology. 2001;43:52-7.

19. Adams ME, Linn J, Yousry I. Pathology of the ocular motor nerves III, IV, and VI. Neuroimaging Clin N Am. 2008;18:261-82, preceding $\mathrm{X}-\mathrm{x}$.

20. Haller S, Etienne L, Kovari E, Varoquaux AD, Urbach H, Becker M. Imaging of neurovascular compression syndromes: trigeminal neuralgia, hemifacial spasm, vestibular Paroxysmia, and glossopharyngeal neuralgia. AJNR Am J Neuroradiol. 2016; 37:1384-92.

21. Kuya J, Kuya K, Shinohara Y, Kunimoto Y, Yazama H, Ogawa T, Takeuchi $\mathrm{H}$. Usefulness of high-resolution 3D multi-sequences for peripheral facial palsy: differentiation between Bell's palsy and Ramsay hunt syndrome. Otol Neurotol. 2017;38:1523-7.

22. Veillon F, Taboada LR, Eid MA, Riehm S, Debry C, Schultz P, Charpiot A. Pathology of the facial nerve. Neuroimaging Clin N Am. 2008;18:309-20, x.

23. Zurawski J, Akhondi H. Tolosa-hunt syndrome--a rare cause of headache and ophthalmoplegia. Lancet. 2013;382:912.

24. Soussan JB, Deschamps R, Sadik JC, et al. Infraorbital nerve involvement on magnetic resonance imaging in European patients with IgG4-related ophthalmic disease: a specific sign. Eur Radiol. 2017;27:1335-43.

25. Smoker WR, Reede DL. Denervation atrophy of motor cranial nerves. Neuroimaging Clin N Am. 2008;18:387-411, xi. 
26. Lee JH, Cheng KL, Choi YJ, Baek JH. High-resolution imaging of neural anatomy and pathology of the neck. Korean J Radiol. 2017;18:180-93.

27. Chhabra A, Bajaj G, Wadhwa V, Quadri RS, White J, Myers LL, Amirlak B, Zuniga JR. MR neurographic evaluation of facial and neck pain: normal and abnormal craniospinal nerves below the skull base. Radiographics. 2018;38:1498-513.
28. Louis D, Ohgaki H, Wiestier O, Cavenee W, editors. WHO classification of tumors of the central nervous System. 4th ed. Lyon: IARC; 2016.

29. Maroldi R, Farina D, Borghesi A, Marconi A, Gatti E. Perineural tumor spread. Neuroimaging Clin N Am. 2008;18:413-29, xi.

30. Borges A, Casselman J. Imaging the trigeminal nerve. Eur J Radiol. 2010;74:323-40.

Open Access This chapter is licensed under the terms of the Creative Commons Attribution 4.0 International License (http://creativecommons. org/licenses/by/4.0/), which permits use, sharing, adaptation, distribution and reproduction in any medium or format, as long as you give appropriate credit to the original author(s) and the source, provide a link to the Creative Commons license and indicate if changes were made.

The images or other third party material in this chapter are included in the chapter's Creative Commons license, unless indicated otherwise in a credit line to the material. If material is not included in the chapter's Creative Commons license and your intended use is not permitted by statutory regulation or exceeds the permitted use, you will need to obtain permission directly from the copyright holder. 
Part II

Brain and Spine 


\title{
Demyelinating Diseases of the CNS (Brain and Spine)
}

\author{
Frederik Barkhof and Kelly K. Koeller
}

\section{Learning Objectives}

- To be familiar with the differential diagnosis of white matter diseases

- Understand the importance of clinical setting, mode of presentation and lab results in idiopathic inflammatory disorders

- Be able to apply MS and NMO diagnostic criteria

- Appreciate the value of spinal cord imaging in the work-up of MS

- Recognize the most important variants and mimics of MS

\section{Key Points}

- Age-related white matter changes are extremely prevalent and affect the deep white matter with sparing of the U-fibers and spinal cord

- The McDonald criteria for MS include cortico/juxtacortical, periventricular, infratentorial and spinal cord lesions

- Longitudinally extensive spinal cord lesions and large/atypical brain lesions should prompt antibody testing to rule out NMOSD

- The differential diagnosis of symmetric white lesions includes toxic and metabolic disorders

F. Barkhof $(\bowtie)$

Department of Radiology and Nuclear Medicine, VU University

Medical Center, Amsterdam, The Netherlands

UCL Institutes of Neurology and Healthcare Engineering,

London, UK

e-mail: f.barkhof@vumc.nl

\section{K. K. Koeller}

Section of Neuroradiology, Department of Radiology,

Mayo Clinic, Rochester, MN, USA

\subsection{Introduction}

Demyelinating disorders of the central nervous system (CNS) that affect the brain and spine have a variety of etiologies and can be separated into primary such as multiple sclerosis (MS) and other idiopathic inflammatory-demyelinating diseases (IIDDs) and secondary (e.g., infectious, ischemic, metabolic, or toxic) diseases. MRI is the imaging modality of choice to assess demyelinating disorders of the brain and the cord and, together with the clinical and laboratory findings, can accurately classify them in most cases [1-3]. This review will highlight the important imaging manifestations of some acquired demyelinating diseases that allow more specific diagnosis.

\subsection{Idiopathic Inflammatory- Demyelinating Diseases (IIDDs) of the CNS}

The term IIDD encompasses a broad spectrum of CNS disorders that can be differentiated according to their severity, clinical course, and lesion distribution, as well as their imaging, laboratory, and pathological findings. The spectrum includes monophasic, multiphasic, and progressive disorders, ranging from highly localized forms to multifocal or diffuse variants [2]. Relapsing-remitting (RR) and secondary progressive (SP) MS are the two most common forms of IIDD. MS can also have a progressive course from onset (primary progressive [PP]). Fulminant forms of IIDD include a variety of disorders that have in common the severity of the clinical symptoms, an acute clinical course, and atypical findings on MRI. The classic fulminant IIDD is Marburg disease but is extremely rare. Baló's concentric sclerosis and acute disseminated encephalomyelitis (ADEM) can also present with severe, acute attacks.

Some IIDDs have a restricted topographic distribution, as is the case with neuromyelitis optica spectrum disorders (NMOSD), which can have a monophasic, but more often follows a relapsing course. 


\subsection{Multiple Sclerosis}

MS is a progressive inflammatory, demyelinating and neurodegenerative autoimmune disease characterized pathologically by perivascular infiltrates of mononuclear inflammatory cells, demyelination, and axonal loss and gliosis, with the formation of focal and diffuse abnormalities in the brain and spinal cord, mainly affecting the optic nerves, brainstem, spinal cord, and cerebellar and periventricular white matter, although cortical and subcortical gray matter damage is also prominent, resulting in chronic progressive disability for the majority of people with the disorder.

The high sensitivity of MRI in depicting brain and spinal cord demyelinating plaques has made this technique the most important paraclinical tool in current use, not only for the early and accurate diagnosis of MS, but also for understanding the natural history of the disease and monitoring and predicting the efficacy of disease-modifying treatments [4].

MRI is the most sensitive imaging technique for detecting MS plaques throughout the brain and spinal cord. Proton density (PD) or T2-weighted MR images (especially acquired using the fluid-attenuated inversion recovery (FLAIR) sequence) show areas of high signal intensity in the periventricular white matter in $>90 \%$ of MS patients (the remainder may have cord lesions only). MS plaques are generally round to ovoid in shape and range from a few millimeters to more than $1 \mathrm{~cm}$ in diameter. They are typically discrete and focal at the early stages of the disease, but become confluent as the disease progresses, particularly in the posterior hemispheric periventricular white matter. MS plaques tend to affect the periventricular and juxtacortical white matter, whereas small vessel ischemic lesions tend to involve the deep white matter more [4]. The total $\mathrm{T} 2$ lesion volume of the brain increases by approximately 5-10\% each year in the relapsing forms of MS.

Both acute and chronic MS plaques appear hyperintense on T2/FLAIR sequences, reflecting their increased tissue water content. The signal increase indicates edema, inflammation, demyelination, reactive gliosis, and/or axonal loss in proportions that differ from lesion to lesion. The vast majority of MS patients have at least one ovoid periventricular lesion, whose major axis is oriented perpendicular to the outer surface of the lateral ventricles. The ovoid shape and perpendicular orientation derive from the perivenular location of the demyelinating plaques noted on histopathology (Dawson's fingers).

MS lesions tend to affect specific regions of the brain, including the periventricular white matter, the inferior surface of the corpus callosum, the cortico-juxtacortical regions, the temporal lobes, and the infratentorial regions. Focal involvement of the periventricular white matter in the anterior temporal lobes is typical for MS and rarely seen in other white matter disorders, especially not in aging/hypertension (see table below). The lesions commonly found at the calloso-septal interface are best depicted by sagittal
T2-FLAIR images-a sequence highly recommended for diagnostic MRI studies.

Characteristic differences between small-vessel disease (SVD) and MS

\begin{tabular}{|l|l|l|}
\hline Involvement & SVD & MS \\
\hline Corpus callosum & Rare & Common \\
\hline U-fibers & Rare & Often \\
\hline Brainstem & Central pons & Peripheral \\
\hline Temporal lobe & Never $^{\mathrm{a}}$ & Often \\
\hline $\begin{array}{l}\text { Gadolinium } \\
\text { enhancement }\end{array}$ & $\begin{array}{l}\text { Exceptional } \\
\text { (subacute infarction) }\end{array}$ & Common \\
\hline Black holes & Rare & Typical \\
\hline Lacunes & Typical & Never \\
\hline Spinal cord & Never & Common \\
\hline
\end{tabular}

${ }^{a}$ With the exception of cerebral autosomal dominant arteriopathy with subcortical infarcts and leukoencephalopathy (CADASIL)

Histopathological studies have shown that a substantial portion of the total brain lesion load in MS is located within the cerebral cortex. Presently available MRI techniques are not optimal for detecting cortical lesions because of poor contrast resolution between normal-appearing gray matter (NAGM) and the plaques in question, and because of the partial volume effects of the subarachnoid spaces and CSF surrounding the cortex. Cortical lesions are better visualized by 2D or 3D FLAIR sequences and newer MR techniques such as 3D double inversion recovery (DIR) MR sequences which selectively suppress the signal from white matter and cerebrospinal fluid (CSF). Juxtacortical lesions that involve the "U" fibers are seen in two-thirds of patients with MS.

Posterior fossa lesions preferentially involve the floor of the fourth ventricle, the middle cerebellar peduncles, and the brainstem. Most brainstem lesions are contiguous with the cisternal or ventricular CSF spaces, and range from large confluent patches to solitary, well-delineated paramedian lesions or discrete "linings" of the CSF border zones. Predilection for these areas is a key feature that helps to identify MS plaques and to differentiate them from focal areas of ischemic demyelination and infarction that preferentially involve the central pontine white matter.

Approximately $10-20 \%$ of $\mathrm{T} 2$ hyperintensities are also visible on T1-weighted images as areas of markedly decreased signal intensity compared with gray matter. These so-called T1 black holes have a different pathological substrate that depends, in part, on the lesion age. Temporary hypointensity is present in up to $80 \%$ of recently formed lesions and probably represents marked edema, with or without myelin destruction or axonal loss. In most cases, the acute lesions become isointense within a few months as inflammatory activity abates, edema resolves, and reparative mechanisms like remyelination become active. Less than $40 \%$ evolve into persisting or chronic "black holes" [5], which correlate pathologically with the most severe demye- 
lination and axonal loss, indicating areas of irreversible tissue damage. Chronic black holes are more frequent in patients with progressive disease than in those with RRMS disease, and more frequent in the supratentorial white matter as compared with the infratentorial white matter. They are rarely found in the spinal cord and optic nerves.

MS lesions of the spinal cord resemble those in the brain. The lesions can be focal (single or multiple) or diffuse, and mainly affect the cervical cord segment. On sagittal scans, the lesions characteristically have a cigar shape and rarely exceed two vertebral segments in length (the so-called shortsegment lesions in contrast to longitudinally extensive lesions in NMO). On cross-section, they typically occupy the lateral and posterior white-matter columns, border the surface but may extend to involve the central gray matter, and rarely occupy more than one half the cross-sectional area of the spinal cord [6].

Acute spinal cord lesions can produce a mild to moderate mass effect with some spinal cord swelling and may show contrast enhancement. Active lesions are rarer in the spinal cord than the brain and are more frequently associated with new clinical symptoms. The prevalence of spinal cord abnormalities is as high as 74-92\% in established MS, and depends on the clinical phenotype of MS. Asymptomatic spinal cord lesions are found in 30-40\% of patients with a clinically isolated syndrome (CIS), even if the presenting symptoms do not involve the spinal cord clinically. In RRMS, the spinal cord lesions are typically multifocal. In secondary progressive MS, the abnormalities are more extensive and diffuse and are commonly associated with spinal cord atrophy. In primary progressive MS, spinal cord abnormalities are quite extensive as compared with brain abnormalities. This discrepancy may help to diagnose primary progressive MS in patients with few or no brain abnormalities.

Longitudinal and cross-sectional MR studies have shown that the formation of new MS plaques is often associated with contrast enhancement, mainly in the acute and relapsing stages of the disease [7]. The gadolinium enhancement varies in size and shape, usually lasting a few weeks, although steroid treatment shortens this period. Incomplete ring enhancement on T1-weighted gadolinium-enhanced images with the open border facing the gray matter of the cortex or basal ganglia is a common finding in active MS plaques and is a helpful feature for distinguishing between inflammatory-demyelinating lesions and other focal lesions such as tumors or abscesses which will have a closed ring of enhancement [8].

Contrast enhancement is a relatively good predictor of further enhancement and of subsequent accumulation of T2 lesions, but shows no (or weak) correlation with progression of disability and development of brain atrophy. In RRMS and early SPMS, enhancement is more frequent during relapses and correlates well with clinical activity. For patients with primary progressive MS, serial T2-weighted studies show few new lesions and less frequent enhancement.
Contrast-enhanced T1-weighted images are routinely used in the study of MS to provide a measure of inflammatory activity in vivo.

MRI-based disease activity is 5-10 times more frequently than clinical evaluation of relapses, suggesting that most of the enhancing lesions are clinically silent. Subclinical disease activity with contrast-enhancing lesions is 4-10 times less frequent in the spinal cord than the brain, a fact that may be partially explained by the large volume of brain as compared with spinal cord. High doses of gadolinium and a long post-injection delay can increase the detection of active spinal cord lesions.

The diagnosis of MS relies heavily on MRI information, certainly when there is a clinically isolated syndrome suggestive of demyelination. MRI can be used to show dissemination in space (lesions 2 of 4 locations - juxtacortical, periventricular, infratentorial or spinal cord) and to demonstrate dissemination in time (enhancement or new $\mathrm{t} 2$ lesions at follow-up).

\subsection{Multiple Sclerosis Variants}

\subsubsection{Baló's Concentric Sclerosis}

Baló's concentric sclerosis is a rare IIDD subtype, considered a variant of MS, with characteristic radiologic and pathologic features. The disease was formerly considered an aggressive MS variant, leading to death in weeks to months after onset, and in which the diagnosis was made on histopathologic findings at postmortem examination. However, with the widespread use of MRI, this MS variant is often identified in patients who later have a complete or almost complete clinical recovery [9]. The pathologic hallmarks of are large demyelinated lesions showing a peculiar pattern of alternating layers of preserved and destroyed myelin. One possible explanation for this pattern is that sublethal tissue injury is induced at the edge of the expanding lesion, which would then stimulate expression of neuroprotective proteins to protect the rim of peri-plaque tissue from damage, thereby resulting in alternating layers of preserved and non-preserved myelinated tissue [10]. These alternating bands are best identified with T2-weighted sequences, which typically show thick concentric hyperintense bands corresponding to areas of demyelination and gliosis, alternating with thin isointense bands corresponding to normal myelinated white matter. This pattern can be also identified on T1-weighted images as alternating isointense (preserve myelin) and hypointense (demyelinated) concentric rings. These bands, which may eventually disappear over time, can appear as multiple concentric layers (onion-skin lesion), as a mosaic, or as a "floral" configuration. The center of the lesion usually shows no layering because of massive demyelination ("storm center"). Restricted diffusion followed by contrast enhance- 
ment is common in the outer rings (inflammatory edge) of the lesion.

The Baló pattern on MRI can be isolated, multiple, or combined with typical MS-like lesions, and the lesion structure can vary from one or two to several alternating bands, with a total size from one to several centimeters. Lesions occur predominantly in the cerebral white matter, although brainstem, cerebellum, and spinal cord involvement has also been reported [11].

\subsubsection{Neuromyelitis Optica Spectrum Disorders}

Neuromyelitis optica (NMO) is an autoimmune inflammatory disorder of the CNS with a predilection for the optic nerves and spinal cord [12]. The discovery of an NMOspecific autoantibody directed against aquaporin-4 (AQP4-Ab), the major water channel in the CNS, clearly identified $\mathrm{NMO}$ as a disease separate from $\mathrm{MS}$ - and requiring a different treatment than MS [13, 14].

This uncommon and topographically restricted form of IIDD is characterized by severe unilateral or bilateral optic neuritis and complete transverse myelitis, which occur simultaneously or sequentially over a varying period (weeks or years). The index events of new-onset NMO are severe unilateral or bilateral optic neuritis, acute myelitis, or a combination of these symptoms. The myelitis attacks appear as complete transverse myelitis with severe bilateral motor deficits, sensory-level, bowel and bladder dysfunction, pain and significant residual neurologic injury. Optic neuritis attacks are generally more severe than those typically seen in MS.

Approximately $85 \%$ of patients have a relapsing course with severe acute exacerbations and poor recovery, which leads to increasing neurologic impairment and a high risk of respiratory failure and death due to cervical myelitis. Patients who experience acute optic neuritis and transverse myelitis simultaneously or within days of each other are much more likely to have a monophasic course. On the other hand, a relapsing course correlates with NMO-IgG seropositivity, a longer interval between attacks, older age at onset, female gender, and less severe motor impairment after the myelitic onset. Although the initial attacks are more severe in patients proven to have monophasic NMO, the long-term neurologic prognosis is somewhat better in this group because patients do not accumulate disability from recurrent attacks.

Clinical features alone are insufficient to diagnose NMO; CSF analysis and MRI are usually required to confidently exclude other disorders. CSF pleocytosis ( $>50$ leukocytes/ $\mathrm{mm}^{3}$ ) is often present, while oligoclonal bands are seen less frequently (20-40\%) than in MS patients (80-90\%). AQP4-Ab detection is best performed using cell-based assays that have greater sensitivity. AQP4-Ab distinguish this form of IIDD from MS and it can predict relapse and conversion to NMO in patients presenting with a single attack of longitudinally extensive myelitis. NMO-IgG is positive in $52 \%$ of patients with relapsing transverse myelitis and in $25 \%$ of patients with recurrent idiopathic optic neuritis [15].

Wingerchuk et al. [16] proposed a revised set of criteria for diagnosing NMO. These criteria remove the absolute restriction on CNS involvement beyond the optic nerves and spinal cord, allow any interval between the first events of optic neuritis and transverse myelitis, and emphasize the specificity of longitudinally extensive spinal cord lesions on MRI and NMO-IgG-seropositive status. More recently the International Panel for NMO Diagnosis developed new diagnostic criteria that define the unifying term NMOSD, which is stratified by serologic testing (with or without AQP4-IgG) [17]. These new criteria require, in patients with AQP4-IgG, core clinical and MRI findings related to optic nerve, spinal cord, area postrema, other brainstem, diencephalic, or cerebral presentations. However, more stringent clinical and MRI criteria are required for diagnosis of NMO-spectrum disorders without AQP4-IgG or when serologic testing is unavailable.

\section{Diagnostic Criteria for NMOSD Without or Unknown AQP4-IgG Status}

1. At least two core clinical characteristics occurring as a result of one or more clinical attacks and meeting all of the following requirements:

a. At least one core clinical characteristic must be optic neuritis, acute myelitis with LETM, or area postrema syndrome

b. Dissemination in space (two or more different core clinical characteristics)

c. Fulfillment of additional MRI requirements, as applicable (see below)

2. Negative tests for AQP4-IgG using best available detection method or testing unavailable

3. Exclusion of alternative diagnoses

\section{Additional MRI Requirements}

1. Acute optic neuritis: requires brain MRI showing

a. Normal findings or only nonspecific white matter lesions

b. Optic nerve MRI with T2w-hyperintense lesion or T1-weighted gadolinium-enhancing lesion extending over $>1 / 2$ optic nerve length or involving optic chiasm

2. Acute myelitis: requires associated intramedullary MRI lesion extending over $\geq 3$ contiguous segments (LETM) or $\geq 3$ contiguous segments of focal spinal cord atrophy in patients with history compatible with acute myelitis 
3. Area postrema syndrome: requires associated dorsal medulla/area postrema lesions

4. Acute brainstem syndrome: requires associated periependymal brainstem lesions

MRI of the affected optic nerve demonstrates swelling and loss of blood-brain barrier integrity with gadolinium enhancement that can extend into the optic chiasm. The spinal cord lesions in NMO typically extend over three or more contiguous vertebral segments and occasionally the entire spinal cord (longitudinally extensive spinal cord lesions); they are centrally located (preferential central gray-matter involvement) and affect much of the cross-section on axial images. During the acute and subacute phase, the lesions are tumefactive and show contrast uptake. In some cases, the spinal cord lesions are small at the onset of symptoms, mimicking those of MS, and then progress in extent over time. The presence of very hyperintense spotty lesions on T2-weighted images ("bright spotty sign") is a specific feature that helps differentiate NMO from MS, particularly in patients without longitudinally extensive spinal cord lesions [6, 11, 12], and likely reflects the highly destructive component of the inflammatory lesion. Spinal cord lesions can progress to atrophy and necrosis and may lead to syrinx-like cavities on T1-weighted images.

NMO was long considered a disease without brain involvement, and a negative brain MRI at disease onset was considered a major supportive criterion for the diagnosis of NMO. However, various studies have shown that brain MRI abnormalities exist in a significant proportion (50-85\%) of patients [18]. Brain MRI lesions are often asymptomatic, but sometimes are associated with symptoms even at disease onset. The brain lesions are commonly nonspecific. They can be dot-like or patchy, $<3 \mathrm{~cm}$ in diameter, and located in the deep white matter, brainstem, or cerebellum. Nonetheless, some brain MRI features appear to be quite characteristic and distinct from MS lesions. These abnormalities may parallel sites with high AQP4 expression adjacent to the ventricular system at any level, such as the hypothalamus, periependymal areas surrounding the third and lateral ventricles, cerebral aqueduct, corpus callosum, and dorsal brainstem adjacent to the fourth ventricle. The appearance of periventricular lesions in NMO is quite characteristic. In contrast to MS, where periventricular lesions are discrete, oval-shaped, and perpendicular to the ependymal lining due to their perivenular distribution (Dawson's fingers), NMO lesions are not oval-shaped, located immediately adjacent to the lateral ventricles following the ependymal lining in a disseminated pattern, and are often edematous and heterogeneous [18]. As opposed to what occurs in MS, NMO lesions do not affect the cortical gray matter.

Involvement of the corpus callosum has been described in $18 \%$ of AQP4-seropositive NMO patients. The lesions are multiple, large, and edematous, show heterogeneous signal intensity on T2-weighted images, and sometimes affect the entire thickness of the corpus callosum.

Lesions may also affect areas where AQP4 expression is not particularly high, such as the corticospinal tracts. These lesions, which can be unilateral or bilateral and may affect the posterior limb of the internal capsule and cerebral peduncle of the midbrain, are contiguous and often longitudinally extensive [19].

Other brain MRI findings described in NMO include extensive and confluent hemispheric white-matter lesions and radial hemispheric lesions (sometimes corresponding to an extension of periventricular lesions), which are likely related to vasogenic edema involving the white-matter tracts. These lesions usually do not show mass effect or contrast enhancement, but there may be a "cloud-like" pattern of enhancement, defined as multiple patches of enhancing lesions with blurred margins [18]. In fact, the finding of large hemispheric lesions in a patient suspected of MS should trigger the option of NMO and testing for AQP4 antibodies.

Some of the typical brain MRI findings may be specific to clinical presentations, such as intractable vomiting and hiccup (linear dorsal medullary lesions involving the area postrema and nucleus tractus solitarius), or a syndrome of inappropriate antidiuretic hormone secretion (hypothalamic and periaqueductal lesions) [18].

Distinguishing NMO from MS is critical, particularly in the early stages, since the treatment and prognosis of these disorders differ. In fact, some evidence suggests that MS-modifying treatments such as interferon- $\beta$, natalizumab, and laquinimod exacerbate NMO. By contrast, several immunosuppressants (e.g., azathioprine, rituximab, mitoxantrone) seem to help in preventing NMO relapses.

NMOSD can be associated with systemic autoimmune diseases such as systemic lupus erythematosus and Sjögren's syndrome). Whether the neurologic manifestations are solely due to NMO-spectrum disorder or are a manifestation of these diseases is controversial, although optic neuritis and transverse myelitis are rare presentations of them, and several studies have shown that patients with systemic autoimmune diseases and NMO-IgG-positive antibodies always have optic neuritis, myelitis, or NMO.

\subsubsection{Acute Disseminated Encephalomyelitis (ADEM)}

ADEM is a severe, immune-mediated inflammatory disorder of the CNS that predominantly affects the white matter of the brain and spinal cord. In the absence of specific biologic markers, the diagnosis of ADEM is based on clinical and radiologic features [20]. This disorder affects children more commonly than adults, and, in contrast to MS, shows no gender preponderance. The estimated incidence is 0.8 per 
100,000 population per year. In most cases, the clinical onset of disease is preceded by viral or bacterial infections, usually nonspecific upper-respiratory-tract infections. ADEM may also develop following a vaccination (postimmunization encephalomyelitis). Patients commonly present with nonspecific multifocal symptoms, which developed subacutely over a period of days, frequently associated with encephalopathy (relatively uncommon in MS), defined as an alteration in consciousness (e.g., stupor, lethargy) or a behavioral change unexplained by fever, systemic illness, or postictal symptoms. Although ataxia, encephalopathy, and brainstem symptoms are frequently present in both pediatric and adult cases, certain signs and symptoms appear to be age-related. In childhood ADEM, long-lasting fever and headaches occur more frequently, while in adult cases, motor and sensory deficits predominate. In general, the disease is self-limiting and the prognostic outcome favorable.

Although ADEM usually has a monophasic course, multiphasic forms have been reported, raising diagnostic difficulties in distinguishing these cases from MS. This multiphasic form, which accounts for $<4 \%$ of ADEM cases, is defined as a new encephalopathic event consistent with ADEM, separated by 3 months after the initial illness but not followed by any further events. The second ADEM event can involve either new or re-emergent neurologic symptoms, signs, and MRI findings. Relapsing disease following ADEM that occurs beyond a second encephalopathic event is no longer consistent with multiphasic ADEM but rather indicates a chronic disorder, most often leading to the diagnosis of MS or NMO [21] and should prompt testing for myelin oligodendrocyte glycoprotein (MOG) antibodies, especially in children.

An ADEM event as the first manifestation of the classic relapsing form of MS occurs in 2-10\%. According to the International Pediatric Multiple Sclerosis Study Group, the diagnosis of MS is met if, after the initial ADEM, a second clinical event meets the following three requirements: (1) it is non-encephalopathic; (2) it occurs 3 months or more after the incident neurologic illness; and (3) it is associated with new MRI findings consistent with the McDonald criteria for dissemination in space [21]. The presence of hypointense lesions and two or more periventricular lesions are MRI features that support an MS diagnosis in children with acute CNS demyelination [21]. Unlike the lesions in MS, ADEM lesions are often large, patchy, and poorly marginated on MRI, especially when there are MOG antibodies [22]. There is usually asymmetric involvement of the subcortical and central white matter and cortical gray-white junction of the cerebral hemispheres, cerebellum, brainstem, and spinal cord. Lesions confined to the periventricular white matter and corpus callosum are less common than in MS. The gray matter of the thalamus and basal ganglia is often affected, particularly in children, and typically in a symmetric pattern [23]. However, the frequency of thalamic involvement in adult ADEM does not differ from that of adult MS. This can be explained by the fact that involvement of this structure is less common in adult ADEM than in childhood ADEM. Four patterns of cerebral involvement have been proposed to describe the MRI findings in ADEM [24]: (1) ADEM with small lesions ( $<5 \mathrm{~mm}$ ); (2) ADEM with large, confluent, or tumefactive lesions, and frequent extensive perilesional edema and mass effect; (3) ADEM with additional symmetric deep gray-matter involvement; and (4) acute hemorrhagic encephalomyelitis. Gadolinium enhancement of one or more lesions occurs in $14-30 \%$ of cases [21]. The pattern of enhancement varies and can be complete or incomplete ringshaped, nodular, gyral, or spotty. Although ADEM is usually a monophasic disease, new lesions may be seen on follow-up MRI within the first month of the initial attack.

Most MRI lesions appear early in the course of the disease, supporting the clinical diagnosis of ADEM. Nonetheless, in some cases, there may be a delay of more than 1 month between the onset of symptoms and the appearance of lesions on MRI. Therefore, a normal brain MRI scan obtained within the first days after the onset of neurologic symptoms suggestive of ADEM does not exclude this diagnosis.

The spinal cord is affected in $<30 \%$ of ADEM patients [24], predominantly in the thoracic region. The spinal cord lesion is typically large, causes swelling, and shows variable enhancement. In most ADEM patients, partial or complete resolution of the MRI abnormalities occurs within a few months of treatment. This course is positively associated with a final diagnosis of ADEM.

\subsection{Infectious Inflammatory Demyelinating Disorders}

\subsubsection{Progressive Multifocal Leukoencephalopathy}

Progressive multifocal leukoencephalopathy (PML) is overwhelmingly a disease of the immunocompromised patient and most (55-85\%) cases are related to acquired immunodeficiency syndrome (AIDS). There is a wide age range of involvement, with the peak age of presentation in the sixth decade. The disease is caused by reactivation a papovavirus (JC virus) that selectively attacks the oligodendrocyte, leading to demyelination. Treatment with monoclonal antibody therapy (natalizumab, rituximab, efalizumab) or other immunomodulatory drugs, commonly used in patients with MS and other disorders has also been linked with PML [25]. Untreated patients with PML have an extremely poor prognosis, with death common in the first 6 months following establishment of the diagnosis. Although there is no specific treatment, combination anti-retroviral therapy (cART) has not only resulted in a lower incidence of PML in AIDS patients, but also substantially improved survival times of afflicted patients, now at 50\% 1-year survival [25]. Unfortunately, 
about $20 \%$ of PML cases are linked to a robust inflammatory response to pathogens associated with recovery of the immune system after a period of immunosuppression. This condition, known as PML-IRIS (Immune Reconstitution Inflammatory Syndrome), has been associated with intracranial masses with generous amounts of surrounding vasogenic edema on MRI. Enhancement may also occur [25].

The lesions of PML are characterized by little mass effect or enhancement. Most lesions involve the subcortical white matter and deep cortical layers of the parieto-occipital or frontal white matter, although gray matter and posterior fossa lesions are also common, occurring in up to $50 \%$ of cases. PML lesions tend to be more confluent in their appearance than ADEM lesions and scalloping of the lateral margin of the lesion at the gray matter-white matter junction is common. Subtle signal intensity changes in the white matter may precede clinical suspicion of the PML-IRIS. While development of mass effect and temporary enhancement in the early phase of cART has been linked to better survival, similar imaging manifestations may also be seen in monoclonalantibody treated PML patients [25].

\subsubsection{Human Immunodeficiency Virus Encephalopathy}

Human immunodeficiency virus (HIV) encephalopathy results from direct infection of the brain by the virus itself. Since the advent of cART, the prevalence of the disease has markedly decreased and the temporal progression has been slowed. Most patients are severely immunocompromised at the time of onset and exhibit psychomotor slowing, impaired mental status, and memory difficulties. Histologically, demyelination and vacuolation with axonal loss are noted, along with occasional microglial nodules. Mild cerebral atrophy is the first and sometimes only imaging feature of AIDS dementia complex, also know as HIV dementia, HIV-associated dementia complex, or as HIV-associated neuron-cognitive disorder (HAND). Involvement of the central white matter, basal ganglia, and thalamus is characteristic. Typically, bilaterally symmetric abnormal $\mathrm{T} 2$ hyperintensity in the basal ganglia and small focal areas in the periventricular regions are noted on T2-weighted MR images [26]. Regression of these findings has been seen following institution of cART.

\subsection{White Matter Disease from Toxic Imbalance}

\subsubsection{Chronic Alcohol Ingestion and its Consequences}

Brain abnormalities in alcoholics include atrophy, Marchiafava-Bignami disease, Wernicke encephalopathy, osmotic myelinolysis, and consequences of liver cirrhosis such as hepatic encephalopathy and coagulopathy [27]. All the reported entities are not specific of alcohol and can be found in many other toxic or metabolic conditions. Ethanol direct brain toxicity is caused by under-regulation of receptors of N-methyl-D-aspartate, abnormal catabolism of homocysteine, resulting in an increased susceptibility to glutamate excitatory and toxic effects. Moreover, immune response occurs mediated by lipid peroxidation products that bind to neurons resulting in neurotoxicity. Neuroimaging studies show a characteristic distribution of loss of volume: initially there is infratentorial predominance with atrophy of the cerebellar vermis and hemispheres. Frontal and temporal atrophy is subsequently evident, followed by diffuse atrophy of the brain. The possibility of partial reversibility of these alterations is also observed. In pregnancy, ethanol determines inhibition of maturation of Bergmann's fibers of cerebellum, with consequential marked cerebellar atrophy.

\subsubsection{Hepatic Encephalopathy}

The term hepatic encephalopathy (HE) includes a wide spectrum of neuropsychiatric abnormalities occurring in patients with liver dysfunction. Most cases are associated with cirrhosis and portal hypertension or portal-systemic shunts, but the condition can also be seen in patients with acute liver failure and rarely, those with portal-systemic bypass and no associated intrinsic hepatocellular disease. Although HE is a clinical condition, several neuroimaging techniques, particularly MRI, may eventually be useful for the diagnosis because they can identify and measure the consequences of CNS increase in substances, which, under normal circumstances, are efficiently metabolized by the liver. Classical MR abnormalities in chronic HE include high signal intensity in the globus pallidum on T1-weighted images, likely a reflection of increased tissue concentrations of manganese, and an elevated glutamine/glutamate peak coupled with decreased myo-inositol and choline signals on proton MR spectroscopy, representing disturbances in cell-volume homeostasis secondary to brain hyperammonemia [28]. Recent data have shown that white matter abnormalities, also related to increased CNS ammonia concentration, can also be detected with several MRI techniques: magnetization transfer ratio measurements show significantly low values in otherwise normal-appearing brain white matter, T2-Flair sequences reveal diffuse and focal high signal intensity lesions in the hemispheric white matter, and DWI discloses increased white matter diffusivity. All these MR abnormalities, which return to normal with restoration of liver function, probably reflect the presence of mild diffuse interstitial brain edema, which seems to play an essential role in the pathogenesis of $\mathrm{HE}$.

In acute $\mathrm{HE}$, bilateral symmetric signal-intensity abnormalities on T2-weighted images, often with associated 
restricted diffusion involving the cortical gray matter, are commonly identified. Involvement of the subcortical white matter and the basal ganglia, thalami, and midbrain may also be seen. These abnormalities that can lead to intracranial hypertension and severe brain injury reflect the development of cytotoxic edema secondary to the acute increase of brain hyperammonemia.

\subsubsection{Marchiafava-Bignami Disease}

Marchiafava-Bignami disease is a rare complication of chronic alcoholism, characterized by demyelination and necrosis of the corpus callosum, with rare involvement of extracallosal regions. Etiology remains unknown but is believed to be caused by toxic agents in red wine of low quality and lack of group B vitamins. Rarely, the disease has also been reported in non-alcoholic patients. Symptoms are mainly represented by cognitive deficits, psychosis, hypertonia, and interhemispheric disconnection, until coma and death. Typical MRI features in the acute phase are corpus callosum hyperintensity on T2-weighted sequences and FLAIR, without significant mass effect, with peripheral enhancement. Diffusion is restricted due to cytotoxic edema. In chronic forms, necrosis of the genu and splenium can be detected [29].

\subsubsection{Wernicke Encephalopathy}

Wernicke encephalopathy is an acute condition first described by the French ophthalmologist Gayet in 1875, and later by the German neurologist Wernicke in 1881, caused by a deficiency of B1 (thiamine). It develops frequently but not exclusively in alcoholics. Other potential causes include extended fasting, malabsorption, digitalis poisoning, massive infusion of glucose without B1 in weak patients. The autoptic incidence is reported to be $0.8-2 \%$ in random autopsies, and $20 \%$ in chronic alcoholics. The classic clinical triad of ocular dysfunctions (nystagmus, conjugate gaze palsy, ophthalmoplegia), ataxia, and confusion is observed in only $30 \%$ of cases. Treatment consists of thiamine infusion, and avoids irreversible consequences including Korsakoff dementia or death. Memory impairment and dementia are related to damage of the mammillary bodies, anterior thalamic nuclei and interruption of the diencephalic-hippocampal circuits. Depletion of thiamine leads to failure of conversion of pyruvate to acetyl-CoA and $\alpha$-ketoglutarate to succinate, altered pentose monophosphate shunt, and the lack of Krebs cycle, with cerebral lactic acidosis, intra- and extra-cellular edema, swelling of astrocytes, oligodendrocytes, myelin fibers, and neuronal dendrites. Neuropathological aspects include neuronal degeneration, demyelination, hemorrhagic petechiae, proliferation of capillaries and astrocytes in peri-aqueductal gray substance, mammillary bodies, thalami, pulvinar, III cranial nerves nuclei, and cerebellum. On MRI, bilateral and symmetrical hyperintensities on T2-weighted sequences and FLAIR are evident at the level of the already mentioned structures, mainly mammillary bodies and thalami [30, 31]. Rarely, cortex of the forebrain can be involved. DWI shows areas of reduced apparent diffusion coefficient (ADC) due to cytotoxic edema, although the ADC can sometimes be high due to the presence of vasogenic component. Rarely, T1-weighted images show bleeding ecchymotic hemorrhages in the thalami and mammillary bodies, a sign considered clinically unfavorable. In $50 \%$ of cases, contrast enhancement is present in periaqueductal regions. Marked contrast enhancement of mammillary bodies is evident in $80 \%$ of cases, even prior to the development of visible changes in T2-weighted sequences and is considered highly specific for WE. In chronic forms, change of signal in T2-weighted sequences becomes less prominent due to the diffuse brain atrophy, more pronounced at the level of mesencephalon and mammillary bodies.

\subsubsection{Osmotic Demyelination Syndrome}

Osmotic demyelination syndrome (ODS) usually occurs in the setting of osmotic changes, typically with the rapid correction of hyponatremia. This causes destruction of the blood-brain barrier with hypertonic fluid accumulation in extracellular space, resulting in a non-inflammatory demyelination. The most common damage is in the pontine fibers [32]. ODS is observed in alcoholics with nutritional deficiency. The most common symptoms include paralysis, dysphagia, dysarthria, and pseudobulbar palsy. Death is frequent. Rarely, ODS affects other regions, especially basal ganglia, thalami, deep white matter. MRI usually shows an area of high signal on T2-weighted sequences in the central part of the pons (central pontine myelinolysis), sparing ventro-lateral portions and corticospinal tracts. The lesion is moderately hypointense in $\mathrm{T} 1$ and may show positive contrast enhancement. If the patient survives, the acute phase can evolve into a cavitated pontine lesion.

\subsection{White Matter Disease Associated with Radiation Therapy and Chemotherapy}

Treatment strategies designed to target cancer cells are commonly associated with deleterious effects to multiple organ systems, including the CNS. As both radiation and chemotherapy alone can be associated with significant toxicity, the 
combination of radiation and chemotherapy may be particularly harmful to the CNS. With advanced treatment regimens and prolonged survival, neurological complications are likely to be observed with increasing frequency.

Neurotoxicity can result from direct toxic effects of the drug or radiation on the cells of the CNS, or indirectly through metabolic abnormalities, inflammatory processes, or vascular adverse effects.

Recognition of treatment-related neurologic complications is critically important, because symptoms may be confused with metastatic disease, tumor progression, paraneoplastic disorders, or opportunistic infections, and discontinuation of the offending drug may prevent irreversible CNS injury.

\subsubsection{Radiation Injury and Necrosis}

It is widely accepted that the white matter of the CNS is prone to radiation-induced injury, compared with gray matter. Radiation encephalopathy has been classically divided into three stages according to its timing after radiotherapy: early, early-delayed, and late-delayed reactions [33]. Within the first several weeks of therapy, patients may experience acute declines with focal neurologic deficits. These effects are possibly related to increased edema, which has been supported by the observation that steroid treatment often results in clinical improvement. Early-delayed adverse effects usually occur within 1-6 months of treatment and are thought to be a result of demyelination. This syndrome is characterized by somnolence, fatigue, and cognitive dysfunction, consistent with dysfunction of the frontal network systems. Late-delayed side effects occur months to years after cessation of treatment and are largely irreversible and progressive. These late changes are commonly associated with progressive cognitive deficits. In more severe cases of late-delayed radiation injury, imaging and histopathological studies may demonstrate leukoencephalopathy and/or focal necrosis.

In all types of white matter radiation-induced damage, CT and mainly MRI may demonstrate variable degrees of white matter signal changes related to an increase in free tissue water in the involved areas. This may result from endothelial damage, causing increased capillary permeability and vasogenic edema, or from demyelination. However, the degree of these white matter changes correlates poorly with the functional deterioration observed in the patients. MRI findings in early reactions occurring during course of treatment are nonspecific. MRI may be normal or demonstrate poorly defined multifocal lesions in both hemispheres that usually disappear spontaneously. MRI in early-delayed reactions also may show signal changes involving not only the hemispheric white matter but also the basal ganglia and the cerebral peduncles, which resolve completely without treatment. These early-delayed changes have been reported in children with acute lymphatic leukemia who have been treated with both whole brain irradiation and chemotherapy. These changes have no correlation with clinical manifestations and have no clear prognostic significance. Late-delayed reactions can be subdivided into diffuse and focal radiation necrosis injury.

Diffuse radiation injury is characterized by white matter changes that are "geographic" in nature, i.e., the areas of abnormal signal intensity or attenuation are limited to the regions of the brain that conform to the radiation portal. This can produce striking differences between the involved zones and the spared surrounding white matter. The involved territories are often symmetric and do not enhance on post-contrast studies. While originally reported in children with leukemia, diffuse necrotizing leukoencephalopathy has also been observed following treatment for many other malignancies in both children and adults. The disease may occur following chemotherapy alone, but the incidence of disease is highest when chemotherapy is combined with radiation therapy. Both the histologic findings and imaging features bear resemblance to radiation necrosis. Axonal swelling, demyelination, coagulation necrosis, and gliosis dominate the histologic picture.

Diffuse white matter changes, with hypoattenuation on $\mathrm{CT}$ and $\mathrm{T} 1$ and $\mathrm{T} 2$ prolongation on MRI, are common and often involve an entire hemisphere. Microbleeds can occur as a sign of vasculopathy. Radiation-induced leukoencephalopathy may be associated with progressive brain atrophy, and patients may present with cognitive decline, gait abnormalities, and urinary incontinence. However, the more common mild-to-moderate cognitive impairment is inconsistently associated with radiological findings, and frequently occurs in patients with normal-appearing scans. Recently, more sensitive tools to quantify the damage to brain tissue, such as diffusion tensor imaging (DTI) have been applied in patients treated with brain radiotherapy and have shown early and progressive diffuse changes in normal-appearing white matter indicating radiationinduced demyelination and mild structural degradation of axonal fibers, which are undetectable with conventional MRI.

Focal radiation necrosis usually manifests as a ring-like or irregular enhancing mass lesion located in the white matter, which may become hemorrhagic. The MRI features commonly seen in radiation necrosis include a soap bubblelike interior and Swiss cheese-like interior. Compared with lesions with the soap bubble pattern, Swiss cheese lesions are larger, more variable in size, and more diffuse. This pattern can be visualized as a result of diffuse necrosis affecting the white matter and cortex with diffuse enhancement at the 
margins with intermixed foci of necrosis. The rim of enhancement is often thinner, more uniform, and more aligned to the gray matter-white matter junction than in malignant tumors. As radiation necrosis progresses, it can lead to severe shrinkage of the white matter and cortex and result in focal brain atrophy with hydrocephalus.

Quite frequently is impossible to distinguish radiation necrosis from recurrent malignant brain tumor, such as glioblastoma multiforme, using conventional MRI. Metabolic imaging (e.g., positron emission tomography) may facilitate differentiating between the two diseases as radiation necrosis is iso-to-hypometabolic while recurrent high-grade tumors are typically hypermetabolic. MR spectroscopy (MRS) may also be useful as radiation necrosis frequently shows a characteristic lactic acid peak and near-normal peaks for $\mathrm{N}$-acetylaspartate (NAA) and choline while recurrent high-grade gliomas typically show elevated choline levels compared to NAA without or with elevated lactic acid levels. Perfusion imaging can identify the areas of increased blood flow associated with tumor recurrence, whereas radiation necrosis is not expected to contain any increased blood flow [34].

\subsubsection{Chemotherapy-Associated Neurotoxicity}

Neurotoxicity has been observed with virtually all categories of chemotherapeutic agents [35]. Neurologic complications may range from acute encephalopathy, headache, seizures, visual loss, cerebellar toxicity, and stroke to chronic side effects, including chronic encephalopathy, cognitive decline, and dementia.

Among the most puzzling aspects of cancer therapyrelated toxicity is the occurrence of delayed and progressive neurological decline, even after cessation of treatment. Anticancer agents affect brain function through both direct and indirect pathways. It is also conceivable that additional variables play important roles, including the timing of treatment, combination of different treatment modalities, patient age, integrity of the blood-brain barrier, and cognitive function prior to treatment initiation.

Imaging studies have provided evidence that structural and functional CNS changes occur in a significant number of patients treated with chemotherapy. Some agents, such as methotrexate or carmustine, are well known to cause a leukoencephalopathy syndrome, especially when administered at a high dose, intrathecally, or in combination with cranial radiotherapy. Nonenhancing, confluent, periventricular white matter lesions, necrosis, ventriculomegaly, and cortical atrophy characterize this syndrome. White matter abnormalities following high-dose chemotherapy have been detected in up to $70 \%$ of treated individuals and usually have a delayed onset of several months.
A delayed leukoencephalopathy syndrome with distinct DWI abnormalities on MRI indicative of cytotoxic edema within cerebral white matter has been recently described. This syndrome appeared to mimic a stroke-like syndrome and has seen mainly in patients receiving methotrexate, 5-fluorouracil (5-FU), carmofur, and capecitabine.

\subsection{Vascular Causes of White Matter Disease}

\subsubsection{Posterior Reversible Encephalopathy Syndrome (PRES)}

Although not a truly demyelinating condition, reversible encephalopathy syndrome is noteworthy because of its affinity for the posterior cerebral white matter territories and is important in the differential diagnosis of demyelinating disease. Under normal circumstances, cerebral perfusion pressure is maintained at a relatively constant level by autoregulation, a physiologic mechanism that compensates for wide changes in systemic blood pressure. Hypertensive encephalopathy is believed to result from loss of normal autoregulation, with competing regions of vasodilatation and vasoconstriction, and endothelial dysfunction. The vessels of the posterior cerebral circulation, lacking less sympathetic innervation compared to those of the anterior circulation, are unable to vasoconstrict in a normal manner and bear the brunt of these vascular changes. Reversible vasogenic edema is the result and is associated with visual field deficits, as well as headaches, somnolence, and an overall impaired mental status. The terms posterior reversible encephalopathy syndrome (PRES) and reversible posterior leukoencephalopathy syndrome (RPLS) have been popularized in the literature to describe this scenario that is most commonly seen in hypertensive states and/or the presence of immunosuppression (particularly cyclosporine A and tacrolimus), chemotherapy, eclampsia, and renal failure. While it commonly involves the posterior cerebral white matter, other sites may also be affected including unilateral cerebral hemispheric or isolated brainstem involvement in patients following aortic valve surgery [36, 37]. Accordingly, it has been suggested that perhaps the terminology should be changed to simply "reversible encephalopathy" [38].

On MR studies, bilaterally symmetric abnormal T2 hyperintensity, representing vasogenic edema, is most commonly seen in the distribution of the posterior circulation, although other sites including the frontal lobes and corpus callosum may be noted as well. Cortical and subcortical lesions may be better detected on FLAIR sequences. DWI may be normal or show restricted water diffusion in regions of infarction that correlate with poorer prognosis [23]. Susceptibilityweighted imaging may show areas of hemorrhage within 
involved territories. With early treatment and limited involvement of the brain, many of these imaging abnormalities will completely resolve and most patients recover within 2 weeks [39]. However, when larger areas or regions of infarction are involved, permanent neurologic deficits or even death are possible. Vascular narrowing has been observed on angiographic studies. Perfusion studies reported in the literature indicate normal to increased perfusion in these zones. When biopsies of these regions have been performed, white matter edema is seen histologically.

\subsubsection{Aging and Ischemic Demyelinating Disorders}

Small focal lesions on T2-weighted images are quite common in the white matter of adult subjects [40]. They are not associated with mass effect, do not enhance, and are typically isointense compared to normal white matter on T1-weighted images. When these lesions have been biopsied, histologic examination reveals a spectrum of findings including gliosis, (partial) loss of myelination, and vasculopathy. They tend to be located in the deep white matter of the centrum semiovale. In contrast to MS, lesions do not involve the corpus callosum or the juxtacortical U-fibers, important distinguishing features [3]. Since the lesions are so ubiquitous and appear to be a part of "normal" aging, various terms have been proposed: senescent white matter changes or disease, deep white matter ischemia, leukoaraïosis, etc. In general, the more lesions present, the more likely it is that the patient will have cognitive problems or difficulties with neuropsychological testing. However, it is not possible to predict a particular patient's status simply based on the imaging appearance alone.

In adult patients between 30 and 50 years of age, the presence of periventricular and subcortical lesions in a patient with a family history of similarly affected relatives should raise the possibility of cerebral autosomal dominant arteriopathy with subcortical infarcts and leukoencephalopathy (CADASIL). A defect in the notch3 gene on the long arm of chromosome 19 has been identified and apparently evokes an angiopathy affecting small and medium-sized vessels. Most lesions occur in the frontal and temporal lobes and less commonly in the thalamus, basal ganglia, internal and external capsules, and brain stem [41].

\subsection{Conclusion}

MRI of the brain and spinal cord is of vital importance in the diagnosis of MS, the most important idiopathic inflammatory CNS disorder [42]. Dissemination in space and time on MRI is not limited to MS and can occur in NMO, and spinal cord imaging is important in the differential diagnosis.
Increasingly, MRI is used to monitor MS treatment and their complications such as PML.

\section{Take Home Message}

- MRI of the brain and the spinal cord should be performed in any patient suspected of MS to show dissemination in space according to McDonald criteria. Additional gadolinium enhancement or new T2 lesions demonstrate dissemination in time and allow a final diagnosis and treatment initiation. Spinal cord MRI with multiple short segment lesions is almost pathognomonic for MS and a negative cord scan is a red flag in MS.

\section{References}

1. Smith AB, Smirniotopoulos JG. Imaging evaluation of demyelinating processes of the central nervous system. Postgrad Med J. 2010;86:218-29.

2. Cañellas AR, Gols AR, Izquierdo JR, et al. Idiopathic inflammatory-demyelinating diseases of the central nervous system. Neuroradiology. 2007;49:393-409.

3. Aliaga ES, Barkhof F. MRI mimics of multiple sclerosis. Handb Clin Neurol. 2014;122:291-316.

4. Rovira À, Barkhof F. Multiple sclerosis and variants. In: Barkhof F, Jager R, Thurnher M, Rovira Cañellas A, editors. Clinical neuroradiology. Cham: Springer; 2018.

5. Bagnato F, Jeffries N, Richert ND, et al. Evolution of T1 black holes in patients with multiple sclerosis imaged monthly for 4 years. Brain. 2003;126:1782-9.

6. Ciccarelli O, Cohen JA, Reingold SC, Weinshenker BG. Spinal cord involvement in multiple sclerosis and neuromyelitis optica spectrum disorders. Lancet Neurol. 2019;18(2):185-97.

7. Cotton F, Weiner HL, Jolesz FA, et al. MRI contrast uptake in new lesions in relapsing-remitting MS followed at weekly intervals. Neurology. 2003;60:640-6.

8. Masdeu JC, Quinto C, Olivera C, et al. Open-ring imaging sign: highly specific for atypical brain demyelination. Neurology. 2000;54:1427-33.

9. Popescu BF, Lucchinetti CF. Pathology of demyelinating diseases. Annu Rev Pathol. 2012;7:185-217.

10. Stadelmann C, Ludwin S, Tabira T, et al. Tissue preconditioning may explain concentric lesions in Baló's type of multiple sclerosis. Brain. 2005;128:979-87.

11. Hardy TA, Miller DH. Baló's concentric sclerosis. Lancet Neurol. 2014;13:740-6.

12. Wuerfel J, Rovira A, Paul F, Barkhof F. Neuromyelitis optica spectrum disorders (NMOSD). In: Barkhof F, Jager R, Thurnher M, Rovira Cañellas A, editors. Clinical neuroradiology. Cham: Springer; 2019.

13. Trebst C, Jarius S, Berthele A, et al. Update on the diagnosis and treatment of neuromyelitis optica: recommendations of the Neuromyelitis Optica Study Group (NEMOS). J Neurol. 2014;261:1-16.

14. Lennon VA, Wingerchuk DM, Kryzer TJ, et al. A serum autoantibody marker of neuromyelitis optica: distinction from multiple sclerosis. Lancet. 2004;364:2106-12.

15. Wingerchuk DM, Lennon VA, Pittock SJ, et al. Revised diagnostic criteria for neuromyelitis optica. Neurology. 2006;66:1485-9. 
16. Wingerchuk DM, Banwell B, Bennett JL, et al. International consensus diagnostic criteria for neuromyelitis optica spectrum disorders. Neurology. 2015;85:177-89.

17. Yonezu T, Ito S, Mori M, et al. "Bright spotty lesions" on spinal magnetic resonance imaging differentiate neuromyelitis optica from multiple sclerosis. Mult Scler. 2014;20:331-7.

18. Misu T, Fujihara K, Nakashima I, et al. Intractable hiccup and nausea with periaqueductal lesions in neuromyelitis optica. Neurology. 2005;65:1479-82.

19. Kim HJ, Paul F, Lana-Peixoto MA, et al. MRI characteristics of neuromyelitis optica spectrum disorder: an international update. Neurology. 2015;84:1165-73.

20. Auger C, Rovira A. Acute disseminated encephalomyelitis and other acute parainfectious syndromes. In: Barkhof $\mathrm{F}$, Jager R, Thurnher M, Rovira CA, editors. Clinical neuroradiology. Cham: Springer; 2018.

21. Verhey LH, Branson HM, Shroff MM, et al. MRI parameters for prediction of multiple sclerosis diagnosis in children with acute CNS demyelination: a prospective national cohort study. Lancet Neurol. 2011;10:1065-73.

22. Hacohen Y, Mankad K, Chong WK, et al. Diagnostic algorithm for relapsing acquired demyelinating syndromes in children. Neurology. 2017;89:269-78.

23. Covarrubias D, Luetmer P, Campeau N. Posterior reversible encephalopathy syndrome: prognostic utility of quantitative diffusionweighted MR images. AJNR Am J Neuroradiol. 2002;23:1038-48.

24. Tan CS, Koralnik IJ. Progressive multifocal leukoencephalopathy and other disorders caused by JC virus: clinical features and pathogenesis. Lancet Neurol. 2010;9:425-37.

25. Wattjes MP, Barkhof F. Diagnosis of natalizumab-associated progressive multifocal leukoencephalopathy using MRI. Curr Opin Neurol. 2014;27:260-70.

26. Gottumukkala RV, Romero JM, Riascos RF, et al. Imaging of the brain in patients with human immunodeficiency virus infection. Top Magn Reson Imaging. 2014;23:275-91.

27. Geibprasert S, Gallucci M, Krings T. Alcohol-induced changes in the brain as assessed by MRI and CT. Eur Radiol. 2010;20:1492-501.

28. Rovira A, Alonso J, Cordoba J. MR imaging findings in hepatic encephalopathy. AJNR Am J Neuroradiol. 2008;29:1612-21.

29. Arbelaez A, Pajon A, Castillo M. Acute Marchiafava-Bignami disease: MR findings in two patients. AJNR Am J Neuroradiol. 2003;24:1955-7.
30. Gallucci M, Bozzao A, Splendiani A, et al. Wernicke encephalopathy: MR findings in five patients. AJR Am J Roentgenol. 1990;155:1309-14.

31. Zuccoli G, Santa Cruz D, Bertolini M, et al. MR imaging findings in 56 patients with Wernicke encephalopathy: nonalcoholics may differ from alcoholics. AJNR Am J Neuroradiol. 2009;30:171-6.

32. Ruzek KA, Campeau NG, Miller GM. Early diagnosis of central pontine myelinolysis with diffusion-weighted imaging. AJNR Am J Neuroradiol. 2004;25:210-3.

33. Soussain C, Ricard D, Fike JR, et al. CNS complications of radiotherapy and chemotherapy. Lancet. 2009;374:1639-51.

34. Baehring JM, Fulbright RK. Delayed leukoencephalopathy with stroke-like presentation in chemotherapy recipients. J Neurol Neurosurg Psychiatry. 2008;79:535-9.

35. Godi C, Falini A. Radiological findings of drug-induced neurotoxic disorders. In: Barkhof F, Jager R, Thurnher M, Rovira Cañellas A, editors. Clinical neuroradiology. Cham: Springer; 2019.

36. Wijdicks EF, Campeau N, Sundt T. Reversible unilateral brain edema presenting with major neurologic deficit after valve repair. Ann Thorac Surg. 2008;86:634-7.

37. McKinney AM, Short J, Truwit CL, et al. Posterior reversible encephalopathy syndrome: incidence of atypical regions of involvement and imaging findings. AJR Am J Roentgenol. 2007;189:904-12.

38. Casey SO, Sampaio RC, Michel E, Truwit CL. Posterior reversible encephalopathy syndrome: utility of fluid-attenuated inversion recovery MR imaging in the detection of cortical and subcortical lesions. AJNR Am J Neuroradiol. 2000;21:1199-206.

39. Post JD, Beauchamp NJ. Reversible intracerebral pathologic entities mediated by vascular autoregulatory dysfunction. Radiographics. 1998;18:353-67.

40. Kloppenborg RP, Nederkoorn PJ, Geerlings MI, van den Berg E. Presence and progression of white matter hyperintensities and cognition: a meta-analysis. Neurology. 2014;82:2127-38.

41. van dem Boom R, Lesnick Oberstein S, et al. Cerebral autosomal dominant arteriopathy with subcortical infarcts and leukoencephalopathy: MR imaging changes and apolipoprotein E genotype. AJNR Am J Neuroradiol. 2006;27:359-62.

42. Rovira À, Wattjes MP, Tintoré M, et al. Evidence-based guidelines: MAGNIMS consensus guidelines on the use of MRI in multiple sclerosis-clinical implementation in the diagnostic process. Nat Rev Neurol. 2015;11:471-82.

Open Access This chapter is licensed under the terms of the Creative Commons Attribution 4.0 International License (http://creativecommons. org/licenses/by/4.0/), which permits use, sharing, adaptation, distribution and reproduction in any medium or format, as long as you give appropriate credit to the original author(s) and the source, provide a link to the Creative Commons license and indicate if changes were made.

The images or other third party material in this chapter are included in the chapter's Creative Commons license, unless indicated otherwise in a credit line to the material. If material is not included in the chapter's Creative Commons license and your intended use is not permitted by statutory regulation or exceeds the permitted use, you will need to obtain permission directly from the copyright holder. 
Part III

Pediatric and Fetal 


\title{
Children with Acute Neurological Emergency
}

\author{
Gunes Orman, Andrea Rossi, Avner Meoded, \\ and Thierry A. G. M. Huisman
}

\section{Learning Objectives}

- To understand common acute pediatric neurological disorders that are encountered in the emergency setting.

- To understand basic neuroimaging findings of acute pediatric neurological emergencies.

Children with acute neurological emergencies frequently present to the emergency room (ER) with various combinations of headache, fever, nausea, vomiting, physical signs of trauma, altered mental status, coma, or focal neurological symptoms. Neuroimaging is often needed in children with acute neurological emergencies due to the difficulties of gathering an adequate and comprehensive patient history or a detailed and reliable neurological examination [1]. The radiologist plays an important role in the ER. Firstly, the radiologist should be able to answer if the child needs neuroimaging emergently. Secondly, the radiologist should be able to answer which imaging modality is appropriate for the child. Different imaging modalities may be chosen based on the institutional availability and clinical status of the patient. Computed tomography (CT) and magnetic resonance imaging (MRI) are the most frequently used imaging modalities in the emergency setting. CT is usually the initial modality of choice in most of the situations. Modern multidetector CT scanners render highly detailed multiplanar submillimeter images of the pediatric brain; however, the main disadvantage of $\mathrm{CT}$ is that it requires

G. Orman $(\bowtie) \cdot$ A. Meoded · T. A. G. M. Huisman Edward B. Singleton Department of Radiology, Texas Children's Hospital, Houston, TX, USA

e-mail: gxorman@texaschildrens.org;

axmeoded@texaschildrens.org; huisman@texaschildrens.org

A. Rossi

Neuroradiology Unit, IRCCS Istituto Giannina Gaslini,

Genoa, Italy

e-mail: andrearossi@gaslini.org the use of ionizing radiation [2]. MRI is a valuable tool to confirm and characterize the entities seen in children with acute neurological emergency. MRI is easy to perform, does not use ionizing radiation, offers multiple different tissue contrasts, superior anatomical details, especially for evaluation of the posterior fossa, and offers multiple advanced, and functional sequences enhancing a sensitive and specific diagnosis $[2,3]$. These advantages made MRI an increasingly preferred modality for the diagnostic evaluation of children with acute neurological symptoms even in time-sensitive settings. Ultrafast MRI protocols should be considered whenever possible as the initial choice of imaging instead of CT [3, 4]. Advanced MRI techniques including susceptibility-weighted imaging (SWI), diffusion weighted imaging (DWI), or diffusion tensor imaging (DTI) with apparent diffusion coefficient (ADC) mapping and perfusion-weighted imaging (PWI) are progressively used in acute pediatric neuroimaging protocols [2]. Last but not least, the radiologist should be able to give a reliable differential diagnosis to guide patient management, in order to reduce morbidity and mortality. In addition, when evaluating brain MRIs of children, the radiologist should be aware that the pediatric brain may be immature and depending on the child's age different states of brain development and maturation may be encountered. A neonatal brain MRI looks very different compared to a teenager's brain MRI. Thus, the radiologist should understand normal brain development and must be familiar with age-specific changes of the developing pediatric brain [2].

The goal of this chapter is to discuss the neuroimaging findings of a number of common acute pediatric neurological emergencies including stroke, hemorrhage, infections, and several less frequently encountered disorders.

\subsection{Stroke}

Arterial ischemic stroke (AIS) is relatively rare in children but can lead to significant morbidity and mortality rates ranging between $4 \%$ and $14 \%$. Understanding that children with AIS 
present clinically differently than adults and often present with unique child specific risk factors will impact the diagnostic approach, treatment, and outcome. The risk factors for AIS in children include cardiac disorders, infection, head and neck trauma, sickle cell disease, vascular malformations, genetic disorders, and autoimmune diseases [5-7]. The clinical presentation in neonates and infants can be very subtle relative to the severity of imaging findings (Fig. 14.1a). In children with suspected stroke, MRI is the preferred imaging modality. Radiologists should embrace an "MRI first" service whenever possible. Recommended sequences include DWI/DTI and ADC mapping to diagnose acute ischemia, gradient-recalled echo (GRE) or SWI to detect intracranial blood and its breakdown products, and T1-weighted imaging (WI) and T2-WI to assess for myelination, extra-axial blood, and edema [8].

Furthermore, combined analysis of DWI/PWI data can identify tissue at risk of progression to infarction, e.g., "ischemic penumbra" [9]. The penumbra shows critical hypoperfusion without matching diffusion restriction (DWI/PWI mismatch), as opposed to the ischemic core represented by matching areas of hypoperfusion and restricted diffusion. Early and reliable identification of potentially salvageable brain tissue is essential and could serve as a guide to treatment [10].

In addition, simultaneous imaging of the head and neck vasculature should always be considered/included in the acute workup if possible. Magnetic resonance angiography (MRA) of the brain is useful to detect vascular abnormalities (Fig. 14.1b). In children with AIS rapid treatment with neuroprotection, antithrombotic medications, and potential use of tissue plasminogen activator (tPA) are recommended to limit infarct progression/severity and stroke recurrence [11].

Hemorrhagic venous stroke (VS) may occur as complication of cerebral venous sinus thrombosis (CVST). The most common predisposing factors are systemic or local infection, hypercoagulable state, and dehydration [1]. CVST should be suspected in any child with hemorrhage or brain injury that cannot be explained by an arterial distribution of infarction or history of trauma or infection. If there are features of hemorrhage, additional MR venography (MRV) may be needed to provide diagnostic confidence (Fig. 14.1c). Post-contrast 3D-T1-WI ultrafast GRE sequence may also be helpful to detect venous filling defects [12]. CVST most frequently affects the superior sagittal sinus followed by the transverse sinus, with resulting VS mostly in these venous drainage regions. VS may not demonstrate the same degree of restricted diffusion as AIS due to associated vasogenic edema. Physicians should hold an increased level of clinical suspicion especially in children with risk factors [1]. Rapid imaging and correct diagnosis is very important.

Clinical mimickers of pediatric stroke include systemic hypoglycemia, hemiplegic migraine, seizure, postictal paresis, acute disseminated encephalomyelitis (ADEM), infection and may be seen in up to $21 \%$ of children with the initial clinical suspicion of an acute stroke [12-14]. The frequency of these disorders underlines the value of MRI in the emergent evaluation of suspected stroke [12].

\section{Key Point}

- Neuroimaging is extremely time sensitive in children suspected of arterial or venous stroke. MRI with DWI/DTI and ADC is the established imaging modality of choice for the diagnosis.

\subsection{Hemorrhages}

Intracranial hemorrhages ( $\mathrm{ICH})$ are a frequent cause of severe neurological morbidity and mortality in children. Excluding head trauma which is an important cause of $\mathrm{ICH}$, other causes of spontaneous ICH include arteriovenous malformations (AVM), aneurysms, cavernous malformations, hematological disorders, and brain tumors in children. In addition, ICH may be observed as a complication of AIS and VS. Children may present with headache, vomiting, impaired consciousness, convulsions, and focal neurological deficits. Spontaneous ICH can also present with non-specific symptoms in children especially younger than 3 years of age. Subacute courses are common and may result in a diagnostic delay which may result in a higher mortality and morbidity, especially for infratentorial hemorrhages, in aneurysms, children younger than 3 years and children with underlying hematological disorders [15].

ICH imaging appearances follow a well-known evolution with five distinct phases identified: hyperacute, acute, early subacute, late subacute, and chronic. Neuroimaging findings and appearance vary with the phase (Table 14.1) [16]. On CT, early hyperacute ICH are isodense to normal brain, could be missed if not carefully observed. However, multiplanar reconstructed CT images increase the sensitivity of CT for detection of ICH [17]. Acute and early subacute ICH reveal hyperdensity due to progressive blood clot retraction, while progressive red blood cell lysis decreases the density in the late subacute phase. In the chronic phase, progressive blood resorption results in a hypodense, cerebrospinal fluid (CSF)-filled brain defect. Similar temporal signal changes are observed for the various MRI sequences (Fig. 14.2). The imaging characteristics of $\mathrm{ICH}$ are summarized in Table 14.1. T2*, GRE, or SWI sequences are most sensitive to blood products and should be included in the MRI protocol when ICH are suspected in the emergency setting. Neuroimaging should not only focus on diagnosis of $\mathrm{ICH}$ and its complications but should also focus on finding the underlying cause of the ICH. MRA and MRV sequences and contrast-enhanced MRI may be necessary to exclude the underlying cause such as vascular malformations and tumors [16]. 

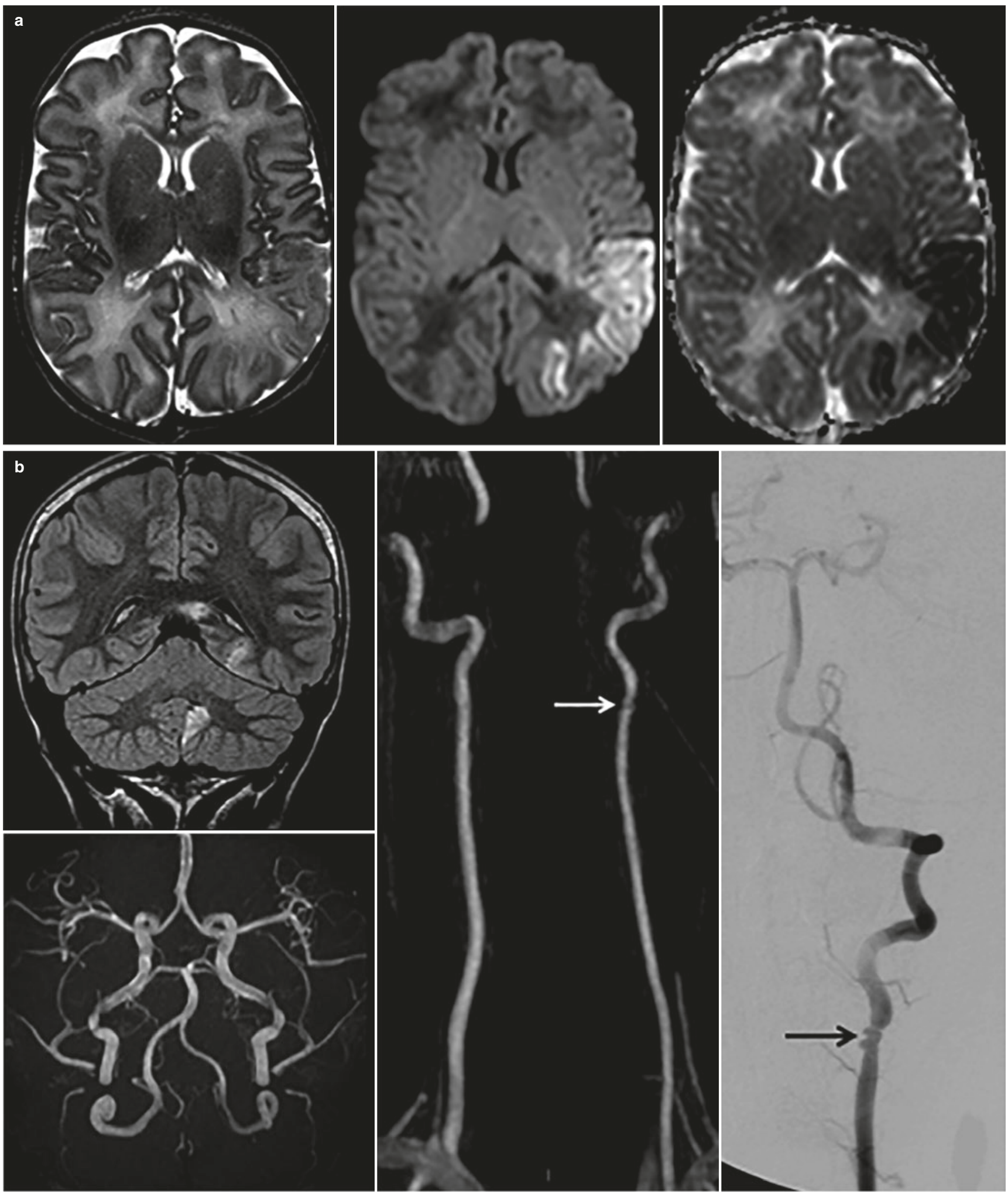

Fig. 14.1 (a, top row) In this 25-day old girl with prior surgery for intestinal atresia and recent onset of clonic fits involving the right limbs, axial T2-weighted MRI shows ill-defined cortico-subcortical blurring of the left parietal lobe. Trace DWI and corresponding ADC map clearly reveal restricted diffusion consistent with an acute ischemic stroke involving the posterior portion of the left MCA territory. (b, middle two rows) In this 13-year old boy with a subacute presentation with ataxia, coronal FLAIR shows multiple hyperintense areas involving the cerebellum and occipital lobe, suggesting a thromboembolic mechanism involving the posterior circulation. Intracranial TOF MR angiography is normal. Cervical
CE-MR angiography, however, shows focal irregularity (arrow) of the left vertebral artery which was confirmed by catheter angiography showing a string-of-beads pattern (arrow) consistent with the eventual diagnosis of fibromuscular dysplasia. (c, lower row) In this 16-year-old girl with recent posterior fossa surgery, sagittal T2-W MRI shows deceivingly normal appearance of the superior sagittal sinus, due to hypointense acute thrombus. Contrast-enhanced axial T1-weighted MRI shows the empty delta sign (arrow) consistent with intraluminal sinus thrombosis, which is confirmed by phase contrast MR venography showing absent flow signal involving the totality of the superior sagittal and straight sinuses 

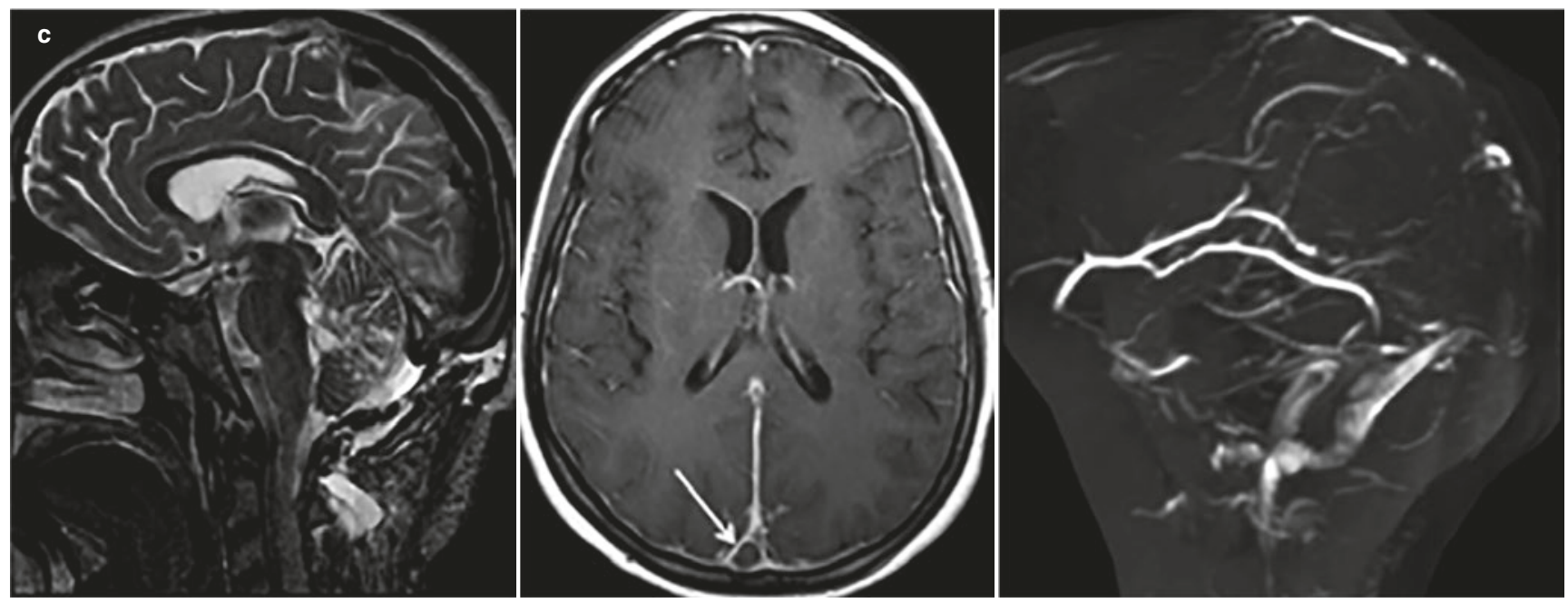

Fig. 14.1 (continued)

Table 14.1 Evolution of ICH characteristics on CT and MRI

\begin{tabular}{|c|c|c|c|}
\hline $\begin{array}{l}\text { Hematoma } \\
\text { age/phase }\end{array}$ & CT & $\begin{array}{l}\text { T1-weighted } \\
\text { MRI }\end{array}$ & T2-weighted MRI \\
\hline $\begin{array}{l}\text { Hyperacute } \\
(<12 \mathrm{~h})\end{array}$ & Isodense & $\begin{array}{l}\text { T1-iso/ } \\
\text { hypointense }\end{array}$ & T2-hyperintense \\
\hline $\begin{array}{l}\text { Acute } \\
(12 \mathrm{~h}-2 \mathrm{~d})\end{array}$ & $\begin{array}{l}\text { Increasing } \\
\text { density }\end{array}$ & $\begin{array}{l}\text { T1-iso/ } \\
\text { hypointense }\end{array}$ & T2-hypointense \\
\hline $\begin{array}{l}\text { Early } \\
\text { subacute } \\
(2-7 d)\end{array}$ & $\begin{array}{l}\text { Increased } \\
\text { density }\end{array}$ & T1-hypointense & T2-hypointense \\
\hline $\begin{array}{l}\text { Late subacute } \\
(8 \mathrm{~d}-1 \mathrm{~m})\end{array}$ & $\begin{array}{l}\text { Decreasing } \\
\text { density }\end{array}$ & T1-hyperintense & T1-hyperintense \\
\hline $\begin{array}{l}\text { Chronic } \\
(>1 \mathrm{~m})\end{array}$ & Hypodense & T1-hypointense & $\begin{array}{l}\text { T2-hyperintense } \\
\text { (hypointense rim) }\end{array}$ \\
\hline
\end{tabular}

$h$ hours, $d$ days, $m$ months

\section{Key Point}

- ICH are a frequent cause of severe neurological morbidity and mortality in children. Depending on the etiology and evolutionary phase of the ICH, CT and/ or MRI imaging characteristics change over time.

\subsection{Infections}

Pediatric central nervous system (CNS) infections are high on the list of differential diagnosis when a child present with fever and altered mental status. Neuroimaging is important in the early diagnosis of CNS infections and should be correlated conjunction with laboratory findings. CNS infections may result in significant morbidity and mortality in children $[2,12]$.

Meningitis is the most common form of bacterial infection of the CNS in children. Causative bacteria vary by age and clinical presentation is different for different age groups.
While older children present with fever, headache, photophobia, nausea, vomiting, and meningismus, neonates may present with less obvious vague symptoms. Meningitis is most commonly caused by hematogenous spread from bacterial infections outside of CNS. Less commonly, it develops from an adjacent infective focus, i.e., sinusitis, mastoiditis, or direct invasion after a skull fracture. In general, neuroimaging is not routinely used for the diagnosis of an uncomplicated meningitis, especially if a lumbar puncture already demonstrated an abnormal number of white blood cells in the CSF confirming diagnosis. If there is no complication, neuroimaging may reveal mildly increased meningeal enhancement. Complications of meningitis may however include areas of adjacent vasogenic or cytotoxic edema, focal ischemic injury, CVST, hydrocephalus, and subdural, epidural, or parenchymal abscesses/empyemas. MRI with contrast is the imaging modality of choice to evaluate the complications of bacterial meningitis (Fig. 14.3a-e) [18].

Parenchymal brain infections include cerebritis and cerebral abscess. Symptoms are variable but typically include fever, headache, nausea/vomiting, focal neurological deficits, and seizures. Visual field defects or personality changes can also be seen. If the abscess collection is large, significant mass effect may cause increased intracranial pressure. Neuroimaging is the key to the diagnosis of parenchymal brain infections. MRI with contrast is the most sensitive and specific modality for the radiology diagnosis (Fig. 14.3a-e). Cerebritis may be treated conservatively with intravenous antibiotics, but cerebral abscess requires surgical intervention or drainage [18].

Pyogenic ventriculitis is a life threatening form of CNS infections. The infectious agents are similar to meningitis and abscess. MRI is key to diagnosis; ependymal enhancement after IV contrast injection is usually very prominent. DWI/DTI may show restricted diffusion with 
Fig. 14.2 In this 17-year old boy presenting acutely with ictal headache, vomiting, and bradycardia, axial CT (a) shows fresh hematoma involving the left parietal lobe with contralateral midline shift and intraventricular blood at the foramen of Monro. CT angiography (b) did not reveal abnormal vessels. MRI performed after 12 hours confirms a large intraaxial bleed which is grossly isointense with gray matter on T1-weighted MRI (c) and hyperintense on T2-weighted MRI (d), consistent with a hyperacute hematoma (prevailing oxyhemoglobin)
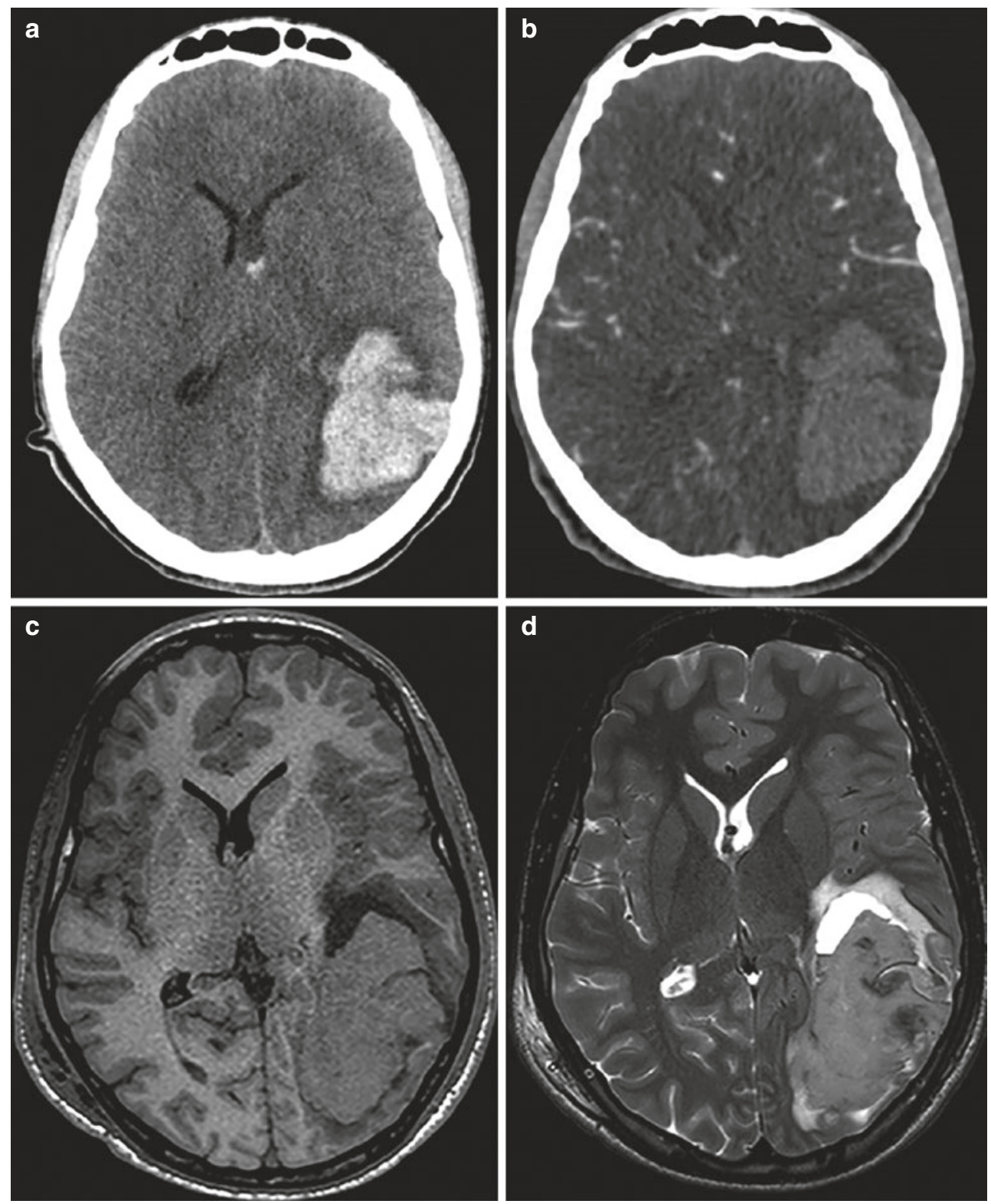

reduced ADC values, especially if pus is collecting within the ventricles [18].

Subdural empyema is an infectious fluid collection between dura and arachnoidea (Fig. 14.3a-e). The acute form develops as a result of spread from, e.g., a paranasal sinus infection and is characterized by high fever, headache, meningismus or focal neurological deficits, more rarely seizures. The complications include CVST, cerebral infarction, cerebral edema, or cerebritis. MRI with contrast is the diagnostic modality of choice [18].

Other acute pediatric CNS infections include: (1) viral meningitis and its complications which include ADEM, transverse myelitis, Guillain-Barré syndrome, and cranial neuritis; (2) fungal CNS infections which are most commonly seen in premature or immunocompromised children; and (3) parasitic CNS infections. Early and correct recognition of the neuroimaging findings of these diseases is critical to guide treatment [19].

\section{Key Point}

- The diagnosis of uncomplicated meningitis is usually confirmed with lumbar puncture by demonstrating an increased number of white blood cells in the CSF. Neuroimaging should be saved to evaluate for life threatening complications of meningitis, including empyema or parenchymal infections. MRI with contrast is the diagnostic modality of choice. 

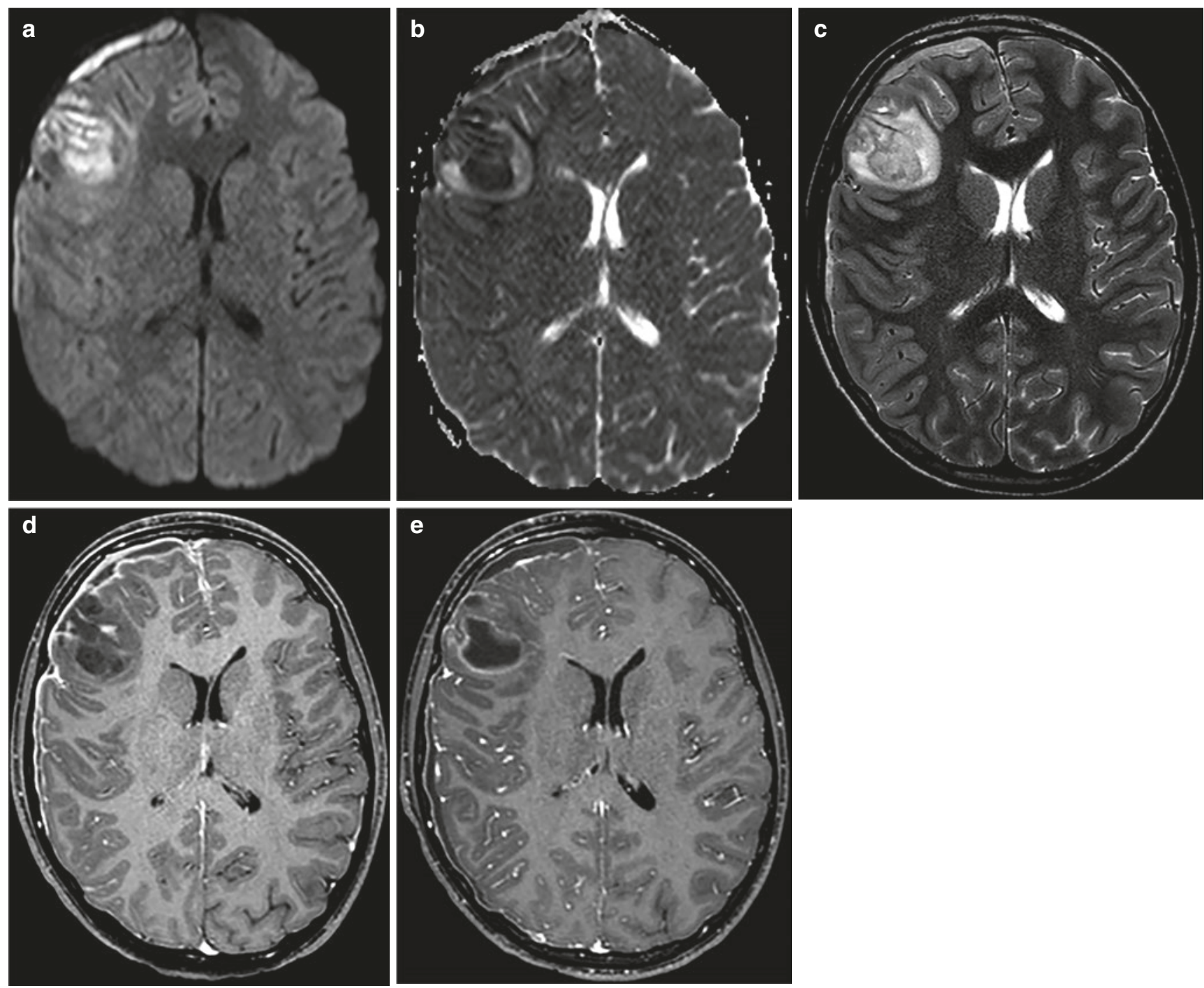

Fig. 14.3 In this 12-year-old boy with suppurative sinusitis (not shown), trace DWI (a) and corresponding ADC map (b) show right frontal subdural empyema and a focal area of restricted diffusion involving the right frontal lobe, corresponding to an ill-defined hyperintense lesion with perifocal edema and mass effect on T2-W MRI (c).
Contrast-enhanced axial T1-weighted MRI (d) shows mild, irregular peripheral enhancement consistent with cerebritis, and also reveals ipsilateral arachnoiditis. Follow-up MRI after 4 days (e) shows development of a well-defined enhancing ring corresponding to evolution towards a frank abscess

\subsection{Trauma}

Traumatic brain injury (TBI) is a significant cause of lifelong disability and mortality in children. The diagnosis of pediatric head trauma requires a multidisciplinary approach. Trauma mechanisms differ from adults and their impact on the developing brain results in unique primary and secondary brain lesions. Birth trauma is responsible for almost all cases of neonatal trauma (parturitional injury); child abuse is a frequent cause of trauma in the first 2 years, whereas falls and motor-vehicle accidents are responsible for the majority of TBI in toddlers and adolescents [20].

Primary TBI lesions include (1) calvarial and maxillofacial fractures; (2) extra-axial: epidural hematoma, subdural hematoma, subarachnoid hemorrhage, and intraventricular hemorrhage; (3) intraaxial: diffuse axonal injury, cortical contusion, and intracerebral hematoma; and (4) vascular: dissection, carotid cavernous fistula, arteriovenous dural fistula, and pseudoaneurysm. Secondary TBI includes both (1) acute: diffuse cerebral edema, brain herniation (subfalcine, uncal, transtentorial), infarction, or infection and (2) chronic injuries: hydrocephalus, encephalomalacia, CSF leak, and leptomeningeal cyst [21]. Different imaging modalities may identify treatable primary TBI and lead to prevention or limitation of secondary TBI. A recommended minimal standard MRI protocol includes (1) a 3D T1-WI, preferably in the sagittal plane, which allows multiplanar reconstruction, (2) an axial T2-WI, (3) an axial DWI/DTI with ADC mapping, 

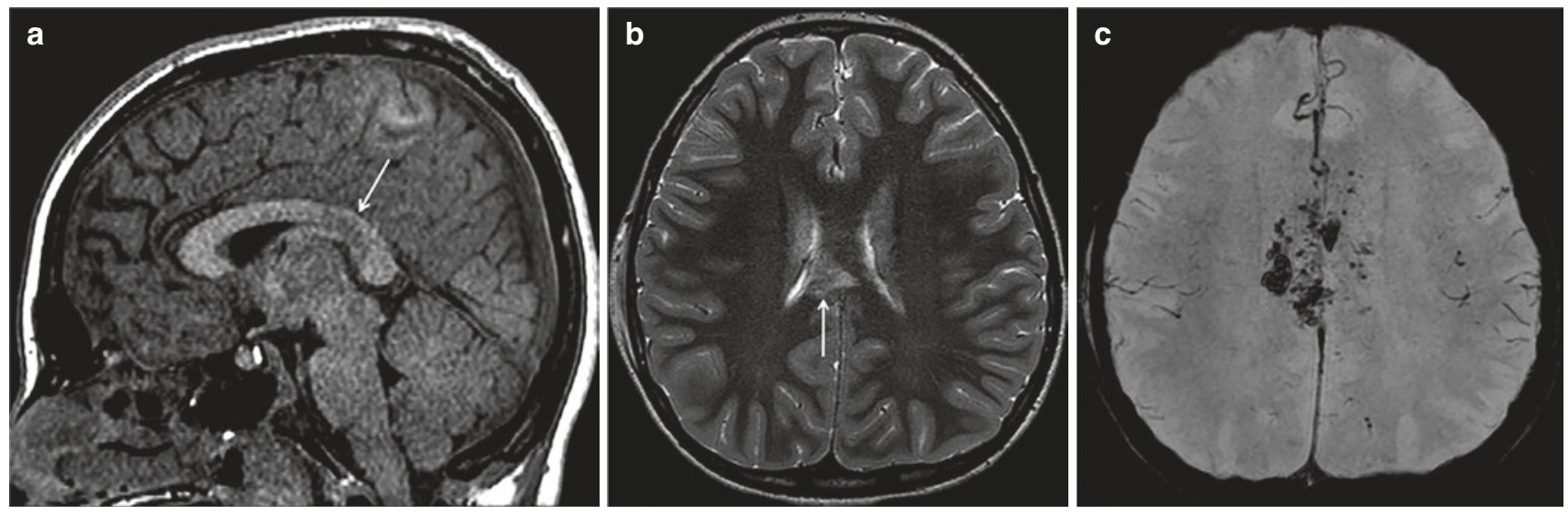

Fig. 14.4 In this 12-year-old boy with a severe blunt head trauma, sagittal T1-weighted MRI (a) and axial T2-W MRI (b) show ill-defined signal abnormality involving the corpus callosum (arrows). SWI (c) reveals more extensive hypointensities consistent with hemorrhagic traumatic axonal injury and (4) an axial SWI from the skull base to the vertex (Fig. 14.4) [20].

It is important to keep child abuse in mind when dealing with trauma in children in the emergency setting. Abusive head trauma diagnosis is challenging both clinically and radiologically; the provided trauma history might not always reflect the truth and no single imaging finding is specific or diagnostic for abusive head trauma by itself. A multiplicity of trauma related findings and severity of findings inconsistent with the provided mechanism of trauma provide clues for the diagnosis. The radiological findings may vary for different types of injury. The common injury mechanisms related to abusive head trauma can be summarized as non-impact shaking injury, direct impact blunt trauma, whiplash-shaking injury of the craniocervical junction, strangulation injury, and stabbing injury. Further diagnostic workup should include a skeletal survey and possible additional cross-sectional studies to exclude other non-CNS solid organ injury $[1,21]$.

Spinal cord injury mechanisms in children differ from adults. Vertebral column fractures and spinal cord injuries are less common in children than adults. Pediatric spinal injuries primarily involve the cervical spine mostly in the proximal part including the craniocervical junction [22]. Thoracolumbar fractures often occur at T11 to L2 levels but may also involve other levels. However, if the injury mechanism is of the seat-belt flexion-distraction type, it typically occurs at L2-L4 levels with high association of accompanying visceral injury. In children of all ages, spinal MRI evaluation is important in the setting of unexplained instability, persistent or delayed symptoms, decreased consciousness, or abnormal X-ray or CT findings. MRI findings including spinal cord transection, intramedullary hemorrhage, edema extending over more than one spinal segment, and persistent compression of the spinal cord are predictive of poor neurological outcome [12].

\section{Key Point}

- TBI is a leading cause of lifelong disability and mortality in children. Birth trauma is responsible for many cases of neonatal TBI; child abuse is a frequent cause of TBI in the first 2 years, falls and motor-vehicle accidents are responsible for the majority of TBI in toddlers and adolescents. CT or MRI are the primary diagnostic imaging modalities of choice depending on the etiology of TBI and the age of the child.

\subsection{Drowning/Near Drowning}

Drowning is a leading cause of accidental death and severe neurologic morbidity. Anoxic brain injury is a common consequence of non-fatal drowning and may cause severe lifelong disability. Irreversible injury develops in the hippocampi, basal ganglia, and cerebral cortex within 4-10 $\mathrm{min}$ following the hypoxic incident. Therefore, early neuroimaging after drowning allows detection of the injury that requires intervention and may also be helpful for the prediction of brain injury severity [23]. CT is usually limited to detect head and neck trauma, but it may detect the loss of gray white matter differentiation and cerebral edema. However a normal looking brain parenchyma on initial/acute CT does not rule out brain injury. MRI especially DWI/DTI with ADC mapping can detect brain injury more reliably, even within minutes after the incident and improves the ability to predict the outcome (Fig. 14.5). Cortical and deep gray matter DWI/ DTI abnormalities and lower ADC values are associated with poor outcomes. 

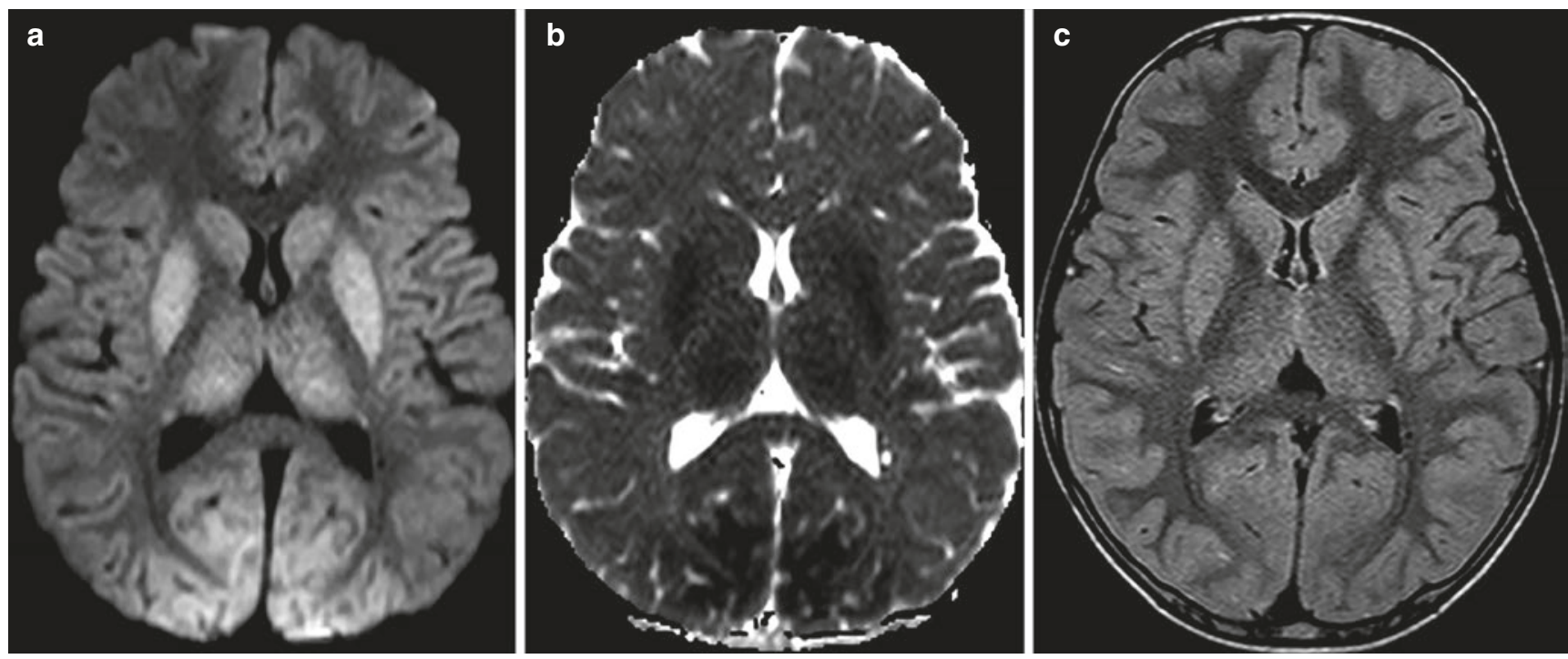

Fig. 14.5 In this 8-year-old boy with severe anoxia due to neardrowning, trace DWI (a) and corresponding ADC map (b) show restricted diffusion involving the basal ganglia, thalami, and parieto-

\section{Key Point}

- The pediatric brain is extremely sensitive to hypoxia and irreversible brain damage develops within 4-10 min after drowning. MRI with DWI/DTI and ADC mapping should be acquired and evaluated promptly for the children with a drowning history.

\subsection{Seizures}

New onset seizures are the most common neurologic emergencies of children presenting to the ER [1,2]. Seizures are abnormal movements or behavior resulting from abnormal electrical activity in the brain. Acute symptomatic seizures occur at the time of a systemic insult or following brain insult with incidence reaching 29-39/100000 per year. Traumatic brain injury, cerebrovascular disease, drug withdrawal, infarction, and metabolic insults are the most common causes $[1,2]$. Unprovoked seizures occur in the absence of precipitating factors, and may be single or recurrent (epilepsy).

A detailed neurological examination with general physical examination and electroencephalogram should be performed initially [1]. Neuroimaging may not be necessary for every child with seizure or epilepsy. For example, neuroimaging is not indicated in children with simple febrile seizures but it should be considered in children with long seizures with focal onset and focal neurological deficits, also it should occipital cortex consistent with cytotoxic edema. Axial FLAIR (c) is relatively unrevealing in the acute phase

be strongly considered in neonates with seizures, because an identifiable cause for seizures can be found in up to $90 \%$ of full term newborns $[1,2]$. When neuroimaging is necessary, MRI is the modality of choice in most of the cases. The aim is to investigate acute focal disorders that need immediate intervention including acute hydrocephalus, acute stroke or hemorrhage, hypoxic ischemic injury in newborns, encephalitis, or tumors. Other relevant disorders include leukodystrophies, metabolic disorders, mesial temporal sclerosis, and malformations [2].

\section{Key Point}

- When dealing with children with seizures in the ER, neuroimaging should be saved for cases with history of long time seizures with focal onset and focal neurological deficits, it should also be strongly considered in neonates with seizures.

\subsection{Autoimmune CNS Diseases with Acute Presentation}

Children with autoimmune CNS diseases may also present to the ER with acute signs and symptoms.

ADEM is a monophasic, immune-mediated demyelinating CNS disorder. Patients usually present 1-3 weeks following an upper respiratory infection or vaccination and may present acutely with significant mass effect due to a tumefactive 

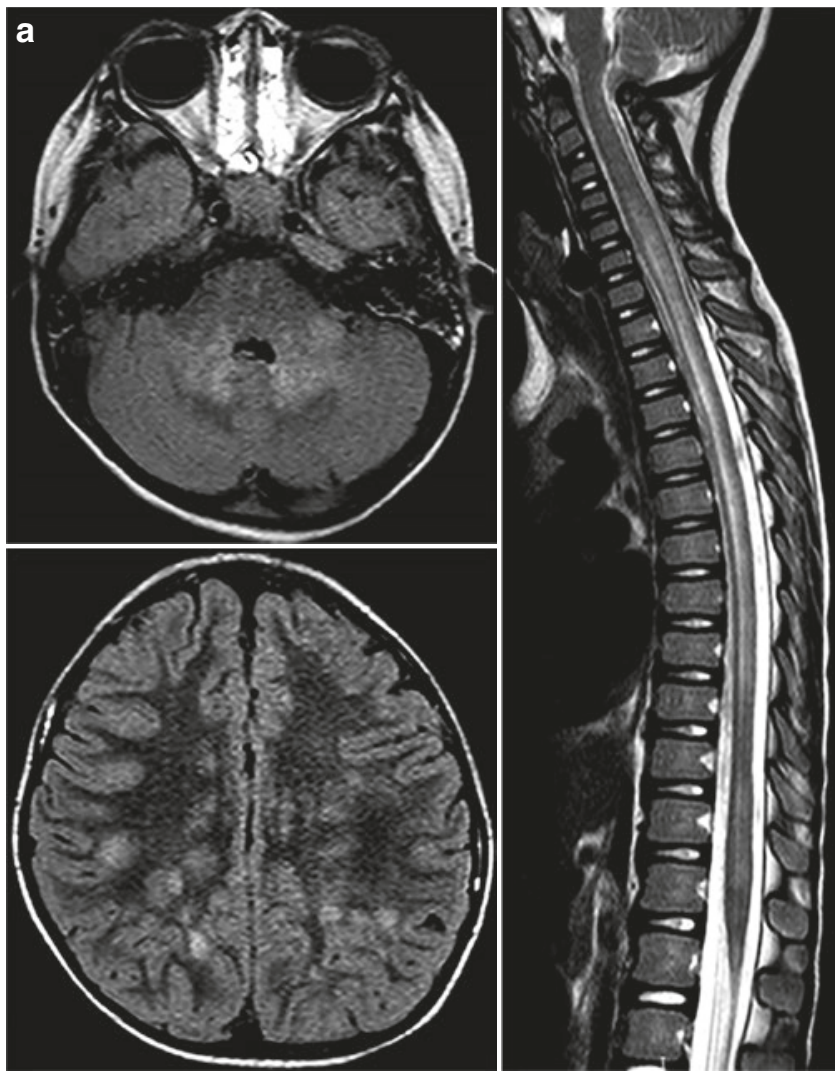

Fig. 14.6 (a) In this 3-year-old girl with a subacute onset of paraparesis and stupor, axial FLAIR shows ill-defined hyperintensities involving the deep cerebellar and supratentorial white matter. Sagittal T2-weighted MRI of the spine shows quasi-holocord swelling and hyperintensity. After treatment with IVIG and steroids, follow-up MRI at 3 months was normalized (not shown), consistent with acute disseminated encephalomyelitis (ADEM). (b) In this 5-year-old girl presenting with headaches and left hemiparesis, trace DWI and corresponding ADC map show a

lesion, with seizures and coma or with behavioral disturbances, headaches, vomiting, fever, or focal neurological signs. Brain MRI typically shows diffuse, poorly demarcated, bilateral, or asymmetrical T2 and FLAIR hyperintense lesions affecting both gray and white matter. The spinal cord may also be involved, showing ill-defined, multiple T2 hyperintense lesions usually involving 2-3 vertebral segments (Fig. 14.6a). ADEM is usually a diagnosis of exclusion.

Multiple sclerosis (MS) is an idiopathic inflammatory disorder characterized by demyelination and degeneration of the CNS, affects most commonly adults, but $3-10 \%$ of patients present their first clinical event during childhood. Pediatric onset MS has higher relapse rates and a less favorable prognosis [24]. Therefore, early diagnosis and prompt treatment of pediatric onset MS is necessary (Fig. 14.6b). Ruling out ADEM and neuromyelitis optica spectrum disorder (NMOSD) is important, because although the clinical presentation of these three disorders
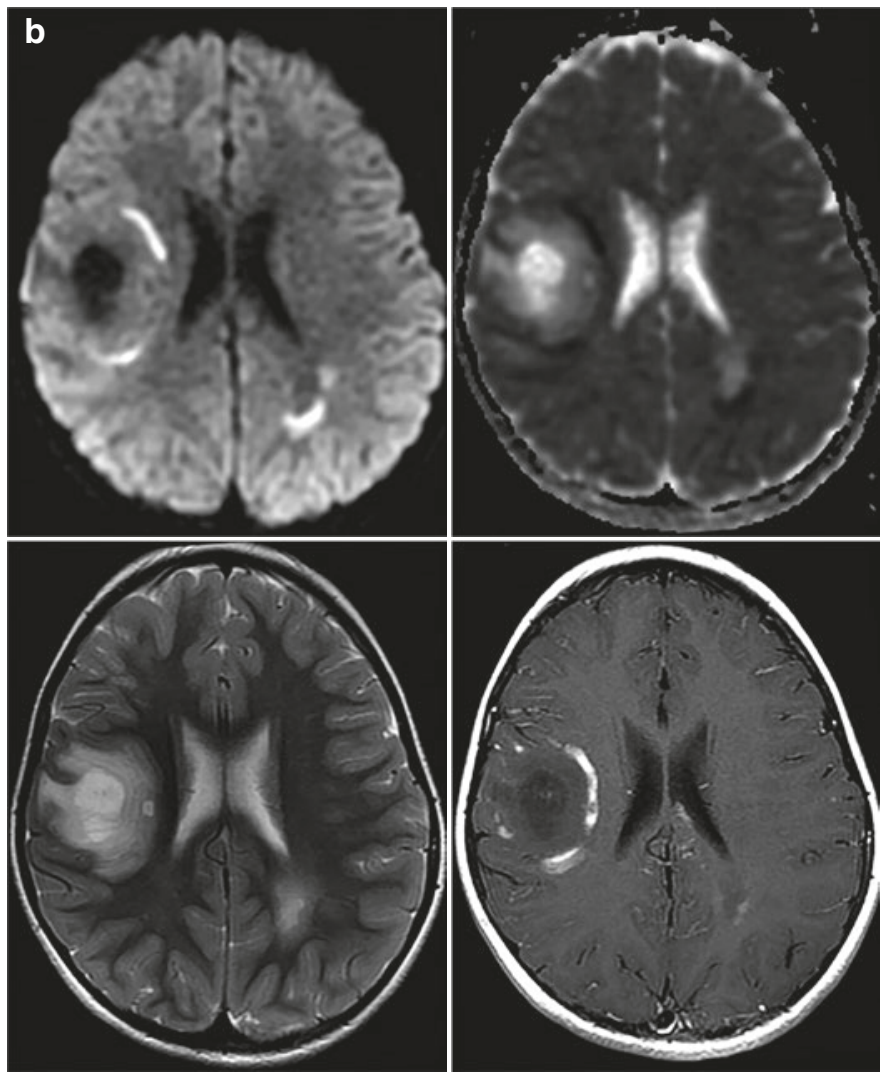

large right inferior frontal lesion with a necrotic center and an incomplete peripheral ring of restricted diffusion. Another small lesion with similar characteristics is seen in the left parietal lobe. On T2-weighted MRI, the larger lesion shows a concentric onion-bulb appearance. Contrast-enhanced axial T1-weighted MRI shows incomplete peripheral ring enhancement corresponding to the areas of restricted diffusion, consistent with tumefactive demyelination

is similar, the treatment is different in each one. Characteristic MRI criteria of MS on brain MRI are helpful to differentiate MS from ADEM and NMOSD but CSF and serum laboratory findings are also required to confirm the diagnosis [25].

NMOSD is an inflammatory demyelinating disease characterized by optic neuritis and acute longitudinally extending myelitis involving at least three vertebral segments. Most clinical, neuroimaging, and laboratory characteristics of pediatric onset NMOSD are similar to those of adult onset disease [26]. Identification of aquaporin-4 immunoglobulin antibodies serves as an important biomarker for the diagnosis. Longitudinally extending myelitis during an acute attack is T2 hyperintense on sagittal spinal cord MRI with central cord predominance and may matching show contrast enhancement on T1-WI. Optic nerve MRI shows unilateral or bilateral T2 hyperintensity or contrast enhancement on T1-WI within the optic 
nerve(s) or optic chiasm. Typical brain lesions are T2 hyperintense involving the dorsal medulla, peri-ependymal surfaces of the fourth ventricle, hypothalamus, thalamus, or peri-ependymal surfaces of third ventricle, as well as large, confluent, unilateral or bilateral subcortical or deep white matter lesions or long, diffuse, heterogeneous corpus callosum lesions or long corticospinal tract lesions [26].

\section{Key Point}

- Children with autoimmune CNS diseases may present to the ER with acute signs and symptoms. In addition to CSF and serum laboratory findings, brain and spinal cord MRI is helpful to differentiate MS from ADEM and NMOSD.

\subsection{Toxic/Metabolic Imbalances}

Diabetic ketoacidosis (DKA) is an acute serious complication in children with diabetes mellitus type 1 . This state may cause significant long-term neurological morbidity and mortality. Brain edema is the most common neuroimaging finding in children with DKA and may cause brain herniation with subsequent cerebral infarctions due to vascular compression. Also, primary ischemic or hemorrhagic stroke during the acute DKA episode may be seen in $10 \%$ of cases [27].

Neonatal hypoglycemia is an important issue in term neonates and can cause severe brain injury. Neuroimaging typically shows focal edema or infarcts most commonly in bilateral parieto-occipital lobes, basal ganglia, corpus callosum, and thalami [12]. Because the urine strip test diagnosis of hypoglycemia can be made simply, neuroimaging is usually used to evaluate the degree of brain injury and estimation of prognosis rather than to confirm the diagnosis.

Osmotic demyelination syndrome is an acute demyelination process that usually occurs several days after a rapid change in serum osmolality and is uncommon in children. It can present with central pontine myelinolysis or extrapontine myelinolysis. MRI is the diagnostic modality of choice. Central pontine myelinolysis is seen with characteristic trident-shaped, bat-winged, or piglet hyperintensity on T2-WI, hypointensity on T1-WI with no mass effect in the central pons, with sparing of the ventrolateral pons, tegmentum, and corticospinal tracts. Extrapontine lesions are usually bilateral and most commonly seen in the cerebellar peduncles, globus pallidus, thalamus, lateral geniculate body, putamen, external and extreme capsule, splenium of corpus callosum, and supratentorial white matter [28].

\section{Key Point}

- Neuroimaging with MRI may be necessary when dealing with children with metabolic imbalances in the ER when brain edema is suspected. In addition, MRI is the diagnostic modality of the choice to evaluate the osmotic demyelination syndrome.

\subsection{Hydrocephalus}

Hydrocephalus in children could be either due to overproduction of CSF by a tumor, ventricular obstruction, or dysfunctional Pachionic granulations along the subarachnoid space. Children can present to the ER with (1) acute findings of hydrocephalus or (2) complications of shunted hydrocephalus including malfunction and infection.

Clinical presentation of hydrocephalus varies with age; in infants abnormally rapidly increasing head circumference, irritability, vomiting, bulging of the fontanelle; in older children headache, vomiting, diplopia, or papilledema can be seen [29]. Neuroimaging should be directed to identify the underlying cause. MRI is the imaging modality of choice in suspected hydrocephalus cases. Next to axial T1-WI or T2-WI, a thin section, high resolution, T2-WI in sagittal plane is the most helpful MRI sequence to evaluate these cases $[1,2]$. Contrast administration would be necessary if a tumor is suspected.

Shunt malfunction is the most common complication of ventricular shunts. Children present with headache, nausea, vomiting, and altered mental status. Multiple radiographs of the skull, neck, chest, and abdomen to evaluate the shunt integrity are often the initial imaging in some institutions $[1,2]$. CT is often used as primary cross-sectional imaging because it is readily available, able to detect changes in the ventricular size and configuration, identify intracranial hemorrhages or infarction, shunt catheter discontinuity and catheter migration [2]. Scout views should not be ignored, because occasionally, axial or reconstructed cross-sectional CT images may miss relevant findings. A possible disconnection of a shunt may be within the neck region below or outside of the scanned region; however, the information may be visible on the scout view which is a standard component of each CT study [30]. Children with infection due to a shunted hydrocephalus present with altered mental status, fever, nausea, and vomiting. CSF cultures are usually sufficient for the diagnosis 
in almost $95 \%$ of the cases and neuroimaging has a limited role in the diagnosis [1]. Uncommonly, infection presents with abdominal symptoms from a peritoneal CSF pseudocyst. Abdominal ultrasound or CT is useful to evaluate these cases [2].

\section{Key Point}

- Children could present to the ER with acute findings of hydrocephalus or complications of shunted hydrocephalus including shunt malfunction and infection. MRI or CT should be used to differentiate the etiology.

\subsection{Concluding Remarks}

Children with acute neurological emergency present to the ER with a wide spectrum of symptoms and signs. Neuroimaging is often needed in children with acute neurological emergency due to the difficulties of gathering a good and reliable patient history or limited physical/neurological examination. The radiologist should be able to answer if the child needs neuroimaging emergently, and which imaging modality is appropriate for the child. Different modalities may be chosen based on the institutional availability and clinical status of the patient. CT and MRI are the most frequently used imaging modalities in the emergency setting. Finally, the prompt diagnosis is essential when dealing with children with acute neurological emergency especially in the time sensitive pathologies, i.e., stroke, drowning.

\section{Take Home Messages}

- Radiology is an integral part of the ER.

- Children, especially infants and young children may present to the ER with non-specific symptoms or subtle findings.

- The threshold for neuroimaging should be held low especially for infants and young children.

- Neuroimaging should be promptly acquired and evaluated when dealing with children with acute neurological emergencies in the ER.

\section{References}

1. Saigal G, Ezuddin NS, Vega G. Neurologic emergencies in pediatric patients including accidental and nonaccidental trauma. Neuroimaging Clin N Am. 2018;28(3):453-70. https://doi. org/10.1016/j.nic.2018.03.007.

2. Prabhu SP, Young-Poussaint T. Pediatric central nervous system emergencies. Neuroimaging Clin N Am. 2010;20(4):663-83. https://doi.org/10.1016/j.nic.2010.07.008.

3. Chang PT, Yang E, Swenson DW, Lee EY. Pediatric emergency magnetic resonance imaging: current indications, techniques, and clinical applications. Magn Reson Imaging Clin N Am. 2016;24(2):449-80. https://doi.org/10.1016/j.mric.2015.11.009.

4. Singh RK, Smith JT, Wilkinson ID, Griffiths PD. Ultrafast MR imaging in pediatric neuroradiology. Acta Radiol. 2003;44(5):550 7. https://doi.org/10.1034/j.1600-0455.2003.00118.x.

5. Stacey A, Toolis C, Ganesan V. Rates and risk factors for arterial ischemic stroke recurrence in children. Stroke. 2018;49(4):842-7. https://doi.org/10.1161/STROKEAHA.117.020159.

6. Goeggel Simonetti B, Cavelti A, Arnold M, Bigi S, Regenyi M, Mattle HP, et al. Long-term outcome after arterial ischemic stroke in children and young adults. Neurology. 2015;84(19):1941-7. https://doi.org/10.1212/WNL.0000000000001555.

7. Mackay MT, Wiznitzer M, Benedict SL, Lee KJ, Deveber GA, Ganesan V. Arterial ischemic stroke risk factors: the international pediatric stroke study. Ann Neurol. 2011;69(1):130-40. https://doi. org/10.1002/ana.22224.

8. Lee S, Mirsky DM, Beslow LA, Amlie-Lefond C, Danehy AR, Lehman L, et al. Pathways for neuroimaging of neonatal stroke. Pediatr Neurol. 2017;69:37-48. https://doi.org/10.1016/j. pediatrneurol.2016.12.008.

9. Gadian DG, Calamante F, Kirkham FJ, Bynevelt M, Johnson CL, Porter DA, et al. Diffusion and perfusion magnetic resonance imaging in childhood stroke. J Child Neurol. 2000;15(5):279-83.

10. Meoded A, Poretti A, Benson JE, Tekes A, Huisman TA. Evaluation of the ischemic penumbra focusing on the venous drainage: the role of susceptibility weighted imaging (SWI) in pediatric ischemic cerebral stroke. J Neuroradiol. 2014;41(2):108-16. https://doi. org/10.1016/j.neurad.2013.04.002.

11. Shack M, Andrade A, Shah-Basak PP, Shroff M, Moharir M, Yau I, et al. A pediatric institutional acute stroke protocol improves timely access to stroke treatment. Dev Med Child Neurol. 2017;59(1):317. https://doi.org/10.1111/dmcn.13214.

12. Lall NU, Stence NV, Mirsky DM. Magnetic resonance imaging of pediatric neurologic emergencies. Top Magn Reson Imaging. 2015;24(6):291-307. https://doi.org/10.1097/ RMR.0000000000000068.

13. Jordan LC, Hillis AE. Challenges in the diagnosis and treatment of pediatric stroke. Nat Rev Neurol. 2011;7(4):199-208. https://doi. org/10.1038/nrneurol.2011.23.

14. Shellhaas RA, Smith SE, O'Tool E, Licht DJ, Ichord RN. Mimics of childhood stroke: characteristics of a prospective cohort. Pediatrics. 2006;118(2):704-9. https://doi.org/10.1542/peds.2005-2676.

15. Meyer-Heim AD, Boltshauser E. Spontaneous intracranial haemorrhage in children: aetiology, presentation and outcome. Brain Dev. 2003;25(6):416-21. https://doi.org/10.1016/ S0387-7604(03)00029-9. 
16. Huisman TA, Singhi S, Pinto PS. Non-invasive imaging of intracranial pediatric vascular lesions. Childs Nerv Syst. 2010;26(10):127595. https://doi.org/10.1007/s00381-010-1203-1.

17. Langford S, Panigrahy A, Narayanan S, Hwang M, Fitz C, Flom L, et al. Multiplanar reconstructed CT images increased depiction of intracranial hemorrhages in pediatric head trauma. Neuroradiology. 2015;57(12):1263-8. https://doi.org/10.1007/s00234-015-1584-7.

18. Nickerson JP, Richner B, Santy K, Lequin MH, Poretti A, Filippi CG, et al. Neuroimaging of pediatric intracranial infection--part 1: techniques and bacterial infections. J Neuroimaging. 2012;22(2):e4251. https://doi.org/10.1111/j.1552-6569.2011.00700.x.

19. Nickerson JP, Richner B, Santy K, Lequin MH, Poretti A, Filippi $\mathrm{CG}$, et al. Neuroimaging of pediatric intracranial infection--part 2: TORCH, viral, fungal, and parasitic infections. J Neuroimaging. 2012;22(2):e52-63. https://doi. org/10.1111/j.1552-6569.2011.00699.x.

20. Pinto PS, Poretti A, Meoded A, Tekes A, Huisman TA. The unique features of traumatic brain injury in children. Review of the characteristics of the pediatric skull and brain, mechanisms of trauma, patterns of injury, complications and their imaging findings--part 1. J Neuroimaging. 2012;22(2):e1-e17. https://doi. org/10.1111/j.1552-6569.2011.00688.x.

21. Pinto PS, Meoded A, Poretti A, Tekes A, Huisman TA. The unique features of traumatic brain injury in children. Review of the characteristics of the pediatric skull and brain, mechanisms of trauma, patterns of injury, complications, and their imaging findings--part 2. J Neuroimaging. 2012;22(2):e18-41. https://doi. org/10.1111/j.1552-6569.2011.00690.x.

22. Meoded A, Singhi S, Poretti A, Eran A, Tekes A, Huisman TA. Tectorial membrane injury: frequently overlooked in pediatric traumatic head injury. AJNR Am J Neuroradiol. 2011;32(10):180611. https://doi.org/10.3174/ajnr.A2606.

23. Topjian AA, Berg RA, Bierens JJ, Branche CM, Clark RS, Friberg $\mathrm{H}$, et al. Brain resuscitation in the drowning victim. Neurocrit Care. 2012;17(3):441-67. https://doi.org/10.1007/s12028-012-9747-4.

24. Tenembaum SN. Pediatric multiple sclerosis: distinguishing clinical and MR imaging features. Neuroimaging Clin N Am. 2017;27(2):229-50. https://doi.org/10.1016/j.nic.2016.12.007.

25. Filippi M, Rocca MA, Ciccarelli O, De Stefano N, Evangelou N, Kappos L, et al. MRI criteria for the diagnosis of multiple sclerosis: MAGNIMS consensus guidelines. Lancet Neurol. 2016;15(3):292303. https://doi.org/10.1016/S1474-4422(15)00393-2.

26. Wingerchuk DM, Banwell B, Bennett JL, Cabre P, Carroll W, Chitnis T, et al. International consensus diagnostic criteria for neuromyelitis optica spectrum disorders. Neurology. 2015;85(2):17789. https://doi.org/10.1212/WNL.0000000000001729.

27. Barrot A, Huisman TA, Poretti A. Neuroimaging findings in acute pediatric diabetic ketoacidosis. Neuroradiol J. 2016;29(5):317-22.

28. Bansal LR, Zinkus T. Osmotic demyelination syndrome in children. Pediatr Neurol. 2019;97:12-7. https://doi.org/10.1016/j. pediatrneurol.2019.03.018.

29. Kahle KT, Kulkarni AV, Limbrick DD Jr, Warf BC. Hydrocephalus in children. Lancet. 2016;387(10020):788-99. https://doi. org/10.1016/S0140-6736(15)60694-8.

30. Orman G, Bosemani T, Tekes A, Poretti A, Huisman TA. Scout view in pediatric $\mathrm{CT}$ neuroradiological evaluation: do not underestimate! Childs Nerv Syst. 2014;30(2):307-11. https://doi.org/10.1007/ s00381-013-2288-0.

Open Access This chapter is licensed under the terms of the Creative Commons Attribution 4.0 International License (http://creativecommons. org/licenses/by/4.0/), which permits use, sharing, adaptation, distribution and reproduction in any medium or format, as long as you give appropriate credit to the original author(s) and the source, provide a link to the Creative Commons license and indicate if changes were made.

The images or other third party material in this chapter are included in the chapter's Creative Commons license, unless indicated otherwise in a credit line to the material. If material is not included in the chapter's Creative Commons license and your intended use is not permitted by statutory regulation or exceeds the permitted use, you will need to obtain permission directly from the copyright holder. 


\section{Part IV}

Head and Neck 


\title{
Evaluation of Tinnitus and Hearing Loss in the Adult
}

\author{
Jenny K. Hoang and Laurie A. Loevner
}

\section{Learning Objectives}

- Identify key anatomical structures in the temporal bone.

- Describe role of CT and MRI for temporal bone imaging.

- Differentiate between diseases of the temporal bone on imaging and describe their clinical presentation.

\section{Key Points}

- Imaging can identify the cause and evaluate the extent of disease for surgical planning.

- The common causes of tinnitus and hearing loss without a mass include otospongiosis, labyrinthitis ossificans, superior semicircular canal dehiscence, and enlarged vestibular aqueduct syndrome.

- Otospongiosis affects the bony labyrinth, while labyrinthitis ossificans affects the membranous labyrinth.

- Vestibular schwannomas often present with nonpulsatile tinnitus and high frequency sensorineural hearing loss.

\subsection{Introduction}

Tinnitus and hearing loss in the adult can have profound effects on the quality of life. Tinnitus is the perception of sound when no external sound is present and may be described as ringing, buzzing, swishing, or clicking sensations. Hearing

J. K. Hoang $(\bowtie)$

Department of Radiology and Radiological Science,

Russell H. Morgan, Johns Hopkins School of Medicine,

Baltimore, MD, USA

\section{A. Loevner}

Department of Neuroradiology, Radiology, Neurosurgery,

Otorhinolaryngology: Head \& Neck Surgery, and Ophthalmology,

University of Pennsylvania, Philadelphia, PA, USA loss ranges from partial to a total inability to hear sounds. Hearing loss and tinnitus can occur concurrently or in isolation. The role of imaging is to help identify the etiology of these symptoms and evaluate for the extent of disease.

\subsection{Causes of Tinnitus and Hearing Loss}

\subsubsection{Tinnitus}

Tinnitus may be categorized as (1) pulsatile or non-pulsatile, (2) primary (idiopathic) or secondary (due to another condition), and (3) subjective or objective. Evaluation of tinnitus starts with otoscopic examination to determine if there is a vascular retro-tympanic mass, audiometric examination, and review of medical history and medications. This evaluation helps to determine if imaging is necessary and if so, what study(ies) are indicated.

\subsubsection{Pulsatile Tinnitus}

Causes include vascular masses (glomus tympanicum), aberrant arterial or venous anatomy, vascular malformations, and intracranial hypertension. Objective tinnitus (auscultation of a bruit on physical examination) is uncommon and has been attributed to turbulent flow in the setting of dural fistulas, atherosclerotic carotid artery disease, jugular bulb abnormalities, and large condylar or mastoid emissary veins.

\subsubsection{Non-pulsatile Tinnitus}

Causes are wide ranging and include cerumen impaction, middle ear infection, mass, medications, noise-induced hearing loss, presbycusis or chronic bilateral hearing loss, hemorrhage, neurodegeneration, and spontaneous intracranial hypotension.

\subsubsection{Hearing Loss}

Clinical assessment and audiometric testing can determine the type of hearing loss as conductive, sensorineural, or 
mixed and guide subsequent diagnostic imaging. Conductive hearing loss results from diseases affecting the conduction of mechanical sound wave energy to the cochlea. Sensorineural hearing loss is caused by diseases that impair the cochlear function or the transmission of electrical signal along the auditory pathway.

\subsubsection{Conductive Hearing Loss}

Causes include otospongiosis (commonly mixed), ossicular erosion or fusion, round window occlusion, dehiscence of the superior semicircular canal, and cholesteatoma or neoplasm with suspected intracranial or inner ear extension.

\subsubsection{Sensorineural Hearing Loss}

Causes include labyrinthine ossificans, vestibular schwannoma, and fractures extending across the otic capsule.

\subsection{Anatomy}

The temporal bone forms part of the middle and posterior cranial fossae and is comprised of five parts: petrous, tympanic, mastoid, styloid, and squamous temporal bone. The first three listed parts form the external auditory canal, middle ear, inner ear, and internal auditory canal. These are the segments to review for hearing loss and tinnitus.

\subsubsection{External Auditory Canal}

The external auditory canal (EAC) extends from the auricle to the tympanic membrane. The lateral one-third of the EAC is fibrocartilaginous, while the medial two-thirds are surrounded by the tympanic portion of the temporal bone.

\subsubsection{Middle Ear}

The middle ear cavity contains the ossicular chain which conducts sound from the tympanic membrane laterally, to the oval window and inner ear structures medially. The roof of the middle ear is the tegmen tympani and the jugular wall is the floor. The middle ear can be subdivided into the epitympanum (attic) superior to the level of the tympanic membrane, mesotympanum at the level of tympanic membrane, and hypotympanum inferior to the level of tympanic membrane. The epitympanum communicates with the mastoid via the aditus ad antrum.

The mesotympanum contains the majority of the ossicular chain. The ossicular chain is composed of three bones: the malleus, incus, and stapes. Since the stapes is anchored to the oval window, a mnemonic for the order of the ossicles is "MISO" representing malleus, incus, stapes, and oval win- dow. The manubrium of the malleus is attached to the tympanic membrane, and the head of the malleus articulates with the body of the incus in the epitympanum forming the incudomalleal joint, which has a characteristic "ice cream cone" configuration on axial sections. The lenticular process of the incus extends at approximately a right angle from the long process of the incus to articulate with the capitulum (head) of the stapes, forming the incudostapedial joint.

An important middle ear structure is the scutum, a sharp bony projection to which the tympanic membrane is attached superiorly. Prussak space, the location for pars flaccida cholesteatomas, is bounded by the scutum laterally and the neck of the malleus medially.

The posterior wall of the middle ear is irregular and includes the sinus tympani, pyramidal eminence, and facial recess.

\subsubsection{Inner Ear}

The inner ear comprises the osseous labyrinth, which includes the cochlea, vestibule, and semicircular canals. The cochlea contains the end organ for hearing while the vestibule and semicircular canals are responsible for balance and equilibrium. The densest portion of the temporal bone that surrounds the osseous labyrinth is termed the otic capsule. The osseous labyrinth encapsulates the membranous labyrinth, which contains endolymph and is surrounded by perilymph.

The cochlea is a spiral-shaped structure with $2 \frac{1}{2}-2 \frac{3}{4}$ turns, including the basal, middle, and apical turns, which are separated by interscalar septa. The lateral aspect of the basal turn of the cochlea bulges into the middle ear cavity, forming the cochlear promontory. The nerve of Jacobson (branch of cranial nerve IX) courses over the cochlear promontory. The cochlear nerve passes from the internal auditory canal through the bony canal for the cochlear nerve (also referred to as the cochlear fossette or cochlear aperture) into the modiolus, which is a crown-shaped structure centered within the cochlea that transmits branches of the cochlear nerve to the organ of Corti. The organ of Corti is the end organ for hearing and is not visible on CT images.

The bony vestibule is an ovoid space located superior and posterior to the cochlea, which connects to the semicircular canals. There are three semicircular canals-superior, posterior, and lateral which are oriented orthogonal to one another. The endolymphatic duct extends from the posterior aspect of the vestibule toward the posterior cranial fossa, and ends in a blind pouch, the endolymphatic sac, at the posterior margin of the petrous ridge. The bony vestibular aqueduct surrounds the endolymphatic duct and normally measures up to $1 \mathrm{~mm}$ at the midpoint and $2 \mathrm{~mm}$ at the operculum, according to the Cincinnati criteria [1]. 
The cochlear aqueduct should not be mistaken for a fracture. It is a narrow bony channel that surrounds the perilymphatic duct and extends from the basal turn of the cochlea to the subarachnoid space adjacent to the pars nervosa of the jugular foramen.

\subsubsection{Internal Auditory Canal}

The internal auditory canal (IAC) is a channel in the petrous bone. It extends from the fundus which abuts the labyrinth to the porus acusticus. At the fundus, a transverse crest (crista falciformis) divides the IAC into superior and inferior compartments. A vertical crest ("Bill's bar") divides the superior compartment into anterior and posterior components. The facial nerve is located in the anterosuperior compartment, the cochlear nerve in the anteroinferior compartment, and the superior and inferior vestibular nerves in the superoposterior and inferoposterior compartments, respectively. A mnemonic for the location of the nerves in the anterior compartment is "Seven (cranial nerve VII) Up Coke (cochlear nerve) down."

\subsection{Imaging Modalities and Techniques}

While CT is often the first modality utilized to assess suspected pathology involving the inner ear structures (cochlea and labyrinth), certain lesions such as a cochlear schwannoma and labyrinthine hemorrhage are better detected with MRI. In the work-up of subjective pulsatile tinnitus, CT is frequently the preferred modality. However, MR imaging with MRA and MRV may be more appropriate in the workup of objective pulsatile tinnitus (audible bruit on auscultation by the clinician). Conventional catheter angiography may then follow to further characterize the vascular abnormality as well as to provide therapeutic management (embolization) of lesions such as dural fistulas. MR imaging is the primary modality for evaluating the non-osseous components of the temporal bone, suspected retrocochlear pathology, and sensorineural hearing loss. Magnetic resonance imaging is required when temporal bone pathology is suspected to involve the intracranial compartment.

\subsubsection{Computed Tomography}

\subsubsection{Temporal Bone CT Technique}

Dual acquisition temporal bone CT (separately acquired direct axial and direct coronal images) has largely been replaced with multidetector row CT (MDCT) in which a single set/volume of axial images are acquired and reformatted in multiple planes. Thoughtfully performed MDCT reduces radiation dose, and its rapid acquisition minimizes artifact from patient motion. Intravenous contrast is typically not used with temporal bone CT because when contrast is necessary (tumors, vascular pathology such as dural fistulas), then higher resolution MRI, MRI with MRA, or CTA are usually obtained.

For temporal bone $\mathrm{CT}$ the patient is placed supine in the gantry with the head angled superiorly and posteriorly. The neck is hyperextended so that the orbits are canted out of the pathway of the X-ray beam to minimize exposure to the lens. Gantry tilt may need to be avoided to facilitate image reconstruction and reformats. Scan coverage is from the roof of the temporal bone (the arcuate eminence) through the mastoid tip. Thin collimation to achieve high resolution is extremely important as the anatomy of the temporal bone involves very small structures. A collimation of $0.6-1.0 \mathrm{~mm}$ provides appropriate resolution. The raw data from each ear is most often reconstructed into $0.6-0.75 \mathrm{~mm}$ thin axial images in bone algorithm at a DFOV of $100 \mathrm{~mm}$ that effectively magnifies the images. Technologists provide reconstructed images including $0.6-1.0 \mathrm{~mm}$ reformats in the axial plane parallel to the lateral semicircular canal created from a sagittal plane, and $0.6-1.0 \mathrm{~mm}$ reformats in the coronal plane. Axial images with $2-3 \mathrm{~mm}$ section thickness of the entire scan volume are also provided in soft tissue algorithm.

Reformats may be obtained in sagittal or oblique planes to improve the detection of pathology in specific clinical settings such as superior semicircular canal dehiscence.

Stenver reformat: On the console the technologist scrolls through the sagittal plane until a view of the lateral semicircular canal is obtained. A reformatted axial plane parallel to the lateral semicircular canal is made. The technologist then scrolls through this axial data set and Stenver reformats are made by tracing a line perpendicular to the long axis of the summit of the superior semicircular canal at submillimetric intervals. This plane is perpendicular to the roof of the superior semicircular canal displaying it in cross section.

Poschl reformat: On the console the technologist scrolls through the sagittal plane until a view of the lateral semicircular canal is obtained. A reformatted axial plane parallel to the lateral semicircular canal is made. The technologist then scrolls through this axial data set and Poschl reformats are made by tracing a line parallel to the long axis of the summit of the superior semicircular canal at submillimetric intervals.

\subsubsection{Magnetic Resonance Imaging}

MRI of the temporal bones is performed in a head coil and should include thin section unenhanced and enhanced axial T1-weighted images, and enhanced coronal T1-weighted 
images. Fat suppression in at least one enhanced plane should be performed to distinguish fat from enhancement, especially in the region of the petrous apex. A maximum section thickness of $3 \mathrm{~mm}$ with no interslice gap and a small FOV are required to provide images able to depict the fine detail of the anatomy and pathology in the temporal bone. Thin section (1 $\mathrm{mm}$ or submillimetric), 3D T2-weighted techniques are valuable in temporal bone imaging to evaluate the relationship of pathologic processes such as a vestibular schwannoma with the surrounding nerves, the patency of labyrinthine structures, the size of the endolymphatic duct and sac, and the extent of cochlear dysplasia in congenital or developmental hearing loss. Reformatted oblique sagittal images perpendicular to the long axis of the internal auditory canal are helpful in identifying cranial nerve hypoplasia or aplasia. Diffusion-weighted imaging (DWI) can help in distinguishing cholesteatoma which typically have restricted diffusion from inflammatory middle ear cavity opacification which has facilitated diffusion.

Axial T2-weighted, flair, and enhanced T1-weighted images of the brain should be performed to assess for intracranial extension of temporal bone pathology, and also to exclude central nervous system pathology that might produce tinnitus, vertigo, hearing loss, or cranial nerve symptoms such as demyelinating disease, stroke, or inflammation/ infection.

\subsection{Tinnitus and Hearing Loss without a Mass}

When no mass is seen on imaging, the radiologist should closely review the inner ear structures. The common causes of tinnitus and hearing loss without a mass include otospongiosis, labyrinthitis ossificans, superior semicircular canal dehiscence, and enlarged vestibular aqueduct syndrome.

\subsubsection{Otospongiosis}

Otospongiosis is an idiopathic progressive disease of pathological bone remodeling that causes conductive, sensorineural, or mixed hearing loss. It results in spongiosis or sclerosis of portions of the petrous bone. Conductive hearing loss is usually secondary to abnormal bone encroaching on stapes with impingement on the stapes footplate. Therefore, stapes surgery is the traditional initial treatment for otosclerosis, but is being augmented or replaced by innovations in hearing aid technology and cochlear implants.

Symptoms typically start in the second or third decade of life and presentation is with progressive conductive hearing loss ( $80 \%$ bilateral) with a normal tympanic membrane, and no evidence of middle ear inflammation. On otoscopic exam-

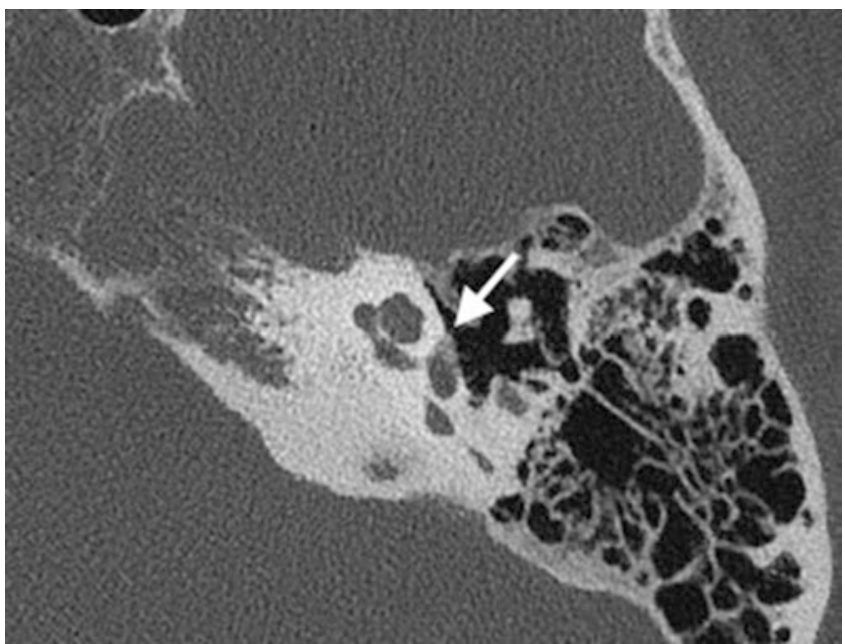

Fig. 15.1 Fenestral otospongiosis on the left with signs of lucency in the fissula ante fenestram (arrow)

ination, the promontory may have a faint pink tinge reflecting the vascularity of the lesion, referred to as the Schwartze sign.

On imaging, the disease is seen as lucency in the otic capsule. The most common location of involvement of otosclerosis is the bone just anterior to the oval window at a small cleft known as the fissula ante fenestram (fenestral otosclerosis) (Fig. 15.1). Involvement of the otic capsule separable from the oval window is referred to as "retrofenestral otosclerosis" or "cochlear otosclerosis" and can give a sensorineural component to the hearing loss.

A later presumably less active phase can occur where the bone becomes more dense and sclerotic. The bone still appears to encroach on the footplate but has a density closer to that of the otic capsule and thus may be difficult to identify.

\subsubsection{Labyrinthitis Ossificans}

Labyrinthitis ossificans is the late stage of labyrinthitis, in which there is pathologic ossification of spaces within the membranous labyrinth. Profound bilateral hearing loss from labyrinthitis ossificans is most commonly due to bacterial meningitis with onset of symptoms 3-4 months after the episode [2]. Other causes include trauma, hemorrhage, autoimmune disease, vascular obstruction of labyrinthine artery, and surgical insult.

Imaging can detect the evolution of labyrinthitis in three stages: acute, fibrous, and ossification. In the acute stage, enhancement of the inner ear is noted on MR images, but the CT scan may appear normal. In the intermediate fibrous stage of labyrinthitis, there is loss of fluid signal intensity on heavily T2-weighted sequence images (Fig. 15.2), while the 


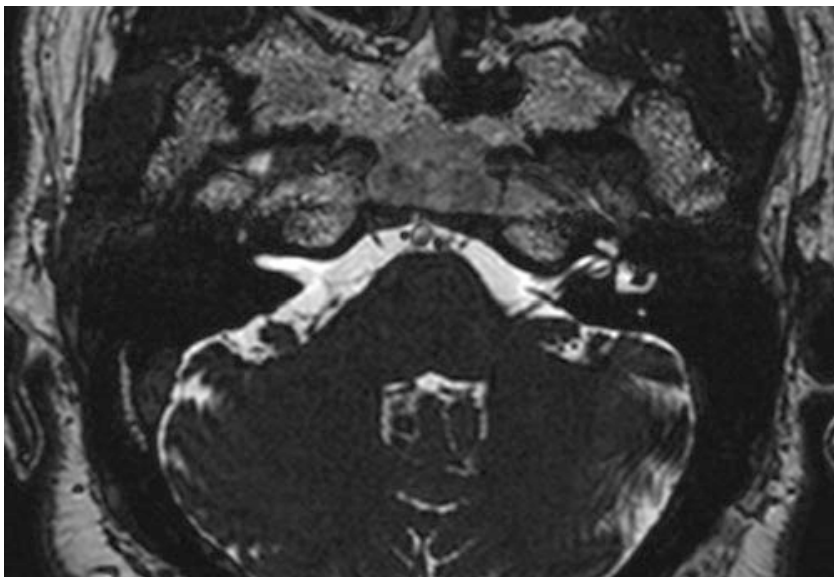

Fig. 15.2 Labyrinthitis ossificans on the right with loss of fluid signal intensity on heavily $\mathrm{T} 2$-weighted sequence images

CT scan may still appear normal. In the late ossific stage, one sees replacement of the normal cochlea, vestibule, and/or semicircular canals by bone attenuation on CT scans.

\subsubsection{Superior Semicircular Canal Dehiscence}

Superior semicircular canal dehiscence is the most common type of a group of disorders known as third window abnormalities. The labyrinth is normally in a closed hydraulic system with the oval and round windows. Defects in the integrity of the bony structure of the inner ear can decompress/dampen the energy of the sound wave resulting in conductive hearing loss.

In addition to conductive hearing loss, superior semicircular canal dehiscence can present with characteristic symptoms of vertigo when exposed to loud sounds (Tullio phenomenon). This is because of movement of fluid in the superior canal without movement in other canals. Other symptoms include autophony and pulsatile tinnitus. At audiometry, there is a characteristic low-frequency air-bone gap due to decreased air conduction and increased bone conduction.

On imaging, there is a defect in the superior semicircular canal, best seen in the coronal plane, and on reformatted oblique images in the Stenvers plane (perpendicular to the canal). The Pöschl plane shows the superior canal as a ring and helps to determine the length of a dehiscence (Fig. 15.3). The defect is typically along the superior arc of the superior canal along the floor of the middle cranial fossa. However, a defect can also occur along the posterior limb of the canal facing the posterior fossa.

Other third window abnormalities present with similar symptoms and include enlargement of the opening of the vestibular aqueduct, dehiscence of the scala vestibuli side of the cochlea, erosion of the lateral semicircular canal by cho-

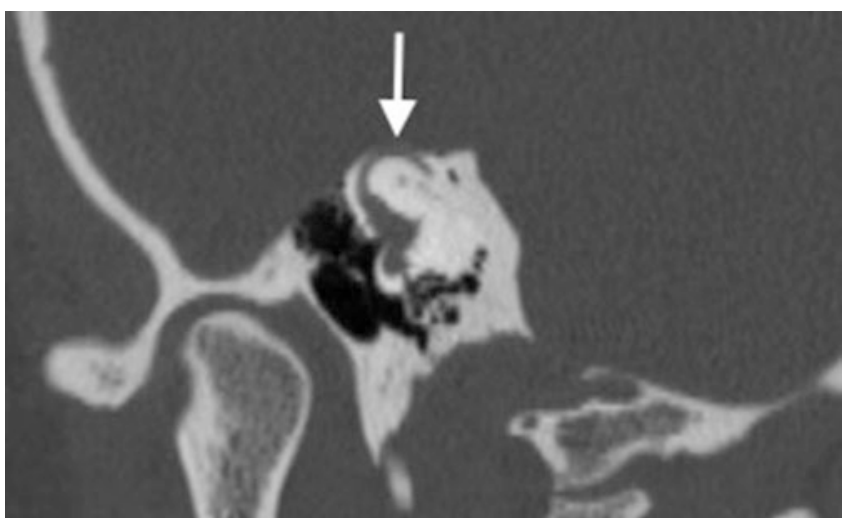

Fig. 15.3 Superior semicircular canal dehiscence on the left. There is a bone defect (arrow) in the superior semicircular canal. Pöschl plane shows the superior canal as a ring and helps to determine the length of a dehiscence

lesteatoma, and abnormal bony thinning between the cochlea and vascular channels. Presence of a defect does not mean that the patient will necessarily have symptoms or will benefit from surgery. Asymptomatic defects are rare (2\% of the population) so further audiometric evaluation is recommended for all patients with imaging findings [3].

\subsubsection{Enlarged Vestibular Aqueduct Syndrome}

Most congenital hearing malformations are detected after birth on newborn hearing screening. The exception is enlarged vestibular aqueduct syndrome (EVA) which can progress in childhood or less commonly early adulthood [4]. Stable hearing is observed in $67 \%$ of ears with EVA of which $34 \%$ will demonstrate fluctuations in hearing. Progression of hearing loss is seen in $33 \%$ of ears of which half will demonstrate fluctuations [5].

During fetal development, the vestibular aqueduct starts out as a wide tube. By the fifth week it narrows, and by midterm it approaches adult dimension and shape. However, the vestibular aqueduct continues to grow and change until a child is 3-4 years old. As yet incompletely understood genetic and/or environmental conditions cause EVA.

It is believed that an EVA does not cause hearing loss, but instead EVA and hearing loss are caused by the same underlying defect (gene mutations). Syndromic EVA includes Pendred syndrome or branchiootorenal syndrome. Syndromic hearing loss associated with EVA can affect other areas of the body. More commonly, the hearing loss associated with EVA is nonsyndromic, affecting only ear function.

On clinical exam, hearing loss can be conductive, mixed, or sensorineural, and the loss may be stable or fluctuating. Imaging is both diagnostic and prognostic (Fig. 15.4). There 


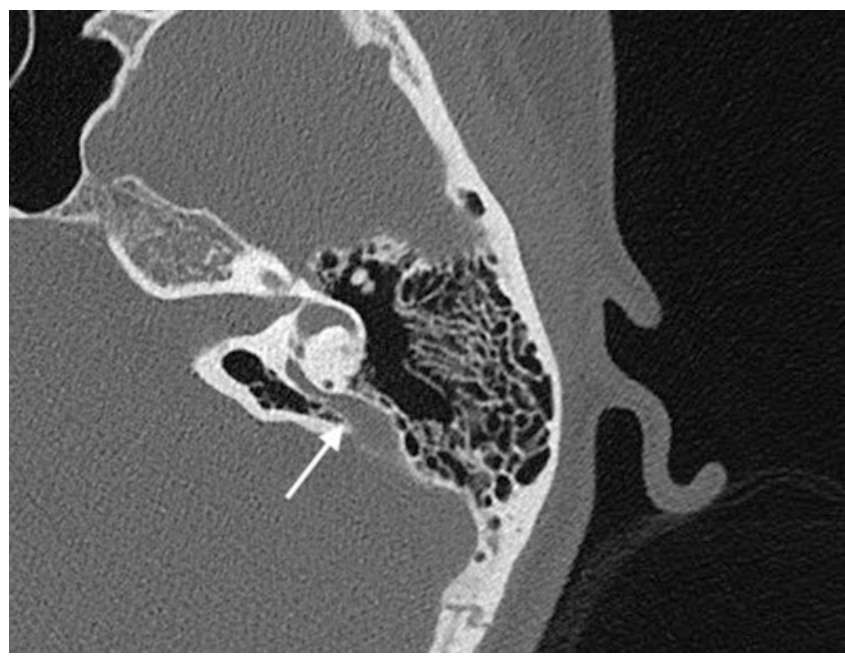

Fig. 15.4 Enlarged vestibular aqueduct. The vestibular aqueduct extends from the posterior aspect of the vestibule toward the posterior cranial fossa (arrow)

are several definitions of an enlarged vestibular aqueduct. The most sensitive criteria is the Cincinnati criteria which defines abnormal as $>0.9 \mathrm{~mm}$ at the midpoint or $>1.9 \mathrm{~mm}$ at the operculum in the axial view [1]. Other criteria are using the semicircular canal as reference-enlarged if width at the proximal intraosseous portion exceeded that of the adjacent posterior semicircular canal. A retrospective study has found the severity of hearing loss in patients with an EVA is influenced by degree of widening of the vestibular aqueduct midpoint width [6].

\subsection{Tinnitus and Hearing Loss with a Mass}

\subsubsection{Vestibular Schwannoma}

Vestibular schwannomas typically arise from perineural Schwann cells of the superior or inferior vestibular nerve in the internal auditory canal near the porus acusticus. They frequently extend into the cerebellopontine angle. Vestibular schwannomas often present with non-pulsatile tinnitus and high frequency sensorineural hearing loss. Less often, they may present with symptoms related to mass effect upon the middle cerebellar peduncle, lateral pons, and/or the cisternal trigeminal nerve. The presence of bilateral vestibular schwannomas is diagnostic of neurofibromatosis type II.

Vestibular schwannomas are typically difficult to identify on CT unless quite large. Expansion of the internal auditory canal may be present. On MR imaging, they are heterogeneous on T1- and T2-weighted images, and enhance following contrast administration. When large $(>2 \mathrm{~cm})$, they often show cystic degeneration. Five to $10 \%$ of vestibular schwannomas may have a co-existent arachnoid cyst. Hemorrhage may be seen in 5\%. Calcification is rare. When evaluating a patient with a vestibular schwannoma, it is important to identify the full extent of the neoplasm including extension to the cochlear aperture and/or cerebellopontine angle, and to describe the presence of mass effect on the middle cerebellar peduncle and brainstem. Approximately $60-75 \%$ of vestibular schwannomas involve the internal auditory canal and the cerebellopontine angle.

The goal of management of vestibular schwannomas over the last decade has shifted from "complete resection" to hearing preservation. Treatment may include surgical resection, stereotactic radiosurgery, and for small, stable tumors clinical and radiologic observation is now common [7].

\subsubsection{Cholesteatoma}

Cholesteatoma is an expansile erosive mass lined by keratinizing stratified squamous epithelium. It may result from a congenital inclusion of squamous epithelium in the middle ear cavity (congenital cholesteatoma), or more commonly from abnormal migration of squamous epithelium into the middle ear cavity through a perforated tympanic membrane as a result of chronic infection/inflammation. Otoscopic examination typically reveals a pearly white mass behind the tympanic membrane. Erosive enzymes and osteoclaststimulating agents within the epithelial debris result in bone destruction which is the radiologic hallmark of cholesteatomas. Acquired cholesteatomas most often occur in the setting of an under-pneumatized mastoid, a perforation of the pars flaccida (Shrapnell's membrane), involve Prussak space, and erode the adjacent bone including the scutum and ossicles (the mallear head and incus body). The ossicles may be displaced medially. Cholesteatomas related to pars tensa perforations are less common. In these lesions the sinus tympani, pyramidal eminence, and facial nerve recess may be involved and eroded. The long process of the incus and the stapes suprastructure may also be involved or eroded.

In the imaging evaluation of patients with a cholesteatoma, it is important to comment on the extent of middle ear cavity opacification. Specifically, it is important to note if there is involvement of the anterior epitympanic recess, round window niche, sinus tympani, and/or facial recess. The integrity of adjacent bone including the roof of the epitympanum (the tegmen tympani), the lateral semicircular canal, and the facial nerve canal should be commented upon. Involvement of important regional anatomy relevant to surgical planning such as the presence of high or dehiscent jugular bulb, or an anteriorly positioned sigmoid sinus plate should also be addressed in the radiologic interpretation. Diffusion-weighted MR imaging may be valuable in distinguishing cholesteatomas (which typically have restricted diffusion) from inflammatory opacification in the middle ear 
cavity. Frequently both are present, and DWI can distinguish the cholesteatoma form the areas of inflammation [8].

\subsubsection{Glomus Tumor}

When imaging the patient with a suspected glomus tympanicum, the radiologist should evaluate for and exclude other causes of pulsatile tinnitus including vascular anomalies such as an aberrant internal carotid artery, high riding dehiscent jugular bulb, jugular vein or sigmoid diverticulum (outpouching of the jugular bulb or sigmoid sinus, respectively, into adjacent pneumatized temporal bone), and large condylar or mastoid emissary veins that traverse air cells.

Glomus tympanicum is a paraganglioma that arises from glomus bodies along the course of Jacobson's nerve (the tympanic branch of CN IX) along the lateral aspect of the cochlea in the middle ear cavity. Glomus tympanicum is the most common primary tumor of the middle ear in adults, and presents in middle age with a slight female predominance. The typical clinical presentation is pulsatile tinnitus. Patients may also have conductive hearing loss. On otoscopic examination a fleshy vascular mass behind the tympanic membrane in the middle ear cavity is most often identified.

The classic imaging appearance is a demarcated mass along the cochlear promontory (Fig. 15.5). These tumors are soft tissue in density on $\mathrm{CT}$, and on MR imaging they are iso- to mildly hyperintense to CSF on T1-weighted imaging, mildly hyperintense on T2-weighted imaging, and enhance intensely following contrast administration [9]. While these tumors are very vascular, the typical "salt and pepper" appearance seen in other glomus tumors/paragangliomas is frequently not seen in glomus tympanicum tumors due to their relatively small size. Once establishing the presence of

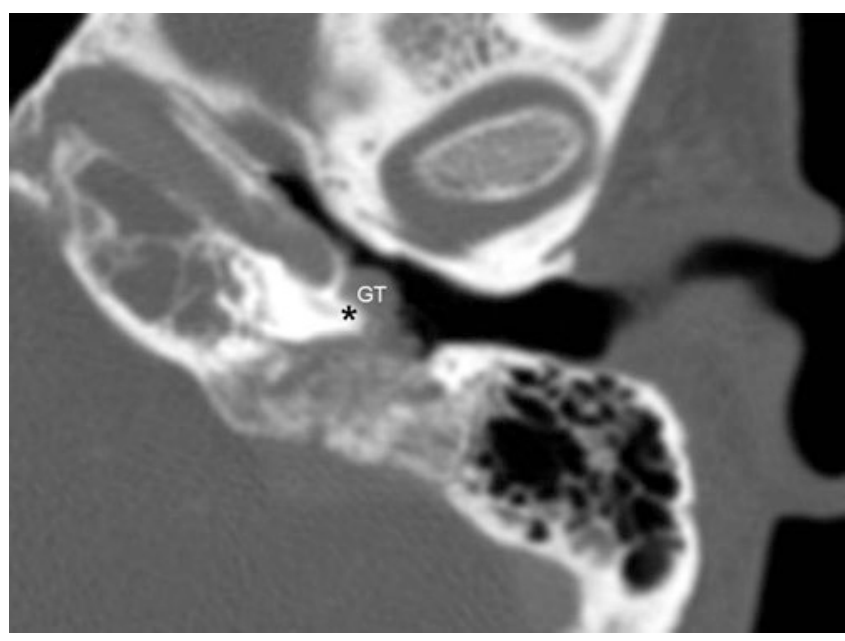

Fig. 15.5 Glomus tympanicum. Axial multidetector CT image of the left temporal bone shows a soft tissue mass (GT) at the cochlear promontory (asterisk) a glomus tympanicum it is important to determine the extent of disease. These tumors are classified based on their spread from the cochlear promontory to the middle ear cavity/ossicles, inner ear structures, external auditory canal, carotid canal, and the mastoid air cells. The radiologist should specify if the tumor is confined to the middle ear cavity (glomus tumors tend to engulf rather than erode the ossicles), identify if there is fistulization to the inner ears structures due to bone erosion, and identify extension of tumor into adjacent structures.

When there is tumor in the jugular foramen it is important to establish if the middle ear tumor is a projection of a larger glomus tumor arising in the jugular fossa-a glomus jugulare-tympanicum. Glomus jugulare paragangliomas arise from glomus bodies in the jugular foramen, either in the adventitia of the jugular bulb, the superior ganglion of cranial nerve CN X, or along Arnold or Jacobsen nerves (auricular branch of $\mathrm{CN} \mathrm{X}$ and tympanic branch of $\mathrm{CN}$ IX, respectively). They frequently grow directly into the jugular vein (Fig. 15.6a, b).

\subsubsection{Cholesterol Granuloma}

Lucent, expansile lesions of the petrous apex (cholesterol granuloma, mucocele, epidermoid, meningocele) are common. They may be incidental lesions detected on CT of the head being performed for unrelated reasons, or they may be identified on a head CT or a temporal bone CT performed for related symptomatology. These lesions may cause symptoms due to mass effect on adjacent structures. The management of each petrous apex lesion is different and clinical history is often of limited use. Biopsy is often difficult to perform, and in certain instances are contraindicated (meningocele) and potentially harmful to the patient. Fortunately, a specific diagnosis can usually be made using a combination of CT and MR imaging together $[10,11]$. On CT the pattern of bone expansion, remodeling, and/or erosion are important. On MR imaging the signal intensity on T1, T2, flair, DWI and enhanced T1 weighting are often diagnostic (e.g., cholesterol granulomas are typically hyperintense on unenhanced T1-weighted imaging, epidermoids classically have restricted diffusion) $[10,11]$. The size, location of the lesion, and its relationship to adjacent critical structures including the petrous internal carotid artery and the internal auditory canal are important to the referring physician.

Cholesterol granulomas typically arise in a pneumatized space, most often the petrous apex of the temporal bone. These develop due to recurrent micro-hemorrhages at the capillary level. This may be due to negative pressures in petrous air cells. The blood incites a mucosal reaction, giant cells, and cholesterol crystal deposition leading to recurrent hemorrhage. The cycle results in petrous apex 

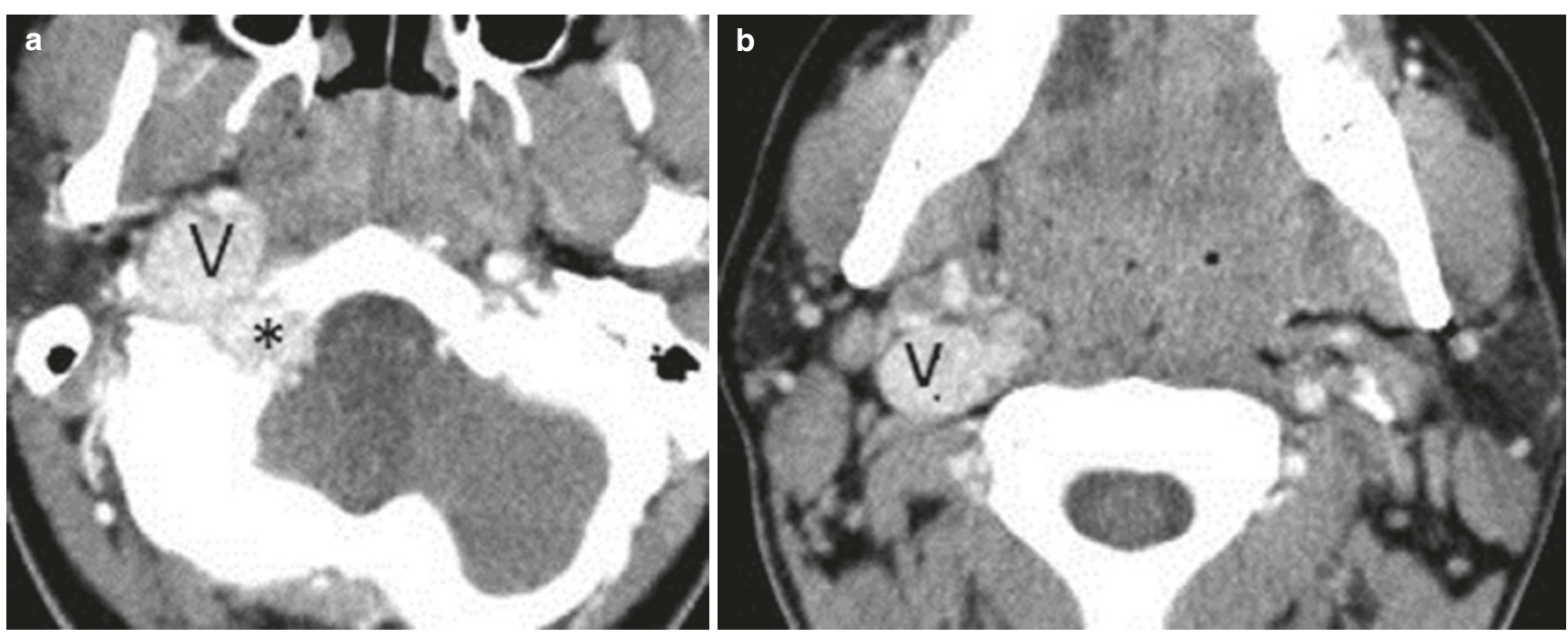

Fig. 15.6 (a, b). Glomus jugulare. Axial enhanced CT images show a vascular avidly enhancing mass in the jugular foramen (*), with direct inferior growth into the internal jugular vein $(\mathrm{V})$

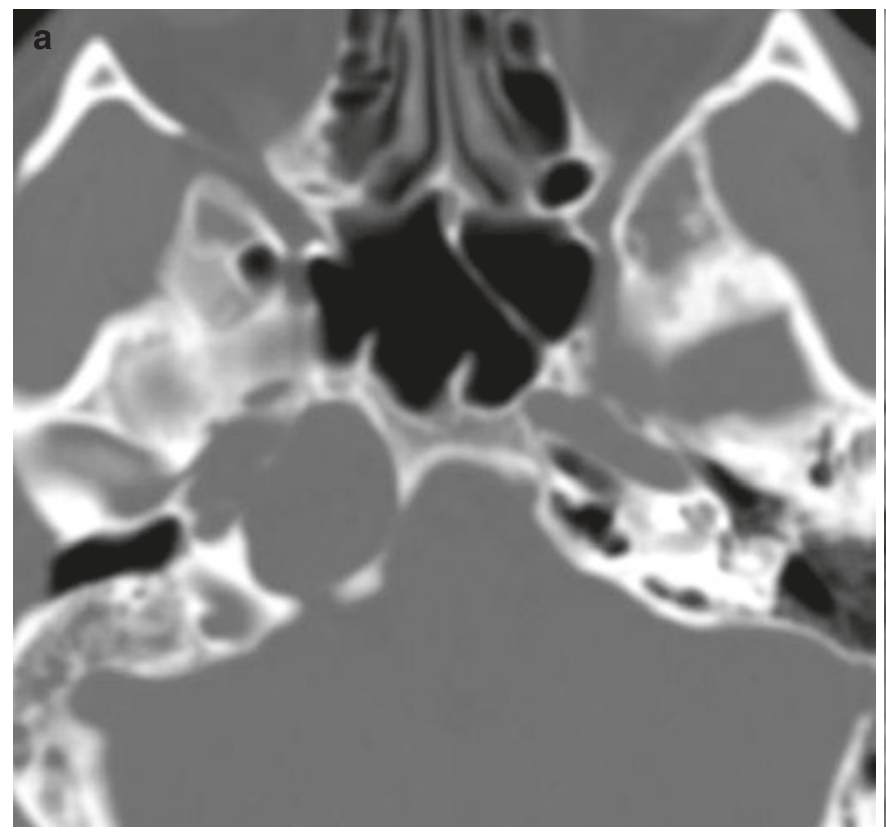

Fig. 15.7 (a, b). Cholesterol granuloma. (a) Axial CT image in bone window shows a unilocular, expansile mass of the right petrous apex. (b) Axial unenhanced T1-weighted gradient image shows the character-

expansion. This can result in a clinical presentation with cranial nerve symptoms (CNs V and VIII) and tinnitus. Cholesterol granulomas are expansile, lytic, circumscribed masses centered in the petrous apex and they are often unilocular (Fig. 15.7a). On MR imaging, cholesterol granulomas while heterogeneous on all pulse sequences due to blood products of varying ages, they are characteristically hyperintense on T1-weighted images related to methemoglobin (Fig. 15.7b).

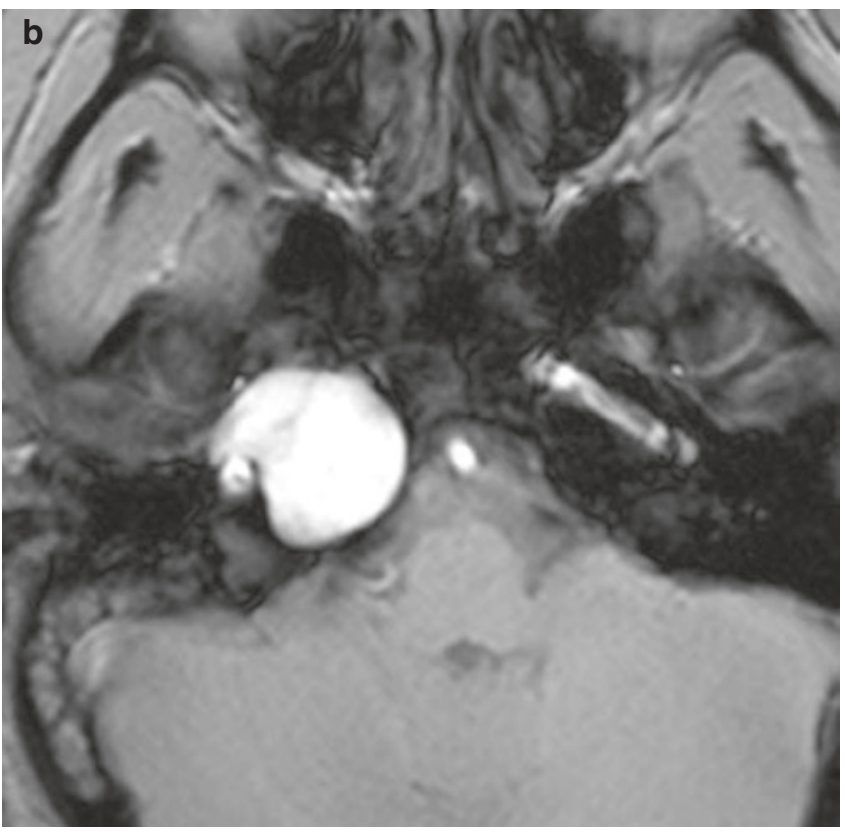

istic hyperintensity of these lesions related to blood products. The right mastoid air cells are opacified

\subsection{Conclusion}

The imaging workup for tinnitus and hearing loss in adults follows otoscopic exam and audiometry testing. Computed tomography and MR imaging have different and often complementary roles in the evaluation of tinnitus and hearing loss depending on the clinical scenario and the suspected underlying cause. Imaging can often identify the cause, and evaluate the extent of disease for surgical planning. 


\section{Take Home Messages}

- The radiologist should be familiar with a few key temporal bone anatomical structures and levels on axial and coronal imaging.

- Imaging approach and differentials for hearing loss and tinnitus can be based on whether a mass is present or absent.

- MR and CT are complementary modalities. The choice of imaging depends on the most likely clinical diagnosis following history and otoscopic examination.

\section{References}

1. El-Badry MM, Osman NM, Mohamed HM, Rafaat FM. Evaluation of the radiological criteria to diagnose large vestibular aqueduct syndrome. Int J Pediatr Otorhinolaryngol. 2016;81:84-91.

2. Hartnick CJ, Kim HY, Chute PM, Parisier SC. Preventing labyrinthitis ossificans. Arch Otolaryngol Head Neck Surg. 2001;127(2):180
3. Berning AW, Arani K, Branstetter BF. Prevalence of superior semicircular canal dehiscence on high-resolution CT imaging in patients without vestibular or auditory abnormalities. AJNR Am J Neuroradiol. 2019;40(4):709-12.

4. Wieczorek SS, Anderson ME, Harris DA, Mikulec AA. Enlarged vestibular aqueduct syndrome mimicking otosclerosis in adults. Am J Otolaryngol. 2013;34(6):619-25.

5. Mori T, Westerberg BD, Atashband S, Kozak FK. Natural history of hearing loss in children with enlarged vestibular aqueduct syndrome. J Otolaryngol Head Neck Surg. 2008;37(1):112-8.

6. Saliba I, Gingras-Charland M-E, St-Cyr K, Décarie J-C. Coronal CT scan measurements and hearing evolution in enlarged vestibular aqueduct syndrome. Int $\mathrm{J}$ Pediatr Otorhinolaryngol. 2012;76(4):492-9.

7. Lin EP, Crane BT. The management and imaging of vestibular schwannomas. Am J Neuroradiol. 2017;38(11):2034-43.

8. Schwartz KM, Lane JI, Bolster BD, Neff BA. The utility of diffusion-weighted imaging for cholesteatoma evaluation. Am J Neuroradiol. 2011;32(3):430-6.

9. Alaani A, Chavda SV, Irving RM. The crucial role of imaging in determining the approach to glomus tympanicum tumours. Eur Arch Otorhinolaryngol. 2009;266(6):827-31.

10. Schmalfuss IM. Petrous apex. Neuroimaging Clin N Am. 2009;19(3):367-91.

11. Chapman PR, Shah R, Cure JK, Bag AK. Petrous apex lesions: pictorial review. Am J Roentgenol. 2011;196(3):WS26-37.

Open Access This chapter is licensed under the terms of the Creative Commons Attribution 4.0 International License (http://creativecommons. org/licenses/by/4.0/), which permits use, sharing, adaptation, distribution and reproduction in any medium or format, as long as you give appropriate credit to the original author(s) and the source, provide a link to the Creative Commons license and indicate if changes were made.

The images or other third party material in this chapter are included in the chapter's Creative Commons license, unless indicated otherwise in a credit line to the material. If material is not included in the chapter's Creative Commons license and your intended use is not permitted by statutory regulation or exceeds the permitted use, you will need to obtain permission directly from the copyright holder. 


\title{
Approach to Masses in Head and Neck Spaces
}

\author{
Ashley H. Aiken and Deborah R. Shatzkes
}

\section{Learning Objectives}

- To understand how the layers of the deep cervical fascia, along with muscles and bones, help to define compartments or "spaces" in the neck.

- To understand the normal anatomy and contents of each of these spaces in the suprahyoid and infrahyoid neck.

- To accurately localize neck pathology into a specific space in order to generate the best differential diagnosis.

\subsection{Introduction to Head and Neck Spaces}

The neck is anatomically complex but can be organized into specific "spaces" or compartments based on fascial planes and individual space contents $[1,2]$. Some authors argue that the use of the term "space" may be an oversimplification and lead to confusion. These authors propose naming fascial layers in functional terms and using the term "compartment" which can be bound by bone, muscle, and/or fascia. For the purposes of clarity and consistency with radiologic literature, this review will continue to use the term "space" but will point out potentially confusing terms and proposed newer terminology to promote interdisciplinary communication. The ability to place pathology within a neck space is the first step to generating a differential diagnosis; then specific imaging features and clinical context can be applied to narrow these different considerations.

\footnotetext{
A. H. Aiken ( ()

Department of Radiology and Imaging Sciences, Emory University

School of Medicine, Atlanta, GA, USA

e-mail: Ashley.aiken@emoryhealthcare.org

D. R. Shatzkes

Department of Radiology, Zucker School of Medicine at Hofstra/

Northwell, New York, NY, USA

e-mail: Dshatzkes@northwell.edu
}

The neck can be divided into suprahyoid and infrahyoid spaces by the hyoid bone. Some head and neck spaces transgress both the suprahyoid and infrahyoid neck. Two layers of fascia, superficial and deep, are commonly used to define the spaces of the neck. The superficial cervical fascia (SCF) is a thin layer, investing loose connective and adipose tissue, platysma, superficial lymph nodes, and muscles of facial expression. For clarification, some surgeons prefer to refer to SCF simply as "subcutaneous tissue" [3] to prevent confusion with the superficial layer of the deep cervical fascia (SLDCF), which is described below.

The deep cervical fascia is subdivided into superficial, middle, and deep layers. The superficial layer of deep cervical fascia (SLDCF) lies between the SCF (or subcutaneous tissue) and the muscles of the neck, attaching anteriorly to the hyoid, superiorly to the mandible, mastoid process, and external occipital protuberance. The "rule of twos" may be helpful to remember that the SLDCF encloses two glands (submandibular and parotid) and two muscles (sternocleidomastoid and trapezius). Some surgeons prefer to refer to subdivisions of the SLDCF as the masticator fascia, submandibular fascia, and sternocleidomastoid/trapezius fascia. In other words, in some cases, it may be helpful to define fascia by function.

The middle layer of deep cervical fascia (MLDCF) extends from the skull base to the mediastinum, and is divided into a muscular layer investing the strap muscles, and visceral layer (also called buccopharyngeal in the suprahyoid neck) investing the larynx, pharynx, trachea, esophagus, and thyroid. The deep layer of deep cervical fascia (DLDCF) surrounds the vertebral column and paravertebral muscles with two distinct components: the alar and prevertebral fascia. The alar layer forms the posterior and lateral walls of the retropharyngeal space and bridges the transverse processes of the vertebrae. The prevertebral layer encloses the paraspinal muscles: the longus colli and longus capitis muscles; the anterior, middle, and posterior scalene muscles; and the levator scapulae. The carotid sheath is generally thought to be composed of all three layers of DCF; however, the thick- 
ness of the carotid sheath varies between individuals and at different levels in the neck.

The suprahyoid neck spaces comprise the area from the base of skull to the hyoid bone, excluding the orbits, paranasal sinuses, and oral cavity. The spaces of the suprahyoid neck include the pharyngeal mucosal space (PMS), the sublingual space (SLS), the submandibular space (SMS), the parapharyngeal space (PPS), the parotid space (PS), and masticator space (MS). The entire neck (suprahyoid plus infrahyoid) spaces include the carotid space (CS), retropharyngeal and danger space (RPS), and the perivertebral space (PVS). In addition to the entire neck spaces (above), infrahyoid neck contains the visceral space (VS), which comprises the thyroid and parathyroid glands and the larynx, hypopharynx, trachea, and cervical esophagus.

\subsection{Suprahyoid Neck: Pharyngeal Mucosal Space (PMS)}

Anatomy and Contents Contents of the pharyngeal mucosal space include the mucosa of the nasopharynx and oropharynx, as well as submucosal structures such as Waldeyer's ring, minor salivary glands (MSGs), the pharyngeal constrictor and levator veli palatine muscles, and the torus tubarius bordering the Eustachian tube orifice [4]. Waldeyer's ring comprises the adenoids and the palatine and lingual tonsils; the ring-like configuration of lymphoid tissue can be thought of as a mechanism of protecting the body from inhaled and ingested antigens. Deep mucosa-lined tonsillar crypts result in a characteristic striped appearance on contrast-enhanced imaging and may hide small primary tumors.

\section{Key Point}

- While squamous cell carcinoma is the most common tumor of the PMS, the presence of Waldeyer's ring lymphoid tissue and submucosal minor salivary glands may result in lymphoproliferative and salivary neoplasms, respectively.

Pathology Squamous cell carcinoma arising from the mucosa represents the most common malignant neoplasm in this space and is covered in a separate chapter. Tumors arising from Waldeyer's ring lymphoid tissue and MSGs are considerably less common. Inflammatory disease of the oropharynx represents a spectrum ranging from non-focal tonsillitis to tonsillar or peritonsillar abscess. (Fig. 16.1) Congenital lesions of the PMS include the central nasopharyngeal Tornwaldt cyst and those related to the embryologic thyroglossal duct, including cysts and the lingual thyroid. (Fig. 16.2).

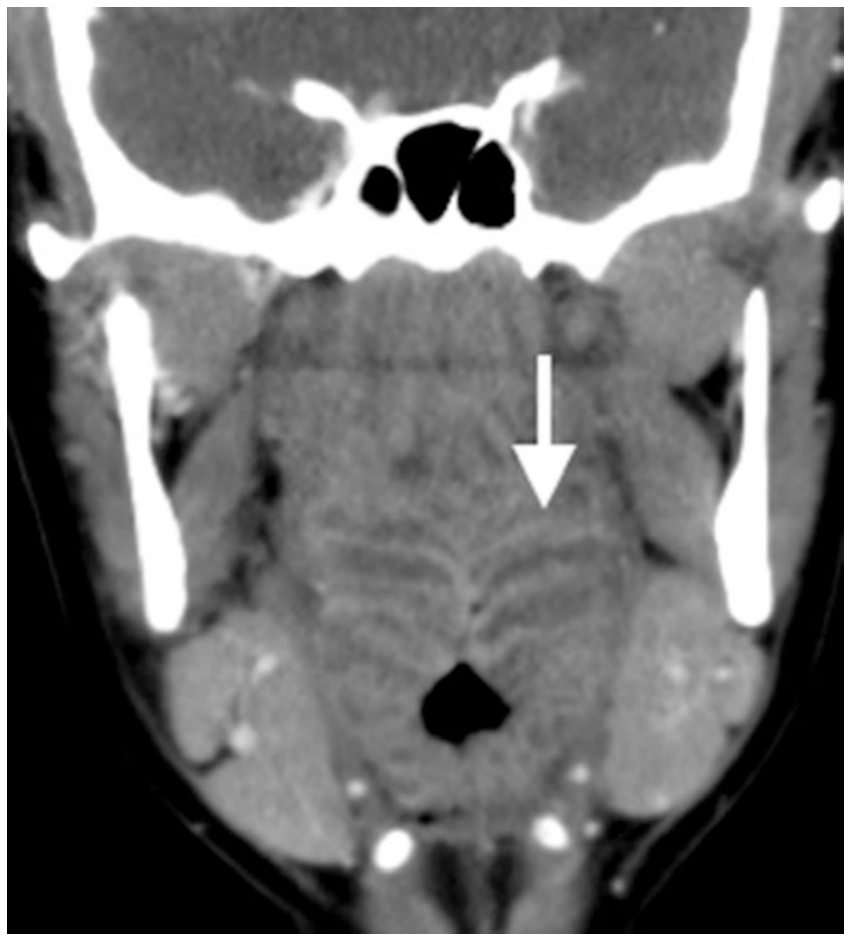

Fig. 16.1 CECT shows characteristic striped appearance of the inflamed mucosa of Waldeyer's ring in a patient with tonsillitis and adenoiditis

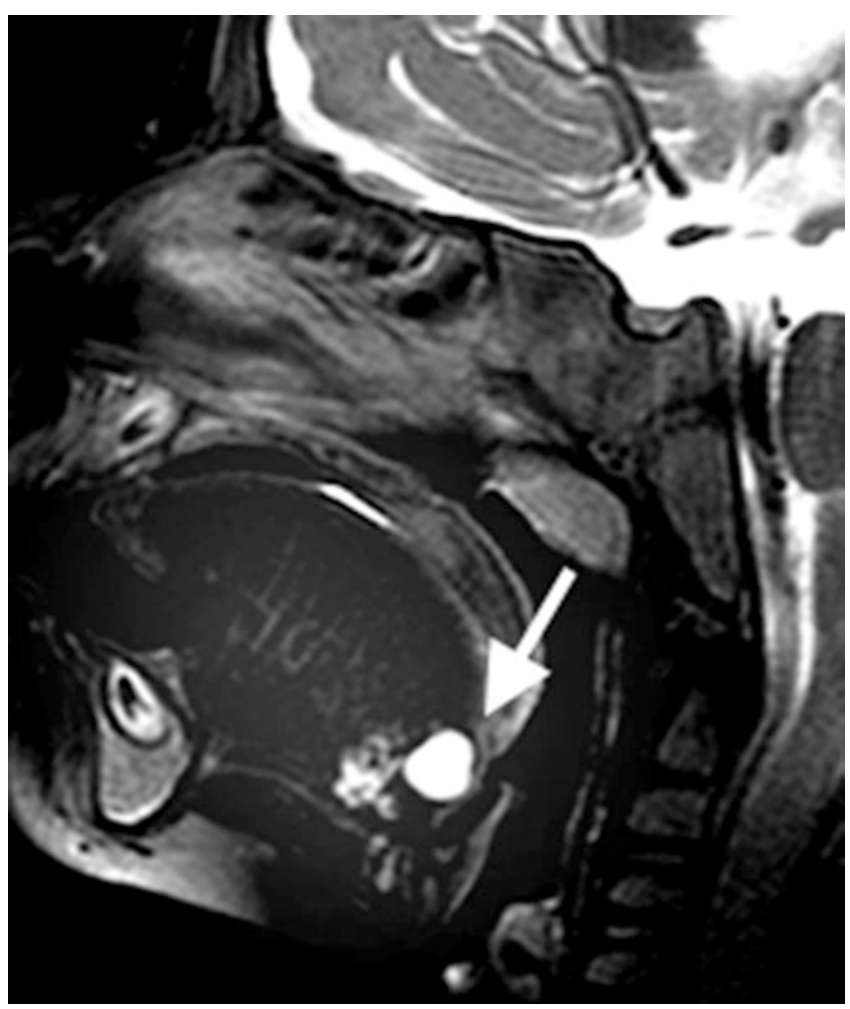

Fig. 16.2 Sagittal T2WI demonstrates a thyroglossal duct cyst at the base of tongue, part of the oropharynx. Adenoidal tissue lines the nasopharyngeal roof 


\subsection{Suprahyoid Neck: Parapharyngeal Space (PPS)}

Anatomy and Contents The PPS represents a triangular fatcontaining space lateral to the PMS, bounded laterally by the masticator and parotid spaces, posteriorly by the retropharyngeal and carotid spaces, and superiorly by the skull base, with inferior extension into the submandibular space. The PPS contents are simple: fat, minor salivary glands, and, rarely, lymph nodes.

Pathology Pathology primary to the PPS is rare, with the majority of lesions salivary in origin and representing benign mixed tumors (BMTs) [5]. The PPS is primarily impacted by pathology of neighboring spaces, and the pattern of deviation or deformity of the PPS fat may be helpful in identifying the space of origin. For example, while a well-defined markedly T2-hyperintense mass bounded entirely by fat is likely a primary PPS BMT, a similar lesion contiguous with the deep lobe of the parotid and displacing the PPS fat anteromedially more likely originates in the parotid space (Fig. 16.3). Similarly, masticator space lesions displace the PPS fat posteromedially, PMS lesions laterally, and carotid space lesions anteriorly.

\subsection{Suprahyoid Neck: Masticator Space (MS)}

Anatomy and Contents The MS is located lateral to the PPS, and contains the muscles of mastication (masseter, temporalis, medial and lateral pterygoids), posterior body and ramus of the mandible and the mandibular nerve, a branch of the trigeminal nerve.
Pathology As with the PPS, most pathology of the MS arises in the neighboring spaces, with the most common being odontogenic infection arising from molar teeth within the neighboring oral cavity [6]. Dental abscesses may extend to the medial pterygoid or masseter muscles and may also result in osteomyelitis of the posterior mandibular body and condyle (Fig. 16.4). Another common non-neoplastic lesion affecting the MS is the venous malformation (VM), which has a predilection for the muscles of mastication and is a frequent incidental finding on

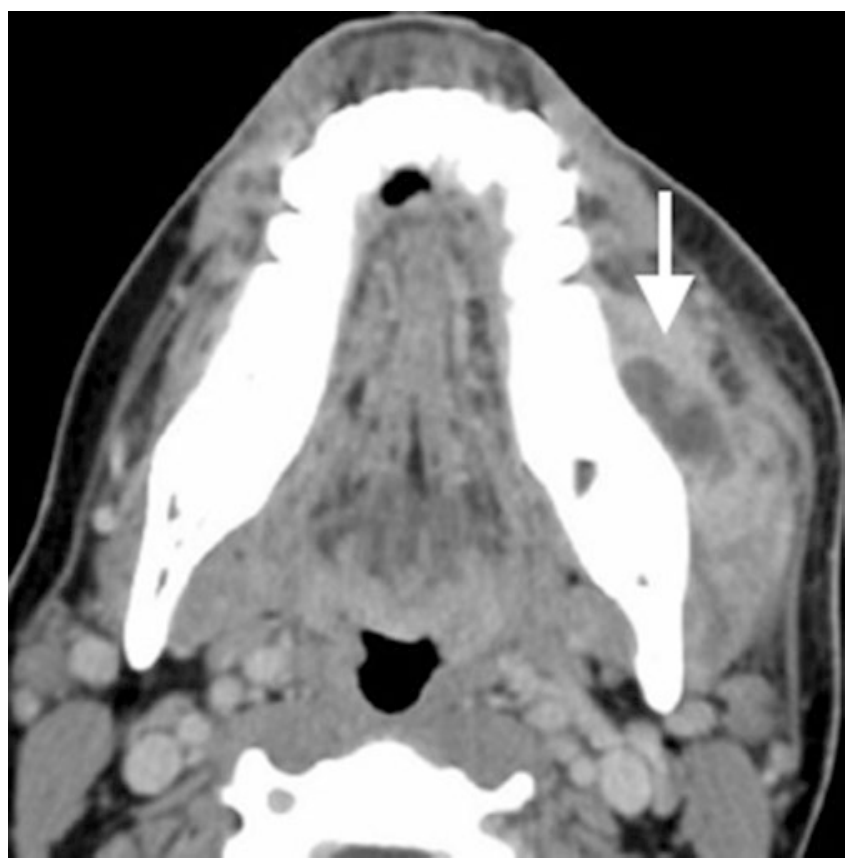

Fig. 16.4 Axial CECT image shows a fluid collection within the enlarged left masseter muscle, representing an odontogenic abscess arising from the left mandibular third molar tooth
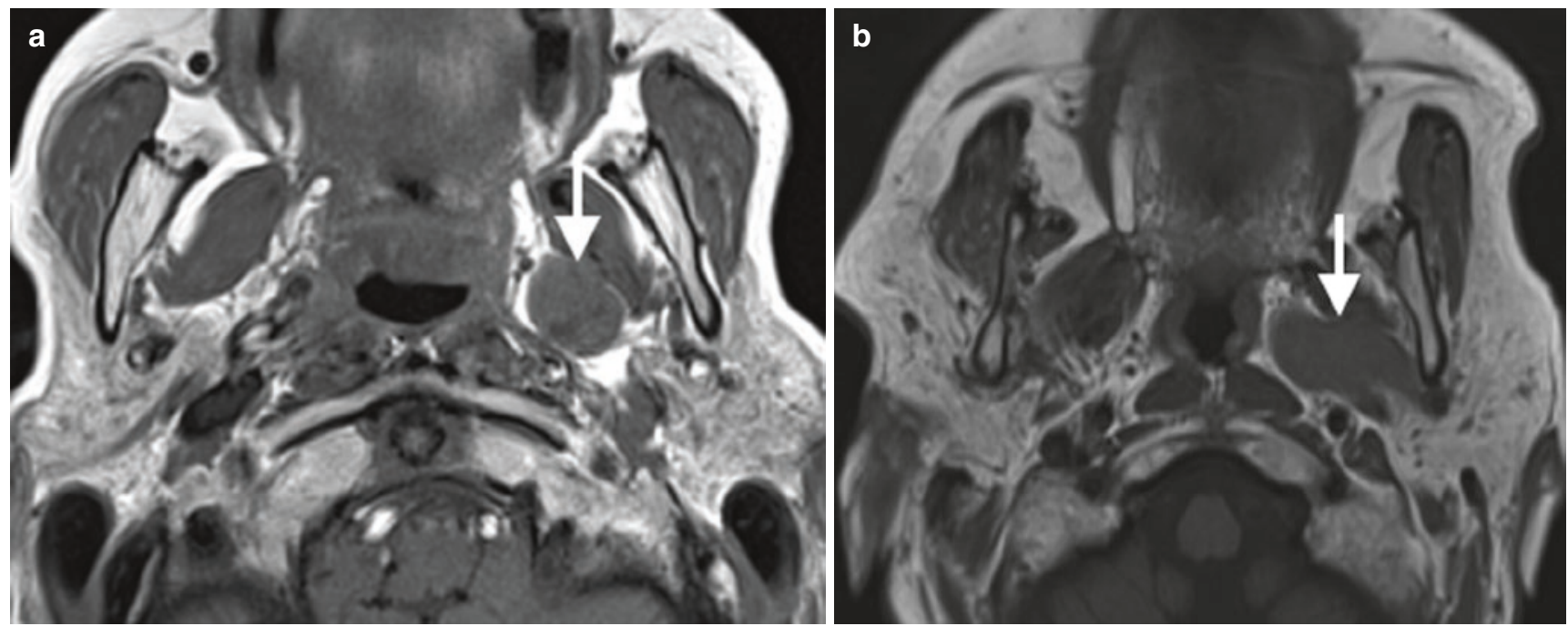

Fig. 16.3 (a) Primary BMT of the left PPS is surrounded entirely by fat on axial T1-weighted image. (b) Axial T1-weighted image shows a primary BMT of the left parotid space inseparable from the deep lobe and displacing PPS fat medially 
imaging studies performed for unrelated indications (Fig. 16.5) The presence of phleboliths within a heterogeneously enhancing and markedly T2-hyperintense mass is virtually pathognomonic for VM. Cranial nerve V3 may be involved secondarily by perineural spread of tumor, or, much less commonly, by primary nerve sheath tumors such as schwannomas. Primary neoplasms of the muscles of mastication, such as sarcomas and

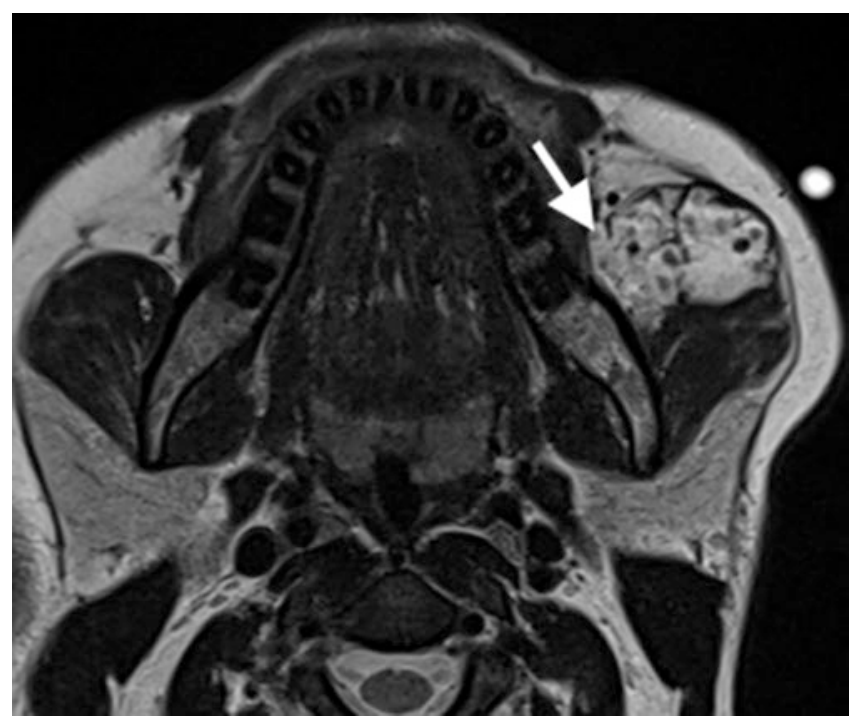

Fig. 16.5 Axial T2-weighted image shows a markedly hyperintense mass in the anterior left masseter muscle with internal round areas of signal void. These represent phleboliths and are virtually pathognomonic for a venous malformation

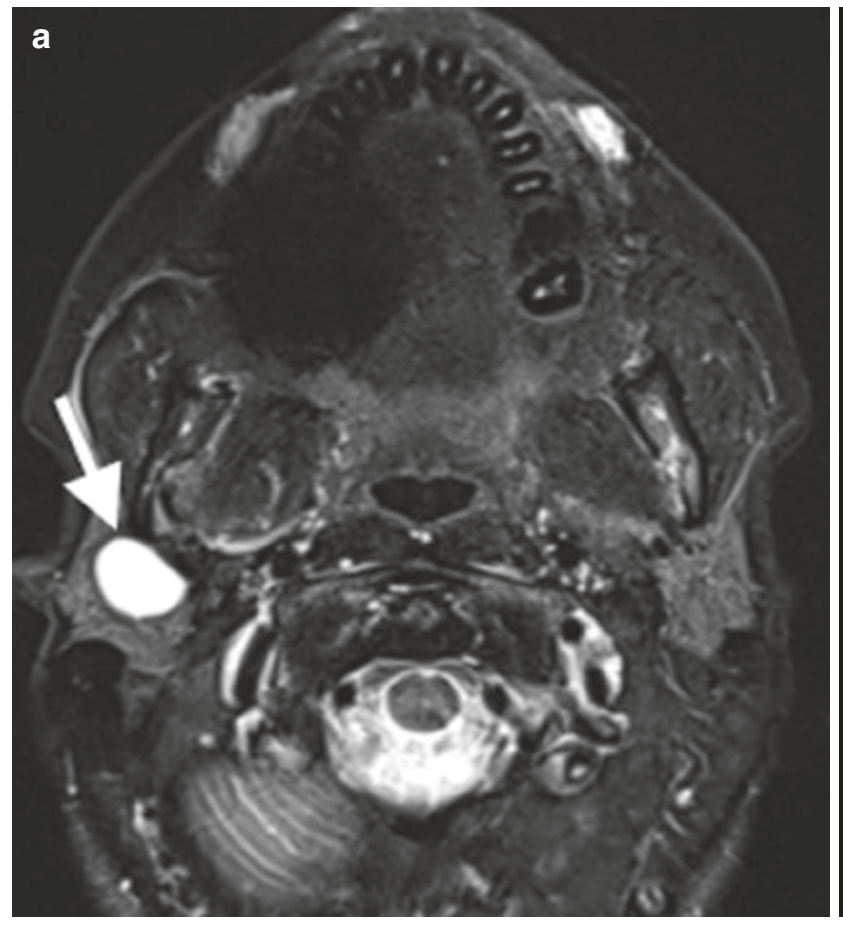

Fig. 16.6 (a) Axial STIR image demonstrates marked T2 prolongation of a well-defined mass in the right parotid superficial lobe found to represent a benign mixed tumor. (b) Corresponding ADC image dem- lymphoproliferative malignancies, are exceedingly rare. More often, malignant MS disease results from spread of tumors arising in the pharynx, oral cavity, or parotid space.

\subsection{Suprahyoid Neck: Parotid Space (PS)}

Anatomy and Contents The PS contains the parotid gland, facial nerve, retromandibular vein, and branches of the external carotid artery. The plane of the facial nerve bisects the gland into superficial and deep lobes; localization of lesions into these lobes is important in the prevention of inadvertent facial nerve injury during resection. This plane may be approximated on cross-sectional imaging by identifying the stylomandibular tunnel, between the styloid process and the posterior cortex of the mandibular condyle, as well as by the position of the retromandibular vein.

Pathology The parotid gland is the only salivary gland to contain lymph nodes, with the important consequence that the differential diagnosis for lymphadenopathy, including neoplastic and inflammatory etiologies, must be considered for any PS mass [7]. The parotid lymph nodes also represent first echelon drainage for cancers of the external auditory canal as well skin cancers of portions of the scalp and facial skin. Fortunately, most primary salivary epithelial neoplasms represent the benign mixed tumor (pleomorphic adenoma), which demonstrates very high signal on T2-weighted and ADC scans (Fig. 16.6). This is one of the few parotid neoplasms that dem-

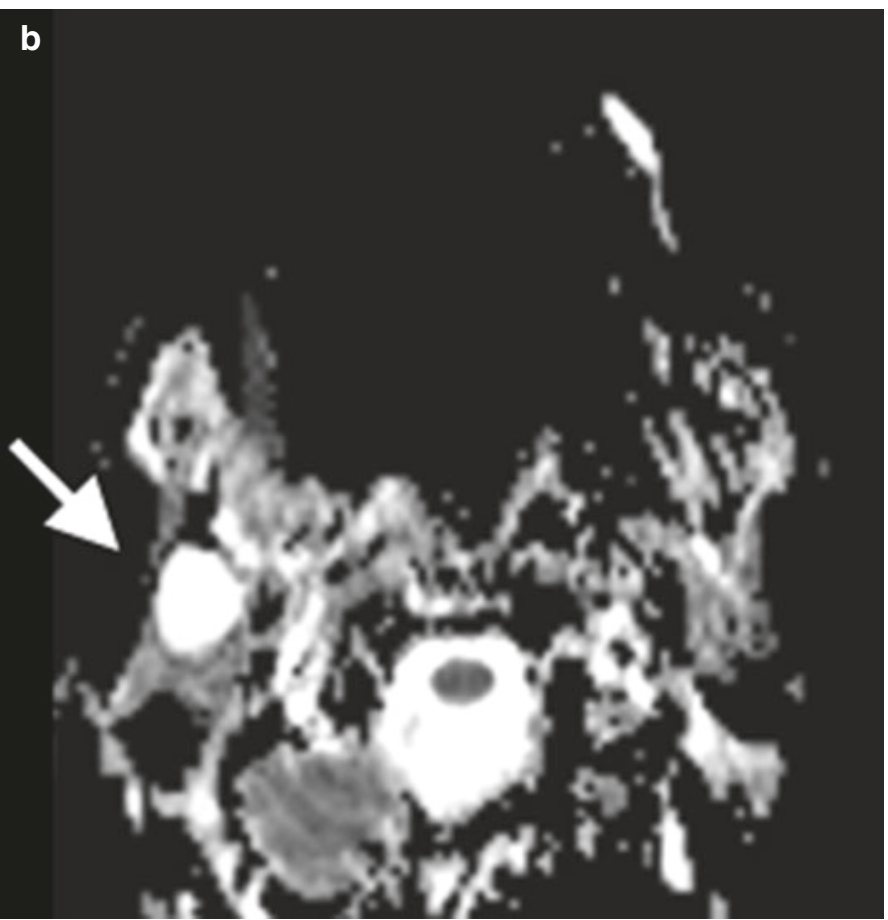

onstrates high signal reflecting the gelatinous nature of these tumors with relative free diffusivity of water 
onstrate characteristic imaging features; in general, given the very diverse neoplastic histology of this region, a specific diagnosis cannot be reliably predicted based on CT or MR imaging. Acute parotitis may occur secondary to obstruction by calculi, retrograde migration of oral flora secondary to poor salivary flow, or hematogenic viral infection. Chronic parotitis is typically bilateral and reflects underlying systemic disease such as Sjogren syndrome or sarcoidosis.

\section{Key Point}

- The parotid gland is the only salivary gland to contain lymph nodes; the differential diagnosis for lymphadenopathy must be considered for any PS mass.

\subsection{Suprahyoid Neck: Sublingual (SLS) and Submandibular Space (SMS)}

Anatomy and Contents The oral cavity is an anatomically complex area with an extensive convoluted mucosal surface covering its constituent structures, including the tongue, floor of mouth, hard palate, cheeks, and gingiva. The SLS is the portion of the floor of mouth below the tongue and above the mylohyoid muscle, a sling-like structure that separates the OC above from the SMS below (Fig. 16.7) [8]. The SLS contains the sublingual glands (SLGs), the deep lobes of the submandibular glands (SMGs) and submandibular ducts, the "neurovascular bundles" (lingual nerves, arteries, and veins), and cranial nerves 9 and 12 . The SMS is relatively simple in composition, containing only the SMG superficial lobes, fat, lymph nodes, and the facial artery and vein.

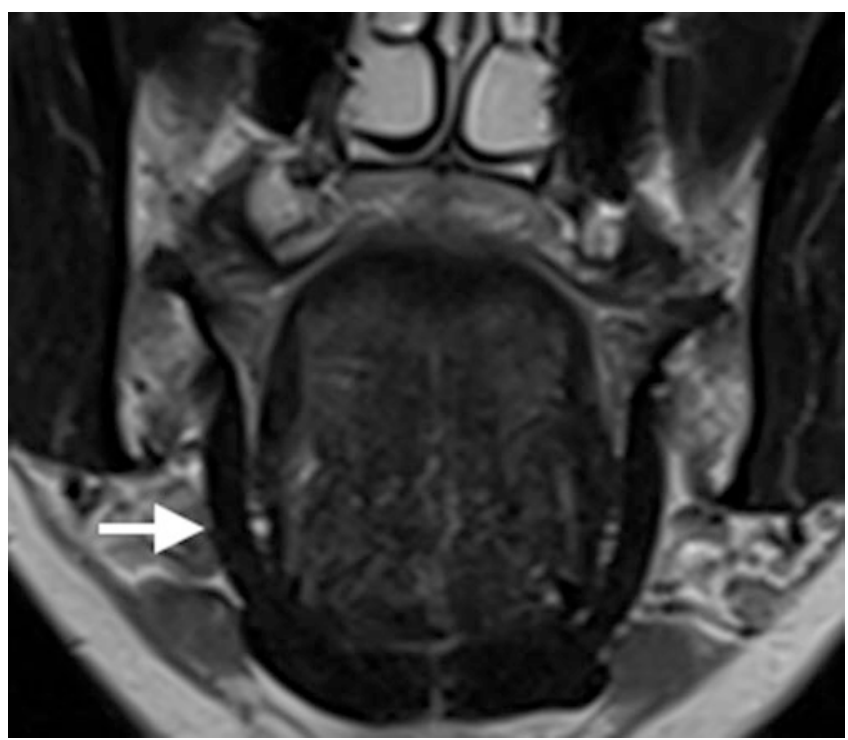

Fig. 16.7 Coronal T2 weighted image shows the sling-like mylohyoid muscle that separates the oral cavity above from the SMS below

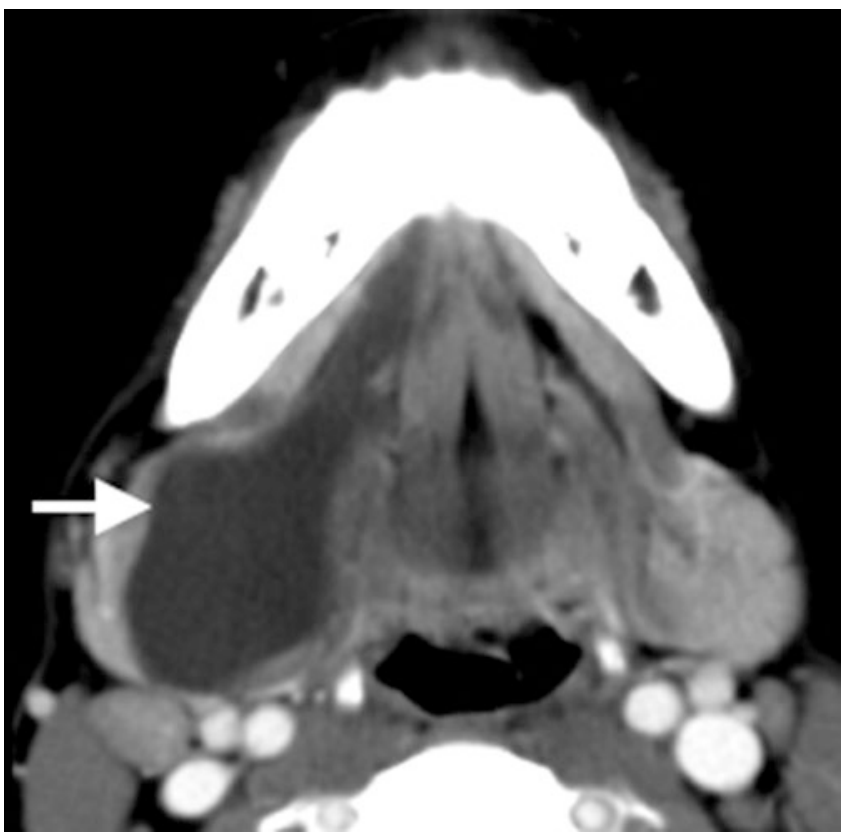

Fig. 16.8 Axial CECT depicts a "diving" or "plunging" ranula extending from the SLS to the SMS behind the posterior free edge of the mylohyoid muscle

Pathology The teeth and salivary glands are important sources of inflammatory disease in both the SLS and SMS, with the vast majority of neoplasms in this region representing squamous cell carcinoma originating from the $\mathrm{OC}$ mucosa. Salivary epithelial tumors may arise from the major salivary glands (SLG, SMG) or from minor salivary glands (MSGs) lying within the SLS submucosal space. Obstruction of the SLG or a submucosal MSG duct may result in formation of a ranula, an epithelial-lined cyst within the SLS. When these rupture, they may form pseudocysts that may "dive" or "plunge" behind the posterior free edge of the mylohyoid muscle into the SMS (Fig. 16.8).

\subsection{Infrahyoid Neck: Visceral Space (VS)}

Anatomy and Contents The VS is a central tubular space extending from the hyoid to the mediastinum, enclosed by the middle layer of deep cervical fascia. It is the only space found entirely in the infrahyoid neck. It contains the thyroid and parathyroid glands, the recurrent laryngeal nerve, the larynx, hypopharynx, trachea, and cervical esophagus. The thyroid gland is located anterior to the prevertebral musculature and posterior to the infrahyoid strap musculature, in close proximity to other structures of the visceral space including the larynx, trachea, and esophagus. The tracheoesophageal groove lies posteromedial to the thyroid lobe, and contains several important structures including the recurrent laryngeal nerve, paratracheal nodes, and parathyroid glands. 
Thyroid and Parathyroid Pathology Abnormalities of the thyroid gland are a major indication for imaging the visceral space. In general, ultrasound is the workhorse imaging modality for inflammatory (Hashimoto's) or infectious thyroiditis and for evaluation of the intrathyroidal nodule. Intrathyroidal nodules may represent colloid cysts, adenomas, differentiated thyroid cancer, or even the rare metastases. Many ultrasound classification systems exist to risk stratify these nodules and decide on biopsy, including ones by the American Thyroid association (ATA) and Society of Radiologists in Ultrasound (SRU), and also new systems such as European (EU-TIRADS) and American College of Radiology Thyroid Imaging and Reporting Data System (ACR TIRADS) are gaining acceptance for its point based approach $[9,10]$.

Thyroid cancer is a heterogeneous group of malignancies, including differentiated thyroid carcinomas (papillary and follicular), medullary and anaplastic carcinomas, and non-Hodgkin lymphoma. Cross-sectional imaging is more appropriate when there is a concern for extrathyroidal extension of tumor [11, 12] (Fig. 16.9) into surrounding structures such as the trachea or esophagus. For rapidly enlarging thyroid masses, anaplastic carcinoma and lymphoma should be considered. For multinodular goiters (MNG), ultrasound is used for surveillance of individual nodules, but non-contrast CT (NCCT) is often the choice for presurgical evaluation if airway compression necessitates removal. NCCT is helpful to evaluate the extent of airway compression, substernal extension for surgical planning and to exclude overt signs of malignancy.

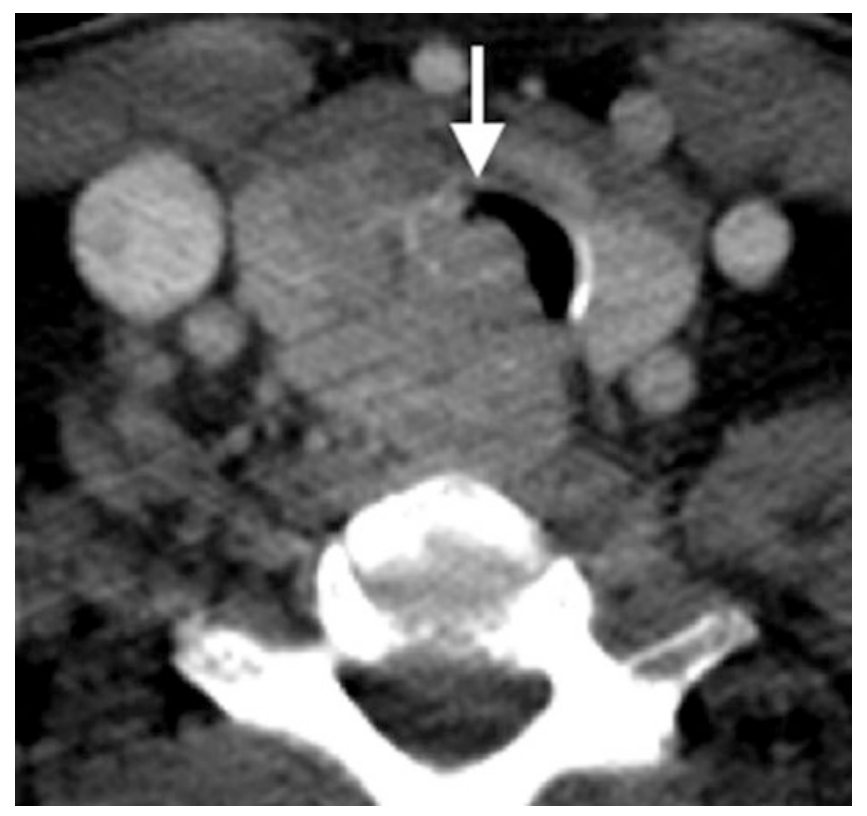

Fig. 16.9 Axial CECT shows a papillary thyroid cancer with extrathyroidal extension and invasion of the trachea (arrow) and esophagus

\section{Key Point}

- US is the primary imaging modality for both inflammatory disease and work-up of thyroid nodules, with cross-sectional imaging used when there is concern for extrathyroidal extension of tumor, or to evaluate potential airway compression or substernal extension.

Congenital lesions in the visceral space include infrahyoid thyroglossal duct cysts or ectopic thyroid tissue along the course of the thyroglossal duct. (Fig. 16.10) Thyroglossal duct cysts are the most common congenital neck masses; most present before age ten as a painless, movable mass that may fluctuate in size after upper respiratory tract infection. When suprahyoid, they are characteristically midline and when infrahyoid, they are often para-midline.

Nodules in the tracheoesophageal groove may represent parathyroid adenomas or carcinomas, lymph nodes or rarely schwannomas of the recurrent laryngeal nerve. Parathyroid adenomas are characteristically hypervascular, demonstrating rapid washin on early arterial phase and relatively rapid washout on venous phase multiphase contrast enhanced CT [13].

Larynx, Hypopharynx, Trachea, and Esophagus Pathology The larynx and hypopharynx are most commonly imaged for staging squamous cell carcinoma (a primary mucosal space abnormality), covered in a separate

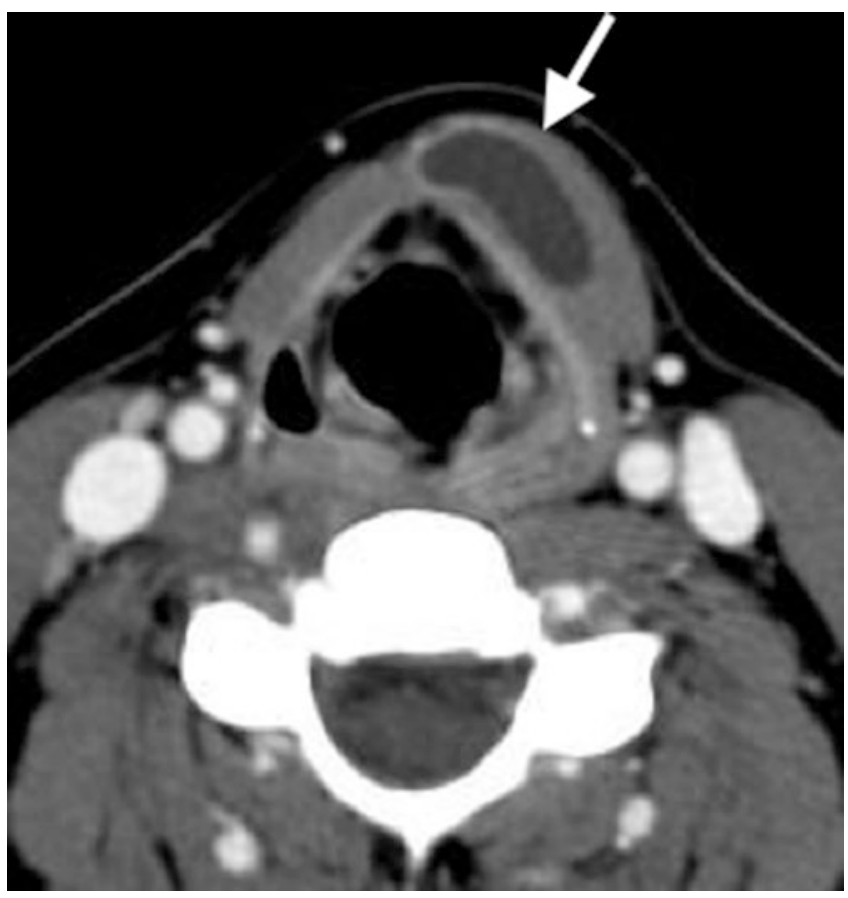

Fig. 16.10 Axial CECT shows an infrahyoid off-midline cystic lesion (arrow), embedded in the strap musculature, compatible with a thyroglossal duct cyst 


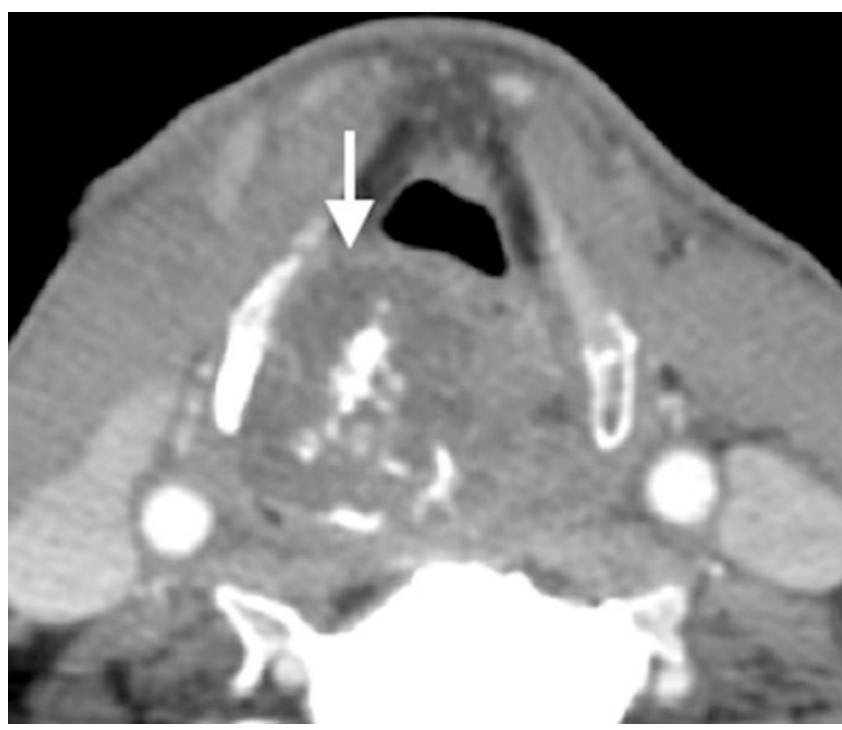

Fig. 16.11 Axial CECT shows an expansile mass replacing the right cricoid cartilage with popcorn or ring-like calcification (arrow). On endoscopy, the surgeons saw a submucosal mass. Findings are compatible with a chondrosarcoma

chapter. More rare submucosal tumors are usually well circumscribed on imaging and will not have an overlying mucosal abnormality on endoscopy. The $\mathrm{ddx}$ for submucosal masses includes minor salivary gland tumors, sarcomas, and lymphoma. Laryngeal chondrosarcomas most often arise in the cricoid cartilage with cartilage destruction, ring-like calcification, and bright T2 signal on MRI (Fig. 16.11). Benign lesions of the larynx, including papillomas and hemangiomas, are also more rare.

Epiglottitis should be suspected in a child with difficulty breathing and swallowing and may initially be evaluated with plain film. When there is a classic "thumb" sign reflecting the swollen epiglottis, treatment may be initiated and CECT may not even be required. Adults may have supraglottis with CECT showing thickened epiglottis and aryepiglottic folds.

\subsection{Entire Neck: Carotid Space}

Anatomy and Contents The carotid space spans the suprahyoid and infrahyoid neck, extending from the skull base to the aortic arch, with all three layers of the deep cervical fascia contributing to the carotid sheath [14]. It is located posterior to the styloid process (and is referred to as the post-styloid PPS by some authors), anterior to the prevertebral muscles, and lateral to the retropharyngeal space. The space can be subdivided into nasopharyngeal, oropharyngeal, cervical and mediastinal components. The suprahyoid CS contains the carotid artery, internal jugular vein (IJV), cranial nerves, IX, X, XI, XII, and the sympathetic chain. The infrahyoid CS differs in that all cranial nerves have exited except the vagus nerve. Lymph nodes are closely associated with the carotid space, along its lateral border. The carotid artery is located medially, IJV laterally, vagus nerve posteriorly along the vessels, and the sympathetic chain posteriorly within the sheath.

\section{Key Point}

- The CS spans the suprahyoid and infrahyoid neck, with all three layers of the DCF contributing to the carotid sheath.

Pathology Masses in the suprahyoid carotid space classically displace the parapharyngeal fat anteriorly and splay the carotid and jugular vein, often displacing the ICA anteriorly and IJV posterolaterally. Vascular pathology, primarily dissection or pseudoaneurysm of the carotid artery or thrombosis/thrombophlebitis of the IJV, may easily be overlooked on routine neck CT or MRI. Specific attention should be paid to the carotid space to exclude dissection in any patient presenting with Horner's syndrome (Fig. 16.12). An IJV thrombophlebitis caused by an extension of oropharyngeal or odontogenic infection is referred to as Lemierre syndrome. CT and MR angiography or venography are studies of choice if there is high clinical suspicion for a vascular lesion.

The most common masses in the high nasopharyngeal carotid space, including glomus jugular paraganglioma, schwannoma and meningioma, often dumbbell inferiorly from the jugular foramen. Close attention to the skull base is important in any patient presenting with hoarseness/ vocal cord palsy or other cranial neuropathy involving $\mathrm{CNs}$ IX-XII. Bony changes on CT are an important clue to the correct diagnosis of a jugular foramen/carotid space mass at the skull base. Permeative destruction favors paraganglioma, smooth remodeling schwannoma, and hyperostosis meningioma. On MRI, paragangliomas are intensely enhancing and can have the classic "salt and pepper" appearance with the "salt" representing microhemorrhages on T1-weighted (T1W) images and the pepper representing flow voids on T2-weighted (T2W) images. Glomus vagale and carotid body paragangliomas are also found in the carotid space. The rare vagale tumors arise along the high extracranial portion of the vagus nerve in the high nasopharyngeal carotid apace and can extend intracranially. Carotid body tumors are located at the carotid bifurcation in the infrahyoid neck and splay the internal and external carotid arteries.

Carotid space nerve sheath tumors include schwannomas and neurofibromas. CS schwannomas are benign tumors of Schwann cells that invest CNs 9-12, most commonly CN 10 (vagus). Neurofibromas are benign spindle cell neoplasms arising from the vagus nerve, hypoglossal nerve, or sympathetic chain and are associated with neurofibromatosis type 1. Schwannomas are typically fusiform enhancing tumors, 


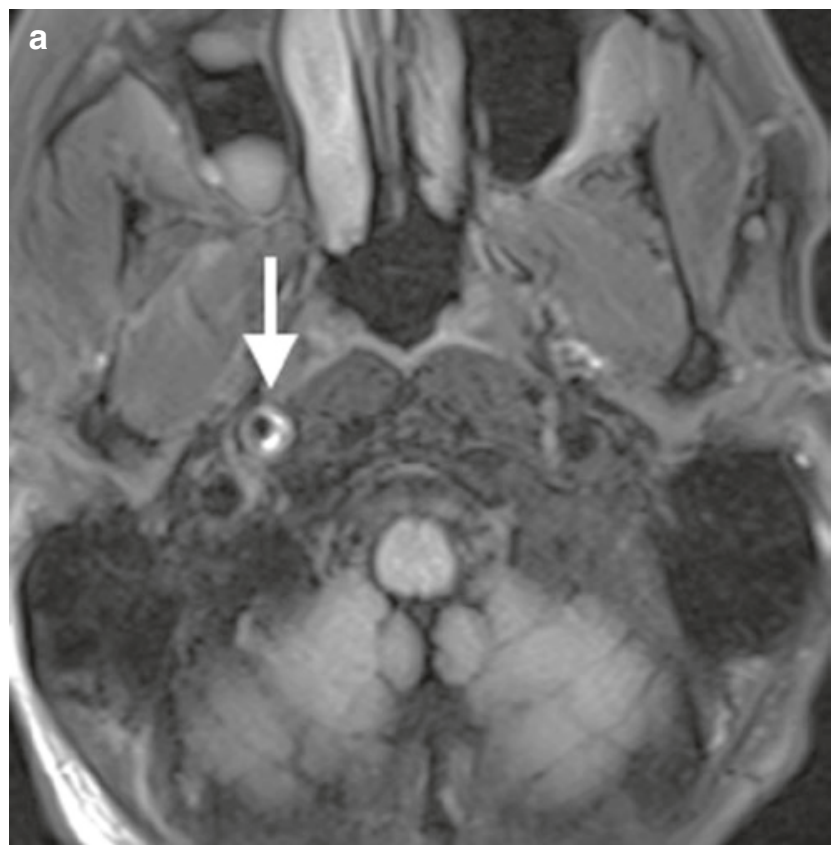

Fig. 16.12 (a) Axial flair sequence shows crescentic shaped mural hyperintensity along the medial right ICA wall (arrow), expanding the caliber of the ICA, but only minimally narrowing the lumen. (b) The source image from a 3D time of flight also shows this crescentic high

sometimes with cystic change, whereas neurofibromas are classically hypodense and hypoenhancing on CT and have a characteristic target sign on MRI. Nasopharyngeal CS schwannomas displace the PPS fat anteriorly, the styloid anterolaterally, the ICA anteromedially, and the IJV posterolaterally (Fig. 16.13). This is in contradistinction to sympathetic chain schwannomas which displace the carotid artery and jugular vein together anteriorly. Oropharyngeal CS tumors also displace the PPS fat anteriorly but also displace the posterior belly of the digastric muscle laterally. Infrahyoid neck CS nerve sheath tumors displace the carotid anteromedially.

\subsection{Entire Neck: Retropharyngeal, Prevertebral and Danger Spaces (RPS)}

Anatomy and Contents As mentioned in the introduction, the DLDCF has two components, the alar and prevertebral fascia, and this results in the formation of three posterior neck spaces: (1) retropharyngeal space (RPS), between the visceral and alar fascia, (2) danger space, between the alar and prevertebral fascia, and (3) prevertebral space, between the prevertebral fascia and the vertebral periosteum (part of the perivertebral space, discussed below). The importance of recognizing the division into these spaces primarily relates to

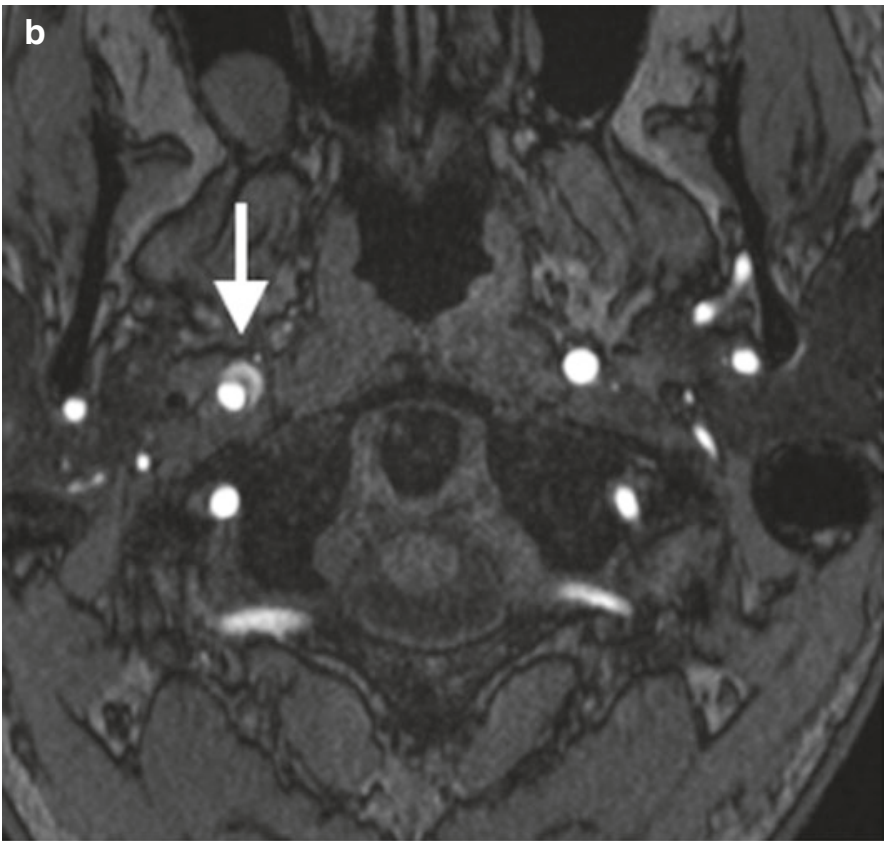

T1 signal (arrow) because of intrinsic T1 shortening, but on the MIP images from the post gadolinium MRA, a caliber change in the lumen could barely be perceived

their inferior extent. While the RPS terminates at the level of the T3 vertebra, the danger space extends more inferiorly to a point just above the diaphragm, and the prevertebral space continues to the coccyx. Though we typically cannot distinguish these spaces on imaging, we must remember to follow a posterior neck space collection to its inferior extent, recognizing that this may lie within the chest or below. Contents of the RPS are simple, consisting of fat and lymph nodes.

\section{Key Point}

- Retropharyngeal collections that have entered the danger or prevertebral spaces can descend to the mediastinum and as far inferiorly as the coccyx.

Pathology Disease processes in the RPS can be categorized as related to nodal pathology and/or the presence of fluid. Fluid within the RPS may be encapsulated and represent an abscess (Fig. 16.14) or, much less commonly, a pseudomeningocele representing a complication of spinal surgery. More frequent is edema or effusion of the RPS and this may occur as a reaction to a wide variety of disease processes, including pharyngitis, lymphatic or venous obstruction, angioedema, and radiation therapy [15]. Nodal enlargement may occur in the setting of inflammatory or neoplastic processes. When 


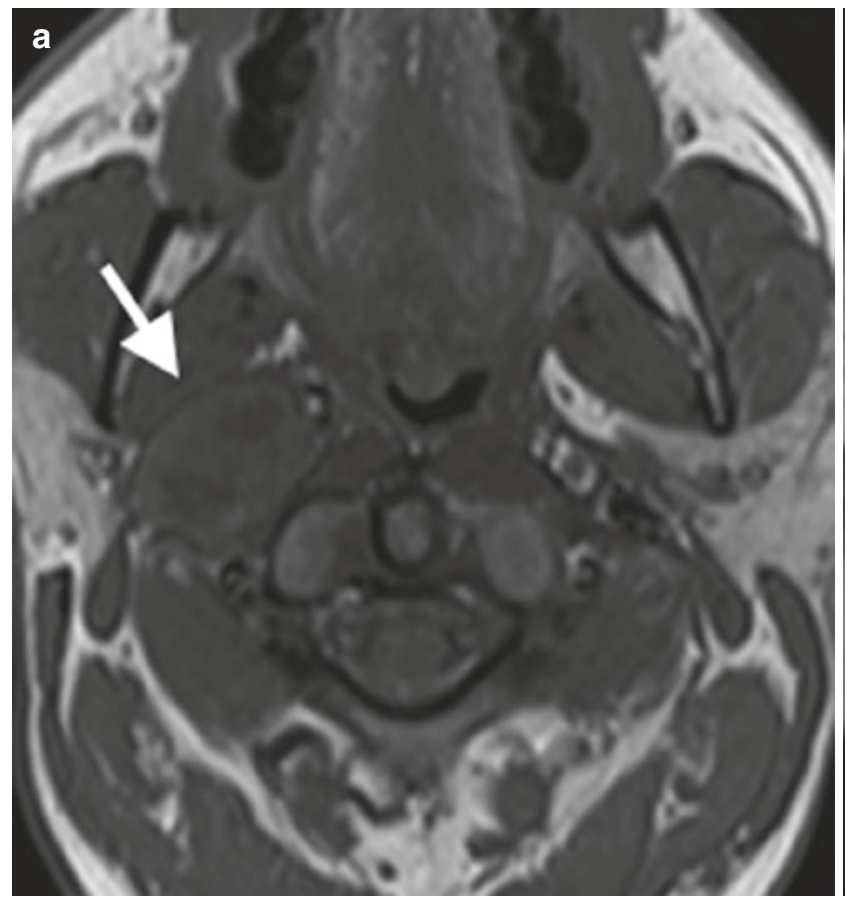

Fig. 16.13 (a) Axial T1-weighted image shows a $\mathrm{T} 1$ isointense mass which displaces the PPS fat anteriorly. This suggests that the mass originates in the carotid space. (b) Axial postgadolinium, fat saturated T1-weighted image shows a fusiform, enhancing mass with central cys-

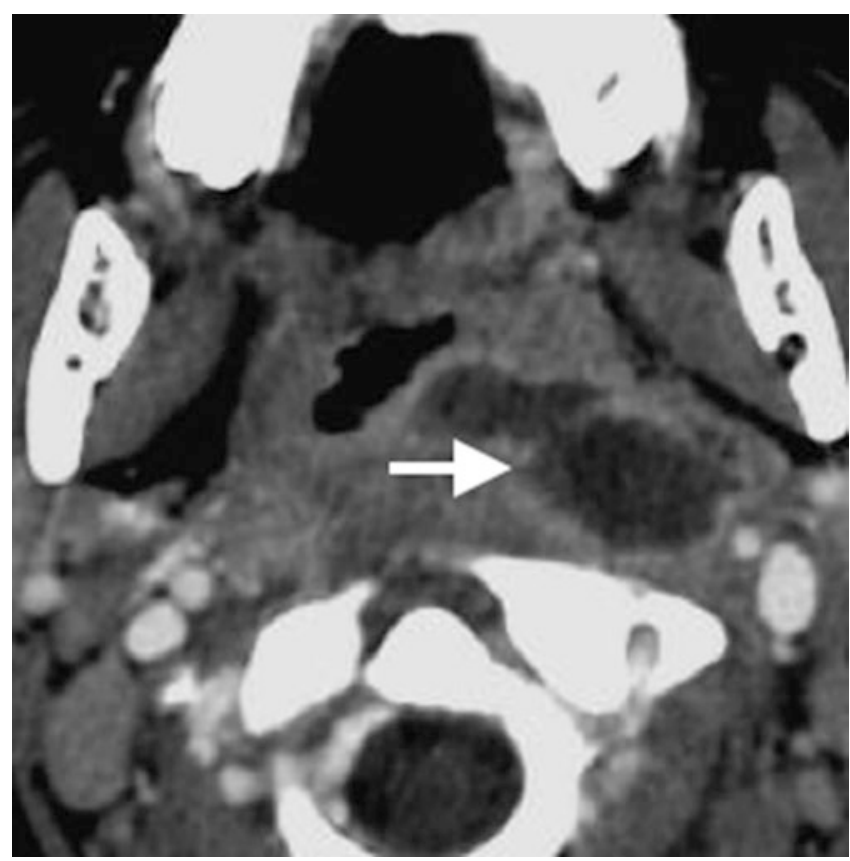

Fig. 16.14 Axial CECT image shows suppurative retropharyngeal lymphadenopathy in a child with streptococcal pharyngitis. Note that the intranodal abscess has ruptured medially and is spilling into the RPS, where it may form a retropharyngeal abscess

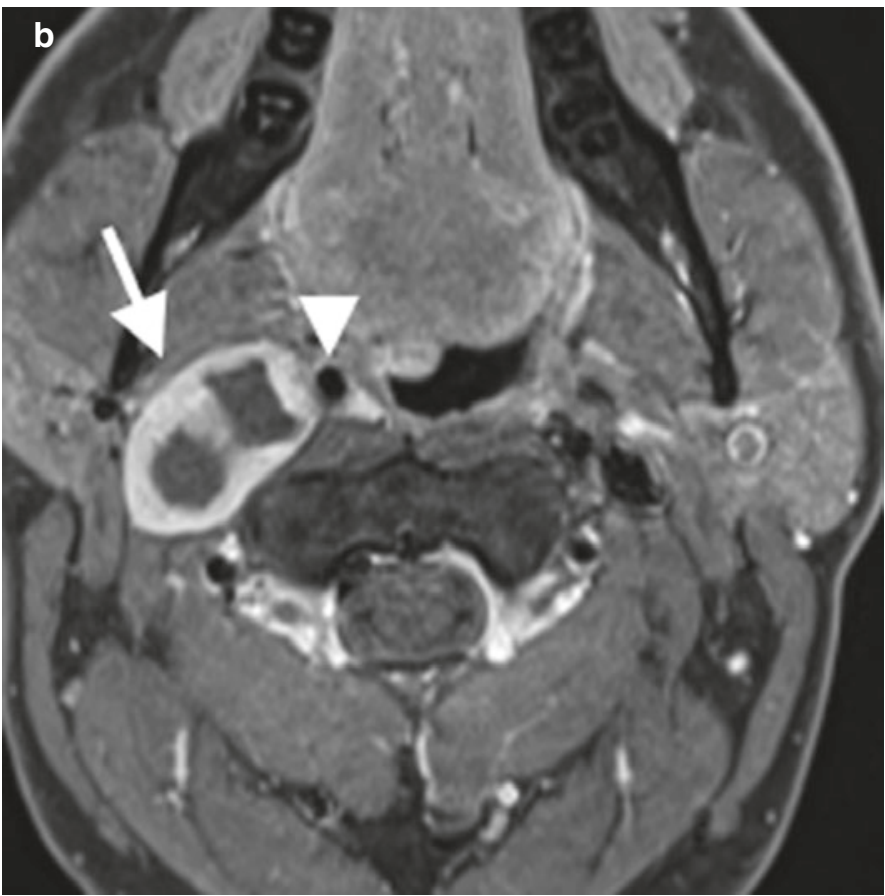

tic change. The ICA flow void is displaced anteromedially (arrowhead) and the IJV is compressed, which further confirms the carotid space origin. Note that retropharyngeal masses will displace the carotid laterally

there is pharyngitis, lymphadenopathy may be reactive and homogenous, or, particularly in children, there may be the development of an intranodal abscess that may rupture into the RPS. RPS lymph nodes may be involved in hematologic malignancy such as lymphoma, or may represent sites of metastatic disease from local malignancies, particularly those of the pharynx, paranasal sinuses, and thyroid gland (Fig. 16.15).

\subsection{Entire Neck: Perivertebral Space}

Anatomy and Contents The perivertebral space (PVS) is a cylindrical space around the vertebral column, invested in the DL-DCF from the skull base to the mediastinum. The PVS can be subdivided into the prevertebral space and the paraspinal space [16]. The contents of the PVS include the vertebral bodies, disc spaces, musculature, and the vertebral arteries in the foramen transversarium. The retropharyngeal space lies directly anterior to the PVS and the paired carotid spaces lie anterolateral. The prevertebral muscles are invested in the anterior DL-DCF, known as the "carpet" by surgeons. It is tenacious and serves as a barrier to infection and neoplasm. Lesions in the PVS tend to lift or displace the prevertebral muscles anteriorly, whereas RPS lesions displace these muscles posteriorly. 
Pathology The vast majority of pathologic lesions in the perivertebral space involve the vertebral bodies and disc spaces, and the minority of lesions are centered in the paraspinal musculature. Therefore, pyogenic discitis/ osteomyelitis and bone metastasis are the most common pathologies found in this space (Fig. 16.16). Atypical infections such as tuberculosis are less common, but have

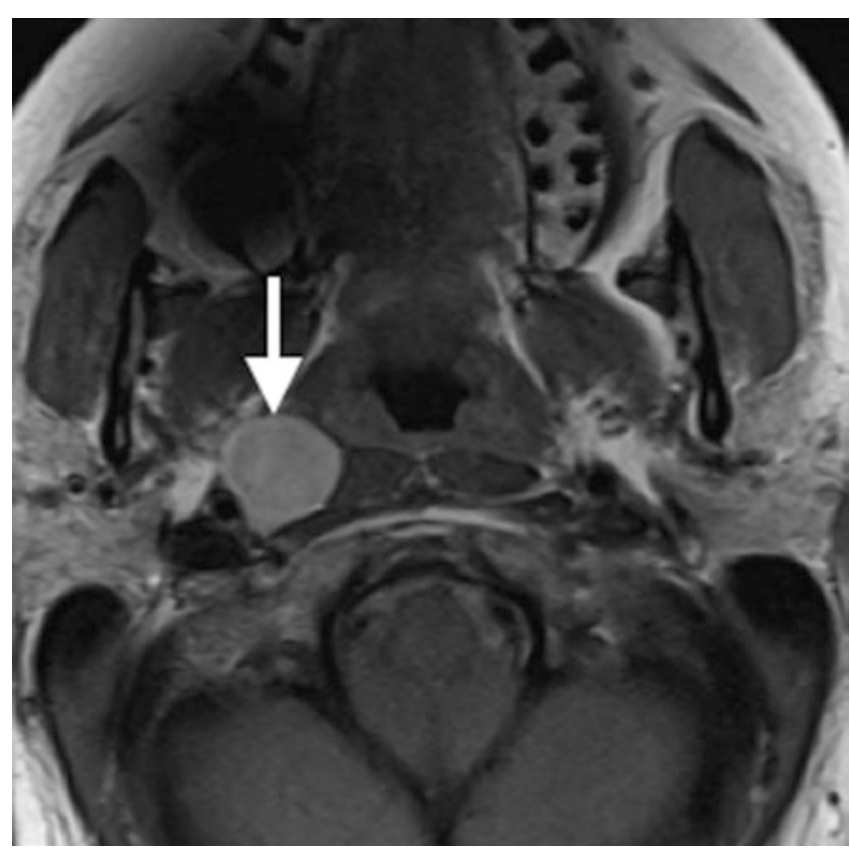

Fig. 16.15 Axial T1-weighted image without contrast shows an enlarged lateral retropharyngeal lymph node in a patient with metastatic papillary thyroid carcinoma. T1 shortening reflects colloid content characteristic imaging features such as sparing of the disc spaces. Primary bone tumors are also uncommon, but have characteristic locations and appearance. For example, chordomas can be suggested when a lesion is found in the upper cervical spine with characteristic bright $\mathrm{T} 2$ signal and enhancement.

\subsection{Concluding Remarks}

The neck is anatomically complex, but can be organized into compartments or "spaces" in order to organize the approach to neck masses. A thorough understanding of fascial planes, the various spaces and their normal contents will help the radiologist generate the best differential diagnoses to appropriately guide management. The parapharyngeal space (PPS) fat is especially important in the analysis of most suprahyoid neck masses, as the mass effect on this triangular fat pad often helps to localize the space of origin. Pathology will displace the PPS in a predictable pattern. For example, masses arising in the deep lobe of the parotid tend to displace the PPS anteromedially, carotid space masses displace the PPS anteriorly, masticator space masses push the PPS posteriorly, and pharyngeal mucosal space masses often push the PPS laterally. Each compartment has common and uncommon pathology, often based on the normal contents. Therefore, once a lesion is localized to the correct space, the radiologist can utilize imaging features and clinical information to provide a tailored list of differential considerations, or in some cases, a specific most likely diagnosis.
Fig. 16.16 (a) Axial CECT shows enlargement and heterogeneous enhancement in the prevertebral space involving the prevertebral muscles and abutting the $\mathrm{T} 2$ vertebral body. (b) Sagittal T1-weighted post gadolinium shows abnormal enhancement of lower cervical and upper thoracic vertebral bodies and a large area of nonenhancement (arrows), compatible with multi-level osteomyelitis. There is also epidural extension of infection (arrowhead)
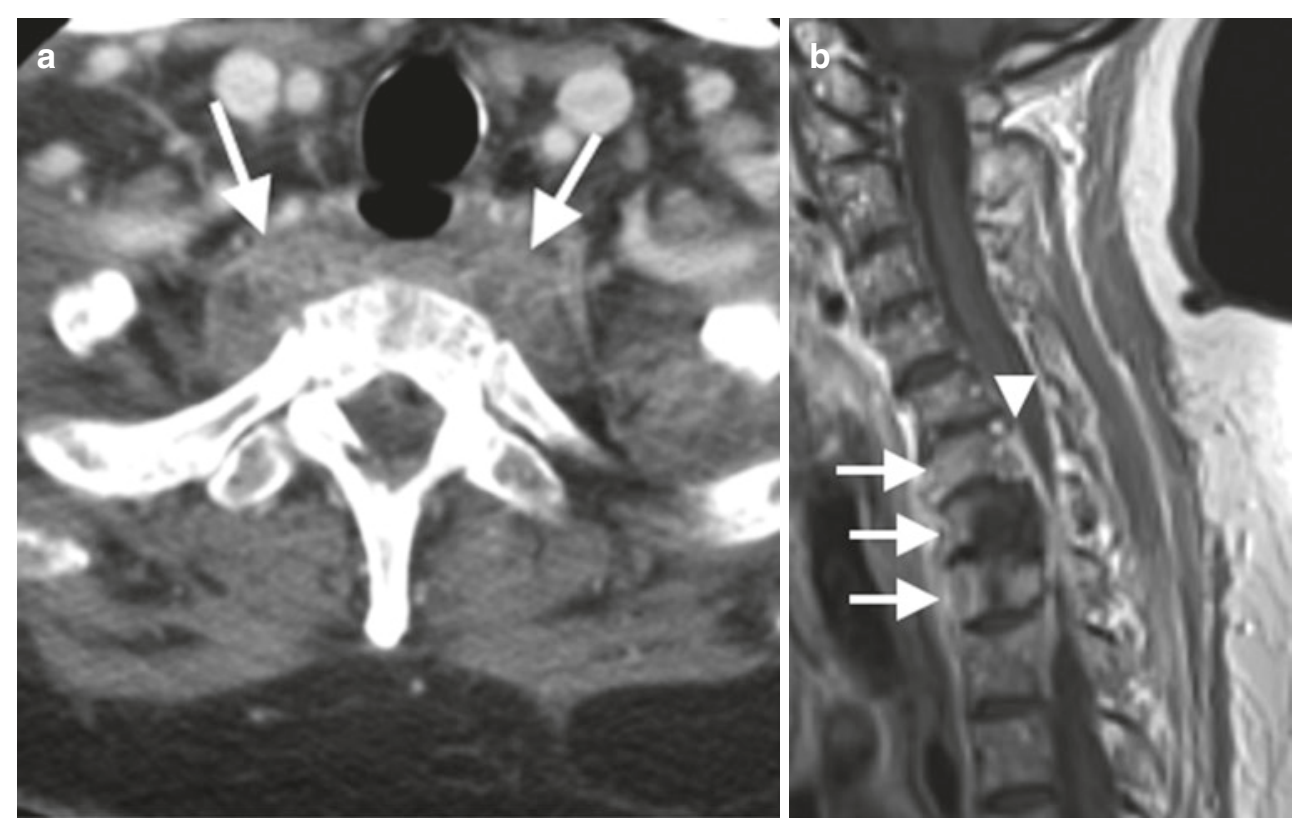


\section{H\&N space contents}

\begin{tabular}{|c|c|c|c|}
\hline Space & Boundaries & Major contents & Common pathology \\
\hline $\begin{array}{l}\text { Pharyngeal } \\
\text { mucosal (PMS) }\end{array}$ & $\begin{array}{l}\text { Mucosa to pharyngeal constrictors } \\
\text { of nasopharynx and oropharynx } \\
\text { (some include hypopharynx and } \\
\text { oral cavity) }\end{array}$ & $\begin{array}{l}\text { Mucosa, Waldeyer's ring, minor } \\
\text { salivary glands, constrictor and levator } \\
\text { palatine muscles, cartilaginous } \\
\text { Eustachian tube }\end{array}$ & $\begin{array}{l}\text { Malignant tumors }(\text { mucosal }=\text { SCC or } \\
\text { NPC and submucosal = minor salivary } \\
\text { tumors or lymphoma) } \\
\text { Pharyngitis, tonsillitis }\end{array}$ \\
\hline $\begin{array}{l}\text { Parapharyngeal } \\
\text { (PPS) }\end{array}$ & $\begin{array}{l}\text { Skull base to mandibular angle, } \\
\text { borders PPS, CS, PS, MS }\end{array}$ & Fat, minor salivary glands & $\begin{array}{l}\text { Benign salivary tumors, rare branchial } \\
\text { cleft cyst or vascular malformation }\end{array}$ \\
\hline Masticator (MS) & $\begin{array}{l}\text { Skull base to mandibular angle, } \\
\text { lateral to PPS }\end{array}$ & $\begin{array}{l}\text { Muscles of mastication, posterior body } \\
\text { and ramus of mandible, CNV3 }\end{array}$ & $\begin{array}{l}\text { Odontogenic infections, venous } \\
\text { malformations, sarcomas }\end{array}$ \\
\hline Parotid (PS) & $\begin{array}{l}\text { Enclosed within the parotid fascia, } \\
\text { lateral to PPS, MS }\end{array}$ & $\begin{array}{l}\text { Parotid gland, } \mathrm{CN} 7, \text { lymph nodes, } \\
\text { retromandibular vein, external carotid } \\
\text { artery branches }\end{array}$ & $\begin{array}{l}\text { Pleomorphic adenoma } \\
\text { Low and high grade malignant salivary } \\
\text { neoplasms } \\
\text { Acute or chronic parotitis }\end{array}$ \\
\hline Visceral (VS) & $\begin{array}{l}\text { Hyoid to mediastinum, anterior to } \\
\text { PVS, medial to CS }\end{array}$ & $\begin{array}{l}\text { Thyroid and parathyroid glands, larynx, } \\
\text { hypopharynx, trachea, esophagus }\end{array}$ & $\begin{array}{l}\text { Thyroid nodules } \\
\text { Thyroid cancer } \\
\text { Parathyroid adenoma } \\
\text { Mucosal SCC } \\
\text { Chondrosarcoma } \\
\text { Diverticula }\end{array}$ \\
\hline Carotid (CS) & $\begin{array}{l}\text { Carotid sheath, enclosed by all } 3 \\
\text { layers of DCF, skull base to aortic } \\
\text { arch (SHN + IFN) }\end{array}$ & $\begin{array}{l}\text { SHN: ICA, IJV, CN 9-12 } \\
\text { IFN: CCA, IJV, CN } 10 \text { (vagus) }\end{array}$ & $\begin{array}{l}\text { Vascular pathology related to carotid } \\
\text { (aneurysm, dissection, arteritis) or jugular } \\
\text { vein (thrombosis or thrombophlebitis) } \\
\text { Nerve sheath tumors } \\
\text { Paragangliomas }\end{array}$ \\
\hline $\begin{array}{l}\text { Retropharyngeal } \\
\text { (RPS) }\end{array}$ & $\begin{array}{l}\text { Skull base to } \mathrm{T} 3 \text {, between visceral } \\
\text { and alar fascia }\end{array}$ & Fat and lymph nodes & $\begin{array}{l}\text { Metastatic lymph nodes (NPC, thyroid, } \\
\text { hypopharynx, lymphoma) } \\
\text { Suppurative lymph nodes (children) } \\
\text { Effusion }\end{array}$ \\
\hline $\begin{array}{l}\text { Perivertebral } \\
\text { (PVS) }\end{array}$ & Skull base to T4, posterior to RPS & $\begin{array}{l}\text { Prevertebral muscles, vertebral bodies, } \\
\text { scalene muscles, brachial plexus roots, } \\
\text { phrenic nerve, vertebral artery and vein }\end{array}$ & $\begin{array}{l}\text { Discitis/osteomyelitis } \\
\text { Longus colli tendinitis } \\
\text { Primary bone tumors (chordoma, ABC, } \\
\text { osteoma, giant cell) } \\
\text { Vertebral metastases } \\
\text { Sarcomas } \\
\text { Nerve sheath tumors }\end{array}$ \\
\hline
\end{tabular}

SCC squamous cell carcinoma, $N P C$ nasopharyngeal carcinoma, $A B C$ aneurysmal bone cyst, $S H N$ suprahyoid neck, $I F H$ infrahyoid neck, $I C A$ internal carotid artery, $C C A$ common carotid artery, $I J V$ internal jugular vein

\section{Take Home Messages}

- The three layers of the deep cervical fascia define spaces, each with unique contents and pathology.

- These spaces can be grouped as suprahyoid or infrahyoid, with some spaces, like the carotid and retropharyngeal spaces, spanning both levels.

- Displacement of the parapharyngeal fat can help determine site of origin of a suprahyoid mass.

- The visceral space is the only space contained entirely in the infrahyoid neck and contains the thyroid and parathyroid glands as well as portions of the aerodigestive tract below the oropharynx.

\section{References}

1. Gamss C, Gupta A, Chazen JL, Phillips CD. Imaging evaluation of the suprahyoid neck. Radiol Clin N Am. 2015;53(1):133-44.

2. Warshafsky D, Goldenberg D, Kanekar SG. Imaging anatomy of deep neck spaces. Otolaryngol Clin N Am. 2012;45(6):1203-21.

3. Guidera AK, Dawes PJ, Fong A, Stringer MD. Head and neck fascia and compartments: no space for spaces. Head Neck. 2014;36(7):1058-68.

4. Parker GD, Harnsberger HR, Jacobs JM. The pharyngeal mucosal space. Semin Ultrasound CT MR. 1990;11(6):460-75.

5. Stambuk HE, Patel SG. Imaging of the parapharyngeal space. Otolaryngol Clin N Am. 2008;41(1):77-10.

6. Meltzer DE, Shatzkes DR. Masticator space: imaging anatomy for diagnosis. Otolaryngol Clin North Am. 2012;45(6):1233-51.

7. Kanekar SG, Mannion K, Zacharia T, Showalter M. Parotid space: anatomic imaging. Otolaryngol Clin North Am. 2012;45(6): $1253-72$. 
8. La'porte SJ, Juttla JK, Lingam RK. Imaging the floor of the mouth and the sublingual space. Radiographics. 2011;31(5):1215-30.

9. Tessler FN, Middleton WD, Grant EG, et al. ACR thyroid imaging, reporting and data system (TI-RADS): white paper of the ACR TI-RADS Committee. J Am Coll Radiol. 2017;14(5): 587-95.

10. Russ G, Bonnema SJ, Erdogan MF, Durante C, Ngu R, Leenhardt L. European thyroid association guidelines for ultrasound malignancy risk stratification of thyroid nodules in adults: the EU-TIRADS. Eur Thyroid J. 2017;6(5):225-37.

11. Loevner LA, Kaplan SL, Cunnane ME, Moonis G. Crosssectional imaging of the thyroid gland. Neuroimaging Clin N Am. 2008;18(3):445-61, vii.
12. Aiken AH. Imaging of thyroid cancer. Semin Ultrasound CT MR. 2012;33(2):138-49.

13. Phillips CD, Shatzkes DR. Imaging of the parathyroid glands. Semin Ultrasound CT MR. 2012;33(2):123-9.

14. Kuwada C, Mannion K, Aulino JM, Kanekar SG. Imaging of the carotid space. Otolaryngol Clin N Am. 2012;45(6):1273-92.

15. Bhatt AA. Non-traumatic causes of fluid in the retropharyngeal space. Emerg Radiol. 2018;25(5):547-51.

16. Mills MK, Shah LM. Imaging of the perivertebral space. Radiol Clin N Am. 2015;53(1):163-80.

Open Access This chapter is licensed under the terms of the Creative Commons Attribution 4.0 International License (http://creativecommons. $\mathrm{org} /$ licenses/by/4.0/), which permits use, sharing, adaptation, distribution and reproduction in any medium or format, as long as you give appropriate credit to the original author(s) and the source, provide a link to the Creative Commons license and indicate if changes were made.

The images or other third party material in this chapter are included in the chapter's Creative Commons license, unless indicated otherwise in a credit line to the material. If material is not included in the chapter's Creative Commons license and your intended use is not permitted by statutory regulation or exceeds the permitted use, you will need to obtain permission directly from the copyright holder. 


\title{
Head and Neck Squamous Cell Cancer: Approach to Staging and Surveillance
}

\author{
Christine M. Glastonbury
}

\section{Learning Objectives}

- To understand the development of HN SCC arising from different areas in the $\mathrm{HN}$ and particularly the contributions of viruses and environmental exposure factors in its pathogenesis.

- To recognize the important features of the AJCC/ UICC staging system and how radiologists can use this system to provide more detailed, valuable reports.

- To develop a systematic method for evaluating all post-treatment scans from baseline through surveillance in order to detect residual, recurrent, and new $\mathrm{HN}$ malignancies.

\subsection{Imaging Approaches for HN SCC}

Across the USA and across the world there are different thoughts and perspectives as to the best imaging modality to use for staging and surveillance, but also as to the ideal timing for use of the modalities after treatment and for ongoing surveillance. The AJCC offers some guidance about the utility of imaging modalities for the staging of specific entities but offers no surveillance plan [1]. In the USA the National Comprehensive Cancer Network [NCCN] guidelines for HN cancer treatment sets out broad strokes but these surveillance protocols are variably used and were developed without radiologist input [2]. The NI-RADS reporting system for

\section{M. Glastonbury $(\bowtie)$}

Department of Radiology and Biomedical Imaging,

University of California, San Francisco, CA, USA

Department of Otolaryngology-Head and Neck Surgery and Department of Radiation Oncology, University of California, San Francisco, CA, USA

e-mail: Christine.glastonbury@ucsf.edu baseline and surveillance imaging after treatment was modeled after the breast imaging BI-RADS system, and aims to standardize an imaging approach to HN cancer with management recommendations. Through ongoing studying of the results of this system, we seek to determine the accuracy of imaging and then further optimize surveillance algorithms in $\mathrm{HN}$ cancer [3-5].

When staging HN SCC some specific tumor sites are better served by either CT or MR. At different hospitals and imaging sites modalities may be favored because of cost, modality, availability, or patient tolerance for the imaging exam. Excellent quality whole neck imaging is more readily reproducible from patient to patient using CT than MR, and this is particularly true for the post-treatment patient who may have difficulty lying on a MR for an extended period while dealing with oral secretions. MR and PET are more expensive and may not be readily accessible in different locations in the USA because of insurance costs. Large field of view, non-optimized MR sequences and lack of familiarity with basic neck anatomy or MR artifacts will make detection of key findings difficult for the less experienced radiologist.

With these caveats in mind, MR offers specific utility in certain areas. For example, it is the preferred staging tool for nasopharyngeal carcinoma because the detection of skull base infiltration (T3) or intracranial extension of disease (T4) is extremely important for staging and treatment planning. Likewise, MR offers significantly better soft tissue contrast for detecting small primary tonsillar tumors and evaluating the deep extent of an infiltrative lesion when planning surgical resection or intensity modulated radiation therapy (IMRT). For this reason it is often used in the oral cavity and oropharynx. In the larynx, MR is so affected by motion artifact that it is largely reserved for determination of cartilage penetration (T4a) when CT is equivocal. Finally, nodal disease at any site is almost equally well evaluated with either CT or MR.

Given the complexity of neck anatomy and intimately located structures, FDG-PET in the HN is best performed as a combined PET/CT examination. It is important to be 
aware of the many tissues with variable degrees of normal FDG uptake, particularly muscles, brown fat, salivary and lymphoid tissue, and recent biopsy sites. These all serve as potential false-positive pitfalls in PET imaging. A potential false-negative finding is the absence of uptake in a predominantly cystic node. Correlation with neck CT imaging will allow correct identification of cystic nodal metastases.

Ultrasound (US) has a limited but important role in $\mathrm{HN}$ SCC. At many practices in the USA it is largely reserved for interrogation of nodes that are equivocal on other imaging modalities, and particularly when a positive finding, such as a contralateral node, might significantly alter staging and management. US can also serve as imaging guidance for fine needle aspiration (FNA).

\subsection{Imaging Anatomy for HN SCC}

It is from the squamous lining of the pharynx, larynx, and sinonasal cavity that mucosal SCC arises. The pharynx is really a muscular tube, extending from the skull base where it is attached by the pharyngobasilar fascia, to the larynx and cervical esophagus. Staging of SCC is individualized to each site, and, in the case of laryngeal SCC, to each of its three subsites $[1,6]$.

The nasopharynx is so-named as it is located posterior to the nasal cavity, extending from the most cranial aspect of the pharynx at the skull base to the level of the soft palate. Most nasopharyngeal carcinomas arise from the lateral pharyngeal recess, also known as the fossa of Rosenmüller, which is adjacent to the opening of the Eustachian tube, and thus nasopharyngeal tumors often result in middle ear effusion at the time of presentation. In the midline of the nasopharynx, immediately caudal to the clivus is the nasopharyngeal tonsillar tissue ["adenoids"] from which nasopharyngeal lymphoma may arise. Adenoidal hypertrophy most often occurs in response to viral pathogens including HIV infection, and can mimic nasopharyngeal neoplasms although there should be symmetry, distinction of this tissue from prevertebral muscles and parapharyngeal fat, and on T2-weighted MR imaging there should preservation of a mucosal stripe [7].

Inferiorly the nasopharynx is contiguous with the oropharynx, which extends caudally to the hyoid bone. The posterior wall of the nasopharynx becomes the posterior wall of the oropharynx. The anterior tonsillar pillars and the circumvallate papillae of the tongue define the anterior limit of the oropharynx. The posterior one third of the tongue is called the tongue base and is the site of the lingual tonsillar tissue. This is part of the oropharynx and tumors arising here are staged using one of two oropharyngeal tumor staging systems depending on their pathologic p16 or high-risk HPV status $[1,6]$. The anterior two thirds of the tongue is known as the oral tongue and lies in the oral cavity. Tumors arising from the inner mucosal surface of the lips, the gingiva of the maxilla and mandible, the hard palate, and the cheek lining [buccal mucosa] are all classified as oral cavity tumors and staged using the oral cavity staging forms.

Below the hyoid bone, the pharynx divides into the more anterior larynx airway, and the posterior hypopharynx for digestion. The posterior wall of the oropharynx continues as the posterior wall of the hypopharynx while the anterior wall of the hypopharynx is the post-cricoid region, the mucosa overlying the posterior surface of the cricoid cartilage of the larynx. The lateral recesses or "pockets" of the hypopharynx are the pyriform sinuses, from which nearly two thirds of hypopharyngeal SCC arise. The aryepiglottic (AE) folds separate the hypopharynx from the supraglottic larynx and tumors arising from these folds are staged as supraglottic tumors. The inferior aspect of the hypopharynx is at the inferior aspect of the cricopharyngeus muscle, which marks the beginning of the cervical esophagus. On cross-sectional imaging this can be determined by the inferior aspect of the cricoid cartilage, and on axial sections the semilunar shape of the collapsed hypopharynx abruptly transitions to the rounded contour of the muscular esophagus [8].

The larynx connects the oropharynx airway to the tracheal airway and is more anterior in the neck. Embryologically, and for tumor staging, it is divided into three subsites, each of which has a separate staging table. The glottis is the true vocal cords; muscular structures which give rise to voice and which extend from the arytenoid cartilages to the midline thyroid cartilage anteriorly. More than half of all laryngeal SCC arise from the mucosal surface of the glottis. The supraglottic larynx is comprised of the structures superior to this including the epiglottis, AE folds, and the false cords. The epiglottis has both laryngeal and lingual surfaces and its most superior aspect extends above the level of the hyoid bone although all tumors arising from the mucosal aspect of the epiglottis are considered supraglottic. The subglottis is the mucosal lining internal to the cricoid cartilage ring below the glottis. The subglottis is contiguous with the cervical trachea and tumors of this area are the least common of all laryngeal SCC.

When talking about HN SCC the nasal cavity and paranasal sinuses are not often included in the discussion; however, they comprise up to $5 \%$ of all $\mathrm{HN}$ cancers and up to $70 \%$ of these are mucosal SCC [9]. The nasal cavity is anterior to the nasopharynx and from this branch the paired left and right paranasal sinuses: frontal, maxillary, sphenoid, and the anterior and posterior ethmoid air cells. Sinonasal SCC are not associated with tobacco, but have been associated with industrial exposure such as wood, nickel dust, or formaldehyde [10, 11]. More recently it has been determined that approximately $25 \%$ of all sinonasal SCC are associated with high-risk HPV [9]. 


\subsection{Staging HN SCC}

In 2018 the eighth edition AJCC/UICC tumor classification system was released after several years of collaborative work between the two committees and from evaluation of interval research that better understood the molecular underpinnings and the prognosis of HN SCC $[1,6]$. While there have been many changes to staging in HN SCC it is not necessary to remember all the intricacies of staging tables. It is more important that the radiologist is aware that such tables exist and that when reporting imaging studies, reference to the specific criteria that determine the tumor $(\mathrm{T})$, nodal $(\mathrm{N})$, and metastasis (M) designation greatly enhances an imaging report by providing critical tumor information $[12,13]$. It is ideal to have on hand a copy of the staging tables to ensure that information is included in the report, keeping in mind that it is not the radiologist's role to provide a TNM stage, but rather to provide all information so that this can be done by the managing physician.

When evaluating the primary tumor $(\mathrm{T})$ the specific criteria in the tables may require distinction by size of the tumor or by specific invasion patterns. When measuring tumor size the longest diameter is used. It is important to refer to $\mathrm{T}$ criteria for specific areas of invasion in order to include such relevant positive or negative information in the radiology report. Some criteria will not be known to the radiologist such as impairment of vocal cord mobility in laryngeal tumors, and depth of invasion (DOI) for oral cavity tumors, which is purely a pathological measurement. Minor changes have been made to the $\mathrm{T}$ criteria for nasopharyngeal carcinoma and for oropharyngeal carcinoma and lip tumors have been removed from the oral cavity staging, and have their own $\mathrm{T}$ criterion under a new chapter of "cutaneous carcinoma of the head and neck."

The detection of perineural tumor (PNT) may significantly alter the extent of surgical resection and/or the radiation treatment field even if it does not change the $\mathrm{T}$ designation and so should be sought on all examinations. Both mucosal and skin SCC exhibit neurotropism as do other skin malignancies, salivary gland tumors, and lymphomas. PNT is usually more readily evident on MR but may be found on CT with careful evaluation of known routes of spread. Evidence of perineural tumor may significantly alter the primary surgical plan and/or the radiation field [14].

The most significant change to the AJCC/UICC staging is the bifurcation of oropharyngeal tumor staging into those tumors which are shown to be 16 positive by immunohistochemistry or shown to be high-risk HPV DNA positive, from those which are not. The former group of $\mathrm{p} 16+/$ HPV-associated oropharyngeal SCC have a markedly better prognosis despite their tendency to have early and extensive nodal metastasis $[15,16]$. The nodal table and the overall prognostic table for these tumors have been markedly modi- fied to reflect this, with up to $80 \%$ of these tumors now being classified as stage 1 with a $90 \% 5$-year survival. This is in contrast to p16-/non-HPV oropharyngeal SCC where most tumors are stage III or stage IV at presentation (Fig. 17.1).

The second major change to the eighth edition of the AJCC/UICC is alteration of the nodal staging table for all nonHPV and non-EBV related SCC and development of separate
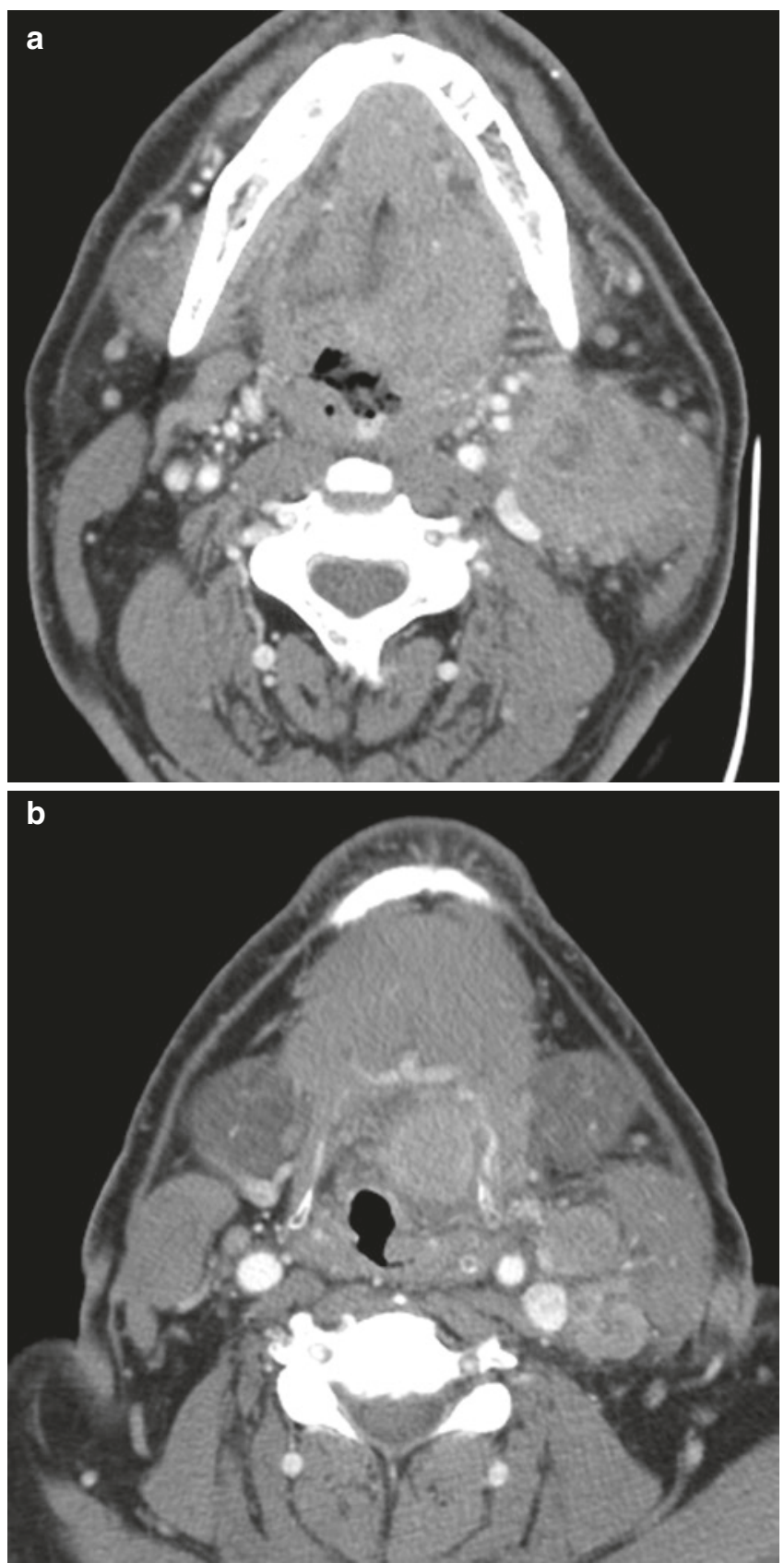

Fig. 17.1 A 51-year-old man with a 30-pack year smoking history presents with a left neck mass. The primary is a large infiltrating tumor at the left tongue base extending into the floor of mouth $(\mathbf{a}, \mathbf{b})$. There are multiple ipsilateral heterogeneous level 2 and 3 nodal masses with illdefined margins (b). As a p16+ SCC this was T4N1M0, stage III. If this had been p16- it would be classified as T4aN3bM0 and stage IVB 

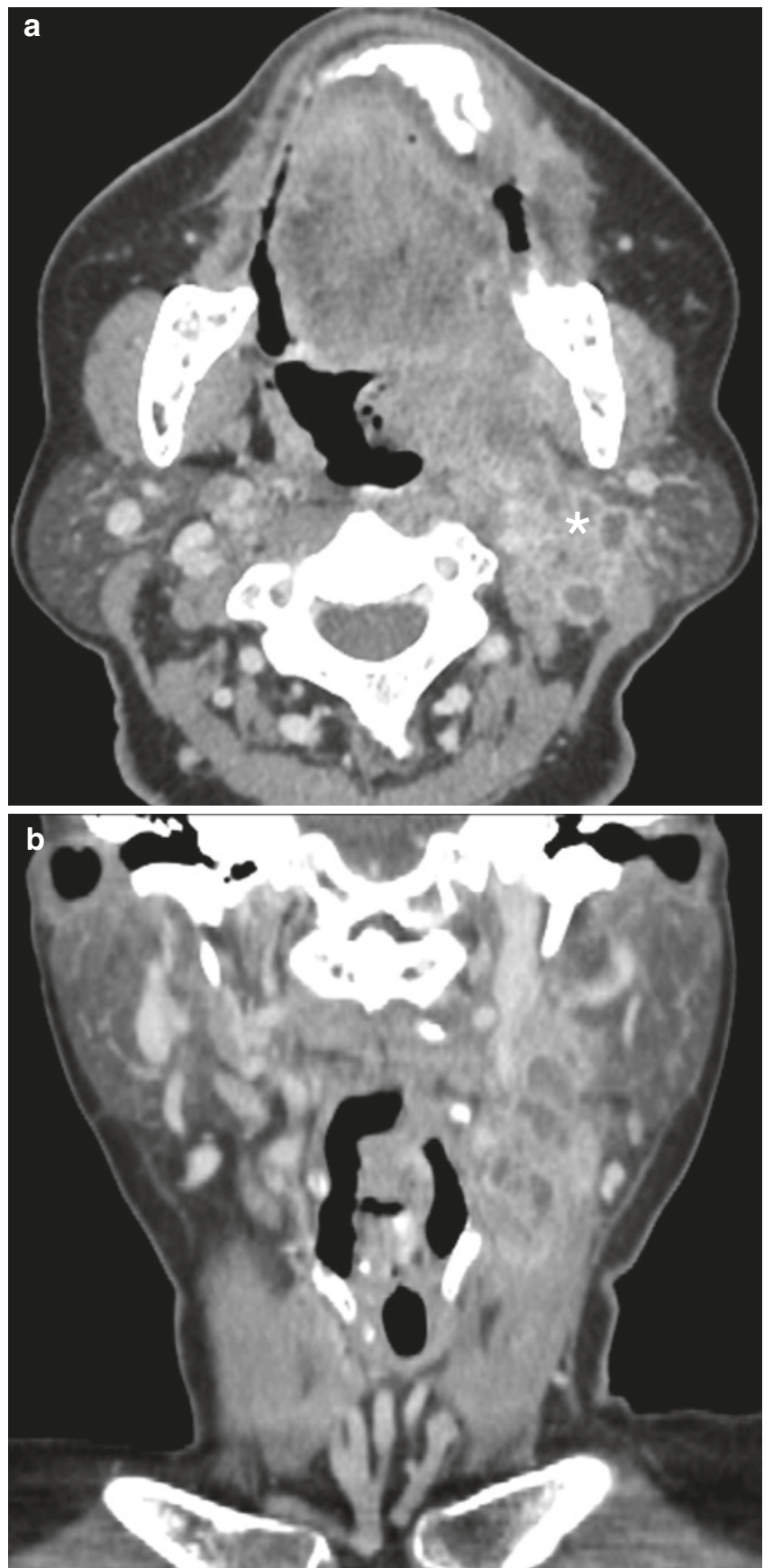

Fig. 17.2 Axial and coronal CECT in a 74-year-old woman presenting with a fixed left neck mass, representing clinical extranodal extension [cENE]. There is a large left tonsillar primary tumor and a nodal conglomerate at the left 2A nodal group (*). The nodal mass has ill-defined margins with infiltration of the adjacent sternocleidomastoid muscle and posterior triangle fat planes. Extranodal extension denotes N3b disease for all HN SCC except NPC and p16+/HPV-associated OPSCC where it is not part of the nodal criteria

pathologic tables for these tumors when neck dissections are performed. This has the potential for significant confusion for radiologists, particularly when the p16/HPV status is unknown for oropharyngeal tumors. In this situation, it is critical to care- fully evaluate the entire neck ipsilateral and contralateral to the primary site for any enlarged, heterogeneous, or frankly necrotic nodes and describe those that appear distinctly positive for tumor or which are highly suspicious. Extranodal extension of tumor (ENE) that is evident on clinical examination by the physician is now an important finding that upstages nodal disease to N3 for p16-/non-HPV and non-EBV lymph node SCC (Fig. 17.2). It has long been recognized that ENE is associated with a poorer prognosis and higher rate of tumor recurrence but it is only in this eighth edition that it has been included in the clinical and pathologic staging tables.

Evaluation for M1 disease (the presence of any metastasis) is best done with PET imaging although patients are always assumed to be M0 if no distant staging imaging/evaluation is performed. At the time of any staging neck scan, the lung apices and the bones should always be evaluated for metastases. Additionally, as many SCC are tobacco and alcohol-related, these patients carry an increased risk of a second primary neoplasm. Second primary tumors are most frequently found with hypopharyngeal SCC and 1/3 are synchronous with the initial SCC. Second primary tumors are most commonly another HN SCC or esophageal or pulmonary primary tumors.

\section{Key Points}

- When staging a new tumor start by locating the primary site and then reference the appropriate table in AJCC/UICC for critical points to include in the report.

- Tumors should always be measured by their longest diameter and then describe the deep extent and evaluate for perineural tumor.

- Evaluate the regional drainage nodes and always look for contralateral nodal metastases.

- Complete the neck report by evaluating for bone and lung metastases.

\subsection{Unknown Primary Tumors}

When a patient presents with a neck mass that is found to be nodal SCC, an initial clinical examination is performed in the ENT surgeon's office. If a primary site is not evident, this is considered to be an "unknown primary tumor" and imaging has an important role in trying to determine the origin so that biopsy can be directed. Radiation can also be targeted to the area of concern, thus reducing treatment morbidity. More than $90 \%$ of unknown primary SCC detected from FNA of a nodal mass in the absence of a clinically evident primary source, are due to $\mathrm{p} 16+/ \mathrm{HPV}$-associated oropharyngeal SCC [17]. It is thus critical to carefully evaluate the palatine and lingual tonsils which may harbor tumor in the depths of the tonsillar crypts where it may not be evident on clinical exam- 
ination. Thus subtle asymmetry of density/signal intensity or of size of the tonsil ipsilateral to a nodal mass suggests the most likely primary site and directs the surgeon to the preferred site for biopsy or tonsillectomy.

It is also important to be aware that nodal metastasis from a cutaneous SCC primary or from a pulmonary SCC can also be p16+ and this must also be considered in the differential when a nodal FNA reveals p16+ SCC. In situations such as these when there is no primary oropharyngeal tumor evident, the node should also be tested for high-risk HPV prior to assuming an occult oropharyngeal source. PET/CT has proven to be extremely useful for determination of the primary tumor site in the presence of an unknown primary nodal metastasis and is frequently the first-line imaging study when a patient presents without a clinical source evident.

If the nodal SCC is p16-, then other sites of origin should be considered including the nasopharynx and hence EBV testing should be performed. If this is still negative and no primary site is evident on cross-sectional CT, MR, or PET/ $\mathrm{CT}$, then no primary $\mathrm{T}$ designation is assigned.

In contrast to the situation where a new imaging study is ordered after FNA has proven that SCC is present, when a scan is ordered for a neck mass, any cystic or solid new mass in an adult should be assumed to be neoplastic until proven otherwise.

\subsection{Baseline and Surveillance Imaging of HN SCC}

Following surgery, radiation, and/or chemotherapy, a posttreatment baseline imaging study should be obtained and should be evaluated with knowledge of the primary site and
AJCC/UICC tumor stage, and the entire treatment plan. Baseline scans, particularly following reconstruction flaps or radiation can be extremely difficult to evaluate, but understanding the expected findings following such treatment is paramount to anticipate the sites and appearances of treatment change and therefore distinguish residual tumor and early recurrence. The baseline scan should show no evidence of residual primary mass or abnormal lymph nodes (Fig. 17.3). It is particularly important to carefully evaluate both the primary site and neck nodal levels when there has been an unexpectedly prolonged delay from treatment to baseline scanning due to complications from surgery or other treatment, or loss of the patient to follow-up.

There is no consensus on the timing of the baseline scan but in the USA it is typically obtained at 8-10 weeks following chemoradiation, while postsurgical scans are often obtained around 10-12 weeks. PET/CT should be delayed to around 12 weeks to minimize false-positive FDG uptake from post-treatment inflammatory changes. The baseline cross-sectional scan also serves as a "road map" of a deformed neck to increase sensitivity for early recurrent disease on the subsequent surveillance imaging. For this reason, it is ideal that the same cross-sectional modality chosen for baseline imaging, either CT or MR, is subsequently used for surveillance.

Radiation therapy has changed enormously over the last two decades with the increasing use of intensity modulated radiotherapy (IMRT) for head and neck cancers. IMRT maximizes dose to the tumor and minimizes radiation to unaffected tissues but requires accurate delineation of tumor margins. This is often best done with MR although some practices use PET/CT or CECT alone, and may also
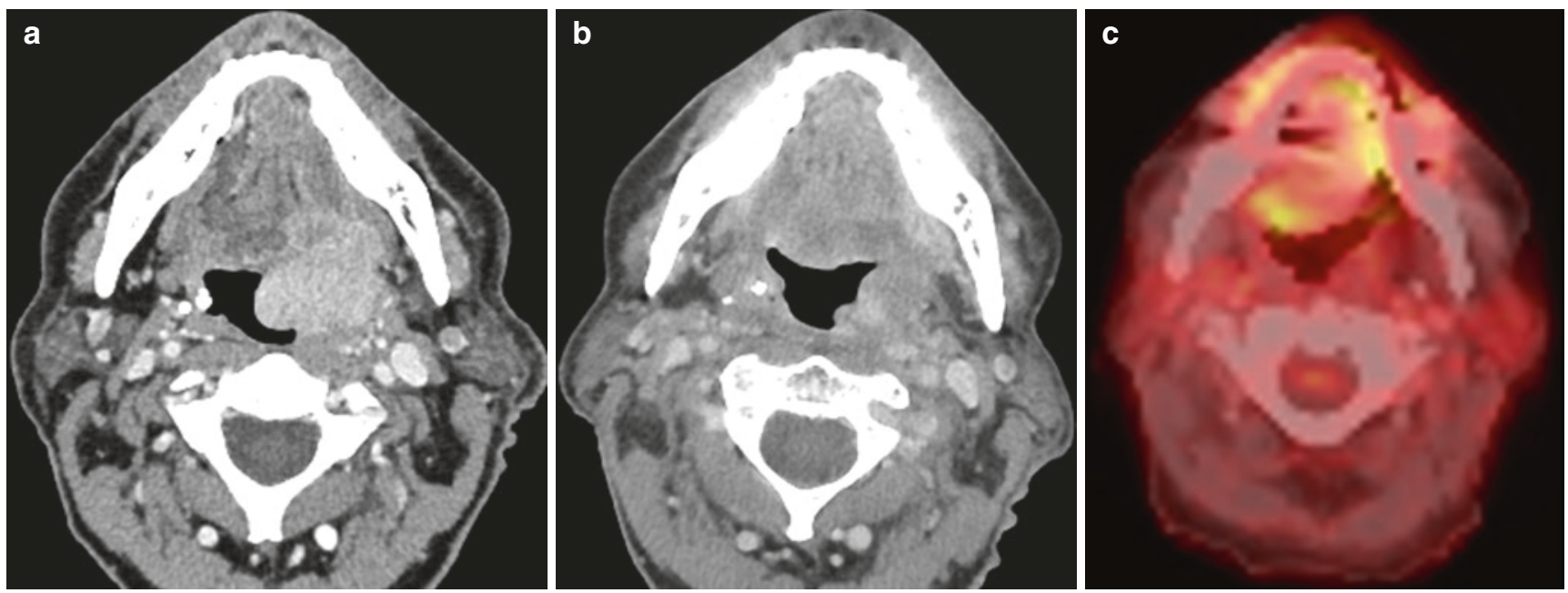

Fig. 17.3 A 66-year-old man with a large left palatine tonsillar mass staged as p16+ SCC cT4N0M0 stage III (a). The patient completed chemoradiation with baseline CT 8 weeks later (b) showing marked decrease in enhancement and decrease in bulk. This is not completely resolved as is hoped for on the baseline scan. Short interval follow-up PET/CT was obtained 4 weeks later (c) showing no significant uptake. The patient has remained disease free 
require greater input from radiologists to ensure accurate treatment volumes [18]. Radiation with or without chemotherapy results in significant changes in the appearance of neck soft tissues [19]. Radiation results in acute inflammation of all tissues in the radiation field with extensive edema. Over time this converts to fibrosis with atrophy and altered signal intensity and texture on MR. Both acute and chronic expected radiation changes can be confusing on $\mathrm{CT}$ or MR, requiring careful evaluation and comparison with prior studies to detect early recurrences.

Surgical resection of a primary tumor and/or nodal drainage regions also results in variable changes to normal neck contours. Familiarity with the types of nodal neck dissections and common flap reconstructions is necessary to evaluate for both complications and recurrence [20, 21]. It is extremely helpful to have knowledge of what surgical procedure was performed prior to evaluating such scans. Relatively simple resections, such as selective neck dissections, can be surprisingly subtle on imaging. Conversely, large resections with flap reconstructions can be quite complex, and imaging is hampered by metallic hardware. MR is less affected by hardware artifact and more sensitive for recurrent tumor, however, the muscular component of a flap reconstruction will undergo denervation change resulting in variable MR signal intensity and enhancement $[22,23]$. It is important on the baseline scan following neck reconstruction to ensure there is no residual or progressive tumor, and having knowledge of the pathology report and of positive tumor margins at the time of reconstruction can be very helpful in this regard.

Recurrent SCC most often occurs during the first 2 years following treatment. The frequency of surveillance imag- ing during this time is variable and may be performed in 3-6 month intervals, depending on the initial tumor staging and histologic prognostic features and the ongoing clinical course and physical findings. At any follow-up imaging examination, the possibility of a second primary tumor must again be considered. Remember on every follow-up study to look for residual, recurrent, and new tumors.

With NI-RADS, the timing for imaging after surgery or chemotherapy for HN cancer is a baseline CT or MRI at $8-12$ weeks with concurrent PET. If this is negative, neck imaging and chest $\mathrm{CT}$ is performed at 6 months, then further neck imaging at 6 months, and if this remains negative then neck and chest imaging at 8-12 months [24]. Any concerning but not definitive findings at any point in the series of follow-up imaging steps is classified as NIR-RADS 2 and leads to either shorter interval imaging (3 months), PET, or direct inspection. Such examples of NI-RADS 2 findings are focal superficial mucosal enhancement or focal mucosal FDG uptake, or discordance between the CT and PET (Fig. 17.4). The neck nodes also receive a NI-RADS score, with NI-RADS 2 most often leading to recommendation for PET imaging. Biopsy of the primary site or lymph nodes is recommended if there is strong concern for residual/ recurrent disease [NI-RADS 3] such as a new or enlarging, discrete nodule with robust enhancement or intense focal FDG uptake on PET. NI-RADS 4 is evidence of an already pathologically proven recurrence or definitive radiologic or clinical progression/recurrence that does not require biopsy. Using NI-RADS in your clinical practice not only allows ready communication with clinicians as to the degree of concern of an imaging finding for malignancy, but also generates data-mineable report. These will allow future optimization
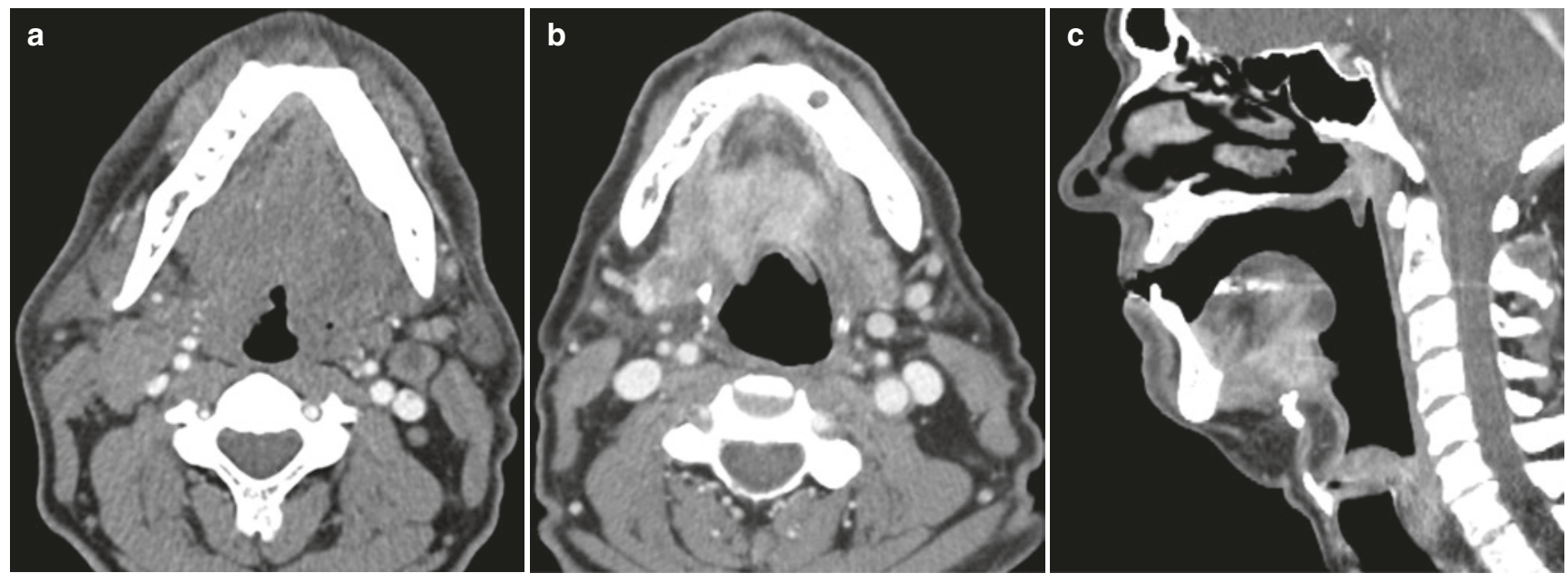

Fig. 17.4 A 59-year-old man diagnosed with p16+ base of tongue SCC T4N2M0 (a) after presenting with bilateral adenopathy and a poorly enhancing but infiltrating mass. The patient was treated with chemoradiation although did not receive all cycles of chemotherapy. Baseline imaging after completion of treatment $(\mathbf{b}, \mathbf{c})$ shows residual enhancing soft tissue mass in the floor of mouth but resolution of the adenopathy. This mass in the floor of the mouth is highly concerning for residual disease and should be biopsied. It is therefore a NI-RADS 3 . The resolved neck nodes are NI-RADS 1 
of imaging surveillance algorithms but also enable the user to determine their imaging accuracy and for practices to determine interobserver variability of reports.

\subsection{Concluding Remarks}

The radiologist has a valuable role in the management of $\mathrm{HN}$ SCC patients from initial staging or evaluation of a new neck mass, to post-treatment baseline imaging and surveillance. Post-treatment scans are often the most-complex examinations to interpret and so having a systematic process of performing the scans and reviewing the scans is important. It is essential for all post-treatment scans to have a history of the initial tumor type, the treatment performed, and the time interval of such. Further clarity can also be added to a report when there is additional knowledge of prior pathology reports, including surgical margins, and any clinical concerns for recurrent disease. The availability of much of this information via electronic medical records can greatly enhance the radiologist's sensitivity for subtle findings and confidence with approaching such cases.

\section{Take Home Messages}

- When reporting staging scans use the AJCC/UICC tables in order that the report includes critical criteria to enable the clinician to correctly stage the patient.

- HN post-treatment scans can be very difficult to read, but starting with a good history of the original tumor and the treatment plan set the stage for the best evaluation.

- Working with your clinicians to set a routine baseline and surveillance imaging plan and consistent imaging protocols is a good practice to standardize patient care and make radiologists most familiar with the expected appearance of treatment changes over time.

- Incorporating NI-RADS to the report can help your team gauge their accuracy and refine their reports.

- A new cystic or solid neck mass in an adult patient is cancer until proven otherwise.

\section{References}

1. Amin MB, Edge SB, Greene FL, et al., editors. AJCC cancer staging manual. 8th ed. New York: Springer; 2017.

2. National comprehensive cancer network guidelines. https://www. nccn.org/professionals/physician_gls/default.aspx\#site. Accessed 22 Sept 2019.

3. Aiken AH, Farley A, Baugnon KL, et al. Implementation of a novel surveillance template for head and neck cancer: neck imaging reporting and data system (NI-RADS). JACR. 2016;13:743-6.
4. Aiken AH, Rath TJ, Anzai Y, Branstetter BF, Hoang JK, Wiggins RH, Juliano AF, Glastonbury C, Phillips CD, Brown R, Hudgins PA. ACR neck imaging reporting and data systems (NI-RADS): a white paper of the ACR NI-RADS committee. J Am Coll Radiol. 2018;15(8):1097-108.

5. Krieger DA, Hudgins PA, Nayak GK, Baugnon KL, Corey AS, Patel MR, Beitler JJ, Saba NF, Liu Y, Aiken AH. Initial performance of NI-RADS to predict residual or recurrent head and neck squamous cell carcinoma. AJNR Am J Neuroradiol. 2017;38(6):1193-9.

6. Brierley JD, Gospodarowicz MK, Wittekind C, editors. TNM classification of malignant tumours. 8th ed. Hoboken: Wiley Blackwell; 2017.

7. Bhatia KS, King AD, Vlantis AC, Ahuja AT, Tse GM. Nasopharyngeal mucosa and adenoids: appearance at MR imaging. Radiology. 2012;263(2):437-43.

8. Schmalfuss IM, Mancuso AA, Tart RP. Postcricoid region and cervical esophagus: normal appearance at $\mathrm{CT}$ and MR imaging. Radiology. 2000;214(1):237-46.

9. Lewis JS Jr. Sinonasal squamous cell carcinoma: a review with emphasis on emerging histologic subtypes and the role of human papillomavirus. Head Neck Pathol. 2016;10(1):60-7.

10. Luce D, Leclerc A, Bégin D, Demers PA, Gérin M, Orlowski E, Kogevinas M, Belli S, Bugel I, Bolm-Audorff U, Brinton LA, Comba P, Hardell L, Hayes RB, Magnani C, Merler E, PrestonMartin S, Vaughan TL, Zheng W, Boffetta P. Sinonasal cancer and occupational exposures: a pooled analysis of 12 case-control studies. Cancer Causes Control. 2002;13(2):147-57.

11. Luce D, Gérin M, Leclerc A, Morcet JF, Brugère J, Goldberg M. Sinonasal cancer and occupational exposure to formaldehyde and other substances. Int J Cancer. 1993;53(2):224-31.

12. Glastonbury CM, Bhosale PR, Choyke PL, D’Orsi CJ, Erasmus JJ, Gill RR, Mukherji SK, Panicek DM, Schwartz LH, Subramaniam RM, Sullivan DC. Do radiologists have stage fright? Tumor staging and how we can add value to the care of patients with cancer. Radiology. 2016;278(1):11-2.

13. Glastonbury CM, Mukherji SK, O’Sullivan B, Lydiatt WM. Setting the stage for 2018: how the changes in the American joint committee on cancer/union for international cancer control cancer staging manual eighth edition impact radiologists. AJNR Am J Neuroradiol. 2017;38(12):2231-7.

14. Bakst RL, Glastonbury CM, Parvathaneni U, Katabi N, Hu KS, Yom SS. Perineural invasion and perineural tumor spread in head and neck cancer. Int J Radiat Oncol Biol Phys. 2019;103(5):1109-24.

15. O'Sullivan B, Huang SH, Su J, Garden AS, Sturgis EM, et al. Development and validation of a staging system for HPV-related oropharyngeal cancer by the international collaboration on oropharyngeal cancer network for staging (ICON-S): a multicentre cohort study. Lancet Oncol. 2016;17(4):440-51.

16. Haughey BH, Sinha P, Kallogjeri D, et al. Pathology-based staging for HPV positive squamous carcinoma of the oropharynx. Oral Oncol. 2016;62:11-9.

17. Motz K, Qualliotine JR, Rettig E, Richmon JD, Eisele DW, Fakhry C. Changes in unknown primary squamous cell carcinoma of the head and neck at initial presentation in the era of human papillomavirus. JAMA Otolaryngol Head Neck Surg. 2016;142(3):223-8.

18. Braunstein S, Glastonbury CM, Chen J, Quivey JM, Yom SS. Impact of neuroradiology-based peer review on head and neck radiotherapy target delineation. AJNR Am J Neuroradiol. 2017;38(1):146-53.

19. Glastonbury CM, Parker EE, Hoang JK. The postradiation neck: evaluating response to treatment and recognizing complications. AJR Am J Roentgenol. 2010;195(2):W164-71.

20. Hudgins PA, Kingdom TT, Weissler MC, Mukherji SK. Selective neck dissection: CT and MR imaging findings. AJNR Am J Neuroradiol. 2005;26(5):1174-7. 
21. Lobert P, Srinivasan A, Shah GV, Mukherji SK. Postoperative and postradiation changes on imaging. Otolaryngol Clin N Am. 2012;45(6):1405-22.

22. Syed F, Spector ME, Cornelius R, Srinivasan A. Head and neck reconstructive surgery: what the radiologist needs to know. Eur Radiol. 2016;26(10):3345-52.

23. McCarty JL, Corey AS, El-Deiry MW, Baddour HM, Cavazuti BM, Hudgins PA. Imaging of surgical free flaps in head and neck reconstruction. AJNR Am J Neuroradiol. 2019;40(1):5-13.

24. Aiken AH, Hudgins PA. Neck imaging reporting and data system. Magn Reson Imaging Clin N Am. 2018;26(1):51-62.

\section{Suggested Reading}

Chegini S, Mitsimponas K, Shakib K. A review of recent advances in histopathological assessment of head and neck squamous cell carcinoma. J Oral Pathol Med. 2019;00:1-5. https://doi.org/10.1111/ jop. 12943 .
Gurudutt VV, Genden EM. Cutaneous squamous cell carcinoma of the head and neck. J Skin Cancer. 2011;2011:502723.

Ouyang YH. Skin cancer of the head and neck. Semin Plast Surg. 2010;24(2):117-26.

Gandini S, Botteri E, Iodice S, Boniol M, Lowenfels AB, Maisonneuve P, Boyle P. Tobacco smoking and cancer: a meta-analysis. Int J Cancer. 2008;122(1):155-64.

Hashibe M, Boffetta P, Zaridze D, et al. Evidence for an important role of alcohol- and aldehyde-metabolizing genes in cancers of the upper aerodigestive tract. Cancer Epidemiol Biomark Prev. 2006;15(4):696-703.

Hashibe M, Brennan P, Benhamou S, et al. Alcohol drinking in never users of tobacco, cigarette smoking in never drinkers, and the risk of head and neck cancer: pooled analysis in the international head and neck Cancer epidemiology consortium. J Natl Cancer Inst. 2007;99(10):777-89.

National cancer institute facts and figures. https://seer.cancer.gov/ statfacts/html/oralcav.html. Accessed 22 Sept 2019.

Open Access This chapter is licensed under the terms of the Creative Commons Attribution 4.0 International License (http://creativecommons. org/licenses/by/4.0/), which permits use, sharing, adaptation, distribution and reproduction in any medium or format, as long as you give appropriate credit to the original author(s) and the source, provide a link to the Creative Commons license and indicate if changes were made.

The images or other third party material in this chapter are included in the chapter's Creative Commons license, unless indicated otherwise in a credit line to the material. If material is not included in the chapter's Creative Commons license and your intended use is not permitted by statutory regulation or exceeds the permitted use, you will need to obtain permission directly from the copyright holder. 


\section{Part V}

Spine, Spinal Cord, Plexus, and Nerves 


\title{
Low Back Pain
}

\author{
Luigi Manfrè and Johan Van Goethem
}

\section{Learning Objectives}

- To understand the very variable causes of low back pain.

- To appreciate the mostly benign clinical course of low back pain.

- To critically assess the critical relevance of imaging findings.

\section{Key Points}

- Section 18.1: Discogenic Low Back Pain (DLBP): The relationship between disc abnormalities and clinical symptoms is still debated.

- Section 18.2: Instability: Microinstability presents with a sensation of instability felt by the patient and objectively observed impaired muscle control.

- Section 18.3: Radicular Pain: Disc fragments may migrate behind the vertebral body and be overlooked in limited imaging protocols.

- Section 18.4: Sacroiliac Joint Disease: Chronic sacroiliac joint arthritis frequently is the cause of low back pain, particularly in women.

- Section 18.5: Failed Back Surgery Syndrome: Epidural fibrosis is one of the most common causes of low back pain after surgery.

- Section 18.6: Vertebrogenic Low Back Pain: Not even Modic 1 abnormalities represent an independent predictor of low back pain. They may be present in asymptomatic individuals.

\section{Manfrè $(\bowtie)$}

Unità Operativa di Interventistica Spinale Mini-Invasiva dell' Istituto Oncologico del Mediterraneo-IOM, Catania, Italy

J. Van Goethem

Department of Biomedical Sciences, Antwerp University Hospital,

Antwerp, Belgium
LBP is one of the most widespread and common diseases, some statistics report that about two-thirds of adults suffer from LBP at some point in their life, being second only to upper respiratory tract [1]. It is the greatest contributor to years lived in disability throughout much of the world and the first cause of everyday life activity limitation as well as absence from work [2]. The 2010 Global Burden of Disease Study considers LBP one of the ten diseases occurring worldwide [3], the prevalence of LBP is considered among $60-70 \%$ in European and US countries, with a recent increase even in younger population and a peak at 35-55 years of age [4-7]. The incidence of LBP has been documented in $50 \%$ of people having light physical activity and in more than $70 \%$ of those performing heavy activities and is the most frequent cause of cessation of activity in patients younger than 45 years old [8]. Moreover, this condition has deep relationship with other pathologies such as depression, anxiety, and sleep disturbances [9].

Only 7-8\% of patients with LBP have symptoms persisting over 2 weeks and just $1 \%$ need a real treatment: among them, symptoms usually improve rapidly in 1 month [8], 1/3 of them having — on the contrary - a persistent moderate to severe LBP after 1 year [10]. The etiology of chronic low back pain remains generally unknown or nonspecific (up to 85\%): there are several known causes of LBP syndrome (i.e., different from LBP) as age, psychosocial aspects (depression, stress, stop working), education (increasing LBP in low-educational status) [11], stress overload (heavy working or sport activities), smoking (the nicotine being a vascular degeneration agent reducing physiological disc nutrition), even genetic cause (74\% heritability in twins), and obesity (body mass index of more than $30 \mathrm{~kg} / \mathrm{m}^{2}$ ) [12]. Obesity in early adult age definitely increases the risk of nonspecific LBP as well as degenerative changes in lumbar spine [13].

Pure LBP conversely is generally related to pathologic degeneration of several structures involved in the spinal unit, as lumbar intervertebral disc, facet joints, muscular fascia, sacroiliac joints, ligaments, nerve root, and muscles directly [14]. Mechanical, traumatic, nutritional, and genetic factors all play a role in the cascade of spine degeneration. 
The role of imaging is to provide accurate morphologic information and influence therapeutic decision making. A necessary component, which connects these two purposes, is accurate natural history data. This is critical as, according to Rego et al. [15], up to $40 \%$ of patients exhibited no indications to imaging. Up to about $59 \%$ of lumbar spine MRI examinations requested among different social and economic settings have been deemed as inappropriate, depending on the evaluation method [16-19]. Of all lumbar and thoracic spine CT examinations in young patients, up to $77 \%$ were judged as inappropriate; therefore, such examinations are responsible for an unjustified cost for individuals or health systems and unnecessary radiation exposure [20].

Any study looking at the natural history of degenerative disc disease, prognostic value of imaging, or its effect on therapeutic decision making will be confounded by the high prevalence of morphologic change in the asymptomatic population [21-23]. Twenty to twenty-eight percent of asymptomatic patients demonstrate disc herniation and the majority have evidence of additional degenerative disc disease [21-23]. These findings are not only non-predictive in the moment, but prospectively as well. In a 7-year follow-up of a patient group with back pain [24], the original MR findings were not predictive of the development or duration of low back pain.

\subsection{Discogenic Low Back Pain (DLBP)}

Studies that demonstrate innervation to the intervertebral disc provide evidence that may account for instances of discogenic low back pain [25]. It was revealed that innervation of the inner disc was observed only in painful discs, not in normal control discs [26, 27]. Moreover, stimulation of peridiscal nerve plexus widely contributes to disc pain generation. Though discography has been used in the past as gold standard in proving the existence of a discogenic pain, today a simple intra-discal injection of sterile solution is considered also [28].

MR imaging findings that correlate with painful discs on discography are those typical for disc degeneration, mainly signal loss of the disc on T2-WI, but also loss of disc height, the presence of a hyperintensity zone (HIZ), and Modic changes [29].

The hyperintensity zone (HIZ) is a localized region of high signal intensity on T2-WI within the annulus fibrosus. Histopathologically, these lesions represent replacement of the normal lamellar structure by a disorganized, vascularized granulation tissue consisting of small round cells, fibroblasts, and newly formed blood vessels around tears that extend from the nucleus pulposus to the outer region of the annulus fibrosus [30]. Originally the presence of a HIZ was strongly correlated with a painful disc on discography [31]. This correlation has confirmed in multiple later studies, but was also questioned in a few other studies. In general, the association between an annular tear on MR images and low back pain is unclear.

\subsection{Spinal Instability}

Lumbar instability is one of the most common causes for chronic low back pain (LBP); in particular, it is the main cause of LBP in young patients, especially in athletes and overweight population, who are subjected to local extra stress.

The biomechanical alterations are related to the intervertebral disc degeneration that loses the physiological capacity to adsorb the applied load and to apply torsion resistance, with consequent change in the vector forces of the spinal unit, and dynamic overload of the posterior arches elements, especially of the zygapophyseal joints.

From a biomechanical point of view, the instability of a vertebral motion segment is defined as an abnormal reaction to applied loads with an increasing range of motion (ROM). The neutral zone (NZ), the displacement between the neutral position and the initiation point of spinal resistance to physiological motion, is considered to be a better indicator of lumbar instability than the ROM $[32,33]$.

The concept of vertebral instability has evolved in the last years, and the new concept of "microinstability" was recently introduced: Microinstability can be defined as a pure motion/ biomechanical dysfunctional syndrome with no or minimal anatomical changes, in absence of spondylolisthesis.

Microinstability lumbar syndromes present with a subjective sensation of instability felt by the patient and an objectively observed impaired muscle control. In the absence of an adequate control by segmental muscles inserting directly on the spine, patients tend to stabilize the dysfunctional MS through muscles which provide a compensatory global trunk stabilization for which high levels of intra-abdominal pressure are generated even during lowload tasks [34].

In flexion microinstability, the most frequent type, signs suggestive of altered movement control within the neutral zone are a good range of spinal mobility, but with a "painful arc" and the inability to return to erect posture from forward bending without assistance. Patients are unable to maintain semi-flexed postures. A loss of lumbar lordosis during standing can be observed at the level of the unstable MS along with a greater flexion during forward bending [35].

Kirkaldy-Willis originally described the cascade of degenerative instability, consisting of three phases: an initial dysfunction, an instability time, and a final restabilization [36]. Instability begins with a dysfunctional time, marked by intermittent nonspecific pain, associated with initial and slight morphological changes, followed by a stage of so-called unstable dysfunction with pathological alterations affecting the constitutive elements of the motor spinal unit. At this stage pain becomes more persistent [36, 37] or chronic. This first phase of the degeneration cascade in absence of spondylolisthesis in flexion-extension radiography can be considered as the equivalent of the clinical syndrome previously defined as microinstability. 
In the final phase of "restabilization," the fibrosis of the joint capsules, the formation of osteophytes, the marked disc collapse, and the radial expansive remodeling of vertebral bodies lead to an overall increase in stiffness along with hypomobility. During this stage functional limitation and stiffness prevail; it can appear as neurological deficits, while spinal pain can eventually fade or subside [36].

Unfortunately, there are no validated clinical signs for diagnosing degenerative instability, with a large overlap of motion patterns between symptomatic and normal individuals, which renders difficult to state any cut-off to distinguish normality and instability [38].

Kotilainen and Valtonen described the three criteria for instability:

Failure to full return from the bent position because of a sudden attack of low back pain (i.e., instability catch); inability to get a raised, straightened leg to move down and suddenly drops the leg due to a sharp pain in the low back (i.e., painful catch); and anxiety resulting from a sensation of collapse of the low back because of a sudden attack of back pain during movement (i.e., apprehension) [39].

Moreover, no exam available allows to detect an active segmental hypermobility, in conditions where a microinstability is suspected.

Lower back pain which originates from the facet joints has not been shown by studies to relate to radiological or pathological changes $[40,41]$. The value of plain radiographs, computed tomography, and magnetic resonance imaging scans in diagnosing facet joint disease remains inconclusive [42]; furthermore, they are not able to predict the patients who would respond to controlled diagnostic blocks of the facet joint so imaging studies have their greatest value in the exclusion of other pathological conditions.

Bone scintigraphy with single photon emission computed tomography (SPECT) is more sensitive in detecting facet joint lesions and allows more accurate anatomical localization. A recent study suggested that SPECT could help to identify patients with low back pain who would benefit from facet joint injections [43].

Facet joint block (FJB) is an indispensable diagnostic instrument in order to distinguish painful from painless facet joints, and to plan the intervention strategy.

The procedure is safe, valid, and reliable; the risk of FJB is very low and complications are rare, although there are case reports on infections [44-46].

Saal describes as the gold standard of diagnostic FJB the highly controlled (CT, MRI) FJB at the median nerve branch (MBB) [47]. The CT or MRI guidance is responsible for the highest specificity of the test [47].

The specificity of the FJB is however not high, with falsepositive rates ranging from $25 \%$ to $38 \%$ [48-50]. Standard blockade injections of the medial branches seem to anesthetize the joint and also the muscles, ligaments, and periosteum they innervate [49].

\subsection{Radicular Pain}

Acute lumbar disc herniation is the most common cause of acute radicular leg pain. After excluding emergent causes, such as cauda equina syndrome, epidural abscess, fracture, or malignancy, a six-week trial of conservative management is indicated [51]. Patients should be advised to stay active. If symptoms persist after 6 weeks, or if there is worsening neurologic function, imaging and invasive procedures may be considered. Most patients with lumbar disc herniation improve over 6 weeks.

If a disc herniation is identified that correlates with physical findings, surgical discectomy may improve symptoms more quickly than continued conservative management. Epidural steroid injections can also provide short-term relief [51].

Herniated discs are more easily detected with MRI than with CT for many reasons. Firstly, MR imaging allows visualization of the complete lumbar (or cervical or thoracic) spine in one examination. Secondly, sagittal images also depict the spinal canal in between intervertebral disc spaces. It is not unusual for a disc fragment to migrate (or extend) into the area behind the vertebral body. Some of these migrated discs can be missed on CT if axial slices are limited to the intervertebral disc spaces examined. Finally, the intrinsic tissue contrast is usually better on MR. Especially the lumbosacral region can be hard to assess on $\mathrm{CT}$ due to beam hardening, especially in larger patients.

Chronic radicular pain can be caused by a disc herniation, but also vertebral osteophytic spurs, degenerative facet spurs and facet hypertrophy, and degenerative foraminal stenosis are an important cause of nerve root irritation. Foraminal nerve root entrapment is best visualized on T1-weighted MRI where the high contrast between fat tissue and the nerve root sheath is of great help. Usually a combination of hypertrophic degenerative facets with osteophytic spurs posteriorly, and vertebral osteophytes and/or disc herniation anteriorly diminishes the anteroposterior diameter of the foramen. Foraminal height is lessened by degenerative disc disease and subsequent disc height loss. Whenever the normal rounded (oval) appearance of the nerve root sheath is lost in combination with loss of the surrounding fat tissue, nerve root compression should be considered.

\subsection{Sacroiliac Joint Disease}

One frequent (5-25\% of all LBP causes) [52-55] and frequently misunderstood cause of LBP in adult males, particularly females, is the chronic sacroiliac joint arthritis secondary to acquired focal instability. The sacroiliac joint (SIJ) is the strongest and richest joint in human body as for ligaments (anterior sacroiliac, interosseous, sacrospinous, and sacrotuberous) and muscles (gluteus maximus, piriformis, and biceps femoris), concurring to stabilize the joint [56]. Despite 
the great stability of this joint, there are several conditions that can reduce the stability of SIJ, generating chronic pain in a patient. SIJ ligaments are weaker in female patients as they are estrogen dependent, preparing the sacrum to the delivery nutation: as a consequence, postpartum chronic pain at the level of the sacrum can occur even after months after the delivery [57]. Another frequent cause of SI chronic pain is biomechanical changes in lumbar spinal unit mobility, secondary to surgical procedures like posterior interbody fixation (PIF)—generally performed in case of lumbar instability but conversely generating sacral instability too: it has been calculated that $75 \%$ of patients underwent PIF treatment suffer from painful SIJ instability in 5 years $[58,59]$. Clinical symptoms related to SIJ disease are numerous, and no specific clinical sign supports the diagnosis. This uncommon uselessness of clinical symptoms analysis is related to the complexity of SIJ innervation, as the posterior surface of the joint receives collaterals from L3 to S4 dorsal rami [60], while anterior articulation is supplied by L2 to S2 nerves [61, 62]. Consequently, SIJ syndrome is responsible for pain referred in several different areas of the body, as the lower limbs, pelvis, coxo-femoral area, buttock, and abdomen, overlaying other common causes of radicular pain. Even if several physical maneuvers are suggested to evocate the pain and propose a SIJ disease, diagnosis and neuroradiological examinations (MRI, bone scanning, SPECT-CT) can show focal SIJ abnormalities proposing a SIJ syndrome, only diagnostic block of the SIJ injecting intra-articular anesthetics (lidocaine) is considered the gold standard method to confirm the disease [63].

\subsection{Failed Back Surgery Syndrome}

Failed back surgery syndrome (FBSS) is one of the most frequent causes of back pain in patient underwent surgical treatment, the etiology being controversial and complex. Epidural fibrosis is generally considered one of the most common causes of LBP after surgery, with patients having extensive epidural scars having more frequent LBP and radicular pain that those with mild epidural fibrosis [64]. Moreover, epidural scars are responsible for neurological impairment demonstrated by electrophysiology and nerve root tethering as well as nerve inflammation induced by high local cytokines, and other inflammatory agent level has been described $[65,66]$. In FBSS, the pain moves from a mechanic/inflammatory origin through a neuropathic pain: patients affected by it suffer from an increased sensitivity and responsiveness of receptors that generate an amplified reaction to mild algogenous stimuli ("hyperalgesia") and/ or misinterpretation of stimuli coming from non-nociceptive receptors ("allodynia"), generally related to abnormal activation of non-neuronal cells as microglia, together with changes in local pain neurotransmitter molecules, increasing pain feeling [67].

\subsection{Vertebrogenic Low Back Pain}

Vertebral body endplates are a significant source of nonspecific chronic low back pain.

Ample histologic, anatomic, and immunohistochemical evidence supports nociceptive role of the basivertebral nerve (BVN) in the pathogenesis of LBP in patients with endplates damage and Modic changes [68, 69].

As described by Antonacci the BVN enters the posterior vertebral body via the basivertebral foramen and arborizes near the center of the vertebral body, sending branches to innervate the superior and inferior endplates. These nerve endings have been shown to proliferate in damaged and degenerated endplates and can be classified as pain fibers: they are PGP 9.5-positive and express Substance P and Trk-A receptors [70].

Many previous studies suggest that Modic type 1, that indicates edema and inflammation, is associated with LBP and has a stronger association with pain in comparison to Modic type 2, since pain decreases as Modic type 1 changes turns into Modic type 2. It is important to consider that Modic 1 is not an independent predictor of LBP, ad like other several degenerative changes have been shown to exist even in asymptomatic individuals [71-73].

Recent introduction of bone HDP SPECT/CT allows to distinguish incidental from clinically relevant findings, it is a hybrid imaging that allows the assessment of both morphology and bone physiology in a single study. It is possible to demonstrate the increased metabolic activity of the suspected MRI findings with high sensitivity: abnormalities become apparent early and sensitivity is higher than with computed tomography (CT) or standard radiography, allowing to treat patients suffering from Vertebrogenic pain with selective intraosseous BV ablation [74].

\subsection{Concluding Remarks}

In conclusion, low back pain remains an enigma with a poorly understood and variable pathogenesis, very often with a favorable outcome. Imaging findings quite often do not correlate with clinical symptoms, although there have been advances in understanding them better. Radiologists need to critically evaluate any findings in order to avoid overtreatment.

Take Home Message

- A small proportion of patients with acute low back pain have symptoms persisting over 2 weeks and just $1 \%$ required active treatment, and the etiology of chronic low back pain remains generally unknown or nonspecific. Therefore, to critically discuss imaging findings in relation to clinical relevance is crucial. 


\section{References}

1. Deyo RA, Weinstein JN. Low back pain. N Engl J Med. 2001;344:363-70.

2. Hoy D, March L, Brooks P, et al. The global burden of low back pain: estimates from the global burden of disease 2010 study. Ann Rheum Dis. 2014;73:968-74.

3. Mazroa A, Mohammad A. Years lived with disability (YLDs) for 1160 sequelae of 289 diseases and injuries 1990-2010: a systematic analysis for the Global Burden of Disease Study 2010. Lancet. 2012;380(9859):2163-96.

4. Jeffries LJ, Milanese SF, Grimmer-Somers KA. Epidemiology of adolescent spinal pain: a systematic overview of the research literature. Spine. 2007;32(23):2630-7.

5. Taimela S, Kujala UM, Salminen JJ, Viljanen T. The prevalence of low back pain among children and adolescents: a nationwide, cohortbased questionnaire survey in Finland. Spine. 1997;22:1132-6.

6. Balague F, Troussier B, Salminen JJ. Non-specific low back pain in children and adolescents: risk factors. Eur Spine J. 1999;8:429-38.

7. Andersson GBJ. The epidemiology of spinal disorders. In: Frymoyer JW, editor. The adult spine: principles and practice Philadelphia: Lippincott-Raven; 1997. p. 93-141.

8. Pengel LH, Herbert RD, Maher CG. Acute low back pain: systematic review of its prognosis. BMJ. 2003;327:323.

9. Stubbs B, Koyanagi A, Thompson T, et al. The epidemiology of back pain and its relationship with depression, psychosis, anxiety, sleep disturbances, and stress sensitivity: data from 43 low- and middle-income countries. Gen Hosp Psychiatry. 2016;43:63-70.

10. Von Korff M, Saunders K. The course of back pain in primary care. Spine. 1996;21:2833-7.

11. Hoy D, Brooks P, Blyth F. The epidemiology of low back pain. Best Pract Res Clin Rheumatol. 2010;24:769-81.

12. Webb R, Brammah T, Lunt M, et al. Prevalence and predictors of intense, chronic, and disabling neck and back pain in the UK general population. Spine. 2003;28(11):1195-202.

13. Frilander H, Solovieva S, Mutanen P, Pihlajamäki H, Heliövaara M, Viikari-Juntura E. Role of over-weight and obesity in low back disorders among men: a longitudinal study with a life course approach. BMJ Open. 2015;5(8):e007805.

14. Manchikanti L, Hirsch JA, et al. Comprehensive evidence-based guidelines for interventional techniques in the management of chronic spinal pain. Pain Physician. 2009;12:699-802.

15. Rego MH, Nagiah S. Over-imaging in uncomplicated low back pain: a 12-month audit of a general medical unit. Intern Med J. 2016;46:1437-9.

16. Jame SZ, Sari AA, Majdzadeh R, et al. The extent of inappropriate use of magnetic resonance imaging in low back pain and its contributory factors. Int J Prev Med. 2014;5:1029-36.

17. Gidwani R, Sinnott P, Avoundjian T, et al. Inappropriate ordering of lumbar spine magnetic resonance imaging: are providers choosing wisely? Am J Manag Care. 2016;22:e68-76.

18. Emery DJ, Shojania KG, Forster AJ, et al. Overuse of magnetic resonance imaging. JAMA Intern Med. 2013;173:823-5.

19. Avoundjian T, Gidwani R, Yao D, et al. Evaluating two measures of lumbar spine MRI overuse: administrative data versus chart review. J Am Coll Radiol. 2016;13:1057-66.

20. Oikarinen H, Meriläinen S, Pääkkö E, et al. Unjustified CT examinations in young patients. Eur Radiol. 2009;19:1161-5.

21. Wiesel SW, Tsourmas N, Feffer HL, Citrin CM, Patronas N. A study of computer-assisted tomography. I. The incidence of positive CAT scans in an asymptomatic group of patients. Spine. 1984;9:549-51.

22. Boden SD, Davis DO, Dina TS, Patronas NJ, Wiesel SW. Abnormal magnetic-resonance scans of the lumbar spine in asymptomatic subjects: a prospective investigation. J Bone Joint Surg Am. 1990;72:403-8
23. Jensen MC, Brant-Zawadzki MN, Obuchowski N, Modic MT, Malkasian D, Ross JS. Magnetic resonance imaging of the lumbar spine in people without back pain. N Engl J Med. 1994;331:69-73.

24. Borenstein DG, O'Mara JW Jr, Boden SD, et al. The value of magnetic resonance imaging of the lumbar spine to predict lowback pain in asymptomatic subjects: a 7-year follow-up study. J Bone Joint Surg Am. 2001;83-A:1306-11.

25. Troyanovich SJ, Harrison DD, Harrison DE. Low back pain and the lumbar intervertebral disk: clinical considerations for the doctor of chiropractic. J Manip Physiol Ther. 1999;22(2):96-104.

26. Coppes MH, Marani E, Thomeer RT, Oudega M, Groen GJ. Innervation of annulus fibrosus in low back pain. Lancet. 1990;336:189-90.

27. Freemont AJ, Peacock TE, Goupille P, Hoyland JA, O’Brien J, Jayson MIV. Nerve ingrowth into diseased intervertebral disc in chronic back pain. Lancet. 1997;350:178-81.

28. Manchikanti L, Hirsch JA. An update on the management of chronic lumbar discogenic pain. Pain Manag. 2015;5(5):373-86.

29. O'Neill C, Kurgansky M, Kaiser J, Lau W. Accuracy of MRI for diagnosis of discogenic pain. Pain Physician. 2008;11(3):311-26.

30. Peng B, Hou S, Wu W, Zhang C, Yang Y. The pathogenesis and clinical significance of a high-intensity zone (HIZ) of lumbar intervertebral disc on MR imaging in the patient with discogenic low back pain. Eur Spine J. 2006;15(5):583-7.

31. Aprill C, Bogduk N. High-intensity zone: a diagnostic sign of painful lumbar disc on magnetic resonance imaging. Br J Radiol. 1992;65:361-9.

32. Panjabi M. The stabilizing system of the spine. Part II. Neutral zone and instability hypothesis. J Spinal Disord. 1992;5(4):390-6. https://doi.org/10.1097/00002517-199212000-00002.

33. Panjabi M, Lydon C, Vasavada A, Grob D, Crisco JJ 3rd, Dvorak J. On the understanding of clinical instability. Spine. 1994;19(23):2642-50.

34. O'Sullivan BP. Lumbar segmental instability: clinical presentation and specific stabilizing exercise management. Man Ther. 2000;5:2-12.

35. Park AL. Instability: clinical manifestations and assessment. In: Slipman CW, Derby R, Simeone FA, Mayer TG, editors. Interventional spine an algorithmic approach. Philadelphia: Saunders; 2008. p. 1109-19.

36. Kirkaldy-Willis WH, Farfan HF. Instability of the lumbar spine. Clin Orthop Relat Res. 1982;165:110-23.

37. Jinkins JR. Acquired degenerative changes of the intervertebral segments at and suprajacent to the lumbosacral junction. A radioanatomic analysis of the nondiscal structures of the spinal column and perispinal soft tissues. Eur J Radiol. 2004;50(2):134-58.

38. Bogduk N. Clinical and radiological anatomy of the lumbar spine. 5th ed. Edinburgh: Churchill Livingstone Elsevier; 2012. p. 207-15.

39. Kotilainen E, et al. Clinical instability of the lumbar spine after microdiscectomy. Acta Neurochir. 1993;125:120-6.

40. Eisenstein SM, Parry CR. The lumbar facet arthrosis syndrome. J Bone Joint Surg. 1987;69B:3.

41. Schwarzer AC, Wang SC, O'Driscoll D, et al. The ability of computed tomography to identify a painful zygapophysial joint in patients with chronic low back pain. Spine. 1995;20:907-12.

42. Schutz U, Cakir B, Dreinhofer K, Richter M, Koepp H. Diagnostic value of lumbar facet joint injection: a prospective triple cross-over study. PLoS One. 2011;6(11):e27991. https://doi.org/10.1371/journal.pone.0027991.

43. Pneumaticos SG, Chatziioannou SN, Hipp JA, Moore WH, Esses SI. Low back pain: prediction of short-term outcome of facet joint injection with bone scintigraphy. Radiology. 2006;238:693-8.

44. Cook NJ, Hanrahan P, Song S. Paraspinal abscess following facet joint injection. Clin Rheumatol. 1999;18(1):52-3.

45. Alcock E, Regaard A, Browne J. Facet joint injection: a rare form cause of epidural abscess formation. Pain. 2003;103(1-2):209-10. 
46. Okazaki K, Sasaki K, Matsuda S, Yuge I, Omiya K, et al. Pyogenic arthritis of a lumbar facet joint. Am J Orthop. 2000;29(3):222-4.

47. Saal JS. General principles of diagnostic testing as related to painful lumbar spine disorders: a critical appraisal of current diagnostic techniques. Spine. 2002;27(22):2538-46.

48. Schwarzer AC, Aprill CN, Derby R, Fortin J, Kine G. The falsepositive rate of uncontrolled diagnostic blocks of the lumbar zygapophysial joints. Pain. 1994;58(2):195-200.

49. Murtagh R. The art and science of nerve root and facet blocks. Neuroimaging Clin N Am. 2000;10(3):465-77.

50. Van Kleef M, Barendse GA, Kessels A, Voets HM, Weber WE, et al. Randomized trial of radio frequency lumbar facet denervation for chronic low back pain. Spine. 1999;24:1937-42.

51. Gregory DS, Seto CK, Wortley GC, Shugart CM. Acute lumbar disk pain: navigating evaluation and treatment choices. Am Fam Physician. 2008;78(7):835-42.

52. Sembrano JN, Polly DW. How often is low back pain not coming from the back? Spine (Phila Pa 1976). 2009;34(1):E27-32.

53. Cohen SP. Sacroiliac joint pain: a comprehensive review of anatomy, diagnosis, and treatment, and treatment. Anesth Analg. 2005;10(5):1440-53.

54. Schwarzer AC, Aprill CN, Bogduk N. The sacroiliac joint in chronic low back pain. Spine. 1995;20:31-7.

55. Maigne JY, Aivaliklis A, Pfefer F. Results of sacroiliac joint double block and value of sacroiliac pain provocation tests in 54 patients with low back pain. Spine. 1996;21:1889-92.

56. Bernard TN, Cassidy JD. The sacroiliac syndrome. Pathophysiology, diagnosis and management. In: Frymoyer JW, editor. The adult spine: principles and practice. New York: Raven; 1991. p. 2107-30.

57. Laslett M. Evidence-based diagnosis and treatment of the painful sacroiliac joint. J Man Manip Ther. 2008;16(3):142-52.

58. Ivanov AA. Lumbar fusion leads to increases in angular motion and stress across sacroiliac joint: a finite element study. Spine. 2009;34(5):162-9.

59. Murata Y, Takahashi K, Yamagata M, et al. Sensory innervation of the sacroiliac joint in rats. Spine. 2000;25(16):2015-9.

60. Grob KR, Neuhuber WL, Kissling RO. Innervation of the sacroiliac joint in humans. Z Rheumatol. 1995;54:117-22.

61. Ikeda R. Innervation of the sacroiliac joint: macroscopic and histological studies. J Nippon Med Sch. 1991;58:587-96.

62. Fortin JD, Kissling RO, O’Connor BL, Vilensky JA. Sacroiliac joint innervations and pain. Am J Orthop. 1999;28:68-90.

63. Cohen SP, Pain SJ. A comprehensive review of anatomy, diagnosis, and treatment. Anesth Analg. 2005;101:1440-53.

64. Ross JS, Robertson JT, Frederickson RC, Petrie JL, Obuchowski N, Modic MT, de-Tribolet N. Association between peridural scar and recurrent radicular pain after lumbar discectomy: magnetic resonance evaluation. Neurosurgery. 1996;38:855-63.

65. Jou IM, Tai TW, Tsai CL, Tsai TM, Yung WS, Jung YC. Spinal somato sensory evoked potential to evaluate neurophysiologic changes associated with postlaminotomy fibrosis: an experimental study. Spine (Phila Pa 1976). 2007;32:2111-8.

66. Cooper RG, Freemont AJ, Hoyland JA, Jenkins JP, West CG, Illingworth KJ, Jayson MI. Herniated intervertebral disc associated periradicular fibrosis and vascular abnormalities occur without inflammatory cell infiltration. Spine (PhilaPa 1976). 1996;20:591-8.

67. Blond S, Mertens P, David R, Roulaud M, Rigoard P. From "mechanical" to "neuropathic" back pain concept in FBSS patients. A systematic review based on factors leading to the chronification of pain (part C). Neurochirurgie. 2015;61(Suppl 1):S45-56.

68. Fras C, Kravetz P, Mody DR, Heggeness MH. Substance P-containing nerves within the human vertebral body: an immunohistochemical study of the basivertebral nerve. Spine J. 2003;3(1):63-7.

69. Bailey JF, Liebenberg E, Degmetich S, Lotz JC. Innervation patterns of PGP 9.5-positive nerve fibers within the human lumbar vertebra. J Anat. 2011;218(3):263-70.

70. Antonacci MD, Mody DR, Heggeness MH. Innervation of the human vertebral body: a histologic study. J Spinal Disord. 1998;11(6):526-31.

71. Luoma K, Vehmas T, Grönblad M, et al. Relationship of Modic type 1 change with disc degeneration: a prospective MRI study. Skelet Radiol. 2009;38:237-44.

72. Luoma K, Vehmas T, Grönblad M, et al. MRI follow-up of subchondral signal abnormalities in a selected group of chronic low back pain patients. Eur Spine J. 2008;17:1300-8.

73. Kääpä E, Luoma K, Pitkäniemi J, Kerttula L, Grönblad M. Correlation of size and type of modic types 1 and 2 lesions with clinical symptoms: a descriptive study in a subgroup of patients with chronic low back pain on the basis of a university hospital patient sample. Spine. 2012;37(2):134-9. https://doi.org/10.1097/ BRS.0b013e3182188a90.

74. Russo VM, Dhawan RT, Dharmarajah N, Baudracco I, Lazzarino AI, Casey AT. Hybrid bone single photon emission computed tomography imaging in evaluation of chronic low back pain: correlation with modic changes and degenerative disc disease. World Neurosurg. 2017;104:816-23. https://doi.org/10.1016/j. wneu.2017.03.107.

Open Access This chapter is licensed under the terms of the Creative Commons Attribution 4.0 International License (http://creativecommons. org/licenses/by/4.0/), which permits use, sharing, adaptation, distribution and reproduction in any medium or format, as long as you give appropriate credit to the original author(s) and the source, provide a link to the Creative Commons license and indicate if changes were made.

The images or other third party material in this chapter are included in the chapter's Creative Commons license, unless indicated otherwise in a credit line to the material. If material is not included in the chapter's Creative Commons license and your intended use is not permitted by statutory regulation or exceeds the permitted use, you will need to obtain permission directly from the copyright holder. 


\title{
Spinal Trauma and Spinal Cord Injury (SCI)
}

\author{
Luc van Den Hauwe, Pia C. Sundgren, \\ and Adam E. Flanders
}

\section{Learning Objectives}

- To understand the prevalence and clinical consequences of spinal trauma.

- To appreciate the utility of radiography, computed tomography, and magnetic resonance imaging in the evaluation of spinal trauma and spinal cord injury.

- To comprehend the grading systems used in spinal trauma.

- To appreciate the soft tissue components of spinal trauma and how they differ in the pediatric population.

- To understand the imaging features of spinal cord injury and traumatic vascular injury.

\subsection{Imaging Modalities for Spinal Trauma}

In the emergency setting the appropriate selection of imaging for spinal trauma depends upon several factors such as, modality availability, the patient's clinical and neurological status, type of trauma (blunt, single, or multi-trauma), and other associated co-morbidities. Clinical factors to consider also include the quality and severity of pain, limitations in motion, or the presence of permanent or transient neurological deficits. MRI is reserved for those patients with post-traumatic myelopathy (spi-

L. van Den Hauwe

Department of Radiology, Antwerp University Hospital,

Antwerp, Belgium

\section{P. C. Sundgren}

Institution for Clinical Sciences/Radiology, Lund University,

Lund, Sweden

e-mail: Pia.sundgren@med.lu.se

A. E. Flanders $(\triangle)$

Department of Radiology, Thomas Jefferson University Hospital,

Philadelphia, PA, USA

e-mail: adam.flanders@jefferson.edu nal cord dysfunction) or in the instance whereupon a patient's symptoms that cannot be explained by findings on radiographs or CT, or when a reliable neurologic exam cannot be obtained.

\subsubsection{Plain Film Radiography}

In the rare circumstance where MDCT is not available, the initial imaging modality is radiography. A minimum of a lateral and anteroposterior view must be obtained for the spinal axis with the addition of an open-mouth odontoid view for the cervical spine. Often additional views such as oblique views and/or the swimmer's view are performed in an attempt to clear the cervicothoracic junction. With the exception of pediatric trauma, in most settings, radiography has been supplanted by MDCT.

\subsubsection{Computed Tomography (CT)}

Thin section multi-detector computed tomography (MDCT) is the preferred method when evaluating the cervical spine for bony injury after blunt trauma. The entire spinal axis can be reliably and expeditiously evaluated with automatic reformatting of the axial dataset into multiple planes allows for better and more exact diagnosis of bone- and soft tissue abnormalities [7-13]. Moreover in the instance of polytrauma, spine images can be reconstructed directly from chest, abdomen, and pelvis datasets with sensitivity that is equivalent to a dedicated spine CT study. This has the added benefit of minimizing radiation dose.

With the introduction of these new MDCT imaging techniques most trauma centers have set up dedicated acute (multi-) trauma protocol(s) which include CT of the brain, cervical spine, thorax, abdomen, and pelvis, with subsequent reformatting of images of the thoracic and lumbar spine. This both expedites the data acquisition for medically unstable patients and serves to minimize radiation dose since the body imaging data can be reconstructed offline into targeted spine reconstructions. 
CT has a higher sensitivity to fractures (especially involving the posterior elements) than radiography. This rapid cross-sectional imaging assessment of the spinal axis has been shown to be more efficient and safer by virtually eliminating the need for repeat radiographs and unnecessary patient transfers in the setting of an unstable spine. Moreover, the diagnostic quality of radiography varies considerably, is more time-consuming to acquire, and may be difficult to perform in a medically unstable patient. While MDCT excels at delineating bony injury, it also can detect many soft tissue abnormalities such as disc herniation, paravertebral soft tissue- and epidural hematoma. A highresolution CT imaging protocol begins with submillimeter overlapping partitions to create an isotropic dataset that yields identical spatial resolution in any reconstructed plane. Axial data can be reformatted into thicker sections for diagnostic display; with reformatted $1.25-2 \mathrm{~mm}$ thin slices in the $\mathrm{C} 1-\mathrm{C} 2$ region, 2-3 $\mathrm{mm}$ thin slices in the rest of the cervical spine, and 3-4 $\mathrm{mm}$ thin slices in the thoracic and lumbar spine are typically chosen for axial presentation. Reformatted sagittal and coronal images of the entire spine are produced from contiguous submillimeter $(0.3-0.75 \mathrm{~mm})$ axial images. Multiplanar reformatted (MPR) sagittal and coronal images of the entire spine are typically produced automatically from the scanning console or from a nearby workstation. Reconstructions are performed with both bone and soft tissue algorithms.

\subsubsection{Magnetic Resonance Imaging (MRI)}

The greatest impact that MRI has made in the evaluation of spinal trauma has been in assessment of the soft tissue component of injury. MRI is today considered the method of choice for assessing the spectrum of soft tissue injuries associated with spinal trauma. This includes damage to the intervertebral discs, ligaments, vascular structures, and spinal cord [14-16]. No other imaging modality has been able to faithfully reproduce the internal architecture of the spinal cord and it is this particular feature that is unique to MRI. Any patient who has a persistent neurologic deficit after spinal trauma should undergo an MRI in the acute period to exclude direct damage/compression to the spinal cord. MRI provides unequivocal evidence of not only spinal cord injury, but will also reliably demonstrate disc injuries/herniations, paraspinal soft tissue edema (ligament strain/failure), epidural hematomas, and vascular injury. In addition, MRI provides the most reliable assessment of chronic spinal cord injury and the imaging analogs of post-traumatic progressive myelopathy (PTPM) which is often manifested with imaging as syrinx formation, myelomalacia, and cord atrophy. The extent with which MRI is able to determine spinal instability is overstated as MRI is unable to provide a reliable assessment of ligamentous integrity in most cases. In fact, MRI falsely overestimates the soft tissue component of injury [17].

An acute spinal trauma MR imaging protocol of the cervical spine shall include $3 \mathrm{~mm}$ thick sagittal T1 (T1-weighted) and T2-weighted (T2W) and short tau inversion recovery (STIR) sequences and $3 \mathrm{~mm}$ thick axial T2*-weighted gradient echo (GRE) images without contrast. In the thoracic and lumbar spine, $4 \mathrm{~mm}$ thick sagittal T1-weighted, T2-weighted, and STIR sequences and axial $4 \mathrm{~mm}$ thick T1-weighted, T2-weighted, and T2*GRE images without contrast is recommended. 3D volumetric axial GRE or T2-weighted partitions at 1-2 $\mathrm{mm}$ thickness are useful in the cervical region. Fat-saturated T2-weighted images are valuable to evaluate for ligamentous and soft tissue injuries, and T2* GRE to evaluate for small hemorrhage or blood products in the spinal cord.

\section{Key Point}

- Radiography has largely been supplanted by MDCT except in the pediatric population for evaluation of bony injury.

\subsection{Different Grading Systems to Evaluate Spinal Injuries}

There are different classic grading scales for determining spinal instability of thoracolumbar injuries based upon the McAfee (two-column) and Denis three column concept [18, 19]. The Magerl classification relies exclusively on CT findings [20]. In recent years a new grading scale that is based on CT and magnetic resonance (MR) imaging findings, like the thoracolumbar injury classification and severity score (TLICS) has been developed by the Spine Trauma Group [21] to overcome some of the perceived difficulties regarding the use of other thoracolumbar spinal fracture classification systems for determining treatment. Also for the grading of the cervical spine a new grading scale and score system-the cervical spine Subaxial Injury Classification and Scoring (SLIC) system [22] - has been developed and is gaining acceptance among spine surgeons. The AO Spine classification system provides a comprehensive classification schema for upper cervical, subaxial cervical, thoracolumbar, and sacral injuries [23].

\subsubsection{Injuries to the Vertebral Column}

Classically, injuries to the spinal column are categorized by mechanism of injury and/or by instability. Instability is defined by White and Punjabi as abnormal translation between adjacent vertebral segments with normal physiologic motion. Unrecognized instability after trauma is a potential cause of delayed spinal cord injury. This is why early stabilization of the initial injury is an imperative to appropriate clinical management. The simplest method to test for instability in a controlled environment is by performing flexion and extension 
lateral radiography to produce a visible subluxation at a suspected level but this is rarely performed in practice.

From biomechanical point of view, the thoracolumbar spine can be divided into three osteo-ligamentous columns: anterior-, middle-, and posterior column [18]. The anterior column includes the anterior longitudinal ligament and anterior two-thirds of the vertebral body and disc including annulus fibrosus. The middle column is composed of the posterior third of the vertebral body and disc including annulus fibrosus, and posterior longitudinal ligament. Finally, the posterior column is composed of the pedicles, articular processes, facet capsules, laminae, ligamentum flavum, spinous processes, and the interspinous ligaments. The mechanism of injury will result in several different types of traumatic injuries to the cervical, thoracic, and lumbar vertebral column and spinal cord, which may result in stable or unstable spine injuries. Although this biomechanical model is often inferred for cervical injuries, there is no similar established model in the cervical spine.

Because of the distinct anatomic differences and the resultant injury patterns, injuries to the cervical spine are divided into subaxial injuries (cranial base to axis) and lower cervical injuries (C3-C7). The mechanism of injury to the cervical column can be divided into four major groups: hyperflexion, hyperextension, rotation, and vertical compression with frequent variations that include components of the major groups (e.g., flexion and rotation). Hyperflexion injuries include anterior subluxation, bilateral interfacetal dislocation, simple wedge fracture, fracture of the spinous process, teardrop fracture, and odontoid (dens) fracture. Of these the simple wedge fractures and isolated spinous process fractures are considered initially stable, while the other fractures are considered unstable such as the bilateral interfacetal dislocation and the teardrop fracture. The odontoid fracture can be considered stable or unstable depending on the type of fracture type. Hyperextension mechanism is less frequent than the hyperflexion and result in the following types of injuries: dislocation, avulsion fracture or fracture of the posterior arch of $\mathrm{C} 1$, teardrop fracture of $\mathrm{C} 2$, laminar fracture, and traumatic spondylolisthesis of C2 (Hangman's fracture). Most of these injuries with the exception of Hangman's fracture are defined as stable fractures; however, this does not imply that these injuries should go untreated. The hyperextension injuries are often associated with central cord syndrome especially in patients with pre-existing cervical spondylosis and usually produce diffuse pre-vertebral soft tissue swelling. Vertical compression results in the Jefferson fracture which involves atlas and is considered unstable or burst fractures. A common site for injuries is the craniocervical junction (CCJ) and the atlantoaxial joint, which is the most mobile portion of the spine as it predominantly relies on a complex ligamentous framework for stability. The imaging findings of important CCJ injuries, such as atlanto-occipital dissociation, occipital condyle fractures, atlas fractures with transverse ligament rupture, atlantoaxial distraction, and traumatic rotatory subluxation, are important to recognize in the acute setting as for the patient management.

Fractures in the lower thoracic and lumbar spine differ from those in the cervical spine. The thoracic and lumbar fractures are often complex and due to a combination of mechanisms. The thoracic cage confers substantial biomechanical protection to the thoracic spine. Therefore, statistically, most injuries occur at the most mobile portion or the thoracolumbar junction where the thoracic cage ends. When injuries occur in the upper or middle thoracic spine it is usually a result of major trauma, e.g., high velocity trauma such as motor vehicular accidents. The most common fracture, at the thoracolumbar junction, is the simple compression- or wedge fracture (50\% of all fractures) which is considered stable. The remaining types of fractures among those the so-called seat belt injury, which can be divided into three subtypes: type I (Chance fracture) involves the posterior bony elements, type II (Smith fracture) involves the posterior ligaments, and, in type III the annulus fibrosus is ruptured allowing for subluxation are considered unstable fractures [24]. With the advent of the three-point restraint system in motor vehicles, these severe hyperflexion-distraction injuries have become uncommon. The most common of all thoracolumbar fractures-the burst fractures account for $64-81 \%$ of all thoracolumbar fractures. The burst fracture, which can be divided into five subtypes, is associated with high incidence of injuries to the spinal cord, conus medullaris, cauda equina, and nerve roots [25]. It is important to remember that a burst fracture involving anterior and middle column can be misdiagnosed as mere compression fracture on plain films and, therefore may be misinterpreted as a simple compression or mild wedge fracture that involves only anterior column. CT has improved characterization of these injuries.

\section{Key Point}

- Different classification schemas are utilized to describe injury patterns based upon mechanism, anatomic and biomechanical differences in unique areas of the spine.

\subsection{Soft Tissue Injuries}

\subsubsection{Traumatic Disc Herniation and Ligamentous Injury}

Traumatic disc injuries are caused by distraction and shearing with failure of the intervertebral disc. A direct injury to the disc is more common than post-traumatic disc extrusion. 
Traumatic disc herniation should be considered when the disc exhibits high signal on T2-weighted images especially when traumatic vertebral body fractures and/or ligamentous injury is present at the same level [13]. Extruded disc material may extend into the epidural or pre-vertebral space. When there is a gap between parts of the vertebrae or by increased signal in the ligament or adjacent structures on fat suppressed T2-weighted or STIR images, a ligamentous injury is suspected. Up to $25 \%$ of all cervical injuries will demonstrate signal changes in the posterior ligamentous complex. This finding does not equate with instability.

Ligamentous injury without underlying fracture in the cervical spine is rare [26]. Disruption of the anterior longitudinal ligament is associated with hyperextension mechanisms with associated injury to the pre-vertebral muscles and intervertebral discs and can be identified as interruption of the normal linear band of hypointense signal of the ligament on T1-weighted images. Hyperflexion and distraction forces may cause disruption of the posterior ligament complex which is manifested by increased distance between spinous processes on lateral radiography and increased signal in the interspinous region on MR sagittal STIR sequences. Abnormal angulation, distraction, and subluxation are often recognized on initial CT study.

\subsubsection{Whiplash-Associated Disorders}

Whiplash injuries represent a separate, relatively common entity (1-4/1000), resulting from an acceleration-deceleration mechanism of injury to the neck, typically from rear-end vehicle collisions. Whiplash injury is among the leading automotive related injuries with respect to burden on patients, the healthcare system and insurance organizations [27]. The pathogenesis of whiplash complaints is still poorly understood. Injury to longitudinal ligaments, facet joints, intervertebral discs, spinal cord, and muscles has been described as possible sources of (chronic) pain. More recently, with the development of more detailed MR imaging techniques, morphologic changes of the ligaments and membranes of the craniovertebral junction, especially the alar and transverse ligaments have been described [28].

Whiplash-associated disorders (WAD) is a clinical diagnosis and describes a variety of clinical manifestations, such as neck pain immediately or $24 \mathrm{~h}$ after trauma, neck stiffness, headache, dizziness, vertigo, auditory and visual disturbances, concentration, and psychological problems. Imaging findings include osseous injuries such as bone contusions and occult fractures, ligamentous injuries (most common finding), and tears. Disc lesions and post-traumatic herniation also can occur [29]. MRI signal changes of the alar and transverse ligaments may be observed. Whether these signal abnormalities are responsible for complaints of patients having WAD remains controversial, as these signal abnormalities have also been observed in asymptomatic individuals and were not significantly associated with clinical testing and prognosis of acute whiplash injury. A recent meta-analysis could not show any association between MRI signal changes in alar and transverse ligaments and WAD [28].

\section{Key Point}

- MRI has unparalleled sensitivity to the gamut of soft tissue injuries associated with spinal trauma; however, signal changes on T2-weighted or STIR MR in the soft tissues due not equate with spinal instability.

\subsection{Injuries to the Spinal Cord}

A majority (80\%) of patients with spinal cord injury (SCI) harbor multisystem injuries [30], typically associated injuries include other bone fractures $(29.3 \%)$ and brain injury $(11.5 \%)$ [31]. Nearly all SCI damage both upper and lower motor neurons because they involve both the gray matter and descending white matter tracts at the level of injury. The American Spinal Injury Association (ASIA) has suggested a comprehensive set of standardized clinical measurements which are based upon a detailed sensory and motor examination of all dermatomes and myotomes [32]. The neurologic deficit that results from injury to the spinal cord depends primarily upon the extent of damage at the injury site and the cranial-caudal location of the damage (i.e., the neurologic level of injury or NLI); anatomically higher injuries produce a greater neurologic deficit (e.g., cervical injury = quadriparesis, thoracic injury = paraparesis). These comprehensive set of standardized clinical measurements have been adopted worldwide. Functional transection of the spinal cord is a more frequent manifestation of SCI compared to true mechanical transection which is relatively rare and confined mostly to penetrating type injuries or extensive fracture-dislocations/translocations. SCI is further categorized clinically into anterior cord syndrome, Brown-Sequard syndrome, central cord syndrome, conus medullaris syndrome, and cauda equina syndrome depending upon the site of injury and the neurologic pattern of injury. Spontaneous neurologic recovery after spinal cord injury overall is relatively poor and largely depends upon the severity of neurologic deficit identified at the time of injury. Of the different spinal cord syndromes the anterior cord syndrome has the worst prognosis of all cord syndromes, especially, if no recovery is noticed during the first $72 \mathrm{~h}$ after injury.

\subsubsection{Spinal Cord Hemorrhage}

Post-traumatic spinal cord hemorrhage or hemorrhagic contusion is defined as the presence of a discrete area of hem- 
orrhage within the spinal cord after an injury. The most common location for hemorrhage to accumulate is within the central gray matter of the spinal cord, and centered at the point of mechanical impact [33-35]. Experimental and autopsy pathologic studies have shown that the underlying lesion most often will be hemorrhagic necrosis of the spinal cord while true hematomyelia will rarely be found [36]. There are significant clinical implications if there is identification of frank hemorrhage in the cervical spinal cord following trauma on an MRI examination. Originally it was thought that detection of intramedullary hemorrhage was predictive of a complete injury. However, the increased sensitivity and spatial resolution of current MRI techniques has shown that even small amounts of hemorrhage are identifiable in incomplete lesions. Therefore, the basic construct has been altered such that the detection of a sizable focus of blood $(>4 \mathrm{~mm}$ in length on sagittal images) in the cervical spinal cord is often indicative of a complete neurological injury [37]. The anatomic location of the hemorrhage closely corresponds to the neurological level of injury and the presence of frank hemorrhage implies a poor potential for neurological recovery (Fig. 19.1) [33-35, 38-42].

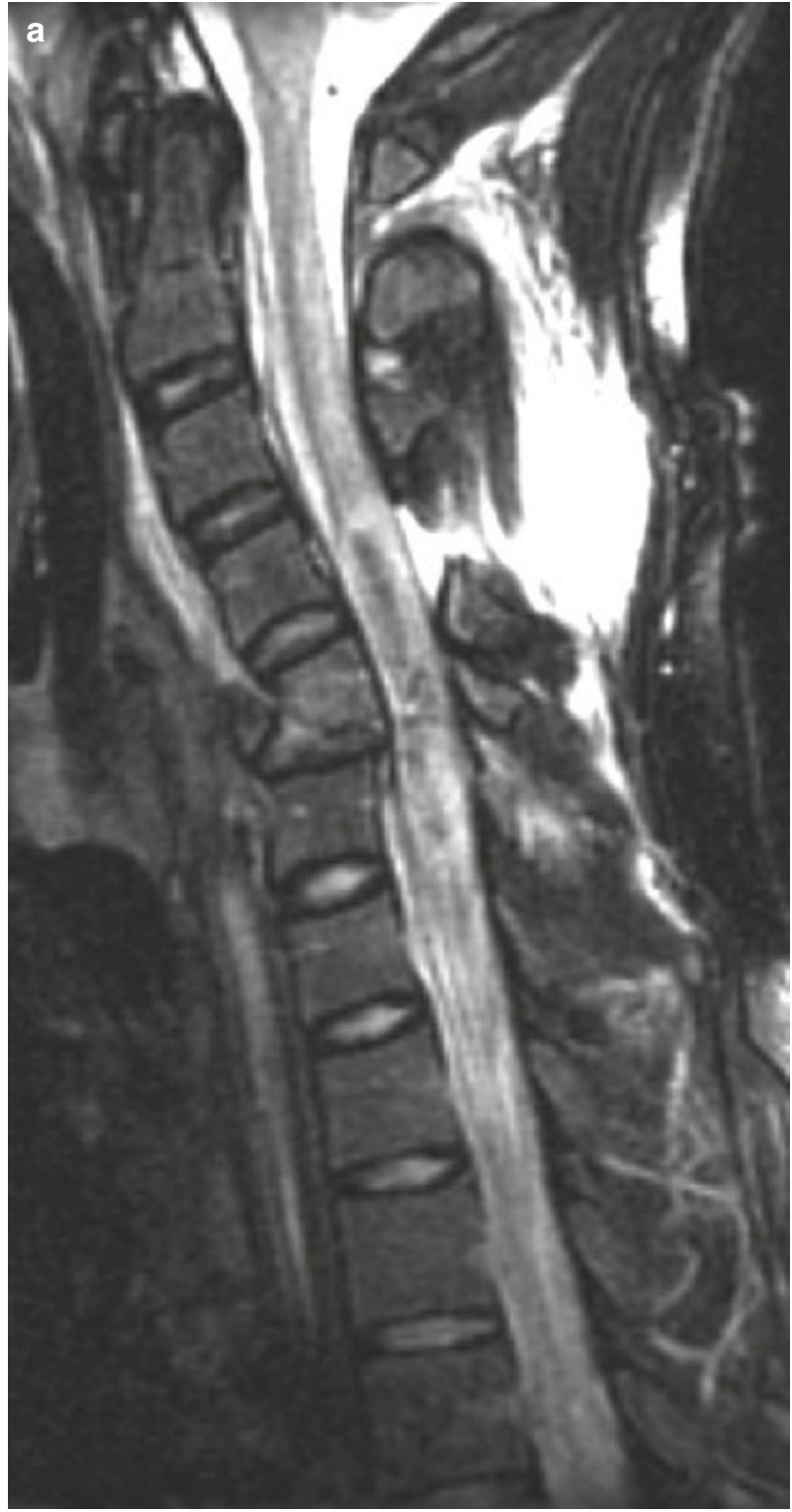

Fig. 19.1 Acute hemorrhagic spinal cord injury. (a) Sagittal STIR sequence shows that there is a flexion type injury of C5 with acute ventral angulation. The spinal cord is markedly swollen with edema spanning the entire length of the spinal cord. There is a central hemorrhagic

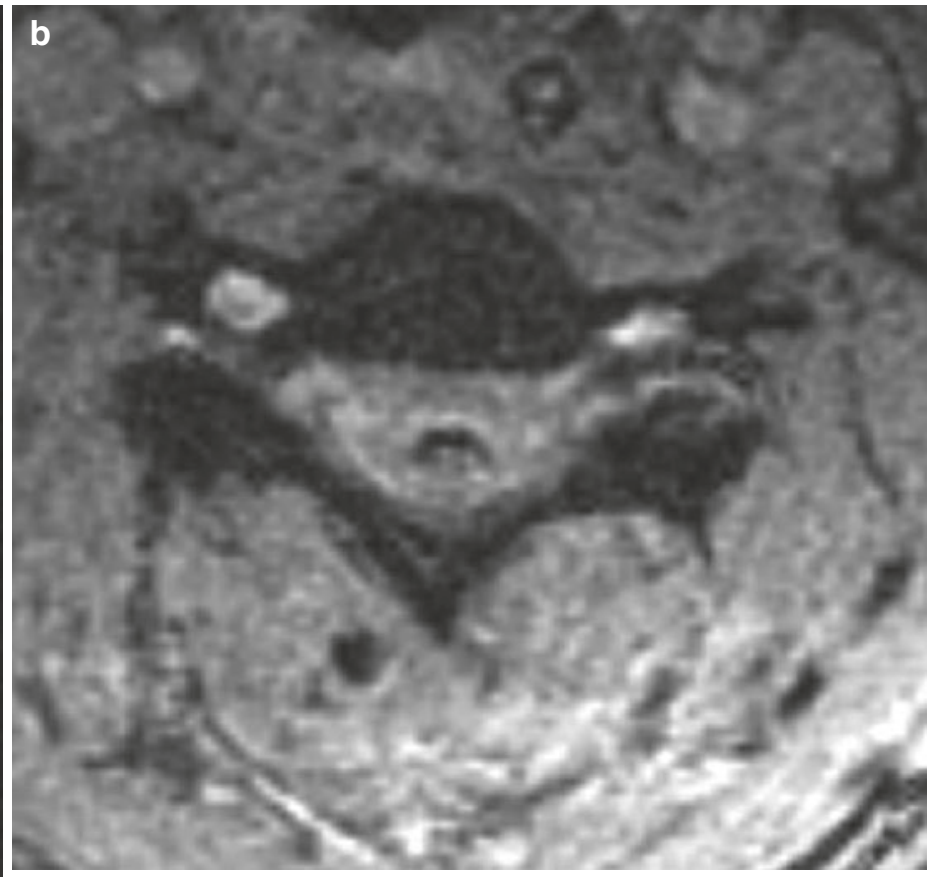

focus which is of low signal intensity that spans from C4 to C6. Note the disruption of the posterior spinal soft tissues. (b) Axial GRE image at the $\mathrm{C} 4$ level shows that the hypointense hemorrhage is confined to the central gray matter 


\subsubsection{Spinal Cord Edema}

Spinal cord edema is defined as a focus of abnormal high signal intensity seen on MRI T2-weighted images [35]. Presumably, this signal abnormality reflects a focal accumulation of intracellular and interstitial fluid in response to injury $[34,35,43,44]$. Edema is usually well defined on the mid-sagittal T2-weighted image while the axial T2-weighted images offer additional information in regard to involvement of structures in cross-section. Spinal cord edema involves a variable length of spinal cord above and below the level of injury, with discrete boundaries adjacent to uninvolved parenchyma and is invariably associated with some degree of spinal cord swelling. The length of spinal cord affected by edema is directly proportional to the degree of initial neurologic deficit [33, 42]. Notable is that spinal cord edema can occur without MRI evidence of intramedullary hemorrhage. Cord edema alone connotes a more favorable prognosis than cord hemorrhage [38].

\section{Key Point}

- The combination of intramedullary hemorrhage and/ or edema correlates with neurologic function and capacity to recover after spinal cord injury (SCI).

\subsection{Blunt Cerebrovascular Injury}

Blunt cerebrovascular injury (BCVI) to the carotid arteries (CAs) and/or vertebral arteries (VAs) is a relatively rare but potentially devastating finding in patients with a high-impact trauma to the cervical spine and/or head. Most complications of BCVI occur hours to days after the initial trauma so early identification and prompt anticoagulation is important to reduce the incidence of post-injury ischemic stroke (Fig. 19.2).

Cervical spine fractures have the strongest association with BCVI, and specific cervical spine fractures are highly predictive of BCVI. These include fractures located at the upper cervical spine $(\mathrm{C} 1-\mathrm{C} 3)$, subluxation, or involvement of the transverse foramina [45]. In the context of a carotid artery injury, it is thought that cervical hyperextension and contralateral rotation lead to stretching of the internal carotid artery (ICA) over the $\mathrm{C} 1-\mathrm{C} 3$ transverse processes, precipitating a vessel wall injury [46]. Nearly all VAIs are associated with cervical spine subluxations and fractures involving the transverse foramen [47]. Other mechanisms of BCVI include hyperflexion, a direct blow, and strangulation.

According to the Denver criteria and Western Trauma Association (WTA) guidelines, radiologic risk factors associated with BCVI include high-energy injury mechanisms with Le Fort II or III fracture patterns; basilar skull fracture with carotid canal involvement; cervical vertebral body or transverse foramen fracture, subluxation, or ligamentous injury; any fracture at $\mathrm{C} 1-\mathrm{C} 3$; closed head injuries with diffuse axonal injury and a Glasgow Coma Score of $<6$; clothesline-type injuries with associated swelling and/ or pain; or near-hanging with anoxia. Urgent screening for BCVI should be performed in these patients that may be still neurologically asymptomatic at this time [46].

With the widespread use of multi-detector CT scanners, CT angiography (CTA) has emerged as the first-line screening modality for BCVI. CTA is a cost-effective screening tool in high-risk populations, ultimately preventing the most strokes at a reasonable cost [46].

Several grading systems exist, based on imaging findings. The Denver grading scale (aka the Biffl scale) is a widely accepted and commonly used system. In grade I injury, subtle vessel wall irregularities, a dissection/intramural hematoma with $<25 \%$ luminal stenosis is observed. In grade II injury, an intraluminal thrombus or raised intimal flap is visualized, or a dissection/intramural hematoma with $>25 \%$ luminal narrowing. Grade III injury corresponds to traumatic pseudoaneurysms, observed as a variable-sized outpouching of the vessel wall. Grade IV injury represents a complete vessel occlusion, usually tapering in the CA and quite abrupt in the VA. Vessel transection represents a grade $\mathrm{V}$ injury, observed as free contrast extravasation into the surrounding tissues, or into the adjacent vein in the form of an arteriovenous fistula (AVF) [46, 47].

\section{Key Point}

- Occult vertebral artery injury (dissection or occlusion) can occur in association with spinal trauma even without the presence of spinal cord injury or fracture.

\subsection{Injuries to the Pediatric Spine and Spinal Cord}

Spinal injuries are generally less common in the pediatric population compared to adults with cervical spine injuries being most frequent spine injury of all spine injuries occurring in up to $40-60 \%$ of all injuries in children. The etiology varies depending on the age of the child. The most common cause of pediatric cervical spine injury is a motor vehicle accident, but also obstetric complication, fall, and child abuse are known causes. In the adolescent, sports and diving accidents are other well-known causes. The specific biomechanics of the pediatric cervical spine leads to a different distribution of injuries and distinct radiological features and represent a distinct clinical entity compared to those seen in adults. Young children have a propensity for injuries to the CCJ, upper cervical injuries (i.e., cranial base to $\mathrm{C} 2$ ) whereas older children are prone to lower cervical injuries 


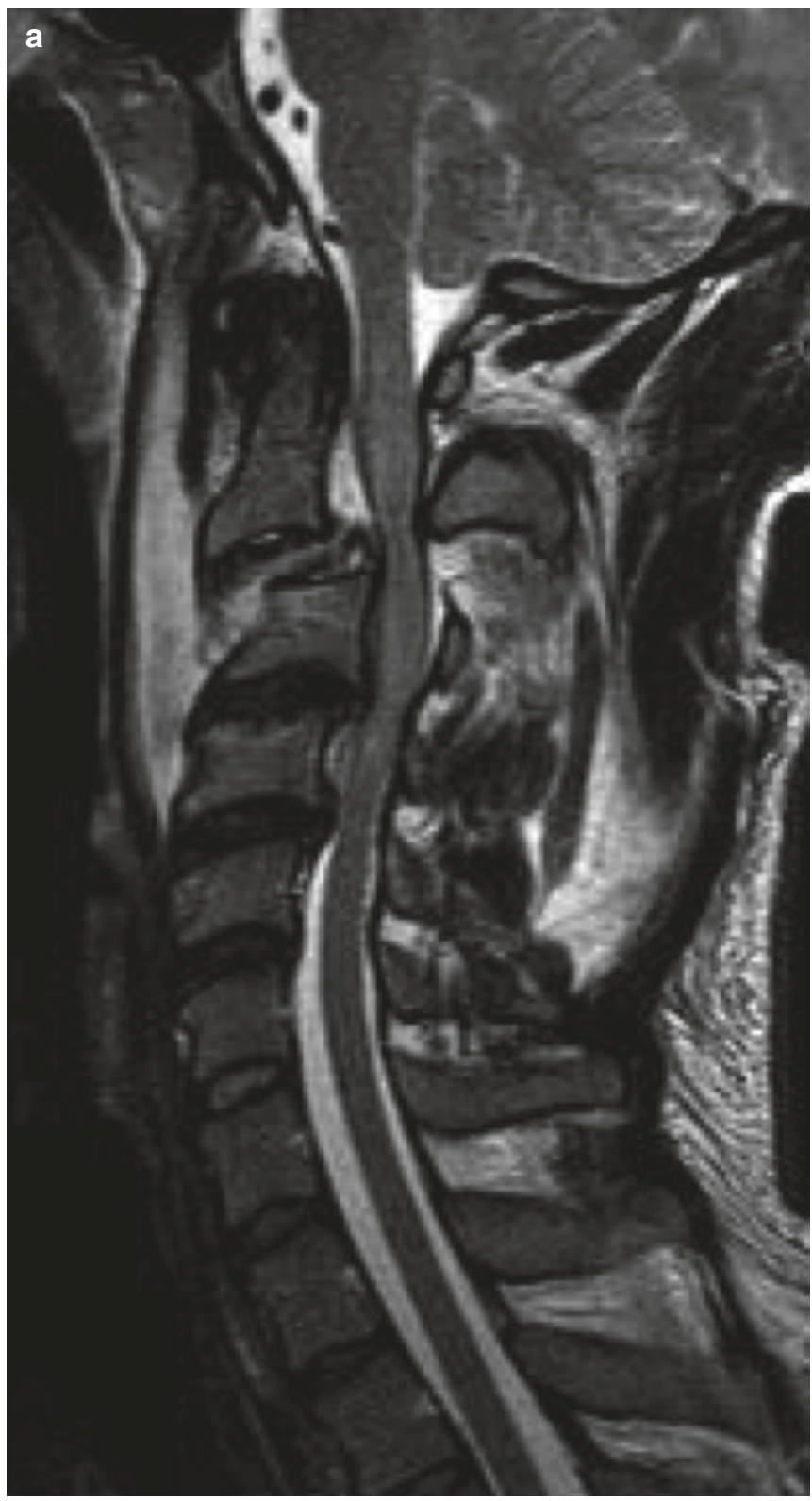

Fig. 19.2 Traumatic thrombosis/dissection of the bilateral vertebral arteries. (a) Sagittal T2-weighted image in a 32 y/o male after a skateboarding accident. Traumatic subluxation of $\mathrm{C} 2-3$ with circumferential disruption of the disco-ligamentous complex and spinal cord compres-

similar to those seen in adults. The spinal cervical injuries in children $<8$ years of age demonstrate a high incidence of subluxation without fractures. The biomechanical differences are explained by the relative ratio of the size of the cranium to the body in the young child, lack of ligamentous stability, poor muscle strength, and increased forces relative to the older child and adult. Children are also more prone to spinal cord injury with otherwise normal radiographs so called SCIWORA (spinal cord injury without radiographic abnormality) compared to adults. This is especially evident in children younger than 9 years of age where there is a high incidence of reported complete cord injuries associated

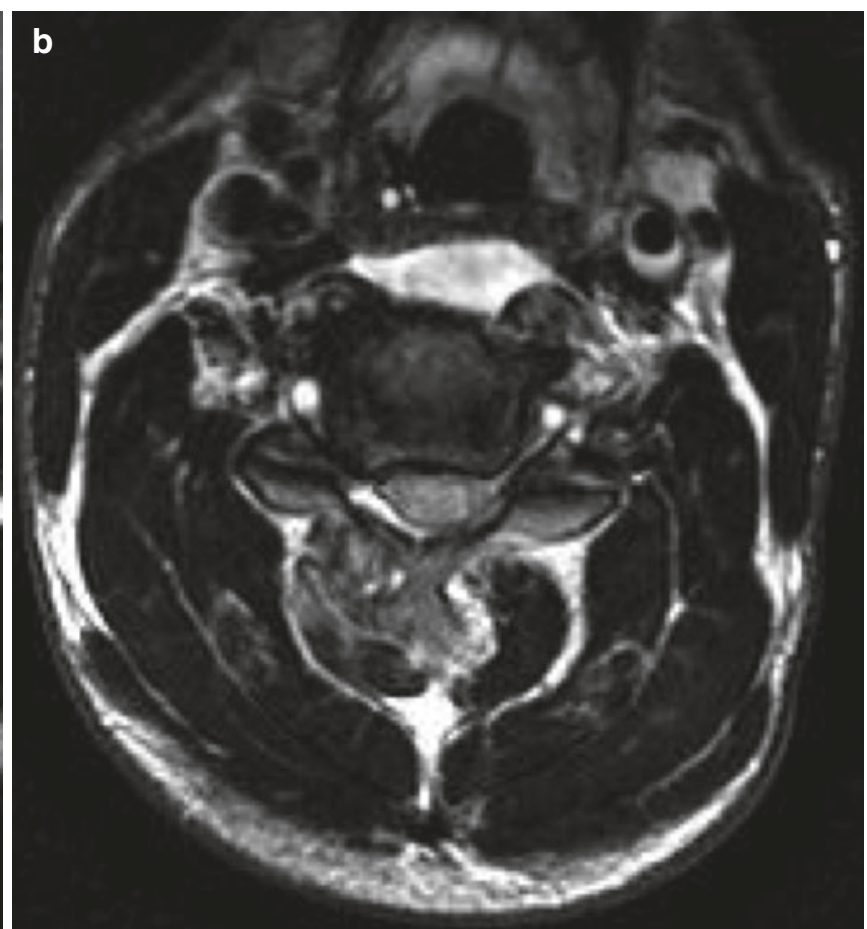

sion/edema. (b) Axial T2-weighted image at the $\mathrm{C} 2-3$ level show high signal in both foramen transversarium from clot/slow flow in both vertebral arteries

with SCIWORA. Suggested mechanisms of the SCIWORA include hyperextension or flexion injuries to the immature and the inherently elastic spine, which is vulnerable to external forces and allows for significant intersegmental movement and transient soft disc protrusion, resulting in distraction injuries, and/or ischemic injury of the spinal cord [48]. The elasticity of the spine allows it to stretch up to $5 \mathrm{~cm}$ before rupture, whereas the spinal cord, which is anchored to the brachial plexus superiorly and the cauda equina inferiorly, ruptures after 4-6 mm of traction [49]. As MRI is readily capable of detecting the soft tissue injury component, the concept of SCIWORA is less relevant. 
The imaging algorithm for pediatric spinal trauma is somewhat different than that for adults. MDCT is used more judiciously due to radiation exposure considerations and at many places lower dose radiography is often utilized initially. MRI is always used if there is a consideration of a pure soft tissue injury or neurologic deficit.

\section{Key Point}

- Patterns of injury in the pediatric population are tremendously different than adults; the imaging algorithm is unique.

\subsection{Neurologic Recovery after Spinal Cord Injury}

Although there are no pharmacologic "cures" for spinal cord injury, spontaneous neurologic recovery after injury can occur and it largely depends upon the severity of the initial neurologic deficit, the neurologic level of injury, patient age, and co-morbidities. Very few patients with a neurologically complete (i.e., no motor or sensory function below the injury level) actually regain any useful function below the injury level although most patients will spontaneously improve by one neurologic level (e.g., a C5 level spontaneously descends to a C6 level). Even these small improvements can have a substantial impact on a patients' capacity to function independently.

The role of MRI to predict capacity for spontaneous neurologic recovery after cervical SCI has been evaluated. Although there is considerable overlap in results some general characterizations about the MRI appearance of SCI and neurologic recovery are evident. Intramedullary hemorrhage four millimeters or greater is equated with a severe neurologic deficit and a poor prognosis. Cord edema alone is indicative of a mild to moderate initial neurological deficit and a better capacity for spontaneous neurological improvement. The length of the cord lesion may also correlate with the initial deficit and in the neurological outcome. Newer MRI techniques such as diffusion tensor imaging (DTI) (Fig. 19.3) have shown great promise not only in stratification of neurologic injury but has also been shown to have benefits in predicting recovery [50-52]. As novel pharmacologic therapies for SCI are developed and tested, MRI will likely play a more essential role in characterizing the injury and helping to select patients for clinical trials.

\section{Key Point}

- MRI findings of SCI have shown promise as a surrogate for the neurologic examination; better prognostic information is attained when MR imaging patterns are used in combination with the clinical examination.
Fig. 19.3 Disruption of the lateral cortical spinal tracts on tractography. Diffusion tractography performed on a lesioned rat spinal cord at 9.4T shows disruption of the fiber tracts in the lateral column following a lesion in the lateral funiculus. (Image courtesy of Eric D. Schwartz MD)

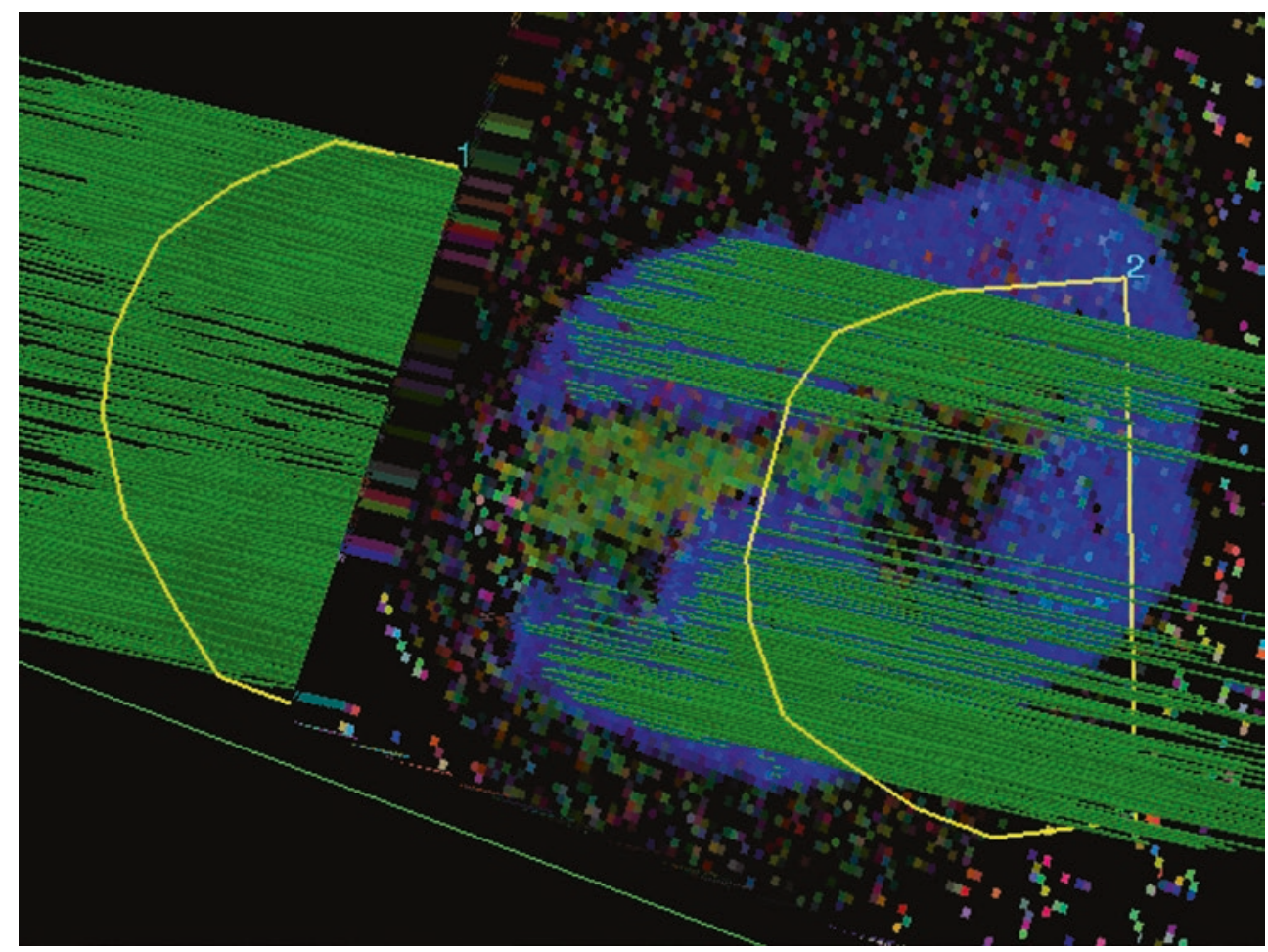




\subsection{Concluding Remarks}

The demographic of adult spinal trauma and spinal cord injury has changed over the last several decades noting a considerable increase in prevalence in the elderly. Safety measures in sports and motor vehicles have drastically reduced the incidence of spinal cord injury in the young adult and middle age population. Imaging algorithms for spinal trauma have also changed in the past few decades with greater reliance on MDCT over radiography in adults and greater emphasis towards minimizing radiation dose in the injured pediatric population. While there have been tremendous improvements in orthopedic stabilization methods through novel instrumentation/fusion techniques, there are still no known cures for paralysis. While spinal cord injury was previously considered to be a fatal disease with patient succumbing to injury at the scene or from long term complications, most SCI patients now survive and many return to meaningful lives while living with their disability. This is principally attributed to revolutionary improvements in rehabilitation and chronic care for the SCI patient.

\section{Take Home Messages}

- Spinal trauma and spinal cord injury are prevalent worldwide.

- Spinal trauma is now more common in the elderly due to falls.

- MDCT has supplemented radiography.

- MRI reveals the soft tissue components of injury including damage to the spinal cord.

- MR signal changes with whiplash-associated disorders are inconsistently present.

\section{References}

1. Riascos R, Bonfante E, Cotes C, Guirguis M, Hakimelahi R, West C. Imaging of atlanto-occipital and atlantoaxial traumatic injuries: what the radiologist needs to know. Radiographics. 2015;35(7):2121-34. https://doi.org/10.1148/rg.2015150035.

2. Hill MW, Dean SA. Head injury and facial injury: is there an increased risk of cervical spine injury? J Trauma. 1993;34: 549-54.

3. Pope AM, Tarlov AR. Disability in America: toward a national agenda for prevention. Washington: National Academy Press; 1991.

4. Riggins RS, Kraus JF. The risk of neurological damage with fractures of the vertebrae. J Trauma. 1997;17:126-30.

5. Castellano V, Bocconi FL. Injuries of the cervical spine with spinal cord involvement (myelic fractures): statistical considerations. Bull Hosp J Dis Orthop Inst. 1970;31:188-98.

6. Rogers WA. Fractures and dislocations of the cervical spine; an endresult study. J Bone Joint Surg. 1957;39:341-51.
7. Diaz JJ Jr, Gillman C, Morris JA Jr, et al. Are five-view plain films of the cervical spine unreliable? A prospective evaluation in blunt trauma in patients with altered mental status. J Trauma. 2003;55:658-63.

8. Griffen MM, Frykberg ER, Kerwin AJ, et al. Radiographic clearance of blunt cervical spine injury: plain radiograph or computed tomography scan? J Trauma. 2003;55:222-6.

9. Holmes JF, Mirvis SE, Panacek EA, et al. Variability in computed tomography and magnetic resonance imaging in patients with cervical spine injuries. J Trauma. 2002;53:524-9.

10. Kligman M, Vasili C, Roffman M. The role of computed tomography in cervical spine injury due to diving. Arch Orthop Trauma Surg. 2001;121:139-41.

11. Schenarts PJ, Diaz J, Kaiser C, et al. Prospective comparison of admission computed tomographic scan and plain films of the upper cervical spine in trauma patients with altered mental status. J Trauma. 2001;51:663-8.

12. Berne JD, Velmahos GC, El Tawil Q, et al. Value of complete cervical helical computed tomographic scanning in identifying cervical spine injury in the unevaluable blunt trauma patient with multiple injuries: a prospective study. J Trauma. 1999;47:896-902.

13. Van Goethem JW, Maes M, Özsarlak Ö, et al. Imaging in spinal trauma. Eur Radiol. 2005;15(3):582-90.

14. Flanders AE, Schaefer DM, Doan HT, et al. Acute cervical spine trauma; correlation of MR imaging findings with degree of neurological deficit. Radiology. 1990;177:25-33.

15. Sliker CW, Mirvis SE, Shanmuganathan K. Assessing cervical spine stability in obtunded blunt trauma patients; review of medical literature. Radiology. 2005;234:733-9.

16. Wilmink JT. MR imaging of the spine: trauma and degenerative disease. Eur Radiol. 1999;9:1259-66.

17. Hogan GJ, Mirvis SE, Shanmuganathan K, Scalea TM. Exclusion of unstable cervical spine injury in obtunded patients with blunt trauma: is MR imaging needed when multi-detector row CT findings are normal? Radiology. 2005;237:106-13.

18. Denis F. The three column spine and its significance in the classification of acute thoracolumbar spinal injuries. Spine. 1983;8(8):817-31.

19. Mcafee PC, Yuan HA, Fredrickson BE, et al. The value of computed tomography in thoracolumbar fractures. An analysis of one hundred consecutive cases and a new classification. J Bone Joint Surg Am. 1983;65(4):461-73.

20. Magerl F, Aebi M, Gertzbein SD, et al. A comprehensive classification of thoracic and lumbar injuries. Eur Spine J. 1994;3(4): 184-201.

21. Lee JY, Vaccaro AR, Lim MR, et al. Thoracolumbar injury classification and severity score: a new paradigm for the treatment of thoracolumbar spine trauma. J Orthop Sci. 2005;10(6):671-5.

22. Dvorak MF, Fischer CG, Fehlings MG, Rampersaud YR, Öner FC, Aarabi B, Vaccaro AR. The surgical approach to subaxial cervical spine injuries: an evidence-based algorithm based on the classification system. Spine. 2007:32(23):2620-9.

23. Kepler CK, Vaccaro AR, Koerner JD, Dvorak MF, Kandziora F, Rajasekaran S, Aarabi B, Vialle LR, Fehlings MG, Schroeder GD, Reinhold M, Schnake KJ, Bellabarba C, Cumhur Öner F. Reliability analysis of the AOSpine thoracolumbar spine injury classification system by a worldwide group of naïve spinal surgeons. Eur Spine J. 2016;25(4):1082-6.

24. Rogers LF. The roentgenographic appearances of transverse or chance fractures of the spine: the seat belt fracture. Am J Roentgenol. 1971;111:844-9.

25. Gertzbein SD. Scoliosis Research Society: multicenter spine fracture study. Spine. 1992;17:528-40.

26. Diaz JJ, Aulino JM, Collier B, et al. The early work-up for isolated ligamentous injury of the cervical spine; does computed tomography scan have a role. J Trauma. 2005;59:897-904. 
27. Sarrami P, Armstrong E, Naylor JM, Harris IA. Factors predicting outcome in whiplash injury: a systematic meta-review of prognostic factors. J Orthop Traumatol. 2017;18:9-16.

28. Li Q, Shen H, Li M. Magnetic resonance imaging signal changes of alar and transverse ligaments not correlated with whiplashassociated disorders. A meta-analysis of case-control studies. Eur Spine J. 2013;22:14-20.

29. Boban J, Thurnher MM, Van Goethem JW. Spine and spinal cord trauma. In: Barkhof $\mathrm{F}$, et al., editors. Clinical neuroradiology. Cham: Springer; 2018.

30. Burney RE, Maio RF, Maynard F, et al. Incidence, characteristics, and outcome of spinal cord injury at trauma centers in North America. Arch Surg. 1993;128:596-9.

31. Dawodu ST. Spinal cord injury - definition, epidemiology, pathophysiology. Emedicine; 2009.

32. American Spinal Injury Association. International standards for neurological classifications of spinal cord injury. Revised ed. Chicago: American Spinal Injury Association; 2000. p. 1-23.

33. Bondurant FJ, Cotler HB, Kulkarni MV, et al. Acute spinal cord injury. A study using physical examination and magnetic resonance imaging. Spine. 1990;15(3):161-8.

34. Flanders AE, Spettell CM, Friedman DP, Marino RJ, Herbison GJ. The relationship between the functional abilities of patients with cervical spinal cord injury and the severity of damage revealed by MR imaging. AJNR Am J Neuroradiol. 1999;20(5):926-34.

35. Kulkarni MV, McArdle CB, Kpanicky D, et al. Acute spinal cord injury: MR imaging at 1.5 T. Radiology. 1987;164(3):837-43.

36. Schouman-Claeys E, Frija G, Cuenod CA, et al. MR imaging of acute spinal cord injury: results of an experimental study in dogs. AJNR Am J Neuroradiol. 1990;11(5):959-65.

37. Boldin C, Raith J, Fankhauser F, Haunschmid C, Schwantzer G, Schweighofer F. Predicting neurologic recovery in cervical spinal cord injury with postoperative MR imaging. Spine (Phila Pa 1976). 2006;31(5):554-9.

38. Flanders AE, Spettell CM, Tartaglino LM, Friedman DP, Herbison GJ. Forecasting motor recovery after cervical spinal cord injury: value of MR imaging. Radiology. 1996;201(3):649-55.

39. Cotler HB, Kulkarni MV, Bondurant FJ. Magnetic resonance imaging of acute spinal cord trauma: preliminary report. J Orthop Trauma. 1988;2(1):1-4.

40. Sato T, Kokubun S, Rijal KP, et al. Prognosis of cervical spinal cord injury in correlation with magnetic resonance imaging. Paraplegia. 1994;32(2):81-5.
41. Marciello MA, Flanders AE, Herbison GJ, et al. Magnetic resonance imaging related to neurologic outcome in cervical spinal cord injury. Arch Phys Med Rehabil. 1993;74(9):940-6.

42. Talbott JF, Whetstone WD, Readdy WJ, Ferguson AR, Bresnahan JC, Saigal R, Hawryluk GW, Beattie MS, Mabray MC, Pan JZ, Manley GT, Dhall SS. The brain and spinal injury center score: a novel, simple, and reproducible method for assessing the severity of acute cervical spinal cord injury with axial T2-weighted MRI findings. J Neurosurg Spine. 2015;23(4):495-504.

43. Goldberg AL, Rothfus WE, Deeb ZL, et al. The impact of magnetic resonance on the diagnostic evaluation of acute cervicothoracic spinal trauma. Skelet Radiol. 1988;17(2):89-95.

44. Wittenberg RH, Boetel U, Beyer HK. Magnetic resonance imaging and computer tomography of acute spinal cord trauma. Clin Orthop Relat Res. 1990;260:176-85.

45. Kopelman TR, Leeds S, Berardoni NE, et al. Incidence of blunt cerebrovascular injury in low-risk cervical spine fractures. Am J Surg. 2011;202:684-8; discussion 688-9.

46. Rutman AM, Vranic JE, Mossa-Basha M. Imaging and management of blunt cerebrovascular injury. Radiographics. 2018;38:542-63.

47. Cothren CC, Moore EE, Biffle WL, et al. Cervical spine fracture patterns predictive of blunt vertebral artery injury. J Trauma. 2003;55:811-3.

48. Kriss VM, Kriss TC. SCIWORA (spinal cord injury without radiographic abnormality) in infants and children. Clin Pediatr (Phila). 1996;35:119-24.

49. Manary MJ, Jaffe DM. Cervical spine injuries in children. Pediatr Ann. 1996;25:423-8.

50. Poplawski MM, Alizadeh M, Oleson CV, Fisher J, Marino RJ, Gorniak RJ, Leiby BE, Flanders AE. Application of diffusion tensor imaging in forecasting neurological injury and recovery after human cervical spinal cord injury. J Neurotrauma. 2019;36:3051. https://doi.org/10.1089/neu.2018.6092.

51. Shanmuganathan K, Zhuo J, Chen HH, Aarabi B, Adams J, Miller C, Menakar J, Gullapalli RP, Mirvis SE. Diffusion tensor imaging parameter obtained during acute blunt cervical spinal cord injury in predicting long-term outcome. J Neurotrauma. 2017;34:2964-71.

52. Schaefer DM, Flanders A, Northrup BE, et al. Magnetic resonance imaging of acute cervical spine trauma. Correlation with severity of neurologic injury. Spine. 1989;14(10):1090-5.

Open Access This chapter is licensed under the terms of the Creative Commons Attribution 4.0 International License (http://creativecommons. org/licenses/by/4.0/), which permits use, sharing, adaptation, distribution and reproduction in any medium or format, as long as you give appropriate credit to the original author(s) and the source, provide a link to the Creative Commons license and indicate if changes were made.

The images or other third party material in this chapter are included in the chapter's Creative Commons license, unless indicated otherwise in a credit line to the material. If material is not included in the chapter's Creative Commons license and your intended use is not permitted by statutory regulation or exceeds the permitted use, you will need to obtain permission directly from the copyright holder. 


\title{
Brachial and Lumbosacral Plexus and Peripheral Nerves
}

\author{
Roberto Gasparotti and Lubdha Shah
}

\section{Learning Objectives}

- Review the anatomy of the peripheral nerves and brachial and lumbosacral plexi.

- Understand the utility of different magnetic resonance imaging (MRI) sequences and magnetic resonance neurography (MRN) for identifying peripheral nerve diseases.

- Describe the imaging characteristics of different peripheral nerve diseases to help narrow the differential diagnosis.

- Discuss the role of advanced imaging techniques.

\subsection{Introduction}

The diagnostic work-up of peripheral neuropathies relies on the patient's clinical history, physical examination, and electrophysiological studies, which usually provide enough information about the location, severity, and the etiology of the underlying nerve injury in the majority of patients. However, electrodiagnostic studies, such as electromyography (EMG), do not display the anatomic detail needed for precise localization and treatment planning because of the deep location of nerves and the variable innervation of regional muscles. EMG sensitivity for lumbosacral radiculopathy ranges from 49 to $86 \%$. Therefore, imaging particularly with magnetic resonance imaging (MRI)/magnetic resonance neurography (MRN) and ultrasound (US) has an increasing role in the evaluation of peripheral

R. Gasparotti

U.O. Neuroradiologia, ASST Spedali Civili, Università degli Studi di Brescia, Brescia, Italy

e-mail: roberto.gasparotti@unibs.it

L. Shah $(\varangle)$

Department of Radiology, University of Utah Health Sciences

Center, Salt Lake City, UT, USA

e-mail: lubdha.shah@hsc.utah.edu neuropathies [1]. Although US is more operator-dependent than MRI and less effective in cases of deep nerves of the pelvis and lumbosacral plexus, it is very effective for dynamic assessment of superficial peripheral nerves abnormalities, such as changes in nerve caliber, continuity, and echogenicity [2].

\subsection{Anatomy of Peripheral Nerves}

Peripheral nerves range from 1 to $20 \mathrm{~mm}$ in size, the largest being the sciatic nerve. Peripheral nerves are formed by multiple axons, grouped into fascicles, the number of which depends on the size and length of the nerve. Endoneurium invests the Schwann cell-axon complex, with the inner border represented by the Schwann cell basement membrane and its outer border by the perineurium. The epineurium, the outermost connective tissue sheath, envelops the nerve and provides mechanical support for the axons. Endoneurial fluid within each fascicle is isolated from the general extracellular space by tightly adherent epithelial-like cells of the perineurium and from the circulating blood by the tight junctions between the endothelial cells of the endoneurial capillaries. The endoneurium and perineurium form a functional, relatively impermeable barrier known as the blood-nerve interface that protects the peripheral nervous system against toxic and infectious agents.

\subsection{Brachial Plexus (BP)}

The ventral rami of the $\mathrm{C} 5$ through $\mathrm{T} 1$ nerves give rise to the BP. The BP structures travel through the supraclavicular fossa paralleling the subclavian artery. Individual ventral rami or roots are best seen on axial and coronal images. The sagittal plane is helpful for following the BP structures from the spine to the axilla as they lie perpendicular to the plane. The upper (C5-C6), middle (C7), and lower (C8-T1) trunks travel between anterior and middle scalene muscles, forming the lateral border of the scalene triangle [3]. In the 
supraclavicular triangle, the trunks divide into anterior and posterior divisions just before passing posterior to the clavicle and going from the lateral aspect of the anterior scalene muscle to the lateral border of the first rib [3]. The cords (lateral, medial, and posterior) course from the mid-clavicle to the inferomedial coracoid process and are named according to location relative to the axillary artery. At the pectoralis minor muscle, just medial to the coracoid, axillary vein, and axillary artery, the cords change into the five terminal branches (radial, axillary, musculocutaneous, median, and ulnar nerves).

\subsection{Lumbosacral Plexus (LSP)}

The ventral rami of the L1-L4 spinal nerve roots and a small contribution from the 12 th thoracic nerve coalesce within or posterior to the psoas major muscle to form the lumbar plexus [2]. The lumbar plexus gives rise to the iliohypogastric (L1), ilioinguinal (L1), genitofemoral (L1-L2), femoral (L2-L4), and lateral femoral cutaneous (L2-L3) nerves, which emerge lateral to the psoas major muscle. The obturator nerve and lumbosacral trunk come forward from the medial border of the psoas muscle. A minor (peroneal) branch of L4 combines with the ventral ramus of L5 to form the lumbosacral trunk. The latter descends over the sacral ala and joins the S1 to S3 ventral rami on the anterior aspect of the piriformis muscle to form the sacral plexus [4]. The sacral plexus gives rise to the sciatic (L4-S3), pudendal (S2-S4), superior gluteal (L4-S1), and inferior gluteal (L5-S2) nerves.

\subsection{Magnetic Resonance Neurography (MRN)}

Although conventional MRI can provide structural information, it is limited in its ability to visualize peripheral nerves due to low contrast resolution between the small nerves, muscles, and vessels, signal intensity variability, and pulsatility artifacts. These disadvantages have largely been overcome with MRN. The MRN technique relies on high magnetic field strength, T2-weighted sequences with fat suppression, dedicated surface coils, and small field of view (FOV). MRN can identify specific characteristics of nerve morphology, such as internal fascicular pattern, longitudinal variations in signal intensity and caliber, and connections and relations to other nerves or plexuses [5]. The most efficient fat suppression is obtained with T2-weighted short time inversion recovery (STIR) sequences, which selectively eliminate the fat signal using an inversion recovery pulse of $150 \mathrm{~ms}$. These sequences, however, are characterized by poor signalto-noise ratio and pulsatility artifacts caused by vessels. Alternative methods for fat suppression are T2 spectral adia- batic inversion recovery imaging (SPAIR) or DIXON-type fat suppression, which both have improved signal-to-noise ratio, at the expense of lower contrast resolution. In order to obtain the best compromise between spatial resolution, FOV, and acquisition time, MRN sequences should be adapted to the anatomical region and the best echo time should be carefully selected in order to obtain a satisfactory differentiation between nerves and muscles, as the signal intensity of the nerve is very sensitive to small changes (Fig. 20.1).

Three-dimensional (3D) MRN is a refinement of conventional MRN. It is typically acquired with isotropic voxels, with the advantage of generating oblique and curved-planar reformations of nerve roots, peripheral nerves, and plexuses. This feature is particularly useful for imaging brachial and lumbosacral plexi, which cannot be fully displayed by $2 \mathrm{D}$ imaging (Fig. 20.2). On MRN axial images, normal nerves are rounded or ovoid structures, typically isointense to slightly hyperintense on $\mathrm{T} 2$, depending on the size of the nerve, on the amount of endoneurial fluid, and the degree of fat suppression. On T1 images, nerves are isointense to the adjacent muscles. The epineurium appears as a thin hypointense rim, and the transverse and longitudinal fascicular pattern may be identified in larger nerves such as the sciatic nerve. Diseased nerves become $\mathrm{T} 2$ hyperintense to muscle and are focally or globally enlarged [6]. The signal intensity change is due to increased water content in the epineurial space caused by blood-nerve barrier damage, axoplasmic flow blockade, inflammation, distal Wallerian degeneration and is relatively independent from

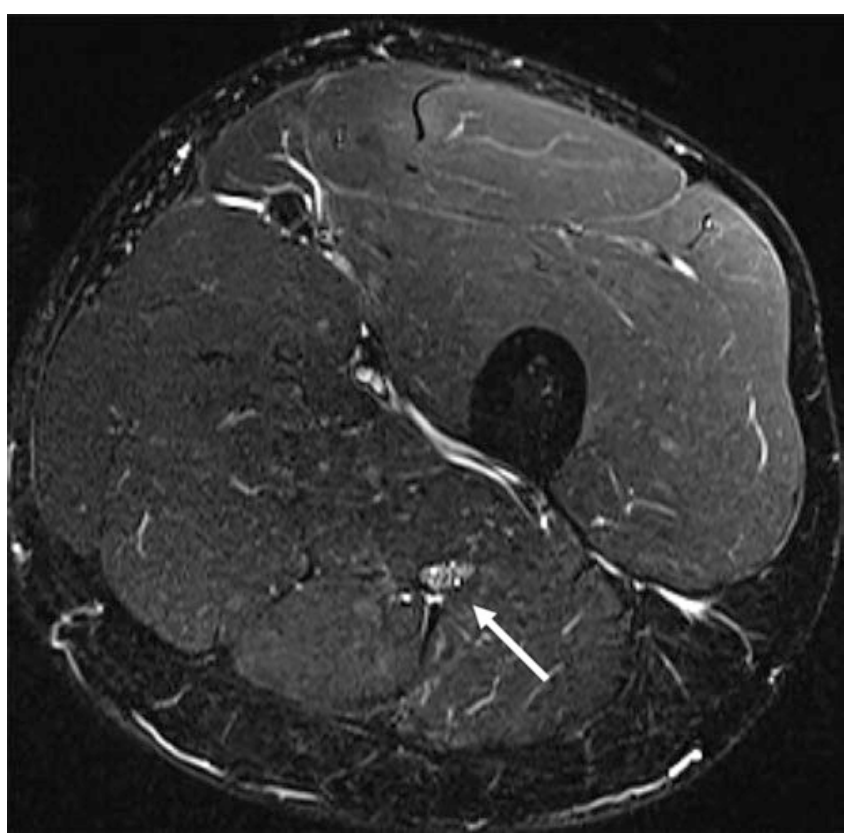

Fig. 20.1 MRN (3 T), axial T2-STIR section at mid-thigh. Normal subject. The right sciatic nerve (arrow) is moderately hyperintense compared to the adjacent muscles and its transverse fascicular pattern is clearly identifiable 


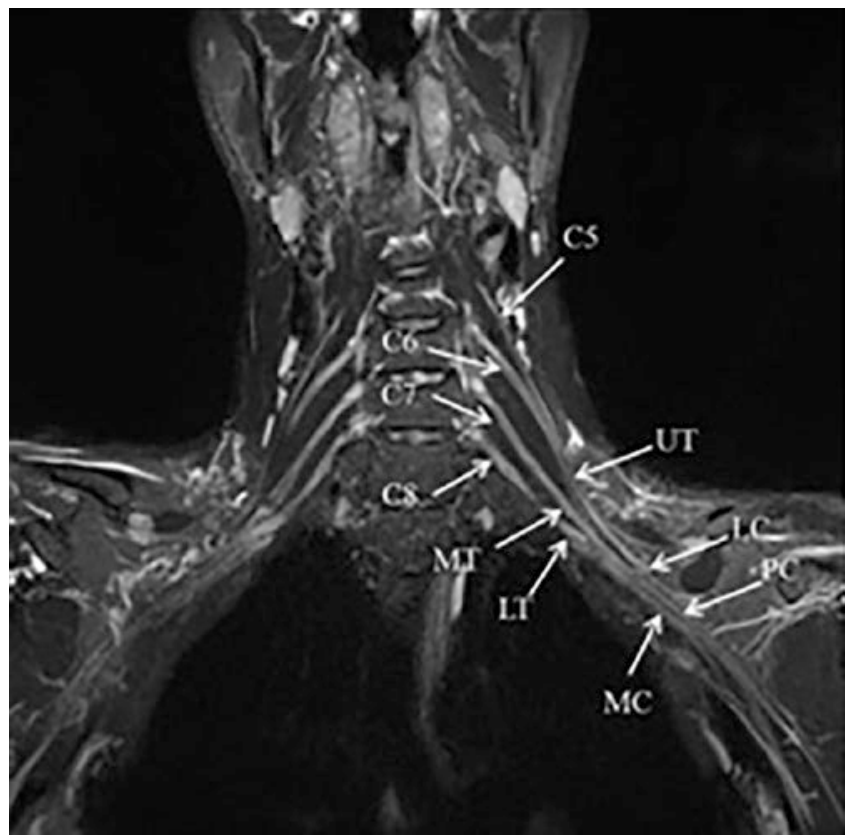

Fig. 20.2 3D MRN (3 T) of the brachial plexus. Oblique coronal reformat. The supra and infraclavicular segments of the brachial plexus are simultaneously displayed in a single image. Upper trunk (UT), Middle trunk (MT), Lower trunk (LT), Lateral cord (LC), Posterior cord PC), Medial cord (MC)

the etiology of the neuropathy. Neuropathies with different etiologies cannot be distinguished only on the basis of signal intensity changes as there are no reliable quantitative methods to differentiate between the signal intensities of normal versus abnormal nerves. MRN has the advantage of a simultaneous exploration of nerves and muscles. Importantly, muscle denervation represents a useful MR sign of peripheral nerve disease. In the acute phase of muscle denervation, increased signal intensity can be observed in T2 sequences as early as $24 \mathrm{~h}$ after nerve injury and lasting for more than 2 months [7]. In the subacute phase, a gradual decrease of $\mathrm{T} 2$ signal intensity is associated with fatty replacement, which progresses to fatty atrophy of the affected muscles, best displayed by T1 images. The MR changes precede the earliest EMG findings of denervation, which are not detectable until the second week.

A comprehensive MRI protocol for the investigation of peripheral nerves should include MRN, which provide both structural and functional information on the nerves and muscle denervation, $\mathrm{T} 1$ sequences which are helpful for a precise anatomical identification of nerves and for the identification of muscular atrophy, and contrast-enhanced T1 sequences for the evaluation of the blood-nerve barrier integrity. MRN is effective in the diagnostic work-up of traumatic nerve injuries [8], nerve entrapment syndromes [9], and nerve tumors [10]. Lumbosacral MRN may demonstrate abnormal intraneural $\mathrm{T} 2$ signal in a substantial portion of patients with clinical symptoms of lower extremity radiculopathy and correlates with findings of active radiculopathy on EMG [11]. Recently MRN has been proposed for the evaluation of hereditary and immune-mediated disorders of peripheral nerves [12].

\subsection{Diffusion Tensor Imaging}

Diffusion tensor imaging (DTI) is a novel technique which has been recently applied to the investigation of peripheral nerve disorders. DTI provides "microstructural" information about nerve integrity. Nerves are characterized by greater water diffusion anisotropy compared to the surrounding tissues, due to a barrier to water diffusion formed by axonal myelin sheaths. Diffusion imaging of the peripheral nerves is challenging because of their small size and course, particularly the BP because of the geometric distortion and artifacts along the course of the nerves between the neck and the shoulder. Additionally, DTI gives quantitative information about the degree and direction of water diffusion: fractional anisotropy (FA), apparent diffusion coefficient (ADC), mean diffusivity (MD), axial diffusivity (AD), and radial diffusivity (RD) [13]. Reconstructing the diffusion information to characterize the integrity of the white matter tracts (diffusion tensor tractography, DTT) yields 3D representations of anisotropic nerve fibers. Successful tracking of the major peripheral nerves can be obtained using the same approach as deterministic tractography of the brain white matter bundles. DTI has been extensively applied to the median nerve at the carpal tunnel and more recently to the BP and LSP, although its overall diagnostic value in clinical routine is still to be ascertained. Although DTI and tractography are not widely used clinically, good reproducibility of DTI quantitative analysis with only small variations in calculated FA and $\mathrm{ADC}$ in the BP of normal volunteers has been shown [14].

\section{Key Point}

- MRN can characterize nerve morphology, longitudinal variations in signal intensity and caliber, and connections and relations to other nerves or plexuses to help identify pathology in correlation with the patient's clinical history, physical examination, and electrophysiological studies.

\subsection{Traumatic Injuries of Peripheral Nerves}

Peripheral nerve injuries are classified into three types: neuropraxia, axonotmesis, and neurotmesis, which are defined by the histopathology and specific anatomic structures of the nerve unit that are damaged (Table 20.1). Neuropraxia 
Table 20.1 MR imaging in peripheral nerve injuries according to Seddon's classification

\begin{tabular}{|l|l|}
\hline Classification & MR \\
\hline Neurapraxia & $-\begin{array}{l}\text { T2 hyperintensity of the nerve (within } 24 \mathrm{~h} \text { of } \\
\text { trauma) } \\
-\end{array}$ \\
\hline Axonotmesis & $-\begin{array}{l}\text { To muscle denervation } \\
\text { with fascicular hypertrophy, with or without } \\
\text { neuroma in continuity }\end{array}$ \\
& - Muscle denervation
\end{tabular}

is the mildest form of traumatic plexopathy, in which there is temporary loss of function, near complete recovery, and the absence of denervation atrophy of the muscle. The nerve is grossly intact but functionally impaired with slowed or absent conduction in the involved segment. More serious injury to the neural axons results in axonotmesis and neurotmesis. In axonotmesis, there is loss of axonal continuity and the endoneurium, with Wallerian degeneration distal to the site of injury. As such, recovery requires nerve regeneration to regain function. Depending on the severity of injury, MRN will demonstrate varying degrees of nerve enlargement, disruption, and effacement of nerve fascicles. In neurotmesis, disruption of the axons, myelin sheath, and surrounding perineurium and epineurial layers typically requires surgical intervention. MRN may reveal a neuroma in continuity or complete transection of the nerve with formation of an end-bulb neuroma [15]. EMG and nerve conduction studies provide functional information but are unable to differentiate axonotmesis from neurotmesis. MRI can be used to differentiate neurapraxia from axonotmesis and neurotmesis through combined assessment of the characteristics of the injured nerve, typically represented by: (a) nerve enlargement with longitudinal variations in size, (b) increase in signal intensity, (c) signal intensity anomalies of the corresponding muscles, reflecting acute or subacute denervation.

\subsection{Traumatic Injuries of the Brachial Plexus}

MR imaging (including MR myelography and MRN) is primarily used to locate the injury level, i.e., the distinction between preganglionic lesions (avulsion of the cervical nerve roots from the spinal cord) and postganglionic lesions (distal to the dorsal root ganglion), which is important for prognosis and therapeutic management [16] (Table 20.2). Multiplanar and curved reformats from 3D MRN are helpful to identify postganglionic lesions. Of note, MRI within 1 month of injury can be challenging to evaluate because hemorrhage and edema can compromise visualization of the thecal sac and nerves. In adults, the majority of brachial plexopathies
Table 20.2 MR imaging findings in different peripheral nerve injuries on MR Myelography

\begin{tabular}{|l|l|}
\hline Brachial plexus injuries & MR \\
\hline Partial avulsion & $\begin{array}{l}\text { Traumatic pseudomeningoceles } \\
\text { Ventral and dorsal roots not identifiable } \\
\text { Denervation edema of the posterior } \\
\text { paraspinal muscles }\end{array}$ \\
\hline $\begin{array}{l}\text { Reduced number of rootlets on MIP } \\
\text { projections } \\
\text { Ventral or dorsal root absent on axial } \\
\text { sections with mild abnormalities of dural } \\
\text { sleeves }\end{array}$ \\
\hline $\begin{array}{l}\text { Pwelling and increased signal intensity of } \\
\text { the roots, trunks and cords of the brachial } \\
\text { plexus } \\
\text { Tortuosity and increased intensity of the } \\
\text { infraclavicular brachial plexus } \\
\text { Post-traumatic neuromas along the course } \\
\text { of the roots and/or primary trunks }\end{array}$
\end{tabular}

are due to traumatic injuries, usually resulting from highvelocity trauma, such as motorcycle accidents. In the pediatric population, traumatic brachial plexopathy is often secondary to excessive severe traction force exerted on the brachial plexus during complicated deliveries. In preganglionic injuries, MRI may reveal spinal cord edema in the acute phase (T2 hyperintensity) [17]. There may be susceptibility effects from hemosiderin, accentuated on $\mathrm{T} 2 *$ gradient susceptibility images. A helpful clue to preganglionic avulsion injury is the formation of a pseudomeningocele, which is seen in $80 \%$ of traumatic avulsions; notably, pseudomeningoceles take time to develop. This lesion is a sequela of traction on the cervical roots and meninges resulting in leakage of cerebrospinal fluid in contiguous areas, anterior epidural space, neural foramina, perivertebral spaces, surrounded by a thin membrane. In complete avulsions, the ventral and dorsal roots are not identifiable, whereas partial avulsions are characterized by rarefaction of the ventral or dorsal rootlets, with mild abnormalities of the dural sleeves (Fig. 20.3). Changes of muscular denervation, including alterations in signal intensities and contrast enhancement, in the posterior cervical paraspinal muscles, particularly in the multifidus muscle, are an indirect imaging finding of a preganglionic injury.

In postganglionic injuries, the nerve may be stretched and remain in continuity. The acutely injured nerve is thickened and typically $\mathrm{T} 2$ hyperintense. The secondary trunks are often characterized by marked tortuosity. Post-traumatic neuromas can be identified along the course of the roots or primary trunks as neuromas "in continuity," characterized by fusiform swelling, or as "stump" neuromas (Fig. 20.4). DTT has been proposed as a diagnostic tool to assess traumatic BP lesions, due to high diagnostic accuracy in the identification of radicular avulsions, especially when extensive dural scar or CSF artifacts limit the usefulness of MRI [18] (Fig. 20.5).

For traumatic injuries of the upper limb, US is the examination of choice and can be performed imme- 

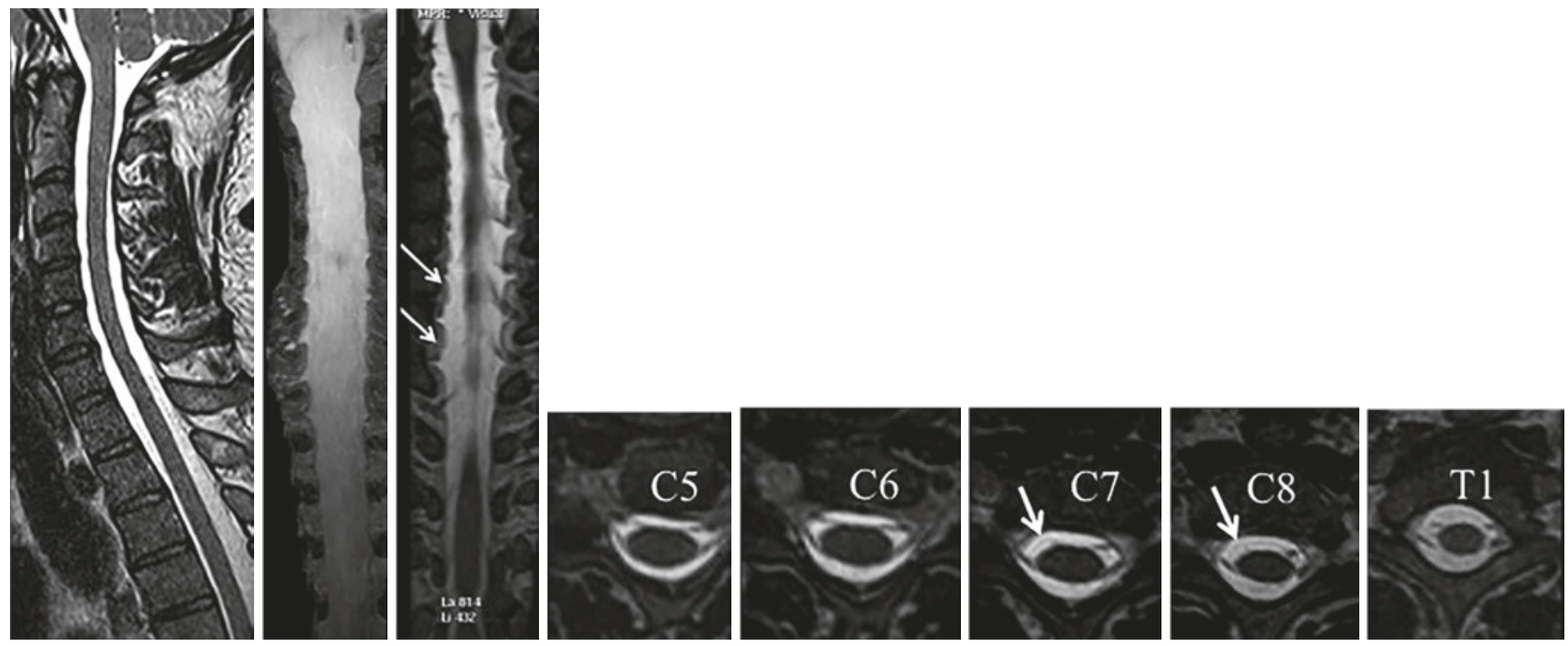

Fig. 20.3 22-year-old male, motor cycle accident, with complete right brachial plexus palsy. 3D MR Myelography. Right C7 and C8 (partial) avulsions of the ventral nerve roots (arrows)

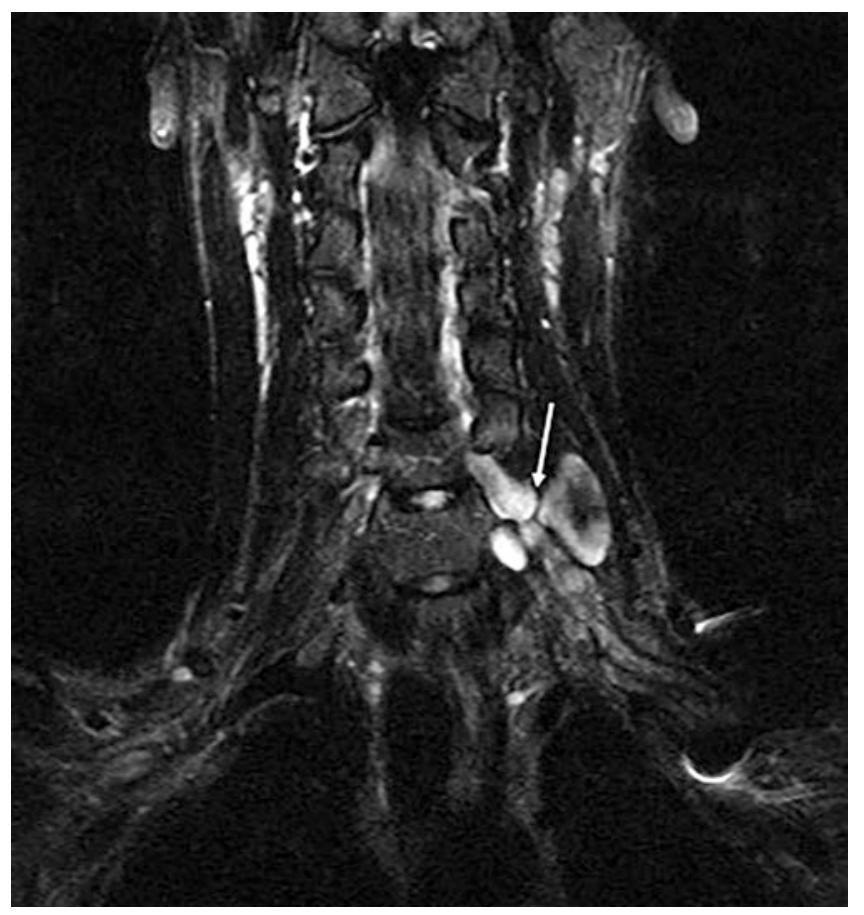

Fig. 20.4 MRN, traumatic injury of the left brachial plexus. Neurotmesis of the left C6 nerve root: stump neuroma (arrow)

diately after trauma to demonstrate neurotmesis-type lesions to show nerve discontinuity. MRN can identify axonotmesis-type lesions, which appear as global nerve enlargement with mild fascicular hypertrophy that extends longitudinally and requires longer time for nerve regeneration.

DTI can be used to assess the nerve regeneration after surgery [19].

\subsection{Traumatic Injuries of the Lumbosacral Plexus, Sciatic and Femoral Nerves}

Indirect injury to the LSP may occur as a part of lumbar spine injuries, pelvic fractures, and hip fractures or dislocations. Pelvic or hip injuries typically cause stretch- or tractionrelated partial plexopathy and, less commonly, nerve avulsions. The avulsions of lumbosacral nerve roots typically involve L4, L5, and S1. MRN can locate the level of the injury and provide information about the associated muscle denervation (Fig. 20.6). Fibular nerve can be injured in fibular fractures and knee dislocations as well as in habitual leg crossing or repetitive exercise in athletes.

\section{Key Point}

- A pseudomeningocele is a preganglionic injury that is seen in $80 \%$ of traumatic avulsions. It results from traction on the cervical roots and meninges with CSF leakage into contiguous areas.

Iatrogenic injury to the LSP may result from surgical gynecologic or anesthetic procedures, compression, traction, and vascular insults [20]. Traumatic injuries of the sciatic nerve can also occur as a complication of prosthetic hip surgery, most frequently involving the fibular division. Injuries of the femoral nerve can be secondary to pelvic trauma or iatrogenic in nature with nerve compression from hematoma or pseudoaneurysm during femoral artery puncture, respectively. The intrapelvic femoral nerve may show increased T2 signal and size or deviation by hematoma, with denervation of the iliopsoas muscle. 

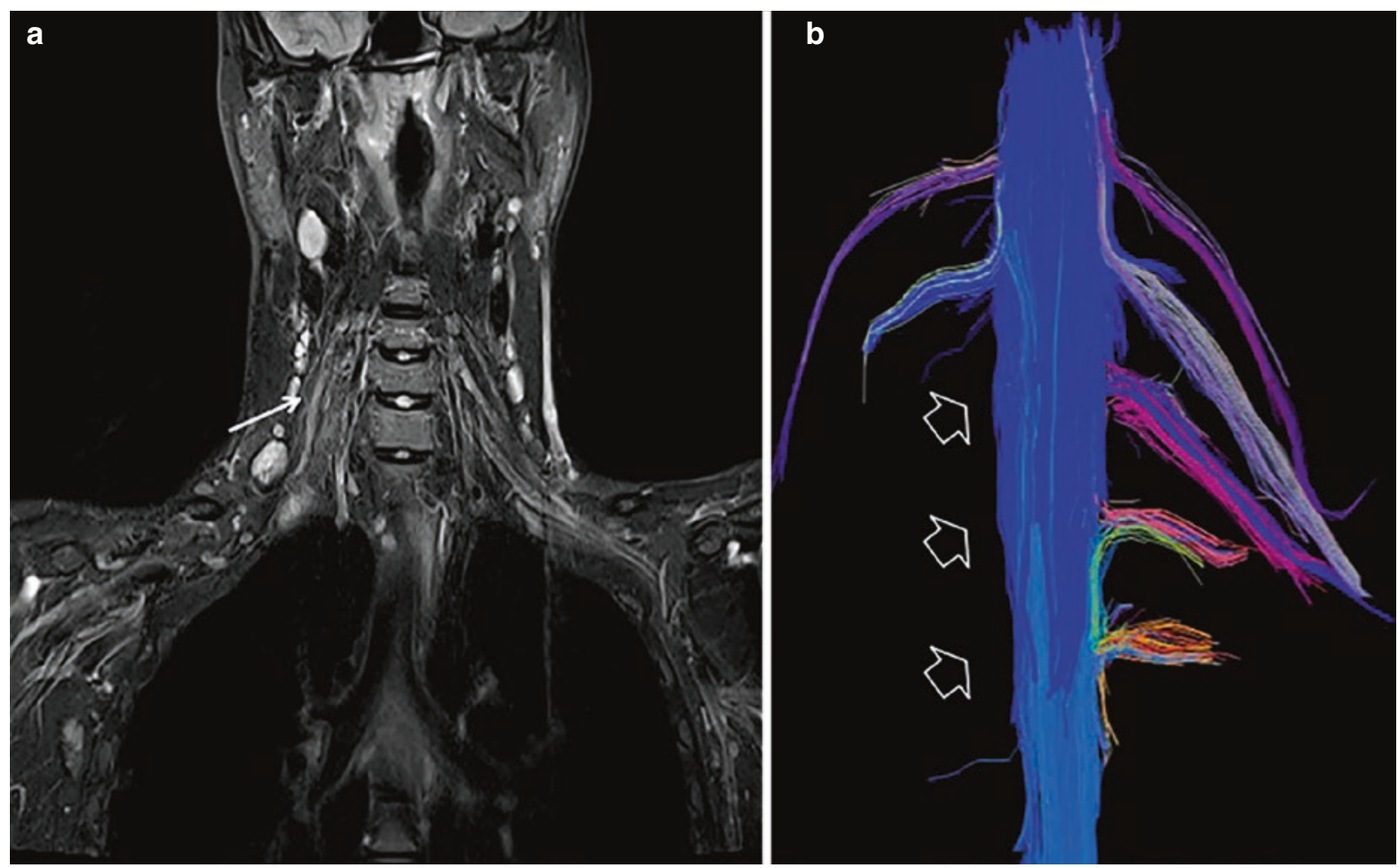

Fig. 20.5 Traumatic injury of the right brachial plexus. (a) MRN: the right brachial plexus is barely identifiable (arrow). (b) Tractography: right $\mathrm{C} 7, \mathrm{C} 8, \mathrm{~T} 1$ nerve root avulsions (open arrows)
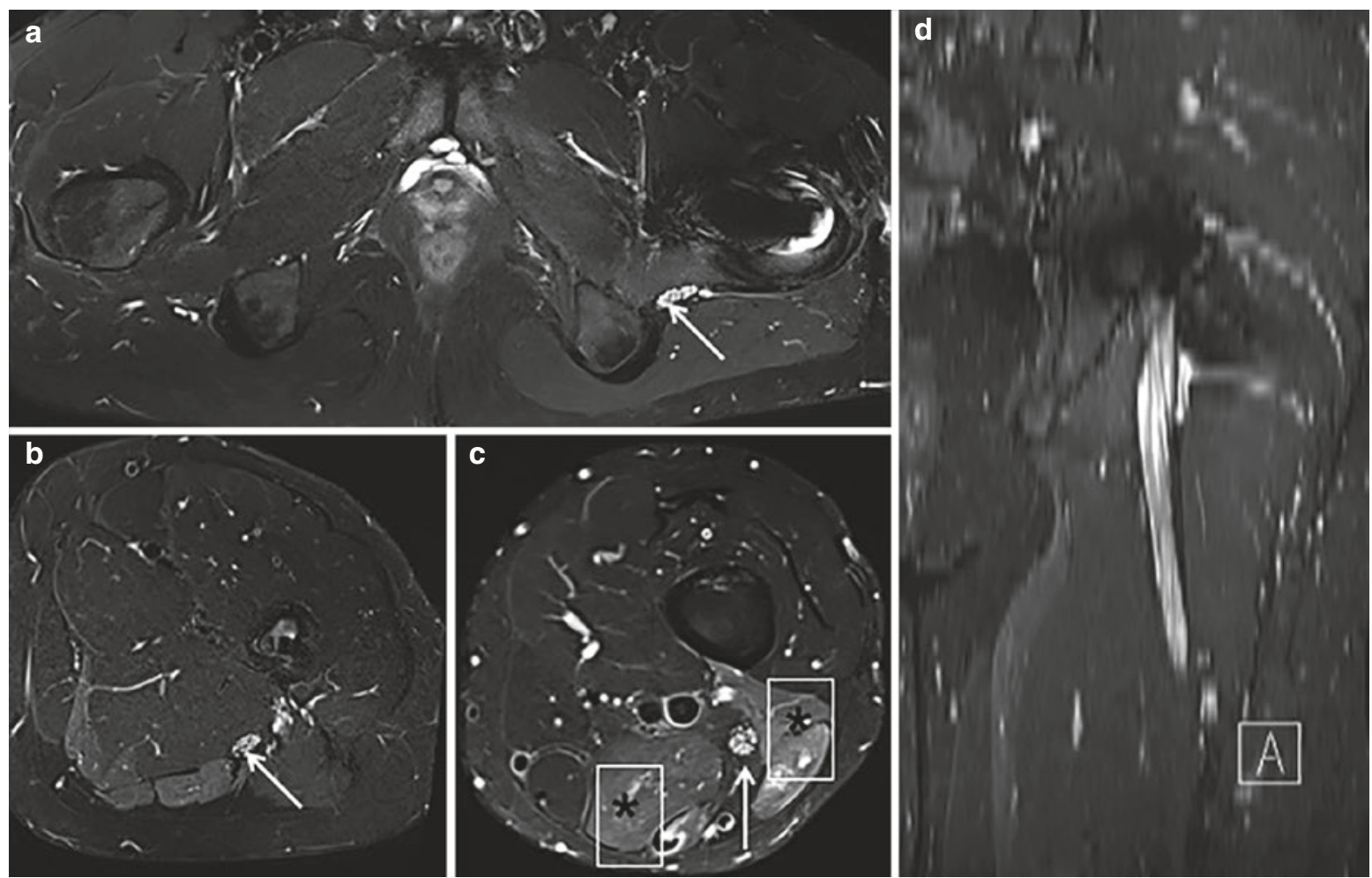

Fig. 20.6 24-year-old male, road accident, with left femoral shaft fracture, sciatic nerve injury. MRN (1.5 T), axial T2-STIR at pelvic floor acterized by fascicular hypertrophy (arrows). Increased signal intensity of semimembranosus, semitendinosus, and long head of the biceps (a), proximal and mid-thigh $(\mathbf{b}, \mathbf{c})$, sagittal oblique reformat (d). femuri, due to acute denervation Enlargement and hyperintensity of the left sciatic nerve, which is char- 
Although nerve abnormalities in the thigh can be difficult to detect, denervation of the quadriceps femoris may be a clue to femoral nerve injury distal to the groin ligament.

\subsection{Entrapment Neuropathies}

Peripheral nerve compression or entrapment usually occurs at specific anatomic locations, often close to limb joints, such as sites where a nerve courses through fibro-osseous or fibromuscular tunnels or penetrates muscles (Table 20.3). US and MRN can provide direct anatomic visualization of the entrapped nerves. US has the advantage of providing dynamic evaluation of nerve entrapment. MRN demonstrates varying degrees of signal intensity within the nerve, proximal to the site of entrapment, and the associated muscle denervation changes. Proximal nerve enlargement and fascicular hypertrophy and distal flattening can develop with increasing severity of neuropathy.

Table 20.3 Common nerve entrapment syndromes

\begin{tabular}{|c|c|c|}
\hline $\begin{array}{l}\text { Nerve entrapment } \\
\text { syndrome }\end{array}$ & $\begin{array}{l}\text { Involved } \\
\text { nerve }\end{array}$ & Site of entrapment \\
\hline $\begin{array}{l}\text { Carpal tunnel } \\
\text { syndrome }\end{array}$ & $\begin{array}{l}\text { Median } \\
\text { nerve }\end{array}$ & $\begin{array}{l}\text { Carpal tunnel, at the level of } \\
\text { hamate (proximal alterations at } \\
\text { the level of pisiform) }\end{array}$ \\
\hline Ulnar neuropathy & $\begin{array}{l}\text { Ulnar } \\
\text { nerve }\end{array}$ & Cubital tunnel \\
\hline $\begin{array}{l}\text { Fibular nerve } \\
\text { entrapment }\end{array}$ & $\begin{array}{l}\text { Fibular } \\
\text { nerve }\end{array}$ & $\begin{array}{l}\text { At fibular head or deep to the } \\
\text { origin of peroneus longus muscle }\end{array}$ \\
\hline $\begin{array}{l}\text { Piriformis } \\
\text { syndrome }\end{array}$ & $\begin{array}{l}\text { Sciatic } \\
\text { nerve }\end{array}$ & Sciatic notch \\
\hline $\begin{array}{l}\text { Tibial nerve } \\
\text { entrapment }\end{array}$ & $\begin{array}{l}\text { Tibial } \\
\text { nerve }\end{array}$ & Tarsal tunnel \\
\hline
\end{tabular}

\subsection{Carpal Tunnel Syndrome}

Carpal tunnel syndrome (CTS) is the most common peripheral nerve entrapment syndrome and has various etiologies, including repetitive trauma, metabolic and hormonal conditions, and ganglion cysts. While US has $77.6 \%$ sensitivity and $86.8 \%$ specificity for diagnosing CTS, sensitivity of MRN is above 90\%. On MRN, abnormal nerve signal length and cross-sectional area at the distal radioulnar joint are the best predictors of the severity [21]. Correlation between median nerve DTI metrics in the carpal tunnel and electrophysiology has been recently reported [22]. MRN is particularly useful for the evaluation of atypical CTS, when assessing for space-occupying lesion, and in patients with persistent symptoms after carpal tunnel release.

\subsection{Ulnar Neuropathy at the Elbow}

Ulnar nerve entrapment at the cubital tunnel is the second most common entrapment neuropathy. The dynamic US evaluation during flexion/extension of the elbow is crucial for showing subluxation of the ulnar nerve. MRN can discriminate between symptomatic ulnar nerve entrapment at the elbow and asymptomatic controls with high diagnostic accuracy. In patients with electrophysiologically and clinically evident ulnar nerve entrapment, DTI may show regional FA decrease at the cubital tunnel, correlating with symptom severity (Fig. 20.7).

\section{Key Point}

- Although both US and MRN offer anatomic delineation of entrapped nerves, US has the advantage of providing dynamic evaluation of nerve entrapment.
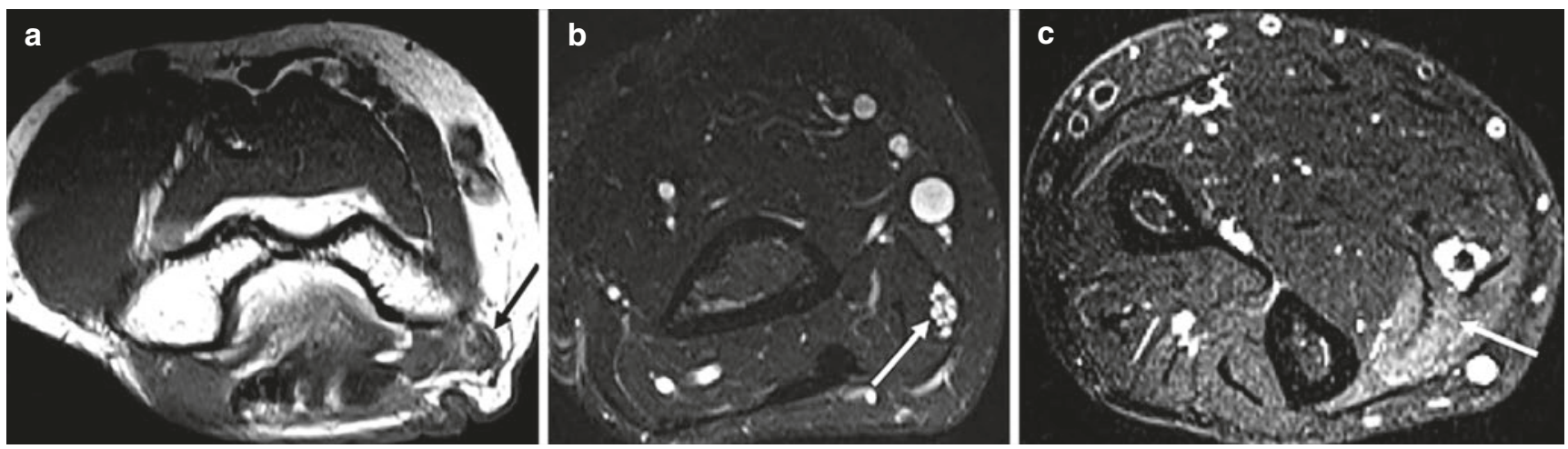

Fig. 20.7 48-year old male with acute onset of right forearm pain and paresthesia. Ulnar nerve entrapment at the cubital tunnel. (a) Axial T1 image at the right elbow (a), MRN at distal arm (b), and forearm (c). Deformation of the right ulnar nerve (black arrow in a). Proximal to the entrapment site, the ulnar nerve is enlarged and hyperintense with fascicular hypertrophy (arrow in b). Denervation edema of the flexor carpi ulnaris (arrow in c) 


\subsection{Non-traumatic Brachial and Lumbosacral Plexopathies}

Idiopathic brachial plexitis or idiopathic neuralgic amyotrophy [INA] ( $a k a$ Parsonage Turner syndrome) is a self-limiting inflammatory disorder usually affecting the unilateral BP, more commonly in males. Acute symptoms include neck, shoulder, and scapula pain. Muscle weakness ensues days or weeks after the initial pain, and the symptoms may last several months up to 3 years. MRN will show diffuse T2 hyperintensity and enlargement of affected neural structures, commonly C5 and C6 nerve roots and the upper trunk, and denervation edema in various muscles that does not follow a typical innervation pattern (e.g., isolated edema in the teres minor muscle).

Although an autoimmune/inflammatory etiology has been hypothesized, a recent alternative theory is fascicular torsion which more frequently occurs in the suprascapular and radial nerves. MRN will reveal the characteristic "bulls' eye sign" of central hypointensity surrounded by a hyperintense rim [23] (Fig. 20.8). MRN is also helpful in distinguishing INA from cervical spondylotic radiculopathy by demonstrating enlargement and increased signal intensity of cervical nerve roots at the level of foraminal stenosis, which correlate with the distribution of radicular pain and muscle weakness.

Idiopathic lumbosacral plexopathy (ILSP) is a self-limiting condition that presents with (sub)acute, severe, asymmetri- cal leg pain, followed by asymmetrical multifocal weakness and atrophy in the subsequent weeks or months. Though some studies have hypothesized an immune-mediated etiology, biopsies of distal cutaneous nerve segments have shown features of an inflammatory microvasculitis causing ischemic damage of the nerves [24]. Diabetic LSP neuropathy has similar clinical and pathological findings, suggesting that inflammation may form part of the final common pathway in both conditions. MRN shows increased signal intensity and mild contrast enhancement asymmetrically involving multiple nerve roots and terminal branches of the LSP as well muscle denervation changes.

LSP may be involved in retroperitoneal disorders, such as psoas abscess, hematoma, retroperitoneal fibrosis, and malignant disease (soft tissue or osseous), either by extrinsic compression or infiltration. Inflammatory sacroiliac joint arthritis may also involve the sacral component of the LSP.

Radiation plexopathy may manifest from a few months to years after brachytherapy or intraoperative radiation therapy, usually with doses exceeding $6000 \mathrm{cGy}$. This type of plexopathy is often painless and progresses slowly, in contradistinction to severely painful tumor-related plexopathy. Expected radiation-induced changes on MRN are uniform, symmetric thickening, T2 hyperintensity, and faint to no contrast enhancement of BP and LSP within the irradiated region. It may be difficult to separate the neural structures from each other. Recurrent tumor often shows a focal or diffuse het-
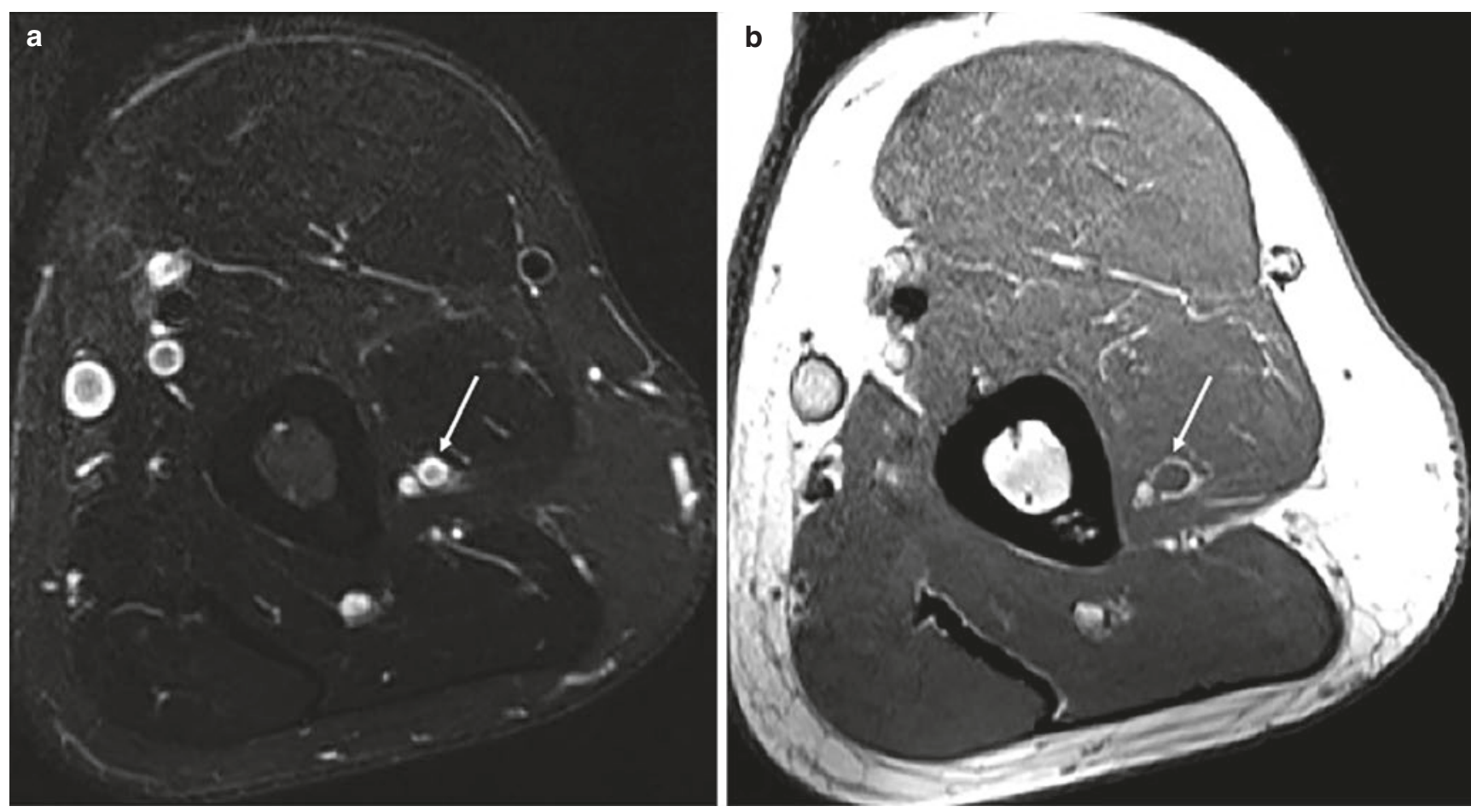

Fig. 20.8 62-year-old male with acute idiopathic neuralgic amyotrophy. (a) Axial T2 STIR, (b) axial T1. Swelling and increased signal intensity of the left radial nerve at mid-arm, with the bull's eye sign (arrow) 
erogeneously enhancing mass [25]. Follow-up imaging is helpful to differentiate radiation-induced inflammation from tumor recurrence as the former will show fibrotic changes over time.

\subsection{Thoracic Outlet Syndrome (TOS)}

TOS includes three disorders involving the neurovascular bundle in its course through the interscalene triangle and costoclavicular space: classic TOS (or neurogenic TOS), vascular TOS, and non-specific TOS. Classic TOS is the most frequent form, affecting middle-aged adults, especially women. It is characterized by chronic neck pain radiating to the supraclavicular region and arm, exacerbated by arm elevation, sometimes associated with paresthesias and numbness in C8/T1 dermatomes. Electrophysiological and clinical provocative tests are non-specific; however, MRI can reveal static or dynamic compression of the BP by structural abnormalities such as cervical or anomalous first rib, long C7 transverse process, muscular anomalies, or narrowed costoclavicular space [26].

\subsection{Immune-Mediated Neuropathies} (Table 20.4)

Immune-mediated neuropathies are diagnosed clinically according to the distribution of deficits and based on electrophysiology examination findings, laboratory parameters, and clinical context.

Guillain-Barre syndrome (GBS) or acute inflammatory demyelinating polyneuropathy (AIDP) is an acute syndrome, typically involving many nerves in a distal-to-proximal ascending distribution pattern. GBS is most commonly seen in the post respiratory or gastrointestinal tract infection or post-vaccination setting. MRI studies are usually not necessary for diagnosis but may be needed to exclude "mimic disorders." Initially, breakdown of the blood-nerve barrier is seen as intrathecal nerve root enhancement on MRI, particularly of the anterior spinal nerve roots [27]. Chronic inflam-

Table 20.4 MRI findings in inflammatory neuropathies

\begin{tabular}{|c|c|c|}
\hline & MRI & Distribution \\
\hline AIDP & Enhancement of spinal nerve roots & $\begin{array}{l}\text { Bilateral and } \\
\text { symmetrical }\end{array}$ \\
\hline CIDP & $\begin{array}{l}\text { Hypertrophy and T2 hyperintensity } \\
\text { of BP and LSP nerve roots, with } \\
\text { gradual normalization in distal } \\
\text { nerves }\end{array}$ & $\begin{array}{l}\text { Bilateral and } \\
\text { symmetrical }\end{array}$ \\
\hline MADSAM & $\begin{array}{l}\text { Hypertrophy and } \mathrm{T} 2 \text { hyperintensity } \\
\text { of peripheral nerve trunks }\end{array}$ & $\begin{array}{l}\text { Multifocal and } \\
\text { asymmetrical }\end{array}$ \\
\hline MMN & $\begin{array}{l}\text { Hypertrophy and } \mathrm{T} 2 \text { hyperintensity } \\
\text { Contrast enhancement of BP }\end{array}$ & Asymmetrical \\
\hline
\end{tabular}

matory demyelinating polyneuropathy (CIDP) is an acquired chronic, immune-mediated, multifocal, demyelinating neuropathy. It is characterized by symmetrical proximal and distal weakness, with sensory loss, impaired balance, and areflexia and affects primarily the spinal nerves, BP and LSP plexi, and proximal nerve trunks but can also involve the cranial nerves. CIDP is characterized by a broad spectrum of clinical phenotypes ( $20 \%$ atypical) [28]. The most frequent MR imaging features are bilateral, symmetric hypertrophy and hyperintensity of the BP and LSP nerve roots, with gradual normalization toward the distal segments [29] (Fig. 20.9). Patients with nerve root hypertrophy usually have a relapsing-remitting course and a longer disease duration, which may be related to demyelination and remyelination.

Hereditary neuropathies such as Charcot-Marie-Tooth disease can mimic the appearance of CIDP on MRI with findings of focal or diffuse peripheral nerve enlargement and fatty degeneration of the involved muscles. The crosssectional measurement of the sciatic nerve area in mid-thigh can be a helpful distinguishing feature [30].

Multifocal acquired demyelinating sensory and motor neuropathy (MADSAM) is characterized by an asymmetric multifocal pattern of motor and sensory loss, conduction block, and other features of demyelination in nerve conduction studies. Nerve hypertrophy is usually asymmetric and multifocal in the peripheral nerve trunks [31].

Multifocal motor neuropathy (MMN) is a chronic, slowly progressive immune-mediated neuropathy, characterized by progressive, predominantly distal, asymmetric limb weakness, mostly affecting upper limbs with minimal or no sensory impairment. The clinical presentation of MMN may mimic motor neuron disease, particularly in patients with predominant lower motor neuron impairment. MRI can be valuable to differentiate MMN from other neuropathies. About 40-50\% of the patients with MMN show asymmetric hypertrophy and signal intensity abnormalities or contrast enhancement on MRI, and the pattern of signal alterations closely correlates with the distribution of muscle weakness [32].

\subsection{Peripheral Nerve Tumors}

The most common neurogenic tumors of the BP and LSP are schwannomas and neurofibromas. On MRI, peripheral nerve sheath tumors are typically fusiform or round-shaped soft tissue lesions in continuity with the nerve and show classic findings such as the target, fascicular, and tail signs on T2 images, split fat sign on $\mathrm{T}$ images, and the bag of worms sign for plexiform neurofibromas [33]. Conventional MRI sequences cannot reliably differentiate solitary neurofibromas from schwannomas, although certain imaging features such as the surrounding T2 hypointense epineurium may be more typical of schwannomas than neurofibromas. 


\section{Key Point}

- When MRN shows diffuse T2 hyperintensity and enlargement of affected neural structures in a patient with acute onset including neck, shoulder, and scapula pain, look for the characteristic "bulls' eye sign" to suggest idiopathic brachial plexitis or idiopathic neuralgic amyotrophy.
A schwannoma arises from a single nerve fascicle, tends to be encapsulated, and grows eccentrically, compressing normal adjacent axons [34]. Multiplanar 3D MRN can accurately display this anatomical relationship of a schwannoma attached to the main trunk of a nerve. Schwannomas are iso- to hypointense on T1 images and hyperintense T2 images and demonstrate variable enhancement, though more homogeneous than neurofibromas (Fig. 20.10). Approximately $40 \%$ of schwannomas may demonstrate internal cystic change and $10 \%$ may show hemorrhage.
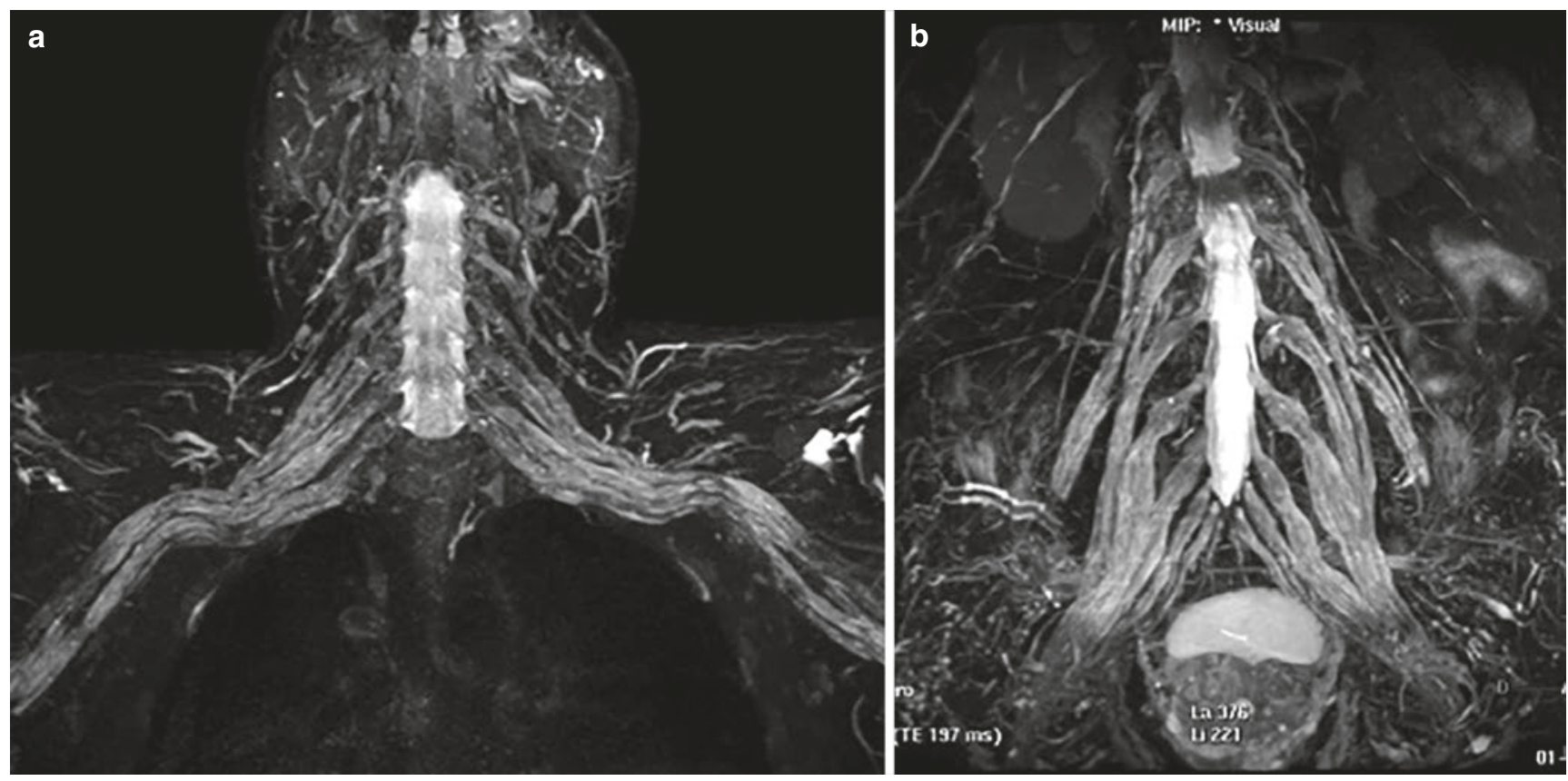

Fig. 20.9 50-year-old male, CIDP: 3D MRN, MIP views bilateral hypertrophy of the brachial (a) and lumbosacral plexus (b)
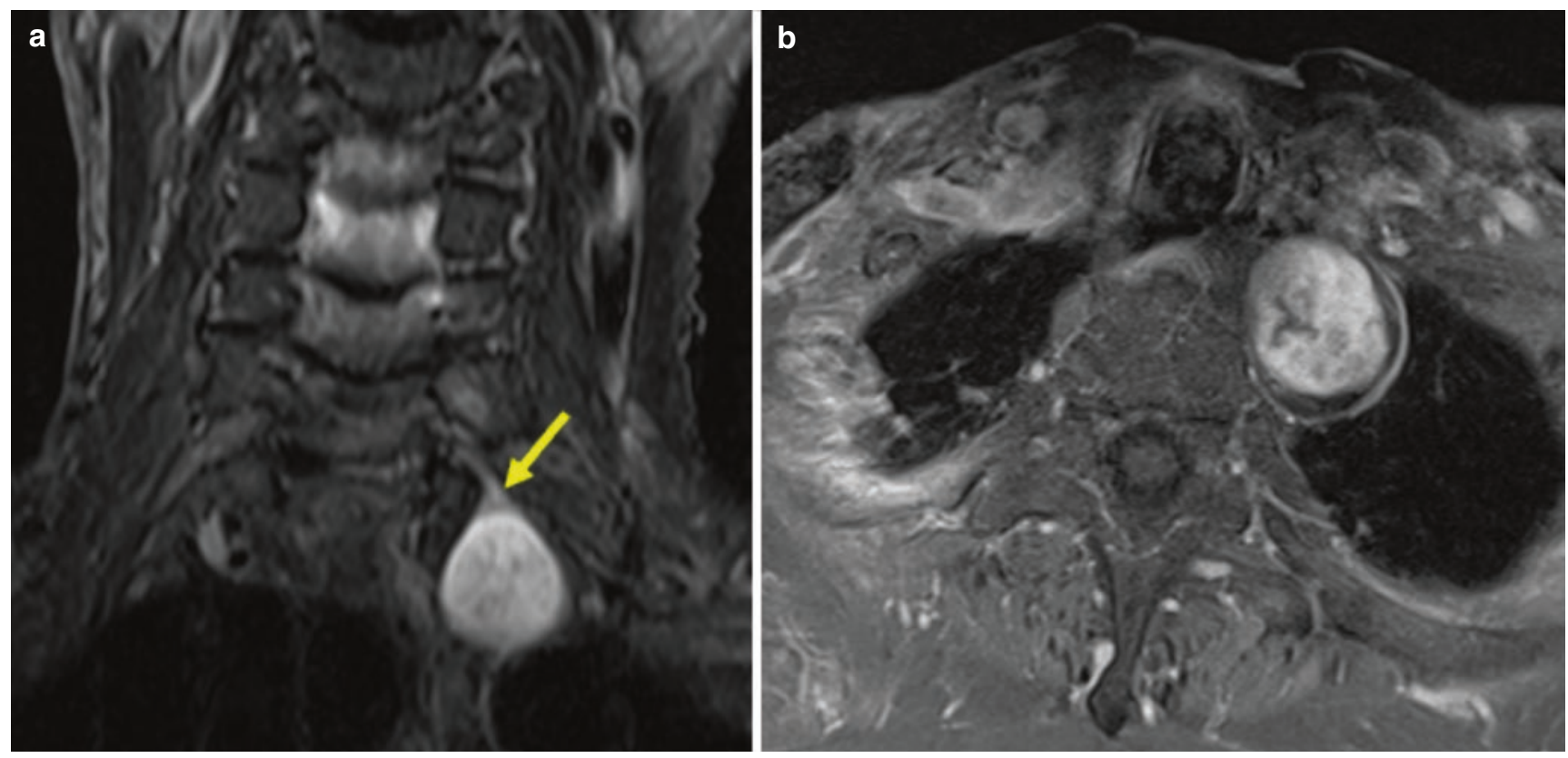

Fig. 20.10 (a) Coronal STIR and (b) axial contrast-enhanced T1 images of a schwannoma demonstrate the origin from a single nerve (arrow) and STIR hyperintensity. The heterogeneous enhancement may reflect internal cystic change 
A neurofibroma is composed of all peripheral nerve cellular elements (Schwann cells, fibroblasts, perineural cells, and axons), grows diffusely within and along nerves, and is not encapsulated. The nerves expand radially while entrapping native neural elements within the substance of the tumor. Approximately one-third of neurofibromas occur in the setting of neurocutaneous syndromes. Plexiform neurofibromas are pathognomonic for neurofibromatosis I (NF1). Neurofibromas are isointense on $\mathrm{T} 1$ images with high peripheral signal and low-to-intermediate signal on T2 images ("target sign"), reflecting the distribution of myxoid material and fibrous tissue (Fig. 20.11). They tend to show variable contrast enhancement. Multiplanar 3D MRN illustrates that neurofibromas are not separated from the normal nerve.

Malignant peripheral nerve sheath tumors (MPNST) have higher prevalence in patients with NF1 and can develop as a long-term side effect of radiation therapy. Malignant degeneration of plexiform neurofibromas can occur in 5-13\% of cases. The imaging distinction between benign nerve sheath tumors and MPNST can be challenging. Imaging features, such as advanced local invasion, bone destruction, poorly defined margins, absence of the target sign on T2 images, and larger size $(>5 \mathrm{~cm})$ suggest malignancy [34]. The combination of MRI findings (e.g., rapid growing, peripheral enhancement, peritumoral edema, intralesional cysts) has been reported to provide $61 \%$ sensitivity and $90 \%$ specificity in detecting MPNST [35]. ${ }^{18}$ Fluorodeoxyglucose positron emission tomography is a complementary diagnostic tool, particularly for identifying tumors with aggressive behavior [36].

Intraneural perineurioma is a rare benign and slowly growing nerve tumor arising from the perineurial cells surrounding the peripheral nerve fibers, most commonly occurring in teenagers and young adults without sex predilection and presenting with progressive muscle weakness.

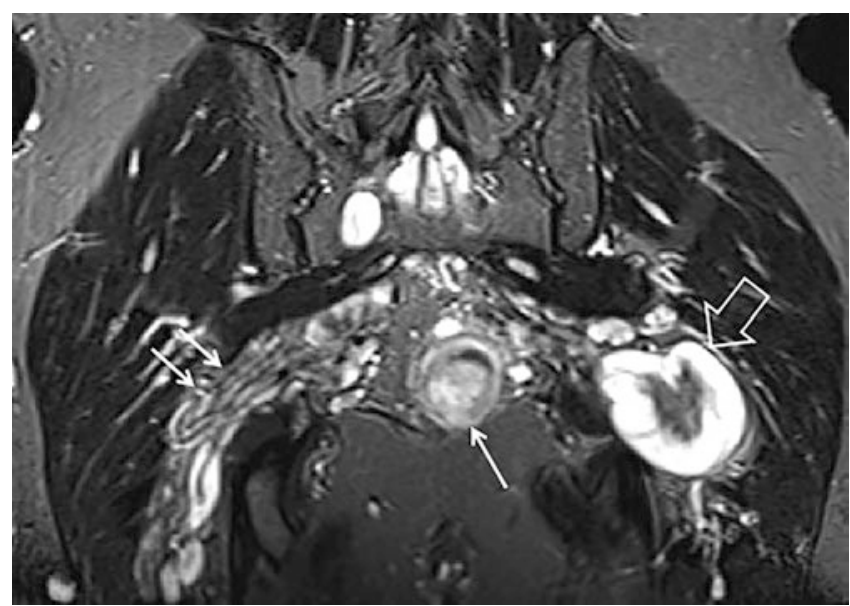

Fig. 20.11 Coronal MRN of a patient with NF1: neurofibroma of the left sciatic nerve with the "target" sign (empty arrow), neurofibroma with intermediate signal intensity of the pelvic floor (arrow), plexiform neurofibroma of the right sciatic nerve (double arrow)
Because of the insidious clinical presentation, the diagnosis is often delayed. Perineuriomas have typical MRI features: enlargement of the involved nerve over a considerable length, fusiform shape, mild increase in $\mathrm{T} 2$ signal intensity, T1 isointense signal, and moderate to marked contrast enhancement. Individual fascicles are uniformly enlarged, and their preserved fascicular architecture gives a "honeycomb" appearance on enhanced T1-fat-sat sequences.

Non-neurogenic tumors can cause plexopathy due to extrinsic compression or infiltration. Desmoid tumors, the most common non-neurogenic primary BP tumors, are isointense to muscle on $\mathrm{T} 1$ sequences and heterogeneously hyperintense on $\mathrm{T} 2$ sequences with areas of low $\mathrm{T} 2$ signal because of increased collagen content. They have infiltrative margins and show avid enhancement. Lipomas are characteristically isointense to fat on all MRI sequences and may externally compress BP and LSP. Lymphoma can encase the plexi due to enlarged lymph nodes and, when primarily involving the nerve roots and trunks, demonstrates increased signal and nerve enlargement. In neurolymphomatosis, there is neoplastic endoneurial invasion, most often associated with non-Hodgkin's lymphoma [37]. Prominent asymmetry, pain, and nodular MRI appearance may help distinguish neurolymphomatosis from paraneoplastic immune demyelinating radiculoneuropathy. MRN shows T2/STIR hyperintensity, focal and diffuse nerve enlargement with fascicular disorganization, and enhancement [37]. Primary neoplasms in close vicinity to the $\mathrm{BP}$, such as bronchogenic carcinoma/Pancoast tumors of the thoracic apex (Fig. 20.12); neurogenic tumors of the vagus nerve, phrenic nerve, and sympathetic trunk; metastatic lymph nodes, and bone tumors can cause nerve irritation/compression or direct invasion into the BP. A helpful clue to identify a Pancoast tumor is to look for intermediate signal effacing the normal $\mathrm{T} 1$ fat signal superior to the lung apex, medial to the scalene muscles, and lateral to the spine. Axial images are useful to demonstrate possible paraspinal extension of tumor in patients with complex symptoms, such as brachial plexopathy and Horner syndrome (second order neuron).

\section{Key Point}

- MRN can accurately display the anatomical relationship of a schwannoma attached to the main trunk of a nerve while a neurofibroma, composed of all peripheral nerve cellular elements, expands radially entrapping native neural elements within the tumor. A plexiform neurofibroma involves multiple nerve roots and has a "bag of worms" appearance. A perineurioma shows fusiform enlargement and mild T2 hyperintensity of the involved nerve and preserved fascicular architecture giving the "honeycomb" appearance on enhanced images. 

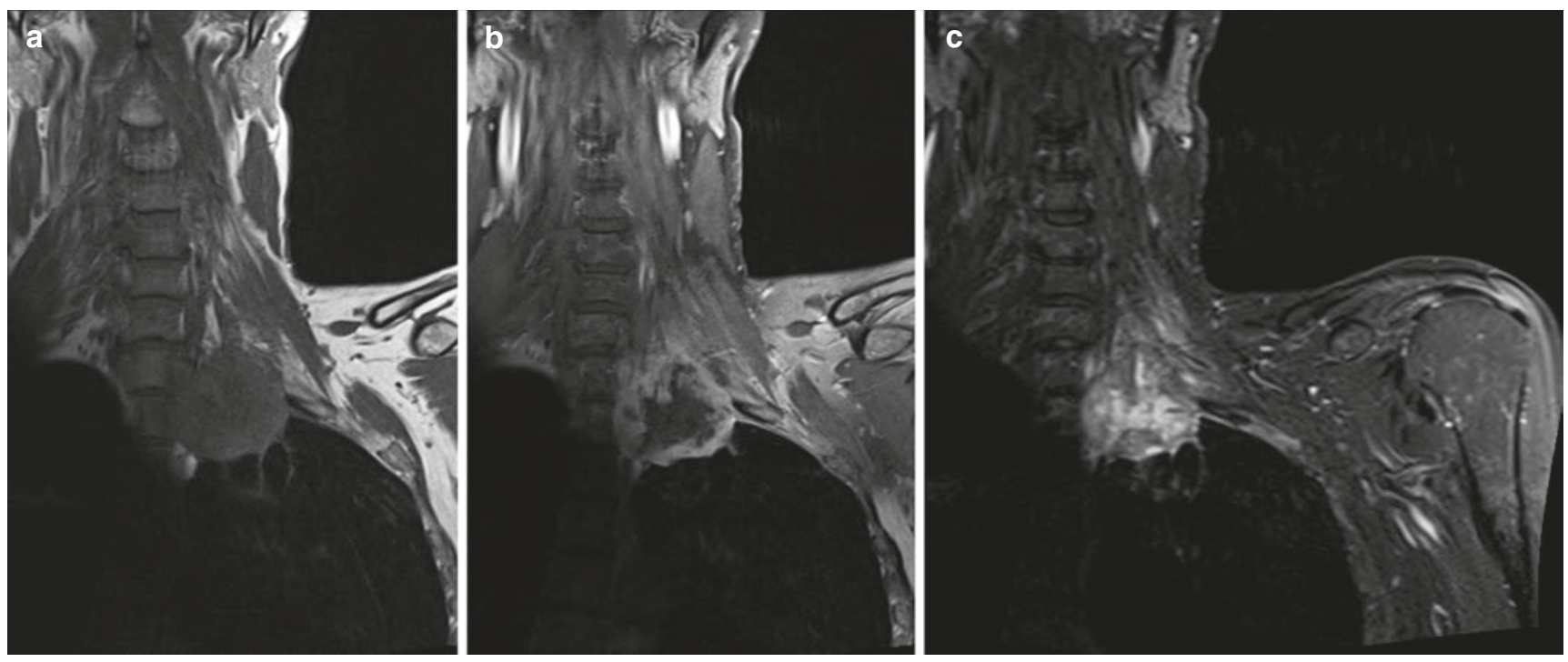

Fig. 20.12 Coronal (a) T1, (b) contrast-enhanced T1, and (c) STIR images reveal a $\mathrm{T} 1$ hypointense, heterogeneously $\mathrm{T} 2$ hyperintense, heterogeneously enhancing lesion in the left lung apex in keeping with a

Non-tumoral masses, such as aneurysms/pseudoaneurysms can cause a compressive plexopathy. DTT can provide relevant topographical information on non-neurogenic soft tissue tumors and adjacent nerves when conventional MRI is unable to depict the course of the involved nerves or distinguish possible nerve infiltration [38]. DTT and low FA provide insight into neural integrity, with low diffusivity values indicating malignancy [10]. As increasing ADC suggests benignity of lesions, this diffusion metric can also be potentially followed as a biomarker to detect tumor response/necrosis if the lesions are being followed longitudinally or after adjuvant medical treatment.

\subsection{Concluding Remarks}

Understanding of the brachial plexus and lumbosacral plexus anatomy is important for the accurate interpretation. Using an anatomic landmark-based approach to interrogate each individual component of the brachial plexus and lumbosacral plexus in combination with the direct and indirect imaging findings enables the development of a relevant differential diagnosis. By incorporating the clinical history, ancillary test results, and physical examination with the imaging findings, one can arrive at the correct diagnosis.

\section{Take Home Messages}

- Diagnostic work-up of peripheral neuropathies should include clinical history, physical examination, electrophysiological studies, and high resolution MRI/MRN (US when nerves are superficial). bronchogenic carcinoma (Pancoast tumor) infiltrating into the interscalene triangle and involving the left $\mathrm{C} 7, \mathrm{C} 8$, and T1 (arrow). This patient presented with persistent left shoulder pain
- MRN affords the advantage of simultaneous exploration of nerves, muscles, and other surrounding tissues which can assess secondary effects of nerve pathology (e.g., muscle denervation), and compressive or infiltrative pathologies.

- It is important to distinguish between preganglionic and postganglionic traumatic plexopathy as it affects treatment options, with the former requiring nerve transfer and the latter often being managed conservatively.

- In peripheral nerve entrapment syndromes, US has the advantage of providing dynamic evaluation of nerve entrapment. MRN is able to identify a variable degree of signal intensity change within the nerve that is commonly higher proximal to the site of entrapment.

- Neurogenic tumors are identified as well-circumscribed, round-shaped soft tissue lesions in continuity with the nerve, and MRI findings of advanced local invasion, poorly defined margins, absence of the target sign on T2-weighted sequences, peritumoral edema, intralesional cysts, and bone destruction, suggest malignancy.

\section{References}

1. Pham M, Baumer T, Bendszus M. Peripheral nerves and plexus: imaging by MR-neurography and high-resolution ultrasound. Curr Opin Neurol. 2014;27(4):370-9.

2. Padua L, Liotta G, Di PA, Granata G, Pazzaglia C, Caliandro P, et al. Contribution of ultrasound in the assessment of nerve diseases. Eur J Neurol. 2012;19(1):47-54. 
3. Rubin JA, Wesolowski JR. Neck MR imaging anatomy. Magn Reson Imaging Clin N Am. 2011;19(3):457-73.

4. Moore KR, Tsuruda JS, Dailey AT. The value of MR neurography for evaluating extraspinal neuropathic leg pain: a pictorial essay. AJNR Am J Neuroradiol. 2001;22(4):786-94.

5. Filler AG, Maravilla KR, Tsuruda JS. MR neurography and muscle MR imaging for image diagnosis of disorders affecting the peripheral nerves and musculature. Neurol Clin. 2004;22(3):643.

6. Aagaard BD, Maravilla KR, Kliot M. Magnetic resonance neurography: magnetic resonance imaging of peripheral nerves. Neuroimaging Clin N Am. 2001;11(1):viii, 131-46.

7. Bendszus M, Koltzenburg M, Wessig C, Solymosi L. Sequential MR imaging of denervated muscle: experimental study. AJNR Am J Neuroradiol. 2002;23(8):1427-31.

8. Chhabra A, Ahlawat S, Belzberg A, Andreseik G. Peripheral nerve injury grading simplified on MR neurography: as referenced to Seddon and Sunderland classifications. Indian J Radiol Imaging. 2014;24(3):217-24.

9. Dong Q, Jacobson JA, Jamadar DA, Gandikota G, Brandon C, Morag $\mathrm{Y}$, et al. Entrapment neuropathies in the upper and lower limbs: anatomy and MRI features. Radiol Res Pract. 2012;2012:230679.

10. Ahlawat S, Chhabra A, Blakely J. Magnetic resonance neurography of peripheral nerve tumors and tumorlike conditions. Neuroimaging Clin N Am. 2014;24(1):171-92.

11. Chazen JL, Cornman-Homonoff J, Zhao Y, Sein M, Feuer N. MR neurography of the lumbosacral plexus for lower extremity radiculopathy: frequency of findings, characteristics of abnormal intraneural signal, and correlation with electromyography. AJNR Am J Neuroradiol. 2018;39(11):2154-60.

12. Trivedi JR, Phillips L, Chhabra A. Hereditary and acquired polyneuropathy conditions of the peripheral nerves: clinical considerations and MR neurography imaging. Semin Musculoskelet Radiol. 2015;19(2):130-6.

13. Jeon T, Fung MM, Koch KM, Tan ET, Sneag DB. Peripheral nerve diffusion tensor imaging: overview, pitfalls, and future directions. J Magn Reson Imaging. 2018;47(5):1171-89.

14. Tagliafico A, Calabrese M, Puntoni M, Pace D, Baio G, Neumaier $\mathrm{CE}$, et al. Brachial plexus MR imaging: accuracy and reproducibility of DTI-derived measurements and fibre tractography at 3.0T. Eur Radiol. 2011;21(8):1764-71.

15. Chhabra ATS, Andreisek G. Nerve anatomy, pathophysiology, nerve injury and entrapment. In: Chhabra AAG, editor. Editor. Magnetic resonance neurography. New Delhi: JayPee Brothers Medical Publishers; 2012. p. 1-9.

16. Doi K, Otsuka K, Okamoto Y, Fujii H, Hattori Y, Baliarsing AS. Cervical nerve root avulsion in brachial plexus injuries: magnetic resonance imaging classification and comparison with myelography and computerized tomography myelography. J Neurosurg. 2002;96(3 Suppl):277-84.

17. Yoshikawa T, Hayashi N, Yamamoto S, Tajiri Y, Yoshioka N, Masumoto T, et al. Brachial plexus injury: clinical manifestations, conventional imaging findings, and the latest imaging techniques. Radiographics. 2006;26(Suppl 1):S133-43.

18. Gasparotti R, Lodoli G, Meoded A, Carletti F, Garozzo D, Ferraresi S. Feasibility of diffusion tensor tractography of brachial plexus injuries at $1.5 \mathrm{~T}$. Investig Radiol. 2013;48(2):104-12.

19. Simon NG, Narvid J, Cage T, Banerjee S, Ralph JW, Engstrom JW, et al. Visualizing axon regeneration after peripheral nerve injury with magnetic resonance tractography. Neurology. 2014;83(15):1382-4.

20. Lang EM, Borges J, Carlstedt T. Surgical treatment of lumbosacral plexus injuries. J Neurosurg Spine. 2004;1(1):64-71.

21. Jarvik JG, Comstock BA, Heagerty PJ, Haynor DR, Fulton-Kehoe $\mathrm{D}$, Kliot $\mathrm{M}$, et al. Magnetic resonance imaging compared with electrodiagnostic studies in patients with suspected carpal tunnel syndrome: predicting symptoms, function, and surgical benefit at 1 year. J Neurosurg. 2008;108(3):541-50.
22. Wang H, Ma J, Zhao L, Wang Y, Jia X. Utility of MRI diffusion tensor imaging in carpal tunnel syndrome: a meta-analysis. Med Sci Monit. 2016;22:736-42.

23. Sneag DB, Saltzman EB, Meister DW, Feinberg JH, Lee SK, Wolfe SW. MRI Bullseye sign: an indicator of peripheral nerve constriction in parsonage-turner syndrome. Muscle Nerve. 2017;56(1): 99-106.

24. van Eijk J, Chan YC, Russell JW. Immunotherapy for idiopathic lumbosacral plexopathy. Cochrane Database Syst Rev. 2013;12:CD009722. https://doi.org/10.1002/14651858.CD009722. pub2.

25. Qayyum A, MacVicar AD, Padhani AR, Revell P, Husband JE. Symptomatic brachial plexopathy following treatment for breast cancer: utility of MR imaging with surface-coil techniques. Radiology. 2000;214(3):837-42.

26. Aralasmak A, Cevikol C, Karaali K, Senol U, Sharifov R, Kilicarslan R, et al. MRI findings in thoracic outlet syndrome. Skelet Radiol. 2012;41(11):1365-74.

27. Byun WM, Park WK, Park BH, Ahn SH, Hwang MS, Chang JC. Guillain-Barre syndrome: MR imaging findings of the spine in eight patients. Radiology. 1998;208(1):137-41.

28. Mathey EK, Park SB, Hughes RA, Pollard JD, Armati PJ, Barnett $\mathrm{MH}$, et al. Chronic inflammatory demyelinating polyradiculoneuropathy: from pathology to phenotype. J Neurol Neurosurg Psychiatry. 2015;86(9):973-85.

29. Van den Bergh PY, Hadden RD, Bouche P, Cornblath DR, Hahn A, Illa I, et al. European Federation of Neurological Societies/ Peripheral Nerve Society guideline on management of chronic inflammatory demyelinating polyradiculoneuropathy: report of a joint task force of the European Federation of Neurological Societies and the Peripheral Nerve Society - first revision. Eur J Neurol. 2010;17(3):356-63.

30. Sinclair CD, Miranda MA, Cowley P, Morrow JM, Davagnanam I, Mehta $\mathrm{H}$, et al. MRI shows increased sciatic nerve cross sectional area in inherited and inflammatory neuropathies. J Neurol Neurosurg Psychiatry. 2011;82(11):1283-6.

31. Rajabally YA, Chavada G. Lewis-sumner syndrome of pure upperlimb onset: diagnostic, prognostic, and therapeutic features. Muscle Nerve. 2009;39(2):206-20.

32. Van Asseldonk JT, Van den Berg LH, Van den Berg-Vos RM, Wieneke GH, Wokke JH, Franssen H. Demyelination and axonal loss in multifocal motor neuropathy: distribution and relation to weakness. Brain. 2003;126(Pt 1):186-98.

33. Thawait SK, Chaudhry V, Thawait GK, Wang KC, Belzberg A, Carrino JA, et al. High-resolution MR neurography of diffuse peripheral nerve lesions. AJNR Am J Neuroradiol. 2011;32(8):1365-72.

34. van Es HW, Bollen TL, van Heesewijk HP. MRI of the brachial plexus: a pictorial review. Eur J Radiol. 2010;74(2):391-402.

35. Matsumine A, Kusuzaki K, Nakamura T, Nakazora S, Niimi R, Matsubara $\mathrm{T}$, et al. Differentiation between neurofibromas and malignant peripheral nerve sheath tumors in neurofibromatosis 1 evaluated by MRI. J Cancer Res Clin Oncol. 2009;135(7): 891-900.

36. Broski SM, Johnson GB, Howe BM, Nathan MA, Wenger DE, Spinner RJ, et al. Evaluation of (18)F-FDG PET and MRI in differentiating benign and malignant peripheral nerve sheath tumors. Skelet Radiol. 2016;45(8):1097-105.

37. Bourque PR, Warman Chardon J, Bryanton M, Toupin M, Burns BF, Torres C. Neurolymphomatosis of the brachial plexus and its branches: case series and literature review. Can J Neurol Sci. 2018;45(2):137-43.

38. Kasprian G, Amann G, Panotopoulos J, Schmidt M, Dominkus M, Trattnig S, et al. Peripheral nerve tractography in soft tissue tumors: a preliminary 3-tesla diffusion tensor magnetic resonance imaging study. Muscle Nerve. 2015;51(3):338-45. 
Open Access This chapter is licensed under the terms of the Creative Commons Attribution 4.0 International License (http://creativecommons. org/licenses/by/4.0/), which permits use, sharing, adaptation, distribution and reproduction in any medium or format, as long as you give appropriate credit to the original author(s) and the source, provide a link to the Creative Commons license and indicate if changes were made.

The images or other third party material in this chapter are included in the chapter's Creative Commons license, unless indicated otherwise in a credit line to the material. If material is not included in the chapter's Creative Commons license and your intended use is not permitted by statutory regulation or exceeds the permitted use, you will need to obtain permission directly from the copyright holder.

(c) (1) 